

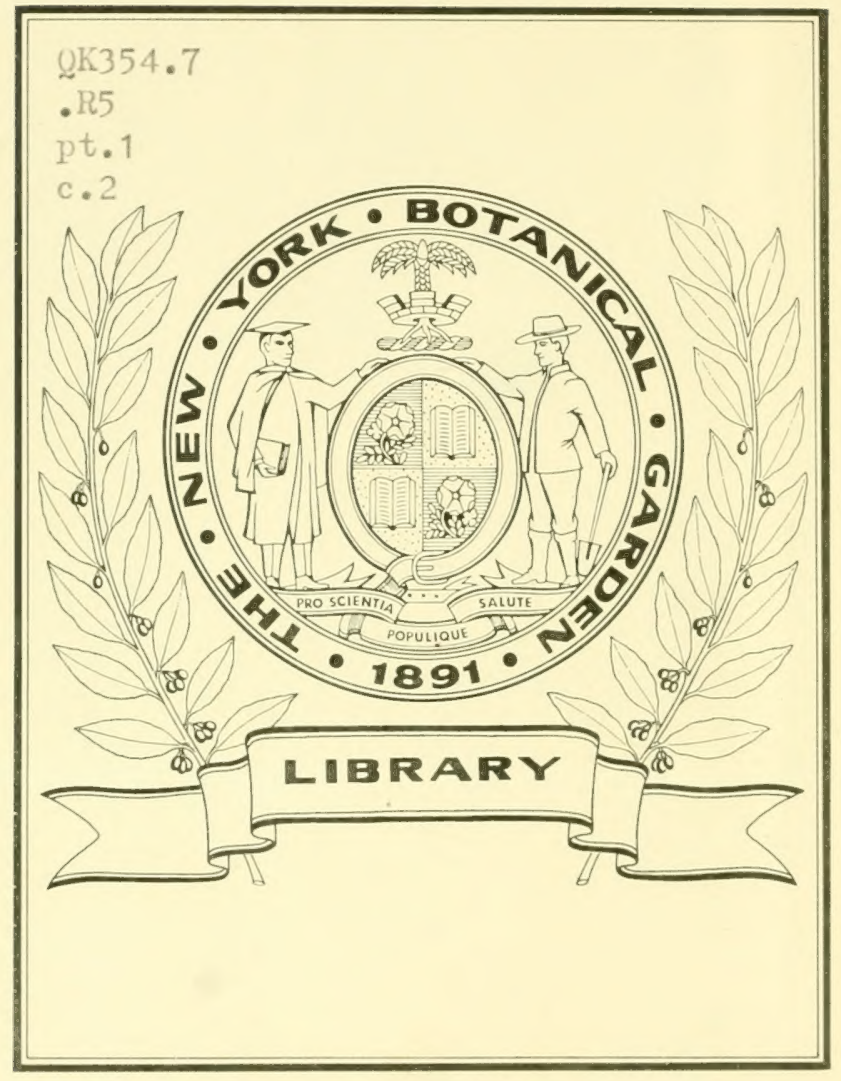






\section{MATERIALS FOR A}

\section{FLORA OF THE}

Malayan Peninsula

\section{PART I}

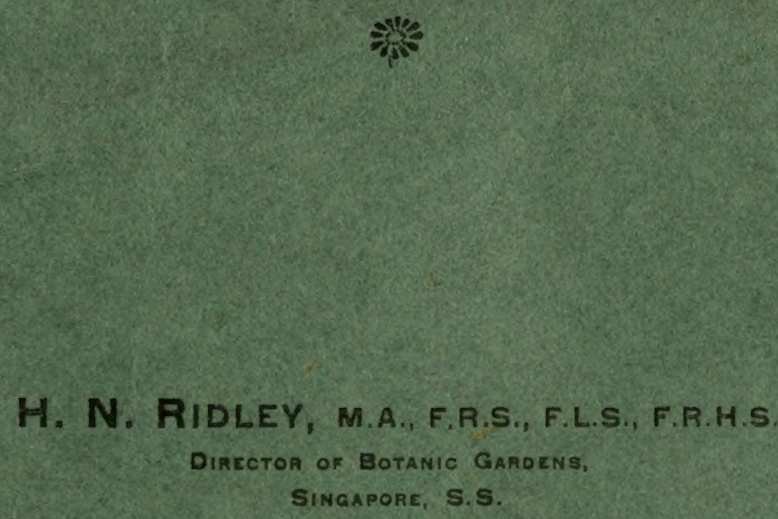

SINGAPORE, S.S. 


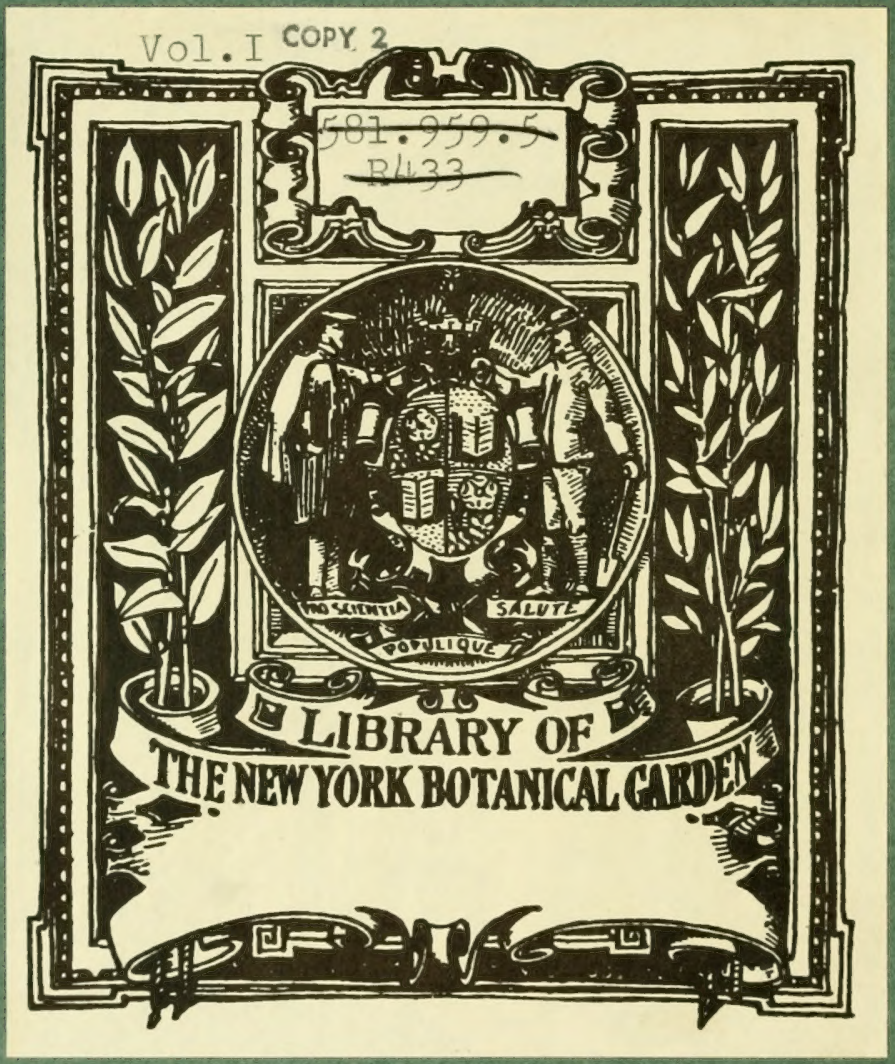




\section{Materials for a Flora of the Malayan Peninsula}

by

H. N. RIDLEY, M.A., F.R.S., F.L.S., F.R.H.S.

Director of Botanie Gardens,

Singapore, S.S.

\section{PART I}





\section{Part I.}

\section{CONTENTS}

$\begin{array}{llllc}\text { Hydrocharideae } & \ldots & \ldots & \ldots & \text { Page } \\ \text { Orchideae } & \ldots & \ldots & \ldots & 7 \\ \text { Apostasiaceae } & \ldots & \ldots & \ldots & 0\end{array}$

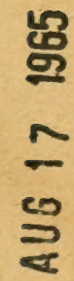





\section{Materials for a Flora of the Malayan Peninsula.}

\section{MONOCOTYLEDONES.}

Herbs, shrubs or trees with endogenous stem structure. Leaves usually parallel-veined, perianth lohes, androceum and pistils in threes or multiples of three. Seeds with one cotyledon only.

\section{Petaloideae Syncapipeae.}

Perianth of two whorls.

Ovary inferior.

Aquatic herbs often unisexual. Stamens 3 to $12 \quad \ldots \quad \ldots \quad \ldots \quad \ldots \quad \ldots$

I. IIydrocharideae.

Terrestrial or epiphytic plants. Flowers irregular.

Stamens and style connate into a column $\quad$. . . . . . .

Stamens 2-3 connate at base only ..

Stamens free to basse, one to five perfect .. .. $\quad$. . .

Flowers regular bisexual.

$\begin{array}{llll}\text { Stamens } 6 \text { excluded } \quad \ldots & \ldots & \text { ₹. A Ameryllideae. }\end{array}$

Stamens 6 included.

Perianth tubular, small herbs often saprophytic ... ..

Large herbs. Perianth campanulate .. . . . . .

Flowers regular unisexual, climbers.

Fruit a winged capsule .. . . VIII. Dioscoreaceae.

(except Trichopus bisexual erect herb).

II. Orchideae.

III. A postasiaceae.

IV. Zingiberaceae.

vi. Burmanniaceae.

vII. Taccaceae.

\section{Ovary superior.}

Perianth segments 4 , climbers .. .. Perianth segments 6 , all similar, stamens 6 equal $\ldots$. . Perianth segments 6, stamens unequal .. Ix. Roxburghiaceae.

x. Liliaceae.

xI. Pontederiaceae. 
Outer perianth whorl sepaline, inner corolline.

Stamen $1 \quad \ldots \quad \ldots \quad \ldots \quad \ldots \quad$ xזr. Philydraceae.

Stamens 3.

Flowers in a terminal hear of brown bracts. Leaves grassy

Flowers in panicles or capitula axillary or terminal. Leaves herbaceous .. . . .

xIIr. Xyrideae.

xw. Commelinaceae. Petaloidfale Apocarpe.le.

Carpels separate usually numerous.

Minute saprophytes, leafless

Aquatic plants leafy.

Perianth, petals conspicuous white .. xr. Alismaceae. Perianth often absent . . $\quad$. $\quad$. $\quad$ xvir. Naiaduceae.

\section{Calyornae.}

Perianth lobes calycine stiff small.

Herbs or elimbers; leaves entire .. .. Trees or shrubs. Leares usually pinnate

\section{NudifLoRAE.}

Perianth none.

Shrubs or trees unisexual. Leaves linear stiff

Herbs inflorescence bisexual, leaves broad herbaceous .. .. .. .. Aquatic floating plants, flowers solitary minute .. .. .. .. ..

\section{Grumaceate.}

Flowers enclosed in dry bracts. Flowers unisexual.

Perianth segments 6 or less minute Flowers usually bisexual in spikes. Perianth none.

Stem solid usually triquetrous. No palere or lodicules.. $\quad . \quad \ldots$ Stem usually fistulous and terete. Prleae and lodicules .. ..
xvirI. Flagellariae. xix. Palmae.

xx. Pandanae.

XxI. Araceae.

xxII. Lemnaceae.

xxin. Eriocaulaceae.

xxiv. Cyperaceac.

xxv. Gramineae. 


\section{HYDROCHARIDEAE.}

Aquatic herbs, stem short, stoloniferous or elongate leafy. Leaves linear lanceolate or broadly ovate. Flowers regular usually unisexual inclosed in a spathe. Perianth usually of two whorls, sepals 3 , petals when present 3 white or yellow, stamens 3 to 12 , filaments short. Ovary inferior one-celled, placentas parietal 3-6, style divided, ovules numerous.

An order of about 50 species extending all over the world.

Freshwater, stems long and leafy.

Leaves whorled $\quad \ldots \quad \ldots \quad \ldots \quad$ 1. Hydrilla.

Leaves alternate $\quad \ldots \quad \ldots \quad \ldots \quad$ 2. Enhydrias.

Stem short leaves radical.

Flowers small, petals linear - .3 . Bly.xa.

Flowers large, petals ovate .. 4. Ottelia.

Marine, rhizome creeping.

Leaves linear. Male flowers very numerous in the spathe .. .. 5. Enhalus.

Leaves ovate. Male solitary... .. 6. Halophila.

\section{Hydrilla, I. C. Rich.}

Submerged stem elongate branched leafy. Leaves short in whorls of 3 or 4 , or lower ones opposite. Flowers unisexual, solitary in axillary spathes. Male shortly pedicelled. Sepals ovate, petals narrower. Stamens three, anthers ovate two-celled. Female sessile, spathe tubular. Perianth as in the male but segments narrower. Ovary enclosed in the spathe except the long beak. Styles 3 rarely 2 linear. Fruit narrow, seeds oblong.

A single species oceurring in Tropical Africa, Mascarene Islands, Asia and Australia.

Hydrilla verticillata, Presl. Bot. Bemerk. 112. Stem 6 inches to a foot long, slender. Leaves $\frac{1}{4}$ inch long oblong acute denticulate. Hook. fil. Fl. Brit. Ind. V. p. 659. Royle Ill. Bot. Himal. 3r6. Serpicula verticillata Roxb. Corom. Pl. t. 164.

Rare, Kelantan in the river at Kamposa (Ridley).

\section{ENHYPRtas, Ridl.}

Stems elongate branched leafy. Leares alternate narrow linear. Flowers solitary base enclosed in a tubular spathe hermaphrodite. Perianth tube long slender. Calyx lobes lanceolate acute 3. Corolla lobes much longer linear. Stamens three arlnate to the mouth of the corolla tube short, anthers linear, a celled. Styles 3 shorter than the 
stamens. Fruit narrow flattened, orules numerous anatropous. Seeds fusiform papillose. Distrib. species one Malaya.

Exhrdris Angustipetali, Ridl., Journ. Bot. 1890, p. 99. Stems 6 inches to 2 feet long 3 inch through, submerged. Leares linear lancelate acuminate alternate, crowded below the flower, or $\frac{1}{8}$ to 1 inch alyart olive green or dull red, minutely denticulate onenerred. Flowers solitary sessile. Spathe 1 inch long, apex bifid, flattened edges keeled, perianth tube above the spathe $1 \frac{1}{2}$ inch long, shenler, greenish or pinkish white. Sepals lanceolate acute, $\frac{1}{x}$ inch long. Petals more than $\frac{1}{t}$ inch long narrow linear lorate obtuse white. Stamens very slender, anthers linear. Fruit an inch long thin walled one-celled. Seeds about 14 fusiform with three longitudinal rows of short blunt processes.

Growing often in large tangled masses in ditches and ponds common. Singapore: Tanglin Garden Lake (Ridley 10i21), Chan Chu Kang: Malacea: (Griffith 60339), Merlimau (Derry 231); Perak: Dindings (Ridley), Kinta (King's Collector ros6) (Scoltechini), Chankat Serdang (Wray i23); Pahang: Pekan (Ridley 149i). Distrib. Borneo.

\section{Blyxi, Du Petit Thouars.}

Sulmmerged stemless usually' stoloniferous herbs. Leaves linear acute crowited, scapes long or short. Flowers unisexual or bisexual, enclosed in a tubular spathe. Male flowers several in a spathe, bisexual and female flowers solitary. Sepals 3 linear, petals 3 longer linear. Stamens 3 to 9 . Styles short, stigmas filiform 3. Fruit very slender many-seeded, seed oblong tailed or not, tubercled.

Species about 15\% Tropical Africa, Mascarene Islands, Asia and Australia.

Bryxa Malayaxa,Ridl, Trans. Limn. Soc. Vol. III. p. 358.

Rootstock short and stout. Teares numerous linear acule 2 to 14 inches long $\frac{3}{8}$ inch wide flaccid deep green. Scapes several axiilary, peduncle 2 to 6 inches long. Flower bisexual 4 inches long. Sipathe flattenerl. Sepals erect linear obtuse. Petals white 1 inch long, margins toother. Stamens is narow, anthers linear. Stigmas rather thick acuminate longer than the stamens. Fruit cylindrie 21 inch long, conclosed in the spathe. Seeds numerous elliptic blunt muricate and ribhed à inch long. J). Ciriffithii, Hook. f. Fl. Brit. Ind. V.p. 661 (in part).

Common in ponds and ditches. Native name, Rumput Lumut.

Singapore: 'Tanglin (Ridley 1625), Chan Chu Kang (Ridley 81); Pahang: Kunala Pahang (Ridley); Malacen: Tanjong Kling

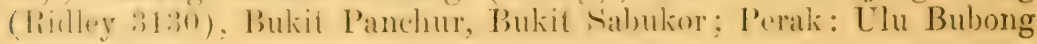


(King's Coll. 10652); Penang: Telok Bahang (Curtis 1888). Endemic.

\section{OtTelia, Pers.}

Submerged fresh-water herbs, stemless. Leares crowded ovate lanceolate oblong or cordate. Flowers showy fugacious solitary in a tubular bific spathe. Sepals 3 linear or oblong. Petals 3 larger white or yellow ovate or orbjeular. Stamens 6 to 1.). Anthers erect. Ovary oblong beaked. Styles linear bifid. Fruit oblong 6-valved enclosed in a spathe. Seeds numerous, testa pulpy. Species about 6 in the tropics of both hemispheres.

O. Alissroides, Pers., Syn I. 400. Leaves ovate or ovate cordate, acute thin undulate petiolate, blade 6 inches long or less 4 inches wide, petiole three angled 6 inches long. Scapes \pm to 6 inches long. Spathe green winged undulate narrow or orate an inch or more long. Perianth tube a little longer. Sepals lanceolate obtuse $\frac{1}{4}$ inch long. Petals broadly ovate unguiculate fugacious, white often tinted with pink or riolet an inch long. Stamens 6 to 10, anthers linear. Orary oblong. Fruit linear oblong subglobose 6grooved. Seeds elliptic light brown very small. Rich. in Mem. Instit. Fr. 1811, II. t. \%, Benth. Fl. Austral. VI. 25\%, Hook. Fl. Brit. Ind.-V. 662. O. indica, Dalz and Gibs. Bombay Flora 278. Damasonium indicum, Willd. Sp. pl. II. 2r6, Boxb. Fl. Ind. II, 216, Cor. pl. II. 45, t. 1S5, Bot. Mag. t. 1201. Stratioles alismoides, Linn. Sp. pl. 535 .

In ponds and ditches.

Perak: Kinta at Tambun (Ridley \%090), 'Tarut (King's Collector t063) 5538; Penang: 'Telok Kumbar (Curtis 851); Province Trellesley; Krian (Ridley); Selangor: Pudol (Goodenough); Lankawi: Kwah (Curtis). Distrib. Tropical Africa, Asia and Australia.

\section{Enhalus, Richard.}

A submerged marine herb with a stout crinite rootstock. Leaves long strap-shaped in twos and threes in a basal sheath. Flower's unisexual. Male very small white numerous in a two leaved spathe. Sepals and petals elliptic or ovate. Stamens 3 filaments very short, anthers broad oblong. Female flower much larger, solitary in a large two leared spathe. Sepals oblong. Petals longer linear. Ovary oroid with 6 bristly ridges, long-beaked 6 celled. Styles 6 bipartite arms slender, ovules few anatropous. Fruit globose oroid beaked with 6 hairy ridges. Seeds few large cone-shaped soft green.

Species one, Madagascar, Ceylon, and Malay seas to Australia. 
Exilulus Toexigi, Rich. in Mem. Institut. Fr. 1811, II. 64. Stem woody far-creeping $\frac{1}{4}$ inch through, covered with stiff black fibres. Leaves subcoriaceous dark green 2-3 feet long, half an inch wide tip rounded. Male inflorescence on a stout peduncle two inches long. Spathes 2 lanceolate acute boat-shaped olive green $1 \frac{1}{2}$ inch long keeled and arm with brown bristles. Flowers very numerous shortly pedicelled white. Sejals and petals similar. Stamens pubescent. Female inflorescence on a peduncle 1-2 feet long, spathes narrower. Fruit $1 \frac{1}{2}$ inch long, 1 inch wide succulent with 6 broad bands of bristles. Miq. Fl. Ind. Bat. III, 2\%. Hook. fil. Fl. Brit. Ind. V. 663. E. acoroides, '/oll. Verz. Ind. Archip. pl. II. 69, Ascherson. Nuor. Giorn. Bot. Ital. III. 299. Stratiotes acoroides, Linn. f. Suppl. 268. Enhalus, Griff. Notul. 175 Ic. Pl. Asiat. t. 249, 250. E. marinus, Griff. l.c. $1 \% 8$.

Common in shallow seas round the coasts. Singapore: Bajau (Ridley 3952).

Natire name Setul. Fruit eaten by children.

The male flowers became detached and float on the surface and the fruit when ripe is raised to the surface also.

\section{Hisophis thouars.}

Submarine plants with slender ereeping stems. Leaves in pairs in the axils of a scarious bract, flowers unisexual solitary in a double spathe. Male pedicelled. Sepals 3 , petals 0 , anthers 3 linear oblong sessile. Female flower nearly sessile. Sepals 3. Orary beaked onecelled. Style 3 filiform. Fruit subglobose. Seeds numerous globular minute.

Species about 6. Tropical seas of America, Asia and Australia.

Hisopintud ovits, Gaud. Freycin. Toy. Bot. 430, t. fig. 1. Leaves elliptic or oborate petioled dark green $\frac{1}{4}$ to $\frac{1}{2}$ inch long, $\frac{1}{8}$ to $\frac{1}{4}$ inch broad, petiole $\frac{1}{2}$ inch long. Male flower shortly pedicelled. Sepals ovate to elliptic ovate. Anthers oblong. Female pedicelled very shortly. Sepals very small, perianth at base tubular. Styles long and slender. Fruit small globose. Seeds $1 \mathrm{~mm}$. across tuberculate and reticulate. Hook. f. Fl. Brit. Ind. V. 663. II. ovalis, Hook. f. Flor. Tasman. II. 45, Miq. Fl. Ind. Bat. III. 230, Aschers. Nuov. Giom. Bot. Ital. III. 301. Diplanthera, Grift. Ic. Pl. Asiat. t. 161 C, f. 2. Caulinia ovatis, Br. Prodr. 359.

Common in shallow seas round the coasts. Singapore: Harbour (lidlly :3iso), ('hangr; Johone Strait; Penang; Lamkawi (Ridley).

Distrib. East African, Indian, Malayan, Australian and Pacific Seas. 


\section{ORCHIDEA}

Herbs or shrubs, either (1) terrestrial with a short stem, herbaceous leaves and a terminal or lateral spike or racenc, rarely climbing plants, often leafless saprophytes, or (2) epiphytes with short or long stems, branches of which may be swollen and fleshy, (pseudobulbs) leaves usually coriaceous, flat, distichous, or terete or (rarely) absent, roots long or short often flattened. Inflorescence axillary or terminal spicate or racemose. Perianth superior irregular, consisting of three Scpals, two laterals free similar to the other one, or connate more or less or prolonged below the ovary into a spur-like sac, the mentum. Petals three, two similar, the third opposite the column and upper sepal very different, the lip, often lobed, and spurred, and bearing processes (calli) of various forms. Stamens and style united into a column, opposite the lip. Column long or short, erect on the top of the ovary, or prolonged below it into a foot to which when present the lip is adnate, and frequently also the lateral scpals also. Anther one terminal (except in Cypripedium, where there are two) adnate to the top of the column, or stipitate by a short filament (sometimes forming the hood of the column). "Polisn grains cohering in 2, 4, 6 or 8 , waxy or powdery masses (pollinia) prolonged or attached to one or two thin flat or terete caudicles, terminating in a variously shaped adhesive disc, or caudicle and disc absent. The other two stamens are represented often by wings or arms of the column or indistinguishable. Stigmas three, the upper one forming a flat rounded or triangular entire or bifid beak below the anther, (rostellum) the other two forming a viscid mass sunk in a hollow of the column, or rarely protruding (stigma). Ovary inferior usually twisted, one-celled. Fruit capsular, or rarely baccate elliptic, pyriform or cylindric. Seeds minute.

Species over five thousand, occurring over the whole wolld, rare in desert and arid regions and most abundant in the tropics.

Tribes ANd Sub-Tribes.

Fertile anther one.

Pollinia waxy 2 to 8 without any caudicle or dise .. .. ..

Pollinia 2 to 4 waxy, with one or two caudicles and one or two dises.

Epiphytic or terrestrial .. . . Tribe II, VANDEAEe

Pollinia mealy with one or two caudicles and one dise.

Terrestrial plants often saprophytic

Tribe I, Epidendreae.

Tribe III, Neottieae. 
Pollinia mealy 2 with 2 caudicles and 2 discs.

Anther adnate to apex of column erect.

Terrestrial herbs .. .. .. Tribe IV, OPHRYDEaE.

Fertile anthers two.

'Terrestrial herlus. Lip siccate . Tribe V, CrTRIPEDIEaE.

No'TE.-King (Ann. Bot. Gard. Calc. Vol, VIII. considers that the "Anther" in Uplirydere is composed of the inner halves of two anthers, of which the outer halves are represented by the small rugulose processes on the outside commonly lnown as stcminodes. This is possible but I ain hardly prepared to enlorse it, especially in view of the structure of the column of Dilochia, in which the three anthers are clenly visible, though the two onter ones are infertile. 'The wings and arms of the column I believe to be the remains of the filaments of these anthers.

\section{EPIDENDieat.}

1. Maldiceato 'Terrestrial or epiphytic, flower's small, inflorescence terminal, column footless. Pollinia $2-t$ with no viseus.

Column short.

Terrestrial leaves flat.. .. ..

1. Microstylis.

Epiphytic, leaves fleshy tistichous .. 2. Oberonia.

Column long, arms short from the top

3. Liparis.

Column usually long, arms from the side

4. Platyclinis.

2. Dexprobieate. Epiphytic rarely terrestrial, inflorescence lateral or terminal. Column with a long foot. Inflorescence lateral or terminal on an elongate stem or terminal on a two-leaved psendobulb $\quad . \quad \ldots \quad \ldots \quad \ldots \quad$..

Inflorescence from the rhizome, pseudobullss one-leaved.

Column short, not hooded arms short subterminal $\quad . \quad \ldots \quad$. . . .

5. Dendrobium.

Column longer hooded, arms long from the side

6. Bulbopleyllum.

Pollinia S.

Sepals forming a mentum .. ..

Sepals comate into a tube .. $\quad$. $\quad$ 9. Porpax.

Sepals free. Column long .. . . 10. T'ylostylis.

Column short

Flowers in long slender racemes, minute .. .. ... .

11. Phreatia. 
Flowers in terminal heads 12. Agrostophyllum.

Flowers in short axillary racemes 13. Poaephyllum.

Flowers few or solitary, axillary 14. Ceratostylis.

3. Bletreae. Terrestrial rarely epiphytic, stems short swollen. Leaves plicate herbaceous. Inflorescence on tall rarely short lateral scapes. Column long.

Pollinia 2, no viscus, column with lateral arms $\quad . \quad$. $\quad$. $\quad$.. . .

Pollinia 2, no viscus, column without

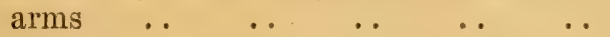

Pollinia 4 or 8 , column with a foot.

Lip not spurred, mobile. Pollinia 4 1\%. Plocoglottis.

Lip not spurred, not mobile. Pollinia

$8 \quad \ldots \quad \ldots \quad \ldots \quad \ldots \quad \ldots$ 18. Tania.

Lip spurred, stems pseudobulbs .. 19. Ascotania.

Lip spurred, no pseudobulbs .. 20. Nephelaphyllum.

Column footless. Pollinia 8.

Stems short swollen. Lip not spurred 21. Spathoglottis.

Stems short swollen. Lip spurred.

Lip convolute round column .. 22. Phaius.

Lip adnate to column .. . 23. Calanthe.

Stems tall leafy. Inflorescence terminal.

Terrestrial. Column with no lateral anthers .. .. .. .. 24. Arundina.

Epiphytic. Column with 2 sterile anthers and one fertile one .. . 25. Diluchia.

4. Coelogratea. Epiphytes, rhizome creeping. Branches or pseudobulbs 2 or more leaved. Lip sessile at column base. Column without foot or arms.

Pollinia 4. Lip not saccate or spurred 26. Coelogyne. Lip saccate at base .. $\quad \ldots \quad$.. $2 \%$ Pliolidota.

Pollinia 2. Lip spurred, branches not pseudobulbs $\quad . \quad$.. $\quad$.. $\quad$..

28. Claderia.

II. VANDEAE.

1. Eulophilate. Leaves herbaccous not articulated.

Lip spurred, column long _. .. 29. Eulophia. 
Lip saccate not spurred, column short

2. Crambidine. Leaves stiff or subeoriaceous articulated, column footless, stems short, scapes long axillary.

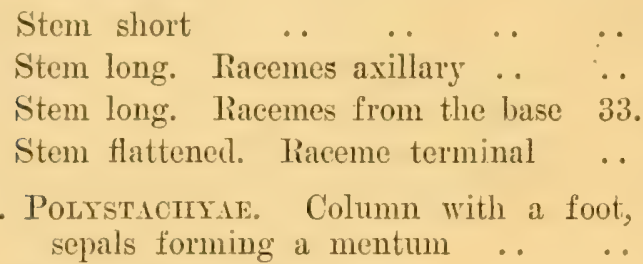

Saprophyte, leafless . . . . .

4. Sarcantineate. Stems terete or slightly flattened, leaves coriaceous lorate or terete, racemes or panicles axillary.

Lip spurless.

Lip entire, leaves terete .. . . $3 \%$ Luisia.

Lip entire, leaves lanceolate .. . . 38. Adenoncos.

Column with a foot.

Lip obscurely 3 -lobed with no callus

Lip distinctly 3-lobed with a rounded callus

Lip distinctly with a forked callus ..

Side lobes of lip forming a mentum with the long column foot ..

Lip 5-lobed . $\quad$. $\quad$. $\quad$. $\quad$.

Lip spurred column without a foot or distinct arms. Stems elongate.

Flowers racemose or panicled.

Column short, midlobe of lip large

Column short, midlobe small .. ..

Column long $\quad . \quad \quad \ldots . \quad \ldots \quad$.. 46 . Renantherella.

Flowers solitary pubescent .. . . 47 . Trichoglottis.

Stem short. Lip entire .. . . 48. Rhynchostylis.

Stems short or long, leaves distant.

Lobes of lip small, spur with callus in the mouth .. .. ..

Leaves crowded. Pollinía $t$.. . . 50. Microsaccus. 
Materials for a Flora of the Malayan Peninsula.

Leafless, stems very short . . 51. Taeniophyllum.

Column with erect arms. Caudicle of pollinia as broad as the large dise Spur saceate horizontal .. ..

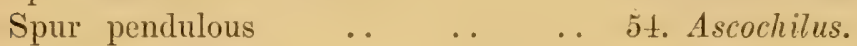

Lip saccate mobile $\quad . \quad \ldots \quad \ldots \quad$ 55. Acrides.

Lip saceate not mobile.

Stems elongate. Bracts flattened distichous $\quad \ldots \quad \ldots \quad \ldots$

Stems short. Bracts not distichous

5. Notrliene. Stems pseudobulbous, epiphytic. Leaves one or few.

Racemes from base of pseudobulbs.

Column long.

Lip spurred. Column foot absent.

Leaves several. Lip adnate half way up to the erect column .. ..

Leaf solitary. Lip forming a tube with the column foot ... . .

6. Podocimlinae. Stems slender or pseudobulbs with distichous leaves racemes laterate or terminal. Pollinia 4-8 attached to 1 or 2 elongate, or calyptriform caudicles. Rostellum elongate.

Stems tall slender. Racemes lateral or

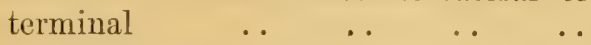

Stems pseudobulbous 1-2 leaved. Scapes from base of pseudobulbs . . ..

Stems short with long distichous leares

56. Thrixspermum.

5\%. Dendrocolla.

58. Acriopsis.

59. Thecostele.

60. Podochilus.

61. Thelasis.

62. Oxyanthera.

iII. Neottiene.

1. Listerieat: Column usually long. Anther terminal. Pollinia 2-4 powdery or granular without any caudicle or gland.

Climbers, leaves fleshy. Fruit baccate

63. Vanilla.

Leafless saprophyte. Fruit capsular 64. Galeola.

Tuberous herbs with one leaf.

Flowers racemose appearing after the leaves . $\quad$.

.. 65. Pogonia.

Flowers solitary in the leaf axil .. 66. Corysanthes. 
Leafless saprophytes.

Lip 3-lobed .. $\quad$.. $\quad$.. $\quad$.. $6 \%$. Aphyllorchis.

Lip entire .. $\quad$. $\quad$.. $\quad$. 68. Didymopleis.

Sepals free. Lip free from column.

Lip adnate column face .. . . 69. Lecanorchis.

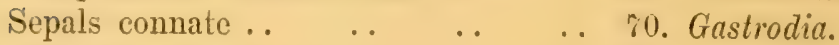

2. Goodyemene. Anther posticous rertical but inverted. Pollinia 2-t granular attached to two caudicles and a disc. Column short.

Stems tall leafy. Inflorescence axillary or terminal. Panicles axillary. Perianth long narrow ... Racemes short axillary or terminal.

Perianth short $\quad$. $\quad$. . . .

Stem short. Inflorescence spicate or racemose terminal.

Lip spurred.

Spur bifid

Spur entire

Lip entire

$\begin{array}{lll}\text { Lip short } 3 \text {-lobed } & \cdots & \cdots \\ \text { Lip long midlobed long clawed } & \cdots\end{array}$

Lip saccate not spurred. Terminal lobe dilated. Stigma entire. Column with a boat shaped ventral process.

Rostellum moderately long. Lip large $\quad . \quad \ldots \quad$.. Rostellum very long curved. $\mathrm{Lip}$ short

Column with no ventral process .. Stigmas 2 distinct .. ..

'Terminal lobe of lip not dilated.

Leares few orate or lanceolate.

Lip inferior, base boat-shaped ..

Lip superior

Sac of lip globose with a short inflexed point .. . . .

Leaves numerous, long and narrow

Lip not saccate.

Lealless saprophyte.. ... ..

Leaves long petioled from a rhizome, scape nearly leafless ... .. i1. Corymbis.

72. Tropidia.

\%. Physurus.

it. Vrydagzynea.

is. Cystorchis.

76. Anaectochilus.

78. Macodes.

79. Haemaria.

80. Zeuxine.

81. Goodyera.

8:. Hetaeria.

83. IIylophila.

81. Lepidogyne.

85. Stercosandra.

86. Cryptostylis. 
inch long. Pedicels twice as long spreading. Dorsal sepal oblong obtuse. laterals orate nhlongr ! inch longr. P'etals rather short ohloner. all pale pink. I.ip large aluricles 3 inch lone lancerolate acute rosy

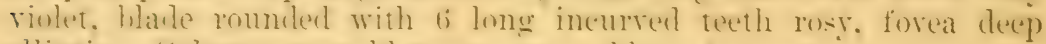

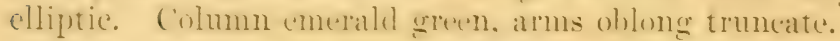

M. plantaginea, Hook. f. Fl. Brit. Ind. T. 6ss (not Steudel).

Perak: Batu Kurau (Curtis), Gunong Buku (Scortechini), Ulu

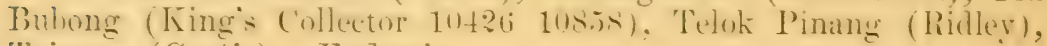
'Iaipeng (Curtis). Endemic.

3. M. Acctanguld, Hook. f. Ic. Pl. t. 1835 Fl. Brit. Ind. V. p. 68. Stem swollen short. Leares orate lanceolate oblique acuminate 4-6 inches long by $1 \frac{1}{2}$ wide. Raceme 6 to 8 inches long base nude. Bracts lanceolate deflesed. Flowers numerous green or white $\frac{1}{9}$ incli across pedicels $\frac{1}{2}$ inch long. Sepals linear oblong. Petals Īonger and narrower. Lip auricles triangular acute. blacle shorter pectinate all round. Arms of columm large rounded.

Perak: Batang Padang, (Wray 1604), Larut Hills (Curtis); Selangor: Bukit Hitam (Kelsall). Endemic.

4. M. Coxgesta, Iichb. f. Walp. Ann. TI. 206. Stem swollen at the base $1-2$ inches long. Leares $t$ or 5 unequal ovate to orate lanceolate acuminate, the largest. 6 to $f$ inches long, by 2 inches wide. Raceme 8 to 12 inches long, base about half nude. Flowers rery small crowded yellow or crimson. Sepals oblong, laterals broader. P'etals linear blunt. Lip without auricles ohlong. three lobed. lateral lobes erect broad blunt, median longer nurrow blunt. Capsule erect $\frac{1}{2}$ inch long, rilos crenulate. Hook. fil. Fl. Brit. Ind. T. 659, Ridley Journ. Limn. Soc. XXIV. 334. King Ann. Roy. Bot. Calc. 8, p. 19, Plate 23.

M. Bernaysii, F. Muell. Fragment XI. 21. M. trilobulata, Kurz Andam. Rep. App. B XIX. M. fusca, Rehb. f. Walp. Ann. VI. 20\%.

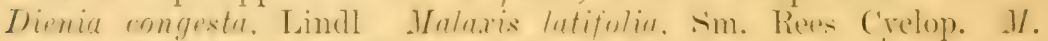
plicata, Roxb. Fl. Ind. III. 456.

Singapore: Kranji, Bukit 'Timah, ete. (Ridley); Malacea: Batu

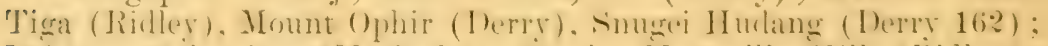

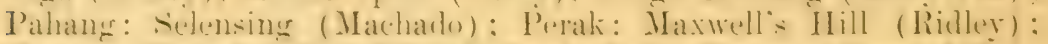

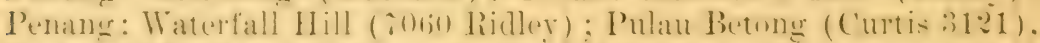

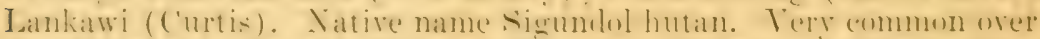
ihre l'eninsula. Jistril. Tronical 11 imalava, Bumah, Antaman isles, Decean, Ceylon, Cambodia, Borneo, China, Australia.

5. M. Micraxtia, Hook. f. Ic. Pl. t. 183t. Fl. Brit. Ind. T. p.

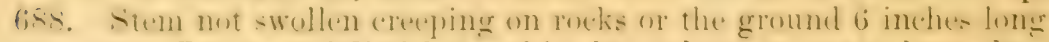
or more. Leaves subdistichous thin lanceolate or ovate lanceolate acute cri-peil copprery shining above, $2-1$ inches long 3 inch wirle, 
petiole purple $\frac{1}{2}$ an inch long. Scapes one or two on a stem, slender, 6-8 inches long, half or more nude. Flowers very small scattered. Bracts linear acuminate recurved. Sepals lorate obtuse purplish. Petals a little smaller. Lip ovate with large lorate obtuse auricles bright yellow, teeth four short acute ones and a larger median obtuse one. Column green arms rounded. Capsule oblong erect $\frac{1}{2}$ inch long.

Damp forests, Singapore: Changi (Ridley 3925), Seletar, etc.; Johor: Batu Pahat, (Ridley); Malacea: (R. Derry); Perak: (Scortechini), Larut Hills at 4,000 feet (Herrey). Distrib. Borneo.

\section{Oberonia, Lindl.}

Small epiphytes, with a very short or moderately long stem, covered with distichous equitant leaves narrow fleshy. Spike terminal-slender covered with minute green yellow or red flowers. Bracts short oblong often toothed. Petals and sepals orate reflexed. Lip entire or lobed with a circular nectariferous depression at base. Column very short, arms short. Anther ovate. Pollinia $t$ adhering by a viscid disc. Capsule globose or elliptic.

About 60 species, Indo-Malayan, with a few in Africa and the Mascarene Islands, Australia, Polynesia and Japan.

$\S 1$. Acaules, stemless.

Lip entire flat ovate .. .. 1. O. Tunata. triangular oblong 2. O. Prainiana.

Lip concave emarginate .. $\quad . \quad 3$. O. subnavicularis.

Lip bilobed denticulate .. . . 4. O. iridifolia.

Lip oblong bifid .. $\quad \ldots \quad \ldots \quad$ 5. O. gracillima.

Lip 3 lobed.

Rhizome long creeping .. . . 6. O. Scortechinii.

Not rhizomatous.

Margins of lip entire . . . \% O. rosea.

Lateral lobes toothed $\quad . \quad \ldots \quad$ 8. O. stenophylla.

Lip 4 lobed.

Lateral lobes short entire .. $\quad \ldots \quad 9.0$. aurantiaca.

Lateral lobes fimbriate ... .. 10. O. insectifera.

§ 2. Caulescentes. Stem elongate.

Lip oblong retuse not toothed $\ldots 11$. O. anceps.

Lip denticulate. Petals entire.

Lip base narrow apex bilobed .. 12, O. miniata.

Lip broad base with large auricles 13. O. porphyrochila. 
Petals toothed. Lip not lobed.

Lip apex bifid .. .. .. 14 O. Bertoldi.

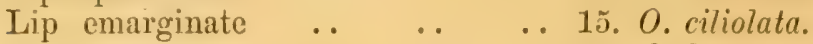

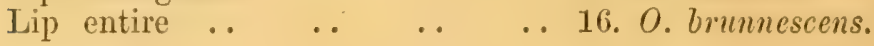

Lip 3 lobed.

Mid lobe cuneate broad .. . . 1\%. O.intermedia.

Mid lobe quadrate bifid .. .. 18. O. gracilis.

Mid lobe with two long narrow lobes 19. O. caudata.

Mid lobe narrow apex bilobed .. 20. O. Rolfeana.

\section{§ 1. AcAULes.}

1. O. Lunata, Lindl., Gen. and Sp. Orch. Pl. p. 1\%. Leaves few ensiform straight or falcate acuminate 3 to 5 inches long, $\frac{1}{3}$ to $\frac{1}{2}$ inch wide. Spike rather stout as long as the leaves. Bracts ovate dentate. Flowers $\frac{1}{4}$ inch across white or yellow sessile in crowded whorls. Sepals ovate acute. Petals linear lanceolate. Lip entire ovate or olsscurely 3 bilobed, apex rounded, dentate. Cipsule sessile triquetrous. Hook. fil. Fl. Brit. Ind. VI. 180. O. biaurita, Hook. f. Ic. Pl. t. 231\%. O. dissitiflora, Ridl. Journ. Linn. Soc. XXXII. p. 218. Malaxis lunata, Bl. Bijdr. 394.

On trees, Singapore: Seletar (Ridley 36t), Kranji, etc., Bukit Timah Road (Ridley 10154, 818\%). Distrib. Java.

2. O Pranniana, King, Journ. As. Soc. Beng. Vol. LXIV. 331, Ann. Roy. Bot. Calc. 8, Pl. 1. 'Tufted. Leaves fleshy falcate $\frac{1}{2}$ to $\frac{3}{4}$ inch long 4 in broad. Spike slender $t$ inches long. Flowers minute distinctly verticillate, bright brown. Bract oblong subentire as long as the orary. Sepals oblong blunt. Petals elliptic lanceolate serrate. Lip triangular or oblong blunt edges eroso-dentate.

Perak: (Scortechini); Penang: Penara Bukit (Curtis). J Jistrib. Sikkim, Siam.

3. O. subsavicularis, King, Journ. As. Soc. Beng. LXTI. II. 5\%9. Leaves ensiform obliquely acuminate 3 to 6 inches long, $\frac{1}{4}$ to $\frac{1}{2}$ inch wide. Spike hardly as long as the leaves, peduncle terete ebracteate. Flowers very densely crowded $\frac{1}{20}$ inch long. Bracts small lanceolate erose. Scepals ovate blunt. P'etals narrowly oblong truncate. Lip about as long as broad very concave from lase to apex edges coarsely retroserrate apex hlunt emarginate with a central tonth.

Perak (Scortechini No. 1202).

4. O. iridifolia, Lindl., Gen. and Sp. Pl. 15, Fol. Oreh. 1. Sitem short. Leares (nsiform acute :3-5 inches long $\frac{1}{2}$ to 1 inch wide. Spike 6-10 inches long densely floriferous. Flower's minute green or yellow. Bracts broarl tootherl sheathing the orary. Sepals ovate torthed. I'etals as large or smaller. Lip orbicular ovate bifid shortly 
at the tip toothed all round. King, Ann. Bot. Gard. Cale. 8, p. 8, Pl. 8, Hook. fil. Fl. Brit. Ind. V. 665. Cymbidium iridifolium, Roxb., Fl. Ind. IIT. 458. Mataxis iridifotia, Rchb. fil. Walp. Ann. VI. 208. Siam

Langkawi, Pulau Hujong Duri (Curtis). Distrib. India and

5. O. gracmuma, Ridl., Jour. Roy. As. Soc. St. Br. Vol. 43, p. 93. Stemless or nearly so. Leaves four or five ensiform acute $1 \frac{1}{2}$ inch long $\frac{1}{8}$ wide. Spike very slender 3 to 4 inches long, glabrous, base nude for $\frac{1}{4}$ of its length. Bracts lanceolate acute entire longer than the ovary. Flowers minute, whorled, 5 or 6 in a whorl. Sepals oblong obtuse recurved entire. Petals broader ovate toothed all whitish. Lip orange oblong tip acute bifid with a wide sinus between the lobes, edges minutely toothed, auricles short rounded.

Tringanu at Bundi (E. Rostado). Endemic.

6. O. Scortechinif, Hook. fil. Fl. Brit. Ind. V. p. 683. Rhizome long creeping stout with the tufts of leaves $1 \frac{1}{2}$ inch apart. Leaves $9-10$ inches long $\frac{1}{4}$ to $\frac{1}{3}$ inch wide ensiform curved narrowed towards the apex. Scape 12 to 18 inches long rather slender, covererl with flowers in distinct whorls to the base. Bracts orate lanceolate $\frac{1}{12}$ inch long. Flowers very small yellow. Sepals oblong obtuse. jetals ilnear. Lip three lobed side lobes lanceolate longer than the small orbicular rhomboid midlobe.

Perak: Kinta district (King's Collector ‘122, 1128), (Scortechini 1588). Endemic.

\%. O. Rose1, Hook. fil. Ic. Pl. t. 2005. Leaves narrowly ensiform 2-3, inches long $\frac{1}{6}$ to $\frac{1}{3}$ wide. Spike as long as the leaves or shorter Bracts lanceolate. Flower's $\frac{1}{30}$ of an inch across sessile pink. Sepals rounderl ovate. Petals elliptic erose. Lip hardly longer side lobes quartrate, midlobe reniform retuse. Capsules subsessile. Hook. fil. Fl. Brit. Ind. VI. p. 180.

Malay Peninsula (W. Norris); Perak: Gunong Batu Putih (Wray 12+6), Larut Hills (King's Coll. 2664). Endemic.

8. O. stexophyld, Ridley, Joum. Limn. Soc. XXXII. p. 218. Leaves ensilorm falcate acute or subacute $2 \frac{1}{2}$ inches long $\frac{1}{8}$ to $\frac{1}{4}$ inch wide. Spike $3 \frac{1}{2}$ inches long slender curved almost entirely corered with flowers. Flowers minute in seattered fascicles. Bracts as long as the ovary lanceolate acuminate. Sepuls and petals equal orate oblong obtuse. Lip lateral lobes widely oblong laciniate, midlobe longer triangular emarginate with acute angles.

Johor: Hulu Sembrong (Lake and Kelsall). On coffee trees Sungei 'T'ebrau (Ridley). 
9. O. aurantiaca, Ridley, Journ. Bot. Vol. 36, p. 210. Leaves about 6 falcate actuninate fleshy dark green 2 inches long $\frac{1}{8}$ inch wide or less. Spike $t$ inches long nodding base corered with lanceolate acuminate bracts. Bracts narrowly linear acuminate. Flowers minute whorled. Sepals ovate sprearling orange. Petals oblong obtuse tips toothed, much narrower. Lip narrow oblong red much Jonger than the sepals, lateral lobes short oblong obtuse narrow, median long narrowly oblong bifid at the apex. C'olumn rather longer than usual.

Selangor: Kajang (Goodenough). Endemic.

10. O. Ixsectiferi, Hook. fil. Ic. Pl. t. 2004. Leaves ensiform straight narrow upwards 2 to + inches long $\frac{1}{3}$ inch wide. Spike about as long as the leaves. Bracts subulate minutely erose. Flowers subwhorled sessile $\frac{1}{20}$ inch across. Sepals ovate subacute. Petals linear. Lip much longer, side-lobes $2-3$ partite with linear lobules, midlobe much longer with two linear lobes. Capsule shortly, pedicelled. Hook. f. Fl. Brit. Ind. VI. p. 180.

Pahang: 'Tahan River (Ridley) ; Perak: Larut Hills at 3,500 feet altitude (King's Coll. 2693). Endemic.

\section{\$2. CAULESCENTES.}

11. O. ANCEPS, Lindl., Sert. Orch. sub. t. 8. Stems tufted 4 to 10 inches long with short cultrate leaves $\frac{1}{4}-\frac{1}{2}$ inch long very numerous and close. Spike cylindrie 1-3 inches long densely covered with flowers. Bracts ovate entire, concealed by the flowers. Flowers ocreous. Sepals ovate. Petals much smaller linear, entire all reflexed so as to be invisible behind the broad lip. Lip ovate or oblong ovate (marginate Jonger than the sepals. Column arms bright green. Capsule sessile globose. Lindl. Fol. Orch. 6, Hook. f. Fl. Brit. Ind. V. 685. O. imbricata, Wight. Ic. t. 1629 (not Blume). O. Griffithii, Wight. Ic. V. 4. Malaxis anceps, Rehb. f. Walp. Ann. VI. 214.

fimgapore: (Aalang (Ridley 9513), Kranji (Ridley); Malacea : Tanjong Kling (Ridley) ; Pahang: neä Pekan (Ridley 1559), Pasir Joyang (lidlley); Solangor: (inting Bislai (Ridley) : Perak: l)indings (livelley) (Seortechini); Penang: Waterfall II ill (Curtis). Distribution 'Tenasserim. Native name, Sakat Lidah Buaya.

Leaves used for poulticing. The largest native species easily distinguished by its long stem and dense spike of yellow flowers.

12. O. Mntat, Lindl., Bot. Reg. 18t3, Mise. S. Stem short 1-11 inch long. ieaves ensiform acute about 8 , one inch long $\frac{1}{4}$ inch wirle. sipile slenter 1 ! -s inches long puberulous base nude. Flowers $y_{20}$ inch acrosis lax perlicelled, red. Bracts lanerolate shorter than the pedicels. Pedicels puberulous. Sepals orate, ciliate. Petals narrower. Laip bate narrow dilaterl towards the anex denticulate bilobed. Lindl. Fol. Oreh. (i. Hook. 1. Fl. Brit. Ind. V. 685. Malaxis miniata, lichb. f. Walp. Ann. VI. 215. 
- Singapore: (Hort. Loddiges). Kranji (Ridley 3\%5, 2034a). Endemic.

13. O. porphyrochils, Ridl., Journ. Linn. Soc. XXXII. 220. Stem rather short. Leaves lorate acuminate flaccid falcate, 6 inches long $\frac{1}{2}$ inch wide. Spide over 4 inches long slender, almost entirely floriferons, rachis angled. Flowers $\frac{1}{20}$ inches across crowded below fascicled towards the apex. Bracts linear acute entire. Sepals short ovate obtuse yecllow. Petals linear much narrower. Lip ovate purple with long crenulate recurved auricles, median lobe bilobed toothed.

Selangor: Bukit Hitam (Kelsall). Endemic.

14. O. Bertoldr, King, Journ. As. Soc. Bengal LXVI, II. 581. Stems very short. Leaves coriaceous narrowly lanceolate acuminate. $\frac{1}{2}-1 \frac{1}{2}$ inch long $\frac{1}{5}$ to $\frac{1}{4}$ inch wide. Spike longer than the leaves, base long ebracteate slender. Flowers $\frac{1}{80}$ inch long in whorls of 6 . Bract lanceolate erose-serrate. Sepals elliptic oblong entire. Petals lanceolate coarsely serrate. Lip longer orate elliptic not lobed with one or two teeth on the sides, apex bifid toothed. Perak (Scortechini 1525). Endemic.

15. O. ciliolata, Hook. f. Fl. Brit. Ind. VI. p. 181, Ic. pl. 2318. Stems tufted 3-6 inches long. Leaves about 9-1 ensiform acute 34 to 2 inches long $\frac{1}{4}$ inch wide. Spike 4 to 6 inches long, floriferous to the base. Flowers $2^{\frac{1}{0}}$ inch wide, white, whorled. Bracts oblong denticulate apiculate. Scpals ovate, ciliate. Petals lanceolate minutely denticulate ciliate. Lip oblong quadrate or ovate retuse serrate. Capsule $\frac{1}{16}$ inch across pedicellate puberulous.

Singapore: Kranji (Ridley 3\%5), Changi, Bukit Timah Roar (Si88, 10153); Malacea: Sungei Kesang, Sungei Rambei (Derry 313) ; Tringanu: Bundi (Rostado). Distrib. Borneo.

16. O. Brunnescens, Ridley, Journ. Linn. Soc. Stem an inch long. Leaves cultrate falcate $\frac{3}{4}$ inch long $\frac{1}{8}$ inch wide. Spike 4 inches long slender base nude. Flowers seattered $\frac{1}{20}$ inch across, reddish brown. Bracts ovate acute. Sepals ovate oblong entire. Petals broad ovate denticulate. Lip ovate laciniate not lobed. Capsule ellipsoid pyriform $\frac{1}{8}$ inch long.

Perak: Larut Hills '(Ridley), Endemic.

1\%. O. ixternedia, King, Journ. Aș. Soc. Beng. LXVI. II. 579. Stem 1 to $3 \frac{1}{2}$ inch long. Leaves acute subfaleate 1 to $2 \frac{1}{2}$ inch long, $\frac{1}{2}$ to ${ }_{20}^{1}$ inch broad. Spike slender decurved about $4 \frac{1}{2}$ inches long. Flowers $\frac{1}{20}$ inch long whorled. Bract lanceolate shorter than the ovary. Sepals ovate acute. Petals linear oblong acute. Lip 3 lobed larger than the sepals, broadly oblong, lateral lobes rounded apex broad and erose.

Perak (Scortechini 1516). Endemic, 
18. O. Gricilis, Hook. fil. Fl. Brit. Ind. V. 685. Stems 6 to 8 inches long flexums. Leares straght lancolate ateme one inch long.

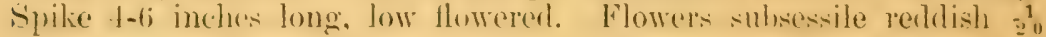
inch across. Bracts very short triangular ovate. Dorsal sepal orbicular, laterals ovate. Petals linear. Lip with two oblong cylindric obtuse lateral lobes, midlobe quadrate flat obtusely 2 lobed.

Perak: Kinta River (King's Collector). Endemic.

19. O. ciupst, King, Journ. As. Soc. Bengal LXVI. II. p. 581. fitems an inch and a hatt long. Leates tor o distant. lineal chsilorm

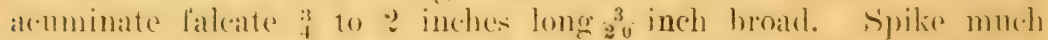
decurved, as long as the longest leaf, lax-flowered. Flowers solitary about 1 inch long. Bract orate entire. Sepals ovate oblong acute pale red. Petals linear ląnceolate acuminate. Lip much longer, lateral lobes ovate atcuminate spreathing, midlobe natrow deeply divided into 2 caudate acuminate lobules.

Perak (Scortechini 315̋). Endemic.

20. O. Rolfiats, King, Journ. As. Soe. Bengal LATT. II, כ81. long and about t inch wile. Spike :3 or t times as longe as the leaves Sitems about an inch long. Leares ensiform alente ladeate $\frac{2}{3}$ to 1 inch slender puberulous rather spatrely flowered. Brated oblong edges arose. Ovary glandular hairy stalked. Flowers inch orange coloured. Sepals subequal ovate obtuse. Petals linear entire. Lip longer base broad and concave, lateral lobes rounded erect, midlobed narrow shortly bilobed at the apex, edges entire.

Perak (Scortechini 2193). Endemic.

\section{Tuparis, Richard.}

'Terrestrial or epiphytic plants. Stems nsually swollen at base. Teares herbaceous (terrestrial species) ol coriaceous (epiphytes) ovate or lanceolate few. Raceme ereet, flowers numerous small, or very small. Sepals and petals narrow linear or oblong. Lip clawed, blade oblong, ovate or reniform, deflexed with a single or double callus at the base. Column long graceful, liee with short arms or wings. Anther terminal orate. Pollinia t. Species over 100. All temperate and topical regions.

$\$$ 1. Moldifolub, terrestrial, leaves herbaceous one ol more on al swollen stem not articulaterl.

Leaves several.

Lip large oblong, dentieulate, Howers deep red ... ..

Lip large ovate, pale veined red 2. L. renosa.

1. I. alrosanguimen. 
Lip small oblong cntire, calli ? Lip small obovate crenulate, callus 1

Lip small apex denticulate bifid

Leares solitary ovate.

Lip oblong bifid denticulate ..

Lip pandurate bifid with two long tails

Lip pandurate bifid lobes fimbriate
3. L. ferruginea.

4. L. transtillata.

5. L. Wrayi.

6. L. Muingayi.

¿. L. parvula.

S. L. furcata.

$\$ 2$ Conmfouse, epiphytic, leaves coriaceots articulate. Raceme lax bracts deflexed.

Flowers large, lip bilobed, toothed Flowers small, lip apex pectinate . Flowers very small.

Lip oblong retuse ... .

Lip oblong bilobed . . . . 12. L. flaccidu.

Lip entire ovate .. . . . . 13, L. longipes.

Lip entire oblong . $\therefore \quad$.. 1t. L. comosa.

5. Disticina, epiphytic, leaves coriaceous articulate. Raceme short compact. Bracts distichous persistent.

Lip half an inch long, connate, flicbelliform .. . . . . . 15. L. compressa.

Lip $\frac{1}{\ddagger}$ inch long obovate retuse .. 16. L. disticha.

\section{है 1. Mollifolia:}

1. L. atroshagerden, Ridl., Journ. As. Soe. Str. Br. Tol. 39, p. 61. Stem stout sheathed $t$ inches tall. Leares ovate lanceolate acute crisped, 8 inches long by 3 inches wide or less. Scape stont over a loot tall. Raceme lax many flowered. Bracts very small, orate lancelate, ovary and pedicel 1 inch long, twisted and the orary with simnate ribs. Flowers as large as those of $L$. venosa entirely deep red-purple. Sepals linear obtuse revolute. Petals much narrower. Lip orbicular oblong $\frac{1}{2}$ inch long subacute denticulate with two short semicireular lamellac at the base. Column arched with narrow wings.

Perak: Gap on Larut Hills 4,0y0 leet alt. (Curtis and Derry).

Allied to $L_{*}$ e cenose but differing in the broader lip, and deeper colouring of the flower. Endemic.

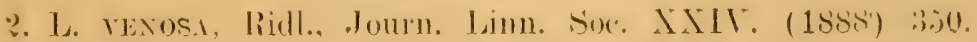
Stem sirollen about 2 inches long, old pseudobulb 6 inches green or purple. Leaves about five unequal ovate lanceolate undulate acute 6 inclic: loug by ? incles wide. Scape stout purple red orer a foot long. 
Raceme lax many flowered. Bracts lanceolate acute deflexed is inch long. Pedicels 1 inch long purple. Flowers large. Sepals oblong convolute: inch long pinkish or green. l'etals nealy as long linear pink. Lip orate obtuse erenulate orer half an inch long and as broad, pale with purple veins, ealli small rounded. Columm rather short nearly straight and thick with raised edges, no arms, purple. Anther ovate purple.

Singapore: Chan Chu Kang (Ridley); Johor (Little); Perak: Bujong Ialacea, (Curtis 3289). Distrib. Sumatra, Borneo.

3. L.Fentivixes, Lindl., Gard. Chron. 18t8, p. 5̃. Stem swollen at the base short. Leares narrow linear lancolate acmunate s to 9 inches long by $\frac{1}{2}$ to 1 inch wide. Kaceme 12 to 16 inches tall stout base nuele, flowers small : inch long, scattered in the upper third. Bracts lanceolate acuminate. Sepals oblong ovate laterals falcate obtuse. Petals linear narrow, all green or purple. Lip fleshy hardly clawed, oblong retuse, green or purple calli two small bosses at the hase. Columm arehed rather stout arms rery obselure, greenish. Anther ovate. Capsule pearshaped an inch long on a pedicel $\frac{1}{4}$ inch long.

L. nervosa, Ridley, Journ. Limm. Soc. XXXII. 22t. Damp grassy spots, ricefields, ete. Singapore: Tanglin. ('hangi (lidere); Ifalacea: (Griffith), Chabau (Ridley); Perak: Larut (King's Collector 2:82), Kampar (Curtis 3108). Distrib. Cambodia.

Probably only a local form of $L$. nervosa, Lindl., difiering mainly in its narow elongate leaves, and long flowerspike.

4. L. Tisistillat从, Ridl:, Journ. Linn. Soc. XXXII. 22J. Stem an inch and a hall long swollen. Leaves about $t$ ovate to ovate lanceolate acute obligue of nerved petioles ? inches long stout blarle di inches long and two wide. Raceme 6 inches long rachis winged, base

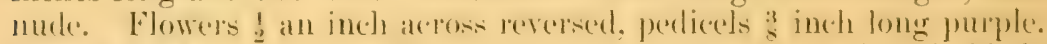
semals lorate purplish. P'etals linear narrower. Lip elawed. Whade oblong obovate slightly retuse, and erenulate dark purple, with a median olive green channel, callus a transverse bar green with two purple spots, at the hatse of the blarle. ('olumm nearly straight alpex hooded yellowish white, arms small oblong trunente.

Perak: Larut Hills (Ridley). Endemic.

5. L. WhiYt, Hook. f. Fl. Brit. Ind. VI. 1) 181. Stem 3-11

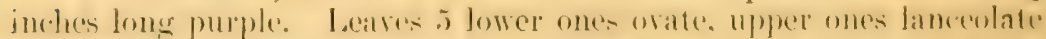
acute undulate bright green six inches long three inches wide or

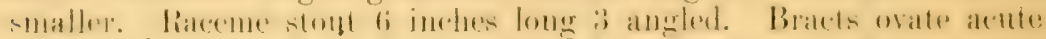
lanceate indulate $\frac{1}{8}$ inch long. Pedicels and oraries slender $\frac{1}{2}$ inch long. Sepals linear obtuse convolute linear shorter all green. Lip oblong apex widely =quare bilubed, lobes 
toothed, green with two purple lines on the dise, no calli. Column gaceful curved green. Capsule elliptic nearly an inch long. $L$. pectinifera, Ridl., Journ. Bot. Vol. 39, p. 210.

Perak: (Wray 3i13, 3631); Dindings on Gumong 'Tungul (Ridley). Endemic.

6. L. Maingayt, Ridl., Journ. Linn. Soc. XXXII. 226. Stem short base swollen subglobose. Leares lower ones reduced to sheaths, upper one orbieular ovate dark green 3 inches long by 2 inches wide. Raceme about $t$ inches lax. Bracts lanceolate acuminate half as long as the pedicel. Pedicel half an inch long. Flowers $\frac{1}{4}$ inch long deep crimson. Sepals narrow lanceolate acute. Petals narrower. Lip Column fairly stout arched, arms short incurved.

Microstylis Maingayi, Hook. f. Ic. Pl. t. 1826, Fl. Brit. Ind. V. p. 689. Wet rock in forest from 2,000 feet elevation upwards.

Malacea: Mount Ophir (Ridley); Perak: Larut Hills (Wray 6\%4), Gunong Batu Puteh (Wray 48\%); Penang: Waterfall Hill (Maingay), Bukit Laksamana (C'urtis 100.5); Kedah: Gunong Jerai (Ridley); Selangor: Pahang Track (Ridley). Endemic.

\%. L. Parvela, Ridl. 1.c.e. Stem short subglobose. Leaves lower ones reduced to sheaths, upper one ovate acute dark green 2 inches long by one wide. Raceme 3 inches base nude. Bracts lanceolate acuminate very small. Flowers rather crowded about $\frac{1}{8}$ inch long deep crimson. Sepals oblong obtuse. Petals narrow. Lip narrow pandurate ending in two narrow tails with a broad sinus between.

Nicostylis parvula, Hook. f. Ic. Pl. t. 182\%, Fl. Brit. Ind. V. 690. Wet rocks, at an altitude of 2,000 feet and upwards.

Perak: Larut Hills (King's Collector 645\%, Ridley 5203). Endemic.

8. L. Furcatı, Ridl., Journ. Limn. Soc. l.c.c. Stem very sinall subglobose. Lower leaves reduced to sheaths, upper one ovate acute 2 inches long by one wide. Raceme very slender 4 to 6 inches long base about one third nude. Flowers numerous very small $\frac{1}{8}$ inch long. Bracts much shorter than the pedicel lanceolate acute. Pedicel slender $\frac{1}{8}$ inch long. Sepals oblong obtuse. Petals linear lorate. Lip not clawed narrow oblong much longer than the sepals, bifid, lobes diverging toothed, with a median tooth. Capsule elliptic $\frac{1}{4}$ inch long.

Microstylis furcata, Hook. I. Ic. PI. 1827 A. Endemic.

Perak: Larut Hills 4,000 feet alt (Ridley), Scortechini 3iob.

\section{Colinfolile.}

9. L. Latrolia, Lindl., Gen. and Sp. Oreh. Pl. p. 30. Stem stout 3 inches long. Leaf solitary elliptic lanceolate very coriaceous 
with about 14 strong ribs 12 inches long 3 inches wide. Raceme 10 to 14 inches long stont. Flowers numerous $\frac{1}{2}$ inch long. Bracts lan-, reolate armminate ! inch long. Penlicel ! inch long. Sepats nartow linear. Petals similar narower yellowish. Lip base narow apex dilated bilobed denticulate, orange, calli two short erect processes. Column slenter arched arms rery short. Anther orbicnlar. Capsule 1 inch long obconic. Ls. robustr, Hook. f. Ic. Pl. 2012. L. Scortechini, Hook. f. Fl. Brit. Ind. V. 508 , Ic. Pl. No. 2009. Malnatis latifolu, Blume, Bijdr. 393. Epiphyte at an altitude of 1,000 to 5,000 leet.

Selangor: Bukit Hitam (Kelsall); Perak: Larut Hills (Scortechini, Ridley). Distrib. Java, Borneo.

10. L. LACERAT, Ridl., Journ. Limn. Soc. XXII. 1886, p. 284.

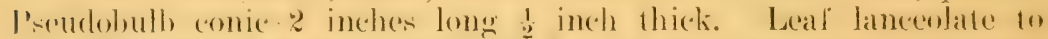

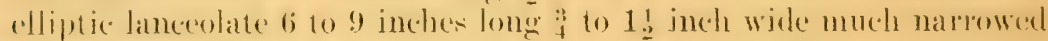

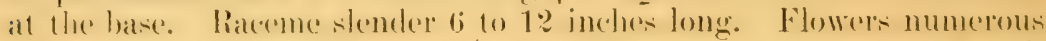
distant if inch long. Bracts narrowly lanceolate acmminate $\frac{1}{\text { in }}$ inch

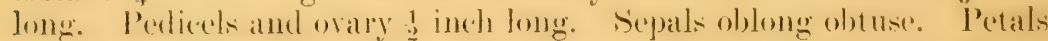

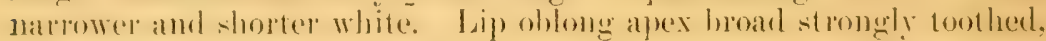
callus obscure single, orange coloured. Column arched, arms rery obscure. Capsule elliptic half an inch long. Hook. f: Fl. Brit. Ind. 1 . . .0\%.

Perak: (Scortechini, King's Collector), Larut Hills (Ridley); Lankawi Isles, Gunong liaya (C'urtis). Distrib. 'J'enasserim, Borneo.

11. L. Elaguss, Lindl., Gen. and Sp. Oreh. Pl. p. 30. Pseudobulb conic cylindric 1 inch long. Leaves? elliptic lanceolate nallrowed at the base 6 to 9 inches long $1-1 \frac{1}{4}$ wide. liaceme $1 \%$ to 18 inches long areed stift base mule. Hlowers rery numeroms small onetenth inch long. Bracts lanceolate acute it inch. Sepals oblong,

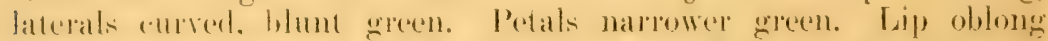

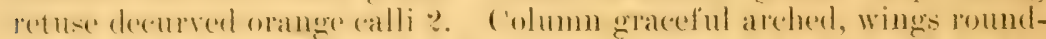
ed, green. Capsule elliptic I inch long. L. gracilis, IIook. I. Ie. I'l. 1. 2011, Fl. Brit. Ind. VI. 182. Epiphyte on trees and rocks, com111011.

Singapore: Kranji, Sungei Buluh (Ridley 23:2) : Johor: Gu-

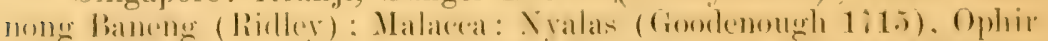

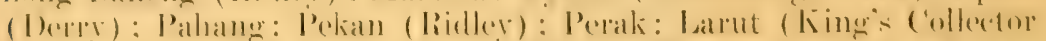

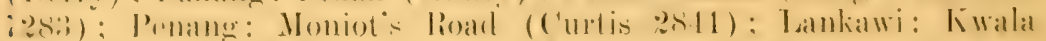
Malacea (Curtis 1695); Kedah: Gunong Jerai (Ridlley). Distrib. Borneo.

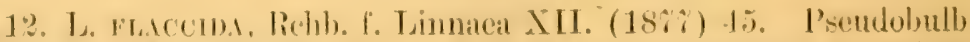

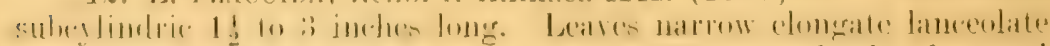
acule (f to 12 inches long : to inch wide liacene slender decurved

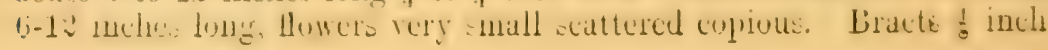


long lanceolate acute. Pedicel and orary 1 inch long. Fepals oblong, laterals falcate obtuse greenish white. Petals narrower and shorter. Lip oblong abruptly decurved in the midlle apex bifid, ederes ciliate, orange, calli 2 small processes. Column graceful arched, arms very short rounded. Capsule elliptic $\frac{1}{2}$ inch long. On lofty trees.

Malacea: Machap (Derry 160); Selangor: Goa Batu (Ridley 8132) ; Perak: Ulu Bubong (King's Collector 10402). Distrib. Sumatra, Jara, Borneo, Siam.

13. L. Loxgipes, Lindl., Wall. Pl. As. Rar. I. 31. t. 35, Gen. and Sip. ()reh. 40. P'seudohull, short oroid, 1-2 inches long or clongate to (i inches, cylindrice, leares linear obovate oblong subspathulate obtuse or acute, t-8 inches long 1 inch wide. Raceme erect about 6 inches long. Flowers copious erowded in the upper part, very small. Bracts linear acuminate $\frac{1}{8}$ inch long. Sepals oblong greenish white. Petals linear. Lip ovate acute decurved in the middle orange or green, no calli. Column graceful curved with short arms. Wt. Leones t. 906. L. pendula, Lindl. Bot. lieg. 1838, Misc. 180. L. spathulata, Lindl., Bot. Reg. 18+2. Rare.

Perak: Larut Hills (Derry), Batang Padang (Wray 14t0); Prnang: Sonkay River (Curtis jo) (Porter in Wallich's Catalogue 1943). Distrib. India, Ceylon, Malay and Pacific Isles, China.

14. L. conosi, Ridley, Journ. Linn. Soc. XXXII. 229. A simall plant with erowded conical pseudobulbs is inch long. Leaves natrowly lanceolate areate narrowed to the base $t \geq 1$ inch long 1 inch wide. Raceme erect 6 inches long. Flowers small numerous green. Sépals lanceolate lorate. laterals broaler. Petals linear. Lip oblong subquadrate apex rounded, no calli. Columm slightly arehed above, arms short obscure. ('apsule ellipsoid subglobose $-\frac{1}{8}$ inch long.

Perak: Larut Hills at 1,000 feet altitude (Curtis). Endemic. :3. Distichat.

15. L. Coveressi, Lidl., Gen. and Sp. Orch. Pl. p. 32. Rhizome (creping, psendoloulbs subgholose at base covered with the sheath of the leaf. Leat solitary colinceous linear lanceolate acute, 9-15 inches long 1 inch wide. Bracts distichous imbricating. Flowers opening singly red brown. Pedicels $\frac{1}{2}$ inch long. Sepals lanceolate. Petals narrow linear. Lip half an inch long, cuneate flabelliform with a narrow claw, apex broal truncate crenulate. Calli 2. Column slender curved, no arms. Hook. f. Fl. Brit. Ind. V. r06, Ridley Journ. Limu. Soc, XIIT. 291. Mulaxis compressa, Bl. Bijdr. 390, t. 54.

Perak: Larut Hills at 1,000 foet (Curtis 2069), Scortechini 1420,

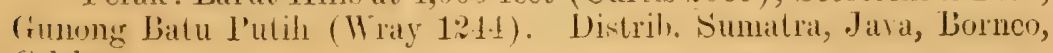
Celebes. 
16. L. Districh, Lindl., Bot. Reg. sub. t. 88\%. Thizome long and slender branched pseurlobulbs pear-shaped f inch long. Leaf linear acute 5 to 10 inches long $\&$ inch wide or less. Scape slender naked, winged 4 to 6 inches long raceme $\frac{1}{2}$ to $\frac{3}{4}$ inch long. Bracts $\frac{1}{6}-\frac{1}{4}$ inch long, distichous. Flowers $\frac{1}{2}$ inch. Sepal oblong narrow rellowish. P'otals linear. Lip \& inch Iong ocre yellow, obovate retuse, with 2 tubereled calli at base. Column slightly eurved broadly winged. Hook. t. Fl. Brit. Ind. Y. 806, Ridley Journ. Linn. Soc. XXII. 291. L. gregaria, Lindl., Gen. and Sp. Oreh. Pl. 33. L. mucronata, Lindl., Ic. 32. Malaxis disticha, I'houars. Orch. Iles. Austr. d'Afrique t. 82. Malaxis mucronata, Bl. Bijdr. 391.

Singapore: Kranji (Ridley 12:), Sungei Buloh (Ridler 2:3i3); Johor: Kwala Kahang, (Kelsall) ; Pahange Kota Glangrgi (Ridley); Prrak (Seortechini 15.59), Bujong Malacea (Riblley 9281), Larut (Kinges ('ollector 1986). Kinta (King's ('ollector ros.)) ; Penang: (Maingay); Lankawi (Curtis). Distrib. Mascarene Isles, Ceylon, 'I'enasserim, Malay Arehipelago.

\section{P'Latrounis, Benth.}

T'ufted epiphytes with numerous obconic pseudobulbs crowded on a rhizome. Leaves solitary petioled lanceolate or linear. Bracts stifl terminal slender. Flowers small green white or brown. Bracts stifl broad convolute or narrow. Sepals and petals narrow spreading. lip sessile or nearly so flat oblong, or pandurate, lobes small, calli various. Column slender or short and broad, with distinct often long arms, tip of column produced into a hood. Inther incumbent 2 celled. Pollinia 4 waxy ovoid.

Species about 20. Malay Peninsula and Islands.

Bracts broad _.. $\quad \ldots \quad$.. $\quad$ 1. P. longifolie.

Bracts narrow, arms of columm long from the middle .. $\quad . . \quad \ldots \quad$ \%. P. gracilis.

A'ms rising from near the base.

Arms much shorter than the column 3. P. linearifolia.

Arms about as long acuminate .. t. P. linearis.

Arms about as long toothed .. 5. P. odorata.

Column and arms very short.

Arms from near the stigma _. 6. P. Kingii.

Arms oblong toothed at base .. \%. P. Sarawalensis.

1. Pe. monghoma, Hemsl. Giard. Chron. (1881) 11. p. 656.

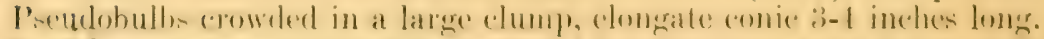

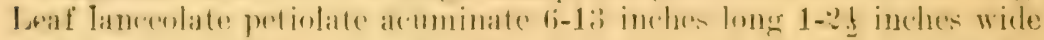
petiolate $2-1$ inches long. Raceme 12 to 15 inches long base nude for about half its leugth. Bracts oblong comolute $\frac{1}{b}$ inch long as long as 
the pedicel and ovary. Flowers about 40. $\frac{1}{4}$ inch across. Sepals and petals lanceolate acute, the latter a little narrow ochreous. Lip pandurate with shorter narrow acute spreading lateral Jobes, midlobe much larger obovate oblong, centre dark brown edges lighter, keels " low ridges brown. Column pale comparatively short arched, arms long and linear from about the middle, erect, hood toothed. Inther conic capsule globose oblong about an inch long splitting into :" equal lobes. Dendrochilum longifolium, lichb. fil. Bonplandia IV. (1856) p. 329 .

Singapore: Kranji, Sungei Jurong (Ridley 4681); Johor: Batu Pahat (lideley); Pahang: Selinsing (Machado); Peak: Hermitage Hill (Ridley). Distrib. Borneo.

2. Pl. Gracilis, Hook. f. Fl. Brit. Ind. V. p. 708 , Ic. Pl. t. 2016. Pseudobulbs crowded one inch long conic. Leaf ellipic lanceolate obtuse 4 to 6 inches long one inch wide petiole $\frac{1}{2}-1$ inch long. Raceme graceful 6 to 9 inches long lower third nude. Flowers numerous small. Bracts lanceolate acute $\frac{1}{6}$ inch long. Sepals and petals lanceolate acuminate acute, pale green. Lip panclurate lateral lobes narrow linear acuminate pale greenish white, median obovate, or cuneate acuminate white with a dull red centre bar and a green line in the centre, or yellowish with a dark brown central patch, keels 3 thick from the base to the middle of the lip, dark green. Column slender curved, arms from about half way down the column long Capsule elliptic, $\frac{1}{2}$ inch long.

Perak: Larut Hills, at 4-5000 feet. (King's Collector 280) (Curtis, etc.).

Var. angustifotia. Leaves narrow linear obtuse, 5 inches long by $\frac{3}{8}$ inch wide.

Perak: Bujong Malacea (Ridley 9816). Endemic.

3. P'L. Lixeanfoli, Ridl, Journ. Limn. Soc. XXXII. 231. Pseudobulbs fusilorm conic $\frac{1}{2}$ inch long densely crowded. Leaf linear lanceolate 2 inches long by $\frac{1}{4}$ inch wide petiole $\frac{1}{2}$ inch long. Racense slender nodding 3 inches long a third to a halt nude. Flowers $\frac{1}{3}$ inch long about 20 yellowish. Bracts longer than the ovary lanceolate acute. Sepals and petals lanceolate acuminate. Lip oblong or obovate, lateral lobes short triangular acute, median ovate oblong, or subquadrate subacute keels three. Column curved, arms linear rising from near the base much shorter, hood round entire. Anther ovate.

Dendrochilum linearifolium, Hook. fil. Fl. Brit. Ind. V. r82, Ic. Pl. t. 1859.

Malacea: Mt. Ophir at 3-4,000 feet alt., (Hullett 862, Derry 626) ; Perak: Batang Padang (Wray), Chosang (Scortechini), Gunong Bubu (Wray 3881). Endemic. 
4. PL. LIxLAlis, Ridl., Journ. Linn. Soc. XXXII. 230. Rhizome thick woody, psendobulls clongate conic $1 \frac{1}{2}$ inch long. Leal over a loot long inch wile linear lanceolate obtuse narrowed at the base inte a petiolate 3 incher long. liacene 12 inches long graceful, lower two-thinels nude. Flowers it inch long, white. Bracts lanceolate atute shorter than the ovary. Sepals linear acute. Petals similar. lip linear obtuse as long, side lobes acute hardly distinct, heels three thick, midklle one lower than the others. Column long arded arms lanceolate actuminate acute rising from near the column bate and projecting about the toothed hood.' Inther long beaked.

Kedah: Gunong Jerai, 3-t,000 feet alt. terrestrial. (Ridley j13S). Endemic.

․ Pl. oporite, Ridl., Journ. As. Soc. St. Br. Tol. 39, p. 92.

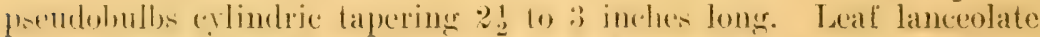
subatute petiolate, bade 9 inches long, in inch wirk. petiole 2 inches long slender. liaceme nodding graceful 1 foot long, lower half mude sender. Flowers greenish white sweet seented, i inch long numernus. Bracts lanceolate acuminate longer than the short ovary. Sepals and petals lanceolate acmminate acute. Lip entire tongue shaped obtusc minutely puleserent, keels. : nearly the whole longth of the lip. Column rather short with broad wings arms free from a little below the stigma as long as the hood, linear apex toothed, hood of column large toothed. Anther with a short broad beak.

Perak: (Curtis No. 285t). Endenic.

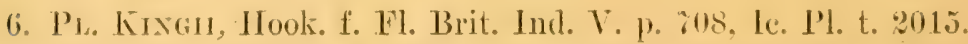
l'scudobulb elliptic oblong, 1 inch long. Leal' lanceolate linear acu-' minate 6 to 10 inches long, $\frac{1}{1}$ inch wide tapering much at base. liacene? inches long lower half nude, graceful. Flower's about '2t, it inch long, pale green, lip darker. Bracts lanceolate nearly twice as long as the ovary. Sepals lanceolate acute. Petals broater. Lip side lobes roumded broad; midlobe orate or lanceolate ovate with two short curred calli at the base. Colmmn very short, broad with rery short toothed arms from the upper part, hood long entire. Anther ellipsoid, with a rounded process on the tip.

Perak: Larut Hills (King's Collector Y45,, Scortechini 608a), (Curtis 2956). Distrily. Borneo.

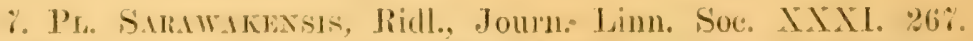
Pseudobulbs short oblong " inch long. Leal lanceolate $\%$ jnches long t) inch wide, petiole 1 inch. Seape s inches long slender nodding.

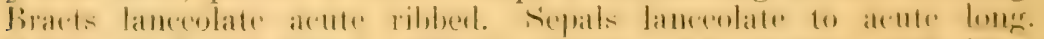

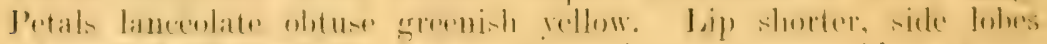

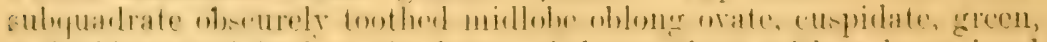
a double rased heel at the ba:e. Column slourt with a large hood 
arms, very short oblong ioothed processes at the base. Anther helmetshaped apiculate.

Perak: (Curtis). Distrib. Borneo.

\section{Dendrobium, Sw.}

Epiphytes rarely terrestrial. Stems elongate, or psendobulbous. Leaves coriaceous, herbaceous or fleshy. Flowers racemose axillary or subterminal. Sepals subernal, lateral ones arlnate to the foot of the column and forming a sac (mentum). Lip adnate to the foot of the column, side lobes erect or rarely spreading, often alsent, terminal lobe longer narrow or broad concave or flat. Rostellum entire. Anther 2 celled scull whaped top thickened, attached by the back to a small tooth, on the tip of the column. Pollinia 4, ovoid, or oblong in pairs in each cell. Capsule elliptic or pyriform.

Species about 300. Tropical Asia to Japan, Australia and Polynesian.

§ 1. Sarcopodiuar. Stems short psendobulbous of one or two internodes on a creeping rhizome. Flowers one to several on a terminal raceme.

Pseudobulb slender crowded one-leafed

Psendobulbs distant, short and thick, leaves 2.

Lip with two rows of tubercles on midlobe

Lip with three keels on dise and $\ddot{2}$ calli on base.

Lip with thickened ridges, no calli

1. D. Tongicolle.

\$2. Bolbidiun. Stems short, tufted, clavate, leaves 2 terminal. Flowers 1 or few from between the leaves, mentum long. Stems stipitate" . . . ..

\$ 3. Desnotrichus. Stems branched, the terminal internode of each branch deveToped into an ovoid or conic, psendobulb bearing a single leaf. Flowers few 1-2 at a time from a tuft of bracts below the leaf.

Terminal lobe of lip not broader than the lateral lobes apex bilobed.

Lip glabrous midlobe oblong base broad ... ... .. ...

Lip midlobe narrowed above horseshoe shaped .. $\quad . . \quad \ldots$

Terminal lobe of lip entire bearded

2. D. longipes.

3. D. macropodum.

4. D. geminatum.

5. D. quadrangulare.
6. D. lonchophyllum.

\%. D. Kelsalli.

8. D. criniferum. 
Terminal lobe much broader than the side lobes.
Margins waved entire. Oblong
9. D. Flabellum.
'I'riangular, apex broad retuse .. 11. D. roseo-punctatum.
Subtriangular almost bilobed .. 11. D. pallidiflorum.
Of two long undulate lobes .. 12. D. bifidum.
'Terminal lobes lacerate or fim- briate
.. 1\%. D. Tnciniosum.

$\S 4$. Aporux. Stems flattened with distichous ensiform or triangular short leaves, sheaths equitant persisting. Flowers small white yellow or pink, in very short racemes usually covered with numerous brown bracts, lateral.

*Terminal portion of stem covered with sheaths only.

Flowers very small.

Lip narrow apex bilobed. Flowers white .. . . . .

Lip narrow apex reniform $\ldots$ 15. D. rhodostele.

Lip apex obovate. Flowers pink 16. D. rosellum. small.

*:Stem leafy to the top. Flowers

Leaves oblong or lanceolate, not imbricating.

Lip cordate side lobes short blunt, midlobe broad .. ..

Tip side lobes long curred, midlobe shorter .. …

Lip cuneate bilobed. Leares $\frac{1}{2}$

inch across .. $\quad \ldots \quad \ldots$ 19. D. grande.

Leaves lanceolate imbricating.

Lip entire oblong spathulate. Bracts numerous ... ..

Lip entire oblong spathulate. Bracts few ... ...

Side lobes of lip distinct. Bracts

very numerous $\quad \ldots \quad \ldots$. ?.?. D. atrorubens.

Stem leafy to top, leaves short, flowers large.

Stems erect or pendulous, lip oblong entire .. .. ..

Stems prostate rooting .. $\quad \ldots$ ?1. D. prostratum.

Stems erect, bracts one or two, lip bilobed .. .. .. ..

1.. D. culophotum.

18. D. rhizophoreti.

20. D. atropurpureum.

21. D. simuatum.

9.). D, Ierminale. 
§ 5. Strongrle. Stems slender not swollen, leaves terete or slightly flattened. Flowers 2 or 3 together from the axils of the leafless terminal portions of the stem, white often reined pink.

Stem short $\ddot{3}$ inches, leaves $\frac{3}{4}$ inch long terete erect .. ..

Stem 4 inches long, leaves thick terete over a foot long .. . . . Leaves flattened narrow short Leaves very short numerous recurved . . . ..

26. D. teres.

2\%. D. plexile.

28. D. kentrophyllum.

29. D. acerosum.

30. D. subulatum.

$\S 6$. Virgatae. Often terrestrial stems slender leafy erect, leaves narrow unequally bilobed. Flowers small in pairs from the upper parts of the sheaths.

Terrestrial, sheaths glabrous when adult

Sheaths covered with black pubescence .

Epiphytic, sheaths short glabrous

31. D. conostalix.

32. D. villosulum.

33. D. pachyglossum.

$\S \%$ Bambusaefoliae. Epiphytic, stems tall erect or pendulons, covered thickly with linear to ovate lanceolate leaves. Flowers in pairs from an internode, with one or more large ovate bracts, yellow unexpanded. Mentum short curved.

Stems erect, leaves linear lanceolate thin

Stems pendulous leaves ovate lanceolate fleshy $\quad$. $\quad$.. $\quad$..

§ 8. Distichophyliat. Stems tufted, rarely scattered on a creeping rhizome, grooved, leafy. Leaves lanceolate bifid, coriaceous or thin. Flowers one or few on axillary racemes mediocre or small, petals and sepals reflexed. Mentum straight, horizontal spur like, lip unusually broad.

Stems tufted.

Lip entire fleshy, flowers small . . Lip 3-lobed emarginate . . . . Lip 3-lobed bilobed ... .. Lip 3-lobed oblong lateral lobes broad

34. D. gemellum.

35. D. pensile.
36. D. bifarium.

3\%. D. IIosei.

38. D. revolutum.

39. D. uniflorum, 
Narrow with a long claw, side lobes small

Stems weak on a creeping rhizome

\$. Cuavatal. Stems with from one to three of the internodes at the base swollen into an ovoid or fusiform pseudobulb, apex of the stem usually leafless. Leares coriaceous lanceolate or terete slender. Flowers produced singly from the tufts of bracts on the mude portion. Flowers mediocre or small white, or pink. Ifentum curved, thick.

Teaves terete.

Stem leafy to top, flowers lateral

Stem nucle at top bearing the flowers . . . . . . .

Leaves flat narrow linear . . . .

Leaves elliptic broad.

Psendobulb oval flattened of $2 \mathrm{in}$ ternodes . . . . . . .

Psendobull fusiform thick of 4 internodes .. . . . . .

$\$ 10$. Breviflores. Stems stout or slender not swollen. T.eaves one or two sul)terminal. Flowers in short racemes or solitary from internodes below the leaves. Tip short with small obscure side lobes. Meniun short.

Stem clubshaped 4 angled above, flowers solitary .. . . .

Stem subterete stout, flowers in a compact raceme. . . . . .

Stem slender, flowers few together raceme lax $\quad . \quad \ldots \quad \ldots$

\$ 11. Pedilonum. Stems terete very rarely flattened. Leaves lanceolate. Flowers solitary or in lateral racemes on the old leafless stems. Flowers large or small, white, pink or orange. Mentum much longer than the sepals straight or curved. Iip with a linear elaw and an orate or oblong limb.

- Stems flattened thin ovoid . sil. D. Inmellatum.

Stems long terete.

Flowers very small, raceme dense
40. D. metachilinum. 11. D. pandaneti.

12. D. gracile.

43. D. clavalor.

t4. D. clavipes.

45. D. tuberifermm.

46. D. srumenatum.
4\%. D. cuphlebium.

48. D. callibotrys.

49. D. moiteshum. .i1. D. serundum. 
Flowers mediocre to large, racemes short, few flowers.

Lip narrow spathulate entire, flowers pink. .
Lip narrow spathulate toothed, flowers pink $\cdots$ ip Howers green ... ... .

Lip short apex pandurate entire, striped red promer...

Lip large obovate, flowers orange

Lip obovate truncate, buff and

white ...

Lip apex entire .. . . .

Lip aper bilobed .. . . . .

Lip blade quadrate erenulate ..

Lip blade abruptly pandurate bilobed . . . . . .

Lip 3-lobed flowers small .. . .

Lip 3-lobed broad, no callus.

Lip 3-lobed, leaves broad ..

Lip 3-lobed, leaves narrow

Lip 3-lobed, callus spur-like.

Midlobe entire .. ..

Midlobe retuse $\ldots \quad \ldots \quad \ldots$

52. D. cornutum.

53. D. Aegle.

j4. D. virescens.

5.). D. serpens.

56. D. crocatum.

5\%. D. Derryi.

.5. D. hymenopterum.

59. D. Calicopis.

60. D. roseatum.

61. D. Foxii.

62. D. tropacoliflorum.

. 63. D. megaceras.

. 64. D. tenuicauli.

. 65. D. kentrochilum.

- 66. D. sanguinolentum.

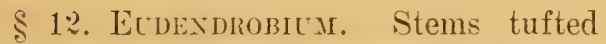
long stout or slender, clavate or nodose. Leaves various. Flowers in short racemes on the old leafless stems. Lip short broad often convolute. Mentum very short.

'This group nearly includes all species not in any other section. It is very poorly represented in this region.

Stems terete. Flowers small.

Lip narrow three lobed .. .. 6\%. D. flavidulum.

Lip narrow lanceolate entire .. 68. D. viridicatum.

Lip boat-shaped . . . . . 69. D. hercoglossum.

Stems terete. Flowers large.

Lip ovate lanceolate, flowers yellow $\hat{0}$. D. aureum.

Stem terete, flowers large.

Flowers purple .. .. .. i1. D. superbum.

Flowers yellow, purple centre .. i2. D. Dalhousieunum.

Stem fusiform, leaf solitary. .. _ . i3. D. aggregatum. 


\section{§ 1. SARCOPODIUM.}

1. D. Loxgicolli, Lindl., Bot. Reg. 1810, Misc. 12\%. Rhizome

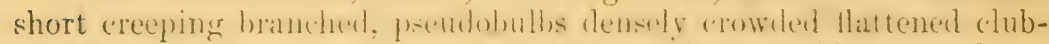
shaped 4 to 8 inches long, $\frac{1}{8}$ inch through at the base, with a few large brown sheaths at the base. I.eaf solitary subterminal lanceolate unequally bilobed 5 inches long one inch wide dark green. Flowers solitary with a long slender pedicel 3 inches long, on a very short rachis. Bracts brown lanceolate acute an inch long. Sepals lanceolate caudate $1 \frac{1}{2}$ inch long $\frac{1}{t}$ inch wide, yellow with dark red tips. Petals similar smaller. Mentum \& inch long obtuse. Lip three lobed

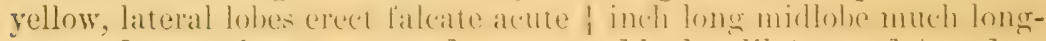
er pandurate, base narrowed, apex suddenly dilate cordate edges crisped. lobulate nerves elevate reticulate, keels 2 straight at the base flexuous on the midlobe. Column thick yellow. Rehb. fil. Walp. Ann. VI. 302. Hook. fil. Fl. Ind. V. 712.

Singapore: (Cuming) Sungei Norai, on tree stumps and the ground in large masses rare (Ridley). Distrib. Borneo.

2. D. LowaIPEs, Hook, fil. F1. Brit. Ind. V. 713. Ic. Pl. 201\%.

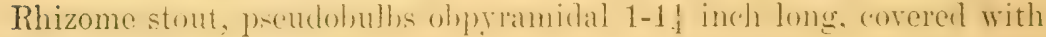
brown sheaths. Leaves 2 elliptic oblong lanceolate, $1 \frac{1}{2}-3$ inches long sessile or shortly petioled stilly coriaceons. Raceme fi to 8 inches long base quite nude, 3-5 flowered. Bracts lanceolate small. Flowers $1 \frac{1}{4}$ to 13 inch across, with red veins, pedicel stout, with ovary $1 \frac{1}{2}$ inch long. Sepals linear or oblong lanceolate subacute 1 inch long. Mentum conic short blunt. Laterals falcate. Lip as long as sepals orate oblong three lobed, side lobes rounded subacute or blunt, midlobe ovate or lanceolate mealy with a few tuhercles in two rows on the disc. Column rather long, arims very obscure. Anther quadrate umbonate.

Perak: (Scortechini $41 \hat{b}$ ) Ulu Batang Parlang (Wray 1616), Gunong Batu Puteh (Wray S89); Selangor, at the Ciap (Curtis). Endemie.

3. D. anchopodur, Hook. fil. Fl. Brit. Ind. T 713. Ic. Pl. 2020. Stem stout covered with sheaths, pseudobullos conic deeply sulcate 2 inches long. Leaves 2 short elliptic obtuse thickly coriaceous $2-3$ inches long. Racemes 6 to 8 inches tall about 6 -flowered. Bracts ovate lanceolate. Orary with the rery short pedicel 2 inches long. Flower's $1 \frac{1}{2}$ inch wide. Sepals lanceolate acuminate, mentum very short. Petals linear acute. Lip three lobed, sicle lobes rounded obtuse midlobe orbicular, keels 3 on the dise and two ear-shaped calli at the base. Column rather long, foot short, arms very obscure. Anther conic. Capsule fusiform.

Perak: (Scortechini) Larut IIills 5,000 feet alt. (Ridley); Pahang: Kluang Terbang (Barnes). 
4. D. Geminatum, Hook. fil. Fl. Brit. Ind. V. 713. Rhizome very long ascending dark purple, over two feet long, pseudobulbs distant, old ones conic 5 angled, 1-1 $\frac{1}{2}$ inch long, $\frac{1}{2}$ inch through deep red or reddish yellow shining. Leaves 2 stiftly coriaceous lanceolate obtuse or elliptic oblong $1 \frac{1}{2}-3$ inches long, $\frac{1}{4}-\frac{3}{4}$ inch wide. Flowers in terminal panicles of few brancher, little longer than the leaves, 3 to 4 inches long, red. Bracts lanceolate acute $\frac{1}{4}$ inch long. Pedicel and ovary $\frac{3}{4}$ inch long pink. Sepals lanceolate acute reddish yellow $\frac{3}{4}$ inch long, narrow. Petals much narrower pale yellow. Lip $\frac{1}{2}$ inch long side lobes broad rounded embracing the column, chrome yellow, midlobe narrow acuminate with an orange red blotch at the base, 2 thick blunt short ridges with a groove between them at the base continued into three elevated veins. Column short thick white edges winged. Anther stipitate, conic. Pollinia \pm oblong pyriform.

Perak: (King's Collector 8011) Larut Hills at 5,000) feet alt. (Curtis 2067) (Derry); Kedah, Gunong Jerai (Ridley). Distrib. Java.

\section{§ 2. Böвidium.}

5. D. Quadrangulare, Par. Hook. fil. Fl. Brit. Ind. V. 714. Pseudobulbs tufted cylindric or dilated upwards 2 inches long yellow. Leaves at the top two, lanceolate obtuse or elliptic apics bilobed $1 \frac{1}{4}$ inch long, $\frac{1}{3}$ inch wide. Flowers one or two small from a tuft of bracts terminal, pedicels $\frac{1}{2}$ inch long. Upper sepal ovate lanceolate acute, laterals ovate, mentum obtuse curved as long. as the ovary. Petals narrower linear. All pale straw colour, or veined. with red. Lip oblong entire yellow with red edges or red veined, apex dilated denticulate retuse with rounded orange process in the centre. Column broad, narrowed at the base, arms short erect. Anther conic. Griffith Notulae, III. 315, Lindl. Journ. Linn. Soc. III. 6.

Singapore: Kranji, Seletar (Ridley); Johor: Kuala Sembrong (Kelsall), Batu Pahat (Riclley); Pahang: Pekan (Ridley); Selangor: Kajang, Bukit Kupariang (Cantler's Coll.): Perak: (S'ortechini); Taipeng (Curtis); Penang: Pulau Tikus (Curtis); Trenganu: (Rostado). Distrib.. Burma, Borneo and adjacent islands. On orchard trees. "Sakat Kalumbai" used in cases of dropsy.

\section{§. Desmotrichum.}

6. D. Lonchophyllun, Hook. fil. Fl. Brit. Ind. V. 714. Ic. Pl. t. 2018. Stems numerous much branched 6 inches to a foot tall, pseudobulbs fusiform cylindric, 2 inches long. Leaf lanceolate coriaceous $2-3$ inches long $\frac{3}{4}-\frac{1}{2}$ wide. Flowers one or two from an excavation in the pseudobull, $\frac{1}{2}$ inch long. Upper sepal ovate lanceolate blunt yellowish with 5 pink stripes, laterals triangular striped pink. Mentum 
rather long. Lip spathulate apex biloberl lobes blunt, base and lisc deep cimson apex yellow with three raised keels on the disc. Column short, arms blunt shorter than the quadrate anther.

Singapore: Sungei Buluh, Kranji (Ridley) : Johor: 'Tana liunto (Ridley 23.9) : Pahang: 'Jahan River (Ridley): Perak: (S'ortechini); Kedah: (funong Jerai (Ridley). Distrib. Rhion.

7. D. Kelsaldu, Ridl., Journ. Linn. Soc. XXXII: 23\%. Stems numerous slender branched 15 inches lomg, psedudobulbs fusiform not flattened 1 inch long, is inch through. Leaves lanceshate sulobtuse $1 \frac{1}{2}$ inch long $\frac{1}{4}$ inch wide. Flowers half an inch across. Upper sepal ovate oltuse, laterals much larger ovate obtuse, mentum large blunt dilate in the middle. Petals lorate smaller pale green. Lip side lobes broad obtuse erect, greenish, disc crimson purple with three equal heels, midlobe horse-shoe-shaped bilober, orange. C'olumn greenish.

Pahang: Pulau Aor (Feilding) Kluang 'lerbang (Barnes 10935 ) ; Malacea: Mt. Ophir (R. Derry 628) (Ridley 21t5) ; Perak: Larut Hills (Ridley), Kedah Peak (Ridley). Endemic.

8. D. Crinifertur, Lindl., Bot. Reg. Mise: 41. A large tufted plant with numerous stems 2 feet tall, pseudolulbs narrow linear oblong 2 inches long $\frac{1}{4}$ inch through flattened. Leaves elliptic, or orate elliptic blunt 3 inches long and as wide. Raceme $\frac{1}{4}$ inch long corered with brown bracts. Flowers in pairs, pedicel and ovary $\frac{3}{4}$ inch long. Sepals $\frac{3}{8}$ inch long spreading lanceolate acute pale rellow. IIentum. very short and broad, spotted with purple. Petals shorter linear yellow. Lip as long as the sepals, side lobes falcate acute pale rellow, with erimson lines, disc vellow or spotted purple, keels '2 crimson running straight to the midiohe and there sinuous, mirllobe linear margins waved, apex covered with yellow filaments. ('olumn short thick white spotted purple. Richh. fil. Walp. Amn. VI. p. 303. Ridl. Journ. Limn. Soc. XXXII. 238. D. comatum Lindl. Gen. and Sp. Orch. Pl. p. 76. D. zollingerianum 'I'eysm. and Binn. 'I'ijdschr. Nederl. Ind. XXIV.p. 313.

Singapore: Kranji (Ridley 39:29), Changi (Ridley 2031) ; Johor: Batu Pahat. Distrib. Rhio and Java.

9. D. FLdBellum, Rehb. fil. Xenia Orch. II. p. 75. t. 118. Large tufterl plant, stems- thick smooth, pieudobulhs ovate flattened olive or purplish 4 inches long, one inch wide, $\frac{1}{2}$ inch thick. Leaf ovate or ovate lanceolate to lanceolate obtuse dark green 5 - to 6 inches long 2 inches wide. Flowers an inch across, pedicel $\frac{1}{2}$ inch long. Bracts 2 ovate lanceolate soon broken up into fibres. Sepals lanceolate acute recurved, mentum as long conic subacute. Petals linear much narrower. All cream coloured with pink spots. Lip base linear, side lobes narrow acute straw color with rosy tips, midlobe pandurate oblong, sides plicate, pale yellow, lieels three, two thick wary on the 
midlobe, median one shorter straight. Column channelled in front. D. Kunstleri Hook. fil. Fl. Brit. Ind. F. p. 71t. Ridley Journ. Linn. Soc. XXXII. 239.

Singapore: Kranji, Changi (Ridley 1.89); Johor: Batu Pahat (Lake and Kelsall); Perak: (Scortechini) Larut (King's Collector' 689\%). Distrib. Siam, Borneo.

10. D. Roseo-punctatuir, Ridley Journ. Linn. Soc. XXXII. 240. Stems 6 to 8 inches long, pseudobulbs fusiform slightly flattened olivaceous, 2 inches long $\frac{1}{2}$ inch wide $\frac{1}{4}$ inch thick. Leat lanceolate lorate obtuse dark green 6 inches long $\frac{3}{4}$ inch wide. Flowers as large as those of $D$. flabcllum, pedicel $\frac{1}{4}$ inch long, a little longer than the bracts. Sepals lanceolate acute $\frac{1}{2}$ inch long $\frac{1}{8}$ inch wide. Mentum short broad obtuse. Petals linear all white. Lip $\frac{3}{4}$ inch long side lobes curved acute white spotted inside with crimson, midlobe fanshaped obcordate emarginate cream colour, keels 2 running on to the midlobe and there plicate sinuate crimson. Column narrowed upwards red spotted arms short. Malay Peninsula (C. H. B. Singapore). Endemic.

11. D. Pallidifloruar, Ridl., l.c.c. p. 240. Stems slender branched, pseudobulbs elongate ovoid, flattened thin $1 \frac{1}{2}$ inch long, $\frac{1}{2}$ inch wide, $x^{3}$ inch thick. Leaves ovate acute thinly coriaceous 3 inches long 1 inch wide. Raceme half an inch long with numerous bracts, pedicels $\frac{1}{4}$ inch long. Flowers small solitary. Upper sepal ovate lanceolate $\frac{1}{4}$ inch long laterals much larger ovate, mentum a little longer obtuse. Petals as long lanceolate. All pale yellow with white. Lip lateral lobes triangular acute, midlobe triangular bilobed yellow edges waved, keels 2 large, wavy on the midlobe, median one shorter straight. Column straight arms ovate. Anther emerald green.

Singapore: Kranji, Pulau Tekong (Ridley); Johor: Hulu Kubong (Kelsall) ; Batu Pahat (Ridley). Distrib. Siam, Sumatra.

12. D.bifidur, Ridl., Jomrn. Roy. As. Soc. St. Br. Vol. 39, p. 73. Plant with the habit of $D$. Habellum Rchbf. Stems a foot or more long slender, pseudobullos 2 inches apart oblanceolate flattened $1 \frac{1}{2}$ inch long. Leaf broadly lanceolate ovate obtuse 5 inches long, 2 inches wide. Bracts lanceolate acute red. Flowers $1 \mathrm{or}^{\circ} 2$ opening at a time, ovary and pedicel $\frac{1}{2}$ inch long. Sepals and petals linear oblong acute recurved yellow spotted with red. Petals smaller. Mentum rather shorter than the sepals acute. Lip longer, claw narrow linear edges and heels crenate, apex with two narrow cuneate undulate truncate lobes half as long as the claw white yellowish at the tip. Column stout conic as long as the foot, anther oblong truncate.

Lankawi Islands (Curtis). Endemic. 
13. D. Lacriosur, Ridl., Journ. Limn. Soe. XXXII. 242. Stems about a foot or more tall branched, pseudobulbs ovoid elliptic flat greenish yellow $1 \frac{3}{4}$ to 2 inches long $\frac{3}{4}$ inch wide and hardly $\frac{1}{8}$ inch thick. Leaf lanceolate obtuse 4 inches long 13 inch wide. Raceme. over an inch long corered with orate lanceolate bracts. Flowers solitary pale yellow. Sepals subequal widely lanceolate obtuse tips thickened $\frac{1}{t}$ inch long. Petals narrower acute. Lip lateral lobes falcate, midlobe with a linear base apex oblong truncate much broader laciniate keels 2 straight to the base of the midlobe then sinuate on the claw. Column white, arms tooth-shaped. Anther sub-quadrate apex rounded margin truncate fimbriate. Capsule elliptic $\frac{3}{4}$ inch long. Endemic.

\section{Singapore: Pulau Seletar (Ridley).}

\section{APORUAI.}

14. D. Serra, Lindl., Journ. Linn. Soc. III. 3. Stems tufted, flattened 6 to $2 t$ inches long sometimes branched, base covered with leares, upper parts with sheaths only. Leaves ovate acute laterally flattened $\frac{3}{4}$ inch long $\frac{1}{4}$ inch wide deep green, sometimes red. Racemes usually from the leafless portion, more rarely from the leafy part, short compact, covered with copious brown acute bracts. Flowers very small $\frac{1}{8}$ inch long, pure white. Pedicel and ovary $\frac{1}{8}$ inch long. Sepals lanceolate orate recurved. Petals linear, smaller. Mentum long, narrow obtuse $\frac{3}{4}$ the length of the ovary. Lip straight, linear oblong, apex of two linear blunt parallel lobes, a small forked callus at the base of the terminal lobes. Column rather long curved, arms erect tooth like. Anther orate green. Capsule $\frac{1}{4}$ inch long inequilaterally pear-shaped. Hook. fil. Fl. Brit. Ind. V. 722. D. micranthum Lindl. Journ. Linn. Soc. III. 3. Rchb. fil. Walp.'Ann. VI. 2\%0. D. Lobbii Lindl. l.c.c. D. aloaefolium Rehb. fil. l.c.c. A porum Serra Lindl. Gen. and Sp. Orch. Pl. 61. A. micranthum Griti. Calc. Journ. Nat. Hist. IV. 3:6, V. 369. Macrostomium aloacfolium Bl. Bijdr. 335, fig. 3\%. Oxystophyllum macrostoma Hassk. 'Tidjschr. Nat. Gesch. X.i 22. Common over the whole Peninsula.

Singapore: (Wallich 2021) Changi (Ridley 185); Johor: Batu Pahat (Ridley 1125\%); Pahang: Pekan (Ridley 1211). Distrib. Borneo, Jara.

15. D. Rmodostere, Ridl., 'T'rans. Linn. Soc. II. Vol. III. (1893) p. 360. Stems 12 to 15 inches long, as in D. serra. Leaves lanceolate or ovate lanceolate acute $\frac{3}{4}$ to 1 inch long $\frac{1}{4}$ inch wide. Flowers very small whitr, wilh mumerous dry lorown hrats. orary and pedicel $\frac{1}{x}$ inch long. Sepals ovate obtuse. Mentum short broad blunt. Petals linear acute. Lip linear oblong, midlobe bilobed rounded broader than the rest of the lip. Column rather large larger than in $D$. serra 
pink. Anther broad flattened. Pollinia narrow linear. Stigma oblong. Capsule pear-shaped $\frac{1}{\ddagger}$ inch long, on an eularged pedicel. Endemic.

\section{Pahang: Tahan River Valley (Ridley). Endemic.}

16. D. Roselluir, Ridl., Journ. Linn. Soc. XXXI. p. 168. Tufter plant with the habit of the prececting. Stems erect branched over a foot long. Leaves orate acute rather distant $\frac{1}{2}$ to $\frac{3}{4}$ inch long, $\frac{3}{4}$ inch broad dark green. Racemes from the bare tips of the stems with numerous brown bracts. Flowers pink 3 inch long. Upper sepals ovate, laterals wider. Mentum long straight apex thickened. Petals smaller ovate lanceolate pink. Lip long narrow, with very short side lobes, midlobe obovate, with an oblong grooved yellow callus on the base apex bilobed lobes broad rounded. Column rather long, arms obscure, anther truncate.

Johor: Gunong Panti (Ridley). Distrib. Borneo.

1\%. D. eulophotum, Lindl., Journ. Linn. Soc. III. 5. Stems several erect 4 to 16 inches tall, leafy to the apex. Leaves lanceolate acute coriaceous not imbricating $\frac{1}{2}$ to one inch long, $\frac{1}{8}$ inch wide, sheaths $\frac{1}{2}$ inch long. Racemes numerous axillary from the mouths of the sheaths $\frac{1}{8}$ inch long. Bracts numerous. Flowers $\frac{1}{8}$ inch across. Upper sepal lanceolate oblong laterals larger triangular ovate. Mentum very short and broad. Petals narrower lanceolate, all yellow with red streaks. Lip cordate 3-lobed yellowish, side lobes short blunt spreading midlobe broad rounded, calli two conic ones or one truncate retuse one in the middle of the base of the lip. Column short thick. Anther small. Hook. fil. Fl. Brit. Ind.' V. ${ }^{2} 23$.

Singapore: Bajau (Ridley 2380), Kranji (Ridley 366) ; Johor: Batu Pahat (Ridley); Pahang: Pekan (Ridley); Perak: (Scortechini); Lankawi, Terutau, (Curtis 1697) ; Trwal (Ridley 8337). Distrib. Tenasserim, Borneo, Java.

18. D. RHIzophoreti, Ridl, Journ. Linn. Soc. XXXII. żâ. Stems one to two feet long oiten branched. Leaves not imbricating lanceolate acuminate $\frac{3}{4}$ to one inch long, 1 inch wide, pale green (dry* ing black). Racemes axillary with few bracts $\frac{1}{t}$ inch long. Flowerg $\frac{3}{8}$ inch long reversed. Upper sepal oblong subacute, laterals mucin wider ovate falcate. Mentum shorter olslong obtuse. Petals smaller linear. All yellowish with a medium red line. Lip claw linear rosy, laterals lobes curved subacute, median indistinct rounded shorter, edge wavy yellowish, calli 2 bases thickened connate pink apices subulate curved. Column foot long linear.

Singapore: Kranji (Ridley) ; Johor: Batu Pahat, Hulu Sembrong (Kelsall 4038). Endemic, 
19. D. GRaNDE, Hook. fil. Fl. Brit. Ind. T. p. 72t, Ic. Pl. 2024. Stems 9 inches to a foot long, broad flattened. Leaves lanceolate acute light green 2 inches long nearly $\frac{1}{2}$ inch wide, corering the stems to the top, reduced to sheaths only at the base. Racemes in the axils of the upper leaves, $\frac{1}{4}$ inch long covered with brown lanceolate acute bracts. Flowers $\frac{1}{3}-\frac{1}{2}$ inch long yellow tinted red, on pedicels $\frac{1}{4}$ inch long. Upper sepal orate laterals broader triangular. Mentum as long obtuse. Petals lanceolate acute. Lip cuneate flabellate bilobed, lobes truncate, lateral lobe short, a toothed lamella on the disc. Column short, arms erect toothed like. Anther conic with a tuft of hairs at the apex.

Singapore: Seletar (Ridley); Johor : Kwala Sembrong (Kelsall); Pahang: 'I'ahan River (Ridley); Selangor: Kuala Lumpor Rawang (Ridley) ; Perak: (Scortechini); Penang: (Ic. in herb. Kew). Not rare on very lofty trees, largest species in the section. Endemic.

20. D. Atropurpureua, Mig. Fl. Ind. Bot. III. 64t. Small tufted plant, 2 to 9 inches tall. Leaves lanceolate or linear lanceolate acute imbricating 1 inch long, nearly $\frac{1}{+}$ inch wide. Racemes short axillary, $\frac{1}{4}$ inch long with numerous dry bracts. Flowers $\frac{1}{1}$ inch long, yellow, dotted with pink, or deep red. Upper sepal lanceolate acute, laterals triangular larger with a short broad blunt mentum. Petals smaller linear. Lip entire oblong-spathulate apex rounderl. pubesent a horse-shoe-shaped callus on the apex and a conic tubercle beneath the tip. Column straight. Hook. fil. Fl. Brit. Ind. V. 724 . D. concinnum Miq. loc. Oxystophyllum atropurpureum, Blume Rumphia IV. 41, t. 193, f. 4 and 198 c. O. camosum, Bl. Bijdr. 336, fig. 38, Lindl. Gen. and Sp. Orch. pl. 72. A porum concimum, Lindl. Gen. and Sp. Oreh. Pl. Ie.

Singapore: (Wallich 2019), Kranji (Riclley 368) : Wohor: Kiwala Kahang (Kelsall) ; Pahang: Rumpin Tiver, P(kan (Ridley) : Malacea: Sungei Rambei; Selangor: Tíuala Lumpor (Ridley); Perak: (Scortechini); Penang: Goverument Hill. Distrib. 'T'enasserim, Siam, Malay islands. Common on trees, mangrove swamps, ete.

21. D. sinuatuar, Jindl., Walp. Ann. VI. 280. Stems long pendulous 8 inches or more long. Leares lanceolate acuminate pungent imbricating fleshy coriaceous 1 inch long $\frac{1}{t}$ inch wide. liacemes from the upper leaf-axils $\frac{3}{8}$ inch long with small and fewer bracts than D. atropurpureum. Flowers larger orange, solitary. Upper sepal lanceolate laterals larger triangular. Mentum short thick. Petals much smaller lanceolate blunt. Lip fleshy cuneate with very short tooth like lateral lobes, apex rounded entire thickened with a horseshoe shaped callus above, dark red, and a conicle tubercle beneath. Column curved, arms erect very inconspicuous. Anther conic. Hook. fil. Fl. Brit. Ind. V. 72t. A porum sinuatum Lindl. Bot. Reg. 1841, Misc. 1, Griflith Cale. Journ. Nat. Hist. V. 368. 
Singapore: Kranji, Seletar (Ridley); Johor: Batu Pahat; Malacea: (Griffith): Perak: Taipeng (Curtis); Penang: Maingay). Distrib. Borneo.

22. D. atrorubens, Ridl., Journ. Linn. Soc. XXXII. $14 \%$. Stems rather stout pendulous or erect, sometimes branched 12 to 24 inches long with numerous. long blackish roots. Leaves imbricating lanceolate acute one inch long $\frac{3}{4}$ inch wide. Racemes subterminal and lateral with very numerous lanceolate acute bracts, forming a tuft $\frac{1}{2}$ to $\frac{3}{4}$ inch across nodding. Flowers $\frac{1}{4}$ inch long deep red. Upper sepal lanceolate acute, laterals larger triangular. Mentum wide obtuse. Petals shorter linear. Lip linear cuneate lateral lobes short toothlike acute, at the case of the thickened rounded terminal lobe, a conical tubercle beneath.

Perak: Larut Hills at 3,000 feet elevation (Ridley); Pahang: Kluang Terbang (Barnes 11230); Kedah: Gunong Jerai (Ridley 5140 ). On stumps and low trees at about 2,000 to 5,000 feet elevation. Endemic.

23. D. Leonis, Rchb. fil. Walp. Ann. VI. 280, Stems crowded in a tuft 4 to 8 inches tall. Leaves thick cultrate or ovate subacute $\frac{1}{2}$ inch long and but little narrower. Racemes terminal short broad with many bracts. Flowers appearing singly fleshy yellowish spotted with pink, scented of vanilla. Upper sepal shortly ovate obtuse $\frac{1}{4}$ inch long, lateals larger widely ovate. Mentum large short and broad. Petals lorate spathulate smaller. Lip oblong nearly entire shorter than the sepals apex bilobed lobes rounded ciliate, an orange yellow central bar ending in small callus. Column short yellow with an orange spot at the base. Anther helmet-shaped. Hook. fil. Fl. Brit. Ind. V. 723. Ann. Roy. Bot. Calc. V. pl. 10. A porum indivisum Lindl. (non Bl.) Gen. and. Sp. Orch. pl 70. A. Leonis Lindl. Bot. Reg. 1840, Misc. 59, Griff. Calc. Journ. Nat. Hist. V. 368.

Singapore: Changi, etc. (Ridely); Johor: Batu Pahat (Kelsall); Pahang: Kwala Pahang, Kota Glanggi (Ridley); Malacea: Jasin (Derry); Sungei Bahru Ulu (Ridley). Distrib. Cochin China, Borneo.

- 24. D. prostratuli, Ridl., Journ. Linn. Soc. XXXII. 248. Stems creeping branched, rooting. Leaves $\frac{1}{4}$ inch long nearly as wide oblong triangular sulsacute or obtuse fleshy, deep green or red. Flowers resembling the preceding but smaller pale yellow, sometimes red punctate. Tpper sepal ovate obtuse, laterals nuch larger ovate subfalcate. Mentum short broad curved. Petals much smaller spathulate. Lip oblong apex dilate retuse, keel on the disc orange, with a small quadrate erect callus.

Singapore: Kranji (Ridley); Selangor: Sepang (Ridley) : Negri Sembilan: Perhentian Tinggi (Ridley). Endemic, 
25. D. Teranyale, Par. and Rohb. fil. Trans. Linn. Soc. XXX. $18 \%$, p. 149. Stems fire to six inches long, flattened numerous. Leares lanceolate acute $\frac{3}{4}$ inch long $\frac{1}{2}$ inch wide. Racemes rery short distinct not tufted, borne on old leafless stems. Flowers in pairs rather thin-textured, pedicels $\frac{1}{4}$ inch long slender. Bracts fow. Upper sepal orate oblong laterals much larger ovate triangular falcate $\frac{3}{4}$ inch long. Mentum thick curved obtuse. Petals much smaller linear lorate. Lip oblong cuncate, lateral lobes deflexed truncate midlobe not much longer liboherl. lobes remmled crisped with an orange spot in the centre, the rest of the flowers primrose yellow. Column narrow an orange spot at the base, no arms. Hook. fil. Ann. Bot. Roy. Gard. Calc. D. Mannii Ridley, Journ. Linn. Soc. XXX. 246.

Malacea: Mt. Ophir District (Derry). Distrib. Assam, Burmah.

\section{§5. STRONGILE.}

26. D. teres, Lidl., Bot. Reg. 1840, Misc. p. 51. Stems sereral slender stiff 10 to 15 inches long. Leaves distant terete obtuse dark green, 2 to 4 inches long $\frac{1}{8}$ inch through, sheaths $1 \frac{1}{4}$ inch long terete. Flowers 3 ind long opening singly from tults of hatets on the ends of the bare portion of the stem. Pedicels and ovary $\frac{1}{2}$ inch long. Sepals lanceolate acute subequal $\frac{1}{2}$ inch long white. Nentum more than $\frac{1}{4}$ inch long straight conic obtuse. Petals a little narrower. Lip shorter 3 -lobed lateral lobes oblong orate subacute, white with orange veins, midlobe narrower lanceolate acuminate, keels on the dise three, mid one shortest, flame-coloured. Colmmn nearly straight chamnelled in front. Anther conic. Hook. fil. Fl. Brit. Ind. V. p. 726 . and Ann. Roy. Bot. Gard. Calc. Vol. V. pl. 11.

Singapore: 'T'oas, (Ridley 1702) ; Selitar (Ridley); Johor: Kota Tinggi (Ridley); Perak: Larut Hills (Curtis); Penang (Curtis). Endemic.

2\%. D. FLEXILe, Ridl., Journ. Linn. Soc. XXXII. 251. Stems numerous 3 inches long slender flexuous pendulous. Leares $\frac{3}{4}$ inch long terete acuminate acute opressed to the stem. Flowers subter-

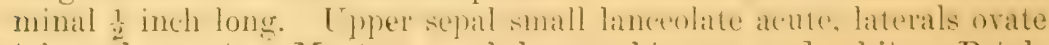
triangular acute. Mentum much larger obtuse curved, white. Petals small linear white. Lip white with a central orange spot, claw linear,

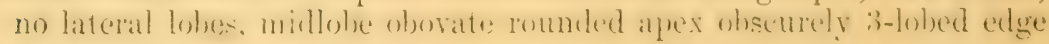
denticulate.

Singapore: Bukit 'T'imah (Ridley); Johor (Ridley); Pulau Aor (Fielding). Endemic.

28. D.кentrophyldua, Hook. fil. Fl. Brit. Ind. V. 725. Ic. Pl. 2021. Stems tufted stout $t$ to 8 inches long. Leaves subterete straight, thick acute $\frac{1}{2}$ to 1 inch long, $7_{6}^{3}$ inch through, numerous, sheatlis $\frac{1}{4}$ inch long. Flower lateral on the ferminal leafless portion 立 inch long. Upper sepal ovate oblong, laterals obtuse. IIentum 
more than twice as long as the dorsal sepal stout incurved, narrowed at the apex. Lip spathulate bilobed with long claw, no side lobes. Capsule pyriform on a slendei pedicel $\frac{1}{8}$ inch long.

Perak (scortechini) : Pahang: Tahan River (Ridley). Endemic.

29. D. acerosum, Lindl., Bot. Reg. 1841, Misc. 86. Stems numerous slender flexuous, 12 to 15 inches long. Leaves recurved laterally flattened linear, pungent 1 to $1 \frac{1}{2}$ inch long $\frac{1}{8}$ inch wide, sheaths $\frac{1}{2}$ inch long, mouth with a black ring. Flowers $\frac{1}{4}$ inch long solitary from the internodes of the leafless portion of the stem. Bracts ovate lanceolate small. Dorsal sepal lanceolate very small laterals triangular blunt spreading. Mentum stout obtuse twice as long as the dorsal sepal. Petals narrower linear. All cream colour with or without pink veins. Lip spreading obcuneate obovate, apex crisped bilobed white with a thickened central bar with a yellow tip. Column very short, arms rounded obscure. Hook. fil. Fl. Brit. Ind. V. 726. A porum subteres Griff. Calc. Journ. Nat. Hist. V. $3 \% 1$.

Singapore: Kranji, Changi (Ridley); Johor: Kwala Kahang (Kelsall 4036), Tana Runto (Ridley); Pahang: Pekan (Ridley 1561) ; Malacea (Griffith); Penang. Distrib. Tenasserim, Siam, Sumatra, Borneo.

30. D.subulatur, Hook. fil. Fl. Bit. Ind. V. 726. Stems tufted numerous 2 to 6 inches long slender. Leaves narrow. pungent slightly flattened recurved $\frac{1}{2}$ inch long, sheaths narrowly $\frac{1}{4}$ inch long. Flowers solitary from the axils of the sheaths of the leafless portion nearly $\frac{1}{2}$ an inch long, pedicels slender $\frac{1}{4}$ inch long. Upper sepal small lanceolate blunt laterals triangular acute, mentum twice as long curved obtuse, all white with pink veins. Petals linear. Lip oblong truncate apex broad shortly 3 lobed, white with a yellow spot at the tip. Column short. Podochilus bicolor Miq. Choix. t. 22, f. 3.

Singapore: Bukit Timah, Kranji, ete., Ridley) ; Johor; Selangor : Batu Caves, In Western Road (Curtis 2408). Distrib. Rhio, Borneo, Java.

\section{§6. VirgataE.}

31. D. conostalix, Rehb. fil. Walp. Ann. VI. p. 292. Terrestria] one to 2 feet tall, stems few wiry terete. Leaves narrow linear apex unequally bilobed, stiff, three inches long $\frac{1}{4}$ inch wide, sheaths $1 \frac{1}{2}$ inch long hispidulous. Flowers one or two together from the axils of the leaves, pendulous $\frac{1}{2}$ inch long. Bracts small ovate. Dorsal sepal ovate green with three olive streaks, laterals rather larger more acute. Mentum rery long straight blunt. Lip base narrow yellowish white laterals lobes short acuté tooth-like, midlobe rounded thick retuse. Column short thick dilate at base, arms erect tooth-like. Capsule one inch long elliptic pendulous on a pedicel $\frac{1}{\frac{1}{4}}$ inch long. Hook. fil. Fl. 
Brit. Ind. T. 726. D. calcaratum Lindl. Bot. Reg. 1840, Mlisc. 89. D. Lobbii Teysm. and Binn. Tat. 'Tijdschr. Tedl. Ind. III. 399, Walp. Ann. VI. 292. Hook. fil. l.c.e.

Terrestrial, always in shallow water, "Rumput Rajah Sari."

Singapore: Changi 23ิ\% (Riclley) (Lobb. ts2); Malacca: Bukit Bruang (Ridley). Distrib. Labuan.

32. D.vrliosulua, Wall. Cat. 2006. Stems graceful terete sometimes branched, 2 to 3 feet tall. Leaves linear lanceolate apex unequally bilobed two inches long nearly $\frac{1}{+}$ inch wide, sheaths terete an inch long covered with black pubescence. Flowers solitary on short perlicels $: 3$ inch long from the mouth of the sheaths, pendulous about $\frac{1}{2}$ inch long. Sepals lanceolate acute 3 inch long yellowish with pale red reticulation. Mentum 2 inch long narrow obtuse straight. Petals narrowly linear recurved, coloured like the sepals. Lip shorter 3lobed, side lobes narrow erect obtuse, nidbohe nearly orbicular edges fleshy primrose coloured. Column short and thick base thickened, ams short obsenre. Anther apex retuse margin emarginate. Hook. fil. Fl. Brit, Ind. V. 728.

Terrestrial on dead leaves from sea level to $t, 000$ feet alt.

Singapore: Bajau (Ridley 23\%8) ; Sungei Buluh (Ridley 368); Malacea: Mt. Ophir (Rirlley); Penang (Porter, Wall. (at. 2006) top of West Hill (Ridley); Kedah: Gunong Jerai (Ridley). Endemic.

33. D. PAchyglossum, Par. and Rchb. fil. 'Trans. Limn. Soc. XXX. 149. Epipliytic. Stems sereral slender, a foot long. Leaves distant linear acute $t$ inches long $\frac{1}{8}$ inch through, whaths $\frac{1}{1}$ inch long. Flowers small in pairs on short racemes, lateral. Bracts small lanceolite ? ats long as the pedicel. Sepals spreading oblong obtuse, laterals broader $\frac{1}{4}$ inch long $\frac{1}{8}$ inch wide. Mentum as long blunt, white. Petals narrowly linear acute. Sepals and petals greenish with red streaks. Lip fleshy spathulate, lateral lobes hardly distinct median longer emarginate blunt pale green, keels 2 on the dise. Column narrow arms tooth-like erect. Hook. fil. Fl. Brit. Ind. V. $82 \%$, Amn. lioy. Bot. Calc. V. 9 8, t. 12. D. abielimum Ridl. Journ. Linn. Soc. XXXII. 252.

Perak: Larut Hills at 2,000 feet (Ridley); Kedah: Gumong Jerai (Ridley); Lankawi: Gunong Jaya (Curtis 2562). Distrib. 'L'enasserim. As this is a hill plant, is it probable that the drawing referred to in the Annals of the Royal Botanical Gardens, Calcutta, from Singapore, was from a cultivated specimen.

\$\%. B.IMUUSAIFOLIAE.

3.. D. Gemeleuar, Lindl., Gen. and Sp. Oreh. pl. 81. Bot. Reg. 1840, Mise. 192, 1814, Mise. 62. Epiphytic. 2 to "3 feet tall terete

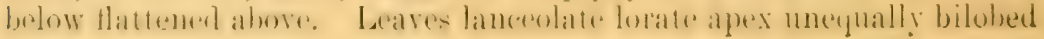


thin light green $t$ inches long $\frac{1}{3}-\frac{1}{2}$ inch wide, sheaths $\frac{3}{4}$ inch long transversely wrinkled. Raceme from the centre of one of the internodes rery short enclosed in ohlong obtuse bracts, 2-flowered. Flowers rather small vellowish unexpanded, pediec $\frac{1}{2}$ inch long ovary thicker $\frac{1}{8}$ inch long. Upper sepal lanceolate obtuse, laterals larger triangular lanceolate. Mentum as long thick obtuse curved. Petals linear lanceolate acute. Lip shorter narrow thin spathulate entire, no side lobes. Column short thick, arms erect, very stout. Capsule ovoid $\frac{1}{2}$ inch long. Hook. fil. Fl. Brit. Ind. V. F2\% Pedilonium biflorum Bl. Bijdr. 322.

Singapore: Serangoon (Ridley); Malacea: Bukit Bruang (Griffith); Perak: 'Taipeng (Ridley); Lankawi (Curtis). Distrib. Siam, Java, Sumatra, Borneo. 'There are two forms, one more slender with narrow leares, the other stouter with broader stiffer leares and rather longer flowers.

35. D. PENSIle, Ridl., Journ. Limn. Soc. XXXII. 253. Stems numerous pendulous 2 to 3 feet long entirely leafy terete. Leares orate lanceolate fleshy distichous bright green 3 inches long, 3 inch wide. Racemes very short from the middle of the internodes with a pair of sessile yellow unexpanded flowers and an ovate triangular broad dry bract. Ovary thick $\frac{1}{2}$ inch long. Sepals lanceolate twisted, laterals subtriangular blunt. Mentum short broad obtuse: Petals narrow lanceolate. Lip much shorter than the sepals, 3-lobed, sicle short erect falcate midlobe longer lanceolate acute pubescent with a red median line. Column thick subglobose narrowed upwards, arms none. Anther thick subglobose. Capsule elliptic an inch long very shortly pedicelled.

Singapore: Changi (Ridley 3930), Selitar; Johor (Ridley). Distrib. Rhio.

\section{§8. Distichophyllat.}

36. D. Bifariun, Lindl., Wall. Cat. 2002. Stems tufted 6 to 12 inches long $\frac{1}{8}$ inch through, channelled. Leaves ellipic oblong shortly unequally bilobed $\frac{1}{2}$ to 2 inches long $\frac{1}{4}-\frac{3}{8}$ inch wide. Flowers solitary axillary $\frac{1}{2}$ inch long expanded, white. Bracts 1-2 minute. Pedicel and orary slender $\frac{1}{2}$ inch long. Lpper sepal lanceolate acute, laterals little larger. Petals linear acute. Mentum short obtuse straight. Lip entire broadly obovate retuse centre papillose yellow. Column short thick with 2 tooth-like erect arms. Capsule an inch long. $\frac{1}{2}$ inch thick pyriform inequilateral. Hook. fil. Fl. Brit. Ind. V. 732. D. excisum Lindl., Bot. Reg. 1841, Mise. $7 \%$.

Singapore: Seletar, Kranji (Ridley) ; Johor: Tana Runto (Riclev): Pahang: Kluang Terbang (Barnes), Tahan River (Ridley); Selangor: Bukit Hitam (Kelsall); Penang (Wallich 2002), Government Hill (Curtis). Distrib. Borneo. 
37. D. Hoser, Ridl., I'rans. Jinn. Soc. III. p. 363. Stems over

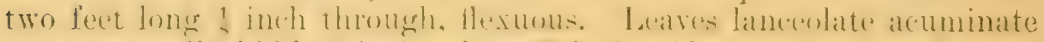
very unegually hilis, ; inches long :

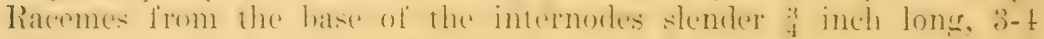
flowered. Bracts ovate acute $\frac{1}{16}$ inch long. Ovary and pedicel $\frac{3}{8}$ inch long. Sepals white oblong orate acute $\frac{1}{2}$ inch long, laterals larger. Ifentum as long as the repals straght. Potals narower linear white.

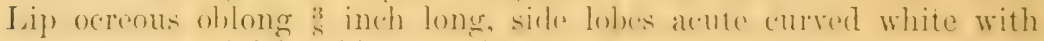
brown veins midlobe oblong reniform retuse edge sinuate ciliate with three brown parallel veins. Column broad narrowed upwards, arms erect acute.

Pahang: Tahan River (Ridley 2362). Distrib. Borneo.

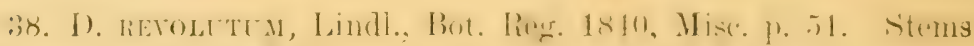

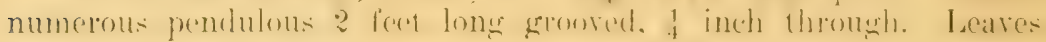
braodly lanceolate thinly coriaceous, unequally bilobed $3-3 \frac{1}{2}$ inches long 3 inch wide. sheathis $\frac{1}{2}-1$ inch long. Flowers solitary on a very short peduncle from the base of an internode. Bracts minute ovate acute. Pedicel and ovary $\frac{3}{4}$ inch long. Upper sepal ovate acuminate $\frac{3}{8}$ inch long, laterals larger broadly ovate. Nentum short blunt $\frac{3}{8}$ inch long. Petals lanceolate shorter. All white recurved. Lip porrect, $\frac{3}{8}$ inch long fleshy, lateral lobes short obtuse deflexed. Median much larger oblong orate truneate retuse, all dull orange with 3 central brown streaks. Column thick white narrowed upwards with two thick keels in front, arms tooth-like erect. Hook. fil. Bot. Mag. t. 6r06. Fl. Brit. Ind. V. 732 (in part) Paxt. Fl. Gard. I. 63, fig. 12.

Singapore: Wayang Satu (Ridley); Johor: Batu Pahat; Malacca: Ayer Panas (Hervey); Kedah: Gunong Jerai (Ridley 5141). Distrib. 'l'enasserim, Rhio.

39. D. CNElonum, Griff. Notulae III. p. 305. Ic. Pl. As. t. 203. Stems about a foot tall, grooved. Leaves oblong lanceolate unequally bilobed 1-1! inch long or less, $\frac{1}{2}$ inch wide, sheaths $\frac{1}{1}$ inch long. Flower solitary white $\frac{1}{2}$ inch across. Peduncle very short $\frac{1}{8}$ inch. Pedicel and ovary over an inch long. Bracts orate lanceolate acute. Upper sepal oblong aemminate $\frac{1}{2}$ inch long, $\frac{1}{4}$ inch wide, laterals

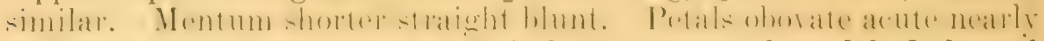
as long as the sepals. Lip 3 inch long cuneate three lobed, lateral

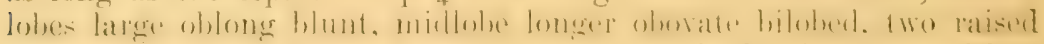
ridges violet run on the dise. Column broad, flat in firont with a raised line, arms very small green.

Malacea: Int. Ophir (Griftith, Hullett 86t, Derry 612) ; Perak: Larut Hills (Ridley); epiphyte $3-1,000$ alt. Endemic.

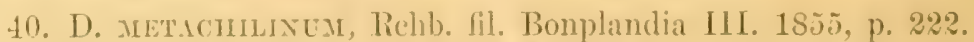
Stems tufted stout over a foot long, 1 $^{3}$ inch thick deeply groored. Leares thee inches long 3 inch wide hroally lanceglate apices very 
unequally bilobed, sheaths an inch long. Racemes very short few flowered from the upper axils. Bracts ovate acute very small. Ovary and pedicel $\frac{1}{2}$ inch long. Flowers moderately large, 3 to 4 . Upper sepal oblong lanceolate acute $\frac{1}{8}$ inch long $\frac{1}{8}$ inch wide laterals similar, mentum $\frac{1}{2}$ inch long straight slender acuminate. Petals linear spathulate $\frac{1}{16}$ inch long. All burnt sienna coloured with darker reticulations. Lip narrow with a long claw and very small tooth-like side lobes whitish, midlobe small ovate fleshy caruncled brown, 2 keels on the dise. Column thick short pale brown with tooth-like erect arms. Anther conic. Hook fil. Fl. Brit. Ind. V. p. 73.

\section{Malacea : Bukit Jelutong (Derry 161). Endemic.}

41. D. Pandaneti, Ridl., Journ. Linn. Soc. XXXII. p. 25\%. Rhizome creeping vertically on stems of palms or Pandani, branched emitting weak stems 8 to 12 inches long, $\frac{1}{8}$ inch through. Leaves lanceolate, acuminate unequally bifid thin herbacenus :3 inches long $\frac{3}{4}$ inch wide sheaths $\frac{1}{2}$ inch long. Racemes in the upper axils $\frac{1}{4}$ inch long, few flowered. Bracts minute ovate. Pedicel and ovary 1 inch long. Sepals lanceolate acute $\frac{1}{2}$ inch long $\frac{1}{4}$ inch wide. Mentum slender obtuse curved shorter. Petals linear acuite all white. Lip base narrow linear, side lobes short blunt incurved, midlobe cordate fleshy orange red, keels 3 on dise orange. Column straight olive brown arms orate incurved. Capsule on a pedicel $\frac{1}{2}$ inch long elliptic pyriform fawn colour.

Singapore: Jurong (Ridley 80r1), Bukit Nandai (Ridley 5029); Johor: Pulau Kukub; Batu Pahat (Ridley). Distrib. Siam.

\section{§9. Clivatae.}

42. D. GRaCile, Lindl. Stems tufted pendulous rery slender with one internode near the base swo!len fusitorm an inch long $\frac{1}{2}$ inch thiick. Leaves setaceous channelled very narrow 3 inches long, sheaths an inch long. Raceme lateral very short $\frac{1}{8}$ inch long eurver with dry browu bracts. Flowers small opening singly on pedicels : Sepals lanceolate nearly $\frac{1}{2}$ an inch long. laterals a little wider. Mentum as long curved obtuse. Petals linear all white. Lip 3 lobed side lobes rather large rounded, white with red veins inside, midlobe ovate clawed white. Column straight narrow, arms tooth-like overtopping the depressed anther. D. setifolium Ridley, Journ. Linn. Soc. XXXI. 270.

Singapore: Chan Chu Kang (Ridley). Distrib. Borneo, Rhio, Java. Lindley writes in Herb Kew on a specimen from Java, (Lobb). "I suppose D. gracile Lidl."

43. D. clavator, Ridl., Joum. Linn. Soc. XXX1I. 255. Stems over a foot long slender, one internode near the base $\frac{3}{4}$ inch long thickened fusiform ten ribhed. Leares terete channelled few distant 3 inches long $7^{\frac{1}{6}}$ - inch thick. Racemes short from the upper (nude) 
portion of the stem. Bracts many brown. Flowers small opening singly wollowish tinted with red. C Proer sejal laneerolate acute go inch long, Laterals widely ovate falcate. Mentum broad curved thick. Pretals 4 inch lone lanerolate shorter and broader. lip side lobes oblonge rommlerl pale with red nerves, midlobe ovate obluse minutely toothed, disc elevated yellowish red. Column short arms obscure.

Perak: 'Taiping (Ridley); Johor: Sungei 'T'brau. D. mellitum, lidll., Journ. Roy. Is. Soc. S. Br. $+13 \%$. Endemic.

4. 1). CLavipes, Hook. fil. Fl. Brit. Ind. Y. p. 728. Ic. Pl. 202\%. Stem crowded 6-12 inches long, slender, one internode at base thickened oroid globose $\frac{1}{2}$ inch long, apex of stem often nude. Leaves narrow linear $1-1 \frac{1}{4}$ inch long $\frac{1}{8}$ inch wide flat, apex bifid. Flowers solitary axillary often on the nude part of the stem, $\frac{3}{8}$ inch long white. Bracts orate acute small. Sepals lanceolate acute veined pink. Mentum as long curved tinick. Petals lanceolate narower. Lip hastate, lateral lobes rounded hardly distinct, midlobe lanceolate acute, callus on the dise oblong yellow.

Pahang: Pulan Chengei (Ridley) ; Selangor near the Batu Caves, Kuala Jampor (Ridley): Perak (Scortechini No. 21st). Distrib. Sumatra.

45. D. tuberiferuar, Hook. fil. Fl. Brit. Ind. V. p. i2S. Ic. 1'l. fig. 1. Stems several 12 to 18 inches tall red, two internodes at the hase swollen into an oral compresied pseudohulb $1 \frac{1}{2}$ inch long one inch wide $\frac{1}{4}$ inch thick, apex of stem leafles: I cares fow hlunt, elliptice ovate minutely unequally bifid, one inch long $\frac{1}{4}$ inch to 3 inch wide, sheaths one inch long. Flowers from the nude portions of the stems lateral solitary $\frac{1}{2}$ inch long. Cpper sepal lanceolate acute $\frac{1}{4}$ inch long white with a riolet-crimson median- line and tip, laterals triangular acute. Mentum a little longer curved blunt. Petals lanceolate acute white with a violet line. Lip three lobes, side lobes rounded white witl violet veins, midlobe longer lanceolate acute apex ciliate, white with a violet crimson central bar to the tip, a retuse callus yellowish on the disc between the side lobes. Column slender.

Singapore: Kranji, Bajau, ete. (Ridley); Johor: Batu Pahat (Ridley); Pahang: Irwala Pahang (Ridley); Perak: Larut Hills, Gunong Hijau (Jlurton). Distrib. Borneo.

16. D. crumbatem, Sw in Schrad. Jomm. ii. p. 23\%. Stems numrous often branched $3-4$ feet long; four internodes at the base swollen into an elongate fusiform ribbed psendobulb three inches long 3 inch through, upper part of stem leafless. Leaves broadly elliptic

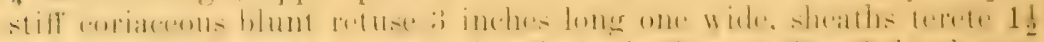

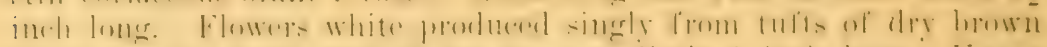

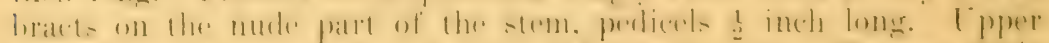

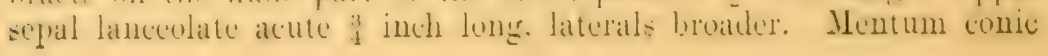


curved as long as the sepal dilated towards the apex. Petals broader, blunter and shorter than the upper sepals. Lip 3-lobed with an ablong base, side lobes elongate romded midlobe oblong-orate cuspidate, keels 3 on the disc between the side lobes rumning to the end of the tip, a pale yellow papillose patch on the midlobe. Column graceful long with a strong central bar, arms tooth-like small. Capsule an inch

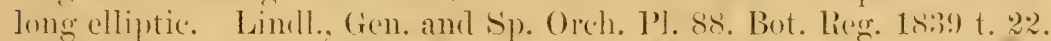
(rill. Notul. iii. 315. Bot. Mag. t. 4013. Miq. C'hoix. t. 22. Hook. fil. I1. Brit. Ind. V. ^29. Onychium crumenulum, Bl. Bijelr. 326.

Whole Peninsula very common.

Singapore: Pulau Tekong (Ridley 2033) ; Johor: Batu Pahat; Selangor, Perak, Kerlah, Penang, Malacea. 1) istril,. Burmals, Andamans, S. China, Siam. Malay Archipelago to Christmas Island. "l'igeon Orchid," native names Poko Merpati, Burga Angin.

\section{$\$ 10$. BREVIFLohes.}

4.. D. euphlebuex, Lindl., Journ. Linn. Soc. ILI. p. \%. Stems numerous from a short slender branched rhizome, 6 to 9 inches long hase slender terete thickened upwards wared: Jeares terminal 2 lanceolate acuminate 3 inches long $\frac{3}{4}$ inch wide narrowed into a very short petioled. Flowers wolitary pendulons on a slender peedicel firom a depression from one of the internodes below the leaves with a small yellow bract. Upper sepal orate oblong blunt, laterals broad triangular orate. Mentum very short. Metals lanceolate spathulate, all white. Lip base narrow, side lobes erect short, midlobe broad orbicular, centre depressed yellow with pink veins and streaks, a small callus at the base of the claw. Column short thick squared. Anther skullshaped. Capsule an inch long obconic. Rehb. fil. Xenia Orch. II. p. 26 t. 110.

Singapore: in mangrore swamps, Kranji, Selitar, ete. (Ridley); Johor : Gumong Panti, Batu Pahat. Distrib. Borneo, Jara.

48. D. Clllibotris, Ridl., Journ. Linn. Soc. XXXII 258. Stems sereral subterete grooved not thickened 6 inches or more long $\frac{1}{4}$ inch thick, internodes $1 \frac{1}{2}$ inch long. Leaves few at the end of the stem lanceolate thinly coriaceous $t$ inches long, one inch wide nerves 6 distinct. Raceme an inch long, from an internode near the top of the stem 6-flowered. Flowers crowded fleshy sweet scented hardly expanding, on pedicels $\frac{1}{4}$ inch long. Bracts ovate acute thin. Upper sepal widely ovate ohtuse $\frac{1}{2}$ inch long ovate acute falcate. Mentum shorter thick. Petals lanceolatrefalcate longer than the upper sepal all white. Lip orate cuneate spoonshaped fleshy yellow ornamented with red streaks. Colum nearly straight. Capsule oblong $1 \frac{1}{2}$ inch long pedicel amb peduncle thickened.

$$
\text { Singapore: Kiranji, Sungei Bulu (Ridley). Distrib. Borneo. }
$$




\section{Materials for a Flora of the Malayan Peninsula.}

49. D. modestua, Ridl., Journ. Bot. 36 (1898) p. 211. Sitens

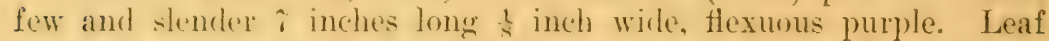
teminal one rarely 2 , lanceolate linear an inch long $\&$ inch wide petiolate. Flowers in pairs on a short peduncle, pedicels $\frac{1}{2}$.inch long. Sepals lanceolate $\frac{3}{8}$ inch long, laterals with a broader base. Mentum as long acuminate acute, all white tinted rose. Petals linear oblong shorter white. Lip orate acuminate, side lobes indistinct, white with violet stripes. Column short arms shorter than the oblong anther violet.

Penang, Hill at 2500 feet alt. (Ridley). Endemic.

\section{\$11. Pedilonuti.}

50. D. Laneldatur, Lindl., Gen. and Sp. Orch. Pl. p. 89. Stems 2 to 4 inches tall, of $4-5$ flexuous internodes, fusiform with a narrow base thin and flat $1-1 \frac{1}{2}$ inch wide ${ }_{1}^{1}$ inch thick. Leaves $2-3$ orate subacute thinly coriaceous on the apices of the young stems $2-3$ inches long one inch wide. Flowers few in short racemes from the upper internodes of the leafless stems. Bracts minute ovate subobtuse. Ovary and pedicel $\frac{1}{2}$ inch long. Flowers nearly an inch long creamcolour withering ochre. Upper sepal ovate subobtuse broad, laterals ovate, mentum long compressed hlunt. Petals obovate ohtuse narrower. Lip shorter than sepal obcuneate, with a broad rounded apex minutely fimbriate, white with a central orange orchre bar of 5 low keels. Column broad rounded arms very obscure thick. Hook. fil. Fl. Brit. Ind. V. 7+9. D. compressum Lindl, Bot. Reg. 18t2. Mise. 6

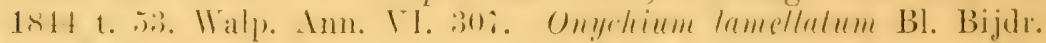
526 fig. 10.

Singapore: C'hangi, Jurong (Ridley); Johor: Batu Pahat; Selangor: Kuala Lumpor (Curtis); Pahang: Kuantan (Durnford); Perak (Stortechini). Distrib. Moulmein. Borneo, daval.

51. D. secuxpua, Lindl., Gen. and Sp. Orch. Pl. 81. Stems terete grooved stout 3 feet long 1 inch through. Leaves elliptic ob-

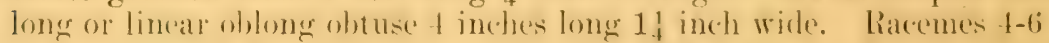
inches long on the old stems, perlunde's $\frac{1}{2}$ inch long. Bratets ovate acole 1 inch long persistent. Flowers very mumerous densely cowded secund $\frac{1}{2}$ inch long, rose pink or white, shortly pedicelled. Upper sepal orate acute, laterals similar. Mentum twice as long, curved thick dilate at the apes. Petals blunt but little smaller than the sepals. Lip acute linear spathulate entire, apex orange. Column thickened upwards. Bot. Reg. t. 1291, and 18tt Mise, 65. Rehb. fil. Exot. Fl. t. 269. Bot. Mag. t. 4352. De Vriese Illustr. t. 6. Veiteh. Manual. Dendrobium 62. Hook. fil. IF. Brit. Ind. V. 830. D. bursigerum Limll., Journ. Limn. Soc. HI. 16. P'edilonium sccundum, Bl. Bijdr. 322. 
Singapore: Long Island (Nanson); Pahang, near Pekan (Ridley 1460) ; Negri Sembilan: Seremban (Dr. Braddon) : Penang (Wallich 1996); Johor. Distrib. Burmah, Sumatra, Java, Borneo, Philippines, Cochin-China.

52. D. Connutur, Hook. fil. Fl. Brit. Ind. V. 820 Ic. Pl. 2029. Stems slender 12 to 18 inches long. Leaves narrow linear lanceolate acuminate $3-4$ inches long $\frac{1}{4}-\frac{1}{2}$ wide thin, sheaths $\frac{1}{2}$ inch long. Racemes short few flowered. Bracts minute. Flowers an inch long rose-pink. Upper sepals oblong obtuse, laterals similar. Mentum eurved obtuse twice as long as the upper sepal. Petals smaller elliptic oblanceolate, obtuse. Lip spathulate with a long claw, and a small callus at base.

Pahang: Kluang 'Terbang (Barnes); Perak: Gunong Batu Putih (Wray). Endemic.

53. D. AEgle, Ridl., Journ. Linn. Soc. XXXII. 260. Stems slender 2 feet long $\frac{1}{8}$ inch thick. Leaves lanceolate subacute coriaceous 3 inches long $\frac{3}{4}$ inch wide. Racemes $\frac{1}{4}$ inch long many flowered. Flowers sinall about $\frac{1}{2}$ inch long, pink on pedicels $\frac{1}{2}$ inch long. Bracts lanceolate acute. Epper sepal oblong obtuse $\frac{1}{4}$ inch long, laterals lanceolate orate subobtuse. Mentum terete obtuse curved $\frac{1}{4}$ inch long. Petals shorter broder and thinner. Lip spathulate narrow blade rounded, margin incurved denticulate. Column short and broad, arms tooth-like rather long. Anther conic obtuse dark crimson on a rather long filament.

Perak: Larut Hills at 2000 feet elevation (Ridley); Johor : Batu Pahat (Ridley). Endemic.

54. D. virescexs, Ridl., Journ. Linn. Soc. XXX1I. p. 259. Stems long over a foot slender weak, internodes $\frac{3}{+}$ inch long. Leaves lanceolate acuminate thin. Racemes lax about $2 \frac{1}{2}$ inch long 12 flowered. Bracts short lanceolate cuspidate. Pedicels one inch long. Flowers an inch long greenish yellow. Lpper sepal ovate acute $\frac{1}{t}$ inch long, laterals wider, mentum $\frac{3}{4}$ inch long cylindric blunt. Petals shorter than the sepals ovate. Lip spathulate with a narrow claw, blade rounded and toothed, margins below the blacte thickened erect with a fleshy transverse bar. Column broad arms thin and broad. Anther capshaped with a long filament.

Endemic.

Legeh at Tomoh (Machado); Perak: Taiping (Curtis).

55. D. skirexs, Hook. fil. Anm. Bot. Roy. Gard. Calc, Vol. V. p. $10 \mathrm{Pl}$. 16. Stems very long $2-3$ feet flexuous weak, internodes $1-1 \frac{1}{2}$ inch long. Leaves elliptic 3 inches long one inch wide apex very unequal. Racemes $1 \frac{1}{2}$ inch long, 6 to 8 flowered. Flowers about an 
incl long shortly pedicelled. Sepals broadly orate obtuse, yellow with red streaks, mentum twice as long as the sepals terete obtuse nearly straiglit green. Petals widely obovate coloured as sepals. Lip claw long linear, blade pandurate, with a scale across the base of the limb. D. penduriferum var serpens Hook. fil. Fl. Brit. Ind. VI. 186.

Perak (Kiunstler). Endemic.

Differs from the preceding in the form of the lip and colouring.

56. D. crocutex, Hook, fil. Fl. Brit. Ind. VI. 185. Amm. Bot. Gard. Calc. Vol. V. p. 9. Pl. 14. Stems 1 to 3 leet long, internodes an inch long $\frac{1}{3}$ inch through. Leaves lanceolate acute very unequally

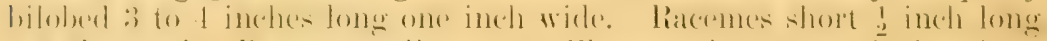

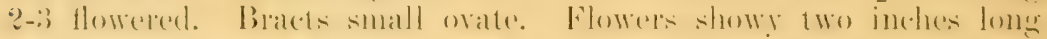
orange. Sepals lanceolate acute $\frac{1}{2}$ inch long. Mentum an inch long

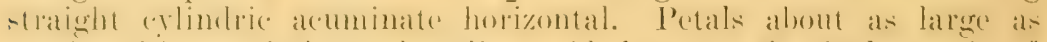

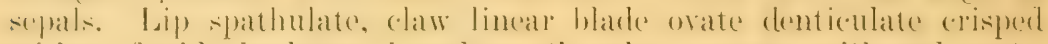
with a double keel running down the claw, orange with red spots. Column dilated above. Anther oroid. D. pyropum Ridley 'T'rans. Limn. Soc. 111. 36\%.

Singapore: Chan Chu Kang (Ridley); Pahang: Tahan Rirel

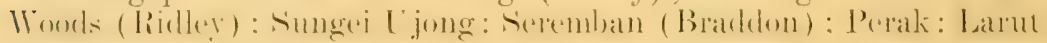
(Kunstle ). Epiphytic sporadically on high trees. I can find no "oblong reversed callus" on the lip as described in Fl. Brit. Ind. but the two ridges join at the base and are a little more elevated at that point. Enclemic.

5\%. D. DERry, Ridl. Stems stout somewhat enlarged upwards red 8 to 10 inches long by 33 inch wide, intrnodes $1 \frac{1}{2}$ inch long channelled. Leaves oblong obtuse 3 inches long or more. Flowers solitary

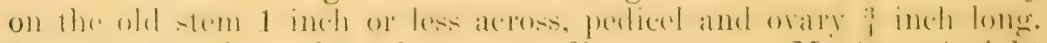
Sepals oblong lanceolate obtuse spreading ocreous. Mentum straight. blunt horizontal thick $\frac{1}{2}$ inch long. Petals narrower ocreous. Lip white with a narow claw, with an elevation double ridge meeting at the base of the lip, blade oblong truncate broad more or less retuse. Column broad short, white, armis erect taller than the depressed flat anther, blunt orange.

Perak: Larut Hills (Derry). Distrib. Borneo, Very variable in the size ol the flower.

is. I), my maxonterum, Hook. fil. Je. Pl. 2032. Stems slender terete or thickened upwards a foot long purple, with short internorles.

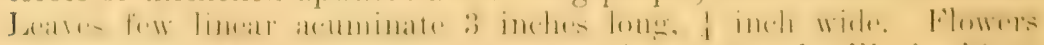
solitary thin $1 \frac{1}{2}$ inch across and one inch long. Sepals elliptic obtuse white oiten tinted with riolet. Mentum nearly as long slender acumi-

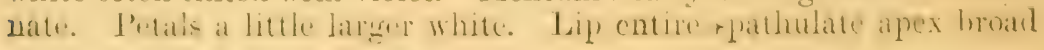


rounded truncate white except the base and nerves pale yellow, keels 2 connate on the claw, divaricate on the blade and 2 parellel raised nerves on the disc. Column short greenish white, orange above. $D$. Hughii Rchb. fil. Gard. Chron. 1882 p. 664. Ridley Journ. Linn. Soc. XXXII. 263.

Pahang: Kluang Terbang (Barnes, Ridl. 110\%6); Perak: Gunong Batu Putih (Wray); Bujong: Malacea (Ridley); Kedah: Gunong Jerai (Ridley) ; Lankawi: Gunong Raya (Curtis 2563). Endemic.

59. D. Calicopis, Rildey, Journ. Roy. As. Soc. S. Br. Vol. 39. p. r2. Stems slender over a foot long, internodes $\frac{1}{2}$ to 1 inch long. Leaves lanceolate acuminate acute 3 inches long $\frac{1}{2}$ inch wide. Flowers an inch cross 3 or 4 on a short peduncle $\frac{1}{2}$ inch long; pedicels with ovary as long. Sepals ovate obtuse, laterals narrower subacute. Petals broader elliptic obtuse all white tinted with rose. Lip entire broadly oblong truncate, apex bilobed, lobes short rounded, raised veins, t, two thicker central ones, and two thinner outside, white with a rose spot at the base. Column short thick, dilated at the stigma, arms erect tooth-like crimson. Anther orate pink large.

\section{Lankawi Island (Curtis). Endemic.}

This resembles 1 ). hymenopterm. Hook. but has the lip bilobed at the tip and the elerated ribs or veins on the disc are very different. The crimson column arms and anther contrast finely. with the pale rose-white perianth.

60. D. roseatum, Ridley Journ. Linn. Soc. XXXII. 261. Stems slender over 2 feet long, internocles 1 inch long. Leaves lanceolate acute 3 inches long $\frac{1}{2}$ inch white. Racemes an inch long about 6 flowered. Bracts ovate persistent ${ }_{1}{ }^{1}$ inch long. Pedicels slender an inch long; flowers larege losy white $1 \frac{1}{2}$ inch long. Sepals $\frac{1}{2}$ inch long ovate. IIentum as long straight thick with a curved and clubbed apex. Petals broader than the sepals widely ovate obtuse. Lip with a narrow linear base, blade abruptly rounded quadrate crenulate. Column short and thick arms short broad incurved.

Perak: Larut Hills (Curtis 2061). Endemic.

61. D. Foxim, Ridl., Journ. Bot. 1900 p. ro. Stems slender 3 feet long reil. Leaves lanceolate acuminate shining dark green, 4 inches long 1 inch wide. liacemes short $\frac{1}{2}$ inch long, f to 10 flowered. Flowers rosy white an inch across, on pedicels an inch long. Sepals orate acute, laterals more obtuse curved $\frac{\pi}{8}$ inch long, $\frac{1}{4}$ inch wide. Irentmm an inch long thick curved dilated towads the acuminate curved point. Petals oblong orate rounded exlges fimbriate, larger than the sepals nearly inch long, white. Lip with a narow linear 
claw, blade abruptly dilated pandurate bilobed lobes rounded fimbriate white. Column broad, arms short blunt orange.

Perak: Larnt Hills at 4000 feet (W. Fox). Endemic.

62. 1). tropaeolifloruar, Hook. fil. Ann. Bot. Gard. Calc. V. p. 11. pl. 1i. Fitem elongate flexuous thichened upwards, 1 foot long. Leaves linear oblong acute $2 \frac{1}{2}$ inches long inch wide. Raceme 1-2 inches long, rachis slender, flowers 6 or more an inch long. Bracts minute, peelices and orary inch long. Sepals triangular ovate acute $\frac{1}{4-\frac{1}{3}}$ inch long. Petals obovate rounded acute all deep purple. Mentum four times as long as the sepals cylindric obtuse yellow. Lip as long as the sepals, claw linear gradually dilated into the lateral lobes hetween which is a large semilunar callus, then suddenly contracted into the obovate, apiculate $\operatorname{limb}$.

Perak (Kunstler). Endemic. More nearly allied to D. serpens Hook. fil.

63. D. Megreeras, Hook. fil. Fl. Brit. Ind. T. F31. Ic. Pl. 2031.

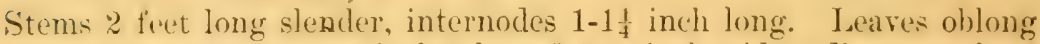
lancerolate acuminate $3-4$ inches long is to 1 inch wide. Racemes short few flowered. Flowers large $1 \frac{1}{2}$ inch long yellowish green. Bracts minute. Lpper sepal elliptic oblong obtuse, laterals smilar. IIentum 2 to 3 times as long cylindric nearly straight. Petals obovate. Lip cuncate three-lobed claw gradually dilated into limb with an clongate keel at the base, lateral lobes subtruncate crenulate, midlobe suborbicular retuse crenate. Column short arms erect rounded. Filament of anther rather long.

Malacea (Maingay 1613). Endemic.

64. D. texuicaule, Ridl., Journ. Roy. As. Soc. S. Br. Vol. 39. p. 73. Stems very slender weak a foot long. Leaves linear lanceolate solitary large is inch across, perlicel and ovary slender hall an inch long. Upper sepal ovate blunt, laterals ovate acute. Mentum very long cylindric apex decurved acute 3 inch lome. Petals broadly ovate. All pink darkest at the tips. Lijp claw very long narrow, lateral lobes broad upeurved, midlobe short ovate at apex bifid edges erisped, white with a central pink line. Column short with a very long foot, arms tooth-like ereet. Anther margin pubescent.

Lankawi: Ayer Hangat (Curtis). Endemic.

65. D. Kextrochilum, Hook. lil. Fl. Brit. And. V. 831. Ic. Pl. 2030. Stem stout eompressed deeply obtuse, 5 to 6 inches long.

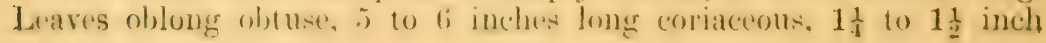


across. Flowers in pairs an inch across, white. Bracts minute, sepals ovate oblong. "Mentum curved as long as the dorsal sepal; stout. Petals suborbicular obtuse. Lip clawed rhombic orate 3 lobed lobes equal, claw with a strong spine. Column short, arms erect curved rather large. Anther hatshaped.

Perak: Batang Padang Valley (Wray). Endemic.

66. D. Sanguinolentum, Lindl., Bot. Reg. $18+2$ Mise. 73. $18 \pm 3$ t. 6. $184 t$ Mise. 52. Stems fairly stout 1-2 feet long flexuous, internodes $\frac{1}{2}$ to $1 \frac{1}{2}$ inch long ${ }_{16}^{3}$ inch thick. Leaves lanceolate subacute few, 2 inches long $\frac{3}{4}$ inch wide. Racemes short 1-1 $\frac{1}{2}$ inch long, 4 to 6 flowered. Bracts ovate acute $\frac{1}{8}$ inch long. Flowers large. showy, an inch across. Upper sepal broadly ovate obtuse $\frac{1}{t}$ inch long, laterals similar; mentum $\frac{3}{4}$ inch long terete apex gibbous curved, white tinted rose. Petals widely orate obtuse $\frac{1}{2}$ inch long $\frac{33}{8}$ inch wide, white. Lip shorter base narrow straight with a recurved spine-like callus, limb suddenly dilate quadrate rounded edges wared. Column thick arms thin rounderl. Anther flat. Veitch Man. Dendr. 72. Hook. fil. Fl. Brit. Ind. V. ;31. Var typica. Flowers cream colour, tip of sepals, petals and lip violet crimson a central yellow patch on the tip.

Var cenina. D. cerinum Rehb. f. Gard. Chron. XII. p. 554. Flowers yolowish ochre, occurs with the other.

Selangor: Pahang track (Ridley 8tr8); Penang Hill (Curtis); Kedah: Gunong Jerai (Ridley 5139). Distrib. Borneo.

\section{§12. EỦDENDRoBIUMr.}

6\%. D. Flavidulur, Hook. fil. Fl. Brit. Ind. VI. 185. Stems rather slender terete 12 to 18 inches tall, internodes an inch long. Leaves lanceolate, $: 3$ inches long by $\frac{1}{2}$ inch wide. Racemes several and inch long or more 3 to 6 flowered. Bracts lanceolate short. Flowers pale yellow or more 3 to 6 flowered. Bracts lanceolate short. Flowers pale yellow ol nearly white $\frac{1}{2}$ inch long. Upper sepal ovate acute, laterals similar. Mentum very short thick straight. Petals lanceolate acute about as big as the sepals. Lip oblong three lobed, lateral lobes short lanceolate acute, median longer orate lanceolate obtuse with a red spot on the tip edges ciliate, 4 low ridges on the dise. Column rather long, arms erect tooth-like acute, anther hatshaped blunt. Capsule elongate elliptic pearshaped $\frac{1}{2}$ inch long.

Singapore: Kranji, etc. (Ridley). Distrib. Rhio. On trees in mangrove swamps. Allied to D. stuposum.

68. D. viridicatux, Ridl., Journ. R. As. Soc. S. Br. 39. p. 'r2. Stem rather slender over a foot long. Leares lanceolate acute $2 \frac{1}{2}$ 
inches long, 1 inch wide, sheaths half an inch long. Flowers numerous on leafless stems in very short racemes of 2 or 3 flower's peduncles $\frac{1}{t}$ inch long. Bracts very small orate sheathing. Pedicels

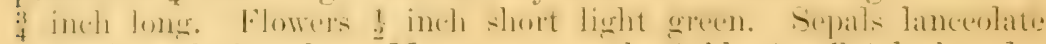
acute laterals broader. Mentum rery short blunt. Petals broader arms erect.

\section{Perak: Ipoh (Goldham).}

69. D. IIercoglossur, Rehb. $\mathfrak{f}$. in IIamb. Gartenz. XIII. p. 5as. Gard. Chron. 1856. II. p. $48 \%$. Stems 1 to $3 \frac{1}{2}$ feet long rery slender pendulous, internodes about an inch long. Leaves few narrowly linear acuminate thin 3 inches long, half inch wide. Racemes hardly an inch long 2-8 flowered. Bracts lanceolate obtuse small. Pedicel and orary

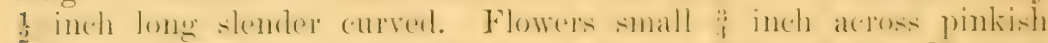

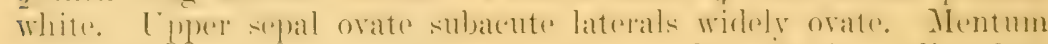
rery short and broad rounded. Petals lạnceolate, much smaller than the sepals. Lip $\frac{1}{ \pm}$ inch long with a very short claw subhemispherie sides enclosing the column, apex beak-shaped acute, a large bearded (allus at the hates of the blake. C'olumm short thick puberent, amms short broad erect toothed. Anther purple papillose skull-shaped grooved on the top. D. aduncum Hook. fil. Bot. Mag. 6rSt. (Not Lindley).

Johor: Pulau Tioman Island, Fast of Johor (Xanson); Batu Pahat (lidlley); Perak: Batang Padang (Curtis). Distrib. China.

\%. D. AUreuz, Lindl., Gen. and Sp. Orch. Pl. p. r\%. Bot. Reg. 1839 t. 20 . Stems subterete thickened upwards 9 to 36 inches tall,

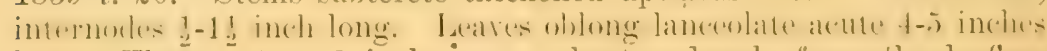
long. Flower's 1 to 3 inches on a short pecluncle from the leafless stems, 2 to $2 \frac{1}{2}$ inches across, fragrant pale yellow. Sepals linear ob-

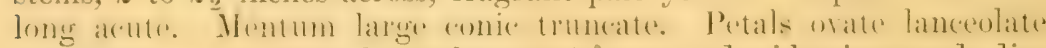
acuminate. Lip osate laneeolate acute reeurved, sides ineurved, dise

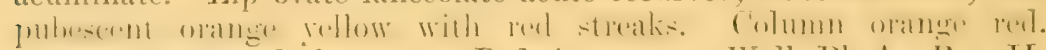
Veitch Man. Dendrob. p. 19. D. heterocarpum Wall. Pl. As. Rar. II. 8t. t. 196. Bot. Mag. t. tros and 4970 FI. de. Serres VIII. t. 842.

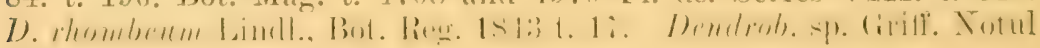
III. 304. Ic. P'. As. t. 306;.

Perak: Latrut Hills (Stephens). Distrib. India, Ceylon, Java, Philippines. 'The form collected (once only) is val. Philippinensis.

i1: 1). screrbuar, Rehb. fil. Walp. Amm. NT. p. 282. Stems terete $2 \frac{1}{2}$ to 4 leet long stout pendant, internodes 1 inch long. Leares oblong laneeolate acute is to finches loner. Flowers in pairs on the old stems $3-1$ inches actoss. Sepals lanceolate acute pink. Petals orate oblong much broader pink. lip ovate sides eonvolute pubescent 
acute pink with a dark purple centre, a three lobed callus at the luare. Misc. 46. Sert. Orch. t. 35. D. macranthum Hook. Bot. Mag. t. Williams Orch. 1. t. 42. D. macrophyllum Lindl. Bot. Reg. 1839? 39\%0. D. Scortechinii Hook. fil. Fl. Brit. Ind: V. $r+1$.

Perak: Larut Hills (Stephens), (Scortechini). . Distril). Philippines, Borneo.

\%2. D. Dalmousmaxur, Wall. Paxt. Mag. Bot. XI. t. 14 . Stems terete stout 3 to 4 feet long, internodes 1-1 $\frac{1}{2}$ inch long. Leaves linear oblong $t$ to 6 inches long base cordate. Flower's very large 4 to 5 inches actoss rosy or vellow with two deep purple blotches on the lip. Sepals oblong acute. Petals much broader. Lip shortly clawed orbicular apex and sides densely villous, dise with two pectinate fringed lamellae. Lindl., Bot. Reg. 1836 t. 10. Journ. Linn. Soc. IIL. 18. Fl. des. Serres TII. t. 698. III. Hort. 1864 t. 423. Veitch. Man. Dendrob. 3อ̃. Hook. fil. Fl. Brit. Ind. V. $7+3$. Burmah.

Singapore on a tree at Chan Chu Kang (Ridley). Distrib. India,

73. D. Aggregatuar, Roxb. Fl. Ind. III. 4\%\%. Stems crowded

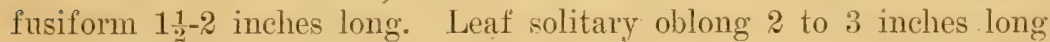
by 1 inch broad. Flowers 1 to 12 in a loose lateral raceme, yellow. Sepals small orate obtuse, nentum subglobose. Petals broader ovate. Lip transerscly ohlong shortly clawed, chisc concare puldescent margins ciliate. Colmon short yellow. Lindl., Bot. Reg. t. 169.). Bot. Mag. t. 36t3. Paxt. Mag. 'VI. t. 145. Veitch Man. Dendrob. 15, II. Hook. fil. Fl. Brit. Ind. I. iso. I). Limlleyi, Steud. Nomencel. I. t!90. D. Jenkinsii Wall. Bot. Reg. 1839 t. $3 \%$. China.

Perak (Scortechini, drawing), Distrib. Assam, Burmah, Siam,

\section{Excluded axd Doubtful Species.}

D. aciculare Lindl., Bot. Reg. $18 \pm 0$ Misc. 81. Singapore Cúming (Hort Loddiges) is doubtlessly wrongly localised, Cuming's herbarium specimens No. 2112 are labelled Bohol Island, Philippines.

D. breviflorum Lindl., Journ. Linn. Soe. Singapore? Hort Toddiges, is stated in the Flora of British India to be D. bicameratum Jinkl. of India.

D. cruentum Rehb. fil. Gard. Chron. 1884, 160t. "Malay Peninsula on the West Coast (Hort Sanders)" is a native of Siam.

D. junceum Lindl. Bot. Reg. 18t2, Mise. 11. "Singapore, Loddiges is doubtlessly wrongly localised. :

D. Il ughii Rehb. fil. Gard. Chron. 1882 II. 764. Singapore, H. Low is altogether doubtful. 'The author says it is allied to $D$. revolutum. It is also (Fl. Brit. Ind. VI. 752) said to be a form of $D$. 
Flowers large expanded.

Sepals ovate obtuse .. .. 10. B.membrani-

Sepals lanceolate acute.. $\quad$.. 11. B. sanguineo-

folium.

Sepals lanceolate caudate $\quad . \quad 12$ B. longiflorum.

Flowers more than one on the peduncle. Flowers glabrous

13. B. subumbellatum.

Flowers hispid.. $\quad \ldots \quad$.. $\quad \ldots \quad$ 14. B. hispidum.

§. Monantha Parva. Rhizome slender filiform. Leaves and pseudobulbs small. Flowers small solitary on slender pedicels.

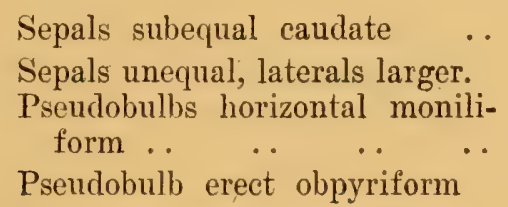

§ 3. Eipicrianthes. Rhizome creeping pseudobulbs. Flowers, solitary very shortly pedicelled. Petals broken up into 6 or 7 threads. .. . . .

§ 4. Hirtula. Small plants, pseudobulbs with a few purple flowers usually pubescent.

Flowers glabrous

Flowers pubescent, rhizome slender flowers few.

Sepals subequal lanceolate .. Sepals unepual ovate lanceolate Rhizome stout flowers numerous

§. Racemosa. Flowers numerous in a dense or open raceme purple or spotted pink.

Raceme elongate lax.

Pseudobulbs absent

Pseudobulbs developed.

Flowers large hairy .. . , ,
15. B. striatellum.

16. B. catenarium.

1\%. B. Avicella.
19. B. tenerum.

20. B. hirtulum.

21. B. cincinnatum.

22. B. limbatum. 
Flower's small glabrous.

liaceme decurved .. ... . . 25. B. alcicome.

\$ 6. Dexsiflors. Raceme dense. Peduncle very long... $\quad \ldots \quad \ldots \quad \ldots \quad$ 26. B. gigas.

liaceme dense, peduncle short.

Psendobulbs absent, raceme very small

$\therefore$ :ล. B. botryophorum.

Pscudobulbs present.

Flowers pubescent ..' . . .

Flowers glabrous.

Leaf oblong obtuse $\quad \ldots \quad \ldots \quad$ 29. B. litacinum.

Leaf lanceolate acuminate .. 30. B. densiftorum.

$\$ \%$ Ixtervallatae. Scape tall wiry, rachis thickened with persistent bracts. Flowers numerous opening singly at long intervals.

Flowers never opening .. $\quad$. . . .

Flowers widely expanded .. ..

8. Oxysepaluar. Stems long pendent from a rooting base. Pseudobulbs appressed to the stem. Flowers small thin solitary or several on a sliort peduncle enclosed in a sheath.

Stems very slender much branched Stems stout, pseudobulbs large ..

31. B. stella.

32. B. cleistogamum.

33. B. clandestinum. :H. B. capitalum.

\$ 9. Biptryes, Rhizome ereeping. Pseudobulbs large, small or obsolete. Racemes short or long. Flowers thin small yellow or orange. Sepals subequal lanceolate acute.

Racemes short, flowers few at the end of the scape.

Seape very short hidden in sheaths

ii. B. brevipes.

Scape slender short.

Sepals equal acute .. . . :3i. B. concinnum.

Sepals unequal obtuse.

Bases not gibhous .. .. :3.. B. modestum.

Bases gilbbous . . . . . . $\quad$ :

Sepals acuminate white .. ... :3:. B. ochrunthum. 
Materials for a Flora of the Malayan Peninsula.

Lateral sepals twice as long as upper sepal .. . . . .

40. B. citrinum.

Raceme elongate. Flowers numerous scattered.

Pseudobulbs obsolete very small.

Sepals acuminate, laterals not gibbous.

Lip entire, ciliate .. .. 41. B. adenopetalum.

Lip 3 lobed .. . . . . 42. B. oblanceolatum.

Sepals acuminate gibbous.

Lip entire pubescent .. _.. 43. B. montigenum.

Lip 3 lobed .. ... ... 4. B. leptosepalum.

Sepals not acuminate.

laceme shorter than leares.

Leavès lanceolate .. _.. .. t5. B. apodum.

Leaves limear .. .. .. 46. B. Tinearifotium.

Raceme much longer than leaves .. 4. . B. odoratum.

Pseudobulbs large globose.. .. \$8. B. globulus.

Pseudobulbs large conic .. .. 19. B. flammuliferum.

§ 10. Cinrhopetulua. Flowers solitary or whorled on a well developed scape, lateral sepals much larger than upper sepals often more or less connate.

Flowers solitary.

Petals smaller than dorsal sepal.

Lateral sepals narrow free ․ 50. B. cphippium.

Laterals bread, partly connate .. 51. B. restrepia.

Petals as large as dorsal sepal.

Laterals comnate twisted ..... 52. B. breviscapum.

Flowers whorled, pseudobulbs flat connate lying on the rhizome . .

Pseudobulbs erect thick.

Lateral sepals free caudate very long.

Flowers fer, 5 inches long ... ... 54. B. maximum. 
Flowers many capitate smaller.

Capitulum $1 \frac{1}{2}$ inch through, flower's spotted pink .. .. . .

5\%. B. medusae.

Capitulum much smaller, flowers yellow .. . . . . .

Lateral sepals caudate connate .. $5 \%$ B. psittacoides.

Lateral sepals linear connate .. 58. B. makoyanum.

Lateral sepals flat free.

Sepals glabrous .. . . . . 59. B. C'urtisii.

Sepals ciliate.

Pseudobulbs conic, sepals acuminate . $\quad$. $\quad \ldots \quad$.. 60. B: acuminatum.

Pscudobulbs globose very small .. 61. B. microbulbon.

Pseudobulbs large oroid, sepals broad blunt .. .

62. B. auratum.

Lateral sepals flat half commate.

Petals glabrous .. $\quad \ldots \quad \ldots 63$. b. perakense.

Petals ciliate _. .. .. 6t. B. semibifidum.

Lateral sepals commate, tips only firee.

Lateral sepals linear acuminate ..

65. B. stenophyllum.

Lateral sepals oblong obtuse.

Scape $2-t$ inches, flowers pink .

66. B. pulchellum.

Seape 8 inches slender, flowers pink . . . . . .

Scape tall stout, flowers large yellow $\ldots \quad \ldots \quad \ldots$

6\%. B. Griffithianum.

68. B. ochraceum.

\section{Sestocinlus.}

1. B. PAtexs, Hook. fil. Fl. Brit. Ind. VI. 18\%, Rhizome stout crinite with dense short roots at calch pseudohulh. Pseudohulhs one inch apart conic one inch long. Leaf elliptic to elliptic lanceolate

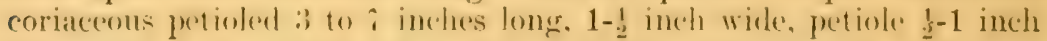
long. Flowers solitary on a rather slender peduncle 1 it inch long yellowish closely red-spotted, abont an inch across, seented of clores. Sepals lanceolate acute, the laterals falcate. Petals as long as upper sepal a little narrower. Lip stipitate on the free part of the column foot linear oblong flat half as long as the lateral sepals crimson. Column dilated alone apex of foot fiee. Arms rery short roumded. 
Singapore: Changi, Bukit Timah, etc., (Ridley) ; Johor: Tana Runto (Ridley); Perak: l)indings (Curtis); no locality (Kunstler); Penang: the Spout (Curtis). Not rare. Endemic.

2. B. imegalanthun, Griff. Notulae III. 86. Ic. Pl. As. t. 292. Thizome fairly stout, pseudobulls small distant. Leaf very coriaceous oblong emarginate petiolate. Flowers solitary dusky with purple spots. Sepals linear acuminate upper $2 \frac{1}{2}$ inches long, laterals scythe-like crossed over the lip. Petals lanceolate long, acuminate rather shorter than the sepals. Lip long narrow slender three lobed, lobes short acute, midlobe long acuminate. Colimn thick arms short rounded. Fruit clubshaped 2 inches long. Walp. Ann. VI. 246. Hook. fil. Fl. Brit. Ind. V. 75 t.

Malacea: on Pulau Besar (Griffith). Only known from Griffith's description and figure.

3. B. pileatua, Lindl. Bot. Reg.-184t, Misc. 73. Rhizome long rather slender $\frac{1}{8}$ inch through crinite, pseudobulbs $1 \frac{1}{2}$ inch apart, cylindric narrow $\frac{1}{2}-1 \frac{1}{2}$ inch long. Leaf thinly coriaceous lanceolate petiolate obtuse 4-6 inches long 1-2 inches across, petiole $\frac{1}{4}-\frac{1}{2}$ inch long. Peduncle very short, pedicel slender 2 inches long. Flowers 1 inch to $1 \frac{1}{4}$ across, yellow. Sepals subequal and similar-lanceolate oblong, obtuse. Petals narrower oblong obtuse, nearly as long. Lip half the length of the sepals flat with a broad rounded base tapering to a blunt point groored at the base yellow with red spots on each side of the groove. Column unusually long green, arms none. Anther conic. Hook. fil. Fl. Brit. Ind. V. p. 754. Sarcopodium pileatum. Lindl. Fol. Oreh. 4.

Singapore: Seletar (Ridley); Johor: Panchur (Ridley); Perak: (Wray) : Penang (Maingay) ; Penara Bukit (Ridley i239); Government Hill (Curtis 17r2). Distrib. Sumatra. Climbing on small trees in forests.

4. B. macrantiuum, Lindl. Bot. Reg. 1844, t. 13. Rhizome very stout crinite, $\frac{1}{\ddagger}$ inch thick woody emitting large tufts of short roots at the nodes. Pseudobulbs an inch apart, oblong wrinkled one inch long. Leaf coriaceous elliptic obtuse petiolate 5 to 8 inches by $14_{4}^{3}-3$ inches wide, petiole stout an inch long. Flowers two inches across, peduncle very short, pedicel thick 2 inches long. I pper sepal lanceolate acute white with purple spots. Lateral sepals larger semiovate acuminate base depressed, connirent, hases rellow with the crimson spots, apices dark brown. Petals resembling the upper sepal but a little narrower. Lip $\frac{1}{4}$ inch long base quadrate with two truncate ears apex acute curved, yellow tinted with red. Column short and thiek yellow, arms porrect tooth-like acute. Anther conic obtuse deep red. Hook. fil. Fl. Brit. Ind. V. 753. Ridley Ann, Bot. IV. p. 328. Pl. XXII. A. 
Bot. Mag. f\%08. D. gelatinosum 'T'eysm. mss. Saropodium macranllım. Lindl. Paxt. Fl. Gard. I. 15̌. Fol. Orch. 3.

, Singapore: Jurong, ete., (Ridley) ; Palıng: Pekan Wroods (Ridley 1:3(i): Sunged l jonge (lidlley): Perak: Sonkaly (Snow). Distrib. l'enasscrim, sumatra, Borneo. Not rare on tree trunks in forests. Flowers clove scented.

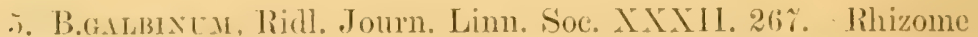

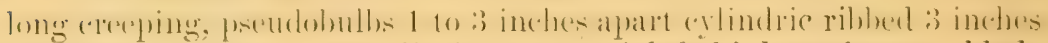

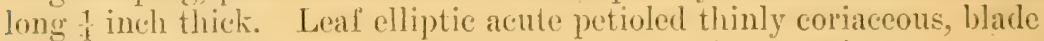
six inclues long $1 \frac{1}{2}$ inches wide, petiole an inch long. Flower's one or two large wide open on a peduncle 3 inches long slender. Bracts orate acute 1 inch long orary and pedicel 3 inches long. Upper sepal lan-

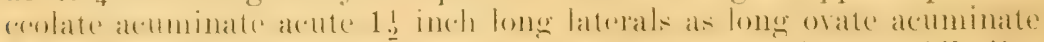

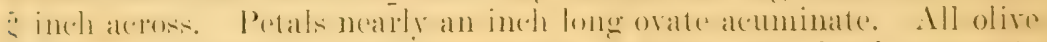

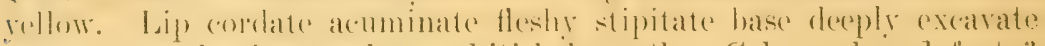
apex recurved crimson above whitish beneath. Column broad foot $\frac{3}{8}$ inch long, arms short angled obtuse. Anther almost three lobed.

Perak: Larut Hills, common (King's Collector', Ridley 2888); Selangor: Bukit Hitam (Kelsall, Ridley). Endemic.

6. B. valablete, Ridl. Journ. Roy. As. Soc. Str. Br. Vol. XXXIX. p. it. Thizome stout woody, pseudobulbs eylindric curved 3 inches long. Leaf elliptic orate acute 6 inches long 2 to 3 inches wide thinly coriaceous petiole an inch long. Seape from near the pseudobulb stout, red with several sheaths at the base and three or four lanceolate red spotted ones scattered on it. Bracts large lanceolate acute spotted red hall as long as the ovary. Flowers one ol two large showy 3 inches across. Upper sepal lanceolate acute, laterals falcate. P'etals lanceolate nearly as long. All yellow with lines of red dots. Lip tongue-shaped recurved with a broader base short apex blunt yellow

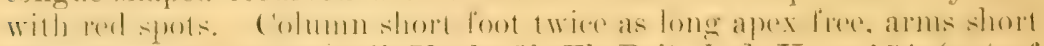
rounded. B. Reinuardlii, Hook. fil. Fl. Brit. Ind. V. p. 75t (not ol Reichenbach). 1122).

Perak: Larut Hills (Ridley; Curtis); Gmong Batu Putih (Wray

Tar. B. rubrum. Sepals and petals erimson with red spots at the base. Lip dark crimson. Column yellow with erimson spots. Both forms oceur in the same locality. lindemic.

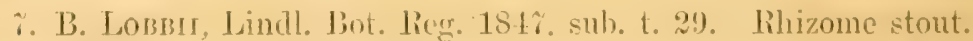
Pseudobulbs close ovoid. Leaf linear oblong acute petioled coriaceous 1. to 8 inches long, one inch wide. Petiole half an inch or long less. Flowers large widely expanded on a very short peduncle with a pedicel 
4 to 6 inches long. Upper sepal lanceolate acute or acuminate yellow. spotted with dark red or plain, laterals deflexed faleate broader acute or obtuse. Petals lanceolate acuminate. All ochreous veined and speckled with red or plain. Lip large broadly ovate recurved yellow or dull reddish, speckled or plain. Column broad yellow arms very obscure. Anther small depressed. Hook. fil. Fl. Brit. Ind. V. 75õ. Bot. Mag. t. 4532. Saunders Refugium Bot. t. 116. B. Henshalli, Lindl. (rard. ('hron. 18.52 422. B. ,iemense, R(chl), fil. Gard. Chron. 186\%. 572. Sarcopodium Lobbii, Lindl. Paxt. Fl. Gard. 1155. S. Lobbii, var Henshulli Henfrey. Card. Mag. Bot. III. 269. S'estochilus uniflorum, Breda Orch. Jav. t. 3.

Perak: Larut Hills (Stephens). Rare. Distrib. 'Tenasserim, Siam, Java.

8. B. pustulatum, Ridl. Journ. Roy. As. Soc. S. Br: Tol. XXXIX. p. it. Stem stout crinite pseudobulbs crowded, oblong conic half an inch long. Leaf elliptic lanceolate acute four inches long by one inch widle, petiole $\frac{1}{2}$ inch. Flowers solitary one inch across, pedicel slender $\frac{1}{2}$ inch long. Upper sepal lanceolate acute, laterals much broader ovate oltuse. $=$ Petals lanceolate acute nearly as large as the upper sepal. All yellow with red stripes. Lip fleshy dark maroon orate cordate obtuse $\frac{1}{4}$ inch long with two raised lobes rounded obtuse at the base and a mass of papillae on the disc. Column short with a long foot, the apex free, arms triangular oblong obtuse.

Malacea: lower part of Mount Ophir (Ridley). Climbing on trees in the forest. Rare. Endemic.

9. B. rugosuar, Ridl. Journ. Linn. Soc. XXXI. 266. Rhizome slender branched pseudobulbs numerous crowded cylindric 2 inches long $\frac{1}{2}$ inch through. ILeaf oblanceolate acute thinly coriaceous 6 inches long $1 \frac{1}{2}$ inch wide petiole. Scape $\frac{1}{2}$ inch long. Bracts few ovate lanceolate. Pedicel and ovary an inch long. Flowers solitary $\frac{1}{2}$ inch across, hardly expanded. Sepals lanceolate acute pale yellow with many grooves on the outer surface, an inch long. Petals shorter yellowish white. Lip thick fleshy oblong with a decurved tip, base excarate reddish pink $\frac{1}{4}$ inch long. Column thick short fuscous, arms rather long decurved obtuse. Anther conic.

Singapore: Chan Chu Kang (Ridley). Endemic.

10. B. membranifolium, Hook. fil. Fl. Brit. Ind. V. 756. Rhizome as thick as a crow quill, pseudobulbs subcylindric narrow rather distant 1-12 inch long. Leaf 6 -10) inches long elliptic lanceolate membranous narrowed into a petiole. Scape stout one-flowered with a single sheath, lanccolate acuminate. Flower $1 \frac{1}{2}$ inch across when expanded. Sepals orate oblong or lanceolate olituse, laterals deflexed. Petals smaller orate lanceolate subacute, all yellow green 
with red spots. Lip small stipitate tongueshaped decurved apex rerrucose purple. Column short, arms blunt rather large, porrect.

Perak: Gunong Batu Putih (Wray). Endemic.

11. B. shangune-Maculituar, Ridl. Joum. Linn. Soe. XXXII. 265. Rhizome slender with numerous roots: Pseudobulbs subterete curved $1 \frac{1}{2}$ inch long $\frac{x}{+}$ inch through. Leaf lanceolate acute thinly coriaceous reticulate 4 inches long, one inch wide. Scape 2 inches long with large thin sheaths. Flowers solitary large 2 inches across. Bracts shorter than the ovary. Upper sepal lanceolate acute fleshy 1 inch lone 1 inch wide, laterals semiovate elliptic 1 it inch long. Peats lanceolate acute base narrowed. All greenish vellow with numerous red spots. Lip short ('urved fleshy blunt rery small stipitate, with distinct erect side lobes, yellowish with red spots, apex and median portion of dise covered with deep crimson papillae, a deep central groove on disc. Column short thick yellow with red spots arms of one erect tooth and another longer porrect curved one. Anther depressed red. Capsule $1 \frac{1}{2}$ inch long green with purple spots.

Selangor: Kíuala Lumpor (Curtis). Endemic.

12. B. Loxgiflondir, Ridl. Journ. Linn. Soc. XXXII. 368. Rhizome woody for creeping $\frac{1}{8}$ inch thick with numerous strong roots. Pseudobulbs cylindric thick 2 inches apart, $1 \frac{1}{4}$ to $1 \frac{1}{2}$ inch long. Leaf lanceolate obovate or elliptic acute subcoriaceous $6-7$ inches long, 2-2 $\frac{1}{2}$ jnch broad, petiole one inch long. Scape an inch long covered with membranous lanceolate acuminate bracts. pedicel $1 \frac{1}{2}$ inch long base enclosed in a long bract. Flowers solitary thin 2 inches long hardly opening. Sepals lanceolate caudate, laterals a little wider $\frac{3}{8}$

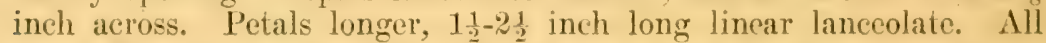
pink with darker veins. Lip 1 inch long broat thick rumed shorty stipitate apex papillose pale orange. Column short greenish. foot long crimson, arms long porrect subterete.

Penang: Government Hill (Curtis); Kedah: Gunong Jerai (Ridley); Perak: Hermitage Hill (Coek). Endemie.

13. B. subumbeldtur, Ridl. Journ, Linn. Soc. IIIII. 2 it. Rhizome long and slender. Pseudobulbs crowded or as much as five inches apart. erlindric 2 inches loner 3 inch thick. Leal laneolate acute petioled $\tilde{5}$ to 6 inches long, 11 jnch wide. Scape rather thick t inches long with 4 or 5 cuspidate ovato sheaths. Flowers 2-3 rather large 2 inches long crowded in very short raceme on slender pedicels $\frac{3}{4}$ inch long. Bracts $\frac{1}{4}$ inch long ovate euspidate. Upper sepal ovate acute nearly an inch loner 3 inch wide. laterals suberpal twisted. Petals much smaller hrobdly orate 3 inch lone to wide. All greenish yellow with red spots. Lipstipitate tongueshaped acute curved ! inch Inng. Column slort with a long slender foot, arms rery short hardly distinct. 
Johor: Hulu Batu Pahat (Kelsall), Tanjong Kupang (Ridley). Distrib. Borneo.

14. B. mispidum, Ridl. Journ. Linn. Soc. XXXII. p. 268. Rhizome stout far creeping with long stiff roots. Pseudobulbs conic thick three inches apart, an inch long. Leaf lancelate obtuse coriaceous shining 3 to 6 inches long, 2 inches wide petiole an inch long. Scapes stout $\frac{1}{2}$ inch long covered with bracts. Flowers about 8 in a dense raceme half an inch long on a thickened rachis, one inch across deep red foetid. Sepals ovate subacute hairy on the edge $\frac{1}{2}$ inch long $\frac{3}{8}$ inch wide, laterals connate. Petals lanceolate falcate $\frac{1}{4}$ inch long red with brown stripes, edges hairy. Lip thick not stipitate with a short thick claw, blade deflexed broad obtuse tongue-shaped. warty with two hooked processes near the base and two keels deep red. Column rather long pale with red spots, foot shorter, arms short erect tooth-shaped. Anther conic obtuse yellow with red spots.

Kedah: Gunong Jerai, alt. 4000 feet (Ridley). Endemic.

\section{§ 2. Monantha pairva.}

15. B. striatelduar, Ridl. Ann. Bot. IV. p. 335. Rhizome filiform creeping, pseudobulbs curved conic $\frac{1}{2}$ an inch long, and half inch apart. Leaf erect lanceolate acute 2 inches long $\frac{1}{3}$ inch wide. Seape filiform little longer than the pseudobulb with one or two sheaths at the base. Flowers solitary small nodding, pedicel an inch long. Sepals similar oblong or oblong lanceolate caudate yellow with three red veins half an inch long. Petals ovate oblong apices rounded, beneath yellow, apex spotted red two red spots at base. Column short arms suberect rather short obtuse.

Singapore: Chan Chu Kang, Kranji (Ridley).

16. B. Catenalium, Ridl. Trans. Limn. Soc. II. Bot. Iv. 235. Rhizome slender branched with oblong internodes long tips upeurved, green. Leaf $\frac{1}{t}$ inch long elliptic obtuse. Scape filiform an inch long, one flowered. Pedicel slender one inch. Flower orange $\frac{1}{t}$ inch long. Upper sepal lanceolate obtuse, laterals longer and broader, orange with red streaks. Petals lanceolate $\frac{1}{3}$ inch length of the sepals yellow with a central red streak. Lip ovate acute fleshy papillose, dark red, channelled. Column short and thick, arms long erect narrow acute acuminate. Capsule elliptic $\frac{3}{16}$ inch long.

Singapore: Kranji on mangroves, Chan Chu Krang (Ridley); Selangor: Bukit Hitam (Kiclsall); Pahang: Kluang Terbang (Barnes); Perak: Larut Hills (Curtis). Distrib. Borneo.

1\%. B. Avicella, Ridl. Journ. Linn. Soc. XXXII. p. $2 \% 0$. Rhizome long and very slender, pseudobulbs an inch apart obpyriform $\frac{1}{2}$ inch long. Leaf $\frac{3}{4}$ inch long $\frac{7}{16}$ inch wide exactly ovate subacute, 
coriaceous dark green. Seapes $\frac{1}{4}$ inch long with solitary reversed small flowers. Bracts very small (eup-shaped mucromate. [Pper sepa] lanceolate actue + inch long green, laterals much longer comnivent into a boat-shape deep crimson. Petals minute cuneate mucronate pallid. Lip shortly stipitate. Claw thickened erect blade lanceolate subohtuse flat narrow orange coloured with a crimson groove purple beneath papillose. Column thick rosy, foot as long, arms long linear truncate

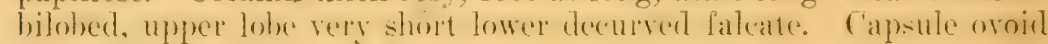
2 inch long.

Singapore: Bajau (Ridley 2361), Kranji; Penang: Govermment IIill 2000 teet (Ridley r00t). Endemic.

\section{$\S 3$. EPICRINTHES.}

18. B. EpICndantmes, Hook. fil. Flor. Brit. Ind. T. 753. Rhizome tolerably thick covered with sheaths pseurlobulls 1 inch alpat half an inch long fusiform enchenel in a sheathing leat. Leal clliptice oblong coriaceous blunt or acute an inch long, half an inch or more wide. Seape very short one flowered. Bracts lanceolate. Sepals $\frac{1}{4}$ inch orate lanceolate acute pink, spreading. Petals represented by 6 or 8 terete chubed threads white at hase grey at tip, less than half as long as sepals. Lip elliptic lanceolate subacute warted deep purple. Column stout foot shorter, pinkish upper part deep purple arms erect acute, taller than anther. Anther depressed with a long up-curved blunt beak.

Perak: Tapah (C'urtis). Distrib. Java.

\section{$\S$ 4. HIRTULA.}

19. B. Texbrum, Ridl. Journ. Roy. As. Soc. S. Br. Vol. IXXIX:

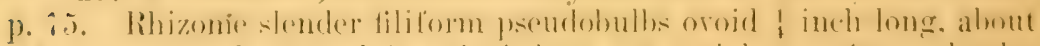

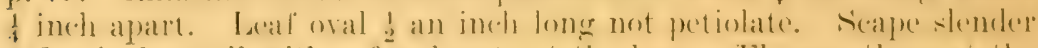
red 2 inches tall with a few bracts at the base. Flowers thee at the apex, small 1 inch long, pedicelled. Upper sepal lanceolate acuminate, latcrals much longer slighly gihbous at base purple with green hases.

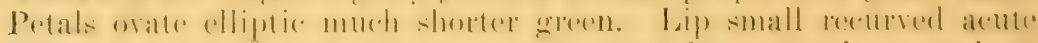
purple. Columi thick curved, green foot as long, purple, arms long linear acute curved.

\section{Lankawi (C'mtis). Indemic.}

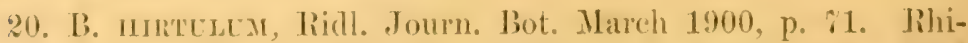
zome slender, pseudobullos globular crowded hardly if inch long. Leaf

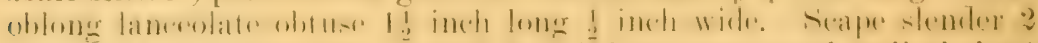
inclies long two flowered, flowers t inch long, ovary and pedicel short red. Lpper sepal ovate lanceolate acute, laterals rider falcate, pubescent white tipped with purple. Petals narow linear shorter, white with brown icius and cilia alumg the crleres. Lip tongue-haped 
straight. subacute deep red hairy. Column short and broad green with a red base, arms very short blunt rounded. Anther hat-shaped orange.

\section{Penang Hill at 2000 feet alt. (Ridley). Endemic.}

21. B. cincrivatuar, Ridl. Journ. Roy. As. Soc. S. Br. Vol. XXXIX. p. 75. Pseudobulb rery small. Leaf eliptic obtuse, closely nerred $t$ inches long by 2 wide. Scape rery slender 2 inches long. Flowers small, inch long, 2. Bracts orate rery short, ovary and pedicel $\frac{1}{8}$ inch long. Sepals lanceolate subacute, nearly equal, brown, hairy. Petals brown linear oblong falcate obtuse hairy. Lip oblong obtuse with long white hairs. ('olumn short foot as long, ar'ms short.

\section{Perak: Batu Tujoh (Curtis). Endemic.}

22. B. Limbatum, Lindl. Bot. Reg. 1840, Misc. 74. Rhizome stout pseudobulb depressed subglobose. Scape stout many flowered, 4 inches long. Flowers $\frac{1}{4}$ inch long, pedicel and ovary very short and thick. Sepals orate obtuse edges ciliate dark purple. Petals spathulate villous purple. Lip fleshy ovate obtuse coneave, dise smooth, margins scabrid, dull green tinted purple. Hook. fil. Fl. Brit. Ind. VI. $\% 63$.

Singapore (Hort Loddiges). This has not been met with again anywhere.

\section{§. RACEMOSAE.}

23. B. Wrayi, Hook. fil. Fl. Brit. Ind. V. \%66. Ic. Pl. 2044. Rhizome woody stout, pseudobulbs conic an inch long, and about an inch apart. Leal elliptic thinly coriaceous obtuse, 8 inches long by $2 \frac{1}{2}$ wide tapering into a petiole 4 inches long. Scape stout 12-18 inches long, with several sheaths at the base and some distant ones above, racemose part 6 inches long. Flowers numerous whorled or subwhorled. Bracts short $\frac{1}{8}$ inch long ovate. Pedicel $\frac{1}{4}$ inch long. Flowers green with pink spots $\frac{1}{4}$ inch to $\frac{2}{3}$ inch (Hooker) long. Sepals lanceolate obtuse laterals falcate. Petals broad oblong obtuse narrowed in the middle, about halt as long as the sepals. Lip three lobed with a short narrow claw, lateral lobes rounded, two elevated bosses on the disc between them, midlobe lanceolate with a narrow base, obtuse fleshy. Column with broad wings, arms acute narrow, foot very short apex free. Anther elliptic large with a long fleshy pustular conic process erect on the top.

Perak: Gunong Berumbun, Pahang (Wray) at 8000 feet alt., Larut Hills at 2500 (Derry). Endemic.

Mr. Derry's plant has flowers only $\frac{1}{4}$ inch long, but the structure which is most peculiar is quite that figured in the Icones. Wray's specimens were incomplete. 
24. B. LAsintury, Lindl. Gard. Chron. 1855, p. 53. Phizome rel'y stout wody 1 inch through, roots copious, pseudobulbs cylindric $2 \frac{1}{2}-3$ inches long, three inches or more apart. Leaf lanceolate acute 9 inches long, three inches wide petiole an inch long. Scape stout t-10 inches long, base (a third) covered with close red sheaths, raceme about 15 thowererl. Bracts lanceolate acute half an inch long. Flowers purple hairy an inch long. Ovary and pedicel $\frac{1}{2}$ an inch long. Upper sepal linear oblong laterals as long base ovate concare. Petals shorter lanceolate acuminate glabrous. Lip glabrous. Capsule cylindric narrowed at the base $1 \frac{1}{2}$ inch long. De Vriese Ill. Orch. Ned. Ind., Miquel Fl. Ind. Bat. III. 649, Hook. fil. Fl. Brit. Ind. V. \%63.

Perak: (Scortechini); Penang: Government Hill (Curtis), Bukit Laksamana (Curtis 1732). Distrib. Sumatra.

25. B. Alciconxe, Far. \& Rchb. fil. Trans. Linn. Soc. XXX. 151. Rhizome long covered with matted roots woody $\frac{1}{x}$ inch thick, pseudobulbs small globose covered with brown sheaths $\frac{1}{8}$ inch apart. Leaf oblanceolate obtuse coriaceous fleshy 3 to 4 inches long half inch across, tapering into a petiole an inch long. Scape graceful 5 inches long peduncle pink nude 4 inches, raceme decurved 1 inch long. Flowers crowded appressed to the rachis about 20. - Bracts very small ovate, ovary and pedicel very short. Upper sepal nearly $\frac{1}{4}$ inch long, lanceolate acute white tipped with purple and with purple reins, laterals a little longer linear oblong obtuse pink. Petals about half as long ovate cuspidate denticulate white, teeth point and nerve purple. Lip shortly clawed, blade straight oblong obtuse elge ciliate, yellowish claw pink. Column stout white foot shorter, arms large bifid lobes linear oblong with two teeth. Fruit elliptic $\frac{3}{6}$ inch long. Hook. fil. Fl. Brit. Ind. VI. 766.

Penang: Government Hill at 2400 leet (Fox), West Hill (Curtis 996). Distrib. 'Tenasserim.

\section{DENSIFLORA.}

26. B. gigas, Ridley Journ. Linn. Soc. XXXII. p. 2\%\% Rhizome

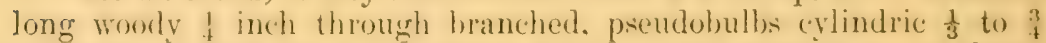
inch long inch thick, six inches apart. Leaf obovate or oblanceolate coriaceous blade 12 inches long, 6 inches wide, with a petiole 6 inches long. Scape stout erect 18 inches tall with large sheaths 4 inches long scattered; raceme 5 inches long many flowered nodding. Flowers $\frac{1}{2}$ inch long. Bracts orate $\frac{1}{2}-\frac{i}{4}$ inch long. Sepals lanceolate acute laterals gibbous at the base. P'etals linear narrower. All white with red spots. Lip small sessile shorter than the column hastate obtuse curved with two parallel keels orange. Column thick white with three purple spots, foot short orange, arms two toothed.

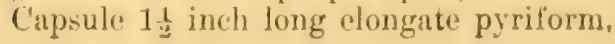


Perak: Larut Hills on rocks and trees (Ridley 5193), (Kunstler 4280). Endemic.

2\%. B. вотryophorem, Ridl. Journ. Línn. Soc. XXXII. 2\%. Rhizome slender far creeping with no pseudobulbs. Leaves $\frac{1}{2}$ inch apart lying flat on the rhizome, elliptic fleshy deep green an inch long, $\frac{1}{2}$ inch across. Racemes pendulous an inch long. Bracts minute ovate. Flowers very small crowded deep purple, $\frac{1}{8}$ inch wide. Upper sepal lanceolate acuminate pallid with three streaks laterals much longer semiovate falcate, deep crimson. Petals quadrate truncate white with crimson edge. Lip fleshy, with an oblong claw an ovate elliptic obtuse deeurved papillose blade with a thick semicircular ridge at the base. Column rather tall, broad, arms large rounded truncate broad crimson. Anther helmet shaped pustular. Capsule $\frac{1}{2}$ inch long oblong elliptic sessile.

Singapore: Kranji, Toas (Ridley); Pahang: near Pekan (Ridley). Endemic.

28. B. trifolium, Ridl. Journ. Linn. Soc. XXXII. 278. Rhizome thick with copious roots, pseudobulbs crowded conoid-ovate $\frac{1}{4}$ inch long. Leaf lanceolate acute 6 inches long one inch wide. Scape an inch long, raceme $\frac{1}{2}$ inch long capituliform. Flowers very numerous pubescent pink $\frac{1}{4}$ inch long. Bracts lanceolate acute pubescent. Upper sepal lanceolate acute white with pink stripes, laterals longer oblique acuminate. Petals shorter-linear lorate obtuse. Lip with a short deflexed claw tongue-shaped blunt, fleshy warted white with pink spots and a central crimson groove. Column green, short, with a large white tooth projecting from the inner face, foot long straight, arms long suberect truncate oblong.

Singapore: Sungei Morai (Ridley); Johor: Tana Runto (Ridley); Pahang: Pekan (Ridley 1428). Endemic.

29. B. LILACINUM, Ridl. l.c. 276. Rhizome stout $\frac{1}{8}$ inch through woody with long stiff roots, pseudobulbs thick conic $1 \frac{1}{2}$ inch tall 1 inch through at the base, distant. Leaf oblong lanceolate thick obtuse 5 inches long one inch wide, petiole $\frac{1}{4}$ inch. Scape 4 inches long thick with sheaths at the base, raceme 3 inches long dense. Upper sepal lanceolate acute, laterals longer lilac with pink spots. Petals much shorter than sepals, claw stout, blade lanceolate obtuse flat straight. Column rather long foot as long arms lanceolate acute erect.

Kedah: Gunong Jerai (Ridley), Lankawi, Niur Stali (Curtis). Distrib. Southern Siam.

30. B. Dexsifloisur, Ridl. 1.e. 2\%\%. Rhizome very long woody thick with long wiry roots. Pseudobulbs narrowly oblong terete, 1-2 inches long $\frac{1}{4}$ inch through, 6 inches apart. Laef lanceolate acuminate acute 12 inches long by $1 \frac{1}{4}$ wide, petiole 1 inch long, coriaceous, 
Raceme dense glabrous pendulous two incles long, perluncle $1 \frac{1}{2}$ inch long, thick, covered with swollen sheaths. Flowers $\frac{1}{2}$ inch long. Bracts nearly $\frac{1}{2}$ inch long lanceolate. Upper sepal $\frac{3}{8}$ inch long ovate lanceolate mucronate, laterals longer acuminate actute, purplish green with darker spots. Petals lanceolate mucronate $\frac{1}{t}$ inch long. Lip fleshy tongue-shaped apex decurved purple. ('olumn nearly globose short white with purple spots foot narrow apex free, arms erect triangular acute. Capsule $1 \frac{1}{2}$ inch long pear-shaped.

Singapore: Chan Chu Kang, Bukit 'Timah, Kranji on trees in forests (Ridley). Endemic.

\section{§\%. INTERVALLATAE.}

31. B. stella, Ridl. Journ. Linn. Soc. XXXT. (1896), p. 2\%. Pseucholulbs crowded oroid 3 inch long, roots copious. Leares lanceolate subobtuse narrowed at the base thick 6 inches long, 11: inch wide. Seapes 18 inches long nude for the greater part except for a few sheaths, raceme about 80 flowered, rachis thickened. Bracts ovate $\frac{1}{8}$ inch long, ovary and pedicel half an inch. Flower large patent. Sepals lanceolate acuminate $1 \frac{1}{4}$ inch long yellow with red streaks. Petals very short $\frac{1}{8}$ inch long, similatly colotired. Lip oblong lancerolate acominate 1! inch long dark pink with two kecels from the base. Column green short, foot as long, arms very short rounded.

B. Vanessa, King. Journ. As. Soe. Beng. LXVI. ii. 3, p. $58 \%$.

Singapore: Bukit Mandai, Jurong (Ridley); Perak: (Scortechini; Kedah: Gunong Jerai (Ridley), Batang Padang River (Ayre). Endemic.

I see no difference between the description of $B$. Tanessa, King, and $B$. Stella.

32. B. cleistoganum, Ridl. 1.c.c. Pseudobulbs densely crowded conic $\frac{3}{4}$ inch long. Leaf oblong or oblanceolate $4-6$ inches long, $\frac{3}{4}$ to 1 inch wide petiole half inch long. S(atpes seroral stiff 12 inches long with a few distant sheaths. Raceme short flattened. Bracts orate acute, 1 inch long. Flowers eleistogamous not or rarely opening. Sepals lanceolate acominate pale yellow, 8 inch long laterals griblous at hase red inside. Peals mimute quadrate tootherl with a long cusp), green. Lip long corved acote lateral lobes short laleate median fong natrow acominate, with two patralled apporoximate lieds, batse crimson, lilade straw coloul. ('olumm short thick geren with erect subulate arms foot very short, rostellum absent:

Perak: Waterloo (Curtis). Distrib. Rhio, Borneo.

\section{§ 8. OXYSEPALUM.}

33. B. Clindistmum, Lindl. Bot. Reg. XXVII. 1841, Misc. 166. Stems tufted pendent branched slender 6 inches to a foot long, 
corered with short close fitting ribbed sheaths, pseudobulbs $\frac{1}{4}$ inch apart, or more distant, oval very small $\frac{1}{x}$ inch long. Leaf elliptic oblong coriaceous $\frac{1}{2}$ inch long by 1 inch wide olstuse narrowed at the base. Flowers borne singly or in pairs in axils of sheaths, on very short perlicels yellowish white $\frac{1}{8}$ inch long. Sepals lanceolate caudate subequal. Petals very much shorter ovate translucent blunt. Lip as long as petals entire orate fleshy. Column short and thick, arms very short erect blunt. Fruit elliptic $\frac{1}{8}$ inch long. Hook. fil. Fl. Brit. Ind. V. 653. B. trisetosum, Grift. Notul. iii. 23, Ic. Pl. Asiat. t. 293. Oxysepala ovalifolia, Wt. Ic. t. 1\%36. Epidendrum sessile, Koenig. Retz. observ. VI. 60.

Singapore: Kranji, ete.; Johor: Kota Tinggi (Ridley) ; Pahang: 'Tahan liver (Ridley); Malacea: (Griffith), Batu Berendam (I)erry); Perak: (Scortechini); Penang: Telok Tikus (C'urtis 1929). Distrib. Borneo, Java, Tenasserim.

34. B. capitatum, Lindl. Gen. and Sp. Orch. Pl. p. 56. Stems stout woody in a tuft 6-9 inches long branched pendent or erect covered with dry ribbed sheaths, pseu(bolulbs conic $\frac{1}{2}$ to an inch long $\frac{1}{8}$ to $\frac{1}{4}$ inch through crowded or $\frac{1}{2}$ inch apart. Leaf lanceolate or elliptic. lancerolate acute or obtuse, 2 to $t$ inches long $\frac{1}{2}$ to 1 inch across coriaceous. Flowers in a short raceme usually half an inch long or less orange scarlet about 6 or 8 in a raceme. Sepals lanceolate acuminate $\frac{3}{16}$ inch long. Petals shorter narrow linear half as long as the sepals, all red. Lip small tongue-shaped yellow. Column short stout arms suberect acuminate. Hook. fil. Fl. Brit. Ind. T. 759. Diphyes capitata, Bl. Bijdr. 314.

On hills 2 to 6,000 feet alt. Johor: Batu Pahat (Ridley) ; Pahang: Kluang Terbang (Barnes 1122s): Perak: Bujong Malacea (Ridley), Gunong Batu Putih (Wray), Larut Hills : (Curtis); Penang: Government Hill (Curtis 2883). Distrib. Borneo, Java.

\section{§. DipHYES.}

35. B. Brevipes, Ridl. Journ. Roy. As. Soc. Str. Br. Tol. IXXTI. p. \%5. Rhizome woody, pseudobulbs $\frac{1}{2}$ to $\frac{3}{4}$ inch apart cylindric conic curved $\frac{1}{2}$ inch long. Leaf elliptic slortly petioled an inch long $\frac{1}{4}$ to $\frac{1}{3}$ inch wide, apex subacute coriaceous. Raceme very short about 6 flowered $\frac{1}{4}$ inch long. Flowers pale yellow. Bracts lanceolate much longer than the orary. Sepals subequal lanceolate acuminate $\frac{3}{8}$ inch long. Petals about $\frac{1}{3}$ as long elliptic blunt. Lip shorter rurved fleshy deeply grooved, base clawed, with two strongly raised ridges from the base. Column short and thick with a short foot. Arms erect narrow acuminate.

Perak: Bujong Malacea (Ridley), loc. incert (Scortechini drawing No. 1\%6). Endemic, 
This is allied to B. Gamb7ei Hook. fil. but with a much shorter peduncle.

36. B. coxcrstur, Hook. fil. Fl. Brit. Ind. VI. 189. Ic. Pl. 2038. Rhizome slender far creeping, with numerous wiry roots, psedudobulbs 1 to 2 inches apart, conic curved $\frac{1}{2}$ inch long. Leaf ovate. or ovate lanceolate or elliptic $1-1 \frac{1}{2}$ inch long $\frac{1}{2}$ to 1 inch wide. Scape close to the pseudobulb very slender $1-1 \frac{1}{4}$ inch Jong. Flowers subterminal 4 or 5 . Bracts lanceolate nearly as long as the short orary. repals narrow lanceolate linear acute $\frac{1}{6}$ inch long connivent, similar yellowish with orange tips. Petals about half as long elliptic blunt pallid. Lip as long as petals lanceolate curved orangerest. ('olumm stont thick, foot shorter, arms erect slender acuminate. Anther depressed.

Singapore: Sungei Buluh (Ridley 2359), Kranji (Ridley); Johor: Kwala Kahang (Kelsall). Distrib. Borneo.

3\%. B. xrodestux, Hook. fil. Fl. Brit. Ind. T. 59 . Ic. Pl. 2038, B. Rihizome slender long, pseudobulls, $\frac{1}{3}$ inch apart narrowly oroid oblique $\frac{1}{2}$ inch long. Leaf linear narrowed at the base apex blunt $\frac{1}{2}$ to 2 inches long, $\frac{1}{6}$ inch wide. Scape slender, 1-1 $\frac{1}{2}$ inch long. Bracts minute lancerlate, at long as the pediced. Flowers :3-5 subterminal very small $\frac{1}{6}$ inch long. Upper sepal orate lanceolate subacute, laterals broader obtuse. Petals two-thirds of the length of the sepals linear oblong. Lip tongue-shaped obtuse recurved with two keels.

Perak (Scortechini), Bujong Malacea (Ridley). Endemic.

38. B. veranculane, Hook. fil. Fl. Brit. Ind . TI. p. 188. Rhizome slender far creeping, psendobulbs in inch apart rery small globose $\frac{1}{x}$ inch long concealed in sheaths. Leaf elliptic oblong to linear obtuse narowed into a petiole 1-3 inches long $\frac{1}{4}$ inch wide or more coriaceous. Scapes $1 \frac{1}{2}$ inch long close to the pseudobulbs, with 5 or 6 scattered flowers, pale green. Bracts lanceolate acute as long as the ovary. Lpper sepal lanceolate, laterals falcate lancenlate areute base giblous, $\frac{1}{16}$ inch long. Petals smallere subspathulate lancesolate. Lip entire lanceolate oldtuse fleshy reelow, oflge ciliate with white hair's. Column short not thick, arms short obtuse.

Singapore: Seletar, Kranji (Ridley 2360); Johor: Kiwala Kiahang (Kelsall); Perak: Bujong Malacea (Ridley 9602 form with very narrow leares. Endemic.

39. B. ocmentucy, Ridl. Joum. Roy. As, Soc. Str. Br. Vol. XXXIX. p. r6. Psendobulbs $\frac{1}{4}$ inch long oblong conic densely crowded. Leaf linear lanceolate acute base narrowed $1 \frac{1}{4}$ inch long $\frac{1}{8}$ inch wide. Seape nearly as long. Flowers 5 or 6 erowded in a head, about $f$ inch long. Braet lanceolate shorter than the ovary. Upper sepal narrow linear lanceolate acuminate, laterals one-quarter longer, 
all white with yellowish tips petals less than half as long as the upper sepal lanceolate obtuse, white. Jip small tongue-shaped acute recurved yellow. Column thick, foot shorter, arms narrow linear acute curved.

Perak: Larut Hills at 3000-4000 feet (Curtis). Endemic.

40. B. citrixum, Ridl. Rhizome covered with fibres of old sheaths pseudobulbs crowded or rarely an inch apart conic hardly an inch long. Leaf oborate obtuse fleshy dark green or purplish $1 \frac{1}{2}$ inch long $\frac{3}{4}$ inch wide. Scape filiform shorter or longer than the leaf. Flower's about ten crowded into a head whorled, lemon yellow, nearly 1 inch long. Bracts linear boat-shaped acute as long as the pedicels. Upper sepal ovate lanceolate acute, laterals lanceolate linear twice as lnng. Petals ovate acuminate or lanceolate acute, shorter than dorsal sepal. Lip fleshy curved thick subacute with two broad keels. Column thick longer than the foot. Arms very short blunt broad. Capsule elliptic $\frac{1}{2}$ inch long, pedicel $\frac{1}{8}$ inch. p. 279 .

Cirhopetalum citrinum, Ridley, Journ. Linn. Soc. XXXI. 1896,

Singapore: Tanjong Gol (Ridley 3932), Sungei Buluh (Ridley 168i); Johor: Sungei Bau (Ridley) : Malaceal : Mount Ophir ; Perak: Thaiping. Distrib. Southern Siam, Borneo.

41. B. andexopetalum, Lindl. Bot. Reg. 1842, Mise. 95. Rhizome stout with copious roots. Pseudobulbs obsolete, very small globose. Leaf thickly coriaceous oblanceolate to lanceolate obtuse 8 inches long by one across, petiole stout enclosed with base of scape in dry brown sheaths. Scape shorter than the leaf 6 inches long rather stout nearly completely floriferous. Flower's numerous scattered pale yellow $\frac{1}{2}$ inch long, pedicel $\frac{1}{8}$ inch. Bracts. lanceolate acuminate longer than the ovary. Sepals lanceolate acuminate, equal hardly gibbous. Petals very short oblong minutely ciliate blunt. Lip tongue-shaped fleshy blunt channelled ciliate. Column short and thick, arms erect short blunt. Hook. fil. Fl. Brit. Ind. VI.

"Singapore" Hort. Loddiges. cult. in Hort. Bot. Singap. without locality Angust 1893. Perak, Kamuning (Machado).

42. B. oblaxceolatud, King. Journ. As. Soc. Beng. LXVI. II. 3, p. 586. Rhizome stout densely clothed with coarse filores and roots, no pseudobulbs. Leares oblanceolate subacute tapering to a stout petiole, blade 6 to 8 inches long, $\frac{11}{7}$ inch to $\frac{12}{5}$ wide, petiole $2-2 \frac{3}{4}$ inch long. Raceme from the rhizome near a leat, peduncle as long as the leaf, with several sheaths $\frac{1}{2}$ an inch long. Flowers straw colour numerous not coloured $\frac{1}{3}$ inch across. Bracts lanceolate as long as the ovary. Sepals spreading linear lanceolate finely acuminate, laterals larger than dorsal, subfalcate. Petals $\frac{1}{3}$ of the length of the 
dorsal sepal linear acmminate. Lip half as long as the dorsal three lobed, side lobes erect rounded gradually merging in front with the narrowly oblong subacute, fleshy midlobe. Column very short, apical teeth small sharp erect.

Perak: (xmong Batu Putih (Tray 9S0). Tamut Hills at 1000 feet eleration (Ridley). Endemic.

43. B. rontigenux, Ridl. Trans. Linn. Soc. Tol. IV. p. 235. Rhizome stout nearly $\frac{1}{4}$ inch through, pseudobulbs none. Leaf oblaneerolate or oblong lanceolate subacute tapering into a long petiole, blade 6 to 8 inches long, $\frac{33}{4}$ inch wide coriaceous, petiole 1 to 3 inches long, enclosed with the base of the raceme in a long brown sheath an inch long. Raceme 5 inches or more long lower portion 1 inch nude. Flowers numerous scattered $\frac{1}{1}$ inch long, ovary and pedicel $\frac{1}{16}$ inch long. Bracts about as long lancerolate. Serpals lancerolate actuminate, laterals gibhous at the base, suberual. Petals less than $\frac{1}{3}$ of the length fleshy papillose lanceolate ohtuse. Lipe as long oblong lanceolate entire obtuse pubescent. Column very small foot longer, arms ereet neute.

\section{Pahang: Kluang Terbang (Barnes 1090). Distrib. Borneo.}

44. B. leptosepalua, Hook. fil. Fl. Brit. Ind. T. $6 \%$ Ie. Pl. 2045. Tufted plant with very small pseudobulbs crowded. Leaf linear often curved acute narrowed at the base into a long petiole, 4 inches long, $\frac{1}{2}$ inch wide. Scape 3 inches long graceful flowers scattered about 15 in number, $\frac{1}{4}$ inch long, pure white. Sepals lanceolate cauclate, laterals gibbous at the base. Petals linear lanceolate much shorter. Lip obseurely 3 lobed lanceolate; side lobes erect, median narrower fleshy pustular. Column short, foot long, arms erect narrow acuminate.

\section{Penang: Government Hill (Curtis 415). Endemic.}

45. B. A Poous, Hook. fil. Fl. Brit. Ind. Y. p. 766 , Ic. Pl. 2043.

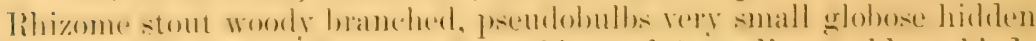
in sheaths. I,eaf stiffly coriaceous oblanceolate or linear oblong blade 3-9 inches long $\frac{3}{4}$ to $1 \frac{1}{2}$ inch wide petiole stont 3 inches long or less, rnclosed in a sheath when young, (whole leat' dries red). Raceme 4 inches long peduncle one inch or less. Flower's numerous, white tinged with yellow, tips orange, $\frac{1}{x}$ inch long seattered. Bracts lanceolate acuminate as long as the very short ovary. Sepals lanceolate

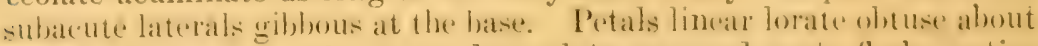
half as long. Lip pale orange lanceolate recurved acute fleshy entire (with small holes rommled at hatse Hook. fil.). Column short with crect acmminate arms as tall or taller than the anther. Capsule dliptic $\frac{1}{2}$ inch long nearly sessile. 
Singapore: Kranji (Ridley 393-t), Chan Chu Kang (Ridley 3933) ; Iralacea: (Maingay), Ayer Panas (Ridley, Merlimau (Derry); Negri Sembilan: Perhentian 'Tinggi (Ridley); Perak: top of Batu Kurau (Scortechini). Distrib. Borneo.

46. B. lineirifolium, King, Journ. As. Soc. Beng. LXVI. ii. :3, $189 \%$, . 586. Rhizome fairly stout, about $\frac{1}{6}$ inch thick through, pseudobulbs obsolete, $\frac{1}{2}$ an inch or more apart. Leaf linear acute or subacute often curved, narowed into a petiole 1-2 inches long, blade \pm to 6 inches long $\frac{33}{8}$ to $\frac{1}{2}$ inch wide. Scape very slender base enclosed with the petiole in a scarious sheath, half an inch long, peduncle 1-1 $\frac{1}{2}$ inch, raceme very lax $3-t$ inches long. Flowers $\frac{1}{8}$ inch long yellow. Bracts lanceolate acuminate as long as the slender pedicel orary dilate. Sepals lanceolate blunt, laterals broader and falcate gibbous at the base. Petals narow linear ahout half as long. Lip oblong fleshy acute or obtuse slightly curved, entire. Column very short, arms erect taller than the depressed anther, broad laineolate blunt. demic.

Perak (Scortechini), Bujong Malacca (Ridley 9524). En-

4\%. B. oporatur, Lindl. Gen. and Sp. Orch. Pl. p. 5t. Rhizome far creeping woody with very small globose pseudobulbs. Leaf 7 or 8 inches long an inch wide oblanceolate obtuse with a petiole 3 inches long, coriaceous. Scape 16 inches rather stout almost entirely covered with flowers.' Flower's very numerous small $\frac{1}{8}$ inch long orange colour turning white fragrant. Bracts lanceolate actminate as long as the ovary $\frac{1}{8}$ inch long. Sepals lanceolate subacute or blunt, laterals similar not gibbous. Petals very small as long as the column ovate lanceolate. Lip very small curved obscurely three-lobed ovate, sides upcurved rather large, midlobe very small tongue-shaped flat obtuse. Column thick, foot oblong, arms entire or two toothed erect acute slender, the back one small subobtuse when present. Diphyes odorata, Bl. Bijdr. 312.

\section{Pahang: Tahan River (Ridley). Distrib. Java, Borneo.}

48. B. Globulus, Hook. fil. Fl. Brit. Ind. T. $76 \%$, Ic. Pl. $20 \pm \%$. Rhizome stout as thick as a swan's quill, pseudobulbs small globose or ovoid crowded $\frac{1}{3}$ inch through. Leaf $1-2$ inches long, linear oblong obtuse coriaceous $\frac{1}{2}-\frac{2}{3}$ inch through. Scape as long as the leaf stiff with two or three distant sheaths. Flowers small scattered. Bracts ovate acute very small as long as the pedicels. Upper sepal linear oblong, laterals lanceolate sulacute. Petals orate oblong subacute half as long. Lip minute lanceolate much recurved. Column short.

Perak (Scortechini). Endemic.

49. B. hedinuliferum, Ridl. Journ. Bot. 36, (1898) 211. Rhizome stout $\frac{1}{8}$ inch through woody, pseudobulbs distant oblong conic often curred flattened two inches long lialf inch wide $\frac{1}{8}$ inch thick. 
Leaf oblong lanceolate obtuse coriaceons narrowed at the base into a short stout petiole, t to 8 inches long 1 inch wide. Seape six inches long, base covered with thin rentricose sheaths, flowers about $25, \frac{1}{2}$ an inch lones. Bracts f inch long. Ionger than the pedicels. sepals lanceolate acuminate yellowish with orange tips, laterals twice as long as the dorsal one not gibbous. Petals very small oblong obtuse yellowish white. Lip fleshy tomgue-shaped obtuse pustulate rellowish with a red tip. Column with bifid arms the upper lobe lanceolate erect, the lower ones shorter rounded obtuse. Endenic.

Selangor on limestone rocks at the caves Kuala Lumpor (Ridley).

\section{¿ 10. Cirrifopétuluar.}

50. B. Eprnprex, Ridl. Rhizome $\frac{1}{4}$ inch thick branched, pseudobulbs conic 1-1 $\frac{1}{2}$ inch long $\frac{1}{2}$ inch thick. Leaf lanceolate acute,

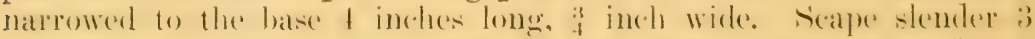
inches long. Flower large. Pedicel and orary $\frac{1}{2}$ inch long. Bract lanceolate. Upper sepal linear lanceolate subulate base ciliate on the edge, one inch long $\frac{1}{8}$ inch wide, laterals $1 \frac{1}{2}$ inch long lanceolate falcate subulate, pink with white edges. Petals small obliquely laneroJite ateute, hases denticulate pink with purple warts. Lip! inch long ovate acuminate thick ciliate with a long point crimson. Column straight rather tall, foot short, arms tooth-like erect.

Ephippium citiatum, Bl. Bijdr. 309. t. 65. Cirrhopetalum Blumii, Lindl. Gen. and Sp. Orch. p. 59.

\section{Singapore: Kranji (Ridley). Distrib. Jara, Borneo.}

51. B. liestrepla, Ridl. 'T'rans. Linn. Soc. II. iii. p. 365. Rhi-

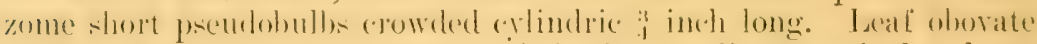
petioled 2 inches long, an inch wide. Scape filitorm 4 inches long.

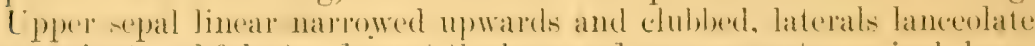
acuminate subfalcate edges at the base and apex commate, an inch long, ereen with brown erleses. Lip mall flesy orate decurved white sproted abose with pink. ('olumn short and thick, foot rery short green, arms bifid, frout lobe largest blint.

Pahang: Pekan on bushes (Ridley 1038). Endemic.

52. B. Bmiscapum, Ridl. Rhizome sheathed, psendobulbs oblong t angled st to 10 lines long covered hy two sheaths. Leat sub)-

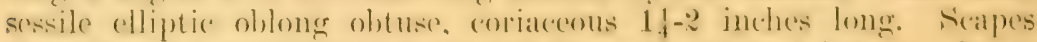

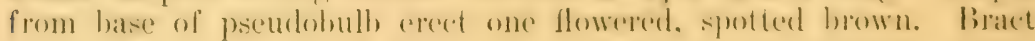
ovate lanceolate acute 2 lines long. Upper sepal lanceolate oblong acute incurved 8-9 lines purple, lateral sepal connate at the base only, oblong subobtuse deflexed, twisted at base 1 inch long, yellow with brown fpots. I'etals lanceolate acute patent s-9 lines loug, purple. 
Lip fleshy 5 lines long base widely cordate obtuse base covered with long hairs, crimson. Column broad at the base narrowed upwards teeth slender acute incurved. Anther puberulous.

Cirhopetalum breviscapum, Rolfe Bot. Mag.t. 8033.

Perak, cultivated at Glasnevin, and Kew Gardens in 1903.

53. B. PLdNibulbE, Ridl. Rhizome long ereeping slender, - psendobulbs elliptic oblong flat lying on the rhizome, purple an inch long $\frac{1}{4}$ inch wide, 3 inches apart. Leaf elliptic oblong obtuse an inch long, $\frac{1}{4}$ inch wide. Scape + inches long slender purple with a ferw sheaths and \pm or 5 flowers at the end. Bracts lanceolate acute $\frac{1}{4}$ inch long. Pedicels slender an inch long. Upper sepal erect lanceolate acute caudate an inch long, laterals deflexed 2 inches long, all yellow with red spots on the back and edges. Petals short obovate subspathulate yellow: Lip reeurved fleshy tongue-shaped acute, sides - elevated, and a median chamnel orange. Column stout yellow foot very short purple, arms long subulate as long as the petals.

Cirrhopetalum planibulbe, Ridl. 'Trans. Linn. Soc. III. p. 364, Pl. LXIV.

Pahang: Pekan (Ridley); Kedah: Yan (Ridley). Endemic.

54. B. Mxinus, Ridl. Cirhopetalum maximum, Ridl. Journ. Bot. March 1900 p. \%0. Rhizome far ereeping stout, pseudobulbs far apart narrow oblong flattened 5 inches long. Leaf lanceolate coriaceous 12 inches long, 4 inches wide petiole $1 \frac{1}{2}$ inch. Scape a foot long with a terminal whorl a foot across of 7 flowers each over 6 inches long including the thick pedicel an inch long. Bracts lanceolate acuminate reddish an inch long. Sepals lanceolate caudate. Lip tongue-shaped fleshy acute large cream colour with a violet centre. Column broad oblong cream colour with mauve markings, arms 4,2 short erect, 2 broad thick horizontal violet.

Perak: Bujong: Malacca on trees and rocks at 2000 feet (Ridley, Curtis 3125). Endenic.

55. B. medusae, Relib. fil. Walp. Ann. VI. 262. Rhizome very stout pseudobulbs an inch long thick angled conic. Leaf 6 inches long one inch wide, oblanceolate very coriaceous deep green, narrowed at base into a short petiole. Scape 4 to 5 inches long stout with large loose sheaths. Flowers in a large dense globose head an inch and a half through with large lanceolate pallid bracts at base half an inch long. Floral bracts lanceolate acuminate much longer than the short ovary. Sepals caudate, lanceolate, white pink spots, laterals six times as long as the dorsal, nearly six inches lone. Petals lanceolate acuminate pinkish much shorter. Lip shorter than the petals nearly straight lanceolate acute vellowish. Column thick, foot as long, arms 
porrect lanceolate acuminate wared long. Hook. fil. Fil. Brit. Ind. V.

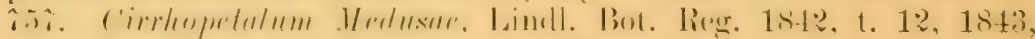
uncter t. 49, Bot. Mag. t. $496 \%$, Hort. P’arad, ii. t. õ.

vingapore: Bukit 'Timah, Bukit Mandi (Ridley) : Johor: Tengarah (Kelsall); Pahang: Tahan River (Ridley); Malacea: Jasin

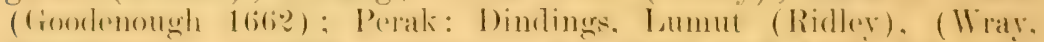
seorlechini): Penang (curtis). On rocks and trees not ralé. Distrib. Borneo, Siam.

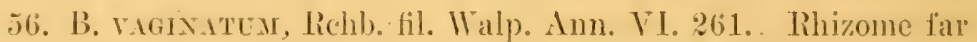
creeping rather stont, pseurlobulbs conic ovoid an inch long an inch apart. Leal rery fleshy and thick, coriaceous, elliptic or oblanceolate obtuse 1 to $t$ inches long $\frac{1}{2}$ to $\frac{3}{4}$ inch wide. Sacpes $3-1$ inches long covered with loose pale sheaths. Flowers numerous erowded into a head pale yellow. Bracts lanceolate acmminate twice as long as the short ovary. Upper sepal ovate obtuse edges ciliate. Lip little longer oblong lanceolate acute recurved fleshy orange. Column short broad, arms tooth-like subulate erect.

Cimhopelahm vaginatum, Lindl. Gen. and Sp. Oreh. Pl. s.), Bot. lieg. 1812 under t. 1\%, and $18+3$ under t. 19. Hook. fil. Fl. Brit. Ind. T1. $9 \% 3$. C. candalum, Wright, Ic. t. 1658.

Singapore (Wallich 1989), Kranji lioad (Ridley $23 \hat{\imath}$ ); Johol: 'l'ana liunto (Ridley 3931); Pahang: Pekan (Ridley 1608 12.14);

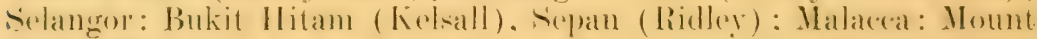
Opliir (lidley), Ching (Derry 589) ; Penang (Curtis 1969), Lankawi (curtis), Distrib. Borneo. Very common often growing in larege masses.

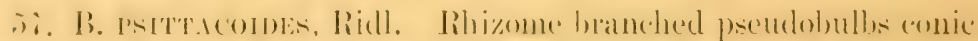

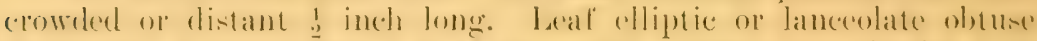

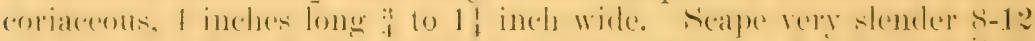

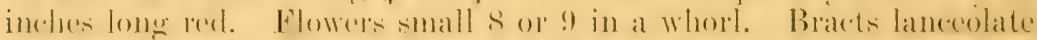
acuminate $\frac{1}{25}$ inch long. Ovary and pedicel $\frac{1}{4}$ inch long. Upper

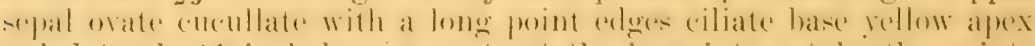
red, laterals $1 \frac{1}{2}$ jnch long connate at the base into a tube the points free setaceous dark ied with two yellow spots in the mouth of the tube. Petals lanceolate acuminate as long as dorsal sepal, with long points edges ciliate. Lip fleshy recurved acute violet. Column conie, loot broad about as long yellow dotted with red, arms rery short romederl. Capsule elliptic fusiform $\frac{1}{2}$ inch long. 280.

Cirrtiopetalum psillacoides, Ridl. Journ. Lim. Soe. IIXII.

C. gracillimium, Rolfe, Kiew Bulletin, 1895, p. 31.

Johor: Iimala Batu Pahat (Kelsall); Malacea: Sungei Hudang (Goudenough liou); legri sembilan: Gunong Ingsi (Ridley 
10356) : Dindings: Telok Sera (Ridley) : Perak: Thaiping (C'urtis), Lankawi (Curtis). Distrib. Siam.

58. B. Marogaxur, Ridl. Rhizome rather slender. Pseudobullse an inch apart oroid nearly an inch long. Leal elliptic obtuse or lanceolate narrowed at the base coriaceous 3 to 6 inches long $\frac{1}{2}$ to 1 inch wide, shortly petiolate. Scape slender red $i-s$ inches long. with one or two sheaths. Flowers 11 in a whorl. Bracts lanceolate acuminate $\frac{1}{8}$ inch long purplish. Ovary and pedicel inch long. Lpper sepal acuminate red edges with yellow hairs, laterals comnate long narrow linear 2 inches long, yellow with red spots. Petals ovate lanceolate acuminate, yellow with red spots or red. Lip small fleshy curved blunt grooved yellow with a red edge or red. Column stout, foot sigmoid, arms broad acute.

Cirrhopetalum makoyanum, Rehb. fil. Gard. Chron. 18\%9, p. 243. Hook. fil. Bot. Mag. t. 7259 .

\section{Singapore: Chan Chu Kang (Ridley).}

Var. Brienianum. Upper sepals and petals brown purple, lower sepals yellow.

C. Brienianum, Rolfe. Kew Bulletin, 1893, p. 62.

Johor: Gunong Panti (Ridley); Perak: Thaiping (Ridley). Distrib. Borneo.

59. B. Curtisir, Ridl. Journ. Roy. As. Soc. S. Br. Vol. XXXIX. p. 76. Rhizome slender creeping pseudobulbs oroid conic $\frac{3}{8}$ inch long, $\frac{1}{2}$ an inch apart. Leaf elliptic oblong obtuse thick $1-1 \frac{1}{2}$ inch long $\frac{1}{2}$ inch wide rery shortly petioled. Sicape slender $2-3^{2}$ inches long: with a lanceolate pointed sheath in the middle. Flowers crowded at the top, about 5. Bracts lanceolate acuminate. Upper sepal triangular lanceolate laterals quite free linear flat narrow $\frac{3}{8}$ inch long yellow. Petals falcate lanceolate, all ghabrous brown. Jip small tongurshaped fleshy eurved. Column broad arms triangular short.

Dindings: in mangrove swamps (Curtis). Endemic.

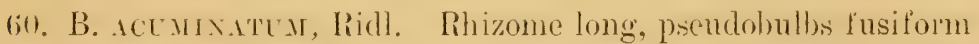
conic an inch apart, $\frac{1}{2}$ inch long. Leaf lanceolate acuminate, 3 inches long nearly one across. Scape 6 inches long slender, 5 flowered in a half circle. Bracts lanceolate acute. Upper sepal ovate aristate ciliate orange with red stripes laterals an inch long narrow elongate acuminate free, reddish with yellow tip. Petals orate ciliate with a long point orange with red stripes. Lip short obtuse slightly curved greenish with purple spots. Column rather long arms short. 282.

Cirropetalum acuminatum, Ridl. Journ. Limn. Soe. XXXII.

Singapore: Chua Chu Kang (Ridley). Endemic. 
61. B. Iricrobejuox, Ridl. Thizome slender pseudobulbs glo-

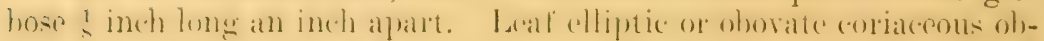
tuse 11 to 13 inch long, 3 inch across. Seape very slender filiform it inches long. Flowers whorled 8, small. Bracts lanceolate acute $\frac{1}{16}$ long. Upper sepal ovate cuspidate edge ciliate laterals very narow

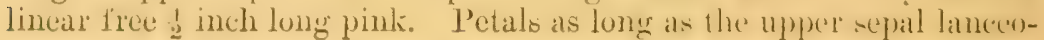
late cuspidate ciliate. Lip minute orate. 283.

Cimhopetalum microbulbon, Ridl. Journ. Limn. Soc. XXXII.

\section{Singapore: Sungei Buru (Ridley). Endemic.}

62. B. Auratus, Ridl. Rhizome short, psendobulbs crowded ovoid grooved and wrinkled $\frac{3}{4}$ inch long, $\frac{1}{2}$ inch thick. Leaf ol)lanceolate petiolate thick obtuse purple six inches long one inch wide. Scape graceful six inches tall nodding rosy, flowers 8 in a whorl. Bracts small lanceolate. Upper sepal orate aristate yellow with 5 deep erimson stripes edges hairy, laterals nearly an inch long free $\frac{1}{5}$

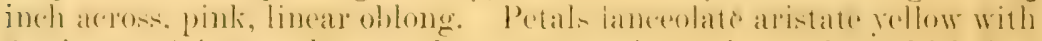

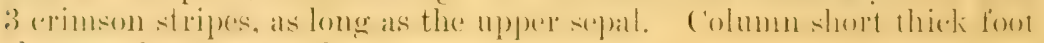
about as long, arms obscure.

Cimpopelatum ammalum, Lindl. Bot. Reg. 18t0, Misc. p. 50. l.c. 1843, t. 61 .

\section{Selangor: Sepan (Ridler).}

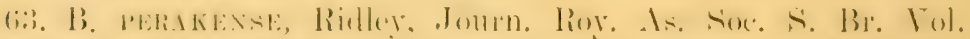

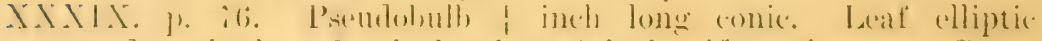
narrowed at the base $2-3$ inches long $\frac{1}{2}$ inch wide coriaceous. Seape 3-4 inches long lairly stout, flowers crowded numerous, glabrous. Bracts lanceolate acuminate. Upper sepal ovate, acute, laterals in inch long, comate for half their length tips acuminate. Petals nearly as latge as the upper sepal onate laneolate ante. Lip tongue slapeel channelled above, but little eurved. Column arms triangular obtuse erect broad.

\section{Perak: Waterloo (Elphinstone). Endemic.}

61. B. svanibifidur, Ridl. Thizome slender pseudolublys conic angled $\frac{1}{2}$ to $\frac{3}{4}$ inch apart $\frac{1}{4}$ inch long. Leal lanceolate coriacens $1 \frac{1}{2}$

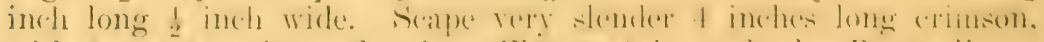

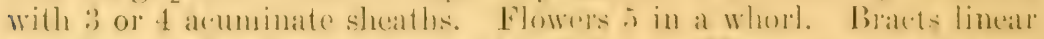

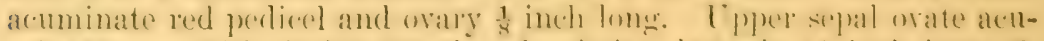

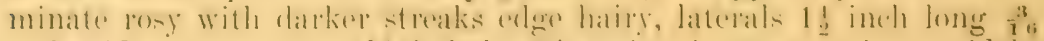

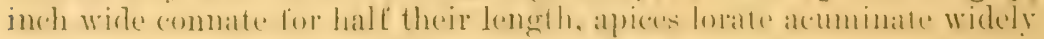
separate, deep crimson. Petals ovate lanceolate falcate acuminate edges hairy. Lip tongue shaped curved y'osy, tip paler. Columm rather long yellow arms short romuded.

C'irrhopetulum bifidum, Ridl. Journ. Limn. Soe, XXXII. 2S4, 


\section{Singapore: Kranji (Ridley). Endemic.}

65. B. straxomyzux, Ridl. Rhizome long slender covered with papery sheaths, pseudobulbs conic cylindric 2 inches apart $\frac{1}{3}$ inch long." leaf narrowly linear lanceolate olutwe 4 inches long 1 inch wide. Scape 6 inches long reddish. Flowers few and small. Bracts 4 inch long linear acute. Upper sepal lanceolate acute greenish with dark purple streakis edge hairy, laterals ahnost completely connate, linear acuminate apices setaceous pale red $\frac{1}{2}$ an inch long. Potals triangular lanceolate acuminate green streaked with purple. Lip tongue shaped subacute curved fleshy purple. 283.

Cirrhopetalum linearifolium, Ridl. Journ. Limn. Soc, XXXII.

Singapore: Changi, Chan Chu Kang, ete. (Ridley). Endemic.

66. B. pulchellus, Ridl. Rhizome slender, pseudobulbs crowdpel conicle 3-angled an inch long. Leaf fleshy narowly oblanceolate obtuse narrowed at the base, 3-6 inches long, $\frac{1}{2}$ inch wide petiolate. Scape slender 2 to 4 inches long, flowers about 12 in a whorl. Bracts narrow lanceolate setaceous half as long as the ovary. Upper ovate aristate yellowish densely spotted with red eilge ciliate laterals almost. completely connate oblong oblanceolate obtuse. Petals lanceolate aristate hairy on the edge. All similarly coloured. Lip thick curved deep purple with a yellow tip. Column thick, foot about as long, arms short broad rounded or truncate.

Cirrhopetalum concinnum, Hook. fil. Fl. Brit. Ind. V. p. 190. Te. Pl. t. 2060 B.

Singapore: Chan Chu Kang (Ridley) ; Johor: Tana Runto (Ridley); Sclangor: Sepan (Ridley).

Var. purpureum, Ridl. whole flower deep crimson, except the yellowish tip. Sepals shorter and broader. Lip more abruptly curved.

Penang Hill (Curtis). Distrib. Islands south of Singapore, Siam (var. purpureum).

Var. brachysepalum. Flowers very small, laterals comnate only in the upper part, tips rounded, hardly $\frac{1}{4}$ inch long, flowers coloured as in var. jupureum. Possibly a distinct species. Lankawi: Kwah (Curtis).

6\%. B. Griffitmindar, Par. Tichb. fil. 'T'rans. Linn. Soc. XXX. 15:). Rhizome moderately stout, psendobulls an inch apart or lesis conic, grooved $\frac{1}{2}-\frac{3}{4}$ inch tall. Leaf oblong lanceolate obtuse or oblanceolate, coriaceous $t$ inches long $1 \frac{1}{8}$ inch wide. Scape 8 inches tall slender, Howers whorled 8-10. Bracts linear lanceolate setaceous, whorter than the pedicel redl. Upper sepal orate artistate hairy, yellowish brown or pink with darker spots laterals sepals connate oblong 
Materials for a Flora of the Malayan Peninsula.

obtuse, $\frac{3}{4}$ inch long $\frac{1}{6}$ inch wide, reddish or pink with dark spots, or

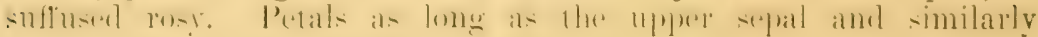
coloured lanceolate aristate edges hairy. Lip fleshy rery small recurved channelled with 3 obseme ribs yellow tipped purple, or brown. Column thick foot hardly as long, yellowish or rosy spotted dark rose, arms short broad romnded.

Cimhopetalum gamosepalum, Griff. Notulac, III. 296. Hook. fil. Fl. Brit. Ind. V. $3 \% 4$. Ann. Bot. Roy. Gard. Calc. Vol. V. p. $1 \%$. Plate 26. C'. Andersonii, Kur\%, liep. Toy. Andam. s1.

Singapore: Bajau (Riclley); Johor: Kwala Kahang (Kelsall); Malacea: Sungei Rambei. Distrib. 'T'enasserim, Borneo.

68. B. ocirnceux, Ridl. Rhizome long, psendobulbs rather crowded conic 4 angled-1 $\frac{1}{2}$ inch long $\frac{1}{2}$ inch thick. Leaf oblanceolate obtuse thinly coriaceous, $\gamma$ inches long one inch wide. Scape rather stout 10 inches red. Flowers about 10 rather large. Bracts lanceolate very acuminate. Upper sepal orate acuminate reddish edge hairy, laterals connate for their whole length oblong lanceolate obtuse an inch long, 1 inch wide orange ochre, with red spots. P'etals lanceolate

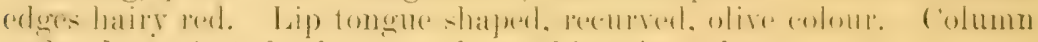
rather long, foot slender, arms large thin triangular.

Cirrhopetahm ochracenm, Ridl. Journ. Bot.:36 1898, p. 212. Selangor: P'ahang 'Track (Rirlley).

\section{EXCLEDE SPIECISS.}

B. crassipes, Hook. fil. Fl. Brit. Ind. T. 660. "Penang H. B. Ker Dec. $+15 \%{ }^{\circ}$ " in Herb. Lindley. Mr. Rolle states this is exactly like the sikkim plant, hence there is a suspicion as to locality. It has not otherwise been known to oceur in the Peninsula.

\section{¿. DENDROCHILUM, Bl.}

Rhizome woody creeping branched, pseudobulbs usually remote leares coriaceous solitary, small. Raceme slender from the thizome near the bulbs. with dry sheaths at the base. Flowers numerous very small white. Sepals narrow equal, laterals adnate to the column foot. Petals smaller. Lip small articulated with the column foot. Column rather tall with lateral arms, apex hooked. Anther two-celled. Pollinia 4 oroid united by a riseus.

Lip lanceolate.
Leal lanceolate ..

$\begin{array}{lll}\cdots & \cdots & \cdots \\ \cdots & \cdots & \cdots\end{array}$
1. 1). album.
Leaf narrowly linear
2. D. angustifotium.

Lip pandurate.

Leaf obovate coriaceous .. $\quad \ldots \quad$. . 3. D. crassum.

Leaf elliptic $\quad \ldots \quad \ldots \quad \ldots \quad \ldots$ 4. D. elliplicum, 
1. D. AlBu ì, Ridl. Journ. Limn. Soc. XXXII. 2S\%. Rhizome long $\frac{1}{8}$ inch thick, pseudobulbs conic an inch apart, one inch long. Leaf elliptic lanceolate obtuse $3-4$ inches long, to one inch wide, petiole $\frac{1}{2}$ inch long. liaceme slender 1 inches long, bases covered with membranous bracts. Flowers small numerous white. Bracts very small much shorter than the ovary. Sepals 1 inch long linear obtuse thickened at the tips. Petals similar smaller. Lip shorter lanceolate subacute, keels 2 thickened. Column arched, upper margin prolonged lanceolate retuse, arms linear acuminate.

Bulbophyllum pteriphilum, Rolle. Kew Bulletin 1894391.

Perak: Larut Hills (Ridley, Derry), (King's Collector 3279) at $t 600$ teet elevation.

2. D. Axgestifolium, n.sp. Ridl. Journ. Roy. As. Soc. S. Br. Tol. IXILX. p. $6 \%$. Rhizome long woody terete, pseudobullss $1-1 \frac{1}{2}$ inch apart or closer subeylindric $\frac{1}{2}$ to $a$ inch long. Leaf narrowly linear laneeolate 2 inches long $\frac{1}{4}$ inch wide blunt mucromulate, narrowed at the base. Scape solitary or Sereral together on a stout short peduncle from the base of the pseudobulb, with numerous basal sheaths $3-1$ inches long, flowers numerous, greenish white $\frac{1}{8}$ inch long. Bracts ovate subacute $\frac{1}{2}$ the length of the orary. Rachis scabrid. sepals linear lanceolate. Petals narrower. Lip narrow lanceolate obtuse with two thick ridges at the base, and a lower one between them. Column short upper margin hooded minutely denticulate, arms linear from near the base. Capsule $\frac{1}{2}$ an inch long subglobose ovoid three angled.

\section{Selangor: Bukit Hitam (Kelsall); Pahang: Kluang 'I'erbang (Barnes). Endemic.}

3. D. crassum, Ridl. Journ. Limn. Soc. XXXII. 288. Rhizome terete $\frac{1}{1}$ inch thick, internodes $\frac{1}{t}$ to $3 \begin{gathered}3 \\ 4\end{gathered}$ inch long. Pseudobulls cylindric is inch long. Leat $t$ inches long $1 \frac{1}{2}$ inch wide elliptic obtuse coriaceous. Petiole $\frac{1}{4}$ inch long. Raceme 3 to 4 inches long completely covered with small flowers, rachis angled. Bracts ovate lanceolate $\frac{2}{3}$ length of the pedieel. Sepals oblong obtuse fleshy $\frac{1}{8}$ inch long and as wide green. Petals obovate obtuse green. Lip small white pandurate, tip broadly obovate with two keels. Column thickened upwards, foot very short, back of the clinandrium broad rounded entire, arms lanceolate acute falcate. Endemic.

Perak: on Hermitage Hill near Krwala Kangsar (Ridley).

4. D. ellipticun, Ridley Journ. Roy. As. Soc. Str. Br. Vol. XXXIX. p. 78. Rhizome long woody branched rellow psendobulbs slender conic cylindric curved $\frac{3}{4}$ inch long. Leaf thinly coriaceous elliptic oblanceolate obtuse is inches long by one wide. Scapes 3 inches long with large sheaths at the base. Bracts orate acute nearly 
as loner as the ovary. Flowers $\frac{1}{8}$ inch long rather fleshy. Sepals lanceolate acute apex thickened terete. Petals similar but narrower. Lip pandurate obtuse pustular, basal ridges obscure, a pustular mass. Column lather long hooded, margin with three tecth, alums from albout half up linear longer than hood.

singapore, Sembawang (Ridley 65:36). Eudenic. Rare.

\section{EXCLLDEN SPIECIES.}

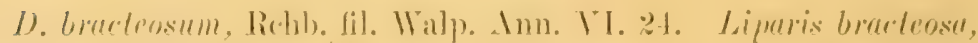
IVall. Cat. ifot. Malay l'eninsula, (Finlayson), Was probably oblaned in Cochin (hina.

D. Tinearifolium, Hook. fil. Plalyclinis linearifoliu, Ridl.

D. pallideforum, Hook. fil. Fl. Brit. Ind. T' is:, error for pallideflacens, Bl. The specimens relerred to this by Sir Joseph Hooker are partly D. album and D. crassum. I have not seen $D$. pullideflacens, Bl., from the Peninsula.

\section{ERLI, Lindl.}

Epiphytes, rarely terrestrial, stems slender terete or more or less swollen. Leaves lanceolate usually corjaceous. Infloresence racemose rarely spicate or panicled. Flowers small white, red or yellow, often tomentose. Sepals free, or rarely the laterals comate forming a

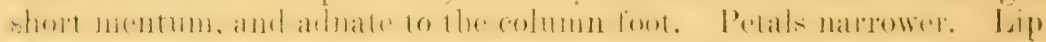
sessile on the end of the column foot, not mobile. Column short, rareJy long, foot louger or shorter, arms short rounded incurved. L'ollinia s, pyriform or ovoid attached to a riseid disc in lours.

Distribution-'lropical Asia. About 200 species.

\$. Stems of several or many internodes, rhizome none.

1. EnItra. Stems tall leaty, leaves long narrow distichous. Flowers very small and numerous in subterminal racemes, woolly. Laterals sepals short and broad, mentum short.

Stems 2-1 inclies tall, leaves narrow . Stems a foot and a half, leaves large.

lip millobe of 2 linear processes. .

Lip midlobe broad denticulate lobes

Lip midlobe subreniform . . .

1. I. obligilu.

2. E. bidens.

3. E. iridifolia.

1. E. major.

ミะ. Mreararturs. Stems Jeafless elongrate rylindric. Leares few terminal. racemes subterminal flowers small woolly.

j. E. longifolia. 
§. Dilocinopsis. Stems tall leafy. Flowers in subterminal panicles. ‥ 6. E. Scortechinii.

$\S$ 4. Banbusifoliate. Stems tall leafy. Leares thin or rarely coriaceons. Racemes short, lateral few flowered. Flowers glabrous.

Racemes 4 to 6 flowered, leaves broad ..

Racemes 1-2 flowered, leares linear, or narrow.

Flowers solitary an inch across $\quad . \therefore$ 8, E. pendula.

Flowers few, $\frac{1}{2}$ inch long _. . . 9. L. pilifera.

$\$$ 5. Thichotosid. Stems lealy terete. Leaves hairy rarely glabrescent. Racemes lateral hirsute. Flowers sessile or nearly so. Bracts longer than the ovary. Mentum long.

Racemes long and pendulous, plants very hairy

Flower's about 20, an inch long .. . . 10. E. iestita.

Flowers about $12, \frac{1}{2}$ inch long $\ldots \quad$.. 11. E. ferox.

Racemes rery short, few flowered, hairy.

Stems a foot or more.

Sepals acute lanceolate .. . . 12. E. cristata.

Sepals oblong obtuse .. .. . 13 . E. velutina.

Stems short very villous.

Leaves lanceolate close . . . 14. E. aporina.

Leaves linear .. $\quad \ldots \quad \ldots . \quad \ldots \quad$ 15. E. hispidissima.

Stems and leaves glabrescent.

Stems tall. Flowers very short numerous .. . . . .

Stems about a foot long or less. Flowers 2 or 3.

Leaves ovate lanceolate $\quad . \quad \ldots 1 \%$. E. oligantha.

Leaves lanceolate. Lip entire .. 18. E. monticola.

Leaves linear lanceolate. Lip 3 lobed.

Stems swollen at the base .. . 19, E. tuberosa.

Stems not swollen .. . . . 20. E. gracilis.

Stems $\frac{1}{8}$ inch long leaves orate heshy :2. E. rotundifolia. 
S 6. Hransent. stems short or long, pseudobulbs or terete crowded. beaves few subterminal. liacemes many flowered from below the leaves. Filowers nearly or quite glabrous. Mentum short.

stems terete. racemes horizontal of many small flowers. Bracts small.

liacemes very dense flowers white or pinkish.

Stem $\frac{1}{2}$ inch thick. Lip apex truncate

stem 1 inch thick. Lip apex retuse

Racemes lax flowers yellow . . . .

stems swollen upwards, leares terete

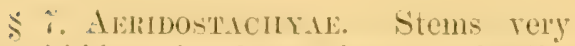
short hidden in large brown sheaths leaves long coriaceous. Racemes with a long nude base. Flowers small densely covered with brown fur, crowded. Mentum long.

Lip entire spathulate.

leaves very large. 18 inches long..

leaves narow at the base. ? inclies long $\quad \ldots \quad \ldots \quad \ldots$. .

lip : lobed. Sheaths of stem coriaceous Lip : lobed. Sheaths thin papery .

s. STELLATAE. Stems swollen prendobulbous, short covered with thin sheaths. Raceme long, flowers large distant, nearly glabrous.

Lip midlobe long acute.

Stems hardly swollen. Flower's white

stems nealy globose. Flowers striped pink $\quad \ldots \quad \ldots$..

Lip midlobe shorter than side lobes

26. Li. Acridostachya.

?人. E. brunca.

28. Li. lorifoliu.

29. L. dasystuchys.

30. E. stellatu.

:31. Li. striolata.

3\%. E. clata.

5. 9. IExdrolnios. Lhizome long. stems distant short. pseudobulbous (exeept li. punnea), Elowers few large woolly.

Scape 6 inches long .. . . . . 33: $E$. albido-tomentosa. 
Scapes short 1-3 flowered.

Pseudobulbs conic crowded. Leaf terete $\quad$.

Stems not pseudobulbous. Laaves 3 linear .. . . . .

Pseudobulbs oborate tapering upwards

Stems swollen or terete. Bracts large rare-

ly small. Hlowers nearly or quite glabrous.

$\$$ 10. Bractescextes. Raceme many flowered with large white or green bracts. Bracts oblong white. Racemes erect .. Bracts oblong, raceme reflexed . . . . Bracts lanceolate short .. _. . . 39. E. Endymion. Bracts ovate. Stems fusiform .. .. 10. W. xanlhochcila.

Stems thick cylindric.

Flowers yellow, purple striped .. 41. L. recurvatu.

Flowers white. Bracts green .. t\%. E. lutibracteala.

11. NuTANTES. Stems terete. Leaves 2 or 3 terminal. Flowers solitary lateral or terminal.

Stems tufted. Flowers terminal: white Stems from branched rhizomes. Flowers terminal

Rhizome far creeping, stems distant.

Flowers lateral below the leaves .. Stems short pseudobulbs covered with sheaths. Scapes erect. Flowers small, pubescent, mentum short.

Leaves oblanceolate.

Lip 3 lobed. Stems short fusiform thick

Lip 3 lobed. Stems with several distant sheaths .. .. . . . . .

Lip entire. Stems cylindric. .. .. 48. E. saccifera.

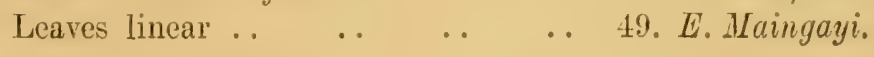

§ 1. ERIURA.

1. E. obliq A, Lindl. Journ. Linn. Soc. III. 1839, p. 55. Small tufted plants, stems $2-4$ inches tall. Leaves linear lanceolate bilobed 


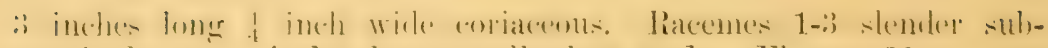
terminal erect, 3 inches long, woolly, base nude. Flowers 20 or more crowded rery small. Bracts ovate acuminate, as long as the ovary. Sepals ovate white woolly. Petals narrower lorate. Lip as long as the sepals three loberl. side lohes wate lalcate acute, michlobe cordate acute hardly longer, with a conic yellow callus in the centre, white with usually a row of violet spots on the side lobes. Columm short and apex abruptly dilate white. Anther pink, arms none. Capsule terete slender half an inch long. Hook. fil. Flor. Brit. Ind. V. 890. ILycamuthes obliqun, Lindl. Bot. Reg. 18t0, Misc. $\%$.

singalpore: (Cuming), Kranji (Ridley 1\%t) ; Johor' Batu P'ahat (Native Collector). Distrib. Borneo.

2. L. BnLis, Ridl. Journ, Limn. Soc. XIXII. 28!) Stens

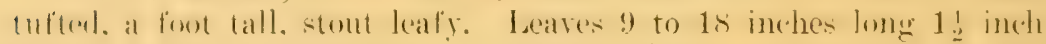
wide distichous subcoriaceous unequally bilobed. Seapes 3 from the upper axils over a foot long woolly. Flowers small numerous. Bracts ovate acuminate $\frac{1}{8}$ inch. Upper sepal orate $\frac{1}{8}$ inch long obtuse, late-

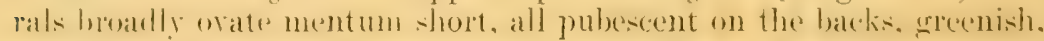
spotted with purple in front and with purple tips. Petals linear pink. Lip small greenish yellow, side lobes rounded blunt, millobe of two linear processes, short, disc white raised, with two red keels. Columu short with a rather long foot. Capsule terete an inch long splitting along one side.

Perak: Larut Hills 2-t000 feet alt. (Ridley 2883). Eudenic.

3. E. InIDHoLA, Ilonk. fil. Fl. Brit. Ind. I. 790. Ic. I'l. $206 \%$. Caespitose, almost stemless or stems a foot long leafy. Leares 9 to is inches long $:$ inch wide, subeoriaceous. Seapes 18 inches from the

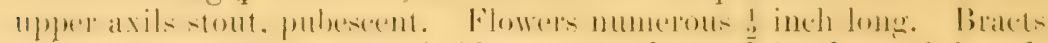
ovate reflexed. Upper sepal oblong ovate obtuse, laterals much broader ovate obtuse oblique, backs chestnut red pubescent, tronts yellow blotched red. Petals linear nearly as long as upper sepal, denticulate yellow, blotehed with red. Lip 3 lobed, lateral lobes oblong ovate hlunt vollow with real spots, midlole bitid lobes broad irrigularly laciniate yellow with red spot, callus at the base of lip 3 lobed, white, a broad elevated band white on the dise, with two narrow yellow ridges on either side, centre of millobe thickened white. Endemic.

P'rak: (iumong Batu Putih (Wray); Bujongr: Malacca (Ridley).

4. İ. MJor, Ridl. 'l'aus. Limn. Soc. IV. p. 23\%. Stems tufted tall over at foot long rather flattened nearly inch through. Leures

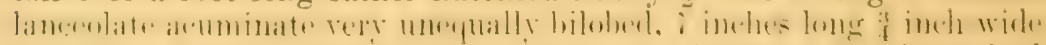
thinly coriaceous, sheaths $1-1 \frac{1}{2}$ inch long. Racemes 1 subterminal slender a loot loug woolly. Flowers rery numerous y inch long, 
pedicels and ovary $\frac{1}{4}$ inch long woolly: Bracts glabrous ovate acuminate $\frac{1}{8}$ inch long. Upper sepal elliptic, laterals broader obtuse woolly on the back greenish yellow inside. Petals oblong obtuse. Lip 3 lobed, side lobes subfalcate oblong white with pink spots, midlobe clawed subreniform deep maroon purple, with a white pulverulent callus, and a lobed callus white powdery at base of the lip. Column short with a long foot, white edged purple. Capsule elliptic fusiform $\frac{1}{4}$ inch long, pedicel as long. E. Kingii, Hook. fil. Fl. Brit. Ind. V. \%90. Ic. Pl. 2066. E. Scortechinii, Stapt. 'Trans. Linn. Soc. IV. p. $23 \hat{\text {. }}$

Perak: Larut Hills 3800-5000 feet alt. (King's (boll. 3311), (Ścortechini, Ridley); Pahang: Kluang T'erbang (Barmes). Endemic.

\section{\$. 2. MYCAIANTHEG.}

j. E. Loxgrfolis, Hook. fil. Fl. Brit. Ind. V. 990. Ic. P'. 2068. Stems several tufted 2 to 8 inches tall clothed in dry white sheaths. Leares $2-3$ terminal narrow linear acuminate very uneqally bitid 4 to 8 inches long $\frac{3}{8}$ inch wide. Scapes solitary shorter than the leares subterminal, 3 to 6 inches long woolly. Flowers very small white verticillate sessile. Lpper sepal oblong obtuse, laterals woolly triangular ovate, much larger, mentum broad rounded. Petals narrow lanceolate. Lip saccate lanceolate orate acute entire fleshy shorter than than the sepals papillose. Column short and broad, foot very short. Capsule elliptic sessile $\frac{1}{8}$ inch long.

Perak: Larut Hills (Ridley); Ulu Batang Padang (Wray); Pahang: Kluang Terbang (Barnes). Endemic.

\section{DILOCHIOH'SIS.}

6. E. Scortectinin, Hook. fil. Fl. Brit. Ind. Y. s09. Ic. Pl. 2082. stems woody 3 to 6 leet tall $\frac{1}{3}$ inch through, base terete sheathed. Leaves linear actuminate very uncqually biloberl, 6 inches long i inch wide, sheaths ribbed felter. Inflorescence panicled 4-5 inches long and broad tomentose, axillary. Bracts broad papery $\frac{1}{4}$ inch long. Floral bracts narrower and shorter, lanceolate. Flowers sessile white or pink. Sepals orbicular, orate obtuse, laterals ovate, pubescent. Petals broad oblong obtuse. Lip 3 lobed, side lobes oblong obtuse large, midlobe shorter rounded or obcordate, dise and centre of midlobe thickly tomentose. Column short, foot longer. Capsule elliptic sessile $\frac{1}{2}$ inch long.

Perak: (Scortechini), Gunong Batu Putil (Wray); Pahang: Kluang Terbang (Barnes). Endemic. 
\& 4. B.MBTSAEYLIAE.

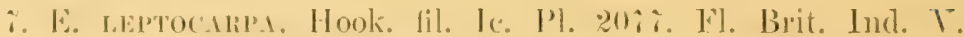
sos. Stems stout robust $2-3$ feet tall. thick, internodes 1 to $1 \frac{1}{2}$ inch long. Leaves linear lanceolate or lorate obtuse, 6-S inches long inch wide thinly coriaceous. lacemes laterals $1 \frac{1}{2}$ inch long, $1-6$ Howered. Bracts orate lanceolate $\frac{1}{1}-\frac{1}{2}$ inch long. Pedicel and ovary 2-1 inch long. I pper sepal lanceolate, laterals orate lanceolate falcate acuminate, $\frac{1}{3}$ inch long, vellow tinted with brown ontside, straw colour within. Petals linear oblong obtuse. Lip spathulate clawed. lateral lobes short, midlobe rounded mealy keels on disc between, side lobes :3. Column very short. Capsule cylindric obtusely angled 2-3 inches long.

P'erak (Scortechini, King's Collector 10339). Distrib. Borneo.

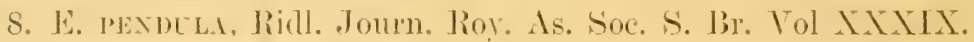

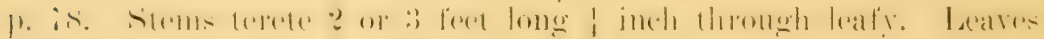
narrowly linera lanceolate acuminate $t$ inches long 1 inch wide sheaths

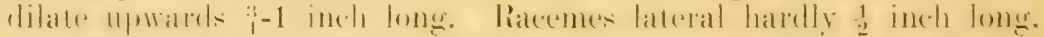
with several lanceolate acute red brown bracts 1 inch long. Flowers

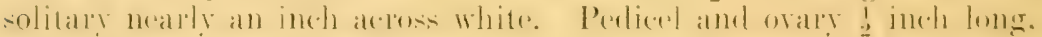

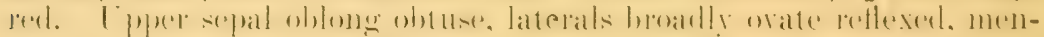
tum short rery broad and blunt. Petals oblong rounded as broad or broader than the upper sepal. Lip shortly clawed loroad oborate rounded, side lobes indistinct, midlobe longer broad, keck two curved laminae on the dise. Column short. foot long.

Selangor: at the caves Krala Lumpur (Kelsall); Perak (Scortechini Drawing). Distrib. Borneo.

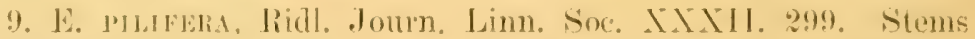
weak subterete leafy 18 inches long. Leaves thin lanceolate acuminate unequally bilobed 3 inches long : inch wide sheaths nearly an inch long. Racemes lateral very short few flowered, with + empty yellow

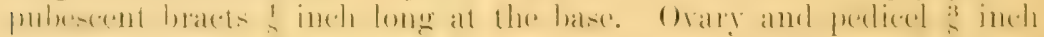
long pubescent. Flowers nearly $\frac{1}{2}$ an inch long. Upper sepal oblong lanceolate obtuse. laterals falcate acute, mentum short obtuse all white. Petals as long lanceolate. Lip shorter narrow euncate, side lobes rounded, midlobe hardly longer obsenrely 3 lobed covered with short yellow hair. Columm curved. Capsule terete 2 inches long.

Perak: Larut Hills (lidley 288\%). Endemic.

\section{ริ 5. 'I'richotosia.}

10. E. restit L, Lindl. Bot. Reg. 1814, Misc. \%6. 1845, t. 2. Stenrs 2 to 3 feet longr. Leaves lanceolate or oblong lanecolate very uncyually bilobed, $t$ in $S$ inches long 1-1 ty we dull green densely

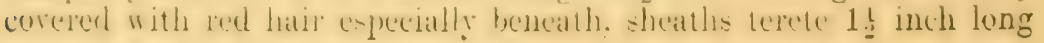


red hairy. Racemes flexuous a foot long, corered with red hair. Flowers an inch long, $\frac{1}{2}$ an inch apart sessile about 20. Bracts orbicular mueronate hairy $\frac{1}{4}$ inch long. Upper sepal lanceolate, laterals similar, mentum about $\frac{1}{2}$ as long stout curved, red. Petals narrow as long lanceolate linear obtuse, pinkish. Lip 3 lobed with a narrow base, side lobes obtuse rounded median cordate longer all crimson except edges of side and midlobe white. Column straight, narrow dilated at the very large stigma, foot as long as column straight crimson.

Singapore: Sungei Brih (Ridley 168\%); Johor; Pahang: Kluang Terbang (Barnes); Pekan (Ridley 1373); 'Telang River' (Machado) ; Malacea: Merlimau (Derry 13\%); Mount Ophir (Ridley); Selangor: Sepang (Ridley); Perak (Scortechini) B. P. D. (King's Collector r9ti); Penang: 'Telok Bahang (Curtis). Endemic.

11. E. perox, Bl. Mus. Bot. Lugd. Bat. II. p. 184. Stems 2-6 feet long $\frac{1}{2}$ an inch through. Leaves lanceolate acuminate to ovate lanceolate 3 inches long $1-1 \frac{1}{4}$ wide covered with stiff hairs sheaths $\frac{3}{4}-2$ inches long, arms with very stift: red hairs. Racemes is inches long hairy, about 12 Howered. Flowers $\frac{1}{2}$ inch long, sessile greenish rellow. Bracts ovate f inch lome. Sepals wate obtuse sparingly hairy, mentum as long as ovary. Petals subspathulate. Lip (uneate spathulate, side lobes waved, crenate, midlobe emarginate with $2-3$ rough calli. Hook. fil. Fl. Brit. Ind. V. 806. Trichotosia ferox, Bl. Bijdr. $3 \pm 2$. Lindl. Gen. and Sp. Orch. Pl. 132. De Vriese 11l. t. 9. and t. 11. f. .).

Perak (Scortechini), Larut Hills (Ridley 5192): Selangor: Bukit Kutu (Ridley); Penang (Lobb. 336), (Maingay); Kedah:

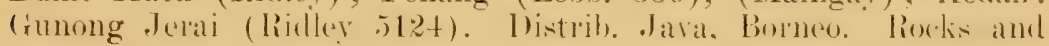
trees at 2 to 5000 feet altitude.

12. E. cristata, Ridl. Journ. Roy. As. Soe. S. Br. Tol. XXXIX. p. 78. Stem a foot tall. Leaves lanceolate acuminate oblique 3 inches long $\frac{1}{2}$ inch wide coriaceous almost glabrous above hairy beneath sheaths glabrescent when old rery hairy when young ? an inch long. Racemes short $\frac{1}{4}$ inch long rery hairy lowest bracts eup shaped, upper ones ovate lanceolate acute, $\frac{1}{2}$ inch long much longer than the short ovary. Flowes's 2 or: $: \frac{1}{2}$ an inch long. Sepals lanceolate acute rovered with red hair, mentum as long biunt. Petals linear obtuse, much narrower. Lip with a rery long claw pubescent at the hase, spathulate, apex rounded retuse glabrous except for the ends of $;$ raised reins which are eovered with short clubbed hairs. Column base pubescent. Endemic,

Penang (Curtis 1696); Lankawi; Terutau (Curtis 1696). 
13. E. velutixi, Lindl. Bot. Reg. 1840 , Misc. p. 86. Stems

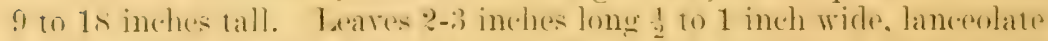

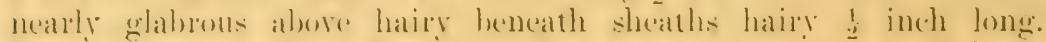
Racemes rery short few flowered. Bracts, lanceolate hairy + inch long. Flowers sessile $\frac{1}{3}$ inch long pale yellow, with red hairs. Upper sepal oblong obtuse, lanceolate larger, mentum short broad blunt. Petals narrower lanceolate blunt. Lip spathulate rounded retuse at apex, side lobes short incurved rounded. Column long slender pink, enlarged at stigma. Hook. fil. Fl. Brit. Ind. V. $80 \%$.

Singapore (Cuming), Kranji: Jurong (Ridley 3\%6) ; Johor: Batu Pahat (Ridley) : Malaceal : Mount Ophir, Nyalas (Gooderough 1320). Endemic.

14. E. Iponri, Hook. fil. Fl. Brit. Ind. V. 808. Ic. Pl. 2081. Stems 2 to 6 inches. densely villously hirsute. Leares lanceolate sub. acute crowded distichous $\frac{1}{4}$ to $\frac{1}{2}$ inch long. Bracts small orate acute. Flowers ! inch long glabresent. Sepals broally wate olutese men$10 m$ as long. P'otals narrowly subspathulate. Lip hroally rawed panduriform ciliolate, side lobes small rounded midlobe emarginate. C'apsule elliptic i inch long.

Selangor: Bukit Kutu (Ridley); Perak (Scortechini), Gunong Batu Putih (King's Coll. 8069), Larut Hills (Rialey). Endemic.

1.. E. mispipiasma, Ridl. Jomm. Bot. XXXIV. 1898, 213. А small slender plant with slender hairy stems 6 inches tall tufted. Leaves linear acuminate densely silky hairy $\frac{1}{2}-\frac{3}{4}$ inch long $\frac{1}{8}$ inch wide. Racemes flexuons hairy inch long, numerous. Flowers

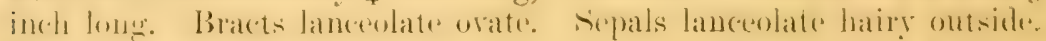
Petals lanceolate glabrous smaller. Lip oblong entire glabrous. Capsule $\frac{1}{8}$ inch long hairy.

\section{Selangor: Ulu Langat (Goodenongh). Endemic.}

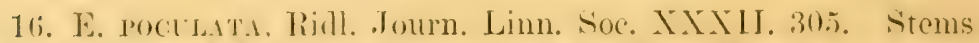

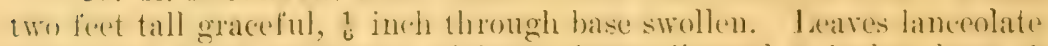

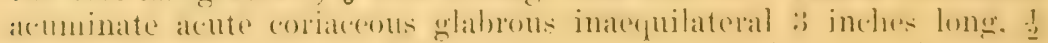
inch wide, sheaths scabrid 1 inch long, mouths red hairy. Racemes hardly $\frac{1}{2}$ an inch long, many flowered with a red hairy enp-shaped bract at the base. Flowers sinall short inch long greenish. Bracts 1 inch long orate pilose nearly as long as the flowers. Pedicel inch

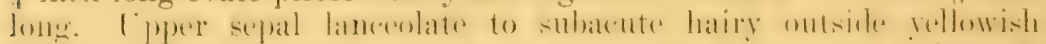
within, laterals broader ovate more acute keeled, mentum as long as the sepals saccate broad obtuse. Petals smaller lanceolate areute thin glabrous. Lip small white with a linear cumeate orange base, apex

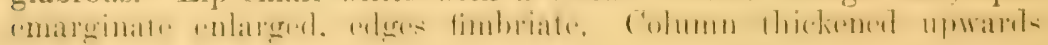
purple. 
Perak: Larut Hills (Ridley 2881); Kelah: Gumong Jerai at 3000 feet alt. (Ridley). Tar. opirenxis. lacemes longer, one inch long. Lip apex acute. Mount Ophir (Ridley 3151). Eindemic.

1\%. F. oligaxtira, Hook. fil. Ic. Pl. 20\%9. Fl. Brit. Ind. V. $80 \%$ Stems 10 to 12 inches tall, as thick as a goose-quill hairy towards the top. I eaves thickly coriaceous orate lanceolate, beneath tomentose or glabrous $2-2 \frac{1}{2}$ inch long obtusc. Flowers $1-2$ on a very short peduncle sessile $\frac{1}{2}$ inch long sparsely pubescent. Racemes $\frac{1}{2}$ to $\frac{2}{3}$ inch long. Bracts widely orate longer than the ovary. Sepals lanceolate, laterals ovate acuminate. Petals oblanceolate acute, mentum spur-like. Lip pubescent with a long narrow claw blade obovate spathulate shortly 3 lobed lobes rounded, median thick crenulate. Column sparsely hairy.

\section{Penang (Maingay). Endemic.}

18. E. มronticoli, Hook. fil. Fl. Brit. Ind. T. S06. Stems 1\% to 18 inches nearly $\frac{1}{4}$ inch long. Leares about 10 , lanceolate atolminate, 3 inches long $\frac{1}{2}$ inch wide, glabrons, sheaths an inch long hairy. Flowers 2 in a shont racene an inch long lower bract cup-like. ftoral hract orate lanceolate. L'prer sepal lanceolate, acute laterals similar mentum short nearly half as long as the sepals, all white. sparingly pubescent outside. Petals narrower oblong, white glabrous. Lip oblong subspathulate pubescent apex rounded whitish base and edges pink apex, orange or pink. Column short broad, narrowing to the pubercent crimson foot. Anther purple. Capsule $\frac{1}{2}$ inch long

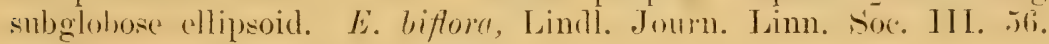
Trichotosia biftora, Griff. Notulae III. 331. t. 315.

Malacea: Mount Ophir (Griffith, Maingar, ete.), Pulau Aor (Feilding); Selangor: Bukit Hitam (Kelsill); Perak: Latrut Hills (Curtis), (Scortechini Drawing 148).

Yar. hirsuta leares softly tomentose bencath, flowers hirsute, lip broader more obeuneate lobès broader. Perak (Scortechini). Endemic.

19. E. Tuberosi, Hook. fil. Ic. Pl. 2080. Fl. Brit. Ind. I. $80 \%$. Stem 6 to 8 inches tall, swollen at the base. glalurescent. Jeares linear lanceolate acute very coriaceous glabrous, 2-;) inches long. Flowers 2 on a very short peduncle $\frac{2}{3}$ inch hairy. Bracts large ovate sheathing. Sepals ovate lanceolate, mentum saccate. Petals sparsely hairy narrow spathulate. Lip pubescent claw long blade obovate three lobed, midlobe retuse with a hairy patch in the centre. Column pubescent.

Perak (Scortechini). Endemic.

20. E. Gricilis, Hook. fil. Ic. Pl. 20\%8. Fl. Brit. Ind. V. 806. Stems several tulted $t$ to 6 inches long curved glabrous. Leares 
narrom linear lanceolate 2 inches long, $\frac{1}{4}$ inch wide glabrous young sheaths pubesecnt. Flower's 2 or 3 in a short raceme. Basal bract cup-shaped, upper ones lanceolate acuminate large + inch long. Sepals laneeolate acute, laterals curved, mentum as long as the ovary, all white covered with red hairs. Petals narrower lanceolate acute. Lip spathulate with a long cha crimson blade shortly three-loberl, midlobe oblong thitkened rellow hairy. Column hairy. Capsule elliptic hairy.

Singapore: Bajau (Ridley); Pahang: Tahan River (Ridley):

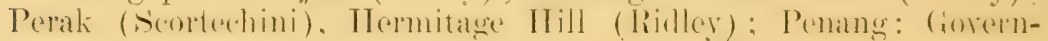
ment Hill (Curtis 1965). Endemic.

21. E. rotrydioli, Ridl. Journ. Roy. As. Soc. S. Br. Tol. XXXIX. p. \%8. Rhizomes forming a matted mass very slender, stems

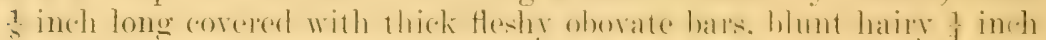

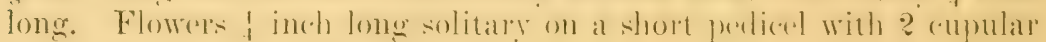
bracts the upper one longer than the ovary. Epper sepal oblong orate, laterals much broader, mentum rather large rounded. Petals oblong obtuse tip broader three lohed side lobes small. midlobe rounded all denticulate greenish rellow with a entral ochreous har and some purple spots on each side. Column short foot long olive green, antirer orange conic 1 celled, lront edge emarginate.

P'enang: above the Waterfall (C'mrtis). Allied to E'. dasyphylla, Par. of India and E. microphylla, Bl. Jara.

\section{HThexeri.}

22. E. Flombrad, lindl. Bot. lieg. 1848, Mise. 43. 18t4, t. 23. Journ. Lim. Sox. III. (60. Stems tufted is to 9 inches tall 7 inch grooved. Leaves subterminal linear lancenlate or lanceolate 5 -S inches Fong at to 1 wide. liacemes laterals from below the leaves 3 inches long, densely floriferous. Axillary bract orate, brown. Floral bracts very small ovate reflexed acute. Wlowers i inch long. Upper sepal orate acute, laterals obliquely triangular. mentum twice as long. l'etals lanceolate acute, midlobe spathulate, apex broadly trumeate. Column short foot long. Capsule terete slender $\frac{1}{2}$ inch long. Hook.

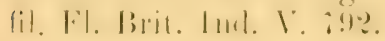

Singapole (Prince, Wállich's coll. : 408 ), Sungei Buluh (Ridley

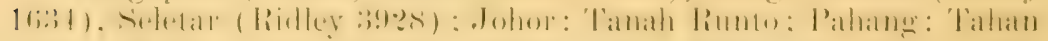
liver (Ridley 205t); Perak (Scortechini); Selangor: Sepan (Rirl-

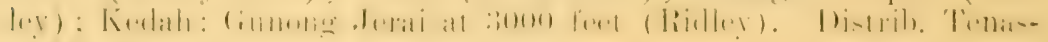
serim, Bolneo.

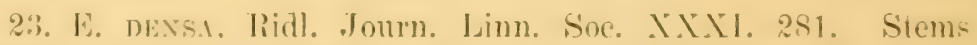
crowded thick cylindric s inches long, one inch thick. Leares four subterninal 11 inches long. 2 inches wide, lanceolate lorate. Racemes 
lateral 9 inches long, rachis pubeicent dense flowered. Bracts ovate a line long. Flowers small. Upper sepal ovate, laterals orate trian. gular. Petals orate narrower, all white or tinted with pink. Lip side lobes falcate acute, midlobe spathulate apex retuse obscurely three lobed. Column thick, foot long white with red edges. Anther red. Borneo.

Perak: Larut Hills (Ridley), Hermitage Hill (Curtis). Distrib.

A large plant with much denser raceme, side lobes of lip longer, midlobe retuse, and stigma smaller, than in $E$. floribunda.

24. E. tenuiflora, Ridl. Journ. Linn. Soc. XXXII. 291. Stems terete, 6 to 8 inches long $\frac{1}{4}$ inch thick. Leaves subterminal lanceolate acute thickened $3-4$ inches long $\frac{3}{4}$ inch wide. Racemes 3 or 4 lateral slender covered with flowers. Flowers thin numerous yellow glabrous, $\frac{1}{4}$ inch long, pedicels $\frac{1}{4}$ inch long. Bracts $\frac{1}{8}$ inch long Petals shorter similar. Lip shorter lanceolate acute, yellow with a red central blotch. Column short.

Singapore: Galang (Ridley 9153), Toas; Johor: Batu Pahat (Ridley); Pahang: Pekan (Ridley); Perak: Hermitage Hill (Curtis). Endemic.

25. E. cepifolia, Ridl. Journ. Linn. Soc. XXXI. p. 282. Stems numerous crowded, 3 inches tall suddenly thickened upwards from a narrow base, green. Leares 4 from the thickened nodes terete acute 6 inches long. Racemes axillary slender 6 inches long pubescent. Flowers numerous small white. Bracts lanceolate $\frac{1}{16}$ inch long. Sepals ovate pubescent, laterals triangular ovate blunt, mentum short, broad. Petals lanceolate narrower. Lip side lobes small acute erect, midlobe longer spathulate cuneate. Column short thick violet.

Perak (Scortechini). Distrib. Borneo.

\section{§ \%. Aeridostachyae.}

26. E. Aeridostachya, Rehb. fil. Lindl. Journ. Linn. Soc. III. p. 48. Rhizome stout stems crowded, 2 inches long thick, covered below with coriaceous sheaths. Leares 3 or 4 stiffly coriaceous, broadly lanceolate 18 inches 2 inches across. Scapes from the upper axils one foot long, lower half nude pubescent stout raceme dense flowers numerous crowded brown pulsescent. Bracts very small lanceolate. Pedicel and ovary pubescent. Upper sepal ovate oblong, laterals triangular orate, mentum twice as long. Petals linear lorate blunt falcate. Lip oblong obtuse apex rounded pubescent within, crisped. Capsule elliptic $\frac{1}{4}$ inch long.

Singapore: Bukit Mandai; Johor: T'anah Runto (Ridley 1653); Malacca, Perak. Endemic. 
2\%. E. BRUXEA, Ridl. Journ. Linn. Soc. XXXII. 29\%. Leares narrowly lanceolate acute narrowed at the base, coriaceous, 9 inches long 3 inch wide. Sicapes $\delta$ to 12 inchw long. lower half mude covered with red wool, raceme very dense flowers small outside brown, inside yellowish. Bracts ovate acute, red tomentose very small. Ovary and pedicel $\frac{3}{18}$ long. Sepals sparingly red tomentose outside, upper one small elliptic obtuse, laterals much larger nvate oblique mentum long. Petals linear obtuse curved. Lip thin, claw long narrow, blade ovate spathulate edges undulate.

Perak: Hermitage IIill (Curtis), Larut Hills (Ridley); Selangor: Bukit Hitam (Ridley). Endemic.

28. E. lorifolia, Ridl. Journ. Linn. Soc. XXXII. 296. Rhizome nearly an inch thick woody psendobulbs an inch long corered with large sheaths. Teaves lorate acule 15 inches long 1-11 inch wide coriaceous. Scapes 18 to 24 inches long, lower half nude glabrous, raceme pubescent rather lax. Flowers very numerous $\frac{1}{4}$ inch long yellow. Bracts minute orate. Pedicel and orary i inch long pubescent. Sepals red pubescent outside, upper one short orate, laterals broader, mentum long pendulous chublexl. Petals wider orate thimner glabrous. Lip shorter oblong, side lobes very short, nidlobe longer oblong obtuse edge undulate. Column very short, foot long.

Kedah: Gunong Jerai (Ridley) ; Sulangor: Bukit Kúutu (Ridley). Endemic.

29. E. Dasystachys, Ridl. Joum. Linn. Soc. XXXIII. 296. Rhizome thick woody, pseudobulbs :; inches long conic cylindric, covered with thin pajery sheatls. Icaves about 4 oblanceolate narrowed into a petiole, 8 inches long an inch wide, thinly coriaceous. scapes 2 erect $i$ to 8 inches long, base stout mude with 6 or $千$ scattered ovate bracts. Flowers crowded in a dense raceme about 3 inches long, rusty pubescent $\frac{1}{4}$ inch long. Bracts inch long, orate lanceolate acute as long as the ovaries. Sepals ovate acute, mentum as long conic thick blunt. Petals shorter linear acuminate, subobtuse. Lip thin, claw linear long, side lobes short rounded crisped, midlobe larger ovate obtuse crisped.

Pahang: Kwala Pahang (Ridley 15\%5). Endemic.

\section{\$. Stellatae.}

30. E. stellata, Lindl. Bot. Reg. t. 904. Stems short hardly pseudobulls shout 2 inches long covered with sheaths. Leaves sereral lancerolate acute or ollanceolate with a long petiole, jointed to a prolonged portion of the sheath, a foot or more 13 to 2 inches wide. Raceme from the side of the stem 18-30 inches long. Flowers scattered 3 inch long. pedierels short. Bracts lanceolate slicathing acuminate $: 3$ inch long. repals lanceolate accuminate acute white with 
brown pubescence on the back, mentum short. Petals narrower and shorter. Lip side lobes erect rounded white edges crimson, with 3 raised reins yellow on the disc, midlobe linear oblong or lanceolate acute yellowish white. Column curved broad white with crimson edges. Anther helmet shaped. Lindl. Bot. Mag. t. 3605. E. fra. grans, Rehb. fil. Bot. Zeit. XXII. 415. Hook. fil. Fl. Brit. Ind. V. 794. Dendrobium peratense, Hook. fil. Fl. Brit. Ind. V. 712. Ic. Pl. 2019.

Singapore: Chua Chu Kang (Ridley); Pahang: Kota Glanggi (Ridley); Perak (Scortechini). Distrib. Sikkim, Tenasserim, Borneo.

31. E. striolata, Rchb. fil. Ill. Hort. (1888) p. 35. t. 48. Pseudobulbs close nearly globose $1 \frac{1}{2}$ inch long $\frac{3}{4}$ inch thick. Leaves 2 thick lanceolate tapering at the base acute, 9 inches long $1 \frac{3}{4}$ inch wide. Scape rather stout about 15 inches tall. Flowers scattered an inch long fragrant. Bracts ovate lanceolate to lanceolate acute, $\frac{1}{2}$ inch long. Sepals lanceolate acute white with pink stripes, mentum short and broad. Petals linear lanceolate greenish white. Lip side lobes blunt rounded white with a pink edge and veins midlobe long narrow linear acute greenish tipped pink. Column broad foot about as long, arms obsolete. Anther helmet shaped. Capsule elliptic half an inch long.

Singapore: Kranji (Ridley); Johor: Batu Pahat (Ridley). Distrib. Borneo and Sumatra.

32. E. Elata, Hook. fil. Ic. Pl. 1848. Fl. Brit. Ind. V. 794. Pseudobulbs elongate thick, covered with sheaths. Leaves two deveioped oblanceolate a foot long, coriaceous. S'cape stout much longer. Bracts large lanceolate longer than the short ovary. Flowers fairly large, numerous ovary and base of perianth pubescent. Upper sepal lanceolate, laterals falcate lanceolate acute, mentum obtuse. Petals linear oblong obtuse falcate. Lip similar, lateral lobes incurved obtuse, midlobe shorter wrinkled, disc with 3 raised ridges. Column slender foot pubescent.

Perak (Scortechini).

\section{§ 9. Dendrolirion.}

33. E. a tubido-Tomentosa, Lindl. Gen. and Sp. Orch. Pl. p. 66. Rhizome far creeping, pseudobulbs ovoid compressed of about 4 internodes 2 inches long, an inch or more apart. Leaves 3 lanceolate acute narrowed at the base 5 inches long, 1.1 inch wide. Scapes from the lowest node 6-8 inches long with numerous close sheathing leaves at the base, rachis woolly. Flowers scattered on the upper part woolly outside. Bracts lanceolate half an inch long. Sepals half an inch long, backs covered with white wool, inner surface sea-green, upper one 
lanceolate acute laterals widely triangular acute spotted with red at the base. mentum short blunt. Petals half as long linear lanceolate acute green. Lip pandurate lanceolate lobes short oblong obtuse, midlobe oblong quite blunt densely spotted purple, a $\mathrm{V}$ shaped callus vellow in the midlobe, with a dark purple spot in the fork. Column long white with a pink spot at the base, arms short rounded. Anther conic obtuse. Ridley Journ. Linn. Soc. XXXII. 301. Rehb. fil. Xenia Orchid ii. t. 136. Dendrobium albido-tomentosum, Bl. Bijdr. p. 345.

Lankawi (Curtis); Kedah: Gunong Jerai (Ridley). Distrib. Siam, Java.

34. E. teretifolin, Griff. Notul. III. 298. Ic. Pl. As. t. 3000. Pseudobulbs conic $\frac{1}{4}$ inch long densely set on a slender rhizome, enclosed in a sheath. Ieaf solitary terete $t$ or 5 inches long. Scape enclosed in the basal sheath woolly 2 inches long. Flowers 1-2. Bracts small orate $\frac{1}{8}$ inch long. Pedicel $\frac{3}{4}$ inch long. Upper sepal lanceolate $\frac{1}{2}$ inch long, laterals broadly triangular, with a large rounded mentum. woolly outside. Petals linear woolly. Lip dull red. E. pellipes, Hook. fil. Fl. Brit. Ind. V. 802.

Malacea : Mount Ophir (Ridley); Perak: Larut Hills (Curtis), Gunong Batu Putih (Wray 469); Penang: Government Hill (Curtis 2429). Distrib. Borneo. On trees at 2 to 6000 feet altitude.

35. E. PANNEA, Lindl. Bot. Reg. XXVIII. 1842, Misc. 39. Rhizome fong and wiry. Stems not swollen half an inch fong 2 inches apart. Leaves 3 succulent narrow linear curred acuminate subterete 3- - inches long. Scape from the axil of one of the upper leaves 1 inch woolly 2 flowered. Bract large ovate. Upper sepal lanceolate acute, laterals triangular half an inch long woolly outside yellow ochre. IEntum short blunt. Petals shorter lanceolate yellow. Lip entire oblong acute fleshy purple with a mealy yellow centre. Column slender green. Capsule narrow fusiform an inch long, perianth persistent. Lindl. Journ. Linn. Soc. III. 50. Hook. fil. Fl. Brit. Ind. V. $80 t$.

Singapore: Kranji, Serangoon (Ridley); Johor: Batu Pahat (Ridley); Malacea: Bukit Bruang (Derry 286); Pahang: 'Tahan River (Ridley); Perak (Scortechini). Distrib. Borneo. Malay name, Kura Kubong.

36. 1E. LeiophylitA, Lindl. Journ. Linn. Soc. III. $5 \%$. Rhizome branching. Stems pseudobulls oborate tapering upwards and covered below with a retirulate sheath, 1 inch through at the base, about 2 inches apart. Leaves 2 lanceolate or lorate coriaceous 4 inches long 3-1 inch broad. Flower solitary on a peduncle enclosed in a long sheath 2 inches long. Upper sepal lanceolate pale ochre yellow, latrals howally triangrular alente half inch long. mentum thick long 
rounded, all ochre covered outside with red pubescence. Yetals narrower light yellow pubescent lorate lanceolate obtuse. Lip fleshy lanceolate side lobes obscure rounded central ridge and disc on midlobe papillose all deep purple. Column tall yellow toot short, arms very short blunt incurved. Anther helmet shaped. Hook. fil. Fl. Brit. Ind. V. 809.

Penang: on rocks Government Hill (Maingay, Curtis). Distrib. Borneo, Java.

\section{\$. Bractescentes.}

3\%. E. BR 1 Ctescens, Lindl. Bot. Reg. 1841, Misc. p. 18. 18tt, t. 29. Stems pseudobulbous sausage-shaped of two internodes 2 inches long $\frac{1}{2}-\frac{3}{1}$ inch thick crowded. Leares two terminal, oblong lanceolate subacute, thinly coriaceous $4-9$ inches long ? inch wide. Raceme from upper part of the pseudobulb 6 inches tall, lax, flowers about 15 . Bracts at the base of raceme oblong broad blunt, floral ones narrower lanceolate oblong obtuse white half an inch long. Rachis and pedicels slender $\frac{1}{2}$ inch long, pubescent. Dorsal sepal lanceolate acute, laterals falcate lanceolate half an inch long mentum conic. Petals narrower lanceolate acute, all white. Lip oblong, three lobed lateral lobes long falcate acute lanceolate rose pink, midlobe cuneate obovate white, ridges on the dise two short thickened forwards, median longer. Column long, foot short white with a pink spot at base. Hook. fil. Fl. Brit. Ind. V. 796.

Singapore: Pulau Tekong. (Ridley 203t), Chan Chu Kang (Ridley, Cuming) ; Johor: Batu Pahat (Ridley); Pahang: Pekan (Ridley 10t6); Kiedah: Pulau Song Song, Lankawi (Curtis). Distrib. Tenasserim, Borneo.

38. E. suaveolens, Ridl. Journ. Iinn. Soc. XXXII. p. 292. Pseudobulbs sausage shaped of 6 internodes, $t$ inches long $1 \frac{1}{1}$ inch through. Leaves $3-t$ lanceolate obtuse coriaceous 6 inches long $1 \frac{1}{4}$ wide narrowed into a petiole an inch long. Raceme stout recurved many flowered, from below the lowest leaf. Bracts $\frac{1}{4}$ inch long, oblong acute sprinkled with black pubescence. Flowers large fragrant. Sepals ovate blunt $\frac{3}{4}$ inch long mentum long oblong compressed. Petals ovate acute nearly as large, all white rose tinted. Lip spathulate claw long side lobes rounded erect blunt, pink, midlobe short broadly ovate fleshy with a central warty yellow callus. Column short foot long.

Johor: Sedili River (E. D'Almeida); Perak: Bujong Malacca (Ridley). Endemic.

39. E. Endynion, Ridley Journ. Linn. Soc. XXXII. 295. Stems connate cylindric of 2 or 3 internodes, crowded 3 inches long. Leaves 8 inches long $1 \frac{1}{2}$ inch wide lanceolate subacute narrowed 
at base coriaceous. Racemes stout erect 6 inches long base nude. Flowers numerous $\frac{1}{2}$ inch across, pedicels thick pubescent. Bracts lanceolate $\leq$ as long. Sepals white backs pubescent, upper widely lanceolate subobtuse, laterals orate acute, falcate, mentum short broad blunt. Petals orate white. Lip, side lobes falcate acute red, with a fleshy callus. Column straight.

Singapore: Seletar (Ridley). Distrib. Borneo.

40. E. Xaxtiocheild, Ridl. f. sp. Stems tufted on a stout rhizome 1 inch through, ahout halt an inch apart evlindric fusiform is inches long, internodes few. Leaves 3 or 4 subterminal lanceolate

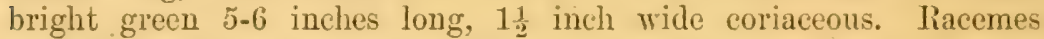
several from the axils below the leaves about 3 inches long. Flowers numerous fragrant $\frac{1}{2}$ inch long, pedicels pubescent $\frac{1}{2}$ inch long. Bracts ovate $\frac{1}{8}$ to $\frac{3}{8}$ inch long, white. Dorsal sepal ovate laterals falcate ovate, mentum twice as long, oblong blunt. Petals lanceolate acute, narrower, all white. Lip clawed long side lobes erect oblong obtuse, or acute curved, rather long, dull red. midlobe orate truncate yellow, keels 3 on dise between side lobes red, apices thickened, median prolonged on midlobe. ('olumn straight foot long anther small.

\section{Selangor: near Klang (Ridley 102\%2). Eudemic.}

41. E. Recurvata, Hook. fil. Fl. Brit. Ind. Y. $79 \%$ Ic. Pl. $20 \% 0$. Stems tufted, thick cylindric of $2-5$ internodes $1-6$ inches tall $\frac{2}{3}$ inch through. Leaves 3 to 5 elliptic lanceolate narrowed into a petiole. 6 to 8 inches long $1 \frac{1}{2}$ to 2 inches wide. liaceme lateral 6 inches pubescent, many flowered. Bracts orate lanceolate as long as the pedicels yellow. Pedicel and ovary pubescent $\frac{2}{3}$ inch long. Flowers $\frac{2}{3}$ inch across. Sepals recurved falcate lanceolate acuminate, yellow with purple stripes, mentum large rounder. Petals lanceolate ateminate yellow with purple stripes. Lip lateral lobes short rounded pale purple, midlobe obcordate rounded yellowish, two keels on the dise.

Perak (King's Collector).

42. E. Latibrac'Testa, Ridl. Journ. Limn. Soc. XXIII. 293. Stems a foot long, crowded, terete 1 inch thick. Leaves 1 terminal lanceolate plicate acute thin $;$ inches long 13 inch wide. Lacemes 2 or 3, compact 2 inches long, sessile. Bracts ovate acute nearly balf an inch long bright green. Flowers about 12 nearly shabrous.

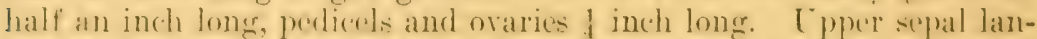
reolate areute. laterals falcate keeled, nentum short and hroat. White with red at bases. Petals shorter and broader obtuse, orate, white. Lip shorter, lonat, lobes from the base, cmoed obtuse, madler pink. midlobe lonere broarlly roumded nvate retuse, white. ? raised lieels, and two elerated nerve: between them on the dise ending in a deep pint 
patch on the centre of the midlobe. Column rather long broad, stigma large oblong, foot rather short.

\section{Negri Sembilan: Seremban (Dr. Braddon). Endemie.}

43. E. nutans, Lindl. Bot. Reg. 1840, Misc. p. 83. Stems tufted, about a foot tall $\frac{1}{2}$ inch through cylindric covered with brown sheaths. Leaves '2 or 3 , subterminal lanceolate acuminate acute, base narrowed, 6 inches long one inch wide. Flowers solitary $\frac{1}{2}$ inch long nodding from above the uppermost leaf, in a large brown bract. $\frac{1}{2}$ inch long. Peduncle $\frac{1}{2}$ an inch, with a small lanceolate bract below the pedicel, which is as long. Upper sepal oblong obtuse, laterals ovate falcate, mentum thick curved blunt. Petals lanceolate obtuse narrower, all white. Lip side lobes falcate acute broad, midlole obovate obscurely 3 lobed at tip, white tinted pink keels three, median running on to midlobe and three crisped laterals only to base of midlobe. Column long curved, foot much shorter, white with an orange cushion at the base of the foot. Capsule eylindric 3 inches long, $\frac{1}{4}$ inch thick. Lindl. Joum. Linn. Soc. III. 58. Hook. fil. Fl. Brit. Ind. V. 800.

Singapore (Cuming), Toas (Ridley) ; Johor: Tanah Runto (Ridley 1639), Ulu Batu Pahat (Kelsall); Malacca: Mount Ophir (Ridley); Perak: Bujong Malacca (Ridley); Penang (Maingay); Kedah: Gunong Jerai (Ridley). Endemic.

44. E. Neglecta, Ridl. Journ. Linn.-Soc. XXXI. p. 283. Rhizome much branched forming dense mats on tree branches. Stems erect base terete thickened upwards 5 inches tall $\frac{1}{1}$ inch thick abore. Leaves lanceolate acute flaceid 1-2, $3 \frac{1}{2}$ inches long, $\frac{3}{4}$ inch wide. Flowers solitary on a short pedicel, hardly projecting beyond the large brown bract, terminal. Sepals lanceolate laterals nearly ovate lanceolate $\frac{1}{4}$ inch long, mentum short broad blunt. Petals shorter lanceolate, all tinted with rose. Lip as long or longer, lateral lobes falcate acute pink, midlobe oblong quadrate or nearly obovate, apex rounded retuse yellow, keels 3 two running to base of midlobe, median one to end of midlobe and there waved. Column rather long, pink with a square papilla on the foot.

Singapore: Seletar (Ridley 203ï); Johor: Tanah Runto (Ridley); Perak (Scortechini). Distrib. Lingga, Borneo.

45. E. LoNgenepex̦s, Riidl. Journ. Linn. Soc. XXXI. 282. Rhizome creeping far $\frac{1}{4}$ inch thick, stems as thick terete, \pm inches long, and 6 or 7 inches apart. Leaves 3 or 4 on the end, lanceolate to ovate lanceolate acute or acuminate petiolate 6 inches long 2 inches wide, petiole $\frac{1}{2}$ inch long. Raceme from below the leares $\frac{1}{2}$ inch long to 3 flowered. Bracts half an inch long fleslry coloured. Sepals orate lanceolate $\frac{1}{2}$ inch long laterals longer with an oblong mentum, pinkish yellow with a darker median line. Petals much smaller lanceolate 
acute yellowish. Lip oblong entire truncate white. Column clubshaped white with a red spot at base. Capsule crlindric 2 inches long. Singapore: Lranji, Sungei Morai (Ridley en:36). Distrib. Borneo. Epiphytic ol terrestrial.

46. E. PUDICA, Ridl. Journ. Linn. Soc. XXXII. 394. Stems crowded crlindric thick of 5 internotes $1 !$ inch long 1 inch through, covered with brown papery sheaths. Leaves: oblancerate subacute 6 inches loug $1:$ inch wide coriaceous. Racenes from the lower nodes 2 inches long. Flowers numerous crowded in the upper half small. Bracts ovate $\frac{1}{3}$ length of the ovary. Ovary and pedicel $\frac{1}{8}$ inch long woolly. Lpper sepal elliptic obtuse pubescent nn the back, laterals much larger ovate obtuse. Petals as long and about as broad as the upper sepal, elliptic obtuse curved, all white. Lip short and broad 3 lobed side lobes short faluate subacute, edges violet, midlobe longel broadly oblong truncate, white with two large olslong tlat pinli-erlged processes on the disc between the lateral lobes. Colmmn broat and long, foot short. Endemic.

Singapore: Changi (Tidley); Johor: Kiwala Kahang (Velsall).

4\%. E. LANCIFolia, Hook. Hil. Fl. Brit. Ind. V. 804. Ic. Pl. $20 \%$. Stems $t$ inches long, rather thick, covered with large sheaths. Leares two oblanceolate subacute 6 inches long, 1 inch wide wide, petiole more than an inch long. Racemes 2 or 3 axillary short sleniler, pubesent. Flowers small about 6 sessile, scattered. Bracts ovate $\frac{1}{8}$ inch long. Upper sepal elliptic or ovate obtuse, laterals much broader ovate falcate obtuse, mentum very short, pubecent. Petals about as long as the upper sepal elliptic. Lip three lobed, side lobes rather large falcate blunt minutely papillowe with two datge blubng tleshy calli on the inner side midlobe longer ovate. Column rather large, foot slender curved. Capsule oblong three angled pubescent $\frac{1}{2}$ inch long, erect. E. dissiliflora, Ridl. Journ. Limn. Soc. XXXI1. p. 292.

Johor: Batu Pahat; Pahang: Tahan River (Native Coll. in Herb. Bot. Sing.) ; Perak (King's Coll.). Lndemic.

48. E. SACCIFErA, Hook, fil. Fl. Brit. Ind. V. $29 \%$ Ic. Pl. t. 20\%1. Stems cylindric 3 inches long crowded. Leaves two or three

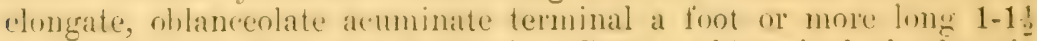
inch wide narowed into a petiole. Seape subterminal slender six

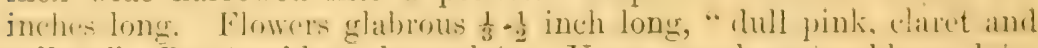
yellow." Bracts oblong lanceolate. Upper sepal ovate oblong, late-

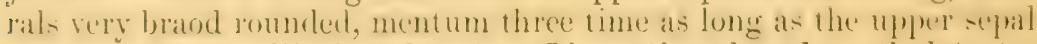
saccate. Petals elliptic subacute. Lip entire clawed spathulate tomentose. Column very short foot long.

Perak: Gunong Batu Putih (Wray 1215). Endemic. 
49. E. Mangayi, Hook. fil. Fl. Brit. Ind. V \%98. Ic. Pl. $20 \% 2$. Pscudobulbs tufted fusiform or cylindric. Leaves ? linear acuminate coriaceous, 3-t inches long $\frac{1}{2}-\frac{1}{3}$ wide, narowed at the base. Racemes much shorter than the leaves, 2-3 inches long. Flowers $\frac{1}{2}$ inch across. Bracts oblong as long as the pedicels. Lpper sepal oblong, laterals triangular orate subfalcate acute, mentum short rounded. Petals as long as sepals linear oblong. Lip 3 lobed obovate, side lobes rounded erenulate, midlobe small rounded, with a woolly callus in the centre, and three keels on the disc between the side lobes.

Penang: Government Hill (Maingay). Endemic.

\section{Excluded Species.}

E. Meirax, N. E. Br. is Porpax Meirax, King.

E. minutiflora, Ridl. is Poaephyllum pauciflorum, Ridl.

E. pulchella, Lindl. is Tylostylis rigida, $\mathrm{Bl}$.

E. pygmaea, Hook. fil. is Ceratostylis eriaeoides, Hook. fil.

\section{Porpıx, Lindl.}

Very small plants, pseudobulbs discoid with reticulate sheaths, crowded. Leaves 2 caducous. Flowers one or two sessile on the top of the pseudobulbs. Sepals connate into a tube. Petals free shorter. Column short wingless, foot adnate to sepals. Pollinia 8.

Species two Indian.

P. Meirax, King and Pantling Ann. Bot. Roy. Calc. VIII. 115. P1. 158. Pseudobulbs clustered nearly $\frac{1}{4}$ inch across discoid covered with thin radiating fibres connected by a membrane, reticulate when dry. Leares 2 elliptic oblong petiolate $\frac{1}{2}-1$ inch long. Flowers $\frac{1}{2}$ inch long from the centre of the bulb, brown. Bracts large ovate acute. Sepals connate into a tube apices ovate, awned. Petals shorter oblong lanceolate blunt. Lip orate orbicular at base, midlobe oblong obtuse. Column short, foot twice as long. Pollinia 8.

Kedah: Gunong Jerai at 4000 alt. on rocks (Ridley) out of flower. Distrib. Sikkim, Tenasserim.

\section{Trlostrlis, Bl.}

Ihizome creeping with distant pseudobulbous stems or several nodes. Leaves coriaceous sessile. Racemes axillary. Flowers medium size. Sepals oblong elliptic pubescent spreading. Petals smaller. Lip articulate to the foot of the column more or less mobile fleshy. Column long curved, loot very short.

T. rigida, Bl. Fl. Jar. Praef. VI. Rhizome fiar creeping, pseudobulbs an inch apart, flattened lanceolate narrowed at the base $1 \frac{1}{2}$ inch 
long, 1 inch across, $\frac{1}{4}$ inch through. Leaves $2-3$ to each pseudobulb broadly lanceolate coriaccous obtuse bificl. Paceme subterminal 3 inches long base mude, flowers an inch across widely expanded 20 or more, opening singly. Bracts orbicular recurved persistent $\frac{2}{6}$ inch long. Upper sepal lanceolate olstuse, laterals broader oblique. Petals spathulate lanceolate falcate smaller, all ocre yellow, finely woolly. Lip heart shaped mobile pubescent blunt $\frac{1}{t}$ inch long maroon edges paler: with three ridges meting in the midlole. ('olumn long arched yellow foot short broad fleshy, maroon at apex, arms very short. Anther conic.

Callostylis rigida, B1. Bijdr. 340. i. it. Lindl. Gen. and Sp. Orch. 129.

Eria pulchella, Lindl. Bot. Reg. 1841, Misc. 52. Journ. Limn. Soc. III. 51. Hook. fil. Fl. Brit. Ind. V. 801.

singapore: S'ungei Morai, P’ulau Tekong (Ridley 1i9s) ; Johor: Batu Pahat (Ridley), Sungei Kihang (Kolsall): Sungei [Jong: Malitera (Cuminer), Bukit Batu Tiga (Ridley); Perak (Scortechini); Penang (Curtis 1.35); Kedah: Gunong Jerai (Ridley). Distrib. Bornco, Jara, Rhio. Native names "Para chinduai;" "Sakat bigus."

\section{Piniedtil.}

small epiphytes tuited. Leares distichous articulate narrow. Scapes axillary slender. Flowers minute green or white. Sepals equal, with a short mentum. Petals smaller. Lip sessile or elawed jointed on the base or foot of the column. Column very short and broad. Anther low, pollinia 8 oboroid, attached to a viseid dise. Capsule very small oroid.

Species about ten. Indian, Malayan and Oceanic.

stems very short or none.

Rhizome stout, leares nearly a loot long .. 1. P. myosurus.

Rhizome none, leaves lanceolate $\ldots \quad \ldots \quad \therefore P$. listrophora.

Thizome none, leaves linear very small .. 3. P. minutiflora. Stems about 2 inches long. . . . . . 4. P. nuna.

1. PII. yryosurus, Lind]. Journ. Linn. Soc. III. 61. Rhizome very stout. Leaves strap shaped unequally bluntly bilobed 8 to 12

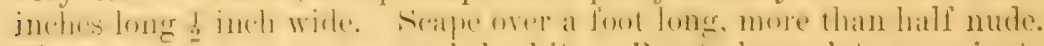
Flowers very numerous crowded white. Bracts lanceolate acuminate $\frac{1}{8}$ inch long. Sepals vbtuse mentum rery short, rounded. Petals similar small. lip sessile oblong or cuneate, apex three lobed. Column short and broad. Rostellum entire. Capsule elliptic fusiform $\frac{1}{8}$ inch long. Hook. fil. Fl. Brit. Ind. V. 810. Erite myosurus, Rchb. fil. Bopplandia V. 54. 
Perak: Larut Hills 3000-5000 feet alt. (Ridley, Scortechini, King's Collector).

Forma minor. Three inches tall. Leaves $\frac{1}{x}$ inch across, same locality (Ridley). Distrib. Borneo.

2. Ph. Listrophora, Ridl. Journ. Linn. Soc. XXXII. 30\%. No rhizome, tufted plants with abundant roots. Stem one inch tall. Leaves 6 inches tall hardly + inch across lanceolate acute unequally bilobed, narrow into a petiole. Scape slender $\%-8$ inches tall more than half nude. Flowers minute white. Bracts lanceolate acuminate 1 inch long. Sepals ovate acute. Petals oblong ovate. Lip with a linear claw and reniform lamina. Rostellum bidentate. Capsule oblong $\frac{3}{16}$ inch.

Perak: Larut Hills at 5000 alt. (Ridley); Lankawi: Gunong Raya (Curtis). Endemic.

3. P. minetiflora, Lindl. Journ. Linn. Soc. III. 82. Rhizome none, stems very short tufted. Leares linear obtuse $1 \frac{1}{2}$ to 2 inches long, $\frac{1}{16}$ to $\frac{1}{8}$ inch wide, distichous. Scapes $1 \frac{1}{2}-2$ inches long slender basal half nude. Bracts lanceolate actuminate $\frac{1}{16}$ inch long. Flowers very minute white, raceme rather lax. Sepals ovate acute, petals lanceolate. Lip ovate cordate concave. Column broad short and thick. Capsule elliptic $\frac{1}{8}$ inch long. Hook. fil. Fl. Brit. Ind. V. 811.

Singapore: Mangroves, Kranji, Jurong (Ridley 46\%9); Johor: (native collector); Selangor: Krrala Lumpor caves (Ridley); Perak: Larut Hills at 1000 alt. (Ridley). ( $A$ form with short recurved very thick leaves crowded together). Distrib. Sumatra, Borneo.

4. P. NAYA, Hook. fil. Fl. Brit. Ind. V. 811. Ic. Pl. Stems tufted 1 $1 \frac{1}{2}-2$ inches long. Leares narrowly ensiform acute, $\frac{1}{2}-\frac{3}{1}$ inch long, $\frac{1}{16}$ inch wide. Spikes capillary as long as the leaves. Bracts ovate acute. Flowers $\frac{1}{20}$ inch across. Sepals broadly ovate. Petals obtuse. Lip ovate obtuse.

Perak (Scortechini, Ǩing`s Collector); Penang: Richmong Pool (Ridley). Endemic.

\section{Agrostophyllum, Bl.}

'Tufted epiphytes, stems tall flattened. Leares long linear, distichous coriaccous articulated numerous. Flowers in dense heads, subterminal with numerous bracts, white rery small. Sepals ovate, no mentum. Petals narrower. Lip base saccate, blade ovate. Column short, foot none arms prominent or not. Pollinia 8 attached to a small riscus. Species about ten, India, Malaya and Serchelles. 
Heads of flowers long compact

Stem broad flattened.

stem terete slender. .

Heads of small flexuous spikelets
1. A. Mujus.

2. 1. callosum.

3. A. glumaceum.

1. A. MlıJ's, Hook. fil. Fl. Brit. Ind. V. S2t. Ic. Pl. 2096. Stems numerous forming a tuft ? or 3 feet through ?-:3 feet tall, $\frac{1}{2}$ inch wide. Leares lanceolate lorate blunt, 8 inches long $\frac{1}{2}$ inch wide, sheaths 2 inches long. Heads of flowers an inch across compact. Bracts broad orate, inner ones smaller. Flowers $\frac{1}{4}$ inch across. Sepals ovate acute keeled. Petals much narrower linear all white. Lip base saccate with a horizontal partition corering the top, and a transwerse one covering the front. white, partitions mathler red, blade orate acute white. Column thickened upwards, arms indistinct.

Singapore: Seletar (Ridley); Pahang: Pekan (Ridley 15t0); Perak (Scortechini), Gopeng (King's Collector 112\%), Larut Hills (Ridley). Distrib. Sumatra.

2. A. callosum, Rehb. fil. Seem. Fl. Vit. 296. Stems woody terete betow slightly thattened above s! feet tall. Leaves linear obtuse 1-i inches long : inch wide slueaths about an inch long edged black. Heads of flowers $\frac{1}{2}-1 \frac{1}{2}$ inch through compact. Flowers inch across, pink. Sepals ovate orbicular. Petals orbicular. Lip 3 lobed midlobe obreniform emarginate, with a large divided subbasal callus.

Selangor: Bukit Hitam (Kelsall), Rawang (Ridley). I have not seen the flowers of this, but the plant so closely resembles A. callosum of India in all other respects, that I do not think it can be distinet. Distrib. Himalaya to Burma.

3. A. GLuatacegr, Hook. fil. Fl. Brit. Ind. V. 821. Ic. Pl. 2095. stems tuftert shert flattened ti inches long ? inch wide. Jeaves distichour lowest sheathe like, uppere two with long flat sheaths ?-3 inches and thin grass like blake linear areminate of feet long half an inch wide. Heard $1 \frac{1}{2}$ inch through lax, racemes very numerous flexuous. Elowers small white, wary short sprinkled with black wool. Bracts ovate mucronate. Sepals short orate. Petals linear. Lip base saccate, laterals lobes erect acute, limb large orate, all white. Column rather tall, arms spreading triangular acute, edyed erimson. Stigma with a tongue shaped process projecting from it. Inther lwak orange. Capsule narrow oblong : inch long.

Solangor: liawang and Pahang Tratek, Sungei Ljonge (Native rollector): Perak (N'cortechini 1810); Tlu Bubong (King's Coll. 10876). Endemic.

\section{Ponepiryluis, Ridl.}

Epiphytes with slender terete stems. Leares barrow. Racemes lateral short, few flowered. Flowers fery small. Sepals lanceolate, 
laterals connate forming a mentum. Petals shorter. Lip clawed claw adnate to the column foot by its edges, spathulate longer than sepals. Column short foot slender, arms long acute. Anther ovate beaked. Pollinia 4 subequal oroid. Species one. Sp. 1.

P. Pauciflora, n. sp. Stems 2 feet long slender few. Leaves linear obtuse unequally bifid 2 inches long, 1 inch wide, sheaths $\frac{1}{2}$ inch long deeply split. Racemes hardly exsert from the sheath, with a few minute tlowers white. Bracts ovate acuminate half as long as the pedicel. sepals lanceolate acuminate keeled, laterals connate at base. Petals linear obtuse. Lip spathulate with a long claw, claw narrow channelled, blade ovate acute with a thickened line on the disc. Column short stout, arms acute. C'apsule cylindric very shortly stalked $\frac{1}{4}$ inch long. Agrostophyllum pauciflorum, Hook. fil. Fl. Brit. Ind. V. 824. Ic. Pl. 209\%. Eri minutiflora, Ridl. Journ. Linn. Soc. XXXII. $29 \%$

Johor: Batu Pahat (Ridley); Pahang: Tahan River (Ridley $2369)$; Perak: Hulu Kwantu (Ścortechini). Endemic.

\section{Ceratostris, Bl.}

Small epiphytic plants, stems not pseudobulbous, simple or branched sheathed, sometimes rush-like. I.tares narrow coriaceous or fleshy, one or two. Flowers very small rarely large, solitary or tufted, white or pink, Lateral sepals forming a mentum. Petals narrower. Lip adnate to the column foot by a claw, blade entire fleshy. Column short, broadest at the top. stigma rery large drawn out upwards in two long arms. Foot of column long. Anther small 2-celled. Pollinia 8 .

Species about 15. Indian, Malayan and Pacific.

Stems erect succulent rush-like .. .. . . 1. C. gracilis.

Stems simple erect, no rhizome .. _. . . 2. C. lancifolia.

Stems very short tufted.

Leaves lanceolate, flowers $\frac{1}{2}$ inch across 3. C. eriaeoides.

Leaves linear, flowers $\frac{1}{4}$ inch .. . . 4. C. cryptantha. Stems branched.

Leaves large lanceolate, sheaths trabeculate 5. C. robusta.

Leares linear, sheaths reticulate ... ... 6. C. clathrata.

Leaves elliptic lanceolate, sheaths punctate \%. C. puncticulata. Stems much branched pendulous .. ‥ S. C. pendula.

1. C. Gracilis, Bl. Bijdr. p. 306. Stems tufted terete dark green enclosed at the base in dry brown sheaths, 3 to 9 inches long. Leaf solitary subterminal crect terete $\frac{1}{2}-1 \frac{1}{2}$ inch long. Flowers in a tuft lateral from the base of the leaf, produced singly inch long 
pinkish, pubescent. Bracts ovate brown. Sepals lanceolate obtuse dull pink pubescnt mentum fairly large serotiform. Petals smaller lancenlate yellowish. Lip claw short. shoe shaped pubescent pale with an orange tip, blunt. Column short foot nearly as long, arms erect hlunt much lareer than the rest of the colmm, ovary pubescent. Capsule subsessile oblong. Rchb. fil. Xenia Orch. II. p. 92. t. 12\%. C. teres, lichb. fil. Bonplanda II. 1854. p. 89. Hook. fil. Fl. Brit. Ind. V 825. C. Malaccensis, Hook. fil. 1.c. 825. Ic. Pl. 2098.

Johol: Batu Pahat (Ridley) ; Selangor: Pahang Track (Ridley) ; Malacea: Ophir (Griffith); Perak: Larut Hills (King's Collector 2333 ) ; Province Vellesley: Bukit Seravah (Ridley); Kectah: Gunong Jerai (Ridley). Distrib. Java.

2. C. LANCIFolia, Hook. fil. Fl. Brit. Ind. V. 826. Ic. Pl. 2102. Sitems 2 to $t$ inches long tufterl erect unbranched covered with shearhs. Leaf solitary narrow linear lanceolate acute. Flowers solitary $\frac{1}{3}$ inch across, pedicel and ovary pubescent. Sepals oblong lanceolate acute. Protals narrower and shorter. Mentum short bilobed. Lip narrow, hase oblong apex thick lagger-shaped. ('olumm arms taller than the anther oblong obtuse.

Perak (Scortechini). Endemic.

3. C. ERtaeoides, Hook. fil. Ic. Pl. 2074 B. Dwarf tufted plant stems $\frac{1}{2}$ inch long covered with sheaths. Leaf solitary $1 \frac{1}{2}-2$ inches long linrar oblanceolate blunt thick. Seape as long filiform one llowered. Flowers $\frac{3}{2}-\frac{2}{3}$ inch across tomentose. Sepals orate lanceolate. Ientum short. Petals oblanceolate acute. Lip much shorter than sepals saccate at the hase, limb tongue shaped with 2 pubescent keels on the dise. Stigma very broad horseshoe-shaped. E. mygmuea, Hook. fil. Fl. Brit. Ind. V. 80 .

Perak (Scortechini, Wray). Endemic.

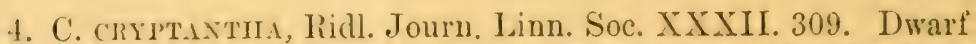
tufted plant stems $\frac{1}{2}$ inch long. Leaves 2 linear subacute 5 inches long $\frac{1}{8}$ inch wide. Seapes filiform $\frac{3}{8}$ inch long woolly one flowered. Flowers $\frac{1}{4}$ inch across on a short woolly stalk. Bract minute orate. Sepals ovate obtuse white pubescent. Petals narrower ovate mueronate oblique deep erimson with the long mucro and a median line white. Lip claw long linear, side lohes blunt broad upeurverl puluscent midlobe reniform, cream colour, a fleshy orate callus on the disc. Column short, foot very long slender arms incurved very obscure. Capsule elliptic erect $\frac{1}{4}$ inch long.

Perak: Hermitage IJill, Larut IIills (Ridley) : Selangor: Pahang l'rack (curtis); Penang: (iovermment Ilill (Curtis. Rilley i?lo). On trees at an altitude of about 2000 feet. Endemic. 
5. C. nobesta, Hook. fil, Fl. Brit. Ind. V. 82\%. Ic. Pl. t. 2103. Stems stout branched 8-10 inches long or more, rooting, sheaths $\frac{1}{2}-\frac{3}{4}$ inch long across nerved. I. caf $1-5$ inches long lanceolate acuminate $\frac{2}{3}$ to 1 inch across. Flowers solitary $\frac{3}{2}$ inch long pale yellow, peduncle slender pubescent. Sepals orate ohutse pulsescent. IIentum spurlike apex cnlarged obscurely bilobed. I'etals elliptic lanceolate obtuse. Lip spathulate with a long narrow claw, apex acute fleshy. C'olumn arms erect large taller than the anther.

Perak at 4900 feet alt. (Wray). Endemic.

6. C. clathrata, Hook. fil. Brit. Ind. V. 825. Ic. Pl. 2099. Stems stout tufted erect or prostrate ascending, branched 3-4 inches long, corered with short olstuse sheaths reticulate with raised nerves. Leaves linear $\frac{1}{2}-1$ inch long fleshy channelled. Flowers minute axillary solitary on a slender pedicel pilose. Bract orate. Sepal upper oblong, laterals ovate gibbous no mentum, pilose. Petals oblong lanceolate. Lip saccate at the base, incurved blade obcordate truncate emarginate with two keels. Column arms short rounded.

Perak (Scortechini), Batang Padang (Wray); Pahang: Kluang Terbang (Barnes). Endemic.

\%. C. Plncticulata, Ridl. Journ. Roy. As. Soc. S. Br. Vol. XXXVII. p. i9. Stems slender weak curved 3-4 inches long, but little branched. sheaths short ampliate mucronulate minutely punetate. Leaves narrowly elliptic lanceolate blunt petiolate, 2 inches long, $\frac{1}{4}$ inch wide. Flowers in pairs on short slender pedicels with minute bracts. Sepals lanceolate acute. Lip spathulate with an acute thickened tip.

Perak: Larut Hills at 5000 feet alt. (Ridley). Endemic. Buds too young.

8. C. PExpula, Hook. fil. Fl. Brit. Ind. V. 826. Ic. Pl. 2100. Stems tufted pendulous and roving, with many wiry roots at the base slender 3-12 inches long, corered with dry chestnut sheaths. Leaves linear narrow obtuse 1 inch long, $\frac{1}{6}$ inch wide coriaceous. Flowers in distant tufts of orate mucronulate brown bracts, white very small. Ovary and pedicel pubescent. Sepals ovate acute or subacute. Petals lanceolate narrower. Lip with a narrow claw blade orate lanceolate, pubescent at base, yellowish in centre. Column arms thick rounded.

Johor: Batu Pahat (Ridley); Pahang: Pekan (Ridley 1400): Selangor: Ginting Peras, Kajang (Ridley); Perak: Larut Hills (King's Coll. 38ty) ; Penang: West Hill (Ridley). On lofty trees. Distrib. Borneo, Celebes, Philippines.

15. Chrisoglossum, Bl.

Terrestrial rhizome creeping. Pseudobulbs cylindric conic, bearing a petiolate leaf. Scape usually tall, from the rhizome. Flowers 
seattered, medium sized. Scepals and petals subsimilar, lateral sepals falcatr. Lip thres lobed, adnate to the short loot of the column, spurless. Column tall arched, with a very short foot, arms linear oblong from the midlobe of the column, anther 2 celled. Pollinia 2.

Species 4, Indo-Malayan.

C. villosum, Bl. Bijdr. 338. Leaves ovate large villous 6.9 inches long, 3-5 wide very shortly petioled, or directly sessile on the pseudobulb. Scapes twice as long as the leaves hairy. Flowers 9 or 10 , distant. sepals and petals oblong obtuse, apiculate yellow with purple stripes. Lip shorter than the column side lobes oblong as long as the widely ovate midlobe, two strong keels in the centre. Lindl. Cien. and Sp. Orch. 13. Bl. Orch. Arch. Ind. 162. Pl. 4\%.

Perak (Scortechini). Distrib. Java.

\section{Collabium, Bl.}

Rhizome creeping, pseudobulbs narrow 1 leared. Leaf plicate. Scape from the rhizome, flowers scattered small. Sepals narrow, laterals connate forming a mentum. Petals narrower. Lip three lobed clawed. Column short, winged, no arms, foot long anther hemispheric. Pollinia 2, no disc.

Sp. 2. Malayan.

C. ncbulosum, Bl. Bijdr. 35\%. Leaf broadly lanceolate or ovate marbled with dark and light green 6-7 inches long 4t inch wide. Scape purple 18 inches. Flowers about 20 small. Ovary and pedicel 3 inch, red. Sepals falcate acute, mentum long blunt. Petals bent in the middle as long as sepals but narrower, all yellow with hroarl red colges. Lip whitr side lobes oblong falcate obtuse erect, midlobe large orbicular undulate, 2 short blunt oblong ovate laminae meeting in middle and 2 low ridges on dise. Column sigmoid short, vellow with transverse red bar, wings low. Lindl. Gen. and Sp. Orch. Pl. 96. Miquel. Fl. Nerterl. Ind. III. p. 6\%8. Choix des Plantes XXVI.

Perak: Larut Hills (Derry), (Ridley). Dense woods at 3000 feet alt. Distrib. Java. Ridl.

Excluded species, C. Wrayi, Hook. fil. is Thecostele marculosa,

\section{1\%. Plocogloters, Bl.}

Terrestrial herbs, with a creeping rhizome. Leares solitary, or several on a stem, ovate lanceolate short or long petioled. Scape tall from the rhizome flowers lew or many in a raceme. Sepals lanceolate. Petals honger or shorter. Iap shorter ('awed, flexible, limb oblong. Column short and broad, foot very short, arms none. Anther orate. Jollinia 1, each pair with a caudicle and rombled dise, both containing pollen grains. 


\section{Species 6 or 7 Malayan.}

Leaf solitary rarely 2 on a stem.

$\begin{array}{lllll}\text { Raceme glabrous .. } & \ldots & \ldots & \ldots & \text { 1. P. Javanica. } \\ \text { Raceme pubescent } & \ldots & \ldots & \ldots & \text { 2. P. porphyrophylla. } \\ \text { ves several on a stem } & \ldots & \ldots & \ldots & \text { 3. P. foctida. }\end{array}$

1. P. JAVANICA, Bl. Bijdr. P. 381. t. 21. Leaf ovate lanceolate acute 9 inches long 3 inches wide, petiole 5 inches long. Scape 1-2 feet tall. Flowers about 30 glabrous. Bracts ovate acuminate $\frac{1}{4}$ inch long, ovary and pedicel twice as long. Upper sepal linear acute $\frac{1}{2}$ inch long, laterals wider falcate. Petals narrow linear, as long, all yellow with red spots. Lip quadrate short truncate edges decurved side lobes minute semiovate obtuse, pale yellow. Column red stout, with large wings. Bl. Orch. Arch. t. 14. Mus. Bot. i. 46. Lindl. Gen. and Sp. Orch. Pl. 132. Hook. fil. Fl. Brit. Ind. VI. 22.

Singapore: Seletar (Ridley); Johor: Tengerah (Feilding), Hulu Batu Pahat (Kelsall 4035) ; Selangor: Ginting Bidai (Ridley 77\%8); Pahang: Kóta Glanggi (Ridley 2055); Halacea: Batang Malacca (Goodenough); Perak: Larut Hills (Curtis), Scortechini 1232); Kiedah: Gunong Jerai (Ridley). Damp woods to an altitude of 3000 feet.

2. Pe. porphyrophylda, Ridl. Trans. Linn. Soc. III. Journ. Linn. Soc. XXXII. 31\%. Rhizome short, pseudobulbs narrow 3 inches long. Leaf 1 rarely two elliptic lanceolate strongly nerved acute, 8-18 inches long 3-31 inches wide, petiole 1-6 inches long, purple. Scape stout 1 to 3 feet tall, raceme lax pubescent. Bracts short ovate acuminate, less than $\frac{1}{2}$ the length of the pedicel. Flowers about 30. Upper sepal lorate more than half an inch long yellow, laterals oblique larger oblong falcate yellow inner edges dark pink polished, pubescent outside. Petals narrow linear acuminate longer than the upper sepal yellow. Lip 4 inch long quadrate, edges at base up curved yellow spotted black, fimbriate, apex very broad, two shining purple horns on disc. Column as long as lip dilate above yellow, crimson above. Capsule $1 \frac{1}{2}$ inch long oblong.

Singapore: Kranji, Seletar (Ridley) ; Johor: Tanah Runto (Ridley 1758), Ulu Kahang (Kielsall 4410 ); Pahang: Pekan (Ridley 3094) ; Negri Sembilan: Perhentian Tinggi (Ridley); Perak: Lumut, Dindings (Ridley 8033); Tiedah (native collector) form entirely green. Sandy places usually near the sea. Distrib. Sumatra, Borneo.

3. Pl. Foetida, Ridl. Journ. Linn. Soc. XXXII. 319. Rhizome stout. Leafy stems 3 feet long. Leares 6 inches long $1 \frac{1}{2}-1 \frac{3}{4}$ inch long broadly lanceolate or elliptic lanceolate acute $3-5$ nerved, petiole very short or none, sheaths terete. Scape tall $2-t$ feet, pubescent. Flowers rather large, about 20 fetid. Bracts $\frac{1}{4}$ inch long ovate. Upper 
gepal if inch long ovate lanceolate acute, laterals twisted deflexed. Potals as long lancoolate acute, all orange with red spots. Jip as long and nearly as wide quadrate fleshy, 3 toothed, yellow. Calunthe gigantea, Hook. fil. Fl. Brit. Ind. V. $\dot{\$} 56$.

Singapore: Bukit 'Timah (Ridley 690\%); Johor: Tanjong Ǩllpang (Ridley) ; Malacea : Jus (Goodenough 1505) ; Selangor: Rawang (Ridley), Kwala Lumpor (C'urtis); Perak: Ulu Bubong (Ǩing's Coll. 102\%) ; Dindings: Clu Bruas (Curtis). Native name "Ginlumbak;" occurs in wet spots in jungle.

\section{Excluded SPeCIES.}

Plocoglottis acuminata, Bl. The plants referred to this species in Fl. Brit. Ind. are $P$. porphyrophylla, lidl.

\section{8. 'I'Aтла, Bl.}

Terrestrial herhs with a shortly creeping stem sometimes slightly swollen, succulent. Leaves herbaceous ovate or lanceolate petiolate solitary on the branches of the stem. Seape tall erect with few rather large flowers, from the creeping stem, lateral sepals adnate to the column foot forming a short mentum. Petals similar. Lip spurlike adnate to the column foot entire or three lobed. Column tall foot short, arms very short. Anther bilobed at the top. Pollinia 8 very unequal.

Species about 10. India and Malaya. Sepals long acuminate.

Flowers pale yellow

Flowers deep purple

. $\quad \cdots \quad \cdots$

1. 'T'. speciosa.

. $\quad$. $\quad \cdots$

2. T. Maingayi.

Sepals lancolate acute.

Lip broad apex rounded .. $\quad \ldots \quad$. . 3. T. latilingua.

Lip apex ovate acute .. .. . . . 1. T. atropurpurea.

1. 'T'. speciosi, Bl. Bijdr. p. 354. Leaf lanceolate acuminate 6 inches long, $1 \frac{1}{2}$ wide, petiole slender 6 inches long. Scapes a foot long. Flowers 5 or 6 pedicels and ovaries $\frac{1}{2}$ inch long. Bracts very narrow linear acuminate 1 inch long. Sepals lanceolate with a very long point $1 \frac{1}{2}$ inch long greenish yellow with red lines. Petals similar about as large. Lip ovate acute entire bright yellow with some red streaks on the inside. Column long arched foot short. Anther tip violet. Hook. fil. Fl. Brit. Ind. V. 822. Eria speciosa, lichb. fil. Walp. Ann. VI. 269, Mitopetalum speciosum, 13l. Fl. Jar. Praef. 8. Mus. Bot. II. 185. Oreh. Arch. Ind. 158. t. 50. f. 1.

Malaceat: Mount Ophir (Ridley) : Perak (Sortechini); Bujong Malacea (Curtis 329), (iunong Batu l'uth (Wray) ; Fedah: Gunong Jerai (liddley 56(0). D). Distrib. Jara. In woods at an elevation of $2-4,000$ feet. 
2. T. Maingayi, Hook. fil. Fl. Brit. Ind. V. 822. Ic. Pl. 2094. Stem stout covered with leaf fibres. Leaf elliptic lanceolate, acuminate, strongly nerved 8 inches long by $1 \frac{1}{2}-2$ wide, petiole short and thick 1 inch long. Scape stout about 2 feet tall. Flowers about $\tau$ deep purple. Bracts lanceolate acuminate $\frac{3}{4}$ inch long. Pedicel and ovary as long. Sepals lanceolate with a long point, 2 inches long. Petals shorter. Lip 3 lobed pubescent, laterals lobes truncate oblong toothed, midlobe ovate acuminate acute sides toothed, and disc covered with short processes, three strong toothed keels in the disc between the side lobes. Column slender long, foot rather short.

Selangor: Bukit Kutu (Ridley); Perak: Bujong Malacea (C'urtis 3290) ; Penang (MIaingay); Kedah: Gunong Jerai (Ridley). In rocky woods, at an elevation of $2-3,000$ feet.

3. T. latilingua, Hook. fil. Fl. Brit. Ind. V. 822. Ic. Pl. 2093. Leaf elliptic acute, 8 inches long, $2 \frac{1}{2}$ inch wide, petiole as long. Scape $1 \frac{1}{2}-2$ feet long, flowers numerous. Bracts narrow as long as the ovary. Flowers an inch across. Sepals and petals and linear blunt about equal, falcate. Lip orbicular, lateral lobes short blunt, midlobe orbicular ridges 3 crenulate on the disc. Column as long as the foot. Anther hemispheric. Pollinia equal.

Perak (Scortechini).

4. T. atropurperea, Ridl. Journ. Linn. Soc. XXXII. 315. Rhizome suceulent. Leares ovate deeply cordate subacute 6 inches long 4 inches wide petiole thick $t$ inches long. Scape a foot tall. Flowers an inch across. Bracts linear-acuminate, longer than the pedicel. Sepals lanceolate acute. Petals as long and broader, all purple, or olive green with red streaks. Lip ovate acute obscurely lobed, lobes rounded, purple with the tip dark brown or white with brown spots base yellow, eight raised nerves and three parallel keels in the centre. Column rather short stout straight, foot longer wings rather large. Nephlaphyllum grandiforum, Hook. fil. Fl. Brit. Ind. VI. p. 192. Ann. Roy. Bot. Gard. Calc. V. 23. Pl. 34. Ic. VIII. 105. Pl. 144. Ipsea Wrayana, Hook. fil. Fl. Brit. Ind. V. 812. Ic. Pl. 2085.

Perak: Larut Hills (Kunstler, Curtis, Ridlley). Distrib. Sikkim.

Apparently various in colour, but all I have seen were of a deep almost uniform purple. Ipsea Wrayena, Hook. fil. cannot I think be an Ipsea. The specimens and figure seem to me to be elosely allied to or identical with this plant.

\section{Ascotainia, Ridl.}

Terrestrial pseudobulbs large conic bearing a lanceolate petiolate leaf. Scape from the rhizome erect tall. Flowers medium size racemose. Sepals lanceolate, laterals free no mentum. Petals smaller. 
Anther oblong apex retuse. Pollinia 8, two pairs larger than the others oblomg obligue lamelliform, fonr lower ones smaller ovate flattened disc small retuse. Sp. 2. Indian and Malayan.

A. Penangiana, n.sp. Psendobulb 3 inches tall, and 2 inches through purple. Leaf lanceolate acuminate 12 inches long 3 inches wide, petiole $t-5$ inches long. Seape 13 foot tall, flowers 9 or 10 1 inch across. Sepals lanceolate acute, petals shorter pale yellow veined purple. Lip shorter 3 lobed, side lobes rounded, midlobe longer ovate, buff and rose colour, spotted with darker rose, spur short rlubbed. Column dilate upwards. Anther obserely 3 lobed. Tainia Penangiana, Hook. fil. Fl. Brit. Ind. T. p. 820. Bot. Mag. t. 7563.

Penang Hill, grassy banks (Curtis).

Tery near A. ILookeriana. Tamia IIookeriana, King and Pantling Ann. Bot. Roy. Gard. Calc. VIII. p. 103, p. 143.

T. viridifusca, Lindl. belongs to the same genus.

\section{Nephelapityluuar, Bl.}

Terrestrial plant with a succulent rhizome, stems ascending. Leaves few cordate or ovate petiolate herbaceous. Scape terminal short. Flowers small racemose. Sepals free lanceolate no mentum. Petals similar. Lip with a clubbed spur, attached to the base of the column entire or three lobed, obtuse. Column short thick, footless. Anther 2 horned. P'ollinia s' unergual on a large oblong linear gland.

Species about 5. Indo-Malayan.

Leaves ovate, raceme dense .. $\quad \ldots \quad$.. 1. N. pulchrum.

Leaves triangular acuminate, raceme lax .. 2. N. tenuiflorum.

1. N. Pelchium, Bl. Bijdr. 333, t. 32. Stems 2 inches long purple. Leares orate cordate acute 3 inches long 2 inches broad brown marble darker. Raceme 2 inches long \& flowered dense, orary

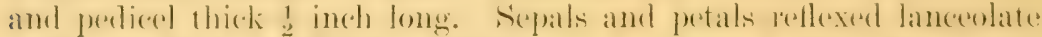

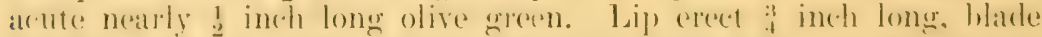
abovate (ntime or ohlone, white with pink reins, with a ralised central bar ending in two or three fimbriate ridges orange colour, spur pink dibare at if). ('olumn hroat white spotterl pink. Anther quatrate with 2 pink knobs. Blume Oreh. Arch. Ind. 1\%1. Pl. 61. fig. 1. Lindl. Gen. and Sp. Orch. Pl. 2t. Rehb. fil. Xenia. Oreh. 1. 216. t. 88, fig. 1. Bot. Mag. t. 5332. Hook. fil. Fl. Brit. Ind. T. 819. Ann. Bot. Gard. Calc. VIII. p. 105. pl. 145.

Singapore: Bukit 'limah, Bukit Mandai (Ridley); Malacea: Mount (j)hir, Bukit fiarlanen (Derry): Perak: Latrut Hills. Distrib. Java, Sikkim. In damp spots among rotting leaves.

2. N. tranurfolumar, Bl. Bijde. p. 373. Stem more slender leawe triangular condate ateminate. 2)-3! inch lone, 1-1 inch wide, brown marbled with darker colour. Șalje graceful $1-\hat{\imath}$ inches tall. 
Flowers 6 or 7 distant pendulous rerersed. Bracts linear. Scpals and petal linear, purplish green. Lip white obscurely 3 lobed, side lobes short blunt; midlobe ovate truncate undulate, with a tuft of purple processes in the centre, spur clubbed purple. Column stout. Anther truncate knobbed. Bl. Orch. Arch. Ind. 172. t. 61. fig. 2. De Vr. Ill. Orch. t. 12. fig. 3. Hook. fil. Fl. Brit. Ind. V. 819.

Perak (Scortechini); Kedah: Gunong Jerai (Ridley). Distrib. Java, Borneo. In mossy spots about 4000 feet elevation.

\section{Excluded Species.}

N. grandiflorum, Hook. fil. Tainia atropurpurea, Ridl.

\section{Spatiroglottis, Bl.}

T'errestrial, pseudobulbs conic 1-3 leaved. Leaves long petioled narrow plicate. Scape lateral. Flowers large showy yellow or erimson in lax racemes. Sepals free subequal. Petals similar or larger. lip. sessile, 3 lobed side-lobes erect midlobe clawed, dise tubereled at base. Column long graceful arehed, above dilate two winged, no foot. Anther 2 celled. Pollinia 8. Isles.

Species about ten India, China, Malaya, Australia and Pacific Petals broad.

Flowers rose, or white, large plants _. 1. S. plicata.

Flowers yellow $\quad . . \quad \ldots \quad \ldots \quad \ldots$ 2. S. aurea. Petals narrow, small plant $\quad \ldots \quad$. 3. S. Handingiana.

1. S. Plicata, Blume Bijdr. 401. t. \%6. Pseudobulb conic covered with fibres of old leaves. Leaves $2-t$ feet long by 1-3 inches wide, lanceolate plicate petiole 6-18 inches long. Scape 12 inches long to 6 feet long, stout. Raceme many flowered. Bracts pink ovate or lanceolate. Flowers an inch across, crimson rarely lilac or white, self fertilized. Ovary and pedicel 1 inch long. Sepals ovate oblong acute. Petals broader. Lip lateral lobes oblong truncate apices thickened: calli, one obcuneate retuse hairy yellow, with two small white lobes on the sides; midlobe, base narrow apex dilated cuneate reniform emarginate. Column slender curved. Capsule oblong pendulous. Lindl. Gen. and Sp. Oreh. Pl. 119. Hook. fil. Fl. Brit. Ind. V. 813. S. Tilacina, Griff. Notul. III. 323. Ic. Pl. As. t. 311 fil. 3. Bletia angustifolia, Gaud. Freyc. Voy. Bot. 421. t. 32. Open country and wet rocks, whole peninsula, common.

Singapore (Wallich $3 \%+3$ ), Common (Ridley, etc.); Johor near the town; Sungei Ujong: Seremban (Ridley); Selangor: Sungei Buluh (Ridley 10513); Malacea (Griffith), Sungei Hudang (Derry 98); Perak: Larut Hills (Ridley 3268); Kemaman (Johnston).

var. alba, flowers and bracts white, Dindings, Pulau Sembilan. Distrib. Malay Islands to Philippines and New Guinea, and Samoa. 
Lsually called Lumbah by the matives from its resemblance to Curculigo.

2. S. Aures, Lindl. Paxt. Fl. Gard. I. 16. Journ. Hort. Soc. V. 34. Preutobulh, globose half an inch through. Leaves lanceolate with a long point plicate 2 feet long 1.1 to 3 inches wirle. S'sape 3-4 feet long. Flowers several, large yellow 3 inches across or less pedicels with ovary $1 \frac{1}{2}$ inch long. Bracts ovate obtuse green half inch long. Sepals oblong lanceolate obtuse $1 \frac{1}{4}$ inch long $\frac{33}{4}$ inch across. Petals bvate lanceolate blunt. Lip shorter, side lobes long obtuse falcate, yellow base pink, midlobe narrow dilated at the apex blunt, yellow with red spots at the base. Calli 2 small horn-shaped ones, and two oblong cuneate fleshy ones, at the base of the midlohe. Column slender arched, dilate above, wings narrow orange. Hook. fil. Fl. Brit. Ind. V. 813. S. plicata, Griff. Notul. III. 325. Ic. Pl. As. t. 311. fig. 1. S. Wrayi, Hook. fil. l.c.c.

Malacea: Mount Ophir (Griffith, Derry 629), (Ridley 3138); Selangor: Bukit Hitam (Kelsall); Perak: Gunong Batu Putil (Wray 202), Larut Hills (Ridley), Gunong Inas (Wray $410 \tilde{)}$ ); Kedah: Gunong Jerai (Ridley 5135). Girassy spots at an altitude of 3-4000 feet. Distrib. Borneo.

3. S. Haxdingiana, Par. and Rehlo. fil. Otia. Hamburg I. 4 รั. Pseudobulbs small globore. J.eaves $2-3$ lanceolate acuminate uncyual, $4 \frac{1}{2}-5$ inches long $3_{4}^{-1}$ inch wide or smaller. Seape graceful 8 inches long purple. Flowers few scattered on slender pecticels $1 \frac{1}{2}$ inch long, purple or pale rose an inch across. Bracts lanceolate acuminate $\frac{1}{4}$ inch long red. Upper sepal lorate lanceolate, laterals larger. Petals narrow lanceolate acute oblique, all crimson or pale rose. Lip side lobes short triangular erect blunt, midlobe linear' acuminate. ('alli 2 club-shaped truncate yellow. (column arehed dilate upwards. Capsule eylindric. Hook. fil. Fl. Brit. Ind. V. 815.

Lankawi Islands (Curtis 2105). Distrib. Burmah, Siam.

\section{Pinsues, Lour.}

Large terrestrial herbs. Leaves thin plaited. Scape lateral or raceme axillary. Flowers large showy racemose. Sepals and petals lanceolate equal free. Lip adnate to the batse of the columm embracing it, base gibbous or spurred. Column long stout foot $O$. Anther 4 celled. Pollinia 8 attached by fours to a viscus.

Species 14. 'Tropies of the old world.

Scape from the base of the psendobulb, tall.

Flowers large sepals and petals acute .. 1. P. Wallichii.

Flowers large sepals and petals obtuse .. 2. P. callosus.

Racenes from the side of a leafy stem .. ‥ 3. P. pallidus. 
1. P. Wallicmir, Lindl. Wall. Pl. As. Rar. II. 46. t. 158. Gen.

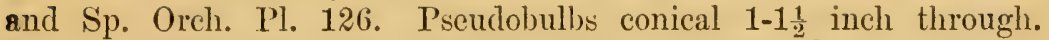
Leaves 1-2 feet long $2 \frac{1}{2}-8$ inches wide, elliptic lanceolate acuminate, petiole 8-10 inches long. Scape $2-t$ feet stout. Flowers four of five inches across, often self-fertilizer. Sepals and petals lanceolate acute white outside, brown, or orange within. Lip tulbular, midlobe ovate lanceolate acute or acuminate crisped, spur $\frac{1}{4}$ inch long. Column long straight. Capsule 2 inches long pendulous. Paxt. Mag. VI. t. 193. Hook. fil. Fl. Brit. Ind. V. 816. P. Blumei, Lindl. Gen. and Sp. Orch. Pl. $127 \mathrm{Bl}$. Oreh. Arch. Ind. 2. t. 1. De Vriese Illust. t. 8. P. bicolor, Lindl. l.c. 128. Sert. Orch. t. 2J. Bot. Mag. t. tor8. Wight Ic. t. 1659-60. Limodorum T'ankervilliae, Roxb. Fl. Ind. III. 466.

Malacea: Ayer Keroh (Ridley), Bukit Sandanen (Derry) ; Selangor: Kiwala Lumpur, Bukit Bintang (Ridley 1051t). Distrib. India, Malay islands. The common form of this here is self-fertilized and does not open its flowers.

2. PH. Callosus, Lindl. Gen. and Sp. Orch. p. 128. Gard. Chron. 1848. p. 28\%. Pseudobulbs short stout. Leaves elongate lanceolate acuminate 2 to 4 feet long 5 inches wide. Seape from the base of the pseudobulb, about 3 feet tall. Flowers 4 inches across. Sepals ohlong or linear oblong half inch across blunt, pinkish or pure white behind, brown in front. Petals narrower. Lip tubular yellow midlobe rounded bilobed with two low processes at the base, pink, spur yellow short curved. Blume Mus. Bot. II. 1r8. Orch. Arch. Ind. 4. t. 2. lichb. fil. Xen. Orch. II. t. 122. Hook. fil. l.c. 81\%. Limodorum callosum, Bl. Bijdr. 3rt.t. 6.

Perak: Larut Hills at 4000 feet on rocks, al)undant (King's Coll. 6413), (Ridley 5191). Distrib. Java. Occurs in dense forests. A very handsome plant.

3. P. PAllides, Ridl. Journ. Lin. Soc. XXXII. 313. Stems 2-3 feet tall $t$ angled stout base covered with sheaths, upper part leafy. Leaves ovate acuminate 9 inches long 3 inches wide. Racemes 3 or 4 from the stem below the leaves, $\%-8$ flowered $2-t$ inches long. Bracts lanceolate acuminate $\frac{1}{2}$ inch long. Flowers 2 inches across, pedicels one inch long. Sepals lanceolate acute rellowish. Petals wider white with violet spots. Lip oblong lanceolate subacute orange yellow with red spots in lines, spur $\frac{1}{2}$ inch long eylindric acuminate pink. Column long. Anther beaked.

Selangor: Bukit Hitam (Kelsall, Ridley vi85) ; Pahang: Tahan Woods (Ridley); Perak: Larut Hills (curtis 2046). Occurs in forests about 3000 feet clevation.

\section{Calditie, R. Br.}

'Terrestrial herbs, sometimes pseudobulbs. Stems short. Leaves plaited. Scape terminal or from the side of the pseudobulb. Flowers 
small or medium-sized, white, pink, or yellow. Sepals subequal. Petals similar. I,ip adnate to the top of the column, 3 lobed, midlobe olten face deeply bifid, disc lamellate or: pectinate. Column usually short. Anther conic, pollinia 8 waxy colnering in pairs by a viseus. Capsules elliptic drooping.

Species about 40, tropical or subtropical regions.

\$ 1. Euchlixtile. Seape appearing with the leares. Bracts persistent.

Lobes of lip linear .. $\quad \ldots \quad \ldots \quad \ldots \quad$. . C. veratrifolia.

Lobes of lip broad oblong, spathulate .. 2. C. Cecitiae.

Bracts caducous.

Leares narrow, lip 4 lobed . $\quad \ldots \quad \ldots 3$. C. angustifolia.

Leaves broad, lip 3 lobed.

Midlobe obovate retuse .. $\quad \ldots$.. $\quad$ t. C. albolutea.

Midlobe ovate small .. ... . . 5. C. Scortechinii.

Mirllobe oblong acute .. $\quad \ldots \quad \ldots .6$. C. curculigoides.

Midlobe linear oblong obtuse .. .. \%. C. aurantiaca.

$\S$ 2. Preptaxtue. Scapes appcaring after the leaves.

Midlobe of lip flabellate obcordate .. 8. C. vestita.

Midlobe of lip linear oblong bilobed .. 9. C. rubens.

\section{§ 1. EuchlaNtine.}

1. C. veratrifolis, R. Br. Bot. Reg. sub. t. 5\%3. Stems short one inch long. Leaves lanceolate or ovate lanceolate acuminate 12-1S inches long 3-6 inches wide, pet jole :3-6 inch long. Sirape 1-2 leet long pubescent, raceme compact or lax many flowered. Bracts lanceolate $\frac{3}{4}$ inch long. Flowers white $\frac{3}{4}$ inch across, ovary and pedicel 2 inches long. Sepals orate acute or elliptic oborate. Petals oblong or lanceolate. Lip adnate to the column 1 inch long side lobes linear oblong obtuse, midlobe longer deeply divided into two linear oblong spathulate lobes, callus on the dise of comnate tuberches in thee rows yellow orange or red, spur slender elubbed is inch long.

Lindl. Bot Reg. t. 720. Gen. and Sp. Oreh. 249. Fol. Orch. S. Bot. Mag. t. 2615. Grifl Ic. Pl. As. t. 283. dig. t. Hook. fil. Fl. Brit. Ind. V.851. C. comosa, Richb. fil. Limnea XIX. 3\%t. C. Perrottetii. A Rich. Ann. Sc. Nat. Ser. ii. XV. 6s. Wt. Ic. t. 166t. Limodorum

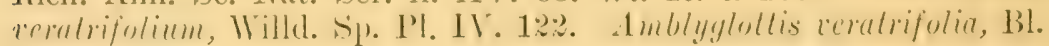
Bijdr. $2 \% 0$.

Johor: Sedili River (Rirlley); Perak: Larut Hills (Hervey, Curtis), Gopeng (King's Coll. 6011). Distrib. India, Malaya, Dustralia 
Rather variable. Plants collected by Curtis on the Perak Hills had a pale yellow lip, and are probably the var Regnieri Rchb. fil. Gard. Chron. II. 188\%. p. 70. The Goping plant is a small compact form described as $C$. diploxiphion, Hook. fil. Fl. Brit. Ind. VI. 849. Ic. Pl. 2111.

2. C. Ceciltae, Rehb. fil. Gard. Chron. 1883. I. p. 432. Stem short, leaves elliptic lanceolate obtuse, ovate, blade 12 inches long by 4 inches wide, petiole 3 to 6 inches long. Scape stout 12-18 inches long, pubescent, raceme short crowded flower's 1 inch across, white tinted violet or all violet. Bracts persistent broadly ovate $\frac{1}{4}$ inch long. Ovary and pedicel 1 inch long. Sepals broadly elliptic ovate. Petals obovate. Lip side lobes oblong obtuse, midlobe deeply bifid lobes spathulate rounded, callus horse-shoe shaped yellow, 3 short papillose ridges on the dise spur long slender curved upwards 2 inches loig. C. Wrayi, Hook. fil. Fl. Brit. Ind. V. 850 .

Selangor: Kwala Lumpur Cares (Ridley 8 486 ), Bukit Hitam (Kelsall); Pahang: Kota Glanggi (Ridley var with violet flowers); Sungei Ljong: Gunong 'Tahan (Native Collector) ; Perak: Hermitage Hill (Ridley); Sungei Siput: Kinta (Curtis). Native name "Suboi." Occurs on rocks and the ground in dense forests.

3. C. Angustifolis, Lindl. Gen. and Sp. Orch. Pl. 251. Fol. Orch. 5. Rhizome creeping long slender. Leaves narrow lanceolate acuminate, 18 inches long by 1 inch wide, petiole a foot long. Scapes 4 inches to a foot long, slender with a large sheathing bract towards the base, raceme two or three inches long, flowers crowded, or lax pure white. Bracts caducous lanceolate acuminate $1 \frac{1}{2}$ inch long, narrow. Pedicel and ovary $\frac{1}{2}$ inch long. Sepals oblong acute. Petals very broad. Lip 4 lobed subequally, midlobe retuse with two triangular lamellae at base, spur clubbed curved $\frac{1}{4}$ inch long. Capsule $\frac{3}{4}$ inch long. Hook. fil. Fl. Brit. Ind. V. 854. C. phajoides, Rchb. fil. Bon. pland. V. 185\%, 3\%. Xenia Orch. I. 20\%. t. 69. Hook. fil. Ic. Pl. 1864. Amblyglottis angustifolia, Bl. Bijdr. 369.

Perak (Scortechini), Larut Hills (King's Collector 5054), (Curtis 2066), Bujong Malacca (Tidley 9826); Pahang: Kluang 'Terbang (Barnes). Distrib. Java. 'Terrestrial; at an altitude of from 3-5000 feet.

4. C. Alboletes, Ridl. Journ. Roy. As. Soc. S. Br. Vol. XXXIX. p. 80. A large plant with broadly lanceolate acute leaves, $2 \frac{1}{2}$ feet long, and 4 inches wide with strong ribs, petiole stout 8 inches long. Seape stout over $1 \frac{1}{2}$ feet tall. Bracts caducous. Flowers about $\frac{1}{2}$ an inch across, pedicel and ovary $\frac{1}{4}$ inch long. Sepals and petals short broad orate acute white. Lip. 3 lobed white with yellow base, side lobes rery short falcate acute midlobe broad renilorm obovate bilobed 
rouncterl: calli 2 rect semiovate ridges, spur shorter than the pedicel thick blunt eurved clubbed. (Ridley).

Perik (Scortechini), Larut IIills (Derry), Bujong Malacea

5. C. Scontecrunit, Hook. fil. Fl. Brit. Ind. V. Sut. Leaves lanceolate acuminate, 2 feet long 3 inches wide. Scape stout $1:$ foot long with several large loose sheaths, raceme dense 2 inches long. Bracts lanceolate acute 2 inches long pale green caducous. Flower's small, ovary and pedicel $\frac{1}{4}$ inch long. Sepals lanceolate acute $\frac{1}{4}$ inch long connivent. Petals broader oblong. Lip small side lobes very short hlunt, midlobe longer ovate edges incurved with wo low kech on the base, spur much shorter than the ovary, clubbed, all yellow. Column white, anther beaked. Capsule $\frac{1}{2}$ inch long elliptic.

Perak: Larut Hills (Scortechini, Curtis). The lip is often retuse with a small decured process in the notch. Forests at $2-4000$ feet elevation.

6. C. curculigoides, Lindl. Gen. and Sp. Orch. Pl. 251. Leaves lanceolate acuminate, $2-3$ feet long. 6 inches wile. petiole $i \frac{1}{2}$ inches long. Scape 1-2 feet tall stout, raceme dense many flowered. Bracts lanceolate acute pale green $1 \frac{1}{2}$ inch long, falling off as the flowers expand. Flowers orange, lip red, perlicel and ovary $\frac{1}{4}$ inch long. Sepals: and petals sub-similar ovate lanceolate acute nearly inch lones. Lip as long as sepals arlnate to column hase, side lobes watved erect obture mitlobe longer entire oblong acute, spur as long as the ovary and pedicel, slender with a hooked tip. ('olumn semiterete. upper edge sinuate dentate. Anther white with a long beak. Lindl. Bot. Reg. 18t\%. t. 8. Fol. Orch. 4. Hook. fil. Fl. Brit. Ind. V. 85t. S'tyloglossum nervosum, Breda Orch. Jav. t. 10.

Singapore (Wallich 7340 ), Bukit 'Timah, Selitar (Ridley); Johor: Kwala 'Tehing 'Tingrgi, Gunong l'ulai (Ridley') : Perak. Distrib. Sumatra, Jara. Damp forest from sea level to about go(n) feet elevation.

Formerly abundant in singapore, but has leeme searece through the destruction of the forests. It is beantiful plant, flowers in September.

\%. C. Aumaxida, Ridl. Journ. Roy. As. Soc. S. Br. Tol. XXXIX. p. 80. Leaves narrow lanceolate acuminate 12 inches long

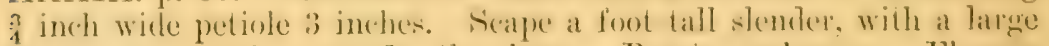
lanceolate sheath towards the base. Bracts eaducous. Flowers t inch across, orange coloured. Pedieel and orary slender $\frac{1}{2}$ jnch long. Sepals ovate much broader, orange. Lip narrow side lobes subtriangrular, ovate midlole narrow lineat oblonger ohtuse, red licels 
2 short semiovate. Spur narrow slender sigmoid blunt. Rostellum long beaked.

Perak: Bujong Malacca (Ridley).

\section{§ 2. Preptanthe.}

8. C. vestita, LindI. Gen. and Sp. Oreh. Pl. 250. Pseudobulbs 2-5 inches long ovoid obtusely angled, leafy after flowering. Leaves 12 to 18 inches long lanceolate, wide. Scape from the side of the pseudobulb 12 to 18 inches or more, villous. Bracts persistent ovate $\frac{3}{4}-1$ inch long. Flowers white large, pedicel and ovary $1 \frac{1}{2}$ inch long. Sepals ovate lanceolate. Petals broadly oblong. Lip side lobes cuneate obovate, mirllobe flabellate obcordate, white with a red or yellow spot at the base. Spur very long. Bot. Mag. t. $46 \% 1$. Flor. des Serres t. 816. Hook. fil. Fl. Brit. Ind. V. 852. Cytheris Grifithii, Wt. Ic. t. 1\%51-2. Preptanthe vestita, Rehb. fil. Bot. Zeit. XVI. 128.

Selangor: Epiphytic on the top of the limestone rocks, near Kwala Lumpur (Ridley, one plant). Distrib. 'Tenascerim, Borneo.

9. C. Rubers, Ridl. Gard. Chron. 1890. I. p. 5\%6. Pseudobulbs conic deeply groored 6 inches long. Leares $12-20$ inches long 5 to 6 inches wicle orate lanceolate. Scape nearly 2 feet long woolly pulsescent except on the sheaths. Flowers rose or white. Bracts ovate acuminate pulsescent, an inch long. Pedicel and orary 2 inches long pubescent. Sepals lanceolate acuminate $\frac{3}{4}$ inch long. Petals shorter. Lip side lobes oblong erect appressed to column obtuse, midlobe linear oblong apex bilobed, lobes excurved obtuse, with a process in the sinus, base of lip darker coloured with three raised lines. Spur filiform curved $\frac{1}{2}$ inch long.

Lankawi Islands (Curtis 2181). Terrestrial. A much smaller plant than C. vestita. Endemic.

\section{Arundixa, Blume.}

Terrestrial herbs, with woody terete stems, and narrow grassy Jeares. Flowers in a raceme simple or sparingly branched, large. Bracts persistent ovate. Sepals lanceolate. Petals broader. Lip 3 lobed, lobes convolute round the column midlobe rounded crisped, 2 or more raised nerres on the dise. Column long dilate upwards, winged. Anther helmet-shaped. Pollinia 8 in superposed pair's cohering by a viscus. Capsule oblong.

Species 6. Indian, Malayan and Chinese.

Flowers 3 inches across . . . . . . .

1. A. speciosa.

Flowers 2 inches across.

Side lobes of lip distinct .. $\quad$. $\quad$.. $\quad$ 2. A. Philippii.

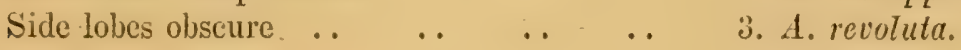


1. A. speciosı, Blume Bijdr. p. 401. t. 73. Stems 3 to $\%$ feet tall slender. leaves narrow lincar acmminate $2-t$ inches long, it inch wide. Raceme 6-8 inches long, few flowered. Bracts ovate acute $\frac{1}{4}$ inch long. Orary and pedicel 1 inch long. Sepals lanceolate $1 \frac{1}{2}$ inch long inch wide, keeled, rose pink. Petals ovate, mucromate as long and twice as broad, pink. Lip longer 2 inches ohlong side lobes short, midlobe bilobed crisped, rose colour with decp erimson apex and edges, a yellow central line, keels 3 on the dise. Column white an inch long. Capsule oblong 3 inches long. A. bambusifolia, Lindl. Bot. Rerg. XXYI. Misc. 2. Journ. Limn. soce iii. 22. Gen. and sip. Oreh. P1. 125. Wight Ic. t. 1661. Grift. Notul. JII. 329. 331. Ie. Pl. As. t. i1t. Hook. fil. Fl. Brit. Ind. Y. Sis: A. dense, Lindl. Bot. Regr. XXVIII. t. 38. Misc. 26. Journ. Linn. Soc. III. 23. A. densiflora, Hook. fil. l.c.c. Cymbidium bambusifolium, Roxb. Fl. Ind. III. 460 . Bletia graminifolia, Don. Prodr. 29.

Johor: Gunong Janeng (Kelsall), Indau River (Ridler); P'it-

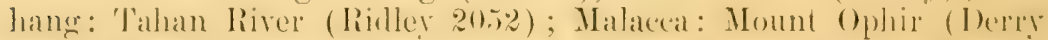

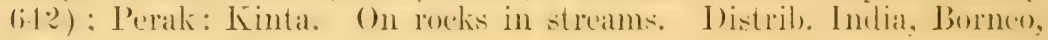
Java.

The locality Singapore given in Flora of British India must be an error. 'There is no place in Singapore likely to have produced it.

2. A. Pinmiprit, lichb. fil. Linnaea XXV. 22\%. var. Malayana.

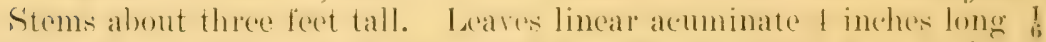
inch broat. Raceme 2 inches long. Bracts orate \& inch long. Flowers 2 inches across, oviry and perlicel one inch long. Sepals lanceolate acute. Petals similar wider, white tinted rose. Lip short"re sirle lobes oblong rounderl. midlobe orbiendar obovatr bilober lobes rounded, pinkish white, with brown streaks on the side lobes, apex of midlobe dark erimson, keels two crested on the disc thickened and connate or the midlobe yellow pubescent with a smaller median one in the centre. Column apex curved thickened, back white, front brown and red wings narrow.

Kedah Peak: Gunong Jerai (Ridley 5128) on rocks. Difiers from A. Philippii typical in its large brown-streaked flowers. Distrib. of type, Java, Hongkong.

3. A. REvolu's, Hook. fil. Fl. Brit. Ind. V. Sõ\&. Stems about 3 feet tall slender. Leaves linear long acuminate rather stifl $t-7$ inches long $\frac{1}{6}-\frac{1}{4}$ inch wide, rather crowded. Raceme 1-2 inches long. Bracts orate acute inch lomer. Ovary and perlicel 1 inch loner.

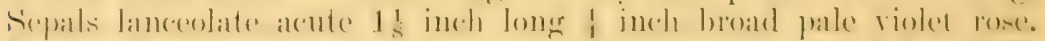

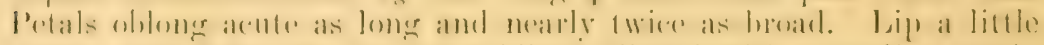

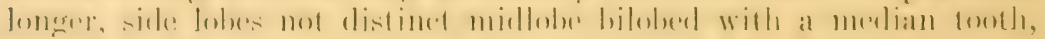
keels 2 low and straight, median one rery low and obseure whole lip 
pale rose elge veined with purple, a pale yellow central bloteh. Column pinkish white. Capsule $1 \frac{1}{8}$ inch long.

Perak: Larut Hills, at Thaiping Waterfall (Scortechini 1504), (Ridley 2882).

\section{Dilocmia, Lindl.}

Epiphytic or terrestrial plants with stout leafy terete stems. Leares coriaceous, broadly lanceolate. Inflorescence racemose or panicled, terminal. Bracts large coriaceous. Flowers mediocre. Sepals lanceolate. Petals similar. Lip three lobed with 5 crests in the centre. Column tall arched with three anthers. Rostellum broad entire. Fruit globose.

Species 2. Malay Peninsula and Islands.
Bracts lanceolate acute .. ... ..
1. D. Wallichii.
Bracts orate blunt
2. D. Cantleyi.

1. D. Wallicimi, Lindl. Gen. and Sp. Orch. Pl. 38. Stems 18 by $2 t$ inches long. Leaves ovate lanceolate acuminate $3-7$ inches long $1 \frac{1}{2}-2$ inches long, sheaths ribbed 1 inch long. Panicle of one or more branches 6 to 9 inches long. Bracts lanceolate acute one inch long. Pedicel and ovary $\frac{3}{4}$ inch long. Sepals and petals oblong lanceolate ochre yellow, half inch long. Lip yellow with red markings sides revolute rounded, midlobe broad rounded. Column large with the three anthers well marked. Capsule globose, opening by slits half way down. Blume Orch. Arch. Ind. t. 8. and 5. A. Arundina WalTichii, Reh. fil. Xen. Orch. II. 13. t. 105. fil. 13-15. Hook. fil. Fl. Brit. Ind. V. 858 .

Singapore (Wallich 1952); Perak: Larut Hills (Derry Herb. Singapore 10818).

2. D. Chxtleyi, Ridl. Journ. Linn. Soc. XXXII. p. 332. Stems as in $D$. Wallichii. Leaves ovate or ovate lanceolate long acuminate, 4 inches long by 1 wide, sheaths $\frac{1}{2}$ inch long. Panicle 3 or 4 inches long with many branches. Bracts ovate boat-shaped $-\frac{1}{2}$ inch long. Flowers $1 \frac{1}{4}-1 \frac{1}{3}$ inch wide. Sepals oblong lanceolate, falcate. Petals linear oblong, all yellow with red nerves. Lip small three lobed side lobes oblong blunt, midlobe flabelliform, keels 5 crenate, traversing the whole lip. Column toothed. Arudina Cantleyi, Hook. fil. Fl. Brit. Ind. 1.c.c. Ic. Pl. 2112.

Perak: Gunong Bubu (Cantley), Gunong Inas (Wray 4098).

\section{Coelogyne, Lindl.}

Epiphytic or rock plants, with a stout rhizome, and two leaved psudobulbs. Leaves coriaceous. Scape lateral from the rhizome or terminal on the top of the pseudobulb. Bracts decidnous papery. 
Flowers white, pale yellow or green often large. Sepals oblnng or lanceolate. Petals narrower. Lip sessile at the base of the column, spurless side lobes embracing the colmmn. Cohmm long footless, top hooded, sides winged. Anther conic 2 celled. Pollinia 4 cohering in pairs by a granular viscus. C'psule large elliptic pendulous winged.

Species about 70. Indo-Malayan.

Raceme pendulous from the rhizome.

Raceme short quite glabrous .. .. .. 1. C. testacea.

Raceme very long many flowered.

Pseudobulbs conic-cylindric. Leaves lanceolate.

Flower's distant nigrohirsute .. .. 2. C. Dayana.

Flowers crowded glabrous .. .. 3. C. densiflora.

Psendobulbs eylindric leaves obovate .. 4. C. Rochusseni.

Pseudobulbs 4 angled, leaves oblanceolate 5. C. quadrangularis.

Tiacemes erect from side of pseudobulbs.

Flowers numerous white large.

Bracts large ovate .. $\quad \ldots \quad$.. 6. C. asperata.

Bracts lanceolate $\ldots \quad \ldots \quad \ldots$

Petals narrower than sepals.. . .

Sepals acuminate. Flowers large .. $\%$. C. Tongibractala.

Sepals acute. Flowers smaller .. S. C. angustifotia.

Petals wider than sepals.

Lobes of lip acute .. .. . . 9. C. Foerstermanni.

Lobes of lip blunt .. ... .. 10. C. Kingii.

Flowers green and black.

Pseudobulbs thin and flat.. .. . 11. C. pandurata.

Pseudobulbs conic, or ovate .. . 12. C. Mayeriana.

Flowers one or two on the scape.

Flowers white. Petals lanceolate .. .. 13. C. Cumingi.

Flowers brown. Petals linear .. ... 14. C. speciosa.

Flowers small lip clawed.

Side lobes acute .. $\quad \ldots \quad \quad \ldots \quad \quad \ldots$ 15. C. sulphurea.

Side lobes truncate $\quad \therefore \quad \ldots \quad \ldots$ 16, C. pusilla.

Scape from the top of a pseudobulb.

Flowers large two inches across.

Lip side lobes blunt, midlobe shorter ․ 1\%.C. anceps.

Side lobes acute, midlobe as long .. 18. C. pallens. 
Flowers small $\frac{1}{2}$ inch across.

Pseudobulbs distant fusiform.

Leaves ovate. Flowers green ․ 19. C. prasina.

Leaves elliptic. Flowers brown .. 20. C. carnea.

Pseudobulbs crowded very small ‥21. C. stenochila.

. 1. C. testace., Lindl. Bot. Reg. 1842. Misc. p. 38. Folia Orchidacea 2. Psendobulbs crowded often forming large masses, conic 2 inches long, 3 inch thick rugose. Leares lanceolate petiolate acute $y$ to 8 inches long, 2 inches wicle, petiole 2 inches long. Racemes short pendulous 6 flowered. Flowers an inch long foetid. Bracts persistent oblong flesh coloured $\frac{1}{2}$ an inch long. Sepals oblong subacute keeled. Petals linear narrow, all flesh colour. Lip $\frac{3}{4}$ inch long, side lobes rounded dark brown edged with white midlobe ovate or oblong orbicular rounded crisped, with 4 crested ridges running the whole length, white with brown marbling at the tip. Column white hood obscurely toothed. Anther conic brown. Botanical Magazine t. 4785. Hook. fil. Fl. Brit. Ind. V. 829.

Singapore: Chan Chu Kang, Kranji, ete., (Ridley); Johor: Tana Runto (Ridley). On the bases of Oncosperma, and other trees near the sea. Endemic.

2. C. Dayana, Rehb. fil. Gard. Chron. 1886. p. 44. fig. 9. Pseudobulbs crowded subcylindric narrowed upwards $t$ inches long. Leaves lanceolate acmminate 20 inches long 3 inches wide narrowed into a petiole 4 inches long. Scape pendulous 20 inches long.

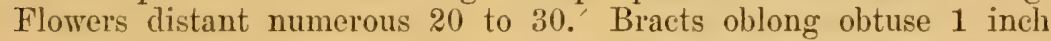
long. Pedicel and ovary 2 inches long, like the rachis covered with a black pubescence. Sepals lanceolate acute keeled $1 \frac{1}{4}$ inch long. Petals lanceolate narrowed. Lip longer, side lobes obtuse brown with white reins inside, white with a brown patch outside, midlobe ollong oroid edges crisped, keels on dise 3 crested, base brown, middle white, apex yellow, with two more outside on the midlobe. Column curved 1 inch long white, brown in front. Hood obscurely toothed. Anther conic. Williams Orchid Album VI. t. 24\% C. tomentosa, Wawra. Bot. Voy. Ps. Saxe-Coburg II. 15t. t. 10. Hook. fil. Fl. Brit. Ind. V. 830. (not of Jindley).

Malacea: Mount Ophir (Hullett 861); Pahang: Kiluang Terbang (Barnes Herb Singap. 10914); Perak: Larut Hills (Derry, Curtis, Kining's Collector); Penang (Curtis). Distrib. Borneo.

var. Massangeana. Pseudobulbs and leaves shorter, keels on midlobe forming an irregular yellow mass. (․ Lassangeana, Rehb. fil. Gard. Chron. 1878. 684.

Perak: Larut Hills (Derry). 
C. tomentosa, Lindl. Folia Orchid 3, is described as having a tomentose ovary and three keels on the lip. It was collected by Lobl), No. $18 \%$ of his collection and has no locality on the specimen. $C$. Dayana is not tomentose at all, but the rachis and ovary are covered with a nigrohirsute pubescence, and has five keels on the lip. $C$. Wasengerene, lichb. fil. has been a very puzzling plant on account of the fact that it has been confuser with typical Dayana. Hooker' (Bot. Mag. t. 6r99) states that it is not nigrohirsute; Williams' Orchid alloum gives it as nigrohirsute (Orehid Album t. 2!)). The original plant had short bulls: and broad leaves, but plants from the Iarut Hills with the flowers of $C$. Mresemgeana, have the psemilobulls and leaves of $C$. Dayana. The coalescence of the ridges on the disc seem to be the only distinguishing point.

3. C. Dexsiflohi, Ridl. Journ. Roy. As. Soc. S. Br. Tol. XXXIX, p. 81. Pesudobulbs long cylindric conic narrow 4 inches long. Leaves lanceolate acuminate 15 inches long, $1 \frac{3}{4}$ inch wide, petiole 2 inches long. Scape pendulous 8 inches long dense, flowers numerous smaller than in $C$. Dayana, rachis and ovaries not nigrohirsute. Bracts red brown oblong truncate half an inch long and as wide. Tipals lanceolate acute. Petals narrower $\frac{1}{2}$ inch long brownish. lip side lohes short acute apices narrow, outside white, insigle brown with white streaks, midlobe orbicular shortly apiculate edge white, centre red brown with a large yellow central papillose masi, keels on the disc between the lober crested. Column hood retuse. Anther white.

Selangor: Bukit Hitam (Kelsall). Endemic.

4. C. Rocirusseni, De Vr. Ill. Orch. t. 12. t. 11. fil. 6. Rhizome stout. Psendobulbs few close eylindric grooved 3-6 inches long. Leaves obovate petiole i-s inches long, 4 inches wide, petiole 1 ! -? inches long. Scapes pendulous 18-2t inches long slender. Flowers distant, numerous. Bracts oblong truncate $\frac{1}{2}$ inch long. Pedicel and ovary slender 3 inch. Sepals lanceolate acute 3 inch long greenish yellow, keeled. Petals linear narrower. Lip as long side lobes long blunt, white outside tawny with white veins inside dise white: midlobe ovate acuminate narrowed at the base, edge crisped white with a yellow centre and 2 tawny spots tewards apex, keels 3 crested, on the dise with two extra outside on the midlobe, tips yellow, bases

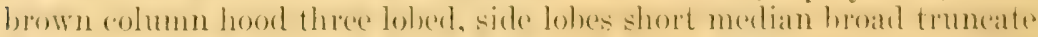
cuneata. Capsule ovoid with three strong keels 2 inches long and one across; Richb. fil. Xenia Orch. 1. p. 212. t. S5. C. macrobulbon, Hook. fil. Fl. Brit. Ind. V. p. 830.

Singrapore: Seletar (Ridley); Johor: Tatu Pahat, Gunong Janeng (Kelsall), Pulau Dayak, Pulau Aor (Feilding); Pahang: 'Jahan River (lidlley); Selangor: Jukit Hitam (Kelsall), Rawang 
(Ridley :is1), Sungei Ujong (Native Collector); Perak: Larut Hills (King's Coll. 6694); Penang (Wallich 196912); Malacea: Mount Ophir (Ridley). Distrib. Sumatra, Tara, Borneo. Native name "Sakat Tulo Ular." (Snake's bones orchid).

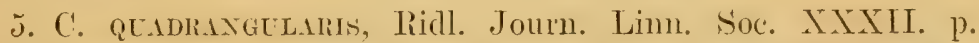
32:3. Rhizome thick, pseudobulbs conic strongly tangled 3 incles long, $1 \frac{1}{2}$ inch thick. Leaves 6 inches long 2 inches wide oblanceolate acute narrowed at the base into a short half inch petiole racemes pendulous 8 inches long 8-12 flowered. Flowers distant, rachis nigrohirsute. Bracts oblong $\frac{1}{4}$ inch long. Sepals lanceolate acute 13 inch long, laterals longer falcate keeler. Petals lancenlate linear narrower. Lip an inch long, side lobes oblong obtuse white outside, inside brown with white veins, midlobe ovate obtuse white with a brown central spot, keels 3 , denticulate with 2 extra ones on the midlobe. Column white with brown markings, hood truncate irregularly toothed. Anther brown.

Perak: Larut Hills 5000 feet (Ridley). Distrib. Borneo.

6. C. asperata, Lindl. Journ. Hort. Soc. IV. 221. Pseudobulbs crowded conic, smooth 5-7 inches long 1-2 inches thick. Leaves oblanceolate obovate cuspidate narrowed at the base dilate upwards, 2 feet long 4 inches across strongly ribbed, petiole 8 inches long. Scape from the rhizome near the bulb drooping, base $t$ inches covered with large ovate bracts erect, raceme 6 inches long. Flower's about 15-20 fragrant. Bracts oblong $\frac{3}{4}$ inch long. Orary and pedicel 1 inch. Sepals oblong lanceolate white or yellowish. Petals linear lanceolate. Lip side lobes long orate, midlobe claw broad limb oblong obtuse crisped with an orange warty ridge. Column stont. Capsule elliptic 2 inches long, wings not very larege. Williams' Orchid Album t. 311. Hook, fil. Fl. Brit. Ind. Y. 835. C'. Lourii, Lindl. Paxt. Mag. 1849. 225.

Perak: Larut Hills on rocks and trees, (King's Coll. $228 \%$ ). Distrib. Sumatra, Borneo.

\%. C. Longrbractata, Hook. fil. Fl. Brit. Ind. VI. p. 194. Ann. Bot. Roy. Gard. Cale. Vol. 8. p. 26. t. 39. Pseudobulbs elongate conic ribbed 3 inches long. Leaves narrow lanceolate acute 8 inches long $1 \frac{1}{4}$ withe. Scape from the rhizome near the bulb, erect at base covered with green sheathing leaves about 3 inches long, two uppermost foliaceous raceme 6 inches long flexuous, 5 flowered. Bracts lanceolate acuminate papery 2 inches long. Flower's 3 inches across, pedicel 1 inch long. Sepals lanceolate acuminate acute. Petals narrower, all white. Lip shorter, side lobes ovate obtuse, yellow veined orange, midlobe short ovate cuspidate edge at base erisped, white with a basal yellow spot, keels three, crisped with 2 outside on the disc. Column dark orange, hood truncate : toothed. 
Anther belt shaped apex thickened. C. caste, liddl. Journ. Limn. Soc. XXX11. 32\%.

Selangor: Bukit Hitam (Kelsall); Perak: (Kunstler), Larut Hills (Derry).

S. C. Angestroma, Ridl. Jomm limn. Soc. XXXII. 322. Jihizome very stout, peeudobulbs $2: 3$ inches long conic smooth. Leares lanceolate acute, narrow is nerved 10 inches long, 1 inch across, petiole 3 inches long. Seape from thizome close to pseudobull, 6 inches long, base 1 inch covered with loose sheaths, racene slender flowers about 6 , small. Bracts Janceolate acute. Sepals lanceolate lorate subacute 1 inch long, cream colour. P'etals much narrower spathulate lineat. Lip it inch long, side lobes long obtuse pale outside brown within, midlobe obovate oblong rounded edge mndulate, keels 33, immer one smaller, sinuate white, edge yellow with brown spots. Column arched cream colour tinted brown, hood rounded. Anther conic.

Lankawi Islands (Curtis).

9. C. Folistermaxi, lichb, fil. Gard. Chron. 1ssa Il. p. 26\%. A large plant with elongate conic pseudobulbs grooved o inches long, 1 through. deaves lanceolate acute, 8-15 inches long $2-2 !$ wide petiole is to $t$ inches long. Seape from thizome near the pseudobulb, \# feet long, base erect covered with sheaths, racene nodeling of seren or more large flowers. Bracts lanceate acute is inches longs Pedicel and ovaly 1! inch long. Sepals lanceolate $1 \frac{1}{2}$ inch long. Petals wider, all jure white. Lip side lobes broad, apoices acute white with orange brown strealis, midlole ovate acute edges erisped leels 5 crested, white centre rellow with a lew red spots. Columm green with yellow edges, hood emarginate denticulate. Anther conic with at boss on the top. C'. Maingayi, Hook. fil. Fil. Brit. Lnd. T. p. 831.

Malacea (Maingay, Derry); Pahang: Kuantan (Durnlord), 'Jelang liver (Machado). Distrib. Borneo. On very lolity trees, a beatilul plant but very dillicult to cultivate.

10. C. Kiskir, Hook. fil. Ann. Bot. Moy. Gard. Cale. V. 5. p. 25. t. 38. lihizome stout, psendobulbs close or distant, elongate conic 1 inches long 1 inch throngh. Leaves lanceolate acute 9. inches long 1: inch wide petiole $1-1$ inch long. Scape from rhizome at base of pseudobulb, base sheathed of inches long raceme flexuous, 1 loot long. Flowers 8 distant white. Bracts $1 \frac{1}{1}$ inch long. Sepals lanceolate acute an inch long. P'etals spathulate lanceolate nearly as long and broater. Lip : inch long, side lobes rounded obtuse, midlobe broaler rounded crisped mucronate, white with a yellow spot on both sides of the side lobes and on base of midlobe brown stealis at base or lip, keels i, merlian shorter all undulate, denticulate, : extra ones on midlobe ontside. Column yellowish, white, 
hood notehed and rounded. Anther buff, helmet shaped, with a boss on top.

\section{Perak: Larít Hills (Kunstler, Derry).}

11. C. Paxdurata, Lindl. Gard. Chron. 1853. p. \%91. Rhizome rather long creeping, psendobulbs orbicular ovoid 3-5 inches long $1 \frac{1}{2}$ inch wide that and thin. Leaves elliptic lanceolate acuminate, narrowed at the base into a long $2-4$ inch petiole 12-18 inches long, 2-3 inches wide. Scape 6-18 inches long basal half enclosed in sheaths, the upper pair foliaceous. Flowers large green and black 8 or 9, laceme lax. Bracts 1 inch long persistent. Ovary and pedicel 2 inches. Sepals linear oblong laterals smaller. Petals subspathulate. Lip pandurate, lobes narrow, midlobe subquadrate, retuse, base narrowed mottled and veined black. Bot. Mag. t. 508t. Richb. fil. Xenia Orch. II. t. 121. Warner Orchid Album t. 63. Lindenia II. t. 86. Hook. fil. 1.c. 835.

Selangor: Rawang; Pahang 'Track (Ridley); Perak: Kinta (King's Coll. r166), (Scortechini). Distrib. Borneo, Sumatra. On trees over streams.

12. C. Mayeriana, Rehb. fil. Gard. Chron. 18\%\%. II. p. 134. Iihizome long and thick, pseudobulbs ovate compressed, 3-t inches apart 2 inches long, 1 inch wide. Leaves lanceolate acute 8 inches long 1 inch wide, with a thick petiole. Scape a foot long distant from the pseudobulb, base 2 inches sheathed $2-3$-upper sheaths foliaceous, raceme rachis stout, lax flowered. Flowers 5-6 pedicel and ovary 2 inches long. Bracts oblong 1 inch. Sepals $1 \frac{1}{2}$ inch long oblong obtuse green. Petals smaller, linear lanceolate acute. Lip green with black nerves and dots, side lobes long obtuse, midlobe pandurate bilobed erisped, keels 3 on dise, 4 on midlobe toothed and crested. Column green, wings wide. Anther conic obtuse. Capsule clliptic 2 inches long keels, low. Ridley Journ. Linn. Soc. XXXII. 325.

Singapore: Kranji, Chan Chu Kang (Ridley); Johore: 'Tana Runto (Ridley). Distrib. Rhio, Sumatra. Low down on trees in sandy places near the sea.

13. C. Cummant, Lindl. Bot. Reg. 1840. Mise. p. \%6. Pseudo-

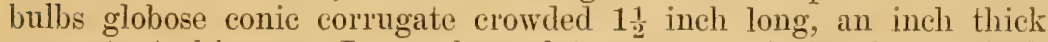
on a stout rhizome. Leaves lanceolate acute, 6 inches long 1 inch wide. Scape erect from among the leaves, 3-5 inches long, few flowered. Bracts lanceolate acuminate 1 inch long. Flowers 3 inches across. Sepals oblong lanceolate subacute. Petals lanceolate much narrower, all white. Lip side lobes oblong obtuse white with orange below the tips, midlobe large broad erose or entire subacute white with an orange centre, lieels 3 erisped on the dise more elerated and denticulate, tecth red brown at the tips on the midlobe. Column 
curved, hood minutely toothed white. Bot. lieg. 18 11. t. 29. Bot. Mag. t. 46t5. Hook. fil. Fl. S. Brit. Ind. T. 834.

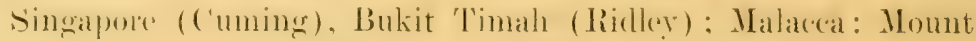
Ophir (Ridley), Pulau Jrawang (Derry); P'ahang: 'Tahan liver (Ridley): l'erak: Walerloo (curtis), Larut Ilills (Ridley).

On the tops of rery lofty trees in the low comntry but often lorming large masses on rocks at an elevation of 3000 feet. Flowers in July. Hlowers liagrant.

14. C'. Sreorosa, Lindl. Gen. and Sp. Orch. Pl. 39. Pscudo-

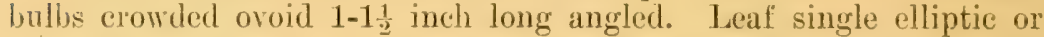
lanceolate acute \pm to 12 inches long, 3 -inches broad, petiole $1-2$ inches fong. liakeme drooping from the axil of the roung leat 1 inches long 1-2) flowered. Bracts lanceolate acmminate, caducous. S'epals oblong lanceolate acute d2-2! inches long greenish or bull. Petals namowly linear. Lip large, sicle lobes obtusc toothed, midlobe broally chased rounded toothed oblong with two fringed crests yellow blotched and marked red brown, margin broad white. (alpsule e? inches long oroid with very latge wings. Bot. lieg. 18t\%. t. 23. Fol. Oreh. 11. Bot. Mag. t. 4889. Hook. fil. Lindl. Fl.

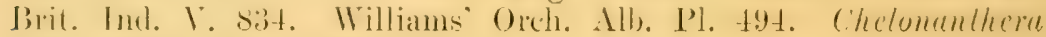
speciosa, B1. Bijdr. 384. t. $\check{1}$.

Singapore: Ulu Sumbawang (Ridley's ('oll.); Johor: Sungei

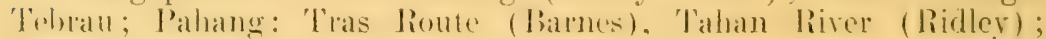
Perak: Lalut Hills common (lidlley 5198), Gunong Batu Putil (Wray); Selangol: Semangko Pass (Ridley); l'enang: West Hill (Curtis). Distrib. Borneo, Java. On trees in dense forest from sea level to 3000 teet eleration.

15. C. sthpulra, Iichb. fil. Bomplandia 185\%. 43. P'sendo-

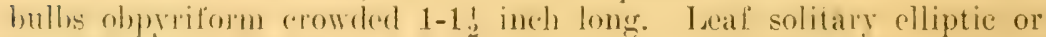
lanceolate, :3-6 inches long $\frac{1}{2}-1$ wide, petiole $1-3$ inches long. Scape from the thizome, base covereel with sheaths $2-3$ inches long, raceme slender 2-3 inches long. Flowers small about 10-12 in number. Sepals oblong lanceolate acuminate nearly $\frac{1}{2}$ inch long. Petals very

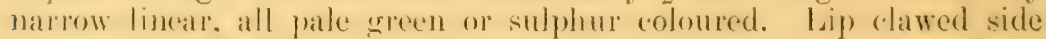
lobes narrow acute, midlobe clawed oblong, blade broad reniform rounded retuse pale yellow with an orange central spot, keels 2 on the dise and elaw of midlobe. Column arehed, hood rery broad rounded Hook. fil. F1. Brit. Ind. V. 833. Chelonanthera suljhurea B1. Bijdr. 38.;.

Palang: Kluang 'Terbang (Barnes 11070); Perak (Scorte(hini), Larut Ilills at joov leet alt. (Curtis), Ulu Batang l’adaug (Iiay 1538).

'I'his and the next species alle almost intermediate between the

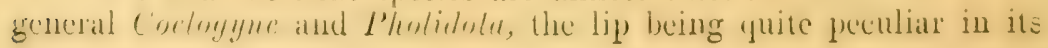


claw and short spreading side lobes and the pseudobulbs being oneleafed only.

16. C. pusilla, Ridl. Journ. Tinn. Soc. XXXII. 32\% Psendobulbs crowded conic 1 inch long $\frac{1}{4}$ inch thick. Leaf solitary $3-4$ inches long $1 \mathrm{inch}$ wide lanceolate acute petiole 1 inch. Scape from the side of the pseudobulb $t$ inches or more long. Bracts lanceolate acute an inch long. Flowers small pedicels $\frac{3}{4}$ inch long. Sepals lanceolate acute flesh colour. Petals narrow linear filiform. Lip clawed, blade deflexed, side lobes small oblong truncate midlobe oblong ochre yellow apex dilated reniform bilobed white with an orange red spot at base, keels 2 between the side lobes. Column arched broadly dilate above with large wings.

Perak: Larnt Hills at 4000 feet (Ridley), (Curtis 2063). Distrib. Java.

1\%. C. Anceps, Hook. fil. l.c. 840. Ic. Pl. 2109. Rhizome stout, psendobulbs distant ellipsoid or fusiform. Leaves 2 elliptic acute $2 \frac{1}{2}-4$ inches long, $\frac{2}{3}-1 \frac{1}{2}$ inch wide, petiole short stout. Seape from the top of the pseudobulb 5-10 inches long base nude compressed raceme stiff with several persistent bracts at the base. Flowers 2 inches across, ovary and pedicel $\frac{1}{4}$ inch long. Bracts $\frac{1}{2}$ inch. Sepals linear oblong acute. Petals narrowly linear. Lip side lobes long apices obtuse, midlobe wider rounded much shorter with 2 short ridges in the centre. Column hood acute.

Perak (Scortechini).

18. C. Pallexs, Ridl. Journ. Roy. As. Soc. S. Br. Vol. XXXIX. p. 81. Rhizome stout, pseudobulbs subcylindric 2-3 inches long wrinkled. Leaves 2 elliptic, or oblanceolate $6-3$ inches long 1-1 $\frac{1}{2}$ inch wide, petiole 1 inch. Scape from the top of the pseudobulb, base mude with 1 persistent bract, raceme long 6 inches flexuous. Flowers 2 inches across. Sepals lanceolate acute pale green or buff. Petals linear filiform. Lip pale buff lateral lobes long, with subacute long denticulate pubescent tips base saccate, midlobe as long with 2 long sinuous brown keels with a short intermediate one. Column hood 3 lobed, central lobe long undulate or nearly entire. Anther conic not beaker.

Perak: Taiping Hills (Curtis), Bujong Malacea (Ridley). On trees alt. 2500 feet.

Closely allied to $C$. anceps, but the scape is terete, and the petals are linear filiform.

19. C. Plisixt, Ridl. Journ. Linn. Soc. XXXII. 326. Rhizome rather slenter long ereeping psendobulls distant fusiform 2 inches long. Leaves orate or elliptic acute 5 inches long $1 \frac{1}{4}$ inch wide, petiole $\frac{1}{2}-\frac{3}{4}$ inch long. Scape from the top of the pseudobulb 6 inches 
Iong flexmons. Bracts lanecolate acmminate an inch long deciduous. Flowers about 5, green $\frac{3}{4}$ inch across, pedicels $\frac{1}{2}$ inch long. Sépals lanceolate acute. Petals shorter filiform from a lanceolate base. Lip much longer pandurate side lobes short rounded, midlobe much longer oblong bate narrowerl, keels 2 thick. Column short. hood short roimder.

Kedah: Gunong Jerai $3-1000$ feet, on trees and the ground (Ridley 5131).

20. C. Carsed, Hook. fil. Fl. Brit. Ind. V. 838. Ic. Pl. $210 \%$. Thirome long stout, bullss distant fusiform $1-2$ inches long. Leave2 elliptic lanceolate $2-4$ inches long $\frac{1}{2}-1$ wide petiole $\frac{1}{2}-1$ inch long. S(alpe lrom top of parudobullus slender $8-9$ inches long, base flattened, raceme (6 inches) flexuous. Bracts lanceolate acute caducous 1 inch long. Flowers small 18-20, $\frac{1}{2}$ inch long yellow and brown. Sepals oblong lanecolate acute, laterals falcate gibbons. Petals linear fililorm. Lip longer, side lobes rounded obtuse, midlobe obovate rounded or retuse, much longer, keels 2 short. Column wings wide. Anther beaked.

Pahang: Kluang Terbang (Barnes 110\%\%); Perak (Scorte(hini). The petak in Ic. Pl. D10: are much too broar.

21. C. stricocirila, Hook. fil. 1.c. $83 \%$, Ic. Pl. 2106. Rhizome rather slender, psendobulbs terete $\frac{1}{2}-\frac{2}{3}$ inches long, close together.

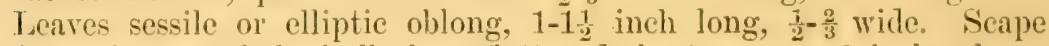
from the top of the bulb, base flattened short, raceme 8 inches long: narrowly linear. Lip narrow side lobes long apices short obtuse, midlobe orbicular, with 3 keels in the centre. Column hood truncate 3 loberl.

\section{Perak: Gunong Batu Putih (Tray).}

\section{2\%. Pholidota, Lindl.}

Epiphytes, with a stout often erect rhizome, pseudolullis distant or crowded. Leares 2. Seapes from the top of the psendobulb, rachis often flexuous. Bracts distichous dry. Flowers small, little expanded pink or white. Sepals concave. Petals flat. Lip sessile on the hase of the column, base saceate entire or $3-4$ lobed. Column very short hooded. Anther 2 celled, beaked. Pollinia 4 subglobose adtiering in pairs by a viscus. Pseudobulbs uninodal sessile on a stout rhizome.

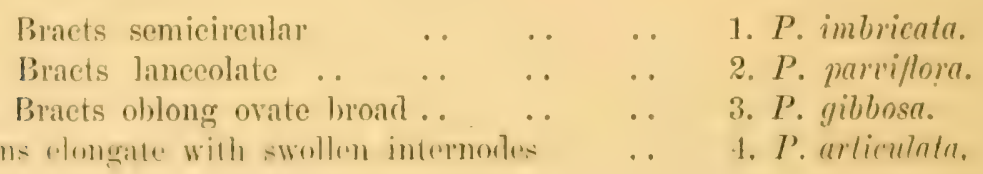


1. Pit. mmbricita, Lindl, in Hook. Exot. Flora II. t. 138. Gen. and Sp. Orch. Pl. 3\%. Bot. Reg. t. 1213 and 1\%\%. Pseudobulbs crowded conic dark green 1-3 inches long. Iseaf solitary elliptic lanceolate 6-12 inches long, petiole 1 inch long, or more. Raceme long decurved 1 foot long, peduncle slender. Bracts distichous semicircular $\frac{1}{3}$ inch broad. Flowers numerous pale flesh colour $\frac{1}{4}$ inch long. Upper sepal orbicular laterals boat shaped keeled. Petals linear oblong falcate. Lip boat shaped, side lobes broad rounded, terminal lobe bifid. Column wings broad. Capsule ellipsoid $\frac{3}{4}$ incli long. Lodd. Bot. Cab. t. 1934. Wight Ic. t. 90\%. Hook. fil. Fl. Brit. Ind. VI. 845. King. Ann. Bot. Gard. Calc. VIII. p. 144. Pl. 201. Ph. pallida, Lindl. Bot. Reg. XXI. sub. t. 1\%\%\% Coelogyne imbricata and $C$. pallida, Rchb. fil. Walp. Ann. VI. 238. Ptilocnema bracteatum, Don. Prodr. 33. Cymbidium imbricatum, lioxb. Fl. Ind. III. 460 .

Selangor caves: Kwala Lumpur (Ridley); Perak (Scortechini, King's Collector); Lankawi Islands (Curtis 2561). Distrib. India, C'eylon, Sumatra, Java, Borneo.

A very rariable plant in small points. The common form in the peninsula is the var. coriacea, Hook. fil. l.c. with coriaceous leaves.

2. Pi. pariflora, Hook. fil. Te. Pl. 1891. P. micrantha, Fl.

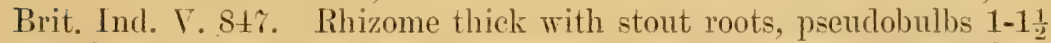
inch long cylindric or oroid, narrow or thick. Leaves elliptic lanceolate acute $1 \frac{1}{2}-3$ inches, $\frac{1}{2}-1 \frac{1}{2}$ inch across or wider. Scape slender 5 inches long flexuous. Bracts lanceolate. Flowers $\frac{1}{8}$ inch across about 35. Sepals ovate or orate oblong acute. Petals smaller, all greenish pink turning pink. Lip boat shaped clawed, midlobe ovate rounded greenish white-yellow with an elevated mass in the centre. Column red. Anther broad and flat, rostellum broad entire. Capsules $\frac{1}{4}$ inch long.

Perak: Gunong Batu Putih (Wray), Larut Hills (Derry).

3. PH. gibsos 1, De Vr. Illustr. t. 10. Rhizome stout branched, pseudobulbs oblong conic, covered with sheaths 2-3 inches long. Leaves lanceolate or obovate with a long point coriaceous $6-7$ inches long 1-3 inches across, petiole 1 inch long. Scape slender nodrling 10-12 inches long, basal half mude, raceme flexuous, flowers about 20 . Bracts broadly oblong ovate, $\frac{1}{4}$ inch long brown. Pedicels short. Cpper sepal oblong lanceolate obtuse, laterals broadly oblong broader $\frac{1}{4}$ inch long very obtuse. Petals linear or broadly oblong, obtuse all white, reflexerl. Lip base oblong boat-shaped, mirlobe oblong bifir lobes rounderl, deflexed, a pair of erect truncate teeth at its base, white, base brneath pinkish. Column curved hood repy broad margin wared brown. Anther red flat. Rostellum long thin bifid. 


\section{Pahang: Kluang Terbang (Barnes), Distrib. Java.}

The Javanese specimens have linear narrow petals, those of Pahang are much broader.

4. P. articulata, Lindl. Gen. and Sp. Orch. Pl. 38. Stems el-

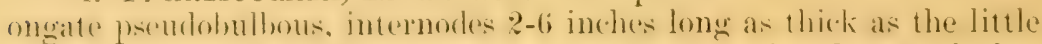
finger. Leaves 2 elliptic lanceolate acnte $4-6$ inches long 2 inches wille petiole ?-1 inch long. Raceme from between the leaver, shorter 4 inches long. Bracts rhombic orate $\frac{1}{2}-\frac{1}{3}$ inch. Flowers small pink, $\frac{1}{4}$ inch long. Sepals upper orbicular or ovate, laterals orate. Petals ovate. Lip boat-shaped, with 5 raised nerves at the base, midlobe bilobed. Column short, rostellum long acute. Capsule $\frac{2}{3}-\frac{3}{4}$ inch long. Hook. fil. Fl. Brit. Ind. V. 844. King. Ann. Roy. Bot. Gard. TIII. 146. Pl. $20 \pm$ 205. P. Khasiana, Rehb. fil. Walp. Ann. VI. 2:is. Lomplamtia 1856, 329. P. Griffithii, Hook. fil. l.c.c. p. 84.5. Is. Pl. t. 1881. P. decurva, Ridl. Journ. Linn. Soc. XXXII. 328. Coelogimme articulata and C. Khasiana, Rehb. fil. Walp. Amn. VI. 328.

Sclangor: Pahang Track (Ridley); Perak (Curtis). Distrib. Himalayas, Burmah. The peninsular form is the small variety Griffithii.

\section{Cuaderia, Hook. fil.}

Terrestrial, climbing a short way on tree trunks. Leares few ovate lanceolate plicate, narrowed into sheaths. Scape terminal erect, simple, or branched, rachis stont with short broad stiff bracts. Flowers large. Sepals obtuse, laterals oblique gibbous at base. l'wals lalcate oblancelate. Lip concave, large three lobed. Column long curved footless, with no arms. Anther helmet shaped. Pollinia 2 with no dise, elliptic acute.

Species 1. Malayan.

C. viridiflora, Hook. fil. Brit. Ind. T. 810. Ic. Pl. 2083. Rhizome slender. Leares green with darker mottlings 6-12 inches long, 1-2. inch long, petiole $\frac{1}{2}$ to 2 inches long. Scape 6 inches long, laceme at length 6 inches, with a long lanceolate acuminate leaf just below it. Bracts $\frac{1}{8}$ inch long. Flowers 1 inch long green reined reticulately with darker. Sepals oblong subaente. Lip side lobes Jong sulacute, midlobe orbicular retuse narrowed at the base broad,

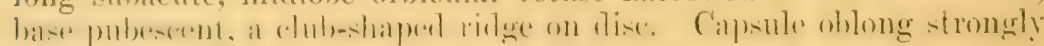
ribhed, 3 inches long $\frac{1}{2}$ inch through.

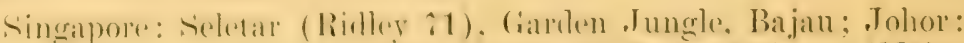

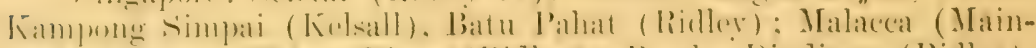

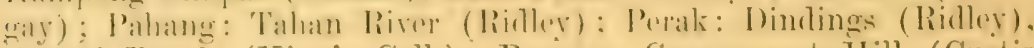
Sungei liayal (King's Coll.) ; Penang: Corernment Hill (Curtis 2969). Distrib. Borneo, Sumatra. 


\section{VANDEAE.}

\section{Eutophia, Br.}

Terrestrial glabrous herbs, rhizome tuberous or psendobulbous. Leaves several, appearing with or after the flowers. Scape lateral or subterminal. Sepals and petals free. Lip from the base of the column base saceate or spurred, side lobes erect. Column long or short with a broad base. Anther 2 celled. Pollinia 2 or 4 sessile or attached by a short caudicle to a discoid gland (disc).

Base of sepals prolonged into a spur .. $\quad \ldots \quad$ 1. E. squalida.

Lip spurred.

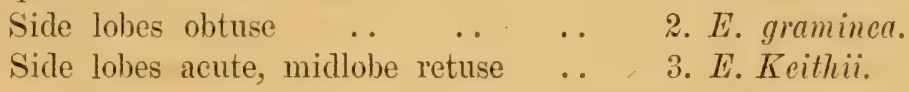

1. E. Squanmı, Lindl. Bot. Reg. 18+1. Mise. 7\%. Pseudobulb conic short. Leaves developing with the flowers, lanceolate acumi-

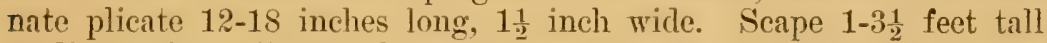
medium size. Bracts lanceolate acuminate half an inch long. Ovary and pedicel one inch. Upper sepal lanceolate cuspidate, laterals prolongerl at the base into a conic cylindric mentum $\frac{1}{4}$ inch long brownish green half an inch long. Petals oblong ovate twice as broad white tinted pink. Lip longer articulated with the base of the column, side lobes rounded, midlobe longer oblong crisped veins in dise + thickened, base of lip ocrenus, apex pale lilae side lobes veined pale ochre. Column broad much shorter than the petals, foot short and broad with a large entrance at the base to the spur wings and arms 0 . Anther orange conic with two whitish knobs at the top. Pollinia 2 globose, caudicle short broad, dise broadly triangular. Capsule pendant fusiform 2 inches long. Hook. fil. Fl. Brit. Ind. VI. 6. E. elata, Hook. fil. 1.c. 3. Cyrtopera squalida, Rehb. fil. Bonplandia 185\% 38. Lindl. Journ. Linn. Soc. III. 31.

Singapore: Tanglin, Chan Chu Kang (Ridley); Johor: Batu. Pahat, Kota Tinggi (Ridley), Sedili River (Nanson); Malacea: Machap (Derry 383); Perak (Scortechini 2023). Distrib. Borneo.

Open grassy country common. It seems hardly distinct from E. nuda, Lindl.

2. E. Graminea, Lindl. Orch. Pl. 182. Pseudobulbs cylindric conic often large, 4 inches long, 1 thick. Leaves grassy linear acute, ahout 6 inches long $\frac{1}{4}$ inch across, produced from the flowers. Scapes lateral 1-:; feet tall slender, brancherl, flowers small numerous an inch long. Bracts $\frac{1}{8}$ inch acuminate. Sepals lanceolate linear acuminate acute. Petals a little broarler green with reticulate brown markings. lip side lobes obtuse, midlobe obovate edge undulate erisped white roined with violet, with white hairs, three raised nerves on the dise, 
spur short clubbed. Column slender ereet, with no foot, nor arms. Anther hroal with two purple processes at the top. Pollinia globose with a short broad caudicle and a rounder dise. Capsule elliptic 3 inches long. Lindl. Journ. Linn. Soc. III. 23. Hook. fil. Fl. Brit. Ind. VI. p. 2. E'. inconspicua, Grift. Notul. 349. Ic. Pl. As. t. 326. lindl. l.c.c.

Singapore (Wallich :372), ('hua ('hu Kang (Ridley) : Malacea (Griffith). (('mung 2059). Bukit Sabukor (B)ery 55\%): Johor: Batu Pallat (Kolsall fo66) ; Pahang: Sungej Meang (Ridley 16:39): Penang Ilill ('urtis): Lankawi: Kwah (Cuntis). Distrib. Siam. Open sandy spots. Native name, Bawang Hantu.

:). E. Keitun, Ridl. Journ. Soc. XXXII. 333. Pseudobulbs :)-5 inchus long 1 inch thich, cylindro-conic. Leaves grassy, linear acuminate $2 \frac{1}{3}$ inches long, $\frac{1}{3}$ inch wide. Scape a foot long not

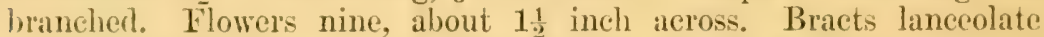
acute $\frac{1}{8}$ inch long, pedicels an inch long. Sepals $\frac{3}{4}$ inch long linear acute. Petals broader, all green. Lip green, disc of midlobe white with brown veins reticulated, side lobes falcate short, midlobe obovate retuse crisped, keels 3 undulate ending in a conical yellow callus, spur clubbed $\frac{1}{4}$ inch long. Column broad winged. Anther with two reenrved red processes.

Lankawi (Curtis). Distrib. Siam.

30. GEodontur. Jack.

Terrestrial herbs, with a tuberous rhizome, and herbaceous plicate leaves. Seape from the rhizome, stout. Flowers small crowded in decurved racemes, pink yellow or white. Bracts narrow. Sepals and petals subsimilar. Lip sessile on the base of the column entire boat shaped, with a central ridge, and callus. Column short stout with low wings. Anther ovate. Pollinia 2 on a strap shaped candicle.

Species 5 or 6 Indian, Malayan and Australian.

$\begin{array}{llllll}\text { Lip oblong obtuse } & \ldots & \ldots & \ldots & \ldots & \text { 1. G. purpureum. } \\ \text { Lip ovate acute } & \ldots & \ldots & \ldots & \ldots & \text { 2. G. citrimum. }\end{array}$

1. Cr.pupureun, R. Br. Ait. Hort. Kew Ed. II. V. p. $20 \%$ TTook. fil. Fl. Brit. Ind. VI. 16. Pseudobulbs short. Stem 3-1 inches tall. Leaves ovate lanceolate acuminate 9-12 inches long 3 inches wide. Scape 18 inches long raceme decurved, 2 inches, sheaths foliaceous. Bracts lanceolate acute $\frac{3}{y}$ inch long. Flowers pink or white I inch long. Perlicels and ovary as longr. Sepals oblong obtuse.

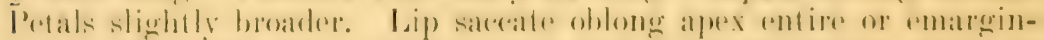

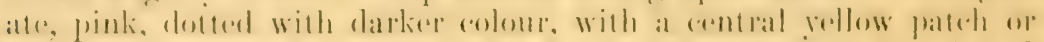
white with pink veins. Callus at hase conic. Colimm broad and 
short, with a short foot. King. Ann. Bot. Roy. Calc. VIII. p. 181, t. 215 (rar. album). G. fucatum, Lindl. Bot. Reg. t. $168 \%$.

Malacea : Rellau, hase of Mount Ophir (Derry), Bukit Sadanen (Derry 385). Distrib. India, Siam. In open grassy spots.

2. G. citrinum, Jack. Andr. Bot. Rep. t. 626. Pseudobulb globose conic. Stem 4-6 inches long. Leaves orate lanceolate to lancelate 8-12 inches long 1-2 inches wide, narrowed into a petiole 3-6 inches long. Scape 10-18 inches long, with foliaceous sheaths. Flowers pure yellow and longer than in preceding species nearly $1 \frac{1}{2}$ inch across. Sepals and petals rery broad ovate acute. Lip ovate acute rellow with brown reins. Column short and broad. Anther orbicular ovoid depressed. Lindl. Gen. and Sp. Orch. Pl. 1\%6. Fol. Oreh. 3. Bot. Mag. t. 2195. Hook. fil. Fl. Brit. Ind. V I. 1\%.

Penang: Balik Pulau (Curtis), 'Telok Bahang (Curtis 354), Pulan Ujong Duri (Curtis 302\%). Distrib. Burmah, Siam.

\section{Crambidiux, Sw.}

Epiphytes rarely terrestrial stems short, often pseudobulbous. Leaves very long and narrow or shorter and broader. Seape erect or pendulous, racemose flowers large. Sepals and petals subequal. Lip sessile at the column base, concare, side lobes ereet, dise with two pubescent or glabrous ridges. Column long footless. Pollinia 2 subtriangular on a broad reniform dise.

Species about 30, tropical and subtropical. Asiatic, African and Anstralian.

Pseudobullos none. Leaves very long lorate.

Scape pendulous. Leares broad ..

Scape pendulous short. Leaves $\frac{1}{2}$ inch

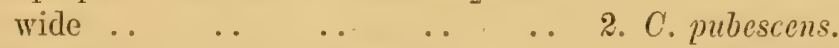

Scape erect, leaves narrow stiff .. 3. C. acutum.

Stem swollen, short leaves lanceolate 4. C. Tancifolium.

Pseudobulbs oblanccolate scape pendulous $\quad . . \quad \ldots \quad \ldots . \quad \ldots \quad$ 5. C. IIuttoni.

1. C. Finlaysonianum, Lindl. Gen. and Sp. Oreh. PI. 164. Stems very short forming large masses leaves lorate coriaceous thick 1 to 3 feet long wide apex bilobed. Raceme pendulous 2 feet long, flowers about 20. Bracts short ovate subacute, $\frac{1}{8}$ inch long. Ovary and pedicel $1 \frac{1}{4}$ inch long. Sepals oblong lorate obtuse an inch or more long, spreading. Petals lanceolate obtuse broader all olive yellow tinted red. Lip shorter, side lobes long acnte dark pink, midlobe oborate retuse blunt white with a rellow line at base and two crimson spots and deep crimson patch at tip, keels 2 crimson purple on the disc. Column curved stout deep pink. Anther broad pustular. C'apsule very large 2 inches long elliptic or oroid. Hook. 
fil. Fl. Brit. Ind. VT. 11. C. Wallichii, Lindl. 1.c. 165. C. pendulum, Bot. Reg. 1840. t. 25. William's Orch. Album X. 43\%. C. pendulum var. brevilabris, Lindl. Bot. Reg. XXX. t. 2t. C. tricolor, Miq̣. Choix t. 19.

Singapore: ('han ('hu Kang, ete., (Ridley), Pulau Chin: Johor: T'mjong Kopang, Batu Pahat (Ridley); Pahang: Kwala l'ahang (Ridley 1640): Malacea: Merlimau (Derry): Selangor: Kiwala S'elangor (Ridley ic8:3): Perak (Seortechini): Penang (Porter Wall. ('at. 63.s8). Telok Bahang (Curt is 35:3). ('ommon near the roast. Xative names, Sakat Panggan daum. Bunga Chandarek. Distrib. Malay Islands.

This is really hardly distinct specifically from $C$. aloifolium, Sw. of India to which species I reduced it in Journ. Limn. Soc. XXXII. 333 , but is a much finer plant.

2. C. Pubescexs, Lindl. Bot, Reg. 1840. Hise. \%o. 1841. t. 38. Habit of C. Finlaysonianum, but leaves very narrow 18 inches long s inch wide. Raceme short a foot long pendulons. Flowers an inch across about 16 in number. Sepals and petals narrow linear lancenlate greenish with a dark purple central bar. Lip base saccate lobes alcute midlobe oblong entire, yellow with puphe markings. pubescent, two median ridges broken in two. Capsule 2 inches long elliptic. Hook. fil. Fl. Brit. Ind. VI. 11.

singapore (C'uming), Sungei Jurong (Ridley) : Johor: Sungei Ban, Batu Pahat (Ridley); Lankawi Islands (Curtis). Distrib. Siam, Borneo.

There are intermediate form between this and $C$. Fintaysonimnum, which are possibly hybrids, but I am more inclined to think this plant is only an extreme form of the preceding one. ('. p'mdulum, Sw. King Amnals Roy. Bot. Gard. Cale. 251. seems to be one of these intermediate forms. King gives it as collected in Perak by Wray No. 3738.

3. C. Acutur, Ridl. Journ. Linn. Soe. XXXII. 33t. A tufted epiphyte with a thick rhizome. Leares narrow grassy not articulated, 12 inches long $f$ inch wide. Seape erect or subereet (nodding in fruit) flowers few. Bracts orate acute. Pedicel and ovary an inch long. Siepals lanceolate acote whitish with a molian purple band, an inch long. Petals shorter. Lip shorter than the sepals. side lobes long obtuse white, eilges and veins crimson, dise yellow with two kinels thiekened at the tips. mirllobe ovate mucronate deene (rim-on with a central orange spot. ('olumn straight violet purple. Anther yellow. Capsule fusiform 3 inches long, beaked. C. Simonsianum, King and Pantling Ann. Bot. Gard, Calc. VIll. 188. P'. 250. 
Perak: Larut Hills, Waterloo Estate (Ridley). Distrib. Sikkim and Assam. C. Simonsiumem appear's to be the same plant.

4. C. Lixcifoliux, Hook. Exot. Flora. t. 51. 'Terrestrial. Stem 1-3 inches long thick cylindrie fleshy. Leaves 3 lanceolate acuminate 6-8 inches long 1 inch wide, base narrowed to a petiole. Scape ereet 6 inches long with a few seattered sheaths. Flower's 5 in a lax raceme, an inch across. Bracts short orate. Sepals oblong lanceolate acute dull green. Petals broader with a central pink dotted line. Lip pandurate entire flat, apex broad white with the edge at the base and some blotehes on the blade crimson, a broad raised ridge from the base, and 2 short blunt ones in the centre. Column broad stout greenish spotted pink. Lindl. Gen. and Sp. Orch. Pl. 16t. Journ. Linn. Soc. III. 30. Lodd. Bot. Cab. t. $92 \%$. Hook. fil. Fl. Brit. Ind. VI. 9. Amn. Bot. Gard. Calc. p. 185. t. 24\%. C. Gibsoni, Paxton Fl. Gard. III. 618. fig. 301. C. javanicum, Bl. Bijdr. 380. Lincll. I.c. 170. Griff. Ic. Pl. As. t. 300. fig. 1.

Malacea: Bukit Sadanen (Derry); Perak: Bujong Malacca (Ridley), (Scortèchini). Distrib. India, Java, China, Japan.

5. C. Hutroxi, Hook. fil. Bot. Mag. 56r6. A large epiphyte with oboroid oblanceolate psendobulbs. Leaves 2 oblong obtuse thickly coriaceous 8 inches long, 4 inches broad. liaceme pendulous (i-8 inches long, with about 10 large flower's $1 \frac{1}{2}$ inch across. Bracts widely triangular. Sepals orate oblong acuminate yellowish denvely spotted in lines by chocolate. P'etals falcate chocolate. Lip 3 lobed lobes broad obtuse, midlobe rounded orbicular with a channel ridge betwen the side lobes, coloured like the sepals. Column thick curved. Anther conic. C. Stephensi, Ridl. Journ. Bot. 1900. $r 1$.

Perak: Larut Hills (Stephens). Rare. Distrib. Java.

\section{Dipodium, Br.}

Stems leafy with equitant leaves in Malayan species, terrestrial and leafless (Australian species). Leares linear articulated. Racemes lateral long peduncled. Flowers moderately large. Sepals and petals similar spreading. Lip adnate to columm base side lobes very small. Column short and broad with large wings. Anther small. Pollinia 2, caudicles 2 linear, disc rounded.

Species 6. Malayan, Australian and Pacific.

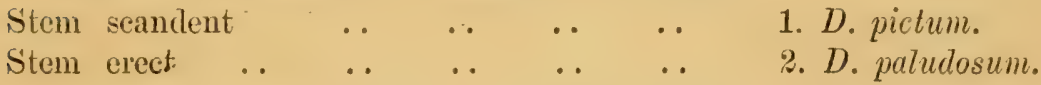

1. D. Pictus, Rehb. fil. Xenia Oreh. II. 15 and 20 t. $10 \%$ Stem climbing liom the ground on trees and clasping with its roots, leaves flaccid linear acuminate distichous 8 inches long by 1 inch wide. Stape 10-12 inches tall. Flowers about an inch and a half 
across. Sepals oblong lanceolate laterals oblicue. Petals broader, all obtuse yellowish in front, blotched with Indian red on the back. Lip shorter base narrow oblong side lobes very small eured acute midlobe oborate, white with pink spots with a white woolly crest at the tip keels at the base 3 pink, woolly. Column short orange with lipoad rounded wings. IHook. lil. Fl. Brit. Ind. TI. 19. Ituilesin picta, Lindl. Journ. Hort. Soc. IV. (18t9) 262. Paxt. Mag. Bot.

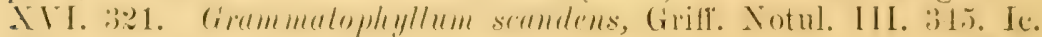
Pl. As. t. 32t. Leopardanthus scandens, Bl. Rumph. IV. 47. Mus. Hot. I. 1\%. t. 1\%.

Singapore: Kranji, Changi (Ridley 3923) ; Malacea (Griflith) ; Jahange: 'Taham Livere (lidlley); Perak: Latrul Jills (curtis); Selangor: Bukit Kuda (Ridley). Distrib. Sumatra, datra In woods not jare.

2. D. Pllubosum, lichb. fil. Yen. Orch. II. 15. Stem erect 1-2 leet tall. Leaves linear erect acuminate 6 inches long, $\frac{1}{4}$ inch wide. Scape one foot tall, racene lax about 15 flowered. Flowers an inch across, ovary and pedicel $\frac{1}{2}$ inch long. Bracts small orate. Sepals oblong obtuse. Petals similar, all white with pink spots. Lip shorter euneate oblong edges dentate, with two short tooth-like side lobes, centre pubescent white blotehed with purple. Column stout yellow at the top. Hook. fil. Fl. Brit. Ind. VI. 19. Williams Oreh. Album t. 422. Grammatophyllum paludosum, Griti. Notul. III. 34t. G. affine, Grill. Ic. 1'. As. t.323. W'ailesia paludosa, Grill. Bomplandia 1851.93.

Malacea (Maingay), Ayer Panas (Griflith); Perak (Wray). In open swamps, now very rare. Distrib. Bornco, Labuan, Cochin China.

\section{Grinditomithum, Bl.}

Lipiphytes, stems pseudobulbous or terete. I teares subcoriaceous. Seapes erect from the base of the short rhizome, racemose. Flowers

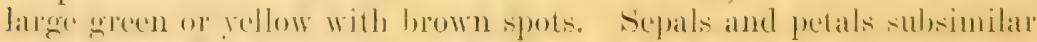

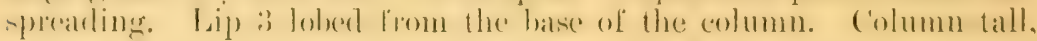
without wings foot very short, thick erect. Anther broad retuse at the top. Pollinia 2 broad, scmioblong rounded, on 2 very short points attached to a broad dise. Capsule large elliptic pyriform.

Species 3-1 Malayan.

G. speciosum, Bl. Bijdr. p. 3\%8. [. 20. Stems not psendobulbous subterete groosed, to to 10 feet long through. Leares flaceid linear 1218 inches long one inch wide acute. Scapes stont 6-10 lect tall. Plowers very numeroms t inches across. Pedicel t inches. Bracts Janceolate lincar $\frac{1}{2}$ inch long. Sepals and petals oblong obtuse.

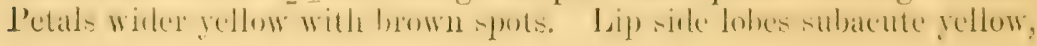


midlobe oblong obtuse pubescent much shorter than the sepal dull pink, keels 3. Capsule oblong pyriform 6 inches long. Bl. liumphia IV. 48 t. 191. Lindl. Gen. and Sp. Oreh. Pl. 628. Miquel Fl. Ind. Bat. 1I. 708. Bot. Mag. t. 515\% Paxt. Fl. Gard. II. t. 69. Fl. des Serres t. 1386. Gard. Chron. 1878. II. 181 fig. 36. 1890. I. 289. fig. 46. Hook. fil. Fl. Brit. Ind. VI. 18. G. fastuosum, Lindl. Paxt. Fl. Gard. II. 159. G. macranthum, Rehb. fil. Xenia Orch. II. 16. Pattonia macrantha, Wight. 1c. t. 1850. Gaberlia scripta, Gaud. Freyc. Voy. Bot. 425. Cymbidium scriptum, Swartz Schrad. Diar. 1799. 228. Epidendrum scriptum, L. Sp. Pl. 1351.

Singapore: Toas, Pulau Ubin (Ridley); Malacca: 'Tanjong Kkling (Ridley), Hulu Chembong (Derry 1126); Sclangor: Kwala Lumpur; Pahang: Talan River (Ridley 23t8); Perak: Dinding. (Finlayson), (Scortechini); Keclah: Gunong Jerai 3000 fect alt. Distrib. T'enasserim, Java, Borneo, Solomon Islands.

\section{BroxhespL, Lindl.}

Stiff herbs terrestrial or epiphytic, rhizome branched stems compressed. Leaves distichous rigid, lanceolate flat or recurved pungent. Paceme terminal or rarely lateral, elongating, or short. Bracts persistent. Flower's medium size to small, white or yellow. Sepals lanceolate. Petals shorter and broader. Lip 3 lobed, spurless, lobes erect. Column elongate curved, without arms, wings or foot, hooded. Anther convex. Pollinia 2 semiovate with a wide emarginate disc. Rostellum emarginate. Capsule cylindric.

Species 8 Malaya, Siam and Burmah.

Terrestrial stems elongate, raceme terminal.

Flowers white $\quad . . \quad \ldots \quad \ldots \quad \ldots \quad$ 1. B. palustris.

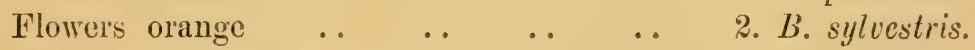

Stems tall raceme lateral .. $\quad . . \quad$.. $\quad$ 3. B. rupestris.

Epiphytic stems tall. Leaves lorate obtuse .. $\quad$ 4. B. alticolia. Epiphytic small leaves lanceolate acute.. $\quad \ldots \quad 5$. B. brevifolia.

Leaves ensiform erect .. .. .. 6. B. pungens.

Leaves ensiform recurved .. $\quad \ldots \quad \ldots \quad \%$ B. aporoides.

Leaves very narrow recurved $\ldots \quad \ldots \quad$ 8. B. scirpoidea.

1. B. Palustis, Lindl. Bot. Reg. XXVII. 1841. Mise. 184. XXX. 184t, 18. Stems tall wiry 1 to 6 feet high flattened. Leaves lanceolate obtuse 5 -6 inches long $1-1 \frac{1}{2}$ inch wide. Racemes terminal gradually developing, "3 to 12 inches long. Bracts distichous boat shaped acute $\frac{1}{8}$ inch long. flower's appearing singly, fragrant. Sepals lanceolate acute white or tinted pink, $1 \frac{1}{2}$ inch long. Petals shorter and broader. Lip side lobes lanceolate subacute white veined with violet, a median raised pubesent line on the dise, midlobe obovate 
whlomg, conter rellow, mealy, erlges white, base spotted violet. ('olumm white hood emarginate. Capsule sessile 23 inch long. Hook. Bot. Mag. t. t001. Wight Ic. t. 1740. Hook, fil. Fl. Brit.-Ind. VI. p. 20. Ridley Journ. Limn. Soc. XXVIII. 336. Br. Finlaysoniana, Richb. fil. Walp. Ann. VI. 630. 882. Miquel Fl. Jav. I. ros. Grammatophy flum Finlaysonianum, Lindl. Orch. Pl. p. 173. Coelogyne caulescens, Grifl. Notulae 1II. p. 28\%.

Singapore common (lidley); Johor: Batu Pahat (Ridley); Maladea (Cuming 20.5), Mount (Ophir (I)erry (ias) ; l'erak: 'Taiping Waterfall (Ridley); Legeh: 'Tomoh (Machado); Kedah: Gumong Jerai (Ridley). Distrib. Cochin China, Sumatra, Java, Borneo. Common in open grass spots, attaining a great size in wet spots on mountain rocks. Native name "Poko Suraman."

2. B. sylvestris, Ridl. Journ. Limn. Soc. XXXIII. p. $33 \%$ Stems slender weak, 1-6 teet tall. Leaves lanceolate thin 5 inches long $:$ inch wide tip unequally bifid. Racemes $1-2$ similar to those of B. pulustris. Flowers smaller an inch long orange coloured. Sepals lanceolate acuminate. Petals wider. Lip shorter, side lobes erect obtuse rellowish with crimson veins, edge white midlobe ovate acute white, centre orange yellow. Column slender curred, yellow, base spotted crimson, hood entire. Anther orate.

Singapore: Kranji in woods (lidlley); Johor: Gunong Panti (Ridley 1146 ), Gunong Pulai.

3. B. meplestris, Ridl. Journ. Limn, Soc. XYX11. 341. 'I'errestrial, stems five teet tall flattened. Leaves lorate lanceolate fleshy, unequally bilobed, $t$ inches long 3 inch wide. Flower's 2 or 3 in a tuft,

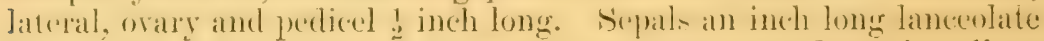
olstuse pale yellow with red tint. Petals shorter broader pale yellow. Lip about as large long as the sepals, side lobes long falcate acute, white with crimson veins, midlobe more fleshy lanceolate yellow with red dots, edge crisped, elevated bar between side lobes yellow. Column yellow hood rounded.

Malacca, Mount Ophir at 4000 feet alt. (Ridley 3143 ); Kedah: Gunong Jerai (Ridley).

4. B. stricold, Ridl. Journ. Limn. Soc. XXYII. p. 398. Pl. XLII. Epiphytic with a mueh branched rhizome, forming large masses of stems 1-3 feet long, flattened. Leaves lanceolate lorate

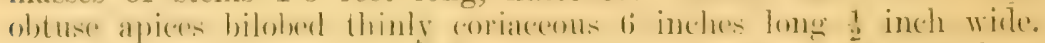
Racemes terminal flexuous $2-3$ inches long. Bracts $\frac{1}{4}$ inch long boat shaped acute. Flowers opening singly white fragrant. Sepals lanceolute acute an jnch long, literals somewhat obligue. Petals lorate lanceolate, smaller, all white. Lip shorter pubeseent narrow side

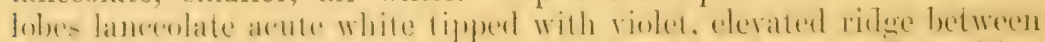
them gellow, midlobe orate subateute centre lleshy gellow edges white. 
Column curved white edges purple, hood ovate entire. Anther white pustular. Capsule erect sessile 3 inches long.

Singapore: Bukit Panjang, Bukit Timah (Ridley); Pahang: Tahan River (Ridley); Mount Ophir (Ridley).

5. B. Brevifolis, Ridl. Journ. Linn. Soc. XXXII. p. 340. Stems several in a tuft 6-8 inches long flattened, covered with distichous lanceolate acute deep green leaves erect $\frac{1}{2}$ inch long, $\frac{1}{4}$ inch wide. Flowers from a terminal tuft of bracts, $\frac{1}{2}$ an inch long, ovary and pedicel red $\frac{1}{+}$ inch long. Sepals lanceolate acuminate, acute, rosy. Petals shorter whitish yellow hairy at the base, disc between them spotted red, midlobe ovate white, centre yellow. Column slender, hood bilobed. Capsule pyriform $\frac{1}{2}$ inch long.

Perak: Hermitage Hill (Ridley), Larut Hills (Ridley). Distrib. Borneo.

6. B. Pungens, Ridl. 1.c.c. Stems 6 to. 8 inches long flattened. Leaves ensiform flat stiff pungent, erect. Racemes 1 inch long almost covered with brown bracts. Flowers $1 \frac{1}{2}$ inch across, pedicels as long. Sepals lanceolate acute yellowish. Petals narrower white. Lip oblong with indistinct side lobes, midlohe emarginate crisped with a yellow lobed thick central callus. Column nearly straight. Anther oblong flat.

Malacca: Mount Ophir (Ridley).

\%. B. Aponoldes, Rchb. fil. Otia. Bot. Hamb. p. 44. Epiphyte, tufted. Stems numerous 4 inches long. Leares ensiform stiff recurred 4 inches long $\frac{1}{4}$ inch wide. Racemes very short crowded hardly 3 inch long with numerous brown lanceolate bracts. Flowers about an inch across. Sepals lanceolate acute yellowish. Petals smaller. Lip as long oblong pandurate, side lobes obtuse with the median line clevated to the base of the midlobe yellow, midlobe oblong obtuse crisped whitish with two purple spots at the base and a central low orange coloured spathulate callus. C'olumn slender hood retuse. Rirlley Journ. Linn. Soc. XXVIII. p. 338.

Singapore: Bukit Timah (Ridley); Malacca: Mount Ophir (Ridley); Perak (Scortechini), Hermitage Hill (Ridley). Distrib. Burmah, Sumatra.

8. B. scirpoides, Ridl. Journ. Bot. 1900. p. \%1. Epiphyte. Stems slender flat, 6 inches long. Leares $t$ to 5 narrow flat curred pungent $4 \frac{1}{2}$ inches long $\frac{1}{16}$ inch wide. Racemes several $\frac{1}{3}$ inch long crowded in a head with numerous ovate imbricate bracts. Flowers an inch across, pedicels $\frac{1}{4}$ inch long. Sepals lanceolate acute, yellowish with a median line. Petals broader pale yellow. Iip side lobes ovate yellowish with purple dots, midlobe yellow with two pink spots. Column thin broad. 
Pahang: 'Tahan River (Ridley); Johor: Tebing Tinggi (Ridley); Perak: Ipoh (Ridley 10129). Distrib. Borneo.

\section{Polystacitya.}

Epiphytes with short stems oblong lanceolate leares thinly coriaccous. Inflorescence racemose or panicled terminal erect, base covered with sheaths. Flowers small green yellow or pink subsessile. Upper sepals lanceolate, laterals triangular, adnate to the foot of column. Petals very narrow. Jip jointed on the column foot 3 lobed, clawed, side lobes small. Column short broad foot long. Anther 1 or 2 celled. Pollinia 4 ovoid usually with a broad disc. Capsules elliptic.

Species 100. S. America, Africa, India, Malaya.

Lip narrow side lobes long, midlobe ovate ..

1. P. singapurensis.

Lip broad three lobed at the apex ... ..

2. $P$. penangensis.

Lip broad side lobes short, midlobe oblong ..

3. P. siamensis.

1. P. singapurensis, Ridl. Journ. Linn. Soc. XXXII. 343. Pscudobullss globose $\frac{1}{4}$ inch long. Leaves $2-4$ inches long, one inch wide, oblong lorate. S'cape 2-6 inches long rachis of racemes pubescent, sheathed up to the flowers, raceme solitary or several. Flowers very small yellowish green. Bracts ovate acuminate half as long as the pedicel. Sepals bullate, upper one lanceolate laterals triangular. Petals narrow linear spathulate. I,ip narrow thin side lobes falcate linear acute, midlole longer ovate julverulent, spotted pink inside. Column short foot long, rostellum absent.

\section{Singapore: Sungei Morai on trees (Ridley).}

2. P. PExangensis, Ridl. 1.c. 344. Pseudobulbs conic one inch long, slender, branches an inch long. Bracts linear setaceous. Flowrrs thin yellow, bullate. Upper sepal lanceolate acute laterals triangular acute. Petals narrower spathulate. Iip broad side lobes short nate midlobe ovate obtuse hardly longer, pubesent yellow with a brown median line. Column thick and short. Anther broad. Capsule $\frac{1}{2}$ inch long, reticulated between the ribs.

\section{Penang: Government Hill (Curtis 1006).}

3. P. sidmensis, Ridl. 1.c. 343 . Pseudobulbs $\frac{1}{2}-1$ inch long conic. Leaves 5 inches long by $\frac{1}{2}-1$ inch wide, lorate or oblong obtuse or subacute. Inflorescence, racemose or panicled, 4-12 inches long. Flowers large and more fleshy than the two preceding species, rachis pubescent. Bracts ovate subulate. Sepals ovate lanceolate, laterals triangular green. Petals much narrow falcate acute white. Lip olovate mealy yellow hroad, side lobes small narrower faleate obtuse. midlobe much longer hroad rouniled apex retuse. Column short. 
Pollinia 4 candicle broad oblong. Capsule narrow ellipsoid $\frac{3}{8}$ inch long not reticulate.

Perak: Dindings at Lumut (Ridley 10293); Lankawi: Kwah (Ridley and Curtis 8301). Distrib. Siam.

\section{Leucolena, Ridl.}

Leafless saphrophyte, with an elongate fusiform rhizome. Stem erect slender with a few scattered sheaths, raceme few flowered. Flowers small, upper sepal lanceolate obtuse, laterals conic. Petals adnate to upper sepal. Lip base narrow apex bilobed with a short median tooth. Column long with very long decurved arms. Anther ovate papillose attached to the top of the column by a very long filament. Pollinia 2 pyriform on a single quadrate disc.

\section{Species 1.}

L. onvati, Ridl. Journ. Linn. Soc. XXVII. p. 340. Pl. 43. Stem 9 to 12 inches tall olive coloured, succulent. Flowers about $\%$. The upper sepal and petals $\frac{1}{4}$ inch long olive green, $\frac{1}{4}$ inch long, laterals sepals olive colour with two darker streaks, and a rosy base. Lip white, centre raised violet. Column white.

Malacea: in dense dark woods Bukit Sadanen (Derry). Lower woods of the Ophir range (Ridley). Rare. The affinity of this plant is altogether obscure.

\section{Luisia, Gaud.}

Epiphytes stems terete rigid. Leaves elongate terete. Flowers few on short lateral spikes, small. Bracts very short thick persistent. Sepals subequal. Petals often narrower, and longer. Lip sessile on the base of the column, lateral lobes small, midlobe oblong or rounded, flat spurless. Column very short. Anther two celled.

Species 15. India, Malaya and Pacific Islands.

Petals not much longer than the sepals .. 1. L. brachystachys. Petals much longer than sepals.

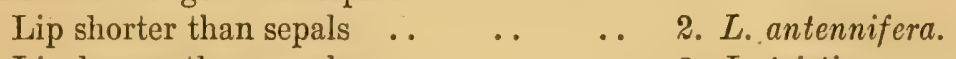

Lip longer than sepals .. $\quad \ldots \quad$.. $\quad 3$. L. tristis.

1. L. BRachystachys, Bl. Rumphia IV. 50. Mus. Bot. I. 64. Stem stout slightly flexuous, 3 inches long $\frac{1}{8}$ inch through, sheaths $\frac{1}{2}$ inch long, roots stout and stiff. Leaves 4-6 inches long blunt. Raceme short $\frac{1}{4}$ inch long with short clasping sheaths. Flowers 3-4 small, pedicel and ovary slender $\frac{1}{4}$ inch long. Upper sepal oblong ovate $\frac{1}{8}$ inch long, laterals lanceolate. Petals lanceolate subacute broad, little larger than the sepals, all green edge pink. Lip fleshy oblong orate blunt entire, with a large depression at the base dark 
crimson tip greenish. Colmmn short crimson, arms romeded blunt. Capsule inch long. Iichh. fil. Xen. Orch. 1. 20.4. t. is. fig. 1. Lindl. Fol. Orch. 3. Hook. fil. Fl. Brit. Ind. VI. 23. Mesoclastes brachystachys, Lindl. Gen. and Sp. Orch. Pl. 45.

Lankawi Islands (Curtis). Distrib. India, Siam.

2. L. Antencifer., Bl. Rumph. IV. 50. Mus. Lugd. Bat. I. 6 . Stem 2 feet tall, stout. Leares obtuse $4-5$ inches long, sheaths $1-1$ inch long. Racemes stout, $\frac{1}{2}$ inch long with orate bracts. Flowers $\frac{1}{2}$ inch across. Sepals oblong sulacute. Petals longer narmo linear from a broader base, all greenish. Lip shorter oblong orate purple with a transverse groove near the base. Column short and thick purple. Anther flat yellow oblong rounded. Pollinia globose, caudiele short and broad, dise as large. Capsule $1 \frac{1}{2}$ inch long narrowed at the base, strongly keeled. Rehb. fil. Xen. Orch. I. 265. t. 78. fig. 2. Hook, fil. l.c. 2ê.

l’ahang: Pulau Chengei (Pidley 2319); Perals (Scortechini). Distril). Java, Borneo.

3. L. Tristrs, Hook. fil. 11. Brit. Ind. VI. 25. Stem stout long. Leaves upeurved $4-5$ inches long, thick blunt sheaths 4 inch long. Racemes short 1-2 inches long thick. Flowers 3 or more. Upper sepal oblong blunt hooded $\frac{1}{2}$ inch long laterals obliquely triangular lanceolate acute, densely spotted pink. Ietals linear base broader twice as long as sepals white tip pink. Lip longer than sepals base oblong nearly straight, terminal lobe much broader orate cordate blunt deep purple. Columm broad and thick. Cymbidium tristo, Willd. Sp. PI. IV. 99. Epidendrum triste, Forst. Prodr. 314.

Penang: Penara Bukit (Curtis 1176).

\section{ADExaxicos, Bl.}

Small epiphytes. Leares distichous linear or oblong fleshy. Racemes very short few flowered. Flowers rery small. Sepals lanceolate. Petals smaller. Lip flat fleshy entire or with rery small side lobes. Column short arms very small. Anther 1 celled. Pollinia $t$ with a linear caudicle and large disc. Capsule oblong or elliptic.

Speeies 3. Malay Islands.

Leaves flat lorate obtuse $\quad \ldots \quad \ldots \quad \ldots \quad$ 1. A. major.

Leaves thick fleshy linear oblong acute $\quad . \quad$ 2. A. virens.

Leaves thick fleshy very narrow subterete .. 3. A. parviflora.

1. ג. M.ton, Ridl. Journ. Jimn. Soc. XIXVII. p. 350. Stems

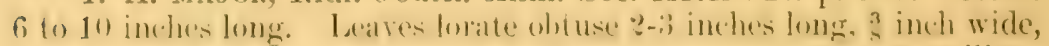
sleaths inch long wrinkled. Flowers sessile solitary axillary 
green. Bracts 3 orate coriaceous, as long as the short orary. Sepals ovate lanceolate acute $\frac{1}{4}$ inch long. Petals much smaller linear acute. Lip cuneate obovate, side lobes minute, midlobe broad apex rounded, keel short papillose. Capsule oblong 1 inch long.

Johor: Batu Pahat (Ridley); Pahang: Kota Glanggi (Ridley); Kedah: Gunong Jerai (Ridley).

2. A. virens, Bl. Bijdr. p. 381. Stems 2-6 inches long. Leaves linear oblong acute recurved very fleshy 1 inch long $\frac{1}{8}$ inch wide, sheaths wrinkled $\frac{1}{8}$ inch long. Thacemes very short 3-4 flowered. Flowers small green scented. Bracts $\frac{1}{16}$ inch long ovate. Pedicels and ovary as long. Sepals lanceolate acuminate, laterals subfalcate. Petals smaller. Lip entire cordate acute with a granular callus at base. Ridley Journ. Linn. Soc. XXXII. 349. Microsaccus virens, Hook. fil. Fl. Brit. Ind. VI. p. $7 \%$.

Singapore: Kranji (Ridley) ; Johor : Tanjong Kopang (Ridley) ; Selangor: Petaling (Ridley); Ginting Bidai: Kwala Lumpur caves (Ridley 81\%1); Perak (Scortechini). Distrib. Java.

3. A. Parviflora, Ridl. l.c. 350 . Stems $2-4$ inches tall. Leaves linear acute thick recurved, 1-2 inches long very narrow. Flowers rery small sessile. Bracts minute orate. Sepals ovate oblong acute hardly $\frac{1}{8}$ inch long. Petals linear smaller, all ochreous. Lip shorter ovate acute purple, side lobes obtuse erect short. Capsule $\frac{1}{4}$ inch long elliptic

Selangor Caves: Kiwala Lumpur (Kelsall).

\section{ACAMPE, Lindl.}

Stems stout, not very tall. Leaves coriaceous lorate. Racemes short flowers medium size. Sepals and petals subsimilar connivent. Lip spurless or near so three lobed often pubescent. Column thick conic. Pollinia with a narrow caudicle and oval disc.

Species 4 or 5. African, Indian and Malayan.

A. Pendingiana, Ridl. Journ. Linn. Soc. XXXII. 358. Stem short and thick. Leaves lorate stiffly coriaceous 7 inches long $1 \frac{1}{2}$ inch wide. Paceme $2 \frac{1}{2}$ inch long, peduncle thick. Bracts ovate obtuse. Flowers crowded at the end of the peduncle an inch across, fleshy connivent peduncle thick. Sepals $\frac{1}{2}$ inch long oblong ovate broad obtuse. Petals narrower spathulate, all yellow with red spots on the back and bars in front. Lip a little longer quite spurless side lobes curred long obtuse, midlobe orate all yellow with three red streaks on the side lobes, pubescent between the lobes. Column very short base broad yellow, with two pink spots at the base. Anther ovate obtuse, arms short erect.

Penang: Government Hill (Curtis). 


\section{Stauropsis, Benth.}

Stems stout tall or short. Leares lorate bilobed at the apex very coriaceous distichous. Racemes lateral, very tall or short flower's large showy fleshy. Sepals oblong, petals a little smaller. Lip adnate to base of column short, spurless, side lobes short, midlobe oblong, an oblong callus at the base. Column short broad footless, without wings or arms. liostellum bifid short, an entire or lobed processes from below the stigma. Anther conic one celled. Pollinia 2 on a broad caudicle with a reniform or triangular disc.

Species 2 or 3. Malayan.

S. GIGanted, Benth. Gen. Plant III. 5\%2. Stem short very stout. Leaves lorate very thick 1 to 2 feet long, $2-3$ inches wide. Raceme short 6 to 15 inches long. Flowers about 12-15 fleshy 2 inches across, orary and pedicel thick 1 inch and long, bracts rery short. Upper sepal oblong obtuse, laterals broader subfalcate, yellow with pale red spots. Petals narrower spathulate obtuse, similarly coloured. Iip half as long as sepals base boat-shaped pink, side lobes very short, midlobe fleshy oblong with three low ridges, yellow. Callus broad conical. Column very short and broad, stigma large, stigmatic process retuse. Pollinia candicle short, dise reniform. Capsule very large. Hook. fil. Fl. Brit. Ind. VI. 2\%. Fieldia gigantea, Rehb. fil. Xen. Orch. II. 39. Walp. Ann. VI. 871. Vanda gigantea, Lindl. fi. l.c. 38. t. 112. Ill. Hort. 1861. 27\%. Vanda Lindleyana, Griff. Notul III. 353.

\section{Lankawi: Kwah; Terutau (Curtis). Distrih. Burmah, Siam.}

\section{Piillanenorsis, Bl.}

Epiphytes stems short, leares oblong or obovate few, coriaceous. Racemes short or long, sometimes panicled. Flowers usually medium size to large showy. Sepals subequal. Petals often larger. Lip spurless, fleshy or thin, clawed or adnate to the column base, side lobes erect, usually with a forked or entire callus. Column with or without a foot, rather long. Anther 2 celled. Pollinia 2 with a linear or spathulate caudicle and a dise.

Species about 25, Indian, Cochin China and Malayan.

Scapes short. Rachis of raceme flattened .. 1. Ph. cornu-cervi.

Rachis not flattened but thickened.

$\begin{array}{llllllll}\text { Lip glabrous } & \ldots & \ldots & \ldots & \ldots & \text { 2. } & \text { Ph. violacea. }\end{array}$

Lip hairy at the tip.. $\quad$. $\quad \ldots \quad$. $\quad$. $\quad 3 . P h$. sumatrana.

Scapes tall slender sometimes branched.

Lateral sepals free from the column foot.

Midlobe of lip narrow acute .. . . t. Ph. muscicola.

Midlobe obovate rounded .. $\quad . \quad$.. $\quad$ 5. Ph. Kunstleri.

Lateral sepals adnate to column foot ., 6. Ph. Esmeralda. 
1. Ph. cornt-cervi, Par. and Rehb. fil. Hamb. Gartenz. 1860. 116. Stem short. Leaves 5-6 lorate obtuse 6 inches long 1 inch wide. Scapes over a foot long or short, base terete, rachis often branched flattened racemes 3 inches long flexuous. Bracts ovate subacute. Ovary and pedicel an inch long. Sepals lanceolate, laterals acute $\frac{3}{4}$ inch long. Petals shorter and narrower, all yellow with brown blotches. Lip $\frac{1}{4}$ inch long, side lobes oblong erect white with violet spots inside, midlobe reniform, or ovate acute, white, callus at base of midlobe tooth-like violet with a long slender forked process at its base. Column with a short foot, tall yellow, two red processes at the base. Anther conic. Pollinia subglobose with a long slender caudicle. Rostellum long and narrowly bifid. Rolfe. Gard. Chron. 1886. II. 276. Hook. fil. Fll. Brit. Ind. VI. 29. Polychilus cornucervi, Breda. Orch. Jav. t. I. Bot. Mag. t. 5570.

Sungei Ujong: Jelebu (Davison) ; Seremban (Braddon); Perak (Scortechini) ; Lankawi (Curtis). Distrib. Burmah, Sumatra, Java, Borneo, Siam.

Very variable in size of flowers and length of scape, a hybrid between this species and $P$. violacea has been found more than once in Perak, and is perhaps the plant described as $P$. Valentini, Rchb. fil. Gard. Chron. 1886. p. 262.

2. P. violacea, Teysm. and Binn. Bat. Tijdschr. XXIV. 10. Leaves elliptic or oblanceolate acute. Scape 3-12 inches long, stout, bracts ovate acute. Flowers 1-3 inches across, rachis thickened sometimes brancheả. Sepals lanceolate, laterals broader oblique. Petals smaller oblanceolate, cream white more or less marked purple. Lip narrow side lobes oblong truncate, midlobe spathulate or obovate violet purple, a single bifid callus at its base. Column rather long. Anther conic. Capsule cylindric 5 inches long. Rchb. fil. 1878. IT. 234. 1881. II. 145. fig. 32. 18\%. Rolfe. Gard. Chron. 1886. II. 27\%. Williams Orchid Album t. 182. (var. MIurtoniana), Ill. Hort. 1855. 173. (var. Schroderi), Hook. fil. l.c.c. 29. Stauritis violacea, Rchb. fil. Hamb. Gartenz. 1802. 34.

var. typica. Sepals and petals carnation with green tips, side lobes of lip yellow dotted purple, lip purple.

Perak (Scortechini).

var. Murtoniana, Rchb. fil. Sepals and petals cream yellow, inner half of sepals purple, side lobes orange.

Perak: Kinta (Murton); Bidor (Curtis). Distrib. Sumatra.

3. Ph. sumatrana, Korth. and Richb. fil. Hamb. Gartenz. XVI. 1860 p. 115 . Leaves five or six oblanceolate obtuse, a foot long, and 4 inches wide, shining. Scapes several a foot long, rachis flatteued 4 ingled, sometimes branched, about 6 inches long. Bracts ovate eymbiform $\frac{1}{4}$ inch long. Flowers 2 inches across fragrant. Upper sepal 
oldanceolate. laterals much broader. Petals lanceolate narrowed at base, white or yellowish barred red. Lip $\frac{3}{4}$ inch long, side lobes oblong truncate, with a tooth on the inner side, white and orange in the inside, dise with 3 two toothed calli in a row, midlobe oblong elevated in the midlobe, white with erimson spols, a woolly tuft towards the end. Column rather tall, hooded, hood toothed, arms short incurved two toothed. Capsule 6 inches long. Bot. Mag. t. 552\%. Flore des Serres t. 16t4. P. zebrina, 'I'eysm. and Binn. Pl. Cult. in Hort. Bogor. p. 320 .

Johor: Batu Pahat (Rirllor). Kwala Sembrong (Técsall) ; Perak: Bidor (Curtis). Distrib. Sumatra, Borneo.

4. Pir. anscicoul, Ridl. 'Irans. Limn. Soc. III. p. 373. Leaves oblanceolate obtuse, 6 inches long, two inches wide. Scape slender b-s inches long sometimes branched, rachis not thickened the flowers often $\frac{1}{4}$ inch apart. Bracts ovate small. Flower's an inch across. Sepals lanceolate acute. Petals subspathulate acute white spotted chestmut. Lip side lobes 4 angled tooth like, yellow spotted with chestnut, midlobe narrow tongue shaped apex decurved, rosy, an oblong bifid callus between the silk ]obes, and a lanceolate blunt one al the base of the midlobe. Capsule linear narrow 3 inches long. jungle.

Pahang: 'Tahan River (Ridley). On mossy trees in dense

5. Pit. Kuxstlert, Hook. fil. Fl. Brit. Ind. VI. 30. Leaves obovate oblancesalate, or clliptic lanceolate acute $4-6$ inches long $2 \frac{1}{2}$ wide. Scape about as long few flowered rachis thickened. Bracts rery small orate. Flowers 1-1 $\frac{1}{2}$ inch across. Upper sepal obovate obtuse greenish yellow with or without a red bar, laterals broader oblique, yellow with a transverse red bar. Petals spathulate or obovate similarly coloured. Lip side lobes oblong truncate denticulate, white, midlobe oborate apex romoled, orange with a white raised central bar and 3 indian red streaks on each side, a bifid callus at the base of the midlote. C'olumn narrowed in the middle greenish, hood ovate. Hook. fil. Ann. Roy. Bot. Gard. Calc. V. 58. Ph. Denisiana, Cogniaux. Gard. Chron. 1899. 82. Dict. Icon. des Orchidees Phal. 6.

Perak (Scortechini, Kunstler). Tambun Hot Springs near Ipoh, (Ridloy: 'T'apah: Sungei Siput (C'urtis); Dindings (curtis). Distrib. Philippines (fide Cogniaux).

6. Ph. Essmeraldi, Rehb. fil. Gard. Chron. 18\%4. 58\%. Leaves 4 to 6 inches long $1 \frac{1}{2}-2$ inches wide oblanceolate obtuse dull green. Seape erect 1 to 2 leet tall simple or branched. Bracts short ovate. Flowers an inch across white rose or crimson. Upper sepal oblanceolate obtuse, laterals oblique orate adnate to the column foot. Petals obovate. Lip with a long claw, with two narrow white lobes, side

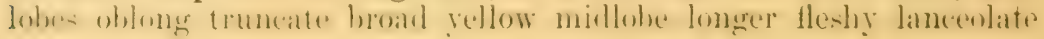


blunt riolet purple. Column olive yellow stout with two bosses at the base. Rostellum rather long ineurved. Stigma large ovoid. Rev. Hort. 18i \%. t. 10\%. Williams Orchiel Album VII. t. 21. Floral Magazine X. S. t. 258. Cogniaux Dict. Icon. Phal. Pl. 5. Hooker. Bot. MIag. 7196. Hook. fil. Fl. Brit. Ind. VI. 31. P. antennifera, Rehb. fil. Gard, Chron. 1878. 398. 1882. 520. P. Buyssoniana, R(hb. fil. Gard. Chron. 1888. p. 295.

Lankawi Islands (Curtis) ; Kedah : Setul (Ellis) ; Patani (Anandale). Distrib. Burmah, Cochin China and Siam.

\section{Doritis.}

Epiphytes, with the habit of Phalaenopsis with usually small flowers, the column sometimes and the foot longer and forming a mentum with the side lobes of the lip.

Species about 5, Indian and Malayan.

D. Wightir, Benth. Gen. Pl. III. 5\%. Leaves few 2-3 oblanceolate thinly coriaceous 6 inches long 2 inches wide. Inflorescence panicled with few branches, $\gamma$ inches long, rachis thickened. Flowers $\frac{3}{4}$ inch across. Bracts very small ovate. Pedicel and ovary $\frac{1}{2}$ inch long. Tpper sepal oblong acute, laterals larger ol,tuse curved. Petals smaller, all white tipped green. Lip side lobes obovate cuneate rounded white with riolet rose streaks, midlobe obcordate retuse violet pink, with a bifid callus white, between the side lobes mentum rery short. Column curved pink. Anther beaked. Pollinia globose, pedicel long dilated upwards dise oral. Hook. fil. Fl. Brit. Ind. VI. 32. Ann. Bot. Gard. Calc. V. 59. King and Prain. Vol. 8. 265. Phulaenopsis Wightii, Rchb. fil. Bot. Zeit. 1862. 214. Ph. alboviolacea, Ridley Trans. Linn. Soc. III. p. 373. Aerides latifolium, Thw. Enum. 430.

Nalacea: Bukit Bangkong (Derry); Pulau Tiuman (W. Nanson); Perak: Larut Hills (Stephens), (Curtis), Lankawi (Curtis). Distrib. India, Burmah, Ceylon. Rather rare.

\section{Staurochilus, Ridl.}

Epiphyte, stem tall climbing with its long roots. Leaves distichous lorate, apex bilobed, coriaceous. Raceme lateral. Flowers large, expanded. Sepals oblong, laterals falcate. Petals narrower. Lip spurless short side lobes short erect, midlohe 3 lobed. Column short thick, with erect arms. Anther ovate beaked. Pollinia 2 on a spathulate caudicle, with an elliptic disc.

Species 1. Siamese and Malayan.

S. Fasciate, Ridl. Journ. Linn. Soe. XXXII. 350. Stem stout. Leaves 5 inches long $\frac{1}{2}-\frac{3}{4}$ inch wide obtuse, sheaths $\frac{1}{2}$ inch long, ribbed. Raceme 4-6 inches long, rachis grooverl. Bracts ovate acute persistent. Flowers $1 \frac{1}{2}-2$ inches across fragrant, orary and pedicel $\frac{33}{4}$ inch 
long. Sepals oblong acute, laterals falcate. Petals spathulate narrower, all yellow banded with red. Lip half inch long, white slightly pubescent side lobes acute erect midlobe of three nearly equal lobes, mid one narrower callus at the base linear oblong toothed. Column red. Anther yellow. Trichoglottis fasciata, Rchb. fil. Gard. Chron. 1872. p. 699. Orchid Album V. t. 208.

Lankawi: Terutau (Curtis 2818) on rocks. Distrib. Siam.

\section{VANDA.}

Epiphytic or terrestrial plants. Leaves coriaceous flat or terete succulent. Flowers large showy racemose or panicled. Sepals and petals subequal. Lip large base saccate or spurred, side lobes large or small, midlobe fleshy or thin. Column short. Anther 2 celled.

Species about 20. Tropical Asia and Australia. The thin flowered species including the only native one would be better excluded from the genus.

V. Hookeriana, Rehb. fil. Bonplandia 1856. 324. Gard. Chron. 1882 II. 488. Scandent stems slender, about 12 feet long. Leaves terete $2-3$ inches long crect. Inflorescence lateral racemose or panicled with few branches, peduncle 6-8 inches long. Flowers 2-3 inches across, thin textured, pedicel and ovary an inch long. Upper sepal obovate half inch long pale rose, laterals much larger oblong oblique, obtuse with a setaceous process on the back, white with a few pink spots. Petals broadly olovate twisted, broader and shorter than the lateral sepals pale rose. Lip large $1 \frac{1}{2}$ inch long, side lobes oblong acuminate obtuse deep purple, midlobe broad large three lobed side lobes oblong dilated at apex and rounded, midlobe oblong obovate white or rose dotted erimson. Callus conic blunt grooved white at mouth of the very short spur. Column fairly long with a long foot pink. Ill. Hort. 1883. t. 48t. Reichenbachia II. \%t. Orch. Alb. t. 73. Hook. fil. Fl. Brit. Ind. VI. 50.

Perak (Scortechini, Wray), Kinta Valley (Ridley, ete.); Johor: Kiwala Batu Pahat (Kelsall). Distrib. Borneo. Scrambling over bushes in open swamps.

\section{Renanthera, Lour.}

Stems tall scandent. Leares oblong obtuse. Inflorescence panieled. Flowers large or small. Sepals and petals spreading. Lip small articulated at base of column side lobes broad truncate, midlobe short tongue shaped base of lip between the lobes spurred, spur short conic. ('olumn short and broad with no foot arms short incurved. Anther broad one celled. Pollinia 2, caudicle broad or rather narrow, disc broad.

Species about 8. Cochin China, Burmah, Malaya. 
Stem tall 10-30 feet, leaves oblong, broad.

Flowers large yellow and brown ..

- 1. R. arachnitis.

Flowers large pink .. . . . .

Flowers large white $\quad \ldots \quad \ldots \quad \ldots \quad 3 . R$. alba.

Flowers very small red .. $\quad \ldots \quad \ldots \quad 4 . R$. elongata.

Stem short, leaves long, narrow $\ldots \quad \ldots \quad 5 . R$. matutina.

1. R. arachnitis, Lindl. Gen. and Sp. Orch. Pl. p. 21\%. Stems 20 to 30 feet long. Leaves lanceolate lorate obtuse, six inches long $1 \frac{1}{2}$ inch wide. Racemes or panicles very long, many flowered. Bracts $\frac{1}{4}$ inch long obtuse. Flowers 3 inches across, scented of musk. Upper sepal linear oblong spathulate laterals shorter falcate. Petals linear oblong spathulate abruptly falcate, all yellow with chocolate blotches. Lip half inch long, side lobes broad quadrate yellow splashed with red edges purple brown, midlobe hardly longer fleshy oblong cuneate acute fleshy pink with a large conic acute callus near the tip. Column short very broad white. Anther very broad flat. A rachnis moschifera, Bl. Bijdr. 365. t. 26. Arachnanthe moschifera, Bl. Rumphia IV. t. 196. 199. Hook. fil. Fl. Brit. Ind. VI. 28. Aerides arachnitis, Sw. Schrad. Journ. 1799. 293. Willd. Sp. Pl. IV. 131. Renathera Flos-Aeris, Rehb. fil. Xen. Orch. I. 88. Walp. Ann. VI. 878. Limodorum Flos-Aeris, Sw. Nov. Act. Upsal 1740. 3\%. Epidendrum Flos-Aeris, Linn. Sp. Pl. 1348.

Perak: Kinta (King's Collector). Distrib. Java, Borneo. Native name "Angrek Bunga Kasturi," and usually known as the Scorpion Orchid by residents.

2. R. Maingayi, Ridl. Journ. Linn. Soc. XXXII. 353. Stems like those of Arachnitis. Leaves thinner 5 inches long $1 \frac{1}{2}$ inch wide. Flowers in panicles or racemose slightly smaller. Sepals linear ob-

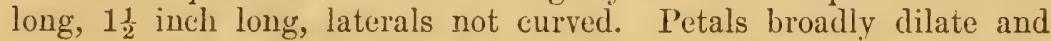
bent at the tip, all mottled salmon pink and white. Lip side lobes broad rounded spreading, midlobe oblong apex ovate, all pink with darker stripes and yellow blotches on the side lobes. Column yellowish. Anther ovate subacute. Pollinia globose caudicle linear at base dilated to the broad disc. Arachnanthe Maingayi, Hook. fil. Fl. Brit. Ind. VI. 28.

Johor: Batu Pahat (Kelsall); Malacca (Maingay). Distrib. Islands south of Singapore, often cultivated in Singapore. The flowers vary much in the proportion of pink and white.

3. R. ALBA, Ridl. l.c.c. Stems 15 to 20 feet long. Leaves lorate obtuse 3 inches long $1 \frac{1}{2}$ inch wide, often hard and brittle and denti(ulate at the edges at the base. Panicle long, flowers 3 inches across. Bracts orate. Sepals linear spathulate obtuse $1 \frac{1}{2}$ inch long all similar. Petals a little smaller, white. Lip side lobes broad rounded yellowish 
white with pink dots midlohe tongue shaped rosy violet with darker median stripes two violet streaks and two violet spots between the lobes. Column white edge violet short and broad. Anther orange. Arachnanthe alba, Ridll. 'T'rans. Linn. Soc. III. p. 369.

Singapore: Pulau Seletar (Ridley); Pahang: Kwala Pahang (Rirlley 16i34). Distrib. Borneo and islands round Singapore. Climbing over trees and bushes in open countries.

4. R. elongata, Lindl. Gen. and Sp. Orch. Pl. p. 218. Stems (i-s feet tall or more. Leaves elliptice, obtuse coriaceous 3 inches long 1 inch wide, sheaths $1 \frac{1}{2}$ inch. Pancle large branches spreading 6-8 inches long. Flowers small numerous. Bracts short orate. Pedicel and ovary one inch long. L pper sepal linear half inch, laterals large deflexed oblong spathulate. Petals linear obtuse smaller all red. Lip very small, side lobes broad obtuse midlobe oblong obtuse, all red except the white base of midlobe, and tips of lateral lobes. Column broad red. Capsule elliptic $1 \frac{13}{2}$ inch long on a pedicel $\frac{1}{4}$ inch. Reichenb. fil.Xenia. Oreh. I. 88. Hook. fil. Fl. Brit. Ind. VI. 48. R. matutina, Lindl. Bot. Reg. 18+3. t. 41. (not of Gen. and Sp. Orch. Pl.). Pescatorea I. t. 11. R. micrantha, Bl. Mus. Bot. I. 60. Rehb. f. l.c. I. 8\%. Saccolabium reftexum, Lindl. Gen. and Sp. 225.

Singapore (Wallich 7309), Bukit 'Timah Road, Pulau Ubin (Ridley); Johor: Kiwala Batu Pahat (Kelsall); Malacea: Jasin (Goodenough 1661) : Selangor: Uhu Selangor (Goodenough); Perak (Sortechini) ; liwala Kangwar (Stephens); Dindings: Lumnt (Ridley 10143); Penang (Curtis). Distrib. Java, Borneo. Rocks by the sea and trees. Native name "Poko Api Api Sesudu."

5. R. Maturina, Lindl. Gen. and Sp. Orch. Pl. 218. Stem short 1-2 feet erect. Leaves lorate $\%-8$ inches long $\frac{3}{8}$ inch wide obtuse. Scape 1; inches long. about half floriferous sometimes brancherl. Bracts ovate $\frac{1}{8}$ inch long. Flowers about 20 distant 2 inches across. Upper sepal linear, laterals clawed broader lanceolate acuminate connate at base or free. Petals lanceolate acuminate, all red with darker hotches on the lateral sepals. Lip very small, side lohes rounded tip tongue shaped deflexed, spur conic rather large, white edges of side lobes, midlobe and spur erimson. Column pink short and broad. Anther crimson. Whole flower more or less pubescent. Rehb. fil. Xenia. Oreh. I. 90. Pl. 35. (very bad). A. angustifolia, Hook. fil. Fl. Brit. Ind. VI. 49. Ie. Pl. 2128, derides matutina, B1. Bijdr. 366. 'Tab. XXIV.

Prak: Bujong Malacea (Ridley 9898) on rocks, Gunong Batu Putih (Wray). Distrib. Java.

\section{Rexantimelila, Ridl.}

Scrambling epiphyte, stems about 2 feet long slender. Leares linear lanceolate semiterele acuminate. lateemes short, flexuous. 
Flowers small. Sepals oblanceolate, laterals oblique. Petals narrower linear. Lip side lobes erect, midlobe linear recurved with 2 quadrate calli, a short conic spur. Column slender long arched. Anther conic. Pollinia 2 spathulate caudicle broad, dise oblong. Capsule obconic.

\section{Species one endemic.}

R. HIstrionicd, Ridl. Journ. Linn. Soc. XXXII. 355. Leaves 3 inches long. Racemes 4 inches. Bracts short ovate. Flowers an inch across. Upper sepal oblanceolate erect, laterals deflexed oblique. Petals linear obtuse, all yellow with red spots, midlobe shorter yellow, with white calli. Capsule $1 \frac{1}{2}$ inch long. Renanthera histrionica, Rehb. fil. Gard. Chron. 1878. p. 74. Hook. fil Fl. Brit. Ind. VI. p. 49.

Singapore: Serangoon (Ridley); Malacea: Mount Ophir (Ridley) ; Perak: Gunong Keledang (Curtis 3343).

\section{4\%. 'I'riciloglottis, Bl.}

Stems rather tall 2-3 feet. Leaves lanceolate lorate coriaceous. Flowers solitary, on short pedicels, small. Sepals and petals spreading similar. Lip shorter side lobes short, base boat shaped or spurred, midlobe entire or 3 lobed, pubescent. Column short thick footless, arms short erect tooth like. Anther ovate beaked. Pollinia 2 caudicle linear dise moderately large.

Species 4 or 5. Malayan and Andamanese, Siam.

Lip midlobe entire .. $\quad \ldots \quad \ldots \quad \ldots \quad \ldots \quad$ 1. T. retusa.

Midlobe trifid.

Base of lip boat shaped .. $\quad . .2$ 2. T. scaphigera.

Base of lip spurred .. .. . . 3. T. tetraceras.

1. T. retusa, Bl. Bijdr. p. 360. fig. 8. Stem stout 3 feet or more. Leares thick lorate obtuse bilobed 5 inches long half inch wide, sheaths reticulately ribbed $\frac{1}{2}$ inch long. Flowers $\frac{3}{4}$ inch across. Pedicel and ovary $\frac{1}{2}$ inch long. Sepals lanceolate acute. Petals narrower, all yellowish marked with pale red. Lip nearly as long, white tinted with pink base boat-shaped side lobes with long recurved points midlobe oblong lanceolate pubescent. Column half the length of the lip, arms erect tooth-like, top dark crimson.

Pahang: Kota Glanggi (Ridley 2358); Selangor: Kwala Lumpur Caves (Kelsall). Distrib. Siam, Cochin China, Borneo, Jara.

2. T. scaphigera, Ridl. Journ. Linn. Soc. XXXII. 35\%. Stem 2 feet long, weak. Leaves lanceolate acute rery shortly subpetiolate, 3 inches long $\frac{1}{2}$ inch or less wide sheaths $\frac{1}{2}$ inch long. Flowers $\frac{1}{2}$ inch across. Bracts orate. Pedicel and ovary $\frac{1}{2}$ inch long. Sepals oblanceolate obtuse pubescent. Petals similar yellow with red blotches. Lip shorter base boat shaped, side lobes short blunt, with a hairy 
callus at the base, midlobe trifid, side lobes linear median one shorter rounded, a large pubescent callus in the centre. Column short, arms long hairy. Anther ovate pubescent.

Penang: Government Hill (Curtis 1961); Perak (Scortechini); Waterloo (Curtis).

3. T. Tetraceras, Ridl. l.c.c. Stem over a foot long weak. Leaves 3 inches long half inch wide lanceolate acuminate, subpetiolate, sheathed $\frac{1}{2}$ inch long. Flower $\frac{1}{2}$ inch across, pedicel $\frac{1}{3}$ inch long. Sepals ohlong orate. Petals smaller ligulate. Lip flat, side lobes tooth like, spur acuminate obtuse, midlobe lanceolate ovate obtuse with two short and two much longer; two keels rumning from the two lobes to the terminal portion ending in a pubescent callus, a fleshy elongate linear truncate callus in the mouth of the spur. Column short arms tooth like.

Lankawi: Goa Chirita (Curtis 281\%).

48. Rhynchostris, Bl.

Epiphytes stem short. Leaves lorate bilobed coriaceous. Racemes short and stont, many flowered. Flowers small. Sepals and petals subequal spreading. Lip adnate to column base, spured, side lobes 1), midlobe clawed. Column short stout footless. Anther flat, beaked. Pollinia 2 semiorate, caudicle linear disc ovate. Rostellum bifid.

Sepals 2, Indian and Malayan.

1. R. gigantea, Ridl. Journ. Linn. Soc. XXXII. 356. Stem short and stout. Leaves broad lorate obtusely bilobed 12 inches long 2-3 inches wide very thick. Racemes 9-12 inches long very dense Howered. Flowers an inch across, white or spotted with pink, and lip violet pink. Bracts boat shaped $\frac{3}{4}$ inch long. Upper sepal ovate obtuse base narrowed, laterals longer. P'etals olblanceolate obtuse. Lip longer pandurate retuse with a blunt tooth in the notch and a conic process beneath, spur straight $\frac{3}{8}$ inch long flattened. Column short. Anther long beaked. Capsule $1 \frac{1}{2}$ inch long. Saccolabium giganteum, Lindl. Gen. and Sp. Orch. Pl. p. 221. Bot. Mag. t. 5635. Williams Orch. Album II. t. 56.

Singapore: Seletar (Ridley); Pulau Aor, East Coast of Johor (Native collector). Distrib. Cochin China, Siam. Only once obtained in the Peninsula.

2. R. Retusa, Bl. Bijdr. 286. t. 49. Stem short. Leaves elonfate lorate obliquely retue apices acute, $10-20$ inches long $\frac{2}{3}-2$ inches wide sheaths short. Racemes 4 to 20 inches long dense. Flowers $\frac{1}{2}-\frac{3}{3}$ inch across white blotehed with rosy purple. Sepals orbicular ovate. Upper one oblong. Petals elliptic obtuse. Lip rosy purple, limb ('uneiform entire or emarginate, spur compressed blunt, puhescent within. Capsule 1-1 inch long clarate. Hook. fil. Fl. Brit. Ind. 
VI. 32. $R$. praemorsa, Bl. l.c. $R$. guttata, Rehb. f. Bonplandia II. 93. R. Garwailca, Rchb. f. Walp. Ann. VI. 888. Saccolabium guttatum, Lindl. Gen. and Sp. Orch. Pl. 220. Journ. Linn. Soc. III. 32. Bot. Mag. t. 4108. Wight Ic. t. 1745-6. De Vriese Orch. t. 14. Griff. Ic. Pl. As. t. 819. S. praemorsum, Lindi. l.c. 221. S. Rheedii, Wight Ic. PI. V. 19. S. retusum, Flor. de Serres XIV. 191. t. 1463-4. S. Blumei, Lindl. Sert. Orch. t. 4\%. Bot. Reg. 1841. Misc. 55. Ill. Hort. t. 545. Williams Orchid Albumat. 169 t. 238. S. Garwalicum, Lindl. Journ. Linn. Soc. III. 32. Gard. Chron. 1879. 102. S. littorale Rchb. fil. Gard. Chron. 1881. II. 19S. Sarcanthus guttatus, Lindl. Bot. Reg. t. 14443. Aerides guttatum, Roxb. Fl. Ind. III. 471. Ae. spicatum, Don. Prodr. 31. Ae. proemorsum, Willd. l.c. Limodorum retusum, Sw. Nov. Act. Upsal VI. 80. Epidendrum retusum, L. Sp. Pl. 1351.

\section{Lankawi (Curtis). Distrib. India, Ceylon, Java.}

\section{SaCCOLABIUM, Bl.}

Epiphytes stems short rarely long. Leaves lorate or oblong coriaceous or terete. Racemes or panicles lateral erect or pendulous. Flowers small. Sepals or petals free or adnate to the column base, subequal. Lip sessile at the column base, side lobes short or almost absent, midlobe small. Spur saccate or conic cylindric, with usually a callus on the back in the mouth, and frequently a ridge more or less developed on the lower face, sometimes meeting the opposite wall and dividing the spur into two. Column short footless, arms very short or wanting. Anther ovate usually beaked, pollinia 2 more or less split, caudicle narrow linear, or broad, dise small or large various.

Species about 200 African, Indian and Malayan, Chinese.

I am quite unable to keep up the genera Cleisostoma and Sarcanthus based on the development of the calli in the spur, closely allied plants after differing in this point so much as to belong to these different genera. The Calceolare section of Saccolabium, with a Eaccate spur, the side lobes quite indistinguishable and forming the complete edge of the sac, and entire absence of any callus, makes a much more distinct genus. Acampe incorporated in the Flora of British India with Sacrolabium as far as the typical species go differs in the lip possessing no real spur at all, and I keep it distinct.

\section{§ 1. UnifLoRA.}

Flowers solitary small or in pairs.

Lip midlobe linear bifid..

Midlobe lanceolate entire

Midlobe 5 lobes.

Midlobe oblong spathulate

$\begin{array}{lll}\ldots & \quad \ldots & \text { 1. S. tenuicaule. } \\ \ldots & \quad \cdots & \text { 2. S. miserum. } \\ \ldots & \quad \ldots & \text { 3. S. Pantlingi. } \\ \ldots & \quad & \text { 4. S. cornigerum. }\end{array}$


ร. 'TERETIFoLi.

Flowers racemose or panicled.

Leares terete long.

Spur short conic not septate .. ..

5. S. halophilum.

Spur conic nearly septate.

Midlobe triangular obtuse . . . 6. S. Machadonis.

Midlobe orate acute $\quad . \quad \ldots . \quad \ldots \quad$ \%. S. Kunstleri.

Spur dilated at base not septate. . 8. S. sacculatum.

Leaves terete short, flowers minute .. . . 9. S. perpusillum.

Leaves lorate or lanceolate.

\$ 3. Calceolaria. Spur large basinshaped side lobes absent, no ealli.

'l'erminal lobe of lip flat denticulate .. 10. S. bigibbum.

'Terminal lobe of lip flat entire ... .. 11. S. catinatum.

Lip entire boat-shaped, acute .. .. 12. S. minimiflorum.

Lip boat-shaped, terminal lobe narrow

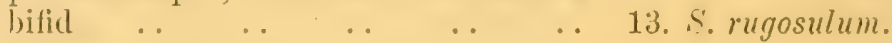

$\$$ 4. Cleisostoma. Spur short broad rounded. Flowers yellow with red spots. A flat callus in the-mouth of the spur.

Stem tall climbing. Panicle wide ..

Stem short and thick panicle lax, callus saddle shaped with 2 legs ... ..

Stem callus quadrate cntire .. ..

Stem short flowers racemose or shortly panicled.

Callus in spur bifid. Scape tall ereet

1:. St horlense.

Callus in spur bifid. Scape rery short

Callus entire semieircular

11. ‘. latifolium.

1.). S. suffusum.

16. S. validum.

$\S$ 5. Salicantios. Spur horn-shaped, midlobe of lip moderately large. Callus entire or lobed on the back of the spur with a ridge more or less developed in front making the spur nearly septate. Flowers greenish with longitudinal red bars, lip white or riolet.

Stem very tall climbing .. . . . ? ?. . Scortcchinii.

Stem stout leares broad .. $\quad \ldots \quad \ldots \quad$ 21. S. densiflorum. 
Stem short or slender, racemes short or slender.

Leaves flat point terete subulate .. . . 22. S. secundum.

Leaves flat blunt.

Midlobe ovate. Spur completely septate

Midlobe ovate notched on each side. Spur not septate .. . . .

Midlobe reniform. Spur with 2 pubescent keels

23. S. pensile.

24. S. fissicors.

25. S. bicarinatum. moderate.

§. 6. Eu-Saccolabiun. Spur small or

Midlobe larger than side lobes.

Spur short side lobes small acute

- 26. S. fissum.

Spur long curved side lobes long rounded

Spur long side lobes rounded midlobe long narrow $\quad \ldots \quad$. . . . .

Spur long side lobes indistinct, midlobe horn like $\quad . \quad$. . . . .

Spur long midlobe smaller than side lobes.

2\%. S. secundiflorum.

28. S. miniatum.

29. S. undulatum.

Scape tall panicled.

Flowers very small $\frac{1}{4}$ inch across $\quad . \quad \ldots \quad 30$. S. ionosmum.

Flowers $\frac{1}{2}$ inch across _. . . . 31. S. Arachnanthe.

Scape racemose.

Flowers very small, spur large curved . 32. S. penangianum.

Flowers moderate spur long nearly straight 33. S. saxicolum.

Spur long clubbed, side lobes large spreading $\quad$.. $\quad$.. $\quad . . \quad$..

Flowers minute

34. S. Angraecum.

35. S. Myosurus.

\section{§. UNIFLORUNI.}

1. S. tenuicaule, Hook. fil. Fl. Brit. Ind. VI. 360. Stems slender 1 foot long. Leaves narrow linear acute 3 inches long $\frac{3}{16}$ inch wide sheaths $\frac{1}{4}$ inch long flexuous. Flowers small $\frac{1}{8}$ inch long $2-3$ on a tubercle with small ovate bracts. Pedicel and ovary very short. Lpper sepal lanceolate subacute, laterals orate acute oblique. Petals ovate acute smaller all yellow or white. Lip side lobes oblong acute, midlobe much longer, linear apex bifid, spur short curved thick blunt constricted above the swollen end. Column rather large curved. Rostellum short. Anther ovate with a long blunt beak. Pollinia crescent shaped, candicle linear dilated into 2 arms above. Hook. fil. Ann. Bot. Roy. Gard, Calc. V. 49, T. 74. 
Selangor: Krwala Lumpur Cares (Ridley 8133) ; Perak (Scortechini 512), (Wray); Penang: Government Hill (Curtis 99. 230\%), (Ridley 7241 ).

2. S. Mrseruur, Ridl. Journ. Linn. Soc. XXXII. 359. Stems over a foot long, stout $\frac{1}{8}$ inch thick. Leaves linear 5 inches long $\frac{1}{4}$ inch wide, sheaths $\frac{1}{2}$ inch long. Racemes very short 3-4 flowered. Flowers very small yellow. Sepals elliptic ovate to ovate laterals a little broader than the upper one. Petals smaller lanceolate oblong. Lip sicle lobes oblong quadrate midlobe ovate obtuse with a minute callus at the base, spur short conic incurved blunt. Column short and thick arms short oval. Capsule elliptic $\frac{1}{2}$ inch long shortly pedicelled.

Penang: Government Hill (Curtis 218t); Lankawi (Curtis); Dindings: (rumong 'l'ungul (Ridley 8396). By a misprint in the original description the stem was given 1 inch thick.

3. S. Panturngr, n.sp. Stems slender pendulous 12 inches long. Leaves thickly coriaceous $-\frac{1}{2}$ inch apart linear oblong apices acute not notcherl. Flowers solitary ? inch atross on a slender pedicel. Sepals and petals theshy suberual orange barred purple brown oblong lancerolate hunt, laterals slightly faleate. Lip fleshy pale rellow narrowly oblong tapering to the acute emarginate apex is lobed, lowest pair near the base small and tooth like blunt, pair at the base of the terminal lobe larger conical pointer outwards, dise between them pubescent. spur $\frac{1}{3}$ length of the stalked ovary horizontal cylindric tapering to apex. Column slont with a linear callus on its anterior surface curved upwards and hairy. Anther broad. Pollinia 4 in two pairs each pair golobose and attached to a narrow catudicle, disc oblong hall as long as the caudicle upper end truncate. Cleisostoma tenuicaule, King. Journ. As. Soc. Beng. LXY I. part II. p. 596.

Perak (Kunstler). I have not seen this, but from its deseription it appears to be allied rather to Trichoglollis.

4. S. corxigenum, Ridl. l.c. 361. Stem long flexuous. Leares lanceolate acute $3 \frac{1}{2}$ inch long, $\frac{1}{2}$ inch wide, sheaths 3 inch long raceme very short 2-3 flowered. Bracts very short orate obtuse. Orary and pedicel $\frac{1}{2}$ inch long. Sepals oblong spathulate $\frac{3}{8}$ inch long. Petals broarler brownish vellow. Lip a litle shorter yellowish white, side lobes erect horn like midlobe oblong spathulate olutuse, spur straight conic 1 inch long. Column short thick, arms rounded.

Penang: West Hill (Curtis).

\section{§ 2. 'I'eretifolis.}

5. S. malophlum, Ridl. Journ. Limn. Soc. XXXI!, 36\%. 'Tufted plant, stems 3-12 inches slender curved. Leaves terete hardly 
$\frac{1}{8}$ inch through pungent recurred, sheaths $\frac{1}{4}$ inch long, pedicel and ovary $\frac{1}{4}$ inch long. Sepals oblong obtuse. Petals smaller, all pale olive tinted with pink. Lip side lobes ovate obtuse rosy violet, midlobe white much longer hastate blunt, spur horizontal not septate, callus obtriangular white. Anther obovate beaked, pollinia globose, caudicle linear disc broad hastate. Column tall. Capsule $\frac{3}{5}$ inch long oblong.

Singapore: Pulau Tekong, Sungei Morai (Ridley); Johor: Tanah Runto (Ridley). Distrib. Sumatra, Rhio, Borneo. Common on trees on the sea shores.

6. S. Machadonis, Ridley Journ, Roy. As. Soc. S. Br. Vol. XXXIX. p. 82. Stems curved 12 inches long. Leaves terete recurved 3 inches long $\frac{1}{8}$ inch thick apex pungent. Racemes 2 inches long. Flowers scattered $\frac{3}{8}$ inch long. Sepals linear oblong obtuse. Petals narrower all recurved olive yellow. Lip pale violet, side lobes erect oblong truncate, midlobe much longer flat hastate triangular acuminate obtuse, spur short curred blunt olive yellow. Upper callus in mouth rounded hemispheric, with an anchor shaped process on the top, lower edge of callus truncate pubescent lower callus conic ending in a lamina running to the back of the spur. Column stout sigmoid olive yellow. Anther 1 celled flattened hemispheric. Pollinia subglobose on a broad elongate caudicle tapering upwards to the point and fixed to a saddle shaped disc. Rostellum lobes broad deflexed parallel oblong.

\section{Johor: Batu Pahat (Ridley).}

\%. S. Kunstleri, Ridl. Stem 4-6 inches long often branching. Leaves terete fleshy stout, $2 \frac{1}{2}-3$ inches long and $\frac{1}{4}$ inch thick. Inflorescence more than twice as long as the leares racemose or panicled. Flowers numerous $\frac{1}{t}$ inch across. Bracts ovate sacuminate. Sepals subequal elliptic obtuse. Petals smaller oblong, white, pink midline. Lip lateral lobes erect broadly oblong subfalcate, midlobe orate acute concave, pink septum imperfect almost catching a large callus on the back wall. Column stout papillose with a short thick foot and a small rounded callus on each side of the anther. Anther truncate in front. Sarcanthus Kunstleri, King. Journ. As. Soc. Beng. LXVI. p. 594 .

Perak (Kunstler). Drawing in Herb. Calc. Leaves remarkably thick.

8. S. Sacculatum, Ridl. Stem slender 8 inches long. Leaves terete obtuse, 6 inches long $\frac{1}{10}$ inch thick, sheaths 1 inch long ribbed and reticulate. Racemes slender 3 inches long. Flowers very small scattered. Bracts minute ovate. Pedicels $\frac{1}{8}$ inch long. Sepals ovate subacute. Petals smaller lorate. Lip shortly clawed, side lobes ovate, midlobe linear lanceolate hastate, spur rather large saccate pinched in at the upper part dilated below, not septate, callus clubbed bilobed, 
Column long. Anther orate not beaked. Capsule sausage shaped, triquetrous inch long. Siarcanthus sacculatus, Ridl. Journ. Linn. Soc. XXXII. 368.

Lankawi Islands (Curtis 2560), (Ridley 8333).

9. S. Perpusillua, Hook. fil. F1. Brit. Ind. VI. 56. Ic. Pl. 2129A. A tufted little plant, stems $2-4$ inches long. Leaves nearly

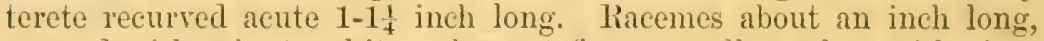
covered with minute white pubesent flowers, yellow when withering. Bracts minute lanceolate acute, as long as the pedicels. Sepals and petals orate oblong. Lip side lobes rounded midlobe ovate fleshy spur eurved clubbed. Columm short. Anther with a long upeurved beak. Pollinia with a linear caudicle and a much longer linear gland. Arms of column short erect. Rostellum very long narrow deeply bifid. Capsule $\frac{x}{8}$ inch long elliptic. Ridley Joun. Limn. Soc. XXXII. 358.

Singapore: Sungei Buluh (lidley 3rt), Jurong (Ridley 4680); Malacea: Sungei Kesang (Derry), Sungei Rambei (Derry 30\%); Perak: 'laiping (Curtis).

\section{§. CALCEOLARI.}

10. S. Bigibibur, Hook. fil. Bot. Mag. 5\%6\%. Stem very short, leares linear oblong or linear falcate acuminate narrow at the base, 3-6 inches long : 8 inch wide, or broader. Raceme short half an inch long. Flower's crowded at the apex, small. Sepals and petals spathulate ohtuse yellow. Jip saceate, terminal bobes reniform denticulate ciliate white, with a yellow red dotted raised mass in the centre. Column short and thick, arms broad incurved. Anther large conie. Rostellum deeply bifir. ('apsule oblong thick hall an inch long. Fl. Brit. Ind. VI.

Pahang: Klameg 'Terbang (Barnes) ; Perak: Larut Ilills (scoltechini), (lidlley). Distrib. Rangoon. The leaves in the original figure are much broader than those of the Peninsula plants.

11. S. catrnatuar, Ridley Joum. As. Soc. Str. Br. Vol. XXXIX. p. 81. Stem hardly 1 inch long. Leaves $2-3$ very coriaceous oblong obtuse broadly bilobed 7 inches long, 2 inches wide. laceme very short rachis stout. Flowers about 6,3 inch across. Sepals obovate spathulate blunt. Petals narower yellowish with red spots. Lip sarcate rombled, side lobes not distinct, terminal lobe oxate triangular entire glabrous blunt all white with violet spots. Column very short and broad at base pink, no arms. Anther obtuse conje in front

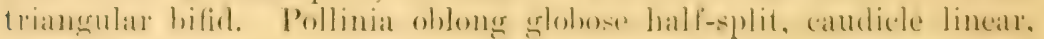
disc oblong hastate. Rostellum bifid. Capsule elliptic narrowed at. base 2 inches long. 
Pahang: Kota Glanggi (Ridley). Distrib. Borneo. This is probably the plant mentioned in the Flora of British India as $S$. calseolare collected in Perak by Carter.

12. S. minmeflonud, Hook. fil. Fl. Brit. Ind. VI. 59. Ic. Pl. 2133. Stem $\frac{1}{1-\frac{1}{2}}$ inch long. Leaves $4-7$ oblong lanceolate oblique narrowed at base 2 inches long $\frac{5}{8}$ wide, blunt. Racemes slender 4 inches long. Flowers numerous minute. Bracts lanceolate acuminate $\frac{1}{16}$ inch long. Sepals narrow lanceolate acuminate red. Petals broader obtuse. Lip saccate hardly spurred, entire acuminate yellow, upeturved a globose tubercle at the mouth (fide Hooker). Anther beaked. Rostellum large, bifid lobes semiovate acuminate. Capsule elliptic. S. cortinatum, Ridl. Journ. Bot. 1898215.

Selangor: Kiwala Lumpur Caves (Ridley); Perak (Scortechini)

13. S. Rugosulua, Ridley Journ. Roy. As. Soc. S. Br. Vol. XXXIX. p. 82. Stem stout 6 inches tall. Leaves linear narrowed at the base, apex bilobed mucronate, 5 inches long $\frac{1}{2}$ inch wide sheaths $\frac{1}{2}$ inch long deeply transversely wrinkled. Racemes short $\frac{1}{2}$ inch long stout, with a few cup shaped sheaths at the base. Flowers $\frac{1}{4}$ inch across on pedicels $\frac{1}{8}$ inch long, yellow spotted with red. Sepals ovate coriaceous. Petals thinner pallid. Lip, boat shaped side lobes very short oblong midlobe large fleshy orate grooved ending in a slender horn bifid at the tip, spur very short conic blunt. Column large arms rounded.

Kedah: Gunong Jerai(Ridley).

\section{Cleisostona.}

14. S. LAtrfolien, Ridl. Stem tall straggling stout. Leaves oblong obtuse bilobed nearly equally, 4 inches long $1 \frac{1}{2}$ inch wide sheaths long. Panicles long spotted purple much branched. Flowers numerous nearly half an inch across. Bracts minute ovate. Ovary and pedicel $\frac{3}{8}$ inch long. Sepals oblong blunt. Petals broader subspathulate, all lemon yellow, backs spotted red. Lip side lobes oblong quadrate midlobe short ovate acute recurved, all yellow sometimes dotted red, spur large scrotiform retuse, walls very thick, lamina oblong apex notched. Column very short rounded. Anther broad ovate beaked. Pollinia globose, caudicle very broad linear, dise large oblong. Capsule cylindric an inch long. Cleisostoma latifolium, Lindl. Bot. Reg. 1840. Misc. 60. Walp. Ann. VI. 889. Hook. fil. Fl. Brit. Ind. VI. 71. C. fuscum, Lindl. Journ. Hort. Soc. 1850. 80.

Singapore (Loddiges); Malacca (Griffith, Maingay); Brisu (Goodenough 1+10); Pahang: Temertoh (Ridley 2350); Selangor: Kiwala Lumpur Caves (Kelsall); Perak (Wray; Scortechini). Distrib. Sumatra. 
15. S. Suffusua, Ridley Journ. Roy. As. Soc. Str. Br. Tol. XXXXIV. p. 189. Stem stout 6 inches long $\frac{1}{2}$ inch through. Leares fleshy light green 6 inches long 1 inch acoss lorate obtuse mequally bilobed rounded. Panicled lax few-branched 18 inches long branches 3 or t. Flowers numerous $\frac{1}{4}$ inch long to the tip of the spur, pedicel $\frac{1}{8}$ inch long. Sepals lanceolate acute yellow suflused with brown. Petals narrower linear. Lip fleshy porrect spur eylindric horizontal obtuse, side lobes short oblong ohtuse midlobe hastate tongue-shaped fleshy obtuse with an erect tooth towards the tip, callus in the spurmouth, thick large decurved fleshy with two crura from the back attaching it to the back wall of the spur, no septum. Column short and broad. Pollinia globose, pedicel oblong linear disc, ovate acute.

Perak: Kamunting, Sungei Siput (Machado). Endemic. A curious plant with a straight horizontal spur and a rery curious callus.

16. S. VAliduri, n.sp. A stout plant with copious thick corky roots, stem $t$ inches long $\frac{1}{2}$ inch thick. Leaves coriaceous lorate 6 inches long $\frac{3}{4}$ inch through unequally bilobed. Panicles from the lower axils 9 inches long, slender, peduncle 3 inches branches two. Flowers numerous but remote. Bracts ovate acute small. Pedicels 1 inch long. Sepals lanceolate subacute. Petals much smaller dull yellow, with reddish purple blotching in the centre. Lip shorter spur nearly as long as the orary horizontal blunt cylimble, sirle lobes short oblong rhomboid blunt, midlobe longer short ovate fleshy: callus in mouth of spur fleshy quadrate large decurved, no septum or callus in spur. Column short. Anther 4 celled orate. Pollinia transversely bilobed pedicel oblong linear, dise oblong truncate. Column wing: short incurved. Rostellum arms oblong truncate with a mimute process on the upper edge.

Perak: Kamunting (Machado July 10th).

1\%. S. molicexse, Ridl. Journ. Roy. As. Soc. Str. Br. Tol. XXXIX.p. 83. Stem stout 1-2 inches long. Leares lorate coriaceous blunt unequally bilobed $t-6$ inches long $\frac{3}{4}-1$ inch wide. Seapes erect taller than the leares, base nude apex lacemose or more usually with a few branches. Bracts small orate. Flowers $t$ inch across. Sepals oblong obtuse, laterals broader. Petals narrower yellow with red edges. Lip yellow side lobes small ereet with two subacute points, midlobe broader orate acute, spur scrotilorm very broad red, callus in mouth of tube a thin lamina bifid at apex. Column short and broat. Anther broarl abruptly truncate beaked. Pollinia elliptic, caudicle linear very narow, disc ovoid. Rostellum entire. Capsule elliptic oblong an inch long.

Singapore: Jurong (Ridley); Johor: Tama Runto (Ridley);

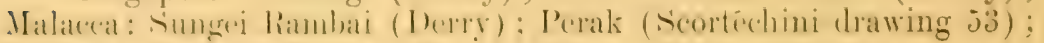
Penang: Tanjong Bunga (Curtis 183t). Distrib. Bornco. 
1S. S. PArvux, Ridl. Stem shortly an inch long with long grey roots. Leaves lanceolate rather thin acute, $1 \frac{1}{2}$ inch long nearly $\frac{1}{4}$ inch across. Raceme very short $\frac{1}{4}$ inch long, few flowered. Bracts small ovate remote. Flowers $\frac{1}{8}$ inch long fleshy. Upper sepal lanceolate, laterals larger ovate. Petals lorate as long. Lip side lobes quadrate, median widely ovate, spur oblong saccate thick, callus lorate bifid. Anther ovate. Rostellum linear. Cleisostoma parvum, Ridl. Journ. Linn. Soc. XXXII. 366.

\section{Pahang: Kota Glanggi (Ridley).}

19. S. uteriferum, Ridl. Stemless leaves lorate apex broad rounded or retuse margins undulate, $\gamma$ inches long by one inch wide. Spike shorter than the leares densely flowered peduncle stout, 3 inches long. Bracts minute. Flowers $\frac{1}{4}$ inch across. Sepals obovate oblong yellow dull red at base. Petals similar, smaller. Lip side lobes truncate, midlobe very small fleshy, spur a large inflated subglobose sac contracted above, callus semicircular conver entire. Column very short. Anther broad. Pollinia globose, caudicle broad, gland oblong. Cleisostoma uteriferum, Hook.' fil. Fl. Brit. Ind. VI. it. Ann. Roy. Bot. Gard, Calc. V. 56. PI. 84.

Perak (Kunstler).

\section{§. Sarcanthus.}

20. S. Scontechinir, Ridl. A tall plant climbing with the habit of a Renanthera about six feet tall $\frac{1}{4}$ inch through. Leaves lanceolate subacute or obtuse 5 inches long one inch wide petiolate sheaths an inch long. Raceme stout deflexed eventually 15 inches long. Flowers numerous opening few at a time, ovary terete grooved nearly sessile, $\frac{8}{16}$ inch long. Flowers $\frac{1}{2}$ inch across. Bracts orate triangular. Lpper sepal oblong, laterals obovate obtuse. Petals narrower, all dark purple with a pale yellowish central bar on the sepals. Lip side lobes rery low sinuous, hardly distinguished midlobe broader triangular liastate blunt violet tip white, spur nearly straight conic, shorter than the orary white, callus in the mouth of the spur clubbed, septum thin almost complete. Column stout white arms obscure. Anther ovate abruptly beaked. Pollinia very small, partly split at back, caudicle very slender dise minute. Capsule oblong, thick $\frac{3}{4}$ inch long. Sarcanthus Scortechinii, Hook. fil. Fl. Brit. Ind. VI. 68. Ic. Pl. 2138.

Pahang: Tahan River (Ridley 234\%), Kota Glanggi (235\%); Perak (Scortechini); Penang (Curtis 3210).

21. S. Densiflorum, Lind1. Gen. and Sp. Orch. Pl. 220. Stem a foot long very stout. Leaves oblong or linear oblong obtuse very unequally bilohed 6 inches long 2 inches wide sheaths an inch long, spike decurved sometimes branched $1-8$ inches long, rachis very stout 
pubescent. Bracts $\frac{1}{2}$ inch long lanceolate acuminate. Flowers $\frac{1}{2}$ inch

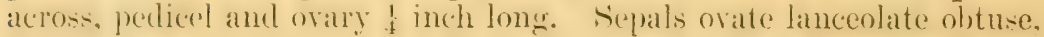
yollnwish with two reel hands or thickly dottert with red. Petals smaller lanceolate. Lip side loles oblong ofotuse, mislobe lanerolate subobtuse yollow tinted red, spur $\frac{1}{4}$ inch long dilated at the apex and biloberl, allus thin forlied septum more or less developer. ('olumn short arms thick. Anther beaked. Caudicle of pollinia slightly dilated upwards, dise minute. Bot. Reg. 1838. Misc. 56. Sarcanthus densiflorus, Par. and Rehb. fil. 'T'rans. Limn. Soc. XXX. 136. S. castaneus, Ridl. 'Trans. Linn. Soc. XXXII. 369. Cleisostoma spicatum, Lindl. Bot. lieg. 184\%. under t. 3\%. Hook. fil. Ann. lioy. Bot. Gard. Calc. Vol. V. p. 55. Pl. 83. King. l.c.c. Vol. 8. Pl. 311.

Singapore: Dalvey lioad (Ridley); Selangor: Rawang (Ridley); l'crak (Scortechini); P'enang (Wallich 8:311), West Ilill (Curtis 995). Distrib. 'Tenasserim, Borneo.

22. S. secuxdur, Ridl. A large plant about a foot or more tall, stem stout more than $\frac{1}{6}$ inch through. Luaves thickly coriacous linear acuminate apex terete pungent, over a foot long $\frac{1}{2}$ inch wicle, sheaths an inch long. Raterne dense pendulous $2-1$ inches long base nude. Bracts ovate very short. Flowers about $\frac{1}{4}$ inch long, ovary and pedicel very short. Sepals oblong obovate obture. Peotals narrower grenen with two red bands. Lip side lobes ereet oblong ending in terete points, midlobe orate fleshy, white tinted violet and yellow, spur horn shaped white, a keel runs from near the apex of the midlobe clown the spur dividing it, callus in mouth of spur short elubbed. Column stout arms rounded incurved. Anther flat quadrate with a narrow acute beak. Pollinia globose, caudicle linear dise orate small. Capsule cylindric $\frac{3}{4}$ inch long $\frac{1}{4}$ inch through. Sarcunthus sccundus, Grift. Notul. III. 362. Ic. PI. As. t. 336. Hook. fil. Fl. Brit. Ind. VI. 63. Ann. Roy. Bot. Calc. 5. p. 51. P1. 7\%. (not Vol. 8. Pl. 321).

Johor: Batu Pahat (native); Malacea: Suugei Kesang (Derry); Sungei Rambei (Ridley); Perak: Larut IJills (Hervey); Lankawi (Curtis). Distrib. India, Assam, Cochin China, Sumatra, Borneo. Native name "Sakat Ular."

23. S. Prisile, Ridl. Stem ỏ inches long thick. Leaves lorate obtusely bilobed, 6 inches long 1 inch wide. Raceme long slender lower lialf nude. Flowers scattered $d$ inch wide. Bracts small ovate. Pedicels $\frac{1}{2}$ incli long. Sepals oblong orate. Petals smaller oblan(eolate obture all greenish linterl with red. Lip pink siele lobers ered horn shapert, mirlobe short ovate ohtuse callus thick blunt, septum (omplete. ('olumn orate heaked, prollinia ovoid hall dividerl, caludicle linear dise small. Sarcanthus pensilis, Ridl. Journ. Limn. Soc. XXXII. 369.

$$
\text { Johor: Batu Palıat (Ridley). }
$$


2t. S. Fissicors, 11.sp. Stem 4 or 5 inches long. Leaves linear oblong rounded emarginate at the tip $t$ to 5 inches long 1 inch acrosi, rather thickly coriaceous. Inflorescence 10 inches long very slender, peduncle 6 inches long purple. Bracts very small ovate. Flowers rather distinct small, rachis faintly black scurfy simply racemose or with one side branch. Pedicel and ovary $\frac{1}{5}$ inch long bright yellow green. Sepals ovate oblong $\frac{1}{10}$ inch long blunt dark red brown. Petals narrower linear similarly coloured. Lip side lobes small erect truncate yellowish midlobe cordate acute, notched on either side, violet with white centre and edge; spur parallel to the pedicel or deflexed and nearly as long cylindric slightly flattened white, callus an oblong truncate fleshy decurved lamina in the mouth, no septum. Columm rather tall violet, wings obscure. Anther white skull shaped truncate emarginate in f'ront. Pollinia flattened subglobose orbicular biloberl, pedicel flat narrowed above and below and slightly dilate in the middle, dise very small oblong. Rostellum lobes short, oblong truncate with deflexed points, stigma cordate.

Perak: Kamunting (Machado). Flowered in H. B. Singapore 1906 December. This species has the habit of $S$. pensile, but the flowers more resemble those of S. Scortechinii.

25. S. Bicarinatuar, Ridl., Stem 6 inches long $\frac{3}{16}$ inch thick. Leaves lorate bilobed, flaceid, 7 inches long $\frac{3}{4}$ inch wide. Panicle of few branches 6 inches long. Flowers about 20, inch across. Pedicels $\frac{1}{4}$ inch long. Bracts minute ovate. Sepals oblong ovate blunt, deep red with a median line and tip green. Petals linear. Lip white side lobes short obtuse, median reniform hastate, spur horn shaped curved blunt $\frac{1}{8}$ inch long, keels in the spur two pubescent upper one and lower one nearly meeting. Column short red arms short erect. Anther yellow. Pollinia oblong, caudicle short and broad, disc oblong with two very low curved arms. Rostellum long deeply bifid. Sarcanthus rostellatus, Ridl. Journ. Bot. 1900 \%3.

Perak: 'Tambun near Ipoh (Ridley).

\section{$\S 6$. Eu-SACCOLABIUM.}

26. S. Fissuar, Ridl. Journ. Linn. Soc. XXXII. 361. Stems stout short. Leares lorate stiffly coriaceons deeply bifid acute, $t$ inches Iong inch wide, sheaths wide $\frac{1}{4}$ inch long. Raceme 3 inches long, base nude, rachis thickened. Flowers $\frac{1}{t}$ inch across, fleshy, pedicel white. Lip side lobes short blunt, midlobe oblong ovate obtuse, white, side lobes and centre of midlobe violet, a hemispheric white callus at the base, spur short blunt thick half as long as the ovary. Column very short, arms rounded incurved. Anther yellow with a violet median line, bealied, caudicle of pollinia spathulate the apes widely triangular, disc ovate.

Lankawi Islands (Curtis). 
2\%. S. secundifloruar, Ridl. Journ. Bot. 1898. p. 210. Stem about an inch or little more tall stout. Leaves lorate coriaceous $1 \frac{1}{2}$ inches long $\frac{1}{f}$ inch wide blunt sheaths short, deep green tinted with purple. Raceme 2 inches long. Hlowers about 6 small secund. Upper sepal lanceolate acute, laterals obtuse white. Petals linear oblong white tipped violet. Lip base saceate, side lobes oblong, yellowish white, midlobe obcuneate apex thickened, truncate with an erect point white, spur nearly as long as the ovary thick curved clubbed, yellowish. C'olumn short and thick green, arms linear erect. Anther' with a long linear bifid upcurred beak. Pollinia globose on a linear caudicle with a much longer linear dise.

\section{Singapore: Chua Chu Kang (Ridley). Distrib. Sinkep.}

28. S. minifum, Lind1. Bot. Reg. 184\%. t. 58. Stem stout woody 3-t inches long, roots very stout. Leares linear obtuse very coriaceous 4 inches long, $\frac{1}{2}$ inch wide recurred, sheaths ribbed $\frac{1}{4}$ inch long. Racemes suberect $\overline{3}-4$ inches long. Flowers densely crowded nearly $\frac{1}{2}$ inch long to end of spur, red or orange yellow (var. citrinum). Sepals obovate. Petals nearly as long and broad. Lip side lobes rounded short midlobe long and narrow oblong acute, spur long subterete slightly arved. Columm stout arms repy short rounded rellow. inther dark red ovate beaked. Pollinia globose pedicel linear broad, disc quadrate much smaller.

var. citrimu, Rehb. fil. Gard. Chron. Ap. 26. 1884. p. $54 \%$.

Lankawi Islands (Curtis). Distrib. Siam.. Distrib. type Jara, variety in Philippines.

29. S. uxdulatur, Ridl. Journ. Bot. 1900. $2 \%$. Stems flattened 6 inches long. Leares lorate undulate flaccid, 5 inches long $\frac{3}{9}$ inch wide. Racemes slender pendulous $t$ inches long densely covered with very small flowers. Bracts minute orate acuminate half as long as the pedicels. Sepals oblong ovate $\frac{1}{10}$ inch long yellowish with two red bands. Petals linear lorate obtuse narrower yellow with four red spots. Lip side lobes hardly distinct midlobe short, hom like decurred, spur as long as the pedicel blunt subcylindric, yellow with 2 red spots in the mouth. Columm short. Pollinia subghohose. caurlicle broadly dilated triangular at alpex base linear, dise oblong. Cinsule oblong pear-shaped.

Perak: at T'elok Pinang near Ipoh (Ridley).

30. S. roxosmus, Ridl. Stem 6 inches long. Leaves lorate apex hilobed obture 6 inches long 3 inch wide. sheathe $: 1$ inch long.

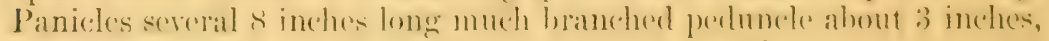
branches 2 inches slender. Bracts ovate acute. Flower's very numerous fragrant, very small f inch across. Pedicels it inch long. Sepals oblong obtuse. P'etals smaller olive yellow with transrerse bars of 
violet spots. Lip side lobes triangular obtuse violet median ovate short with two conic calli. Spur $\frac{\text { i }}{8}$ inch long straight blunt pinkish white with a white tongue-shaped callus in the mouth. Column short violet. Anther flat tip bilobed. Pollinia deeply divided, caudicle linear disc small orate. Rostellum bluntly bifid. Cleisostoma ionosma, Ridl. Journ. Linn. Soc. XXXII. 366. (not of Lindley).

Perak: Larut Hills (Ridley).

31. S. Arichnantine, Ridl. Joum. Roy. As. Soc. Str. Br. Vol. XXXIX. p. 83. Stem tall scandent. Leares oblong obtuse 4 inches long $1 \frac{1}{2}$ inch wide, sheaths $\frac{1}{2}$ inch long. Panicle $2 \frac{1}{2}$ feet long peduncle long nude except for a few sheaths purple, bracts 3 or 4 across, pedicels longer slender. Bracts small ovate. Sepals and petals spreading spathulate obtuse, lateral sepals falcate, white with purple spots at base. Lip fleshy side lobes indistinct forming a wall round the entrance of the spur, midlobe ovate broad short, spur broad saccate rounded large all white, callus in the mouth oblong notehed. Column short and broad, rostellum short. Anther thin depressed, hemispheric. Pollinia 2 globose, caudicle broadly linear disc half as long oblong.

Perak and Kedah (Curtis). This plant has the habit and column of a Renanthera with the flowers of a Cleisostoma.

32. S. Pendxgianum, Hook. fil. Brit. Ind. VT. p. 5\%. Ic. Pl. 2129 B. Stems short, $4-5$ inches long tufted. Leases linear or narrowly linear lanceolate $2 \frac{1}{2}-4$ inches long $\frac{1}{8}$ inch wide. Racemes 1-1 $\frac{1}{2}$ inch long. Bracts subulate. Flowers very small $\frac{1}{6}$ inch long, crowded yellow or whitish speckled red. Upper sepal oborate oblong, laterals oblique. Petals much smaller. Lip erect side lobes ovate midlobe much smaller ovate rounded, spur large curved swollen at base narrowed to tip. Column arms rounded. Pollinia caudicle spathulate. Hook. fil. Ann. Bot. Gard. Calc. V. 4\%. Pl. 71.

Perak: Sungkei river (Curtis), (Kunstler); Kedah: Gunong Jerai (Curtis).

33. S. saxicolum, Ridl. Trans. Linn. Soc. III. p. 3r4. Stem a foot or more long $\frac{1}{8}$ inch thick. Leaves linear obtuse, equally bilobed coriaceous, narrowed at the base 3 inches long $\frac{1}{8}$ inch wide, sheaths ribbed 3 inch long. Racemes slender 2-3 inches long, base nude except for some short amplexicaul sheaths, rachis thickened. Bracts small ovate acute, deflexed. Flowers many on pedicels 1 inch long, half an inch long to end of spur. Sepals oblong ovate obtuse rellow with a red keel. Petals very small oblong acute oblique. Lip side lobes rounded midlobe very small triangular ovate with a tongue shaped callus in the mouth of the spur, spur long nearly straight narrored to the tip. Column short arms long rounded. Anther long beaked. S. flaveolum, Ridl. Journ. Linn. Soc. XXXII. 360. 
Pahang: Kota Glanggi (Ridley); Perak: 'Tambun (Ridley $9830)$; Kedah: Gunong Jerai (Curtis).

34. S. Axgruecur, Ridl. Journ. Bot. 1898. p. 214. Stem an inch long. Iatres corlateous lanceolate falcate oblique blunt, 2 inches long, $\frac{5}{5}$ inch wide. Raceme short $\frac{3}{4}$ inch long, rachis thickened flattened. Bracts ovate acute very short. Flowers half inch across, white, pedicel $\frac{1}{4}$ inch long. Sepals elliptic acute. Petals narrower. Lip with a short (daw, side lobes large obliguely obovate, minutely toothed, midlobe minute cordate, spur nearly half an inch long clubled. Column short. Pollinia ovoid globose, pedicel narrow, disc shield shaped. Rostellum elongate. Capsule triquetrous $1 \frac{1}{2}$ inch long.

\section{Selangor: Kiwala Lumpur Cares (Ridley 8131).}

3\%. S. Mrostius, Ridl. Journ. Roy. As. Soe. Str. Br. Tol. XXXIX. p. St. Stems short 1-2 inches crowded together, with copious roots. Leaves lanceolate falcate subacute 3 inches long, $\frac{1}{4}$ inch wide, sheaths inch long. Scapes slenter 3 inches long, scabrid at base, racemes 1 inch long thickened. Bracts ovate, very numerous blunt. Flowers minute. Sepals lanceolate oblong falcate. Petals narrower. Lip side lobes oblong erect, midlobe ovate lanceolate shorter, spur dependent broad blunt as long as ovary. Column short and broad, footless. Capsule cylindric $\frac{1}{2}$ inch long, pedicel $\frac{1}{8}$ inch.

Pahang: Kwala 'Tembeling (Ridley). A most curious plant with the habit and racemes of a Dendrocolla, but the flowers very minute and fleshy of a Saccolabium.

\section{Microsicces, Bl.}

Stems short tufted, corered with distichous ovate equitant leares. Racemes very short. Flowers 1-2 white. Bracts ovate rather large. Sepals and petals suberpal. Lip almost entire fleshy, spur saccate obtuse. Column short arms short bjunt. Anther conic, one-celled. Jollinia 4, caudicle narrow dise small. Tostellum rather long entire.

Species one. Burmah and Malaya.

M. Jutexsis, B1. Bijdr. 36\%. Stems curved t-6 inches long. Leares scalpelliform, obtuse 1 inch long, crowded. Flowers white $\frac{3}{3}$ inch across. Bracts white. Sepals and petals ovate lanceolate acute. Petals a little smaller. Lip side lobes very indistinct midlobe obtuse, a yellow spot at the base, spur stont shorter than the

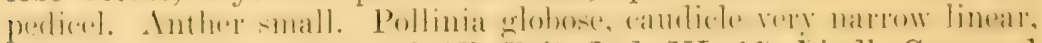
disc orate acute. Wook. fil. Fl. Brit. Ind. VI. \%\%. Lindl. Gen. and Sp. Orch. 1'l. 219. Saccolubium Griffithii, Par. and lichb. fil. 'I'rans. limn. Soc. XYY. 1 145.

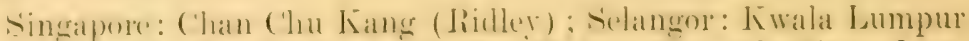
Cares (Kelsall); Peral (Scortechini). Distril, Burmal, Siam, Jara. 


\section{ExClUded SPeCIES.}

M. virens, Hook. fil. is Adenoncos virens, $\mathrm{Bl}$.

\section{Taexiophyluude, Bl.}

Stems very short roots copious flat, long. Leaves very small or usually none, racemes short slender. Flowers minute. Bracts distichous persistent. Sepals and petals free or conic. Lip entire or three lobed, spurred. Column short, with broad incurved arms. Anther beaked pollinia t. Rostellum elongate. Capsule large sausage shaped.

Species about 6. India, Malaya, Pacific.

$\begin{array}{lllll}\text { Sepals and petals free .. } & \ldots & \ldots & \ldots & \text { 1. T. serrula. } \\ \text { Sepals and petals connate } & \ldots & \ldots & \ldots & \text { 2. T. rubrum. }\end{array}$

1. T. serrula, Hook. fil. FI. Brit. Ind. VI. $7 \%$ Roots very long, stem exceedingly short, leaves none. Racemes $\frac{1}{2}-1$ inch long, almost entirely floriferous. Bracts acute pubescent. Flowers minute white or yellowish. Sepals narrowly lanceolate obtuse. Petals narrower. Lip boat shaped nearly entire, side lobes rounded, spur scrotiform. Column arms obtuse. Anther large with two purple spots, beak truncate. Pollinia 4 globose, caudicle linear. Capsule an inch long sausage shaped. Ridl. Journ. Linn. Soc. XXXII. 363.

Singapore: Tanglin, Chan Chu Kang, etc., (Ridley); Johor: Kota 'Tinggi (Riclley); Pahang: Pekan (Ridley); Selangor: Kiwala Lumpur (Ridley); 'Trengganu: Cherating River (Ridley); Penang: Western Road (Curtis); Perak: Larut (King's Collector), Ipoh (Curtis 3129).

2. T. rubrun, Ridl. l.c. Roots few slender terete, stem minute. Leaves none. Raceme an inch long very slender, upper half only floriferous. Bracts ovate distichous pubescent red. Flowers minute red, ovary with short nearly sessile. Sepals, petals and lip connate. Sepals lanceolate acute. Petals linear. Lip as long triangular lanccolate, spur broad saccate shorter than the ovary.

Sungei Ujong: Linsum (Ridley).

\section{Pelitantheria, Ridl.}

Stems tall climbing. Leaves oblong, obtuse. Racemes short. Sepals and petals similar. Lip side lobes adnate to column, midlobe large flat, spur short a callus in the mouth of the spur, and a tooth like one on the midlobe. Column very short and thick with long erect arms. Anther 2 celled. Pollinia 2, caudicle short and broad subquadrate, disc as large or reniform.

Species three, India, Cochin China and Malaya. 
P. cristata, Ridl. Journ. Linn. Soc. XXXII. 3\%3. Stems 8 inches long. Leares 2 inches long $\frac{1}{2}$ inch across thick blunt. Racemes 4 inches long rachis thickened. Bracts orate. Flowers $\frac{3}{8}$ inch across with short thick pedicels. Upper sepals ol,long spathulate, laterals falcate, with a green crested keel, yellowish white with red stripes. Petals oblong white striped red. Lip white with pinkstripes, side lobes long incurved, midlobe cordate acuminate spur short conic blunt, callus in mouth of spur clubbed, large. Column white, arms long incurved. Anther with an oblong truncate beak. Pollinia elliptic, pedicel rery short and broat, much shorter than the disc of two large curved processes. Rostellum bifid. Cleisostoma cristatum, Ridl. Trans. limn. Soc. III. 380.

\section{Pahang: Kota Glanggi, and Tahan River (Ridley).}

\section{Sarcocinllus, Br.}

Stem usually short. Leares oblong coriaceous. Racemes short or long often thickened upwards. Flowers medium sized fugacious. Sepals and petals subequal, or petals smaller, free or rarely the two lower sepals adnate to the lip. Lip attached to the column foot, lateral lobes small or fairly large, midlobe very small or almost absent spur horizontal porrect, large. ('olumn fairly long usually dilated upwards, arms none, foot always present and often long. Anther ovate. Pollinia 2, with a narrow rarely broad pedicel and small dise.

Species 30. Indian, Malayan and Australian.

In some descriptions of the species of this genus the column foot has been taken for the claw of the lip, and the spur which is cllriously pushed forward and horizontal or upeurred taken for the midlobe, this organ being taken for a callus, but it is I think quite clear as to the so-called fleshy midlobe of the lip being really the spur. The genus Ornitharium, Lindl. restored by King in the Ann. Bot. Gard. Calc., I would keep under Sarcochilus. It chiefly differs in the complete suppression of the midlobe, and even of the side lobes.

Stems short, leaves crowded.

Flowers orer an inch across .. . . 1. S. un!nuiculatus.

Flowers less than an inch across.

lachis not thickened..

Rachis thickened, spur globose .. 3. S. cladostachys.

Rachis thickened, spur long clubbed.. 4. S. stenoglottis.

Rachis thickened, spur conic.. ‥ 5. S. tanyphyllus.

Rachis thickened, spur conic very short 6. S. virescens.

Rachis flat, bracts distichous.

Flowers very small..

ก. S. biserratus.

Stem tall. Leaves distant.

Lateral sepals adnate to lip. .. . 8, S. adnatus, 
1. S. uxguiculatus, Lind1. Bot. Reg. 1840. Mise. 6\%. Stem short stout, about. 2 inches long. Leares lorate thickened coriaceous blunt, 6 inches long $1 \frac{1}{2}$ inch wide, sheaths about $\frac{1}{2}$ inch long. Raceme 5 inches long thickened upwards. Bracts orate small. Flowers large over an inch across, pedicel and ovary 1 inch long. Sepals and petals lanceolate, petals subacute, acute white or yellow. Lip short lateral lobes ineurved oblong large, falcate, purple midlobe broad, at base and abruptly narrowed to the blunt apex, a low callus between the side lobes, and a large double one behind, all yellowish spotted purple, spur short globose, narrowed in at the base. Column broad narrowed at base dilate upwards, font long white. Anther ovate shortly beaked. var. aureus. Sepals and petals yellow. Sarcochilus aureus, Hook. fil. Fl. Brit. Ind. VI. p. 35. Ann. Roy. Bot. Gard. Cale. V. 64.

Perak (Kunstler). Distribution Borneo, Philippines.

2. S. Chligaris, Ridl. Trans. Linn. Soc. III. 372. Stem very short. Leares lorate falcate acute unequally bifid $3-5$ inches long $\frac{3}{4}$ inch wide. Raceme half inch long, rachis not thickened. Flowers 3 or $4 \frac{3}{4}-1$ inch across fugacious. Bracts ovate acute. Sepals lanceolate acute. Petals subspathulate narrower, all white. Lip side lobes quadrate or-linear erect crimson, midlobe very small retuse white, with two yellow spots at its base, spur short broad clubbed. Column beaked. Pollinia elliptic, pedicel broadly triangular spathulate disc triangular small. liostellum bifid with acute lobes. Capsule linear 4 inches long. Phalaenopsis fugax, Kranzlin Gard. Chron. 1893. II. p. 360. (e deser).

Singapore in dense forest: Bukit Timah, Chan Chu Kang, etc., (Ridley); Pahang: Pekan (Ridley); Selangor: Kiwala Lumpur Caves (Ridley); Perak (Herb. Bot. Gard. Singapore).

3. S. Cladostachys, Hook. fil. Fl. Brit. Ind. VI. 35. Stem short, leaves crowded lorate apex bifid, $6-12$ inches long $1 \frac{1}{2}-2$ inches wide. Peduncle 1-2 inches long, rachis thickened, few flowered. Bracts ovate acute. Flowers $1 \frac{1}{2}$ inch across, cream colour. Sepals and petals lanceolate actminate. Lip shorter, side lobes oblong obtuse pale purple midlobe bifid lobes quadrate, spur globose spotted with purple. Column short. Anther acute in front. Foot of column spotted purple. Ann. Roy. Bot. Gard. Cale. p. 43. t. 64.

Perak (Kunstler, Scortechini). Differs from S. caligaris in its large leaves, thickened rachis, and acuminate sepals and petals.

4. S. stexoglottis, Hook. fil. Fl. Brit. Ind. VI. 34 . Stem short and stout about 3 inches long. Leaves oblong lorate coriaceous broadly obtuse, 8 inches long $2 \frac{1}{2}$ inches wide. Raceme 3 inches long thickened upwards many flowered. Flowers half an inch across opening one and 2 at a time fugacious. Bracts small ovate. Sepals oblong obtuse, laterals broader. Petals lanceolate obtuse, all white, 
bases yellow, with crimson transrerse streaks. Lip shorter narrow sigmoid, flattened laterally, side lobes low with two short points, midlobe ovate small, spur long curved upwards, clubbed, all white. C'oJumn foot very short, dilated upwards. Inther orate beaked. Pollinia subreniform, caudicle oblanceslate cuneate, disc small shield shaped. Hook. fil. Ann. Roy. Bot. Gard. Cale. V. 41. Pl. 62. S. sigmoideus, Ridl. Journ. Linn. Soc. XXXI. 298.

Perak (Scortechini). Distrib. Borneo, Sumatra.

5. S. TaNypirylus, Ridl. Trans. Linn. Soc. II. 3. 372. Stem very short hardly an inch long stont. Leaves 4 or 5 lorate 1 to nearly 2 feet long, an inch and a half across, unequally bluntly bilobed. liacemes $4-5$ inches long hardly dilate above. Bracts persistant lanceolate $\frac{3}{16}$ inch long. Flowers white rather large pedicels one inch long. Sepals lanceolate acuminate acute. Petals broader lanceolate subacute. Lip shorter, side lobes lorate falcate blunt small, spur conic blunt. Column short foot with a narrow erect base then abruptly deflexed. Anther beaked. Pollinia elliptic pedicel cuneate oblong, disc broad triangular. Rostellum with two long linear acuminate points. Capsule rather short oblong $1 \frac{1}{2}$ inch.

Pahang: limestone rocks Kota Glanggi (Ridley).

6. S. vinescens, Ridl. Journ. Roy. As. Soc. Str. Br. Vol. XXXVII. p. 85. Stem very short. Leaves lanceolate subacute $1 \frac{1}{2}$ inch long $1 \frac{1}{2}$ inch wide or less. Raceme 1 inch long, bracts orate. Flowers 1 inch across. Sepals orate acute. Petals narrower lanceolate, all light green. Lip very short white, side lobes short rounded, midlobe represented by an orange callus, spur short broad conic. Column short and thick, foot as long curved. Anther orange beak triangular.

Perak: Tapah (Aeria) drawing in Herb. Penang. Endemic. This little species is allied to $S$. viridiforus, Hook. fil. of Ceylon which has a larger lobed callus on the lip and blunt sepals and petals.

\%. S. Bisenratus, Ridl. Journ. Bot. 1900. p. i3. Stems crowted slender 3-4 inches long. Leaves narrowly linear lanceolate acute fleshy 4 inches long $\frac{1}{4}$ inch wide, sheaths $\frac{1}{8}$ inch long. Racemes flattened 6 inches long $\frac{1}{8}$ inch wide. Flowers dist irhous $\frac{1}{2}$ inch actoss, very numerous but appearing singly. Bracts ovate very small. Pedicel $\frac{1}{4}$ inch long. Upper sepal lanceolate acute, laterals oblique. Petals narrower, all yellow white, Lip side lobes linear acute curved wutwarls midlobe minute, spur long conice horn shapeed porrect i inch long, all white except the mouth of the spur violet. Columm slender straight white loot short. Anther ovate orange. Pollinia globose, caudicle linear dise very small orate.

Perak near Ipoh (Ridley 10156). Endemic. 
8. S. ADNitus, Ridl. Journ. Linn. Soc. XXXII. 3\%3. Stem tall and stout. Leares lorate coriaceous, unequally bifid, 6 inches long 1 inch wide. Racemes 3 inches long, with a stout purple peduncle. Flowers 12-16 fleshy, 1 inch long on pedicels the same length. Bracts ovate small. Upper sepal elliptic obovate obtuse laterals adnate to the column foot and lip, beneath apices rounded free, all yellow with red spots. Petals lanceolate yellow. Lip with a narrow claw side lobes oblong obtuse, midlobe shorter ovate, spur porrect broad obtuse entrance partly closed by a process, yellow tipped with reddish brown. Column rather with a short foot. Anther ovate obtuse. Pollinia 2 globose, caudicle linear very short joined at an angle with a rery much longer linear on disc rer'y small. Rostellum very long subulate acute. Endemic.

Singapore: Sungei Toas (Ridley); Johor: Kota Tinggi (Ridley).

\section{Exçluded Spectes.}

S. amplexicaulis, Rchb. fil. is Thrixspermum lilacinum, Rchb. f.

S. archnites, Rehb. fil. is Thriaspermum Arachnites, Rchb. $f$.

S. brachystachys, Hook. fil. is Thrixspermum calceolus, Rehb. f.

S. filiformis, Hook. fil. is Dendrocolla filiformis, Ridl.

S. hirsutus, Hook. fil. is Ascochilus hirsutus, Ridl.

S. hirtulus, Hook. fil. is Ascochilus hivtulus, Ridl.

S. lilacinus, Griff. is Thrixspermum lilacinum, Rchb. f.

S. notabilis, Hook. fil. is Thrixspermum notabile, Ridl.

S. recurvus, Hook. fil. is Dendrocolla pardalis, Ridl.

S. scopa, Hook. fil. is Thrixspermum Sropa, Ridl.

S. Scortechinii, Hook. fil. is Thrixspermum Scortechinii, Ridl.

S. trichoglottis, Hook. fil. is Dendrocolla trichoglottis, Ridl.

\section{Ascochilus, Ridl.}

Small epiphytes stem short with lorate or linear falcate leaves crowded, or elongate with terete leaves. Scapes short muricate or hairy flowers small in a short raceme. Bracts short quaquarersal, rachis not or hardly thickened. Lateral sepals larger than the upper sepal and decurrent or adnate to the column foot. Petals narrower. Lip 3-4 lobed, side lobes large, midlobe large or very small, spur cylindric pendulous or porrect thick. Column usually tall and slender, foot narrow as long or longer. Anther beaked. Pollinia 2 globose, caudicle linear disc small. Capsule narrow cylindric.

Species 5 or 6 . Andamans, Malaya and Siam. 
Stem elongate leaves terete .. $\quad \ldots \quad \ldots \quad$ 1. A. teres.

Stem short leaves broad.

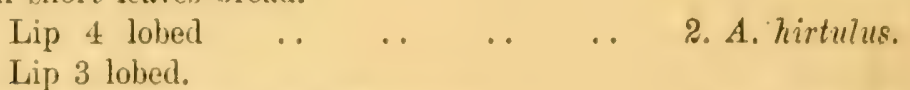

Sepals narrow acute yellow glabrous .. 3. 3 . siamensis.

Sepals broad barred red, hairy .. .. 4. A. hirsutus.

Siepals lanceolate flowers very small glabrous 5. A. minutiflorus.

1. A. Teres, Journ. Roy. As. Soc. Straits Br. Vol. XXXIX. p. 85. Stem 6 inches or more tall, slender. Leaves terete acute 4.5 inches long, $\frac{1}{8}$ inch thick, sheaths half an inch long ribbed and transversely wrinkled. liaceme slender 4 inches long. Flowers few scattered $\frac{1}{4}$ inch across. Bracts very small ovate. Ovary and pedicel 3 inch long. Cpper sepal oblanceolate hooded, laterals oblique much larger oblong ovate. P'etals broadly spathulate oblique shorter. Lip side lobes ereet lanceolate faleate, mirllohe hastate basal lobes rounded apex subacute, spur hall the length of the lip curred obtuse. Column as long as its foot nearly as long as the petals arms short and broad.

. Johor: on Bukit Banang, Batu Pahat (Ridley).

2. A. mintulus, Ridl. Journ. Limn. Soe. XXYII. p. 375. Stem short half an inch long. Jeaves falcate linear acute $2-3$ inches long. Scapes half an inch or little more long muricate raceme very short. Bracts orate, minute. Flowers nearly $\frac{1}{2}$ inch across. Upper sepal lanceolate, lateral sepals adnate to column foot much larger oblanceolate obtuse. Petals linear lanceolate, all cream with pink spots. Lip side lobes erect linear curved, midlobe bilobed lobes as long linear, spur short curved blunt. Column tall slender, foot narrow linear. Inther long beaked, acute. Capsule narrowly eylindric shorter pedirelled $1 \frac{1}{2}$ inch long $\frac{1}{\gamma}$ inch through. Sarcochilus hirtulus, Hook. fil. l. Brit. Ind. TI. P. 89. Ic. Pl. t. 2121.

Pahang: Kota Glanggi (Ridley 236t); Malacea (Maingay); fielangor: Kwala Lumpur ('aves (Ridley) ; Pename: Watrefall (Ridley) ; Perak (Scortechini) ; Lankawi (Curtis).

3. A. stanexsis, Ridl. Joum. Limn. Soe. XXYII. p. 375. Stem an inch long, with long slender roots. Leaves j inches long, hardly I inch wide lorate falcate bilobed acute, scapes 3 inches long muriculate, racemes very short. Bracts minute orate. Pedicels $\frac{1}{8}-\frac{1}{4}$ inch long. Flowers t inch across. Epper sepal elliptic laneenlate acute, lutcrals much larger rhomboid ohlique lieeled acute. Petals linear lancmlate curved, all ochre vellow. Lip white, side lobes oblong apex rounded midlobe tongue shaped small blunt, spur long slender rylindric blunt. Colmmn sigmoid, with a long slender foot, and short erect blunt arms. Anther bllong shortly beaked. Pollinia globose, caudicle narrow linear. 


\section{Lankawi (Curtis). Distrib. Siam.}

4. A. Hinsutus, Ridl. Stem short stout. Ieaves lorate narrowed at the base $t-8$ inches long, 1-11 inch wide, sheaths short. Scapes pendulous short 1-1. inch long hairy. Flowers crowded at the end fleshy 4-8, pedicels $\frac{1}{4}$ inch long hairy. Bracts $\frac{1}{8}$ inch long ovate hairy. 'Upper sepal oblong obovate laterals oblique larger, all hairy, yellow with Indian red bars and blotches. Petals lanceolate smaller. Lip side lobes oblong erect, with two horn shaped processes between, midlobe very small truncate notched, spur large dilated at the base then abruptly narrowed, whole lip white with red blotehes on the side lobes and spur, shorter than the sepals. Column rather short, foot fairly long. Anther large ovate beaked, two celled. Pollinia 2 globose caudicle linear disc orate. Sarcochilus hirsutus, Hook. fil. Fl. Brit. Ind. VI. 38. Ann. Roy. Bot. Calc. V. p. 41. Pl. 6\%.

Perak (Kunstler), Teluk Anson (Curtis). A curious plant connecting Sarcochilus and Ascochilus. The sepals are adnate to the foot of the column and oblique, the lip and spur are rather those of Sarcochilus.

5. A. Minutiflorus, Ridl. Journ. Roy. As. Soc. S. Br. Vol. XXXIX. p. 85. Stem very short. Leaves linear lanceolate falcate acute, 3 inches long $\frac{1}{2}$ inch wide or less, sheaths very short. Scape very slender 1 inch long pubescent raceme very short. Bracts cucullate ovate. Flowers $\frac{1}{8}$ inch across. Upper sepal lorate oblong, laterals lanceolate all keeled yellow with red spots. Petals oheuneate yellow with a red spot at the base. Tip side lobes large oblong truncate, midlobe very short truncate entire, spur short blunt rather thick saccate serotiform obtuse. Column tall curved slender, foot hardly as long. Anther long beaked.

Pahang: Kwala Tembeling (Ridley).

\section{Afrides, Tour.}

Stems long. Leaves linear unequally bilobed or terete. Flowers in long lax or dense racemes, or few. Sepals broad and spreading, laterals adnate to the foot of the column. Petals broad. Lip adnate to the end of the column foot, side lobes usually large sometimes absent, midlobe usually larger spur pendent, calli none. Column short foot short or long. Anther two celled. Pollinia 2 globose caudicle long or short. Rostellum bifid.

Species 10. India, Malaya and Cochin China.

Af. odoratir, Lour. Fl. Cochin Ch. 525. Stems about a foot or more stout, leares lorate coriaceous a foot long inch wide, unequally bilobed blunt. Scape a foot long or less, base nude except for small distant sheaths. Raceme lax. Bracts ovate lanceolate acute. Flow. ers 1 inch across, fragrant pedicels 1 inch long. Upper sepal obovate 
obtuse, laterals much larger. Petals narrower oblong all pinkish white darker at the tipss. Jip large saccate side lobes long nuevrved obtuse, midlohe narrow acute. white or pinkish dotted pink, spur rorved horn staped usually tipped green. Column short straight with a long broad foot. Anther ovate bluntly beaked. Pollinia globone caudicle linear. Capsule fusiform 1-1t inch long. Jindl. Fen. and Sp. Orch. 11. 239. Journ. Linn. Soc. III. 41. Bot. Mag. t. 4139. Paxt. Fl. Gard. II. t. 143. Maund. Botanist IV. t. 180. Hook. fil. Fl. Brit. Ind. VI. 4\%. King. Ann. Roy. Bot. Gard. Calc. VIII. 212 pl. 282. Ae. cornutum, Roxb. Hort. Beng. 63. Fl. Ind. III. 472. Bot. Reg. t. 1485. Ae. suavissimum, lindl. Journ. Hort. Soc. IV. 263. Paxt. Fl. Gard. II. 141. t. 66. Hook. fil. Fl. Brit. Ind. I.c.c. Ae. Reichenbuchii, Lindl. Foch and Fintelm. Wochenchrilt. 18.5. 61. Rehl,. fil. Xen. Orch. II. 11. t. 10t. Ae.nobile, Warner Sel. Oreh. Ser. I. t. 11. Gartenflora 40. t. 641., Ae. Rohanianum, Iichb. fil. Gard. Chron. 188t. I. 206. Ae. flavidum, Lindl. Paxt. Fl. Gard. II. 101. Ae. Ballantimianum, Rehb. fil. Gard. Chron. 188.). II. 198. Ae virens, Lindl. Bot. Reg. 1843. Mise. p. 48. Bot. Reg. t. 41. Paxt. Fl. Garden 18\%. p. 19\% Le. suareolens, B1. Mus. 1. p. 112. Liumphia p. 53. t. 193. f. I.

Johol: P'ulau 'T'ingrgi (Nammn) : Malatea (Maingay) : Felangor : Bukit Itam (Kelsall); Perak (Ic. in Hort. ('alcutta); P'nang (ficle IIooker) ; Kélah: Ian (Ridley) ; Lankawi (Curtis). D)istrib. India, China, Cochin ('hina. Malay Lylands. Varialle in depth of colour and form of the small midlohe.

\section{6. 'Thrisipermua, Jomr.}

Terrestrial or epiphytic plants, stems generally long, rooting at intervals. Leaves amplexicaul orate or oblong or lorate coriaceous, with rather or very long sheaths. Pertumeles of salpes long with short flat racemes of distichoms bracts bersistent. Flowers larger fugacious opening at considerable intervals. Sepals and petals similar ovate obtuse or caudate. Lip saceate side lobes falcate, midlobe usually fleshr, one of two calli on the dise. C'olumn with a short font. Anther ovate. Pollinia 2 elliptic with a small or large disc, no caltdiele. Capsule clongate.

Species 11. India, Malayan and Cochin China.

Sepals and petals caudate.

Stem tall. Leaves orate amplexicaul .. 1. T. lilacinum. Stems long leaves lanceolate.

Midlobe large conic .. $\quad \ldots \quad \ldots$ 2. $T$. calceolus.

Midlobe small obtuse $\quad \ldots \quad$. $\quad \ldots \quad$ 3. T. pauciflorum.

Stem short. I.ip lobes indistinet .. 4. T. brachyglottis. 
Sepals and petals candate. Lip 3 lobed.

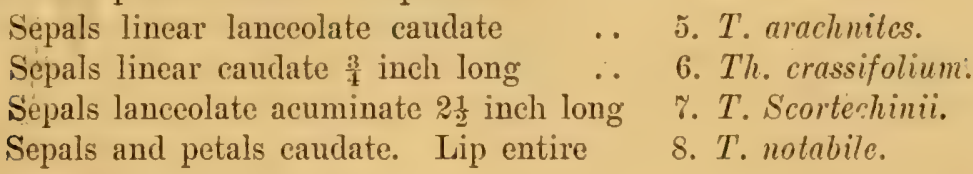

1. Th. Lildencex, Rehb. fil. Xenia. Orch. Il. p. 121. Stems several feet long 3-6. Leaves ovate amplexicaul unequally bilobed an inch long $\frac{3}{4}$ inch wide. Peduncles stiff \pm to 10 inches long, racemes 3-6 inches long. Bracts ovate $\frac{1}{4}$ inch long. Flowers an inch across, white or pale lilac, pedicels nearly an inch long. Sepals aul petals ovate obtuse. Petals smaller all spreading. Lip shorter, side lobes falcate acute, midlobe blunt conic, with an oblong truncate callus on the dise, base of lip saccate. Column short broad, foot short. Anther small conic. Pollinia deeply bifid oblong, with a triangulat disc. Capsule subeylindric 4 inches long $\frac{1}{t}$ inch thick. Ridl. Journ. Linn. Soc. XXXII. Sarcochilus lilacinus, Grift. Notul. HI. p. 33t. Ie. Pl. As. t. 320. f. 2. Hook. fil. Fl. Brit. Ind. Vol. VI. p. 40. Bot. Mag. t. 765t. Rehb. fil. Walp. Ann. VI. p. 499. Dendrocolla amplexicaule, Bl. Bijdr. p. 288. Aerides amplexicaule, Lindl. Gen. and Sp. Orch. Pl. p. 239. Orsidice amplexicaulis and O. lilacinus, Richb. fil. Bonplandia II. p. 93.

Singapore: Holland Road, Seletar (Ridley 1692); Pahang: Pulau (hengei, Pahang River (Ridler 2:351). Pelan (Ridley 138.5); Malacea : Ayer Panas (Derry 284), Chabau (Native Coll.); Selangor: Pudu (Goodenough); Perak: Taiping (Ridley), Changkat Jerin (Fray 18:2); Penang (H. Singap.). Distrib. Borneo.

In open grassy swamps. Blume's description of Dendrocolla amplexicaule does not agree with this plant, and it is perhaps not conspecific. Native name "Rumput Sesudu Paya."

2. T. C.Ilceolus, lichb. fil. Yenia. Orch. II. p. 12\% Stems stout 10 to 12 feet long, slightly flattened. Leares lanceolate coriaceous $2-4$ inches long unequally bilobed, sheaths ribbed $\frac{1}{2}$ inch long. Racemes an inch long, 1 to 3 together numerous, sessile. Bracts orate cucullate $\frac{1}{4}$ inch long. Flowers few opening singly or in pairs on each raceme, 2 inches across white fragrant, pedicel and ovary $\frac{1}{2}$ an inch long. Sepals widely linceolate acute keeled. Petals narrower oblanceolate. Lip shoe shaped base siceate, side lobes falcate acute, midlobe conic obtuse, callus oblong truncate excarate at apex, all white except an orange spot round the callus, and small orange and crimson dots at the base of the lip. Column short white. Anther thin orate quadrate. Pollinia scmipreriform 1 dise reniform. ('apsule evindric 6 inches long. Sarrochilus Calccolus, Lindl. Bot. Reg. (18t6) t. 19. S. brachyslachys, Hook. fil. Fl. Brit. Ind. VI. p. 41. 
Sinfapere: Jurong River (Ridley so69), T'ulau 'Tekong (Ridley 20132): Johor: 'Tama Romto (lidley), Batu l'aluat (lidelley 1121:3); Pahane: Liwala 'lenok (Ridley 2:363) ; Sungei T jong: Gunong Angsi (Ridley); Dindings: 'Telok sera (Curtis); l'enang ILill (Maingay). Distrib. Sumatra.

3. Tur. P.luciforuar, Ridl. l.c. Stem rigid $\frac{1}{5}$ inch in diameter, flexuous. Leaves oblong obtuse rather distant $2 \frac{1}{2}-3$ inches long 1 t to 1. inch wide corlaceous. Racemes short an inch long, few flowered, basal sheaths imbricate. Bracts subacute $\frac{1}{8}$ inch long. Sepals oblong lanceolate acute $\frac{2}{3}$ inch long, white. Petals nearly as large obovate ollong obtune. Lip stipitate yellow side lobes broad rounded obtuse midlobe small fleshy obtuse with a conic callus at the base, and a broad lamella between the side lobes, saccate base of lip conic. Column short. Anther ovate obtuse depressed. Pollinia 2, deeply hitid, caucticle short. Surcochilus punciflorus, Hook. fil. Fl. Brit. Ind. VI. 41.

Perak: Larut Hills at 3000 feet (Scortechini). Allied to T. (inlceolus, Rehls. fil. but ditlers in the smaller flowers, straight blunt side lobes and short blunt midlobe of the lip.

1. 'Tir. Bracmyglotris, Ridl. Stem rather slender short. late's congate oblanceolate acuminate bifid or entire $4-8$ inches long by one inch to $1 \frac{1}{3}$ inch wide. Scape short stout an inch long few flowered. Flowers white tinted pink $\frac{1}{2}$ inch across. Sepals lanceolate acute mucronate. Petals oblong obovate ohtuse. Tip not halt as long sessile at the base of the columm subcumeate truncate laterally compresed hollow with a short spur below the blunt fleshy tip. Column short footless, hoad with large erect triangular acute arms. Anther orate acuminate. Pollinia 2 globose caudicle linear dise shield shaped. Sorrorhilus brachyglotlis, Hook. lil. Inn. Bot. Loy. Gard. Calc. V. 42. Pl. 63.

Perak (Kunstler). Endemic. I have only seen the figure of this plant. It is difficult to refer it to any genus. The column and lip to some extent suggest an infinity with Thridspermum C'ulceolus, lichb. fil. The pollinia and habit suggest a sarcochilus.

5. 'Tir. Ar.cnxites, Rehb. fil. Xen. Orch. II. 121. Stems 4-6 inches long. Leatres lorate oblong 6 inches long 3 inch wide apices bilobed, sheaths 1 inch long. Scalues soveral, peduncles $6-8$ inches long, racemes 3-12 inches long. Bracts close set acute $\frac{1}{4}$ inch long.

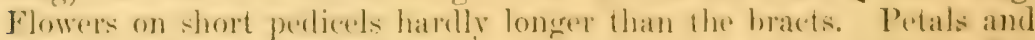
sepals similar 2 inches long linear lancelate caudate, yellow. Lip

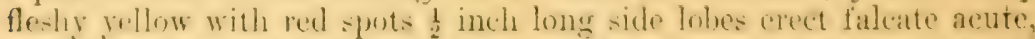
midlobe obtues thick, a quadrate truncate callus on the dise, base of lip saceate almo-t sum like blunt. Colmum narrow sllaight. Antlers conic. I'ollinia vioid, caudicle transterscly oblongr, dici as broad. 
Capsule cylindric 3 inches long $\frac{1}{2}$ inch through. Sarcochilus arachniles, Rchit. fil. Walp. Ann. VI. 498. Hook. fil. Fl. Brit. Ind. VI. 41. Dendrocolla arachnites, Bl. Bijdr. 28\% t. 6\%. Aerides arachnites, Lindl. Gen. and Sp. Oreh. Pl. 238. S. Scopa, Hook. fil. Fl. Brit. Ind. VI. 45 .

Singapore: 'Tanglin, Seletar (Ridley); Johor: Batu Pahat (Ridey); Selangor: Bukit Hitam (Kelsall); Perak: Larut (King's Coll. 5\% \%) Penang (Kunstler). Distrib. Borneo, Sumatra, Java. Often scrambling on low bushes, or on trees. I can see no rharacters to distmguish S. Scopa, Hook. fil. which appear's to be merely the elongite form found on trees.

6. 'Th. Chassiforius, Ridley Journ. Roy. As. Soc. Str. Br. Vol. XLJ. p. 32. Whole plant 6 inches tall with a stout stem and close set leaves, sheaths under half an inch long purple blade oblong obtuse thick leathery apex rounded 4 inches long $1 \frac{1}{4}$ inch thick. Scaves 3 to 5 inches long stiff base purple. Inflorescence $1 \frac{1}{2}$ to 4 inches long. Bracts $\frac{1}{4}$ inch long close set flattened orary and pedicel $\frac{1}{4}$ inch long. Sepals linear caudate from a broader base 3 inch long bright yellow. Petals narrower linear caudate. Lip $\frac{1}{1}$ inch long pubescent orange passing into cherry scarlet at the tip, npur saceate blunt side lobes arched blunt midlobe fleshy blunt ovate, callus tongues shaped rounded at the tip depressed in centre. Column white with rounded broad wings. Anther hemispheric. Fruit angled linear 3 inches long $\frac{1}{4}$ inch through.

Johor on old coffee trees, at Castlewood (Ridley). Endemic. A plant of the habit of Th. Scortechini with much smaller flowers even than those of $T h$. arachnites.

\%. Th. Scontechini, Ridl. l.c. Stem 6 inches long rery stout internodes $\frac{3}{4}$ inch long. Leaves oblong lorate bilobed coriaceous $4-6$ jnches long 1-1 $\frac{1}{2}$ inch wide. Scape 9 inches long, raceme 2 inches. Bracts close set $\frac{2}{3}$ inch long. Sepals and petals lanceolate acuminate, $2 \frac{1}{2}$ inch long $\frac{1}{4}$ inch wide at the base, yellow. Lip saccate side lobes short midlobe conical obtuse fleshy, yellowish thick mottled with rusiy red inside. Pollinia 4 disc lunate caudicle oblong. Surcochitus Scortechinii, Hook. fil. Fl. Brit. Ind. VI. 40. demic.

Perak (Scortechini); Kedah: Gunong Jerai (Ridley). En-

8. T. Notabile, Ridl. Journ. Limn. Soc. XXXII. 3\%9. Stem short 2 inches long. Leares broad lorate or oblong, green or deep purple 3 inches long $\frac{1}{3}-1$ inch wide. Seapes very slender about 3 inches long, purple, with a few distant sleatls, raceme an inch long. Bracts acute, ovate purple. Flowers 1 inch across. Sepals linear rery narrow acuminate caudate. Petals similar, rellow or reddish. 
Lip saccate ovate cuspidate side lobes not distinct, yellow, nith pink spots. ('ulumn short broad. foot indistinct adnate to the lip. Anther randicle tranversely ohlons, dise orate. Surcochilus (Ridleya) notabilis, Hook. fil. Fl. Brit. Ind. Vr. p. 42. Ic. Pl. 2126.

Singapore: ('han C'hu Kang, Bukit Mandai (Ridley); Julbr: Batu Pahat (Ridley). Endemic.

\section{5\%. Dexdrocolla, Bl.}

Small epiphytes, stems short. Leaves lorate coriaccous or terete. Sicapes erect, with a long peduncle, rachis of short raceme thickined or not. Bracts persistent not distichous. Flowers few opehing singly fugacious. Sepals and petals usually sprearling free. Lip broad often (onvolute and pubescent side lobes small or indistint, spur none. ('olumn short and thick foot narrow. Pollinia 2, cendicle very small or absent, disc large. Capsule linear.

Species about 20. Bumah, Ceylon, Malay Islands.

\section{Leares filiform}

1. D. filiformis.

Leares lorate. Lip pubescent.

Bracts subulate recurved

Bracts ovate acute.

Lip midlobe very small truneate. Flowers pale yellow side lobes short .. 3. D. trichoglottis.

Flowers pure white side lobes large .. 4. D. alba.

Lip midlobe linear .. $\quad \ldots \quad \ldots \quad$ 5. D. maculata.

Lip midlobe ovate no callus .. $\quad . .6$ 6. D. ciliatum.

Lip midlobe orate with a woolly callus .. \% D. carnosa.

Lip glabrous, side lobes distinct linear.

Stem and scape long $\ldots \quad \ldots \quad \ldots \quad$ 8. D. carinatifolia.

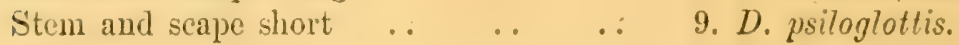

1. D. Filiforans, Ridl. Journ. Linn. Soc. XXXII. 382. Stems 2-4 inches long, slender. Leaves few terete slender acute 6 inches long sheaths half an inch long. Scapes slender 3 inches long, raceme across, pedicel and ovary $\frac{1}{2}$ inch across. Sepals lanceolate obtuse. P'etals a little smaller, white. Lip much shorter, side lobes erect romuled orange, midlobe oblong elliptic fleshy rellow, pulverulent. Column rather long foot short. Anther oblong quarlate, thin flat "celled, dise transierse connecting the fwo subreniform pollinia. Sarochilus filiformis, Hook. fil. Fl. Brit. Ind. VI. p. 39. Ie. 1'l. t. 212t.

Singapore: Buhit Mantai, tete. (Litlley); Jerak (King's Collector). Eudewic. 
2. D. Pardalis, Ridl. Journ. Linn. Soc. XXXII. p. 382. Stems 10 inches long or less with numerous long roots. Leaves lanceolate lorate unequally bifid lobes acute 2 inches long $\frac{1}{4}$ inch wide. Scapes six to ten inches long slender, racemes 2 inches long, rachis thickened with crowded subulate bracts. Flowers bardly $\frac{1}{2}$ inch across. Sepals orate obtuse. Petals much smaller linear, all white with pink spots. Lip obcuneate truncate sides involute yellowish with reddish tint corered with white wool, callus oblong linear obtuse. Column short thick. Anther ovate. Pollinia elliptic on a rounded disc. Sarcochilus pardalis, Ridl. 'Trans. Linn. Soc. II. iii. 3\%1. S. recurvus, Hook. fil. Fl. Brit. Ind. VI. 39. Ic. Pl. 2122.

Pahang: Pulau Chengei, Pahang River (Ridley 2365); Perak: Gopeng (King's C'ollector 6040), "Limbo Ilills" Larut (King" is Collector). On low trees and shrubs. Native name "Suttong Terbang." Hooker"s S. resurvus is probably this plant but the flowers are not described. Distrib. Borneo.

3. D. trichoglottis, Ridl. Journ. Linn. Soc. XXXII. 381. Stem 2 to 6 inches long erect, roots long. Leaves stiff lorate obtuse unequally bilobed $1 \frac{3}{4}-2$ inches long $\frac{3}{8}-\frac{1}{2}$ inch wide. Scapes stiff peduncle $\frac{1}{2}-2$ inches long, raceme $\frac{1}{2}$ inch long thickened. Bracts ovate mucronate. Flowers $\frac{1}{2}$ inch across, pedicels shorter. Upper sepal lanceolate acute, laterals much wider orate lanceolate keeled. Petals narrower spathulate, all pale jellow. Lip much shorter base saccate, side lobes very short broad truncate erect hairy midlobe rery small truncate, a small oblong retuse callus between the lobes all yellowish, with an orange spot near the callus. Column white foot narrow orange. Anther ovate obtuse. - Rostellum entire, flat. Capsule cylindric slender 5 inches long. Sarcochilus trichoglottis, Hook. fil. Fl. Brit. Ind. VI. p. 39. Ic. Pl. t. 2123.

Singapore: very common, Tanglin, Bukit Timal (Ridley); Pahang: Pekan (Ridley 2366, 1303) ; Perak: Kiwala Kíangsa (Ridley), Dindings near Bruas (Ridley), Chanderiang (King's Collector 5934 ) ; Tringganu: Bundi (Rostado). Distrib. Borneo.

4. D. ALBA, Ridl, Journ. Roy, As. Soc. S. Br. Vol. XLIV. p. 191. Stems 3 to $t$ inches long roots copious long and slender. Leaves lorate lanceolate fleshy dark green 1 to 2 inches long $\frac{1}{4}$ inch wide obtuse. Scapes 4 inches long slender green, racemes $\frac{1}{4}$ inch long thickened. Bracts ovate acuminate quaqua versal. Flowers one or two open at once on pedicels $\frac{1}{4}$ inch long white 3 inch across. Sepals lanceolate acute. Petals narrower and shorter narrowed at the base, lip $\neq$ inch long saceate, lateral lobes short distinct curved blunt edged with short thick clubbed hairs, midlobe little longer rounded blunt excavate, callus in the centre at the base of the midlobe oblong truncate white with light brown blotches inside the pubescent sac and similar 
spots on the side lobes and midlobe and hip of callus. Column white oblong, font narrower blotched bown, anther skull shaped. Pollinia oroid, pedicel very short and broad.

Perak: Kamuning near Sungei Siput on coflee trees (Machado).

5. D. Mculdts, Ridl. Journ. Limn. Soc. XXXII. 381. Stems 3 inches long. Leaves linear coriaceous bilobed. Scape 3 inches long stiff, raceme :3 inches long, rachis thickened. Bracts ovate acute. Flowers $\frac{1}{2}$ inch across, pedicels slender. Sepals lanceolate spathulate, laterals dilated at the base yellow. Petals narrower. Lip shorter side lobes romoled tootherl pubeserent yellow with crimson spots, midlobe linear yellow pubesecent, base of lip saccate long, an oblong retuse callus in the centre. ('olumm short hroad with hroad involute wings, foot rather long. Anther with rather a long truncate beak. Pollinia oblong reniform, dise reniform.

- Singapore: Bukit Mandai, Chua Chu Kang (Ridlej).

6. D. cildatur, Ridl. Journ. Roy. As. Soc. S. Br. Tol. XLIV. p. 192. Stem about an inch long. Leares erowded 6 elliptic obture narrowed at the base fleshy dark green es inches long 3 inch wide. Pedunde slenter e inches long raceme hardly thichened. Bracts ovate acute rather distinct minute. Flowers opening singly pedicels $\frac{1}{4}$ inch long. Sepals patent oblong obtuse ochreous vellow 1 inde long. Petals narrower and shorter. Lipe as lomer side

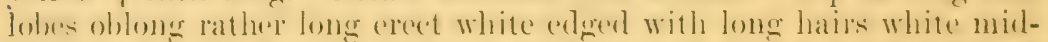
lobe short conic Heshy. Column stout straight anther helmet shaped beaked orange red. Pollinia oroid shortly stalked orange, pedieel oblong flat indented at the sirles dise ahout 1 is length triangular pale orange fellow. ('linambium flat with two orange triangular flaps.

Perak: Kamuning, Sungei Siput (A. D. Machado).

․ D. Cumasi, Ridl. Journ. Bot. 1898. Stem 2 inches long.

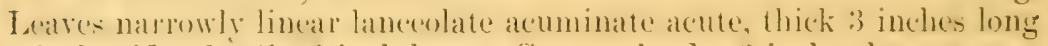
A inch wide, sheaths $\frac{1}{4}$ inch long. Scapes slender 3 inches long raceme thickenerl an inch longer. Bracts ovate acute. Flowers small pediech 1 inch lone. semals and petals oblong ovate pellow. Lip shorter side lobes long and broad, batice saccatc, midlobe fleshy orate obtuse with a woolly papilla at base.

Penang (Curtis).

8. D. Chisatrold, Rid!.Journ. Jimn. Soc, XXXII. 382. Stems 6 inches long flattened. Leares fleslyy oblong blunt, 2 inches long $\frac{1}{2}-1$ inch wide. Scapes stender 6 inches long, racene an inch

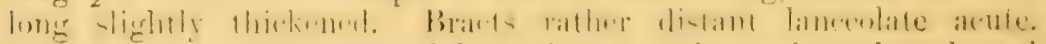
Flowers white. Lipper sepal lanceolate acuminate, laterals enlarged at base. Petals shorter linear lanceolate. Jip base saceate side lobes long lincar ateminate midlole shorter orate thick blunt wilh a blunt 
rounded callus, all white except a yellow spot on the base of the lip outside. Column straight thick. Anther quadrate. Pollinia oval decply bifid disc oval. Capsule slender linear cylindrie 3 inches long. Sarcochilus carinatifolius, Ridl. Journ. Straits As. Soc. 1891. p. 136.

Johor: Kwala Sedili Besar, Pulau Aor (Feilding). Distrib. Christmas Island.

9. D. rsiloglottis, Ridl. Stem short $1 \frac{1}{2}$ inch long. Leaves lorate 2 inches long 1 inch wide apex rounded bluntly bilobed, sheaths short. Scapes erect 3 inches long slender, raceme short 4 inch long not thickened. Bracts lanceolate acuminate subulate recurved. Flowers $\frac{1}{4}$ inch across. Upper sepal lanceolate acute, laterals triangular acute, pale yellow with an orange spot at base. Petals subspathulate yellow. Lip short side lobes long linear white, midlobe obtuse recurved fleshy white with an orange central spot. Capsule cylindric linear 2 inches long. Sarcochitus psiloglullis, Ridl. Trans. Linn. Soc. III. 372.

Pahang: on trees Kiwala Pahang (Ridley).

58. Acriopsis, Reinwdt.

Pseudobulbous epiphytes, with linear or oblong articulated leares. Scape racemose or panicled, with small pink and white or yellow flowers. Petals and sepals spreading narrow laterals sometimes connate. Lip spurless adnate to the column by its claw half way up, 3 lobed with a central callus. Column tall arched apex hooded, curved, arms long porrect. Anther orate. Pollinia 2 narrow with a long slender caudicle and a very small disc. Rostellum ovate entire. Capsule globose.

Species õ. Malay Peninsula and Islands.

Leaves linear. Scape panicled.

Lip lanceolate, side lobes broad .. . . 1. A. javanica.

Lip very narrow linear, side lobes small .. 2. A. indica.

Leaves linear. Scapes racemose.

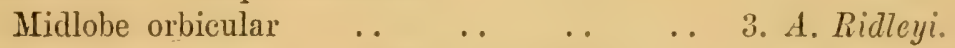
Leaves oblanceolate broad. Scape racemose .. 4. A. purpurea.

1. A. Javaxica, Reinwdt. Flora Literat. 1825 II. 4. Syllog. Ratisb. 1828. Pseudobulbs crowded ovoid, about an inch long. Leares 2-3, narrowly linear 3-6 inches long $\frac{1}{3}-\frac{1}{4}$ inch wide. Panicle over a foot long; slender with few or no long slender hranches. Flowers half an inch across, pedicel and ovary $\frac{1}{8}$ inch long. Bracts very small. Upper sepal lanceolate, laterals connate, lanceolate, white tipped crimeon. Petals spathulate white with erimson central bar. Lip side lobes broad flat rounded, midlobe lanceolate blunt with two 
little triangular keels in the centre white with a central violet crimson spots. Column rosy, arms long. Capsule globose $\frac{1}{2}$ inch long. Blune Bijdr. 3\%\%. Lindl. Gen. and Sp. Oreh. Pl. 140. Walp. Ann. TI. 492. Hook, fil. Fl. Brit. Ind. VI. 79. A. picte, Lindl. Bot. Reg. XXIX. Misc. 105. A. Griffithii, Rehb. fil. Bomplandia II. 92. A. crispa, Griff. Notul. III. 333. Ic. Pl. Asiat. t. 318. Spathoglottis trivalvis, Lindl. Gen. and Sp. Orch. 120.

singapore (Wallich 3it?), Tanglin, Changi (Ridley); Johor: Bukit Ifurlom, Batu labat (Kelsall foo6); Malacea (Cuming 2060 ) ; Pahang: Pekan (Ridley) : Selangor: Pahang Track (Ridley); sumere C jong (native collector) : Perak: Iant Hills. Hermitage Hill (Ridler) ; Penang: Balik Pulau (Cutis 24:3). Distrib). Tenasserim, Sumatra, Java.

Common on orchard trees ete., growing also in tult's of grass at Pekan. Native names "Angrek Darat," "Sakat Bawang," "Saliat Ubat Kapialu." A decoction of the plant is used for fever.

2. A. IxDica, Wight Icones. t. $1 \% \pm 8$. Pseudobulbs crowded $\frac{1}{2}$ inch long covered with old sheaths. Leaves very narrow linear 3 inches long less than 1 inch wide. Scape $6-8$ inches long panicled with several long 4 inch branches peduncle $2-3$ inches long. Flowers less than $\frac{1}{6}$ inch across pedicels nearly $\frac{1}{+}$ inch long. Bracts minute. ripals narrow linear oblonge, laterals romnate. Petals broater oblong retuse shorter, all yellowish spotted red. Lip very narrow linear with two short lobes at the base, callus bilobed near the lip base. Column hood large, arms slender long. Capsule 3 inch long globose. Walp. Anu. VI. 492. Hook. fil. Fl. Brit. Ind. VI. 89.

Penang: Government Hill (Curtis 2ti2).

3. A. Ridelis, Hook. fil. Fl. Brit. Ind. TI. 79. Pseudobulbs wate subcompresed crowded an inch long. Leares ?-3 linear obture \pm inches long 1 inch wide. Scape racemose, a foot long. Flowers few, about 16, larger than those of $A$. javanica. Upper sepal linear, laterals spathulate commate. Petals spathulate broader, all yollow with red spots. Lip white side lobes oblong, midlobe orbicular with a narrow base, callus of two short keels on the claw of the midlobe crimson. Column hood long upeurved, white with crimson spots, arms long clubbed, porrect. Anther ovate obtuse. with.

Singapore: Bukit Manmiai (Riclley). A single plant only mel

4. A. Punuma, Ridl. Trans. Linn. Soc. 11. iii. p. 406 . Psen-

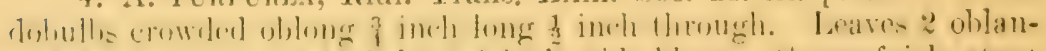

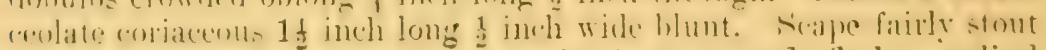
5 inches long racemose. Flowers about 13 secund, fleshy, pedicel and vary ? inch long volet aud orrecen. Bracts rery small orate. 
T'pper sepal narrow apex decurved, laterals similar connate. Petals spathulate blunt, all dark purple. Lip violet rose edged with white, spathulate oblong truncate side lohes 2 small ears, callus entire suptare in the centre of the midlobe white. Column purple arms long hood decurved broad. Capsule oblong $\frac{1}{2}$ inch long.

Pahang: Pekan (Ridley) rare. Distrib. Borneo.

\section{Timecostele, Rehb. fil.}

Epiphytes, pseudobulbs oroid, crowded. Leaf solitary large. Raceme pendulous from the side of the psendobulb. Flowers numerous small pink and white, pedicels short. Sepals free, spreading laterals oblipue. Petals smaller. Lip base adnate to the base of the column and forming a tube with it blade clawed with two short or large side lobes and a larger midlohe. Column upper part slender curved with well developed arms. Base of foot adnate to lip. Anther thin. Pollinia 2, with one or two caudicles and a broad dise. C'apsule subglobose beaked three-valved.

Species 6. Malayan, Tenasserim to Java.

Flowers small, numerous.

Side lobes of lip very small .. ... 1. Th. masulosa.

Side lobes of lip large clubbed $\quad . \quad$... 2. Th. Zollingeri.

Flowers few, large an inch across.

Lip midlobe large, side lobes smaller .. 3. Th. secunda.

Widlobe small, side lobes large falcate no extrá linear processes .. .. ..

A pair of linear processes before the side lobes

4. Th. Waingayi.

5. Th. quinquefida.

1. Tu. maculosi, Ridl. Trans. Linn. Soc. II. p. 3\%t. Psendobulbs crowded conic $t$ inches long. Leaf lanceolate oblong 12 inches long, 3 inches wide. Scape pendulous 12-18 inches long, flowers numerous small. Bracts small orate. Pedicel short and thick. Cpper sepal oblong subacute, laterals broader oblong oblique. Petals very narrow lanceolate acuminate falcate all yellowish with chestnut purple spots. Lip tube thick straight, side lobes rery short eurved white midlobe broad pandurate retuse pubescent white with dark crimson tips and a horse-shoe shape crimson central spots. Column slender curved, arms linear lorate. Anther flat, pollinia 2 globular with short pedicels and a large quadrate disc. Capsule oroid beaked f inch long three valved. Collabium Trayi, Hook. fil. Fl. Brit. Ind. i. p. r84. Ic. Pl. t. 2065.

Malacea (Goodenough) ; Sungei Ujong: Burunang (native collector Herb. Singap.); Pahang: Kwala Tahan (Ridley); Perak: 
Taiping (Rirley), (Wray 255\%), (Scortechini); Kedah: Yan (Tidley). Tative name "Sakat Belimbing."

2. Tir. Zohlisgent, Rehb. fil. Bonplandia V. $185 \%$ p. 3\%. Xen. Oreh. II. 133. t. 14\%. Pseudobulbs oroid conic $1 \frac{1}{2}$ inch long. Leaf lanceolate obtuse 8 inches long 3 inches wide. Scape pendulous of inches long lax flowered. Flowers $\frac{1}{2}$ inch across, pedicels short. Bracts $\frac{1}{2}$ as long orate acute. Upper sepal lanceolate, laterals adnate to columm base orate rhomboid, subacute much broader. Petals very narrow linear, all white tipped and blotehed erimson, base orange. lip tule long, lateral lobes large obovate spathulate twisted spoonshaped pubescent yellowish red, midlobe orate with a narrow base and deeply bilobed apex glandular pubescent pink. Column slender arched, arms long curved. Anther 2 celled. Pollinia globose on rery short caudicles with a broad oblong dise. Th. alata, Par. and Rehb. fil. Trans. Linn. Soc. XXX. 135. Cymbidium alatum, Roxb. Fl Brit. Ind. III. 459.

Penang: Government Hill (Curtis); Perak: Waterloo Estate (Curtis 2308, 2309). Distrib. Borneo, T'enasserim.

3. Tr. secuxda, Ridl. Journ. Linn. Soc. Bot. XXXI, p. 299. Psendolutbs ovoid ol, long $1 \frac{1}{2}$ inch long 1 inch through. Leaf lanceo* late subacute, 8 inches long $1 \frac{1}{2}$ inch broad. Raceme 3 inches long pendulous. Flowers about 10, secund, an inch across. Upper sepal boat-shaped curved acute green, laterals broadly ovate acuminate light green with a median red stripe. Petals linear lanceolate acuminate acute, green with 3 red stripes. Lip tube fairly long, side lobes arehed monded. olive green, with two curved processes between hate ovate white with a yellow spot edged crimson in the centre and a purple spot near the tip. Column dark maroon long, arms long curved. Anther conic with a broad base, pollinia 2 fusitorm with a single caudicle tapering to the apex, dise small.

Perak: Taiping (Ridley), Tapa (Aeria), (Scortechini drawing 80). Waterloo (Curtis). Distrib. Borneo.

4. Tir. Mangayi, Hook. fil. Fl. Brit. Ind. VI. 20. Ic. Pl. 2118. Pseudobulls ovate oblong ribbed $\frac{2}{3}$ to 1 inch long. Leaf elliptic or olsorate oblong $3-4$ inches long, $1-1 \frac{1}{4}$ inch wide narrowed into a petiole. Raceme 1-, inches long, flower's seattered an inch across. Tpper sepal lancerlate acutr, laterails much broader orate acuminate. l'etals lancenlate arcuminate narrower. Lip small velvety, tube long, claw wide and half as lone, side lobes large curved with two curved elubbed processes between, midlobe small ovate. Column tall curved. Pollinia $t$ on a long narrow strap dilated upwards, npex glandular.

Malacca (Maingay). Only known from Maingay's description and specimen. It is clearly near secunda but has a much smaller lip, with larger side lobes in proportion to the midlohe and 4 pollinia. 
5. Tit. Qtinquefida, Hook. fil. Fl. Brit. Ind. VI, 20. Ic. Pl. 2119. Pseudobullss ovate oblong 1-1 $\frac{1}{2}$ inch long. I Leaf linear elongate narrowed to a petiole, $5-i$ inches long 1-1 $\frac{1}{2}$ inch wide. Raceme 2-3 inches long decurved. Bracts small ovate. Flowers $\frac{2}{3}$ inch across. Upper sepal lanceolate boat-shaped, laterals ovate acute. Petals linear lanceolate. Lip pubescent with 2 erect linear auricles at the base, side lobes large curved obtuse, midlobe ovate.

Malacca (Maingay).

\section{Podocinlus, Bl.}

Epiphytic or rupestrial herbs, stems usually short tufted not swollen terete or flattened, leafy. Leaves distichous, oblong, lanceolate or linear, articulated (in Malayan species). Racemes lateral or subterminal, short flowers usually very few small, white or green rarely pink. Upper sepal lanceolate, laterals broader prolonged into a mentum. Petals smaller. Lip little longer base saccate adnate to the column foot, usually entire, side lobes absent, with or without a callus on the disc or at the base of the sac. Column short and thick with a foot, arms absent or very obscure. Anther ovate beaked, pollinia 4 to 8 clubbed, narrowed to a long or short caudicle or pair of caudicles, or caudicles absent, disc usually one rarely two. Rostellum ovate or lanceolate erect acute, entire or bificl. Capsule small elliptic.

\section{Species $4 \%$ Indian, Malayan.}

I have followed Sehlechter in amalgamating Appendicula with Podochilus, which has long been obviously necessary.

§ 1. Apista. Very slender mossy plants with small leaves and minute flowers.

Stems creeping.

Lip sagittate at base $\quad . \quad \ldots \quad \ldots \quad$ 1. P. tenuis,

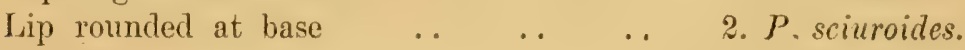

Stems tufted ascending.. $\quad \ldots \quad \ldots \quad$.. $\quad$ 3. P. lucescens.

§ 2. Appendicula. Erect usually stout plants, leaves broad or narrow long.

Stems flattened winged..$\quad \ldots \quad \ldots \quad \ldots \quad 4$. P. anceps.

Stems terete. Bracts small.

Leaves narrow linear . . $\quad \ldots \quad \ldots \quad$ 5. P. mipestris.

Leaves broad.

Stems long pendulous. Lip spathulate .. 6. P. unciferus. Stems erect stiff.

Lip with a callus on the disc. Flowers glabrous. 


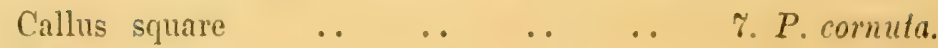

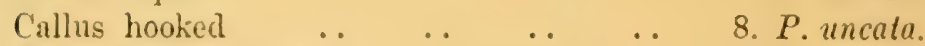

Callus conic. Leaves thick shining .. 9. P. lucida.

Leaves oblong, close _. . . . . 10. P. densifolia.

Flowers hairy _. $\quad . \quad \ldots \quad \ldots \quad 11$. P. muricata.

Lip with no callus.

Stem short stout, leaves oblong.. .. .. 12. P. refiexa. Stem tall.

Lip oblong pandurate ... $\quad \ldots \quad$.. $\quad$ 13. P. pendula.

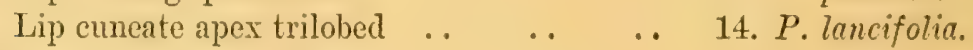

Inflorescence a terminal head.

Bracts larger than flowers distichous $\ldots$ 15. P. torta.

Bracts numerous forming a tuft.

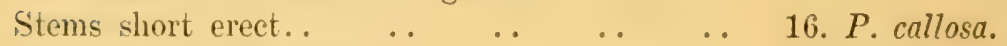

Stems very long, pendulous ... $\quad \ldots \quad \ldots \quad$ 1\%. P. Hasselti.

\section{\&1. APISTA.}

1. P. TExurs, Lindl. Orch. Pl. p. 235. A slender tufted moss like plant, stems often brancherd 3-12 inches long. Leaves linear acute $\frac{1}{8}$ inch long sheaths shorter. Racemes subterminal very short flowers minute white. Upper sepal ovate obtuse, laterals larger oblique, mentum. Petals oblong ligulate obtuse. Lip oblong obtuse concave. Column short foot long. Anther orate cordate. Pollinia lanceolate compresised attatedred to two separate dises, restelun 3 lobed. Miq. Fl. Ind. Bot. III. wis. Schlechter Monographie der Podochilinae p. 1\%. $P$. acicularis, Hook, fil. Fl. Brit. Ind. VI. p. 82. Ic. Pl. $21+1 \%$

Pahang: Tahan River (Ridley) ; Selangor: Pahang Track (Ridley 846 (63); Perak: Sungei Rayah (Kunstler 982), Larut Hills (Ridley), (11 ray 2916); Bujong Malacen (Ridley 982.), (Scortechini 1298); Redah: Gunong Jerai (Ridley); I'enang (Maingay), (Ridley). Distrib. Java. On wet mossy trees and rocks.

2. P. scrurones, Rehb. fil. Bonplandia V. p. 41. A slender much branched plant, stems 4 to s inches long tufted and often forming large mats roots thicker than the stem. Leares oblong elliptic f inch long mucronate. Racemes or panicles subterminal hardly $\frac{f}{4}$ inch long, slender. Flower's very small white. Bracts ovate half as long as the ovary. Sepals ovate, laterals oblique, mentum bilobed broad bunt. Petals nearly as long and broader very blunt, with a median violet line. Lip oblong apex blunt decurved, no calli. Column short. Anther lanceolate acute, pollinia 6 elongate pyriform, caudicle solitary, disc oroid minute red. Iostellum triangrilar lanceolate minutely 
bifid. Schlechter I.c. 21. P. microphyllus, Hook. fil. T1. Brit. Ind. VI. 81. Miq. Fl. Ind. Bat. III. 688. (not Lindley). Cryptoglottis serpyllifolia, Rehb. fil. l.c. p. 41. not of Blume.

Singapore: Chan Chu Kang (Ridley), Kranji (Ridley 362); Jolor: Bukit Pengarani, Gunong Pulai (Ridley); Pahang: Pekan River (Ridley 23 22); Malacea: Mount Ophir (Ridley); Perak: Bujong Malacea (Curtis 312t) ; Penang: Bukit Laksamana (Curtis 1733); Kedah : Gunong Jerai (Ridley). Distrib. Java. Common on mossy trees.

3. P. Lucescens, Bl. Bijdr. 295. t. 12. Stems tufted 6 inches or more tall erect unbranched. Leaves narrow elliptic $\frac{3}{8}$ inch long $\frac{1}{8}$ inch wide apex unequally bilobed obtuse. Racemes subterminal $\frac{1}{2}$ inch long slender. Bracts subdistichous ovate acute. Flowers minute white. Sepals and petals lanceolate subobtuse, mentum very short. Lip distinctly 3 lobed, side lobes broad rounded, median linear oblong with three raised ribs on the disc. Column very short arms elongate acuminate. Anther orate obtuse, pollinia 4 elongate pyriform with a calyptrate dise. Rostellum 3 lobed median lobes longest, acuminate. Lindl. Orch. Pl. p. 234. Miq. Fl. Ind. Bat. III. 68\%. Hook. fil. Fl. Brit. Ind. VI. 82. Schlechter l.c. p. 1\%.

Kedah: Gunong Raya (Curtis 2559); Penang Waterfall (Curtis). Distrib. Tenasserim, Java, Borneo.

4. P. Axceps, Schl. l.c. 33. Stems 6 inches to 18 inches flat pale green. Leaves lanceolate or elliptic base rounded blunt unequally bifid light green $1 \frac{1}{3}-2$ inches long $\frac{1}{2}-\frac{3}{4}$ inch wide, sheaths $1-1$ inch long flattened. Racemes lateral or terminal half inch long. Bracts lanceolate blunt deflexed. Flowers small greenish white. Sepals ovate obtuse, mentum short rounded. Petals similar to the upper sepal but smaller. Lip shorter than sepals rounded entire orate blunt, no callus, white with two purplish streaks in the spur. Column short with a very broad oblong foot, purple above base green. Anther aciniform crimson pustulate acute. Pollinia 6 prriform, disc very small lanceolate red. Rostellum ovate acute bitid. A ppendiruln anceps, Bl. Bijdr. 299. Lindl. Orch. Pl. p. 228. Miq. Fl. Ind. Bat. p. 702. A. Lewisii, Griff. Calc. Journ. Nat. Hist. IV. p. 378. t. 19. Notul. III. p. 360. Ic. Pl. As. t. 33\%. Hook. fil. Fl. Brit. Ind. VI. p. 83. A. complanata, Ridl. Journ. Linn. Soc. XXXI. p. 389. MLetachilum cyathiferum, Lindl. Orch. Pl. p. 74.

Singapore (Wallich 2022); Johor: Gunong Banang, Batu Pahat (Ridley), (iunong Pulai (Ridley 3173); Selangor: (aves, Kwali Lumpur (Rilley 8130), Rawang (Rirley G.90), Bukit Hitam (Kelsall) ; Malacea : Batu 'Tiga (Derry 97\%) ; Perak: Relau, 'Tujor (Wray 4030), (Scortechini), larut Hills (King s coll. 2069); Penang: Tlest Hill (Curtis 993), (Ridley 7242). Distrib. Jara. 
5. P. RUpestris, Schlechter 1.c. p. 56. Stems tufted on a short stnut rhizome slender erect, unbranched 8 inches tall. Leaves linear lanceolate 1 inch long $\frac{1}{8}$ inch wide, sheaths $\frac{1}{4}$ inch long. Racemes subterminal an inch long or less. Bracts lanceolate subacute deflexed. Flowers very small white. I Tpper sepal ovate lanceolate larger, mentum short obtuse. Petals smaller lanceolate. Lip oblong elliptic blunt. Column short, hood long, no arms. Anther rounded cordate hlunt. Appendicula rupestris, Ridley Journ. Linn. Soc. IXXII. 391.

Pahang on rocks in the 'Tahan River (Ridley 2368); Perak: Bujong Malacea (Curtis 3127),

6. P. uxchferus, Hook. fil. Fl. Brit. Ind. VI. p. 81. Ic. Pl. XXII. t. 2145. Stems 2-3 feet long pendulous slender branched. Leaves elliptic blunt 1 inch long $-\frac{1}{2}$ inch across sheaths $\frac{1}{4}$ inch long. Racemes numerous axillary slender filiform an inch long flexuous. Flowers pink scattered $\frac{3}{16}$ inch long. Bracts lanceolate acuminate $\frac{1}{8}$ inch long. Lpper sepal lanceolate subacute, laterals much broader orate, mentum long clubbed $\frac{1}{8}$ inch long. Petals shorter oblong lanccolate orate acute, all whitish tipped violet rose. Lip base linear spathulate truncate, entire white tipped violet rose, callus at hase of lip longer strap shaped retuse. Column short, arms very small. Anther long beaked. Pollinia 6 in two bundles each enclosed in a calyptrate pedicel, dise orbieular. liostellum very short bifid. Capsule $\frac{t}{4}$ inch long fusiform. Ridley Journ. Linn. Soc. XXXII. 385. $P$. longicalcarata, holfe. Kew Bulletin 189t. 186. P. undulatus, Schlechter l.c. 4\%. Appendicula purpurascens, De Vriese Illustr. t. 12. f. 1. (not of Blume).

Pahang: Kota Glanggi (Ridley 2369); Perak: Larut Hills $2-5000$ feet (Curtis 206i2), (IV. Fox), (Ridley 10626). 1)istrib. Borneo, Java, Sumatra, Philippines.

7. P. consut, Schlechter 1.c. 35. Stems tufted terete 6-12 inches tall. Leaves oblong, bilobed nearly equally, base half amplexicaul, $3-1$ inch long 3 inch wide, sheaths terete striate $\frac{1}{4}$ inch long. liacemes subterminal and lateral inch long. Bracts orate longer than the ovary. Flowers white 1 inch long. Sepals ovate, laterals obligue mentum as long. Petals ovate clliptic or obovate smaller. hip base oblong with an innex horse-shoe shaped lamina, hardly 3 lolbed, ferminal lobe ovate deflexed or nearly lanceolate with a square callus in the centre. Column short foot long with long erect acuminate arms. Pollinia 6 to 8 elongate pyriform with a small red lanceolate dise. Rostellum ovate atcminate bifid. Capsule oblong A inch long. Appendicula cornuta, B1. Bijalr. p. 302. Lindl. Orch. Pl. 229. Mi.. Fl. Ind. Bat. II I. 303. .1. bifarii, I indl. Hook. Kew Journ. VIL. 3j. Benth. Fl. Hongt. 3js. Walp. Ann. VI. 393. Hook, 
fil. Fl. Brit. Ind. VI. p. 82. Ridl. Journ. Linn. Soc. XXXII. $38 \%$. King. Ann. Roy. Bot. Cale. VIII. 248. Pl. 330. A. reduplicata, Rchb. fil. and Par. Ot. Hamb. p. 45. A. manillense, Rchb. fil. Miq. 1.c. p. ¡63. Dendrobium manillense, Schau. Nat. Act. Acad. Cur. XIX. Suppl. p. 430 .

Singapore: Kranji, Bukit Timah (Ridley 373) ; Malacca: Mount Ophir (Ridley); Selangor: Bukit Hitam (Kelsall), Gap (Curtis); Pahang: Tahan River (Ridley 2370); Perak: Dindings, Lumut (Ridley 6244 ); Taiping (Scortechini); Penang: Government Hill Kedah: Gunong Raya (Curtis), Gunong Jerai (Ridley). Distrib. Rhio, Sumatra, Java, Borneo, Philippines, Hongkong, India.

8. P. uxcata, Ridl. Stems over a foot tall tufted rather stout. Leaves lanceolate acute $2-3$ inches long rather thin $\frac{1}{2}$ inch wide sheaths $\frac{1}{2}$ inch long ribbed. Racemes lateral or subterminal $\frac{1}{3}$ inch long rachis thick. Bracts ovate strongly ribbed reflexed. Flowers white Sepals ovate acute, mentum rather large blunt. Petals ovate lanceolate smaller. Lip oblong with a rounded apex, a claw shaped callus on the disc. Column thick short. Anther elongate lanceolate acute. Thostellum rather long triangular bifid. Capsule oblong $\frac{1}{2}$ inch long. A ppendicula uncata, Ridl. Joum. Linn. Soc. XXXII. 390.

Singapore: Bukit 'l'imah Road (Ridley 6931); Selangol: Petaling (Ridley).

9. P. Lucida, Schlechter l.c. 3\%. Stems crowded 6 inches long, leares cordate ovate amplexicaul unequally bifid polished bright green $\frac{3}{4}$ inch long $\frac{1}{2}$ inch wide, sheath terete $\frac{1}{4}$ inch long. liowers whitish green in short axillary panicles. Bracts small ovate brown. Sepals ovate blunt, mentum thick short. Petals lanceolate smaller all green with a pale purple median line. Lip base oblong apex ovate orbicular white with a conic callus in the centre. Column thick, arms hardly distinct. Anther ovate beaked truncate. Pollinia 6 narrowed to the ovate dise. Rostellum long bifid. Appendicula Tucida, Ridl. Journ. Linn. Soe. XXXII. p. 392.

Singapore: Bukit Timah, Kranji, Chan Chu Kang (Ridley); Selangor: Sepang (Ridley). Distrib. Rhio, Borneo.

10. P. Denstfoli, Ridley Journ. Roy. As. Soe. S. Br. Tol. XXXIX. p. 86. Stems over a foot long, covered with close distichous leaves, oblong obtuse bases broad an inch long : inch broad, sheaths

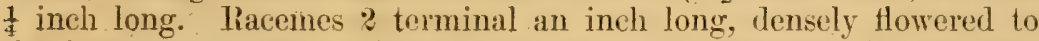
the base, rachis stout, bracts orate reflexed. Flowers $\frac{1}{6}$ inch long white. Sepals ovate obtuse mentum rather long. Petals ovate but little smaller. Lip ovate acute fleshy an irregular fleshy callus in the centre with a thickened ridge running to the tip. Column short. Rostellum long decply bifid acuminate. Anther lancelate subacute. 
Pahang: Tahan River (Ridley 23\%0). This has the raceme of nne of the pendule section but the column with a long rostellum and the callus of the lip is rather that of $P$. cormuta.

11. P. arcricita, Schlechter ].c. 6t. Stems tufted 6 inches tall slender terete. Leares oblong obtuse rounched i inch long and half as wide, sheaths very short. Flowers lew terminal white. Sepals lanceolate acute covered as is the ovary with hairs, mentum very short hardly distinguishable. Petals lanceolate narower. Lip violet oblong rlibated romeded at the tip with a short conical tooth. Column stout arms long and broad acuminate. Stigma crimson. Anther with a long green beak. Pollinia 4 nearly equal lanceolate fixed to a spoon-shaped pedicel, disc elliptic. Rostellum long acuminate bifid. Capsule $\frac{1}{4}$ inch long elliptic hairy. Appendicula muricala, Teysm. and Binn. 'Tijdschr. Ned. Ind. V. XXIV. 322. Ridley Journ. Linn. Soc. XXXII. p. 392. A. cehinocarpa, Hook. fil. Fl. Brit. Ind. VI. 83. Ic. Pl. t. 2152.

Perak (Scortechini), Bujong Malacea (Ridley), Gunong Batu Tutih ( ITay 968); Kedah: (xunong .Jerai (liclley j112); Schangor: Bukit Hitam (Ridley).

12. P. REFLExA, Schlechter 1.c. p. 31. Stems rather stout over a foot long. Leaves close thinly coriaceous oblong elliptic an inch fong half ineh across dark shining green equally bilobed, sheaths nearly half inch long. liacemes axillary half inch long with a few lanceolate acuminate bracts at the base. Hlowers erowded very small. bracts lanceolate obtuse. Upper sepal narrow lanceolate ovate obtuse, laterals much larger ovate, mentum as long rounded. Petals smaller linear all light green. Lip white, sides adnate to columm base with a horse-shoe shaped ridge at the base an orate acute rexurved blade. Column short. Anther pyriform beaked. Pollinia 6 pear shaped on a small brown dise. Arms of column very short. Capsule sessile oblong $\frac{1}{4}$ inch long. Appenticula reflexa, Bl. Bijdr. 301. Lindl. Oreh. Pl. 229. Miq. Fl. Ind. Bat. HI. 803. Ridl. Journ. Limn. Soc. XXXI. 390. A. cordalu, Hook. fil. Fl. Brit. Ind. VI. 83. Ic. Pl. t. $21+8$.

Johor: Batu Palsat (Ridley) ; Pahang: 'Tahan River (Ridley); Perak (Scortechini). Distrib. Java.

13. P. PExde LA, Schlechter 1.c. 48. Stems erect 1-2! feet tall. Leaves lanceolate thin $2-2 \frac{1}{2}$ inches long $1-\frac{1}{2}$ inch wide, apex very unequally bilobed acute, sheaths joch long. Racenes axillary or subterminal pendulous 1-8 inches Jong. Bracts ovate reflexed persistent. flower's small. Upper sepal banceolate laterals ovate mentum short and broad. Petals narrow lanceolate, all pale green. Lip oblong pandurate blumt, with an imner semilunar lamella, white elged purfle, landla purple. Colmun short and thick jellowish marticed purple 
in front. Anther orate orange. Pollinia 8 very unequal on a small rounded dise. Column arms rery short. Rostellum shortly bifid. Appendicula pendula, Bl. Bijdr. 298. Lindl. Gen. and Sp. Orch. Pl. p. 228. Miø. Fl. Ind. Bat. III. r01. A. Chalmersiana, Fv. Muell. Wing. South Science Record 1885. A. Maingayi, Hook. fil. Fl. Brit. Ind. VI. 85. Ic. Pl. 2151. Conochilus distichus, Hassk. Tijdsch. Nat. Ges. IX. p. 146.

Perak (Scortechini) ; Penang (Maingay 161\%), Government Hill (Curtis 2822), (Ridley 6243). Distrib. Java, Borneo, Philippines, New Guinea.

14. P. LANcrioura, Schlechter 1.c. 51: Stems tall over two feet sometimes branched. Leaves thin dark green $1 \frac{1}{2}-1$ inches long $\frac{1}{4}$ to 1 inch wide, apex subacute, sheaths $\frac{1}{4}-\frac{1}{2}$ inch long. Racemes terminal 2-3 inches long. Bracts orate lanceolate reflexed. Flowers small $\frac{1}{8}$ inch long green or yellow. Sepals orate acute mentum short broad. Petals obovate oblong blunt. Lip free cuneate oblong, apex 3 lobed lobes rounded short midlobe ovate subacuminate, a curred horse-shoe shaped lamina running as far as the side lobes. Column short, foot long. Rostellum triangular bifid. Column arms short truncate. Appendicula lancifolia, Hook. fil. Fl. Brit. Ind. VI. 84. Ic. Pl. XXII. 2150.

Selangor: Bukit Hitam (Ridley); Perak: Gunong Batu Putih (King's Coll. 8032), Larut Hills 5000 alt. (Ridley 2886). This is very near $P$. pendula Schl.

15. P. тоRтA, Schlechter 1.c. 60. Stems tufted 6-8 inches long, flattened, seldom branched. Leaves oblong rounded $\frac{1}{2}$ inch long wide, sheaths $\frac{1}{8}$ inch long. Spike terminal $\frac{1}{4}$ inch long. Bracts distichous ovate white leaf-like $\frac{1}{4}$ inch long recurved. Flowers hidden in the bracts yellowish white small. Sepals lanceolate acute, laterals very oblique acuminate, mentum long parallel to the column. Petals lanceolate subacute broader. Lip base oblong apex broadly and orbicularly bilobed, lobes rounded. C'olumn short arms rounded foot long: Anther orate acuminate crimson. Pollinia $t$ connate into a caudicle with an oblong disc. Appendicula torta, Bl. Bijdr. p. 303. Lindl. Gen. and Sp. Orch. Pl. 230. Miq. Fl. Ind. Bat. III. 20t. Rehb. fil. Xen. Orch. 11. 11\%. t. 138. Hook. fil. Fl. Brit. Ind. VI. p. 8t. A. rhodiola, Rchb. fil. Xenia. II. p. 118. t. 138. A. Peyeriana, Kranzl. Gard. Chron. X. 1891. p. 669.

Selangor: Rawang, Kwala Lumpur Caves (Ridley); Perak (Scortechini); Larut Hills (Ridley). Distrib. Java, Borneo.

16. P. Callosa, Schlechter 1.c. 62. Stems tufted 6-18 inches long terete. Leaves distichous twisted at the base so that the one edge is uppermost oblong obtuse, unequally bilobed, $\frac{1}{4}$ inch long $\frac{1}{8}$ inch 
wide sheaths hardly i inch long truncate the angles prolonged into setaceous points. Capitulum terminal of numerous brown crowded bracts. Flowers small white. Sepals orate lanceolate acute mentum rather long obtuse. Petals narrow linear. Iip base oblong rounded, limb orate orbicular with a yellow transversely oblong callus at its base. Column stout, foot long, arms ereet broad ineurved pink. Anther ovate cordate. Pollinia 8 narrow clubbed attached to a cireular dise. Rostellum long nbtuse entire. Capsule ressile eylindrie $\frac{1}{4}$ inch long. A ppendicula callosa, B1. Bijdr. p. 303. Lindl. Orch. Pl. p. 230. Miq. Fl. Ind. Bat. III. ‘0). Griff. Cale. Journ. Nat. Hist. V. p. 362. t. 62. Rehb. fil. Ot. Hamb. p. 45. Hook. fil. Fl. Brit. Ind. TI. 83. Ridl. Journ. Linn. Soc. XXXII. 388. A. stipulata, Grift. Sotul. III. p. 358. Ic. Pl. As. t. 335. fig. II.

singapore: Sumbawang, Bukit 'limah (Ridley); Johor: Kwala Kahang (Kelsall), (Ridley 4039); Pahang: 'Tahan River (Ridley); Malacea, Mount Ophir (Ridley); sungei Ljong; Perak: Larut Hills (Ridley), (Scortechini): Penang: Gorermment Hill (Curtis 358). Distrib. Tenasserim, Sumatra, Java, Borneo, Celebes. The commonest species growing on rocks or trees.

1\%. P. Hasselti, Schlechter l.c. 62 . Stems three feet long pendulons flattencel. Lraves oblong obtuse base broad apices rounded bilobed, is inch long and hall as wide, sheaths $\frac{1}{4}$ inch long truncate. Flowers white crowded in a terminal head of many bracts $\frac{1}{2}$ inch Inng. Sepals orate subaeute laterals willer. Petals lorate subobtuse smaller. Lip sigmoid is lohed, margins adnate to rolumn edge, side? Inhes rounder midlobe longer crisped concave, a callus ruming longrtudinally from the base to the midlobe. Column short, foot slender arms long erect. Capsule sessile oblong $\frac{1}{4}$ inch long. Appendicula Hasselti, Bl. Bijdr. p. 304. Lindl. Orch. Pl. 230. Miq. Fl. Ind. Bat. 1I1. 704. A. clongato, Rid1. Trans. Iinn. Soc. II. 375. Jяบล.

Pahang on trees over the Tahan River (Ridley 2371). Distrib.

\section{1. 'THelasis, Bl.}

Epiphytes with subghoboce pecurbobulbs on at shorter or long rhizome. Leaves one or two, the lower one being more or less developed, ligulate, fleshy, obtuse. Scape from the base of the pseudobulb bearing a short or long raceme or spike. Flowers very small greeni-le pellow, perianth comnivent. Sepals lancentates petals slightly smaller. Jip ovate lanceolate entire with no callus. Column short rostellum long acuminate bifid. Anther lanceolate. Pollinia 4 to 6 globose on a slender caudicle. Capsule small fusiform.

Species 6. Indian and Malayan.

Raceme short erowded.

Pseudobulbs crowded, rhizome short ..

1. Th. elongata. 
Pseudobulbs distant, rhizome long stout Raceme long and lax.. .. . . .
2. Th. capitata.

3. Th. macrobulbon.

1. Th. elongata, Bl. Oreh. Arch. Ind. p. 23. Pl. t. 7. fig. 2. Pseudobulbs oroid depressed $\frac{1}{2}$ inch long on a short rhizome. Leaf ligulate blunt narrowed at the base $1 \frac{1}{2}-3$ inches long $\frac{1}{4}-\frac{1}{2}$ inch wide. Scape $3-4$ inches long racemose portion $\frac{1}{2}-3$ inches long, covered with reflexed ovate acuminate persistent bracts. Flowers $\frac{3}{16}$ inch long greenish yellow. Sepals oblong acute keeled. Petals thinner and shorter spathulate. Lip as long, ovate lanceolate. Column short. Rostellum lanceolate bifid. Capsule very small oblong. Miq. Fl. Ind. Bat. III. 701. Hook. fil. Fl. Brit. Ind. VI. 86. Schlechter Monogr. Podochil. p. 80. Th. Lriptera, Rchb. fil. Bonplandia III. 219. Th. Zollingeri, Rehb. fil. 1.c. V. 3\% Th. hongkongensis, Rolfe. Kew Bull. 1896. 199.

Singapore: Kranji (Ridley 1.3) ; Johor: L'u Batu Pahat (Kelsall); Pahang: Pekan (Ridley); Perak (Scortechini); Lankawi (Ridley). Distrib. Malay Islands as far as the Philippines, Hongkong and Admiralty Islands.

2. Th. Capitata, Bl. Bijdr. 386. Orch. Arch. Ind. 20. t. \%. fig. 1. Rhizome very stout, pseudobulbs rather distant nearly an inch long, oroid globose. Leaf 4 inches long $\frac{1}{2}$ inch wide, lorate ligulate blunt. Scape fairly stout 6 inches long with a sheath $\frac{1}{4}$ inch long in the middle raceme stout 1 inch long. - Bracts ovate acute. Flowers green $\frac{3}{16}$ inch long. Sepals lanceolate acute keeled. Petals similar blunt. Lip orate lanceolate concave. Lindl. Orch. Pl. 253. Miq. Fl. Ind. Bat. 700. Hook, fil. Fl. Brit. Ind. VI. 86. Schlechter l.c. 69. Th. ochreata, Lindl. Journ. Linn. Soc. III. 63. Th. elongata, var. major, Ridl. Journ. Linn. Soc. XXXII. 393.

Pahang: Tahan River (Ridley); Selangor: Kwala Lumpur Caves (Ridley); Perak (Scortechini). Distrib. Sumatra, Java, Borneo.

I doubt very much that this is more than a form of $T h$. elongata Bl. except for the stout long rhizome, larger leaf and thicker scape, with a rather shorter raceme, the plants are quite similar. It occurs in dense wet woods, while $T h$. elongata frequents more open lighter spots.

3. Th. yacrobulbon, Ridl. Journ. Linn. Soc. XXXII. 393. Roots copious and slender, pseudobulbs flask shaped of two internodes, the lower one globose the upper cylindric, an inch long. Leaves two lower one an inch long $\frac{1}{4}$ inch wide, the upper one 4-6 inches long 1 inch wide lanceolate or oblong petioled. Scape 6-10 inches long, lower half nude except for a few acuminate sheaths raceme lax many flowered. Flowers sessile green. Bracts $\frac{1}{x}$ inch long lanceolate cuspidate. Sepals oblong obtuse not keeled. Petals smaller. Lip fleshy shorter 
lanceolate subacute. Anther lanceolate, pollinia 4. Rostellum ovate acuminate larger deeply bifid.

\section{Perak: Larut Hills and Bujong Malacea (Ridley).}

\section{Oxinanthera, Brngn.}

Epiphytes, stemless. Leares distichous ligulate thin textured apices bifid. Scapes axillary about as long as the leaves. Flowers small racemose, periant connivent green or yellow. Sepals and petals lanceolate. Lip lanceolate nearly entire about as long as petals base concave, no callus. Column rery short, no foot, arms indistinct. Rostellum much longer than column, deeply bifid. Anther acuminate two celled. Pollinia 6-8 globular on a very slender caudicle. Capsule fusiform.

$\begin{array}{llll}\text { Raceme elongate straight } & \ldots & \ldots & \text { 1. O. elata. } \\ \text { Raceme short decurved .. } & \ldots & \ldots & \text { 2. O. micrantha. }\end{array}$

1. O. ELATA, Hook. fil. Ic. Pl. 2156. Leaves lorate obtuse -12 inches long 1 inch wide, articulated sheathing base two inches long. siape slender a foot long base nude racemose portinn $2-4$ inches long. Flowers numerous. Bracts lanceolate $\frac{1}{8}$ inch long ovary and pedicel $\frac{1}{4}$ inch long. Sepals lanceolate connivent at base, acute. Petals smaller, all green at the base tips white. Lip as long as the petals side lobes elevated green, midlobe oblanceolate mucronate white. Column rery short green no arms. Rostellum much longer acuminate deeply bifid, white. Anther lanceolate yellow. Pollinia 6 semiglobose, on a long slender pedicel. Capsule $\frac{1}{8}$ inch long fusiform terminated by the remains of the perianth. $O$. carinata, Schlechter l.e. p. ז.. Thelasis carinata, Bl. Bijdr. 386. Orehideae Arch. Ind. p. 25. Pl. \% fig. 3. Lindl. Orch. Pl. p. 253. Th. elata, Hook. fil. Fl. Brit. Ind. VI. p. $8 \%$

Singapore: Sungei Puluh, Kranji (Ridley) ; Pahang near Pekan (Ridley), Kota (Hanggi (Ridley); S'elangor: Kwala Lumpur Caves (lidley 8465) ; Perak: (ioping (King's Coll. 82.13), Hermitage (Cock, Curtis 2852); Penang: Corernment Hill (Maingay). Distrib. Java, Borneo, Sumatra.

Species 2. Malayan and Pacific.

2. O. muchaтtia, Brngn. Duperry Voy. Bot. p. 198, t. 38 B. Leaves 6 inches long 3 inch wide lorate obtuxe, sheathing portion 1-1 th inch long. Seape very slender 3-t inches long fawn colour, with one or two distant sheaths below racemose portion balf an inch long or less recurved. Flowers $\frac{1}{8}$ inch long fawn colour. Bracts ovate obtuse. Sepals and petals lancenlate ohtuses. whitish with fawn coloured bases. Lip lanceolate acute fleshy with thinner edges. Column very short. Anther ovate acmminate. Pollinia 8 globose 
with a long slender candicle. Rostellum very long deeply bifid. Capsule $\frac{1}{8}$ inch long fusiform. Schlechter l.e. $7 \%$ O. decurva, Hook. fil. Ic. Pl. 215\%. Thelasis contracta, Bl. MIus. Bot. Lugd. Bat. II. 18\%. Orch. Arch. Ind. 24. t. 5 B. Th. decurva, Hook. fil. Fl. Brit. Ind. VI. $8 \%$.

Singapore: Kranji (Ridley 16t0, 36\%); Pahang: Tahan River (Ridley); Penang: Government Hill (Maingay). Distrib. Sumatra, Java, Rhio, Borneo, Pulau Condor, Waigiou. Common in mangrove swamps.

\section{Neottieat.}

\section{VANILLi, Sw.}

Climbing shrubs with fleshy ovate leaves or none. Racemes short stout axillary. Flowers large and showy. Sepals and petals subequal spreading. Lip large three lobed or subentire adnate to column base, with the sides wrapping the column or explanate. Column long. Anther broad incurved. Pollinia granular. Rostellum broad entire or nearly so. Capsule long fleshy terete. Seeds minute globose.

Species about 20. All tropical cointries.

V. Griffithil, Rehb. fil. Bonplandia II. 88. Stem about 20 feet long. Leaves fleshy lanceolate to ovate lanceolate 6 inches long, 3 inches wide or less. Raceme erentually 2 inches long thick. Bracts ovate. Flowers numerous opening singly over 2 inches across. Sepals orate. Petals linear obovate, greenish white. Lip explanate three lobed side lobes rounded white, dise channelled with chestnut red raised nerves, midlobe broadly bilobed lobes rounded fan-shaped plicate white with a white woolly ball at the base. Column arched white. Anther broad truncate. Fruit $2 \frac{1}{2}$ inches long $\frac{1}{2}$ inch through succulent eatable. T'. albida, Hook. fl. Fl. Brit. Ind. VI. 91. (Not Blume) V. sp. Griff. Notulae III. 20\%. Ic. Pl. As. t. 281. V. tolypephora, Ridl.. 'Trans. Linn. Soc. III. 376.

Singapore: Changi (Ridley 3924), Pulau Lbin (Ridley); Johor: Batu Paliat; Pahang: Pekan (Ridley); Nalacea: Panchor (Goodenough 1520, 1969); Selangor: Bukit Hitam (Kelsall); Perak: Larut Hills (Stephens), (King's Coll. 2537, 2996, 7528); Dindings: Bruas (Ridley), (Scortechini); Penang Waterfall (Curtis 11:9, 22:1), Telok Bahang (Curtis), (Wallich 7401). Distrib. Karimun Isles. Native names, "Akar Punubal," "Telinah Kerbau Bukit." 'The flowers pounded with water are used to rub on the body for fever, the leaves are mashed and applied to the hair for strengthening it. The fruits are sweet and catable. The stem and leaves contain a whitish irritating latex. 


\section{GALEOTA.}

Terrestrial leafless saprophytes with thick fleshy roots. Stems short or very long yellow branched. Panicles large spreading or a simple raceme with persistent orate bracts. Flowers moderately large. Lateral sepals larger than upper one. Lip saceate entire. Column long curvel or nearly straight almost or quite footless. Anther oblong decurved, with very shallow cells, and a rather long filament. Pollinia 2, ovoid curved no caudicle. Rostellum very short. Fruit capsular long. Seeds minute globular with a broad circular wing.

Species about it. India, Malaya, Australian and Japanese.

\section{Stem stout furfuraceous .. .. . 1. G. Hydra.}

Stem slender glabrous, lip with a woolly callus 2. G. altissima.

1. G. Hypra, Rehb. fil. Xenia. Orch. II. $7 \%$ Stems long and stout many feet long yellow branched furfuraceous. Sheaths ovate $\frac{1}{2}$ inch long. Inflorescence panicled branches 8 inches long. Bracts ${ }_{8}^{2}$ inch long. Flowers $\frac{3}{4}$ inch across. Sepals ovate acute, laterals oblique larger yellow. Petals lanceolate oblipue white. Lip obscurely 3 lobed denticulate, papillose within yellowish white with dull red streaks. Column stout narrowed upwards curved, with a very short foot, yellowish. Anther oblong yellow streaked red. Fruit eylindric stout 6 inches long $\frac{1}{2}-1$ inch through. Hook. fil. Fl. Brit. Ind. VI. 89. King. Ann. Bot. Gard. Cale. VIII. 36t. Pl. 351. Vanilla pterosperma, Lindl. Wall. Cat. 7402 . V. rubiginosa, Grifl. Notulae III. 24\% Erythrorchis altissima, Lindl. Gen. and Sp. Orch. 438. (partly).

Singapore ( Wallich $\% 402$ ), (Hullett 1\%), Kranji (Ridley 1791, 4037 ) : Malacea: 'Tabong (Grillith). Bukit Besar Ophir (Rirlley); Selanger: Kwala Lumpur ('aves (Rilley 8458), (Curtis 351, 2390). Kajang (Ridley s198); Johor: (funong Janing (Kelsall); Perak (Seortechini), Larut Hills at 5000 feet (Curtis 2060), (King's Coll. 26.5i), Waterloo (Ridley 2859); Penang (Curtis). Distrib. Himalaya, T'enasserim, Siam, Java and Sumatra.

2. G. Altissiana, Rehb. fil. Xen. Oreh. II. 7\%. Stem long yellow very slender 30 to 40 feet long or more. Panicle large, branches slender 8 inches long. Bracts ovate small. Flowers half an inch across yellow and brown. Sepals and petals linear oblong blunt. Lip boat-shaped, apex rounded erose waved, yellow barred purple inside, with a broad fleshy pubescent central bar terminating in a bilobed woolly mass. Column rather long. Capsule narrow linear 8-10 inches long. Hook. fil. Fl. Brit. Ind. VI. 89. Cyrtosia altissima, B3. Bijdr. 396. Rumph. I. t. 70. Lindl. Gen. and Sp. Orch. Pl. 26. Erytrorchis altissima, Blume. Rumphia. I. 200. Lindl. l.e. 438 (in part). Haematorchis altissima, Bl. 1.c. IV. t. 200. B. 
Johor: Base of Gunong Panti (Ridley 4143), Bukit Murdom (Tielsall) ; Province Wellesley: Bukit Juru (Ridley); Perak (Scortechini) ; Dindings: Lumut (Ridley $725 \%$ ) ; Penang: Telok Bahang (Curtis 352).

\section{Pogonia, Jussieeu.}

\section{(Section Nervilia).}

Tuberous herbs with short stems and a solitary cordate petioled leaf, appearing before the flowers. Racemes solitary or several on the stem, lax. Flowers solitary or few nodding. Perianth connivent. Sepals and petals narrow. Lip entire or three lobed spurless or with a short spur. Column long. Anther helmet shaped.' Pollinia 2 or 4 , without dise or caudicle.

Species of section about 12. Tropies of old world.

$\begin{array}{llll}\text { Stem tall many flowered .. } & \ldots & \ldots & \text { 1. P. fabelliformis. } \\ \text { Stem short, flowers } 1 \text { or } 2 & \ldots & \text {. } & \text { 2. P. puncata. }\end{array}$

1. P. flabelifforars, Lindl. Gen. and Sp. Orch. Pl. 415. Leaf orbicular cordate many-nerved glabrous, 4 to 10 inches across green, margin waved, petiole $4-8$ inches long. Flowering stem 6 to 18 inches tall, lax flowered. Bracts linear acuminate $\frac{1}{2}$ inch long. Flowers pale green 1 inch long, pedicel $\frac{1}{8}$ inch, ovary $\frac{1}{4}$ inch long. Sepals and petals lanceolate acute petals narrower obtuse. Lip 3 lobed about the middle, side lobes obtuse, midlobe ovate acute hairy. Hook. fil. Fl. Brit. Ind. VI. 121. P. Nervilia, Bl. Mus. Bot. I. 32. Orch. Arch. Ind. P. crispata, Wight. Ic. t. 1720. Nervilia Aragoana, Gaud. Freye, Vol. 7. Bot. 42\%. t. 35.

Perak: Padang Rengas in long grass (Ridley); Pahang: 'Tembeling (Ridley). Distrib. India, Malaya, Siam. 'The Pahang plant is in leat only but I believe it is this species. 'The leaves were green with a row of black blotches.

2. P. Punctati, Bl. Mus. Bot. Lugd. Bat. I. p. 32. Orch. Archip. Ind. p. 150. t. 49. f. 2 . Base of stem tuberous, one inch long with a few sheaths. Leaf appearing before the leaves ovate or orbicular cordate 1-2 inches loug $1 \frac{1}{2}-2$ inches wide green, undulate. Flowers solitary nodding on peduncles, $2-3$ inches long with close fitting pink spotted sheaths. Bracts shorter than the very short pedicel ovate. Ovary nearly $\frac{1}{2}$ inch long purple. Sepals an inch long narrow linear acuminate pinkish with dark spots. Petals similar. Lip white with violet spots 3 lobed, side lobes short midlobe lanceolate shorter than the sepals. Column tall with broad thin wings tapering upwards, arms winglike ovate acuminate points pointed downwards. Anther small hemispheric pink. Stigma large. Ridley Journ. As. Soc. Straits Branch Vol. 23. p. 146. 
Singaunre: Bukit T'imah, Chua ('hu Kang (Ridley); Penang Waterlall ('urtis); Lankawi: Terutau and Kwah (Curtis 2809).

\section{Corysantines, Br.}

Tery small tuberous herbs, with one orbicular or orate leaf, and one large flower. Dorsal sepal falcate broad laterals and petals filiform or petals absent. Lip large erect with a tubular base dilated into a broad recurved limb. Columm short erect stout with a broad stigma. Anther 2 celled reniform or oroid not beaked. Pollinias 2 elliptic with no caudicle or disc. Rostellum short and broad. Capsule erect elliptic on a long suceulent pedicel.

Species 15. Australian, Malayan and Indian. Lateral sepals and petals filiform long.

Lip broad rounded .. $\quad \ldots \quad \ldots \quad$.. $\quad$ 1. C. picta.

Lip ovate acuminate $\quad . \quad$.. $\quad$.. $\quad$ 2. C. mucronata.

Sepals and petals shorter than lip.. .. 3. C. fornicata.

1. C. PICrA, Lindl. Gen. and Sp. Orch. Pl. 394. Whole plant 3 inches tall. Leaf cordate acute undulate dark green with white reins $\frac{1}{2}$ inch long. Flowers deep red $\frac{1}{2}$ inch long. Upper sepal ('uneate 3 tootherl. I ateral sepals and petals $1 \frac{1}{2}$ inch long very slender. Lip ovate with a tubular base recurved, margin of limb toothed. Column short and broad. Capsule elliptic. Blume Orch. Arch. Ind. 17. t. 64. fi. Hook. fil. Fl. Brit. Ind. VI, 118. Calrearia picta, Bl. Bijdr. t. 33. part.

Pahang: Kluang T'erbang (Barnes); Malacca: Mount Ophir (Ridley); Perak: 'Tambak Batak (Scortechini). Blume's figure in the Orehideate Archipelagi Indici is coloured violet, and looks very different from the peninsular species which may be distinct.

2. C. Mucroxata, B1. Oreh. Areh. Ind. 175. t. 66. C. Whole plant 2 inches tall. Leaf ovate cordate $\frac{1}{2}$ inch long. Flower less than half an inch sessile red with darker spots. Upper sepal entire or nearly so mucronate. Lateral sepals and petals 1 inch long. Lip white with red spots, much smaller than in preceding speeies, hase tubular limb ovate denticulate. Cipsule elliptic $\frac{1}{4}$ inch long. pedicel 3 inches long.

Kedah: Gunong Jerai (Ridley). Distrib. Java.

3. C. Fonncata, Lindl. Gen. and Sp. Orch. Pl. 394. Stem $\frac{1}{2}-2$ inches. Leaf $\frac{1}{2}-\frac{2}{3}$ inch long wate cordate margin not wared. Flowers sessile. Bracts subacute lanceolate. Dorsal sepal ganch long blade spathulate is ribbed rose colonured, lateral sepals and petals shorter than the upper sepal. Lip with a hong ereet tulue produced downwards into two spurs, limb fanshaped with short blackish purple ribs. 
Blume Orch. Arch. Ind. 155. t. 64. fig. 2. Hook. fil. Fl. Brit. Ind. VI. 118. Calcearia fornicata, Bl. Bijdr. 41\%. t. 33.

Perak: Gunong Enal and Tambak Batak (Scortechini). Distrib. Java. These little plants are to be found in wet moss usually at about an altitude of $3-4000$ feet. 'The pedicel of the capsule elongates as it ripens to a considerable length as in Didymoplexis.

\section{6\%. Aphyllorohis, Bl.}

Leafless saprophytes, with long roots. Raceme long lax, flowers mediocre to small white or yellow. Bracts broad deflexed. Sepals and petals subsimilar or the latter, narrower or broader. Lip entire or 3 lobed without calli free from the column. Column slender footless with no wings or arms. Anther eapshaped 2 celled. Pollinia 2 hemispheric or subpyriform with no caudicle or riscus. Rostellum entire.

Species 4 or 5 . Indian and Malayan.

Stem tall. Flowers an inch across .. .. 1. A. striata.

Stem short. Flowers $\frac{1}{2}$ inch wide .. .. 2. A. pallida.

1. A. striata, Ridl. Pogonia striata, Ridl. Trans, Linn. Soc. III. 37\%. Stem abuot 3 feet tall, white with violet streaks stout, sheaths numerous with acuminate points. Bracts deflexed $\frac{1}{3}$ inch long lanceolate. Flowers about 12 pedicel and orary $\frac{1}{2}$ inch long. Sepals half an inch long lanceolate acute white streaked violet. Petals broader ovate white. Lip narrow lanceolate acute white a purple median line. Columm violet, apex and anther yellow. Anther rounded. Pollinia hemispheric. IRostellum elongate triangular. Stigma broad. Capsule pendulous cylindrie 1 inch long.

Pahang: Tahan Woods (Ridley); Johor: Gunong Banang, Batu Pahat (Ridley). Endemic. Allied to A. montuna, Richb. fil. Rare only two specimens found.

2. A. PALLida, Bl. Bijdr. t. 7\%. Mus. Bot. Lugd. Bat. I. p. 30. Orch. Arch. Ind. p. 52. t. 13. Stems solitary 10-18 inches tall slender yellow spotted purple with long stout roots. Sheaths numerous limb bluntly lanceolate. Raceme lax 3-4 inches long, many flowered. Bracts oblong ovate, blunt $\frac{1}{8}-\frac{1}{4}$ inch long. Ovary and pedicel $\frac{1}{2}$ inch long slender yellow dotted purple. Sepals $\frac{1}{+}$ inch long oblong obtuse, laterals deflexed straw colour. Petals narrower lanceolate acute. Lip paler, about as long ovate obtuse side erect. Columm straight stout hooded. Anther conic. Pollinia 2 reniform grooved.

Singapore: Tanglin, Buhit Timah (lidley 812\%); Pahang: Tahan River at Kiwala Tenok (Ridley); Perak: Bujong Malacia (Ridley), Larut Hills at 4300 feet alt. (Fox), Bataug Padang (Wray 
14ii); Penang (King's Collector 4s61). Distrib. Java. In rather dry parts of woods, often on jungle tracks.

\section{Drizaroplizis, Grilt.}

Suculent leafless saprophytes, hizome crindric. Stem short deeply sunk in the ground. Flowers small racemose. Lpper sepal and petals connate apices free, laterals sepals connate a short mentum. Lip inserted on the column foot short broarl entire. Column long two winged, with wo short erect arms. Anther ovate low shortly stipitate. Pollinia reniform. ('apsules elliptic on very long succulent pedicels much longer than the stem.

Species 2-3. Indian, Malayan and Oceanic.

D. PALLENs, Grift. Cale. Journ. Nat. Hist. IV. 383. t. 1\% Rhizome $\frac{1}{2}-3$ inches long. Stem $3-6$ inches long. Racemes few flowernd. Flowers f inch long pinkish or yellowish white. Brats small orate. Upper sepal oblong ovate obtues. Petals shorter lateral sepals united half way, apices blunt. Lip cunéate oblong papillose. C'apsule half to $1 \frac{1}{2}$ inch long, pedicel 6 to 12 inches long. King. Ann. Bot. Gard. Calc. VIII. p. 260. Pl. 346. Hemsley Journ. Linn. Soc. XX. 311. t. 28. Kur\%. Journ, Bot. 1866. 40. Hook. fil. Fl. Brit. Ind. VI. 122. Leucorchis sylvatica, Bl. Mus. Bot. I. 31. Orch. Arch. Ind. $14 \%$. A petalon minulum, Wight. Ic. t. 1758. Arethusa erristata, Grift?. Notul. III. 378. Ic. Pl. Asiat. t. 343. 344. Epiphanes pallens, Rehb. fil. Seem. Fl. Vit. 296 .

Singapore: Bukit Timah, Chan Chu Kang, etc. (Ridley); Johor: (iumong Panti (Rirlley); Perak (Soortechini), (Wray 3.s8s) : Kedah: Gunone Jrai (Ridley) ; Penang: Richmond Pool (Ridley). Distrib. India, Java, Christmas Island.

\section{LECINORCIIS, Bi.}

Lealless saprophyte with wiry black stems, roots elongate fusiform. Raceme tew flowered, rarely branched. Hlowers thin ovary slender terminated by a toothed cup. Sepals and petals subequal. Lip adnate for most of its length to the column as long as the sepals spurless. Columm slender dilated upwards. Anther oblong a celled terminal. Pollinia 2 elliptic gramular with no caudicle.

Species 3. Malayan and Japanese.

L. Malcowsis, Ridley 'Trans. Isinn. Soc. II. iii. 3\%. t. 65. Stems solitary or several 8 inches to nearly 2 leet tall, slender, with a few sheaths often branched. Flowers an inch long pale flesh colour or yellowish white. Sepals lanceolate spathulate. P'etals similar, all comivent. Lip blade spathulate bearded yellow with 2 low keels from the base to the limb. Column slender. Rostellum absent. Anther broad. Capsule long narrow terminated by the cup blact an inch long. 
Singapore: Bukit Timah, Bajau (Ridley) ; Johor: Pengaram, Bukit 'Tenabang (Kelsall); Pahang: Sungei 'Tahan (Ridley); Malacea: Lower slopes of Mount Ophir (Ridley), Bukit Sadanen (Derry 342); Negri Sembilan: Gunong Angsi (Ridley); Kedah: Gunong Jerai (Ridley 3126); Pahang: Government Hill (Ridley). Distril. Borneo, Siam. Not rare in dry woods, very inconspicuous.

\section{\%0. Gastrodia, Br.}

Leafless saprophytes with a cylindric rhizome and a solitary succulent brownish stem. Flower's in lax racemes. Sepals and petals connate into a tube more or less split. Lip short aclnate to the foot of the column and perianth, entire or obscurely 3 lobed. Column usually long 2 winged foot short or 0 arms short erect. Lostellum small. Anther hemispheric. Pollinia in two granular masses.

Species 8. India, Malaya, Japan, China, Australia.

Stem tall, perianth cleft nearly to base ‥ 1. G. Ialayana. Stem short, perianth campanulate .. .. 2. G. Hasselti.

1. G. Malayana, Ridl. Rhizome cylindric 6 inches long ringed, $\frac{1}{2}$ inch through. Sten stout 9 inches tall purplish brown, ringed at the base, with short sheaths with orate blades. Raceme few flowered. Flowers shortly pedicelled, ovary short conicul. Bracts ovate $\frac{1}{4}$ inch long. Upper sepal lanceolate lorate blunt, laterals prolonged into a short mentum, apices pustular purple brown, mentum green. Petals lorate obtuse free to near the base, green and purplish. Lip base narrow linear, margins rolled in, plicate, cuneate subtrilobed, side lobes broad blunt, midlobe narrow straight blunt orange yellow, with a raised central bar. Column semiterete broally grooved, foot long. Anther conical broad. Pollinia mealy. Rostellum flat broad entire. Capsule 1 inch long elliptic, pedicel $\frac{1}{4}$ inch.

Singapore: Bukit 'T'imah, Pulau Damar, Seletar (Ridley); Johor: Sungei Tebrau (Ridley). Grows on rotten wood. Rare and difficult to find, often growing in rotten wood, in dense forests. This plant I took for $G$. javanica, Bl. but it differs in the form of the perianth of which the parts are nearly free to the base, and the lip is more blunt and broader

2. G. Hasseltit, Bl. Mus. Bot. II. 175. Orch: Arch. Ind. 145. t. 52. f. 5-8. A small plant 6 . inches tall. Bracts ovate lanceolate. Flowers $3-1$ broadly campanulate $\frac{1}{2}$ inch long. Sepals and petals all adnate. Lip free, claw narrow, limb suborbicular with apex truncate. Hook. fil. Fl. Brit. Ind. VI. 123.

Perak (Drawing by Seortechini). This is only known as a native of the Peninsula from Scortechini's shetch which certainly resembles Blume's figure. 


\section{Conysis, 'Thouars.}

Tall terrestrial plants with leafy stens. Leaves plicate broad Flowers white in short sesile axillary panicles. Sepals and petals narrow linear spathulate, at first cohering in a tube with sprealing tips. Lip linear spathulate erect from the column lasede. Column as long as the petals. ereet terete with two ears at the apex. Anther lancoolate acuminate. Pollinia 2 narowly priform on a linear catulicle with an ovate disc. Rostellum shortly bificl. Capsule fusiform, with the persistent columm.

Species 5 or 6 described, all closely allied,-and possibly nearly all one species. Africa, India, Malaya and Australia.

C. LoxglitorA. Hook. fil. Fl. Brit. Ind. VI. 9\%. Stem 6 to 10 feet tall slender, with large roots. Leares broadly lanceolate to ovate lancerlate, deep errens. l'anicle 3 inches long flowers white fragrant. Bracts ovate acuminate $\frac{1}{+}$ inch long. Orary erect $\frac{1}{2}$ inch long. Petals and sepals linear spathulate acuminate ? indes long. elges of petals crisped. Lip nearly as long spathulate claw tubular. Column shorter. Anther loown cuncate. Capsule an inch long subtriangular with wared ridges when ripe. C. rhiylidocarpa, Hook. fil. l.c. p. 92.

singapore: P'ulau L'bin (Ridley 2013:). Chan Chu Kang (Ridley 6r56) ; Malacea: Selandor (Native Coll.); Porak: Larut Hills (King's (oll. 69sos). ( Wray f!o and 3906), Batang Parlang (King's

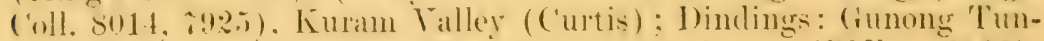
gul (Ridley); Selangor: Kwala Lumpur Caves (Ridley s128). Native Name "Lulumba Paya." In forests. Only diflers from C. rentrifolie, B1. in the proportions of the flower and the undulation of the ribs of the fruit.

C. Brevistruis, Hook. fil. Fl. Brit. Ind. Tol. VI. 91. Stem 1 to 2 leet slender. Jeares elliptic acuminate subpetioled, $;$ nerved $5-6$ inches long, $2-3$ inches broad. Panicles few flowered. Column $\frac{1}{3}$ inch long. Fruit 1 inch long.

Perak: Kiwala Dipang on limestone rocks (King's Collector S248). Endenic.

\section{\%2. 'T'ropidi, Bl.}

Stems erect wiry, leafy. Leares lanceolate acuminate. Panicles or racemes short axillary or terminal. Flowers small green or white. Sopals and petals suberual comnivent. Lip entire saccatte at base. Column short. Anther lanceolate. Pollinia + elongate pyriform with a long slender candicle and orate dise. Rostellum triangular bilid. Capsule ceylindrie.

Species 6 to $\%$. Indian, Malayan, Chinese. 


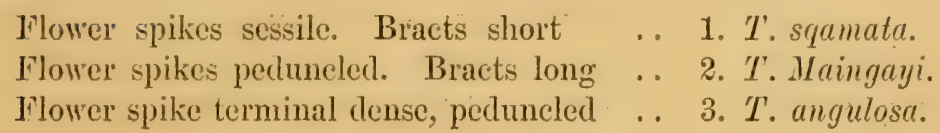

1. 'I'. Squhate, Bl. Oreh. Arch. Ind. p. 123. t. 41. Stems slender about a foot or 18 incher tall. Leaves lanceolate acuminate plicate variable, 6-8 inches long $1-1 \frac{1}{2}$ inch wide. Racemes axillary or subterminal $\frac{1}{2}$ inch long, crowded with broad orate ribbed bracts. Flowers 1 inch long greenish-white, scenterl. Sepals lanceolate falcate. Petals narrower linear faleate. Lip saceate tip lanceolate. Anther lanceolate acuminate. Pollinia elongate pyriform. T. gremminea, Hook. fil. Fl. Brit. Ind. XXXII. 63.

singapore: Bukit 'Timah, Bukit Mandai, Chua C'hu Kang (Ridley 8is6); Johor: Batu Pahat (Ridley); Malacea: Mount Ophir (Grillith), (Ridley 3132); Selangor: Ginting Bidai (Ridley rist), Bukit Hitam (Kelwall); Perak: Larut Hills (Ridley .519.5), (Wray 322\%); Kedalr: Gunong Jerai (Ridley 5129). Distrib. Borneo. Not rare but seldom found in flower. Dense wooks to about 3000 feet alt. Flowers are scented of vannila.

2. 'T. Mningit, Hook. fil. Fl. Brit. Ind. VI. 93. Stem 12-18 inches long. Leaves elliptic lanceolate acuminate. Spikes terminal

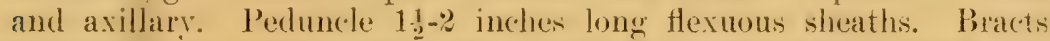
variable lower ones $1 \frac{1}{2}$ inch long. Flowers $\frac{1}{4}$ inch long. Sepals oblong orate acute. Pétals obliquely oblong. Lip with median and intramarginal lamellae from the base to the acute tip.

Malacea: Mount Ophir (Maingay).

3. 'T. AMElosi. B1. Orch. Arch. Ind. 1\%\%. Stem slender brancherl $8-12$ inches long. Leates 2 or :3 terminal broarl orate arenminate many nerved base rounded $3-5$ inches long, 1-3 inches wide. Spike terminal dense, peduncled $3-t$ inches long. Bracts $\frac{1}{2}-\frac{3}{4}$ inch long narrow. Flowers nearly of inch long greenish white. Liper sepal linear lanceolate acute, laterals lanceolate connate to near the acuininate tips. Petals nalrower, blunt. lip oblong orate blunt, two lamellate nerves in the centre, hase spurred. Column shorter than the long, narrow acuminate rostellum. Anther lanceolate acuminate. Pollinia clubbed with a long caudicle and linear gland. Capsule $\frac{1}{2}$ inch long. Hook. fil. Tl. Brit. Ind. VI. 92. King Ann. Roy. Bot. Gard. Cale. VIII. 235. P1. 365. T. Govindovii, 'T. semilibera, Bl. l.e.c. C'nemidir angulose and C. semilibera, Lindl. Gen. and Sp. Orch. Pl. 463. Govindovie nervose, Wight. Ic. t. 2090.

Perak: Ipoh (C'urtis) Iurc. Distrib. India, Burmah. 


\section{Phrsulies, Richard.}

'Terrestrial herlos with ovate or lanceolate petiolate leaves, and terminal spilie of pubescent or hairy small flowers. Petals adnate to upper sepal or free. Lip short almost oblong or quite entire, with a spur cylindrie usually clubbed, and bifid at the tip. Column very short. Anther long-beaked. Pollinia 2 elongate pyriform on a small disc. Rostellum arms long erect. ican,

Species about 20, two or three. Indo Malayan the rest Amer-

PII. LAtifolius, B1. Orch. Arch. Ind. p. 95. PI. 2\%. Stem about 8 inches long. Leaves distant orate acute 3 inches long by two wide with 5 conspicuous veins petiole 1 inch long. Scape 18 iuches long base nude with a few acuminate sheaths pubescent, racemose portion 6-\% inches. Flowers numerous erowded erect sessile. Bracts lancolate acuminate hisped, as long as the orary. Sepals hairy $\frac{1}{6}$ inch long lanceolate obtuse. Petals adnate to upper thin linear spathulate. Lip shorter oblong obtuse entire with 5 sinuate reins on the dise, spur $\frac{1}{2}$ the length of the ovary curved elubbed apex bifid. Anther very long acuminate, rostcllum deply bifid with long linear acuminate lobes. Ovary hairy: Capsule elliptic $\frac{1}{2}$ inch long.

Perak: Larut Hills (Curtis). Distrib. Java.

\section{\%4. Vixdagzial, Blume.}

Small herbs with an ascending stem. Leares ovate or lanceolate petioled. Flowers small spicate erect. Sepals and petals comnivent. Lip entire short, spur rather long pendulous with two pediecelled glands, (rudiments of the two lower stigmata) inside. ('olumn short, with thick arms erect. Anther orate. Pollinia thick club-shaped on a large dise. Capsule ovoid.

Species 5 or 6 . Indo-Malayan.

Spike peduncled longer than the leaves Spike nearly sessile conie short.

Leaves lanceolate acute green ..

Leaves ofvate olive with pink stripes
.. 1. V. lancifolia.

2. V. albida.

- 3. V. tristriata.

1. V. Lancrmolia, Ridl. Journ. Linn. Soc. XXXII. 398. Stem 6 to 9 inches tall erect, rhizome long. Leaves lanceolate acute oblique $1 \frac{1}{2}-3$ inches long i to $: 4$ inch wide petiole $1-1$ inch long. Poluncle variable usually rather long, raceme densely cylindric. Flowers glahrous or glandular pubesent white tipperd with pink. Brats lancenlate actuminate $\frac{1}{8}$ inch long. Sepals lanceolate laterals falcate broader. Petals shorter adhering to the upper sepal. Lip much shorter oblong obtuse, sides intulute spur suberlindric obtuec grlands globose 
on short free pedicels. Column arms short broad. Anther bluntly oroid. Pollinia short and thick. Capsule ovoid nearly $\frac{1}{2}$ inch long.

Singapore: Bukit Timah (Ridley 1686, 2033, 392\%, 6905); Johor: Batu Pahat, Gunong Panti (Ridley); Perak (Scortechini $141 \%)$.

2. V. ALBIDA, Bl. Orch. Arch. Ind. Pl. 75. t. 19. f. 2. Stem 3-4 inches long. Leares tufted at the top lanceolate acute undulate green 3 inches long by $\frac{1}{2}$ inch wide, petiole $\frac{1}{4}$ inch long. Spike very denre conic almost sessile, an inch long. Bracts lanceolate acuminate glandular pubescent longer than the ovary. Flowers numerous small, green and purplish at the base white above withering yellow, fragrant. Upper sepal and petals narrow lanceolate obtuse, lateral sepals with a gibbous base. Lip narrow, shorter, glabrous oblong lanceolate obtuse edges incurved spur swollen in the middle tapering to the point with two short clubbed glands (stigmata). Column short and thick arms erect short blunt. Anther ovate. Pollinia 2 thick clubbed with a large shield-shaped dise. Hook. fil. Fl. Brit. Ind. VI. p. 96. Etaeria albida, Bl. Bijdr. 410. Lindl. Gen. and Sp. Orch. Pl. 491.

Singapore: Chua Chu Kang, Bukit Mandai, Bukit Timah (Ridley 6906); Johor: Batu Pahat (Ridley); Perak: Batang Padang (Wray). Distrib. Java. Common in damp woods by streams.

3. V. tristiata, Ridley Journ. Limn. Soc. XXXII. 398. Stem 6 inches long. Leaves ovate acute $1 \frac{1}{2}$ inch long $\frac{1}{2}$ inch wide, olive coloured with three pink stripes. Spikes subsessile shorter than the leares dense. Bracts lanceolate acuminate reddish. Flowers small white glabrous. Sepals lanceolate oblong, laterals somewhat oblique. Petals adnate to the upper sepal. Lip shorter lanceolate tongueshaped obtuse, with a pustular cushion in the middle, spur elongate saccate, glands yellow globose pedicelled, adnate to the spur for half its length.

Singapore: Chan Chu Kang in wet woods (Ridley); Perak: Horum (Scortechini V. gracilis, Hook. fil.) is also this plant I believe. Endemic.

\section{Crstorchis, Bl.}

Terrestrial, rarely saprophytic plants. Leaves ovate petioled or absent. Flowers small pubescent. Upper sepal adnate to petals laterals larger. Lip clawed side lobes inrolute, midlobe distinct or not, base spurred. Column short, stigma broad. Anther ovate. Pollinia 2 deeply grooved on an ovate dise.

Species 3 or 4 . Malayan.

Leaves ovate lanceolate .. $\quad \ldots \quad$.. $\quad \ldots$ 1. C. varicgata. Leafless saprophyte $\quad \ldots \quad \ldots \quad \ldots \quad \ldots$ 2. C. aphylla, 
1. C. VARIEGATA, Bl. Orch. Alch. Ind. 89. t. 24. f. 3. and 38. c. Whole plant 6-18 inches tall. Leaves ovate lanceolate acute crisped, olive green with lighter patches 3 inches long 1 inch broad or smaller, petiole 1 inch or less. Inflorescence spicate pubescent. Flowers crowded. Bracts lanceolate acuminate red $\frac{1}{2}$ inch long. Ovary shorter. Upper sepal with petals oblong lanceolate, laterals oblong elliptic. Sepals green petals white, with an orange transverse bar pubescent. Lip as long, side lobes involute orange, midlobe orate narrowed at the base, white. Hook. fil. Fl. Brit. Ind. VI. $9 \%$.

Singapore: Kranji, Chan Chu Kang (Ridley 371); Johor: Gunong Panti (Ridley) : Selangor: Kwala Selangor (Ridley), Negri sembilan (Ridley 1001t); Pahang: 'Tahan River (Ridler'): l'erak (Scortechini); Batu Kurau (C'urtis); Kedah: Gunong Jerai (Ridley).

rar. purpurca Ridley. C'. javanica, Bl. l.c. p. 8\%. Hook. fil. l.c.c. IIetacria jactnica, Bl. Bijdr. p. 410. Lindl. Gen. and Sp. Orch. Pl. 191. (Letheria). Leares deep purple, usually a small plant.

singapore. Chua Chu Kang (Ridley); Pahang: 'Tahan Woods (Ridley). Distrib. Jara, Borneo.

2. C. Aphyla, Ridl. Journ. Jimn. Soc. XXX. 400. A saprophyte 6 inches tall, with thick roots, leafless. Stems covered with orate acuminate sheaths. Flowers $\$$ or 5 in a short terminal spike. Bracts lanceolate acuminate acute, shorter than the orary. Sepals lancerolate ateminate f inch long. Protals shorter and narrower. Lip Janceolate hase salceate, side lobes involute hardly distinct. midlobe blunt. Anther orate beaked curved upwards. Rostellum absent.

Malacear: Merlimau (Ridley) : Selangor: Pahameg Track (Ridley 8\%80). Endemic.

\section{:6. Axalectocinges, Blume.}

Terrestrial herbs, with ascending stem, and ovate or lanceolate leaves petioled. spoikes glandular pubesent. Flowers molium or

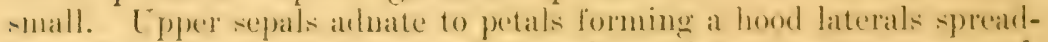
ing. Lip arlnate to the base of the columm, base saceate or spurred, sirle lobes erect or spurading, midlobe clawed, the claw often peetinate, blade broadly bilobed lobes divaricate or nearly entire rounded. (olumm short with two appendages or wings in front. Inther lanceolate. Pollinia elubbed on a shield-shaped dise. Liostellum bifid long. Stigmata a pustular on either side of the rostellum.

Species about 20. Indo Malayin and Chinese. This genus ats now cnended includes Odontochilus, Bl. and Wyrmechis, Bl. 
Claw of midlobe of lip fimbriate.

Leaves brown with gold reticulations. Flowers few.

Spur entire, claw of lip horizontal . 1. A. Reinwardtii.

Spur entire, claw geniculate .. . 2. A. geniculata.

Spur bifid at apex .. $\quad$. $\quad \ldots \quad$.. 3. A. brevilabris.

Leaves green. Flowers numerous . . . . 4. A. pectinatus.

Claw of midlobe toothed .. $\quad . . \quad \ldots$.. A. brevistylis.

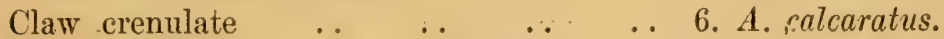

Flowers large solitary or 2. Lip spathulate .. 7. A. macranthus.

1. A. Remwiridti, Bl. Oreh. Arch. Ind. 48. t. 12. f. 2. Stem 1-2 inches long. Leaves orbicular or ovate deep purple brown reticulated gold, 1-2 inches long 1 inch across. Scape $t-6$ inches tall with 3 or 4 flowers at the top, pubescent, bracts lanceolate half an inch long reddish. Sepals dull red pubescent upper one greenish attached to the white petals half inch long, laterals ovate lanceolate. Lip straight white spur horizontal with two masses of papillae inside, claw narrow with numerous slender filaments, blade narrowly transrersely oblong, lobes rounded with an intermediate tooth. Anther ovate lanceolate. Hook. fil. Fl. Brit. Ind. VI. 95. Belg. Hort. 1861. t. 18 .

Perak: Larut Hills (Ridley, Scortechini); Kedah: Gunong Jerai (Ridley).- Distrib. Java, Sumatra,

2. A. gleiculata, Ridl. Journ. Linn. Soc. XXXII. 406. Stem and leaves resembling those of the preceding. Flower's as large, lateral sepals gibbous at the base. Lip base narrow erect parallel to the column, then abruptly deflexed forming a knee, white with two red spots below the knee claw of midlobe with 18 filaments, lobes obcuneate erect truncate, spur $\frac{3}{4}$ inch long curved oblong laterally compressed with two ovoid papillose calli inside. Anther elongate acuminate. Pollinia clubbed long.

Singapore: Bukit Timah, Selangor (Ridley); Johor: Gunong Panti (Ridley); Malacea: Mount Ophir (Ridley 3146); Penang: West Hill (Curtis 1063). These two plants grow in dead leaves in forests and are called Bunga Tulis by Malays. Endemic.

3. A. Brevilabris, Lindl. Gen. and Sp. Orch. Pl. 499. Whole plant about 6 inches tall. Leaves ovate oblique $1 \frac{1}{2}$ inch long $\frac{1}{2}$ inch wide, deep purple netted with gold, with a brown pale bar down the -eutre, petiole short $\frac{1}{4}$ inch. Scape thick reddish. Flowers about $y$. Sepals reddish or dark brownish red, pubescent. Petals adnate to upper sepal thin white. Lip white horizontal, claw short with a pair of oblong calli at the base, filaments few $6-8$ on each side, limb 
of two short oblong truncate lobes. Spur curred rather long, apex bifid. Column with broad triangular wings and a fork process from the centre. Anther ovate pubescent. Pollinia eurved pyriform on a slender caudicle. Hooli. fil. Fl. Brit. Ind. VT. 96. A. albo-linealus, Par. and Rehb. fil. Trans. Linn. Soc. XXX. 141.

Selangor: Bukit Iritam (Késall); Perak: Taiping (Derry). Distrib. India and Burmah. I believe this is not rare in the Peninsula but I have seldom seen flowers.

4. A. Pectixates, Ridl. Odontochilus pectinatus, Hook. fil. Fl. Brit. Ind. TI. 99. Ic. Pl. 2165. Stem tall a foot long, internodes an inch long. Leaves crowded above orate oblique 3 inches long by 1 wide or less, green, petiole half an inch. Spike 2 inches long, nude below. Flowers $\frac{1}{2}$ an inch long about 10. Bracts lanceolate nearly as long as the ovary pubescent. Upper sepal and petals adnate ovate pubescent, laterals ovate hardly gibbous at the base, green. Lip much longer white base saccate with two curved clubbed processes inside, side lohes short rounded, claw of midlobe long with numerous short filaments limb bilobed lobes falcate obtuse. Column short wings triangular, stigma 2 papillose. Anther long acuminate. Pollinia obovoid on a single stout eaudicle with an ovate dise.

Perak: Larut Hills (King's Collector 6398, 213\%), (Ridley 5195). Endemic.

5. A. BRevistruis, Ridl. Odontorhilus brevistylis, IIook. fil. Fl. Brit. Ind. VI. 100. Ic. Pl. 2166. Stem tall about 6 inches long. Leaves few at the top orate acuminate $1 \frac{1}{2}-2$ inches long petiole $1-\frac{1}{2}$ inch long. Perduncle 1 inch long pubescent. Flowers $3-4 \frac{1}{2}$ an inch long white. Bracts lanceolate. Upper sepal ovate, laterals larger oblique ovate with a tuft of hairs at the tip. Petals semiorate, beaked. Lip $\frac{2}{3}$ inch long, hase saceate, side lobes hardly distinct claw with "; or + falcate teeth, limb bifid lobes semiovate rounded. Anther ovate, pollinia ovoid on a broad trianugar eaudicle with a small oblong gland.

Perak: Gunong Batu Putih (Wray 1210). Endemic.

6. A. Calcaratus, Ridl. Odontochilus calcaratus, Hook. fil. Fl. Brit. Ind. XII p. 90. Ic. Pl. 2162. A very slender plant 2 to 5 inches tall. Leaves ovate sessile or shortly petioled $\frac{1}{4}-\frac{1}{3}$ inch long, brown (ereen dashed with reimson and reined gold. Wray) dark purple "(rortechini). Hlowers 2 of $3, \frac{1}{2}$ inch across white. Sepals lanceolite (onnate at the base into a mentum. Petals linear lanceolate free. L.in base sacrate spurred, side lobes (urved, (daw cremulate, limb lobes fyladlate truncate. Column with a hifid elongate process descending into the spur. Anther ovate lanceolate, pollinia pyriform with two stout caudicles and an ovate disc, 
Perak (Scortechini 1339), (Wray), Hermitage Hill on a rock (Ridley). Endemic.

\%. A. macranthus, Ridl. Odontochilus macranthus, Hook. fil. Fl. Brit. Ind. VI. 98. Ic. Pl. 2161. Stem nodose ascending about 5 inches long. Leaves lanceolate petiole 1 inch long by $\frac{1}{2}$ inch wide or much less. Flowers 1 or 2 terminal $\frac{3}{4}$ inch long white sessile. Bracts leaf-like lanceolate, $\frac{1}{2}$ inch long. Upper sepal lanceolate obtuse, adnate to the semiovate petals, laterals lanceolate gibbous at base. Lip spathulate sides involute base saccate with two small processes at the base, terminal lobes rounded crenulate. Column short with two processes. Anther long lanceolate acuminate. Pollinia clubbed with a slender base dise long. Capsule oroid acuminate sessile an inch long.

Perak: Larut Hills (Scortechini, Curtis). Very near if not identical with Cystopus Hasselti, Bl.

\section{7\%. Dossinia, Morren.}

Terrestrial herbs, leaves orate petioled. Scape tall flowers small. Upper sepals adnate to the petals laterals free. Lip adnate to base of column basi alcate with two glands inside side lobes short, midlobe clawed apex dilated. Column short with a boat-shaped process in front, projecting into the lip sac. Anther acuminate. Pollinia 2 on a single caudicle. Rostellum bifid.

Species 1. Malayan.

D. alarirorata, Morren, Ann. Bot. Gaud. IV. 1\%1. t. 195. Stem ascending. Leaves ovate or ovate elliptic $2-3 \frac{1}{2}$ inches long $1 \frac{3}{4}-2 \frac{1}{2}$ inch wide deep velvety green marbled and reined with lighter green, petiole short broadly sheathed reddish. Scape a foot or more pubescent. Flowers long numerous. Bracts lanceolate acuminte. Sepals pink ovate, laterals larger falcate. Lip longer white apex bilobed.

Perak: Telok Bahang (Curtis). Distrib. Borneo.

78. MACODES, Lindl.

Terrestrial herbs with a short stem, leaves ovate petioled. Spike tall pubescent. Fowers small. Bracts lanceolate. Sepals and petals free connivent. Lip saceate 3 lobed spurless, with two calli at the base. Column short thick. Anther elongate 2 celled. Pollinia elongate long stalked with a very small dise. Rostellum bilobed lobes very long curved. Two very large curved laminae on the column below the stigma.

Species 2. Malayan,

II. Petola, Lindl. Gen. and Sp. Oreh. Pl. p. 49\%. Stem ascending glabrous. Leaves ovate petiolate 2 inches long $1 \frac{1}{4}$ inch wide, acute, 
dark green reticulated with gold, petiole $\frac{1}{4}$ inch long. Flowering strm $2-6$ inches long pubescent. Flowers ahout 1\%, small pubescent. Bracts lanceolate as long as the orary. Sepals lanceolate blunt redclish glandular puberent. Petals linear shorter and narrower glabrous. Lip as long pandurate twisted, side lobes curved up rounded midlobe narrow spathulate blunt, with 2 rather large fleshy hooks, at the base of the lip. Column short with two large parallel laminae below the stigma. Anther elongate lanceolate beaked 2 culled. Pollinia long and narrow long stalked $\frac{1}{6}$ inch long. Blume Oreh. Arch. Ind. 119. t. 31. 1. 2. t. 36 D. Rehb. fil. Xenia. Orch. II. 96. Miq. Choix, des Plantes XVII. fig. 1 Neottia Petola, Bl. Bijdr. 40\%. fig. 2 .

Singapore: Chua Chu Kang (Ridley); Johor: Gunong Panti (Ridley) : Selangor: Pahang 'Track (Ridley); Malacea : Mount Ophir (Ridley); Perak: Gumong Keledang (Goldham); Kedah: Gunong Jerai (Ridley 5132). Distrib. Java, Lingga.

\section{i9. Haemaria, Lindl.}

Creeping herbs, with ovate or lanceolate petioled leaves. Flowers fairly large, racemed, white. Upper sepal adnate to petals, forming a large ovate galea lateral sepals oblong orate spreading. Lip spurless saceate aduate to sides and base of the column, midlobe bilobed. Column dilate upwards. Anther orate acuminate 2 celled. Pollinia long narrow pyriform.

Species 1 or 2. Malayan and Chinese.

UI. DIscolor, Lindl. Gen. and Sp. Orch. Pl. 190 . Stem $t-12$ inches tall, fleshy. Leaves orate or lanceolate acute 1-3 inches long $\frac{3}{4}-1 \frac{1}{2}$ hroad deep velvety brown with pink midrib and 2 onter reins. Spike 3-S inches tall white pubescent. Bracts lanceolate acuminate $\frac{1}{2}$ inch long. Overy and pedicel white woolly : inch long. Flowers fragrant white. Lip twisted side lobes short rounded erect, base saccate with 2 small glands, midlobe broarly oblong. Jobes divaricate. Anther yellow. Miq. Chox. Pl. t. 16. Hook, fil. Fl. Brit. Ind. VI. 101. Ludisia discolor, Rich. Dict. Class Nat. Hist. VI. 45\%. Blume Orch. Arch. Ind. 113, 114. t. 34. 1. 2, 3 and t. 42. Goodyera discolor, Ker Bot. lieg. t. 271. Bot. Mag. 2055. Lodd. Bot. Calc. t. 143. Myoda rufescens, Jindl. Gen. and Sp. Orch. Pl. 489. Anoeclochilus Dausonianus, Low Gard. Chron. 1868. 1038.

Pulau 'Tluman, Pulau Aor, Pulau 'Tinggi (Ridley) ; Penang Hill (Wallich 7390), Pulau Betong (Curtis).

var. Ordiam, Williams. Teaves green with golden reins. var. Otletue, II. otletie, Rolfe. III. Hort. XXXVIII. p. 31. t. 12t. witl, narow leaves, oceus in the Pulau 'Tiuman group of Islands. Distrib. Cochin China, Hongkong. Native name "I)am Low." 'The plant usually grows on the bare rocks in streams. 


\section{S0. Zeuxine, Lindl.}

Terrestrial herbs, stem shortly creeping. Leaves linear lanceolate to ovate. Flowers small spicate. sepals subeymal, dorsal forming a hood with the petals. Lip athate to the column saccate with calli inside, apex not dilated. Column very short with lamellar or conical processes on the front. Stigmas 2 distinct. Pollinia prriform with an oblong gland.

Species about 15. Tropical Asiatic and African.

Leares linear sessile

Leares ovate or lanceolate.

Lip midlobe bilobed, not elawed.

Flowers $\frac{1}{4}$ inch long, lip yellow

Flowers very small ..

Lip midlobe with a denticulate claw

Lip midlobe entire rounded

Lip midlohe reniform
.. 1. \%. sulcata.

.. 2. Z. affinis.

.. 3. \%. clandestina.

.. 4. Z. mpestris.

.. 5. Z. violascens.

.. 6. Z. reniformis.

1. Z. sulcata, Lindl. Gen. and Sp. Oreh. Pl. 485. Stem erect 2-16 inches tall. Leaves narrow linear lncelate acuminate 1-2 inches long $\frac{1}{8}$ inch wide. Raceme nearly sessile dense, subglabrous. Bracts lanceolate acuminate $\frac{1}{4}-\frac{1}{2}$ inch long. Flowers white, yellow or pink. Sepals $\frac{1}{6}$ inch long oblong obtuse. Petals similar. Lip yellow boatshaped with a short pubescent claw ending in a hammer headed or bilobed limb. Column short, rostellum short. Anther ovoid pollinia pyriform oroid on a broad oroid candicle and small disc. Capsule $\frac{1}{4}$ inch long. Lindl. Journ. Linn. Soc. I. 186. Griff. Notulae III. 396. Ic. Pl. As. t. 349. Blume Orch. Areh. Ind. 6\%. Miq. Flor. Ind. Bat. III. 223. Hook. fil. Fl. Brit. Ind. TI. 106. King. Ann. Bot. Gard. Cale. VIII. p. 286, Pl. 381. Z. bracteata, brevifolia and robuste, Wight. Ic. 1724, 1725, 1726. Z. membranacea, Lindl. Gen. and Sp. Orch. Pl. 486. Z. Tripleura, Lindl. Journ. Limn. Soc. I. 186. Z . integerrima, Lindl. Gen. and Sp. Oreh. Pl. 486. Bhume Fl. Jav. 55. t. 19. and 23. C. Orch. Arch. Ind. $6 \%$ Z. emarginata, Lind. Gen. and Sp. l.e. $Z$ : procumbens, Bl. Fl. Jav. t. 22. f. 3. and t. 23 B. Tripleura pallida, Lindl. Gen. and Sp. 452. Adenoslylis emarginata, and integerrima, Bl. Bijdr, 414. t. 1\%. Pterygodium sulcatum, Roxb. Fl. Ind. III. 452.

Malacea: Sungei Rambai (R. Derry 306). Distrib. India, Mala! Islands, China.

2. Z. Affinis, Benth. Gen. Plan. t. III. 600. Stem elongate stout, leaves ovate to ovate lanceolate acute 1 inch long $\frac{1}{2}$ inch wide. scape 6 inches long many flowered pubsecent. Flowers $\frac{1}{4}$ inch long. Coper sepal oblong obtuise, laterals orate pubescent green. Petals 
oblong glabrous. Lip sac broad with two curved processes inside midlobe base narrow, limb bilobed lobes spreading blunt yellow. C'olumn short. Anther longer lanceolate, rostellum arms long narrow. Pollinia prriform caudicle narrow disc ovate. Ilook. fil. Fl. Brit. VI. 108. King Ann. Bot. Gard. Calc. VIII. p. 290. t. $38 \%$. Monochilus affine, Lindl. Gen. and Sp. Orch. Pl. 48\%. Aetheria mollis, Lindl. Journ. Linn. Soc. I. 184.

Penang: Government Hill (Maingay), Distrib. India.

3. Z. clandestina, Bl. Orch. Arch. Ind, p. 70. Pl. 39. fig. 4. Plant slender erect about a foot tall. Leaves distant about 5, lanceolate ovate thin green, $1 \frac{1}{4}$ inch long by $\frac{3}{8}$ inch wide, petiole $\frac{1}{4}$ inch long. Scape slender hispid 6 inches long, base nude, above spicate with very small flowers. Bracts lanceolate acuminate half as long as the ovary. Upper sepal adnate to petals forming an acute galea, laterals free oblong all green hisped tipped white. Lip saceate at base green with two curved processes in the sac, side lobes involute, terminal lobe white bilobed spreading lobes obtuse. Column short. Anther lanceolate ovate. Rostellum arms long curved. Capsule $\frac{1}{4}$ inch.

Singapore: Garden Jungle (Ridley s.'s.), Bukit Timah. Distrib. Javi. A rery inconspicuous little plant occurring in dry woods.

4. Z. Rupestris, Ridl. Journ. Roy. As. Soc. Str. Br. Tol. XXXIX. p. 86 . Whole plant 6-8 inches tall slender, leaves few lanceolate narrow blunt $\frac{1}{2}-\frac{3}{4}$ inch long $\frac{1}{8}$ inch wide. Scape slender pubesent. Flowers 2, terminal $\frac{1}{t}$ inch long white. Sepals ovate hairy, petals adnate to the upper sepal. Lip base saceate, with two linear subulate processes inside, limb clawed, with a terete, minutely denticulate claw, hlade hifid lobes oblong truncate. Column short, rostellum lobes linear blunt incurved. ('apsules ereet 1 inch long.

Penang: Tocks on Govermment ITill (C'urtis 2823). The long narrow claw of the lip is more like that of an Anoectochilus.

5. Z. violascens, Ridl. Plant slender 6-10 inches tall. Leaves lancenlate or ovate lanceolate acute $\frac{1}{2}-1$ inch long petiole $\frac{1}{8}$ inch long. purple, with or without a central pink vein. Scape slender pubescent, with seattered aleuminate sheaths. Flowers o or 6 terminal crowled, white. Bracts narrow lanceolate acuminate $\frac{1}{6}$ inch long. Orary longer. Sepals oblong obtuse $\frac{1}{6}$ inch long. Petals adnate to the upper sepal. Lip as long as the sepals, base saceate sides involute terminal lobe broader retuse monded, two short corred processes in the sac. Column short rostellum arms long eurved. Netoeria purqurascens, Bhume Oreh. Arch. Ind. 1. 10). P1. :32. Psycherhilus purpurascens, 13. Pl. 9. fig. 3 . 
Johor: Tanjong Kupang very rare (Ridley). Distrib. Sumatra, Java. Inhabits very wet woods.

6. Z. Reniformis, Hook. fil. Fl. Brit. Ind. VI. p. 10\%. Ic. Pl. 21\%3. Stem below the leaves 6-10 inches tall. Leaves ovate lanceolate acuminate $2-3 \frac{1}{2}$ inches long base rounded, petiole $\frac{1}{2}$ inch long slender. Scape 2 inches long pubescent. Bracts $\frac{1}{3}$ inch long, longer than the ovary. Sepals $\frac{1}{4}$ inch long ovate subacute glabrous. Petals semiovate. Lip sac hemispheric with 2 toothed calli inside, terminal lobes reniform crenate. Column short 2 winged in front. Rostellum arms trumpet shaped. Anther lanceolate. Pollinia pyriform, on a large cuneate quadrate candicle with a triangular dise.

Perak (Scortechini 1\%\%0).

\section{Goodyera, R. Br.}

Terrestrial herbs. Leaves ovate or lanceolate petioled. Flowers small spicate. Upper sepal with petals forming a hood, lateral sepals free. Lip saccate attached to the column, with procestes or lamellae inside the sac, apex small acute decurved. Column short. Anther pyriform beaked. Pollinia $2-4$ on a disc. Stigma solitary.

Species about 40. Europe, Asia, Mascarene Islands and North America.

Flowers very small, 2 calli in the sac .. 1. G. gracilis.

Flowers few large, sac pubescent within.

Leaves oblong lanceolate. Pollinia 2 .. 2. G. rubens.

Leaves ovate. Pollinia 4 .. $\quad \ldots \quad$. $\quad 3$. G. cordata.

Flowers few large, sac with 2 digitate pro-

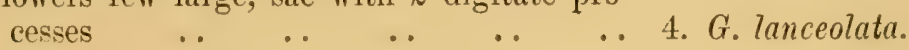

1. G. GRacilis, Hook. fil. Fl. Brit. Ind. VI. 112. Ic. Pl. 2183. Whole plant 8 to 12 inches tall, stem short. Leares tufted ovate deep green 1 inch long $\frac{3}{4}$ inch wide, petiole $\frac{1}{4-\frac{1}{2}}$ inch long. Scape tall, with scattered sheathing acuminate bracts, flowers white fragrant in a spike an inch long. Bracts longer than the ovary, acumina glabrous. Sepals $\frac{1}{6}$ inch long linear oblong or lanceolate, laterals gibbous at the base, glabrous. Petals linear lanceolate obtuse. Lip ovate concave shortly beaked with 2 calli at the base and a broad raised ridge in the centre. Column short, arms of rostellum long. Pollinia narrowly pear-shaped narrowed into a short eaudicle with a short linear gland.

Perak: Larut Hills at 5000 feet (Scortechini, Ridley, Derry). Endemic.

2. G. Rubens, Bl. Orch. Arch. Ind. p. 43. Stem tall about 8 inches. Leaves oblong lanceolate, or elliptic acuminate oblique 3-\% 
inches long $112-21$ inches wide. Spike 12 inches redlish pubescent.

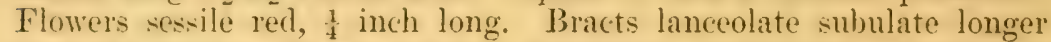
than the ovary. Sepals obtusely acuminate puberulons. Petals adnate to upper sepal. Lip boat-shaped with a short linear acute limb white villows inside. C'olumn rather long. Anther shorter lanceolate beakerl. L'ollinia 2 clavate tapering to the small disc. Stigma large, rostellum short bifid. Hook. fil. Fl. Brit. Ind. VI. 111.

Perak: Ipper part of Batang Padang Valley (Wray). Distrib. Tava, Philippines.

3. G. CondtTA, Hook. fil. Fl. Brit. Ind. VI. 11t. Ic. Pl. $218 \%$. Stem rery slender ascending. Leaves about 4, ovate acute petioled wisped 1 inch long :3 inch wide, petiole $\frac{1}{2}$ inch long slender. Scape 3-t inches long pubescent with 2-3 flowers. Bracts lanceolate acuminate. Flowers $\frac{1}{2}$ inch long dull red. Sepals lanceolate acute acuminate greenish tipped red. Petals adnate to the upper sepal. Lip short oblong subacute saccate, apex recurved with two hairy patehes inside at the base. Columm short, broad. Anther lanceolate acklminate nearly as long as the lip. Pollinia 4 elongate clubbed with a narrow caudicle and linear gland. Lostellum lanceolate deeply bifid. Georchis cordata, Lindl. Gen. and Sp. Orch. 496. Rehb. fil. Gard. Chron. 189\%. II. 520.

Penang: Fern Hill, 2500 feet alt. (Ridley, Curtis) ; Perak (Scortechini drawing 14). Distrib. India. Scortedhinis drawing shows $\%$ or 8 flowers. My specimen and Curtis drawing two only.

4. G. Indeeolata, Ridl. Journ. Roy. As. Soc. S. Br. Tol. XXXIX. p. 86. Stem slender 9 -inches long. Leaves lanceolate acuminate $1 \frac{1}{2}$ inch long nearly $\frac{1}{2}$ inch wide. Scape $3 \frac{1}{2}$ inches long puberent fer flowered. Bracts lanceolate arominate 3 inch long. woolly pubescent. Flowers $\frac{1}{2}$ inch long. Sepals lanceolate, laterals oblique, ac:mminate woolly pubescent redilish. P'etals adnate to the I1pper sepal thin glabrous reddish. Lip base saccate adnate to the columm, ly the elges glabrous within with a raiked central keel, and a tult of digitate processes on each side, apex of lip acuminate subulate. Columm short. Anther very long acuminate. Pollinia $f$ inch long clubbed with a pair of yellow caudicles comnate alout half way down and a small linear dise. Rostellum long shortly bifid, lobes acute. Stigma large with thin walls.

Selangor: The Gap, Pahang 'T'rack (Curtis). A single specimen.

\section{צ.. HeTAERIA, B1.}

'lerrestrial herbs, with a short creeping ascending stem, ovate or lanceolate petioled leaves and a slender dense spike or small flowers. Epper sepal usually armate to petals, pubescent. Lip 
generally uppermost (i.e. Hower reversed). Lip base boat-shaped with two small calli at the base, apex narrow sides rolled in and often comnate forming a tube. Column fairly large, with long rostella arms.

Species about 15. India, Malaya and Pacific. Differs from Zeuxime in the absence of a dilated end to the lip.

Leaves ovate.

Spike 10 inches long, 2 calli in sac of lip .. 1. H. alta.

Spike $3-4$ inches.

Flowers very small, calli numerous .. 2. II. elata.

Flowers $\frac{1}{8}$ inch long, calli in 2 rows .. 3. H. nitida.

Leaves lanceolate, broad, calli $2 \quad \ldots \quad \ldots$ 4. H. obtiqua.

Leares lanceolate $\frac{1}{4}$ inch wide, calli numerous .. 5. $I$. parvifolia.

1. H. ALTA, Ridley (by a misprint alba), Journ. Linn. Soc. IXXII. 40t. A tall plant nearly two feet high, with long. woolly roots. Leaves seattered numerous orate acuminate acute oblique 4 inches long $1_{4}^{*}$ inch wide, petiole an inch long, sheaths large ampliate. Spike 10 inches long pubescent. Flowers yellow glabrous. Bracts lanceolate acuminate $\frac{1}{4}$ inch long orary $\frac{3}{4}$ inch long pubescent. Sepals lanceolate-obtuse glabrous. Petals free as long. Lip shorter base boat-shaped, with two linear calli inside, blade lanceolate sides inrolute. Column short. Anther lanceolate linear. Column arms large oblicuely euneate oblong. Rostellum lobes worter than anther lanceolate linear. Capsule ellipsoid $\frac{1}{2}$ inch long. Endemic.

Perak: Hermitage Hill, Kiwala Kangsa (Ridley), and Kiamuning.

2. H. Elated, Hook. fil. Fl. Brit. Ind. VI. 116. Ic. Pl. 2191. Stem 2 or 3 inches long with long stout woolly roots. Leares seattered on the stem orate acuminate 3 nerved, 4 inches long, $1 \frac{1}{4}$ inch wide petiole slender orer an inch. Seape slender orer a foot long with lanceolate acuminate sheaths. Spike 3 inches long, flowers rery small spreading. Bracts lanceolate acuminate with long points. Sepals ovate. Petals linear falcate. Lip boat-shaped ovate not adnate to. the column except at the base, with numerous short processes in the sac, apex subacute. Column short, rostellum long deeply bifid. Anther ovate arms of column rather long.

Malacea : Mount Ophir on Gunong Tundok (Ridley); Perak (Scortechini), Batang Padang (Wray 151\%). Endemic. I have described this from the Nount Ophir plant in which I find the petals rather narrower than in the figure I. P'. 2191, and some slight diflerences in the lip. There is a good sketeh No. 15 by Scortechini labelled. Goodyera consimilis, Hook. fil. 
3. H. Nitrd., Ridley Journ. Lin. Soc. XXXII. p. 404. Stem creeping 1 inch through succulent. Leares orate acute $3 \frac{1}{2}$ inch long 1.t inch wide, dark polished green, petiole 1 it inch long, sheaths $\frac{1}{4}$ inch long. Scape 9 inches long pubescent, with numerous scattered sheathis, spike close many flowered. Flowers $\frac{1}{4}$ inch long. Bracts lanceolate acuminate red it inch long. Sepals orate equal subobtuse pubescent red. Petals lree spathulate white. Lip shorter adnate to the column ovate boat-shaped edges at the tip involute so as to form an acute point, a row of linear processes on each side in the lip base. Column short and broad, arms linear ereet. Anther ovate beaked. Pollinia pyriform on a single caudicle, with a linear disc. Rostellar lobes linear rather short.

Singapore: Bukit 'Timalı, Chan Chu King (Ridley); Penang: Pulan Betong (Curtis). Endemic.

4. H. obliqui, Bl. Orch. Arch. Ind. p. 10t. t. 34. fig. 1. Whole plant about a foot tall with a faily stout creeping stem. Leaves obliquely lanceolate 2-:3 inches long by -1 inch wide rather distant dark green sometimes with a median silver bar, petiole $2-1$ inch long. s'ape graceful pubescent. Spike fairly dense :3-10 inches long. Flowers numerous reversed appressed. Bracts half as long as the ovary lanceolate acminate pubereent. Tpper sepal nearly $\frac{1}{4}$ inch long comate with the petals for $\frac{1}{2}$ its length white. Sepals pubescent, laterals lanceolate obtuse dull red pubescent base giblous. Lip rery small base boat-shaped with the edges meeting apex narrow rolled up into a tube, tip open spoon-shaped white, 2 calli in base of lip triangular toothed. Column large yeliow. Anther lanceolate red. l'ollinia 2 clubbed shaped narrowed into 2 caudicles on an oblong dirc. liostellum arms long linear curved, erect. Capsule elliptic sessile 8 inch long.

Singapore: Serangoon lioad, Bukit 'Timah. Pulau 1)amar (líilley) ; Johor: Panchor (Ridley) ; Sungei L jong: Bukit Danan (Native Collector); l'erak (Scortechini drawing 19) ; P'enang: P'ulau Betong (Curtis). Distrib. Borneo, Sumatra. Native name "Polio 'Tumbah Hutan." Leaves used for poulticing sores.

5. H. Parvigolia, Ridl. Journ. Roy. As. Soc. Str. Br. Vol. XXXIX. p. s\%, stem 2 inches long. Leares small lancevlate acute nearly sessile 1 inch long inch wite, sheaths i inch long ampliate. Sorape slender puberent of or 6 inches long. with several rather longr distant acuminate sheaths. Raceme \& inches long. Flowers very small $\frac{1}{8}$ inch long appressed. Bracts narrow lanceolate acminate nearly as long as the ovary. Upper sepal adnate to petals ovate acuminate pulrereent latteral- lanceolate alcute. Lip bare salceate with

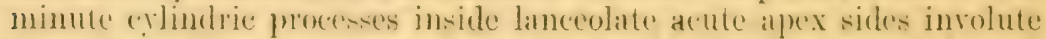
forming a tube, not longer than the sepals. Colmen short dilated 
above. Rostellum arms nearly as long lincar truncate. Anther with a long narrow beak.

Penang: Government Hill (Ridley). Endemic.

\section{Hriophili, Lindl.}

Terrestrial herbs, stem long creeping. Leaves lanceolate petioled. Flowers small pubescent spicate, connivent. Lpper sepal hooded adnate to petals. Petals falcate. Lip a subglobose sac with a small linear limb and 2 calli inside. Column very short, no arms. Rostellum very long bifid. Anther long beaked. Pollinia \& pyriform with a tapering to a narrow disc.

\section{Species 2. Malayan.}

Sepals $\frac{1}{4}$ inch long glabrous .. . . 1. H. lunceolata.

Sepals $\frac{1}{8}$ inch long viscid pubescent .. 2. II. mollis.

1. H. Laxceolati, Hook. fil. Fl. Brit. Ind. VI. 110. Stem stout long and creeping. Leaves lanceolate caudate acuminate or acute wared t-5 inches long, petiole 1 inch long. Scape shorter than the leaves or not much longer 3 inches long dense flowered. Bracts lanceolate acuminate. Flowers numerous white nearly or quite glabrous. Sepals $\frac{1}{4}$ inch long. Petals obliquely ovate. Lip globosely saceate with a short narrow limb. Column very short. Rostellum lobes and anther much longer. Dicerostylis lanceolata, Bl. Orch. Arch. Ind. 116. t. 38. f. 1.

Perak (Scortechini), Bujong Malacea (Curtis). Distrib. Java.

2. H. MoLlis, Lindl. Gen. and Sp. Orch. Pl. p. 490. Rhizome creeping for some distance. Stem $6-8$ inches tall, leaves numerous thin lanceolate acute polished green $t$ inches long $1 \frac{1}{4}$ wide, petiole 2 inches. Scape slender glandular pubescent 1.1 feet tall, with a lew sheathing acuminate leaves. Spike about 6 inches long with small crowded sticky green flower's. Bracts shorter than the ovary $\frac{1}{4}$ inch long narrow lanceolate acuminate. Sepals $\frac{1}{8}$ inch long. Upper one saccate beaked pubescent, laterals ovate oblique. Petals adnate to the upper sepal. Lip saccate adnate to the column, with a linear terminal lobe. Column very short. Anther very long beaked. Rostellum lobes long waved. Capsule elliptic sessile $\frac{1}{4}$ inch long. Blume Orch. Arch. Ind. 115. t. 35, 36 F. Hook. fil. Fl. Brit. Ind. VI. 110.

Singapore (Wallich 7396 ), Teban, Bukit Timah, Toa Payoh (Ricley 3926); Malacca (Griffith); Perak (Scortechini), Larut (King's Coll. 218:). Distrib. Sumatra. Often very abundant in wet woods.

\section{8t. Lepidogyxe, Bl.}

A large plant with a stout creeping stem and long linear acuminate leares. Scape tall covered with sheaths. Spike long and 
dense with linear acuminate bracts. Upper sepal adnate to petals. Lip saceate base adnate to the column with several short processes inside sirle lobes short, terminal lober linear. Column short, rostellum very deeply bifid, lobes linear. Inther elongate lanceolate acuminate. Pollinia pear-shaped, narrowed into catcdicles with a narow linear disc.

Species 1. Malayan.

L. Loxiffolid, Bl. Orch. Areh. Ind. 94. Pl. 25. Leares 1 foot long lanceolate or linear 2 inches across. Scape nearly 3 leet long, spicate portion 1 loot long. Flowers pink. Sepals lanceolate acuminate $\frac{1}{4}$ inch long. Capsule elliptic $\frac{1}{2}$ inch long.

Penang: Penara Bukit (Curtis 308t). Distrib. Lingga, Java.

\section{S.j. STEREOSANDRA, Bl.}

A leabless saprophyte with a swollen tuberous rhizome, a tall slender stem and secund raceme of pendulous flowers. Bracts linear or linear lanceolate. Pedicels short sepals and petals subsimilar lanceolate connivent. Lip as long similal entire with 2 rounded pulvini at base no spur. Column very short anther large. Pollinia elongate priform. liostellum absent. Stigma transwersely obloner on a short ilistinet style, ovary alliposid not twisted. Capsule elliptic splitting into 3 ribs held together by the perianth at the top.

Species 1 or 2. Jara and Malay Peninsula and Siam.

S. J.IXICA, Bl. Mus. Bot. 2. 186. Oreh. Arch. Ind. 30. 1. 10. fig. i. 11. fig. 9. lihizome ringed $\frac{1}{2}-\frac{3}{4}$ inch long 8 inch throngh. Stem 1.) inches tall theshy pale with t-5 distant papery sheaths. Rareme nodiling or suberect. Flowers about 10 sceund deflexerl. Bracts $\frac{1}{12}$ inch long. l'edicel purple sepals and petals + inch long acuminate.

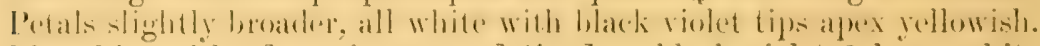
Lip white with edges sinuate and tip deep black violet 2 large white round cushions at the base. Anther elliptic oblong white with two violet central blotehes and base bilobed yellow filament distant as long as the style. Pollinia elongate pyriform orary inch long ribs deep violet. Citpsule elliptic tinch long.

Singapore on the banks of a stream at Bukit Panjang (Ridley for. !. 1!n:i) ; Johor near C'astlewool (Ridley) : Penatng Hill (curtis).

Iery rare and diflicult to find. Hitherto only known from the specimen collected in Java. I found $2 \mathrm{in} \mathrm{singapore} \mathrm{and} \mathrm{Mr}$. Curtis is in l'enaner. The flowers are self-fertilised the tip of the pollen reaching down to the stigmat, and there being no rostellum. 
86. Criptostruis, R. Br.

Terrestrial herbs with thick woolly roots. Teaves basal long petioled. Scape lateral with several membranous, sheaths at the base, tall. Spike many flowered lax. Sepals and petals very narrow. Jip superior erect lanceolate entire base embracing the column. Column very short. Anther oblong obtuse. Pollinia 2 pyriform, deeply lobed with a small dise or free, rostellum broad thick. Stigma large rounded. Capsule elliptic.

Distribution India, Malaya and Australia.

C. ARACHnites, Bl. Orch. Arch. Ind. 132. t. 45. Leaves elliptic or ovate acute $2 \frac{1}{2}-6$ inches long $1 \frac{1}{2}-3$ wide, petiole slender $1-1$ inches long. Scape 6-30 inches tall, racemose portion 1-6 inches long. Sepals $\frac{1}{2}$ inch long green. Lip ${ }_{x}^{3}-\frac{1}{2}$ inch long narrow or wide light brown with darker red brown spots. Capsule $\frac{3}{4}$ inch long elliptic. Hook. fl. Fl. Brit. Ind. VI. 118. Zosterostylis arachnites, Bl. Nijdr. 419. t. 32. Z. zeylanica, Lindl. Gen. and. Sp. Orch. Pl. 446. Z. Walkerae, Wt. Ic. t. 1748. f. 4.

Singapore: Bukit Timah, Pulau Damar (Ridley); Malacea: Merlimau (Derry 565) ; Selangor: Bukit ITitam (Kelsall); Perak (liriy), (Soortechini), Larut Hills (Ridley; Penang: Moniot's Road, (iosermment Hill (Curtis) ; Kedah: Gunong Jerai (Ridley). Distrib. India, Ceylon, Java.

Very rariable especially in the breadth of lip and height of scape. Banks and woods from the plains to 3000 feet alt. Local but not rare. Native name "Bunga Bangkong."

\section{OPHRTDEAE.}

\section{8\%. HABENARIA, L.}

Terrestrial herbs with lobes or undivided tubes. Leaves sheathing at the base herbaceous. Flowers in a terminal spike or raceme. Sepals subequal or laterals longer spreading. Petals simple or divided. Lip adnate to the column base, simple or 3 lobed, spurred. Anther adnate to the column 2 celled, hases of the cells sometimes prolonged into a tube. Pollinia 2 clubbed or pear-shaped with a long or short slender caudicle and dise to each. Staminodes one or two viscid processes on the face of the column below the anther. liostellum entire erect below the anther cells.

Species about 400 , scattered all over the world.

Lip entire ... .. ... .. 1. H. zosterostyloides.

Lip trifid. Spur long. Petals undivided.

Lip lobes broad, midlobe deeply bifid yellow

2. H. xanthocheila. 
Lip lobes broad, midlobe retuse pink or white .. $\quad . . \quad \ldots \quad$. . . 3. H. rarnea. Lobes of lip very narrow linear.

Upper sepal as long as the laterals .. 4. H. Kingii.

Upper sepal much shorter .. ‥ 5. II. Murtoni. Lip trifid. Spur short. Flowers small.

Stem 4-5 feet tall .. .. . 6. H. gigas.

Stem under 3 feet.

Lip shortly 3 lobed, white $\quad . \quad \ldots \quad$ \%. H. lacertifera.

Lip lobes linear green .. $\quad . . \quad$.. $\quad$ S. H. monticola.

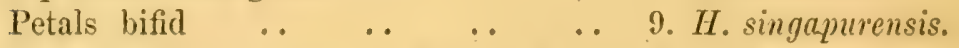

1. H. zosterostyloides, Hook. fil. Fl. Brit. Ind. VI., 155. Whole plant $1 \frac{1}{2}-2$ feet tall, with long stout roots. Leares orate acute lower ones radical $t$ inches long and 2 inches wide, petiole 2 inches long, cauline leares lanceolate sessile. Raceme lax. Flowers rather small green. Bracts lanceolate acute shorter than the orary $\frac{1}{4}-\frac{1}{2}$ inch long. Tpper sepal orate, laterals linear oblong rather larger. Petals triangular orate. Lip entire linear as long as the sepals, spur slender cylindric acute as long as the ovary. Anther cells divergent with very short processes. Pollinia with stout candicles and large orbicular disc. Capsule nearly sessile elliptic oblong $\frac{1}{2}$ inch long.

Malacea : Momnt Ophir (Griffith, Ridley 314i); Perak: (imnong Brumber Pahang (Wray 15is), (Scortechini 560 B). In mossy spots at an altitude of $f(000$ feet remarkable for its radical leares like those of Cryptostylis.

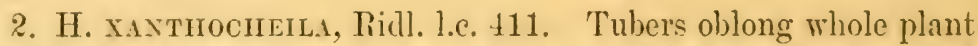
a font tall. Leaves laneeolate acuminate hasal 6 inches long, 1 it inch wide. Raceme lax about 10 flowered. Flowers 1 inch across showy. Bracts an inch long acuminate. Orary twice as long. Upper sepal orate obtuse green laterals orate oblong deflexed. Petals spathulate ollong. Lip 11 inch long vellow, 3 lobed side lober oblong spathulate faleate truncate, midlobe obovate bifit. spur decurved slender 2 inches long. Inther with hroad processes. Pollinia fusiform. Rostellum large ovate.

Penang: Pulau Betong (Curtis) and near the spout.

3. H. CARxea, N. E. Br. Gard. Chron. 1891. II. p. 729. fig. 105; 1892. II. p. 300. Stem tuberous rery short, leaves hasal oblong or lanceolate acute:" inches long by 1 inch wide dull green with white spots or plain. Scape 6-12 inches tall with orate almminate bracts. Flowers i-20) large rose pink or white, 1 inch across. Sepals ovate upper one smaller than the other's. Petals orate as larwe as the upper sepal. Lip 1 inch long, side lobes resy broad roumdent midlobe reniform or almost orlicular retuse with a narrow base. Inther galcate 
processes stout rostellum triangular larger. Pollinia conic with very long slender caudicles.

Lankawi: Limestone Islands, Kwah, Terutau (Curtis 2104). var. minor, smaller plant with white flowers and unspotted leaves. Same locality. Distrib. Siam.

4. H. Krvair, Hook. fil. Fl. Brit. Ind. VI. 14t. Whole plant $1-2 \frac{1}{2}$ feet tall usually stout, leaves crowded at the base subpetioled lanceolate or elliptic lanceolate, 6-10 inches long $1 \frac{1}{2}$ inch across undulate. Spike base flowered. Flowers numerous green. Bracts lanceolate acuminate as long as the ovary $\frac{3}{4}$ inch. Upper sepal hooded $\frac{1}{4}$ inch long oborate, laterals cultrate obtriangular falcate, base narrow. Petals linear. Lip three lobed, lobes narrow linear, equal. Spur nearly as long as the ovary, slender cylindric. Anther yellow with very long processes. Rostellum small. rocks.

Perak: Ipoh (Curtis 3342), (King's Coll. 7172) on limestone

5. H. Muntoni, Hook. fil. l.c.e. Plant 2-3 feet tall, leaves crowded oblanceolate acute 6-10 inches long $2-3$ inches wide narrowed into a petiole. Stem with numerous ovate lanceolate sheath leaves. Flowers $\frac{1}{2}-\frac{3}{1}$ inch across. Bracts as long as the ovary. Upper sepal orbicular hooded, much shorter than the laterals which are bluntly triangular. Petals small triangular. Lip trifid with linear filiform lobes, subequal, spur longer than the ovary slender dilate at the apex. Anther cells short, processes very long. Stigmatic processes globose. Rostellum yery small.

Perak on limestone rocks (Scortechini 552 B), limong Pondok (Murton).

6. H. GIGAS, Hook. fil. Fl. Brit. Ind. VI. p. 160. Stem 4-5 feet tall hollow at the base and nearly an inch through, nearly 3 feet before leafing. Leares elliptic acuminate $8-10$ inches long, 3 to \pm inches wide, narrowed into a petiole. Spike dense cylindric 8 inches long. Flower's small pale green. Bracts longer than the flowers $\frac{3}{4}$ inch long. Upper sepal linear oblong obtuse, laterals longer lanceolate. Lip as long as the sepals obtusely trifid, spur shortly oblong. Anther orbicular processes upcurved. Pollinia clarate subsessile on the large oblong glands. Stigmatic processes large. Rostellum small. Capsule linear oblong $\frac{3}{4}$ inch long:

Perak: Batang Padang at 2000 feet alt. (Wray 1449).

\%. II. Licertiferi, Benth. Fl. Hongkong p. 362. Whole plant $8-18$ inches tall, roots tuberous. Leaves 2 or 3 at the base of the stem lanceolate acute or elliptic lanceolate green. Spike 6 to 9 inches long. Flower's erect appressed fragrant white. Bracts lanceolate acuminate 
Jonger than the orary. Lpper sepal ovate, laterals lalcate oblong obtuse longer nearly a inch long. Petals oblong lanceolate obture shorter. Hip short broad three lobed lobes blunt short, central one longest, spur very short conic green. Anther cells parallel. Capsule oblong strongly ribbed $\frac{1}{2}$ inch long. Hook. fil. Fl. Brit. Ind. VI. 163. II. Maingayi, King Journ. As. Soc. Beng. LXVI. II. $189 \%$. 1). 601. Cocloglossum lacertiferum and acuminatum, Lindl. Gen. and Sp. Oreh. Pl. ¿0\%. Peristylus chloranthus, Lindl. Kew. Journ. Bot. VII. 3\%. Chacradoplectron spiranthe's, Schauer P'. Meyen. 136. t. $13 . \mathrm{C}$.

Singapore: 'T'anglin, Jurong, etc., (Ridley) ; Johor: ncar Castle Wood (lielley) ; Malacea: Sungei Ujong (Derry 101), Nyalas (Goodenough 1516); Penang: Govermment Hill (Curtis 2105), (Ridley 7061), (Wallich 7055).

Common on road sides and in grassy spots. 'The var. robustior, Kramzl. Engl. Jahrh. XVI. (1892). p. 160. var. robusta, Hook. fil. l.c.e. is a common large form. H. Maingayi, King. being the most abundant road side form. The plant raries but little except in size. I have met a form in which the outer lobes of the lip were longer than the central one. Distrib. India, Lingga, Hongkong.

8. H. moxticoLA, Ridl. Joum. Linn. Soe. XXXII. 413. Whole plant 15 inches tall, tubers $\frac{1}{2}$ inch long oblong. Leaves basal $\ddot{z}-1$ lanceolate acute an inch long inch wicle, dark green. Stem slender with a few acuminate bracts, jaceme lax. Flowers numerous very small geren. Bracts lanceolate acute $\frac{1}{8}$ inch long nearly as long as the pedicel. Serepals oblong ovate ${ }^{1}$ inch long. Petals a little shorter clliptic oblong. Lip trifid with a short claw side lobes \& inch long fililorm midlobe much shorter spur as long as sepals straight bifid. Capsule elliptic $\frac{1}{4}$ inch long. (liidley).

Malacea: Mount Ophir (Ridley 9994); Kedah: Gunongr Jerai

9. H. singapurexisis, Rial. Journ. Linn. Soc. XXXII. 410. Whole plant about : fect till lower part of stem covered with sheaths only. Leaves about 12 lanceolate acuminate dark green, 6 inches long 4 inches wide, subpetiolate, sheaths 1 inch long. Scape glateeseent, with scattered acuminate sheaths. Flowers numerous crowded.

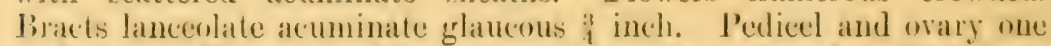
inch longr. Lpper sepal lanceolate oblong boat-shaped, with a longer fuint : 8 inch longr, laterals oblong obluse obligue with a loner point, all greenish white. letals bilid with long linear olire green arms. lipe : bobed to nearly the hase iobes linear, midlobe a little shorter, frur slenter eglindric 3 inch long. Anther with long straight processes. 
Singapore: Chua Chu Kang (Ridley); Johor: Batu l'ahat (lidley 1194). In dense wet jungle swamps, local.

\section{CYTIRILDIE.AE.}

\section{8\%. Cypripledum.}

Herbs usually terrestrial. Leaves distichous radical olten tessellate. Seape 1 to many flowered. Flowers large. Sepals spreading laterals connate under the lip. Petals free narrower and usually longer. Lip sessile lateral lobes inflexed short, midlobe large saecate inflated. Columm short terete. Anthers? globose with a large disclike staminode between and covering them, stigma rounded discshaped. Capsule long and narrow.

Species about 40. Europe, Asia and North America.

Leaves oblong tessellate.

Petals as broad as the sepals, not warted .. 1. C. niveum.

Petals narrower than sepals warted on the edge 2. C. barbatum.

Petals narrower than sepals warted all over 3. C. superbicus. Leaves narrow coriaceous not tessellated .. .. 4. C. Lowii.

1. C. Niveun, Rehb. fil. Gard. Chron. 1869, 1038. Xen. Orch. 11. 155. Leaves linear oblong green above with darker blotehes, dark purple beneath. Scape 6-8 inches tall 1-2 flowered. Bracts short close-fitting. Flowers 38 inches across white dotted with purple. Dorsal sepal broal orbicular, laterals ovate oblong smaller. Petals broadly oblong obtuse longer. Lip sac ovoid mouth contracted. Staminode reniform oblong yellow with a white edge. Bot. Mag. t. 5922. Ill. Hort. 18:1. t. 83. Flor. Mag. 18\%1. t. 543. Reichenbachia I. t. 3t. Gard. Chron. 1883. 1I. fig. 1. Hook. fil. Fl. Brit. Ind. VI. $1 \% 1$.

Lankawi Islands on limestone rockis (Curtis). Distrib. 'I'cubilan Islands.

2. C. BАвватum, Lindl. Bot. Reg. 1841. Misc. 53. 1842. t. 1\%. Leaves oblong or linear oblong $4-8$ inclies long 1 inch wide acute pale green with darker spots. Scape 10-1\% inches tall pubeseent. Bracts shorter than the ovary. Flower's 1-2, 2-3 inches across. Upper sepal broad, orate or orbicular acute or obtuse lolded in the middle white with purple veins, base greenish reined purple. Petals greenish brown ¿ inches long, lincar oblong curved with a few black warts and cilia along the cdge. Lip reddish purple helmet-shaped. Staminode reniform rotten deeply emarginate in front. Capsule oblong tapering at base 1 inch loner. Bot. Mag. t23 H. Fl. der serres JII. t. 190. IVIII. t. 18\%9. Belg. IIortic. XIXIII. 96. t. \%. Warners sel. Orehid 
Ser. 3. t. 11. (var. Warneri) Gard. Chron. 1866. II. 308. f. 63. Hook. fil. Fl. Ind. VI.18. C. purpuratum, Wight. Ic. 1860. (not of Lindley). C. Warnerianum, Rehb. fil. Gart. Zeit. 1883. fase. IX. C. vilum, lichl. fil. Gard. C'hron. 1ssi. II. is. ('. C'rossii, Belg. Hort. XV. 22\%. Veiteh. Man. 12. fig.

Johor: Gunong Panti (Ridley); Malacca : Mount Ophir (Griffitl: and all ('ollectors): Penang: Gorermment Hill (Maingar, Curtis, ete.) ; Kedah: Gumong Jerai (Ridley 5130). Distrib. Siam. On rockis and in grassy slopes at an altitude of 2-1000 feet. Tative name "Bunga Kasut." It is somewhat variable in depth of colour.

3. C. Superibiexs, Richb. fil. Bonplandia 1855. 22\%. Allgem. Gartenz. 1856. 323. Xen. Orch. II. 9. t. 103. Leares oblong tessellate $5-7$ inches long by $1 \frac{1}{2}$ inch broad. Scape 9-12 inches long. Bracts much shorter than the ovary. Flowers $t$ inches across solitary. Dorsal sepal orbicular acute white veined with green. Petals broad oblong greenish white ciliate along the edge and dotted all over with purple warts. Lip helmet-shaped reddish. Staminode suborbicular notehed in front 2 lobed, green. Garten. Fl. 1863. 49. Warner Sel. Orch. II. t. 12. Williams Orchid Album XI. Pl. 486. Flore des Serres t. 1996. Hook. fil. Fl. Brit. Ind. VI. 173. C. Teilchianum, IIl. Hort. XII. t. 429. Rev. Hort. 18:1. 595. fig. 78, 89. C. barbatum Veitchii, Fl. des Serres t. 1t50. C. barbatum superbum, Belg. Hort. 1883. 9\%.

\section{Malacea: Mount Ophir (Lobb).}

'I'his plant seems never to have been seen on Mount Ophir again. It came with a lot of $C$. barbatum, and should be looked for again. It is easily distinguished from C. barbulum by its green reined upper sepal and the petals dotted all over with purple warts.

4. C. Lowis, Lindl. Gard. Chron. 18t\%. p. 765. Epiphytic. Leares lingulate plain green 9-1, inches long apex bilobed coriaceous. Scalpes 201.3 feet tall, 2 to 5 flowered. Bracts $\frac{1}{3}$ the length of the ovary. Flowers $3-t$ inches across pubescent. Upper sepal broadly oval acute yellowish green, base purplish lower ones similar but smaller. Petals 3 inches long spathulate twisted decurved, base rellow with black spots, apex bilobed purple. Lip cylindric helmetshaped hown purple. Staminode obcoldate edged with puple hairs green with a tooth on the base and one in the simus. Fl. des Serres IV. t. 375. Williams Orehicl Alhum 9. Pl. 428. Veitch's Manual p. 38. C. cruciforme, Zoll. and Miq. De Vr. 111. Oreh. 1851.

Selangor: The Gip), Pahang 'Track (C'urtis). Distrib. Borneo and Simatia.

C. sp. I have twice found plants of a small Cypripedium, with faintly tessullated leaves in sindy spots on the sea shore in Johore. 
It seems to be allied to C. barbatum, Lindl. No flowers were met with, and further search was in vain as the place was burnt later. The locality for a Cypripedium was most unusual, and the plant should be sought again.

\section{APOSTASIACEAE.}

Erect herbs with a leafy stem bearing a terminal raceme or panicle of moderate or small white or yellow flowers. Perianth superior of three sepals and three petals lanceolate cuspidate. Stamens three or one aborted. Anthers dorsifixed, two celled, pollen powdery, filaments connate below for half their length. Style cylindric. Stigma capitate obseurely 3 lobed. Ovary triquetrous 3 celled. Fruit capsular, rarely baccate. Seeds minute with reticulated testa.

Flowers moderately large. Perfect sta-
$\begin{aligned} & \text { mens } 3 \quad \cdots \\ & \text { Flowers very small. Perfect stamens 2 }\end{aligned}$

1. Neuwiedia, Bl.

Herbs with erect or ereeping stems. Leares numerous plicate lanceolate. Flowers racemose with long bracts, yellow or white. Sepals and petals equal and similar lanceolate cuspidate. Stamens 3 fertile. Anthers linear oblong dorsifixed, filaments connate at the base with the style, upper third only free. Style stout stigma capitate. Fruit capsular or baceate. Seeds numerous minute.

Species 6 Malayan to the Louisiades.

Fruit capsular, flowers yellow .. .. 1. N. Lindleyj.

Fruit capsular, flowers white .. .. 2. N. Griffithii.

Fruit a red berry, flowers yellow .. . . 3. N. Curtisii.

1. N. Lindleyi, Rolfe. Journ. Linn. Soc. XXV. 232. t. 48. f. 10-12. Stem 4-5 feet tall, erect leaves lanceolate acuminate many nerved tapering below into a petiole, 18-2t inches long, 2 inches wide. Upper part of stem covered with appressed narrow lanceolate bracts. Raceme 12-2t inches long dense. Flowers numerous shortly pedicelled bright yellow. Sepals $\frac{1}{2}$ inch long lanceolate pubescent. Capsule $\frac{1}{2}$ inch long, with 3 broad obovate reflexed valves. Hook. fil. Fl. Brit. Ind. VI. 175. Bot. Mag. t. 7368.

Singapore: Chua Chu Kang, Bukit Timah (Ridley); Malacea: Bukit Lintang (Derry 10J); Sungei Ujong (Cantley); Penang (Curtis 469). Distrib. Borneo. Native name "Jamba."

2. N. Grifremmin, Rchb. fil. Xen. Orch. II. 215. Rhizome long crecping with long branched woolly roots. Leaves lanceolate petiolate acuminate, 8 inches long $1 \frac{3}{4}$ inch wide, petiole 2 inches long. Raceme 
shorter than the leares $4-5$ inches long dense peduncle short. Flowrers white. Sepals laneeolate pubescent mucronate ${ }_{16}^{3}$ inch long. Petals broader thinner keeled. Lip lanceolate with a raised median bar. Stamens 3 white filaments connate at base. Anther cells at base devaricate. Style longer stigma davate obscurely 3 lobed. ('ilpsule $\frac{1}{4}$ inch long is angled pubeseent. Rolfe. Journ. Isinn. Soe. Hook. fil. Fl. Brit. Ind. VI. p. Bot. Mag. t. \%.425.

Singapore: Sungei Morai (Ridley 23r5); Bukit Timah (Ridley $\$ 896)$; Malacea: Bukit Sadanen (Goodenough 1510), (Griflith, Maingay): Perak: Ulu Bubong (King"s Coll. 11128).

¿. N. Curtisin, Rolfe. Jomrn. Linn. Soc. XXV. 233. t. 48. f. 13. Stem not creeping a loot or more tall acuminate 18 inches long $2-2 \frac{1}{2}$ inch wide. liaceme pubescent t-r) inches long shortly acuminate. Pedicel $\frac{1}{x}$ inch long. Sepals yellow linear lanceolate. P'etals broarder. Ovary +1 inch long, pubescent. Fruit a red succulent berry. T'upistre singapureana, Wall. List 5195. Bak. Journ. Linn. Soc. XIV. 581. Hook. fil. l.c. 325.

Singapore (Wallich), Changi (Ridley); Tohor: Gunong Panti (Ridley +145); Negri Sembilan, Gunong Angsi (Ridley 9998); Perak: Goping (King's C'ollector 601.) : Penang IIill (C'urtis 118.; 2826). Distrib. Sumatra.

\section{Apostisia, Bl.}

Stems slender woody ascending. Leaves linear acuminate sheathing. Inflorescence terminal panicled, or racemose, nodding. Flowers small sessile or shortly pedicelled. Ovary long narrow. Petals and sepals similar ennirent or sometimes recurved. Stamens perfect 2, the third reduced to a staminode or absent. Style slender. Stigma obseurely 3 lobed. Capsule linear.

Species 5 or 6. India, Ceylon, Malay Islands to Australia.

Filament of third stamen persistent .. .. 1. A. Wallichii.

No trace of third stamen.

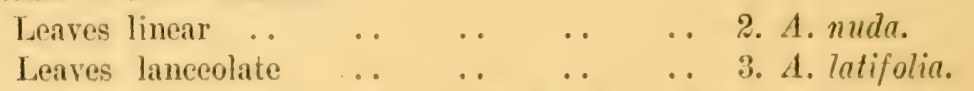

1. A. Wathicini, Br. Wall. Pl. As. Tar. I. \%6. t. S5. Stem fi-s inches tall. Lataves linear acuminate $6-8$ inches long 1 inch wide. Racemes stendre :-?) inches Inng recurved spreading with few or no bracts at the hase. Flowers secured yellow. Floral hadets lanceolate acuminate. Orary and pedieel slender t inch long. Sepals and petal. narrow linear acuminate recurved. Anthers oblong acute.

Johor : Gunong Panti (lidley) ; Pahang: 'Tahan Rivor (Ridley):

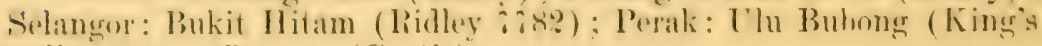
Coll. 10629) ; Penang (Curtis). 
2. A. NUDA, R. Br. Wall. Pl. As. Rarior. I. 1830. 76. t. 85. Stems several or solitary 1-2 feet tall. Leaves linear acuminate 5-8 inches long $\frac{1}{4}-\frac{1}{2}$ inch wide. Panicled decurved 2-4 inches long with numerous linear crowded bracts at the base of the branches. Bracts of flowers lanceolate acuminate $\frac{1}{8}$ inch long. Ovary and pedicel $\frac{1}{4}$ inch long. Sepals and petals white (rarely yellow) $\frac{1}{10}$ inch long lanceolate cuspidate. Stamens filaments short. Anthers basifxed cells equal. Blume. Ann. Sc. Nat. Ser. II. 2. 93. Miq. Fl. Ind. Bat. III. 748. Rolfe. Journ. Linn. Soc. XXV. 239. Hook. fil. Fl. Brit. Ind. VI. A. Brunonis, Griff. Notulae III. 1851. 243. Icones t. 282.

Singapore (Lobb), Changi (Ridley 23\%6), Jurong (Ridley 3871) ; Johor: Bukit Murdom (Kelsall); Negri Sembilan: Perhentian Tinggi (Ridley); Malacea (Maingay), Mount Ophir (Ridley $3133)$; Perak: Dindings, Lumut (Ridley); Penang: Moniot's Road (Ridley); Kedah: Gunong Jerai (Ridley 5125). Common in dry woods up to 3000 feet elevation. Native names "Pelampas budak," "Sen Juan Hutan Jantan" and "Kenching Pelandok." Used medicinally for diarrhoea and hydrophobia.

3. A. Latifolia, Rolfe. Journ. Linn. Soc, XXV. p. 242. Stom 6 inches to 3 feet tall. Leaves lanceolate acuminate petioled 3-6 inches long $\frac{3}{4}-1$ inch wide. Racemes 1-5 inches long. Bracts subulate lanceolate. Ovary sessile. Flowers not secured small. Petals and sepals linear oblong short. Anthers linear cordate, no staminode.

Malacca: Mount Ophir (Ridley); Perak (Scortechini 868), Ulu Batang Padang (Wray 1605). 





\title{
Materials for a Flora of the Malayan Peninsula
}

\author{
by \\ H. N. RIDLEY, M.A., F.R.S., F.L.S., F.R.H.S. \\ Director of Botanic Gardens, \\ Singaporc, S. S.

\section{MONOCOTYLEDONS$$
\text { VOI.I. }
$$ 



\section{Introductory Note.}

The three parts of the Materials for a Flora of the Malay Peninsula dealing with the Monocotyledons were written in the same style and form as the other parts of this work which were brought out in the Journal of the Asiatic Society of Bengal, by Sir George King, aided by other botanists. The first part was published in July 1889 and the work has continued to the present day, being now nearly completed to the end of the Gamopetalae, lcaving now only the Apetalae, to be completed. The three volumes of the Monocotyledons were published respectively on.

\section{Errata Vo1. I.}

P. 18 lines 33. Gnting read Ginting.

75 , 22. Andenopetalum read Adenopetalum.

82,42 . bifidum read semibifidum.

$109 \%$ 5. panciflora read panciflorum.

14. Eri read Eria.

125, 35. Arudina read Arundina.

159 \% 2. Garwailca read Garwalica.

213,24 . Gleiculata read Geniculata.

\section{Addendum.}

Page 84 under Excluded species, mention should have been made of

Cirrhopetaluar longescapun, Teysm. \& Binn. in Tijdschr. Neder. Ind. XXIV. (1862) p. 310. "Penang Lobb."

The description given excludes it from the genus Bulbophyllum and as the type, in the Buitenzorg herbarium has perished, it is impossible to guess what plant was intended. 



\title{
Materials for a flora of the Malayan Peninsula.
}

\author{
I N D F X.
}

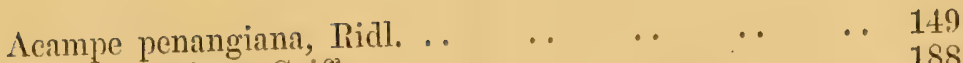
Acriopsis crispa, Griff. .

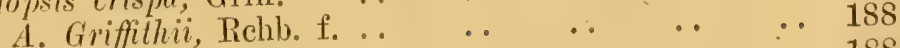

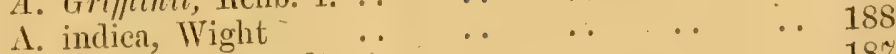

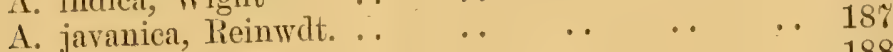

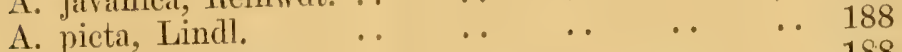

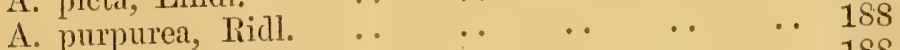

A. Ridlevi, Hook. fil. . $\quad$.. $\quad$.. $\quad$.. $\quad . .188$

Adenoneos major, Ridl. .

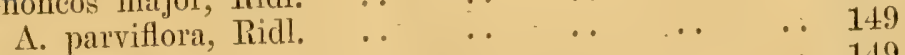

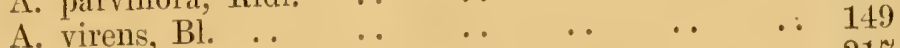

Adenostylis emarginata, Bl. $\quad$.

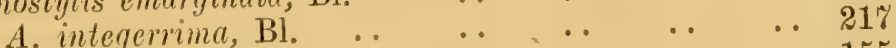

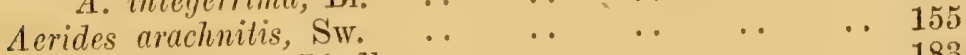

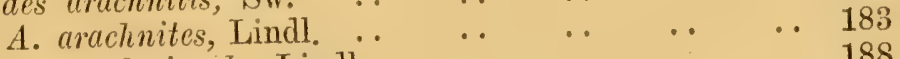

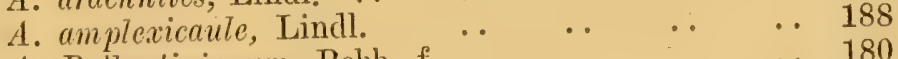

A. Ballantinianum, Rchb. f. .. $\quad$. $\quad$. $\quad$.. 180

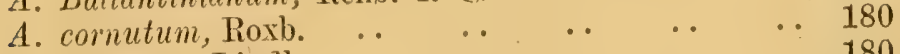

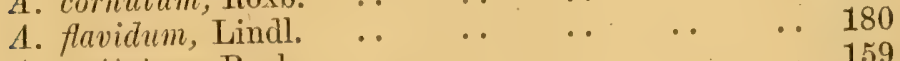

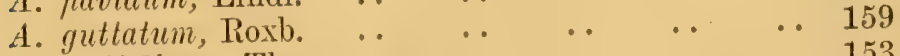

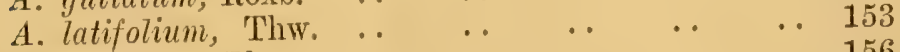

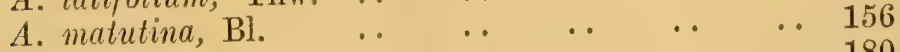

A. nobile, Warn.

A. odoratum, Liur. $\quad \begin{array}{llllll} & \ldots & \ldots & \ldots & \ldots & 179\end{array}$

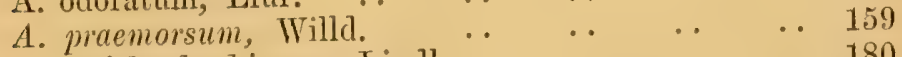

A. Reichenbachianum, Lindl. . . $\quad$. $\quad$. . $\quad$.. 180

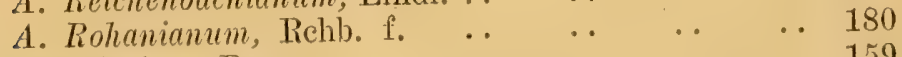

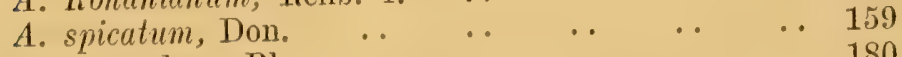

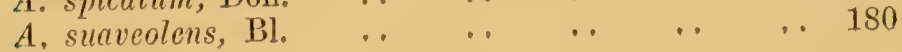


A. suavissimum, Lindl. $\quad \ldots \quad$.

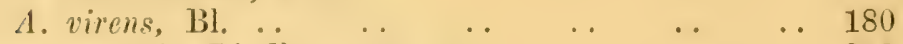
Aetheria mollis, Lindl. $\quad . \quad$. Agrostophyllum callosum, Richb. f. $\quad$. $\quad \ldots \quad \ldots \quad \ldots \quad 108$

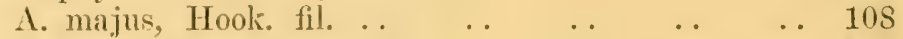

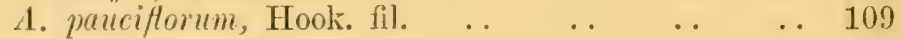
Amblyglottis angustifolia, BI. .

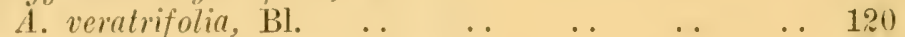

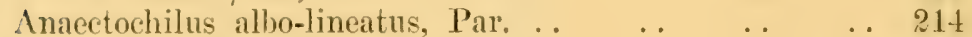

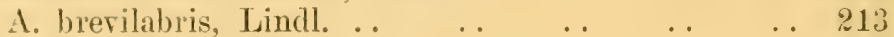

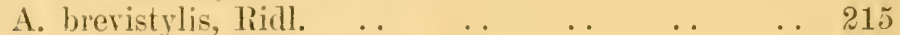

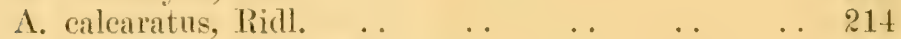

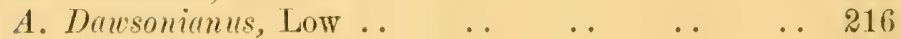

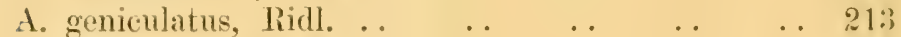

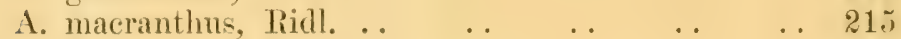

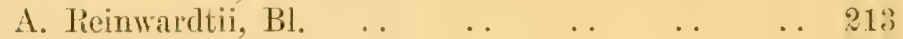

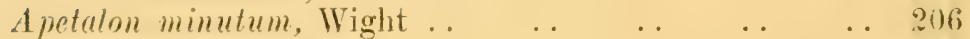

$\begin{array}{lllllll}\text { Aphyllorchis pallida, BI. } & \ldots & \ldots & \ldots & \ldots & \ldots & 20.5\end{array}$

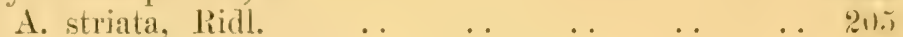

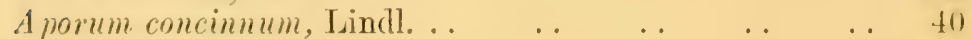

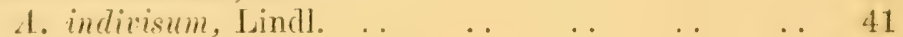

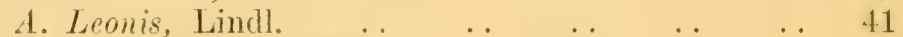

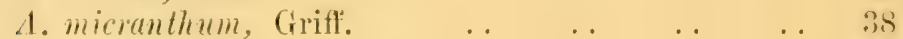

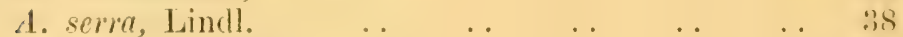

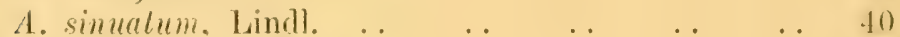

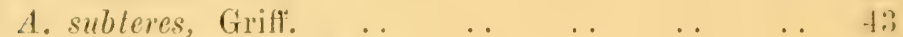

$\begin{array}{llllll}\text { A postasia Brunonis, Grifl. . . } & \text {. } & \text {. } & \text {. } & \text {. } & 238\end{array}$

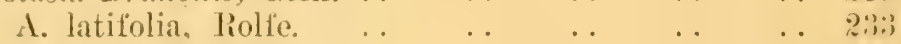

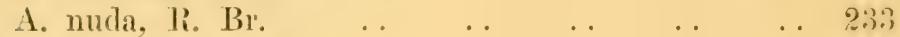

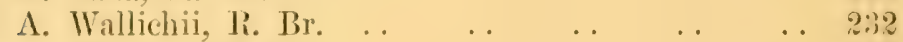

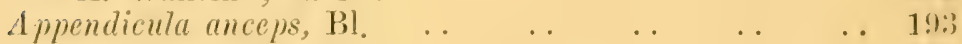

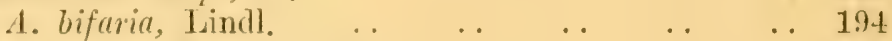

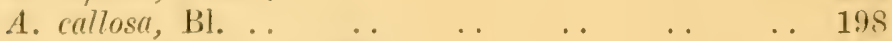

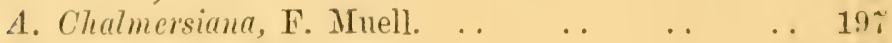

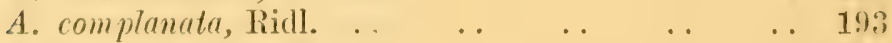

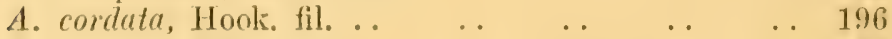

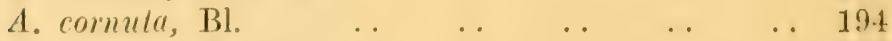

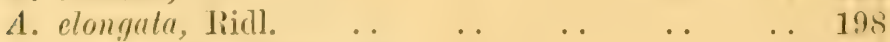

A. echinocarpa, Hook, fil. .. $\quad$.. $\quad$.. $\quad$.. 196

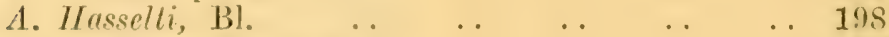

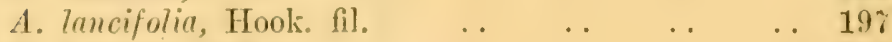

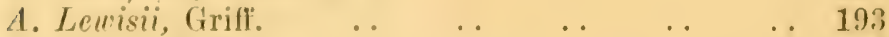

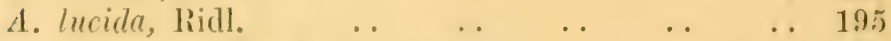

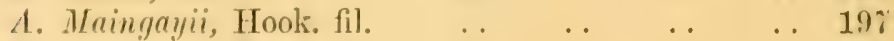

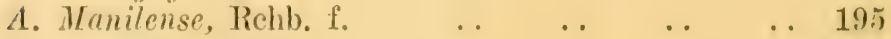

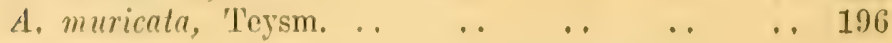


Materials for a Flora of the Malayan Peninsula.

A. pendula, $\mathrm{Bl}$.

A. Peyeriana, Kranzl.

A. purpurascens, De Vr.

A. reduplicata, Rehb. $\mathrm{f}$.

A. reflexa, Bl.

$\begin{array}{llll}\ldots & \ldots & \ldots & \\ \ldots & \ldots & \ldots & \end{array}$

- 194

A. rhodiola, Rehb. f. .

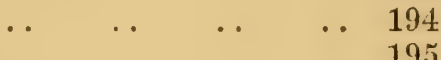

A. rupestris Ridl

1. stipulata, Griff. ..

A. torta, Bl. .. . .

A. uncata, Ridl.

Arachnanthe alba, Ridl.

A. anqustifolia, Hook, fil

A. Maingayi, Ridl.

A. moschifera, $\mathrm{Bl}$.

Arachnis moschifera, $\mathrm{Bl}$.

Arethusa ecristata, Griff.

Arudina bambusifolia, Lindl.

A. Cantleyi, Hook. fil.

A. densa, Lindl.

A. densiflora, Hook. fil.

A. Philippii, Rehb. fil.

A. revoluta, Hook. fil.

A. speciosa, Bl.

A. Wrallichii, Rehb. fil.

Ascochilus hirsutus, Ridl. . .

A. hirtulus, liidl.

A. minutiflorus, Ridl.

A. siamensis, Ridl.

A. teres, Ridl...

Ascotainia penangiana, Ridl.

Bletia angustifolia, Gaud.

B. graminifolia, Don.

Blyxa Griffithii, Hook. fil.

B. Malayana, Ridl.

$\begin{array}{lllll}\cdots & \cdots & \cdots & \cdots & 195 \\ \cdots & \cdots & \cdots & \ldots & 196\end{array}$

$\begin{array}{lllll}\cdots & \ldots & \ldots & \ldots & 19 \%\end{array}$

$\begin{array}{lllll}\text {. } & \ldots & \ldots & \ldots & 194\end{array}$

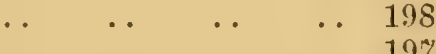

$\begin{array}{lllll}\text {. } & \ldots & \ldots & \ldots & 197\end{array}$

$\begin{array}{lllll}\text {. } & \ldots & \ldots & \ldots & 195\end{array}$

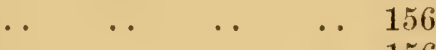

$\begin{array}{llllll} & \ldots & \ldots & \ldots & \ldots & 156\end{array}$

$\begin{array}{lllll}\ldots & \ldots & \ldots & \ldots & 156\end{array}$

. $\quad \ldots \quad \ldots \quad \ldots \quad \ldots 156$

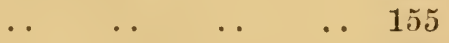

$\begin{array}{lllll}\text {. } & \ldots & \ldots & \ldots & 206\end{array}$

. $\quad \ldots \quad \ldots \quad \ldots \quad \ldots 12 t$

.

.

. $\quad \ldots \quad \ldots \quad \ldots \quad \ldots 12 t$

. $\quad \ldots \quad \ldots \quad \ldots 124$

. $\quad \ldots \quad \ldots \quad \ldots 12 t$

$\begin{array}{lllll}\ldots & \ldots & \ldots & \ldots & 121 \\ & \ldots & \ldots & \ldots & 12 \pm\end{array}$

$\begin{array}{lllll}\cdots & \ldots & \ldots & \ldots & 125\end{array}$

$\begin{array}{lllll}\cdots & \cdots & \ldots & \ldots & 125 \\ \cdots & \ldots & \ldots & \ldots & 169\end{array}$

. $\quad \ldots \quad \ldots \quad \ldots 1 \% 8$

. $\quad \ldots \quad \ldots \quad \ldots 1 \% 9$

$\begin{array}{lllll}\ldots & \ldots & \ldots & \ldots & 178\end{array}$

$\begin{array}{lllll}\ldots & \ldots & \ldots & \ldots & 1 \% 8\end{array}$

$\begin{array}{lllll}\ldots & \ldots & \ddots & \ldots & 116\end{array}$

$\begin{array}{lllll}\ldots & \ldots & \ldots & \ldots & 11 \%\end{array}$

$\begin{array}{lllll}\ldots & \ldots & \ldots & \ldots & 112\end{array}$

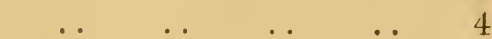

․ . $\quad \ldots \quad \ldots 144$

B. aporoides, Rehb. f... . $\quad \ldots \quad \ldots \quad \ldots 145$

B. brevifolia, Ridl. .. $\quad \ldots \quad \ldots \quad \ldots \quad \ldots 145$

B. Finlaysoniana, Rehb f. .. $\quad \ldots \quad \ldots \quad \ldots \quad \ldots 14$

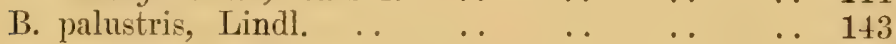

B. pungens, Ridl. . . $\quad \ldots \quad \ldots \quad \ldots \quad \ldots \quad \ldots 145$

B. rupestris, Ridl. . $\quad \ldots \quad \ldots \quad \ldots \quad \ldots \quad \ldots 14$

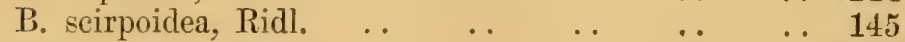

B. sylvestris, Ridl. . $\quad \ldots . \quad \ldots \quad \ldots . \quad \ldots 144$

Bulbophyllum acuminatum, Ridl. . $\quad \ldots \quad \ldots \quad \ldots \quad \ldots 81$

B. adenopetalum, Lindl. $\quad \ldots \quad \ldots \quad \ldots \quad \ldots \quad \ldots 75$

B. alcicorne, Par. \& Rchb, .. . . .. .. 70 
$\begin{array}{lllllll}\text { B. avicella, Ridl. } & \ldots & \ldots & \ldots & \ldots & \ldots & 6 \%\end{array}$

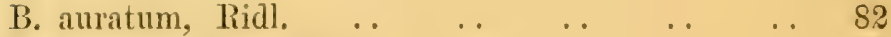

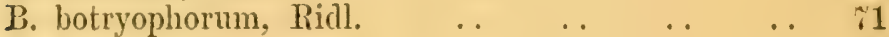

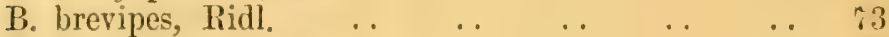

$\begin{array}{llllll}\text { B. breviscapum, Ridl. } & \ldots & \ldots & \ldots & \ldots & \text { is }\end{array}$

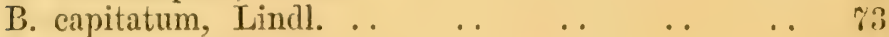

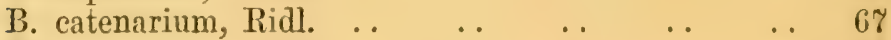

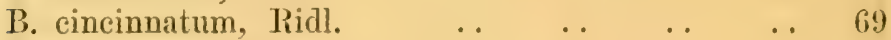

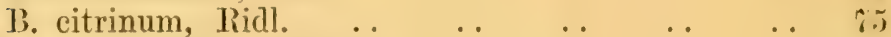

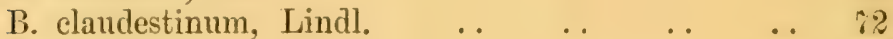

$\begin{array}{llllll}\text { B. cleistogamum, Ridl. } & \ldots & \ldots & \ldots & \ldots & \end{array}$

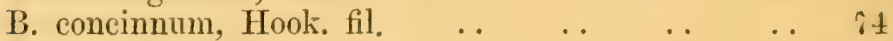

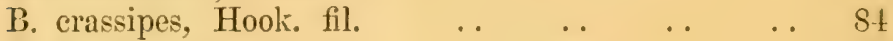

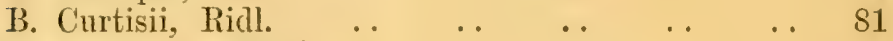

B. densiflorum, Ridl. $\quad . \quad$.

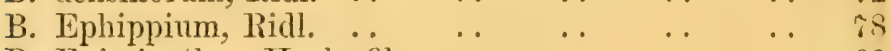

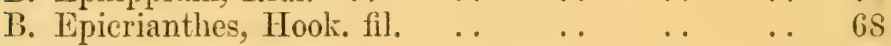

B. flammuliferum, Ridl. $\quad \ldots \quad$.

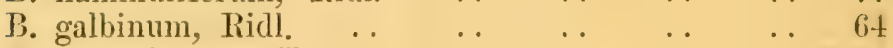

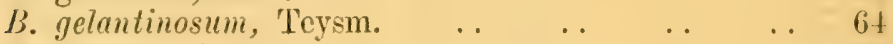

$\begin{array}{lllllll}\text { B. gigas, Ridl. } & \ldots & \ldots & \ldots & \ldots & \ldots & \text { r } 0\end{array}$

B. globulus, Hook. fil. $\quad \ldots \quad$.

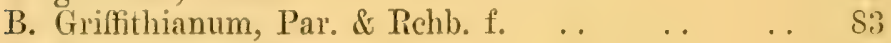

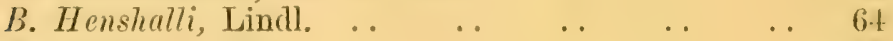

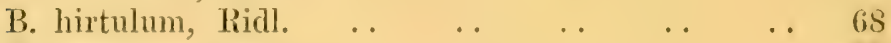

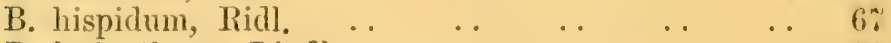

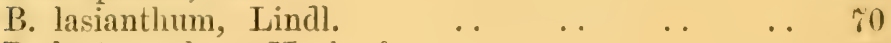

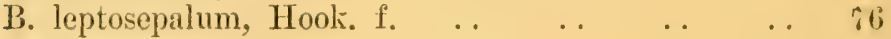

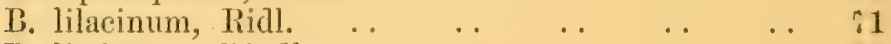

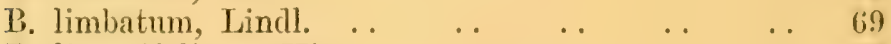

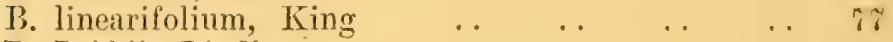

B. Lobbii, Lindl. $\quad \ldots \quad$.

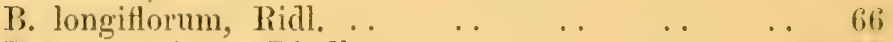

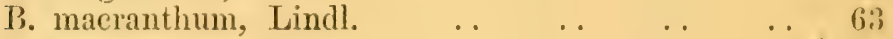

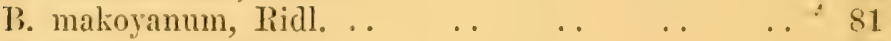

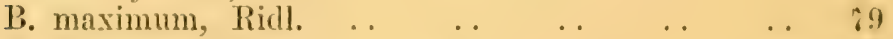

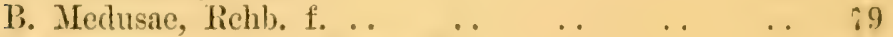

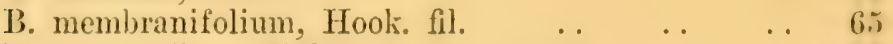

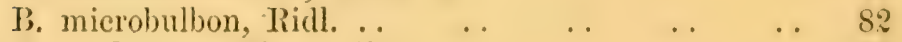

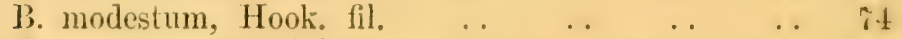

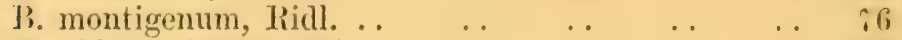

l3. oblanceolatum, $\begin{array}{llllll}\text { King } & \ldots & \ldots & \ldots & \ldots & \text { is }\end{array}$

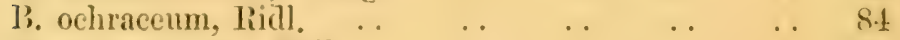

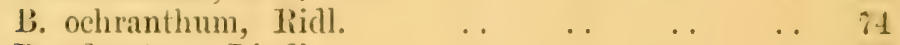

B. odoratum, Lindl. $\ldots$.

13. patens, Hook. fil, .. 
Materials for a Flora of the Malayan Peninsula.

B. perakense, Ridl. $\quad \ldots \quad$.

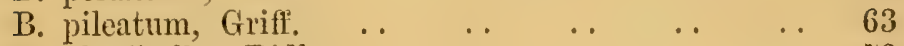

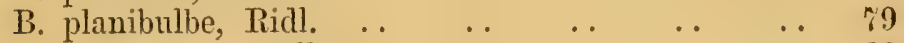

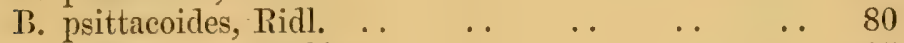

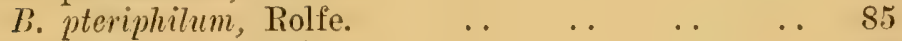

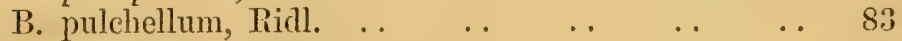

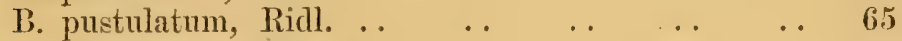

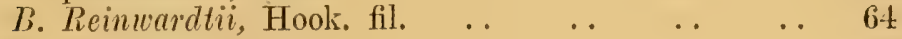

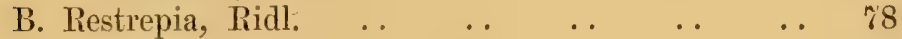

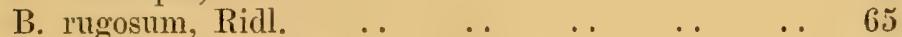

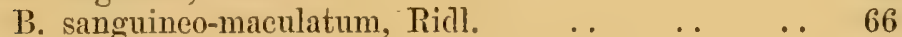

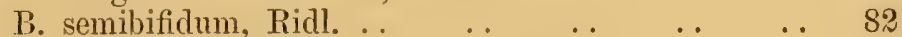

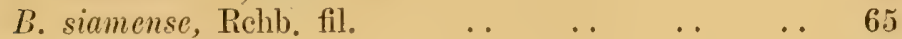

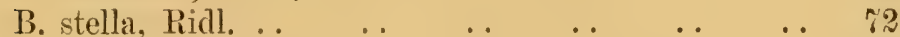

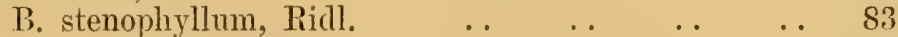

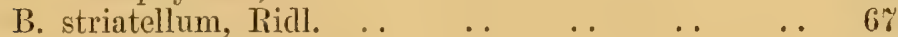

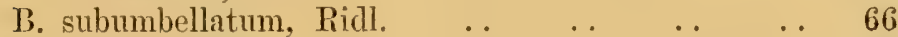

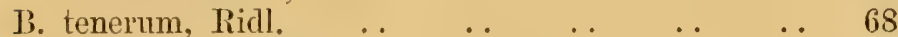

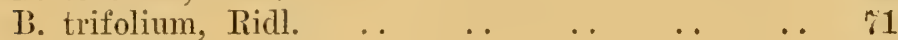

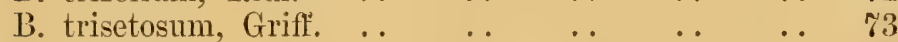

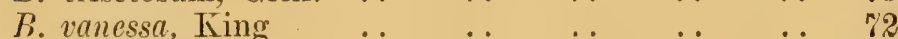

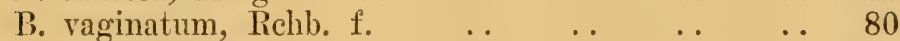

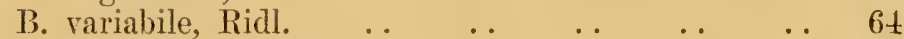

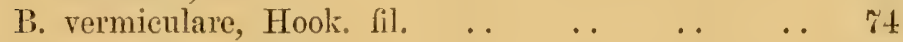

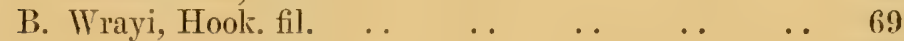

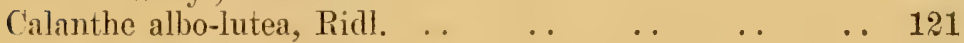

$\begin{array}{llllll}\text { C. angustifolia, Lindl. } & \ldots & \ldots & \ldots & \ldots & 121\end{array}$

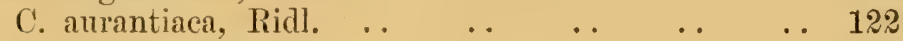

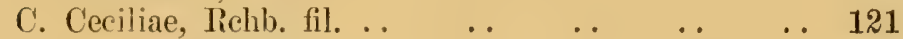

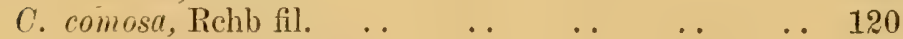

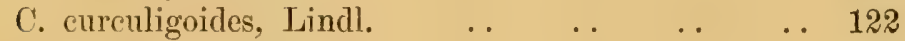

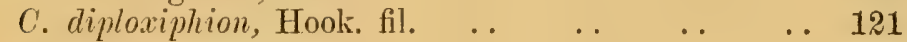

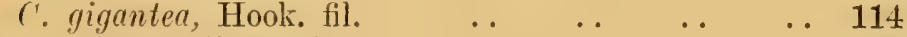

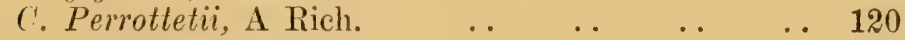

r. phajoides, Rchb. f. . $\quad$. $\quad$. $\quad$.. $\quad$.. 120

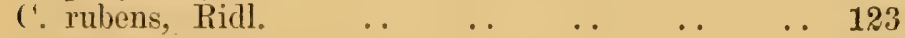

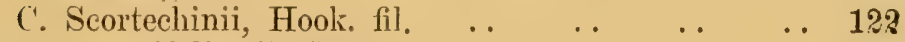

$\begin{array}{llllll}\text { (. veratrifolia, R. Br. } & \ldots & \ldots & \ldots & \ldots & 120\end{array}$

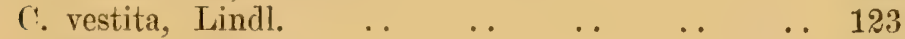

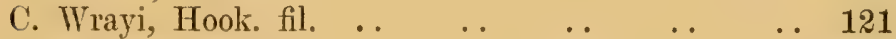

$\begin{array}{lllllll}\text { Calcearia fornicata, Bl. } & \text {. } & \text {. } & \text {. } & \text {. . } & \text {.. } & 205\end{array}$

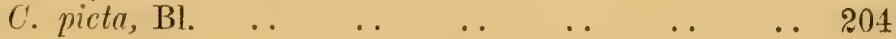

$\begin{array}{lllllll}\text { Callostylis rigida, } \mathrm{Bl} . & \text {. } & \text {. } & \text {. } & \text {.. } & \text {.. } & 106\end{array}$

$\begin{array}{lllllll}\text { Caulinia ovalis, } \mathrm{R}, \mathrm{Br} . & \ldots & \ldots & \ldots & \ldots & \ldots & 6\end{array}$

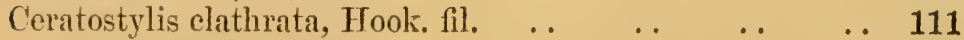

C. eryptantha, Ridl, .. . . . . $\quad$.. $\quad$.. 110 
C. eriacoides, Hook. fil.

C. gracilis, Bl... .

C. Iancifolia, Ilook. fil.

C. malaccensis, Hook. fil.

. 109

C. pendula, Hook. fil. .

C. puncticulata, Ridl.

C. robusta, Hook. fil.

C. teres, Rehb. fil.

Chaeradoplectron spiranthes, Schau.

Chelonanthera speciosa, Bl.

Ch. sulphurea, Bl.

.. 110

.. 110

. 111

. 111

.. 111

. 110

Chrysoglossum villosum, $\mathrm{Bl}$.

Cirrhopetalum acuminatum, Ridl.

C. Andersonii, Kurz. .

C. auratum, Lindl.

C. bifidum, Ridley

C. Blumei, Lindl.

C. breviscapum, Rolfe.

C. Brienianum, Rolfe .

C. caudalum, Wight ..

C. concinnum, Hook. fil

C. gamosepalum, Grill.

C. gracillimum, Rolfe

C. linearifolium, Ridl.

C. Makoyanum, Rehb. fil

C. maximum, Ridl.

C. Medusae, Lindl.

C. microbulbon, Ridl. .

C. ochraceum, Ridl.

C. planibulbe, Ridl.

C. psillacoides, Ridl. .

C. vaginatum, Lindl

Claderia viridiflora, Hook. fil

Cleisostoma cristatum, Ridl.

C. fuscum, Lindl.

C. ionosma, Ridl.

C. Tatifolium, Lindl . .

C. parvum, Ridl.

('. spiralum, Lindl.

C. tenuicuule, King

C. uteriferum, Hook. fil.

Cnemidia angulosa, Lindl. .

C. semilibera, Lindl.

Coeloglossum acuminalum, Lindl. C. lacertiferum, Lindl. 
Coelogne anceps, Hook. fil. . .

. 133

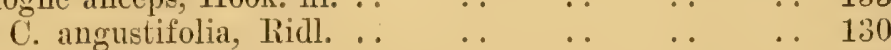

C. articulata, Rehb. fil. . $\quad \ldots \quad \ldots \quad \ldots 136$

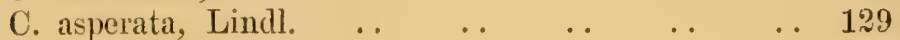

C. carnea, Hook. fil. . $\quad \ldots \quad \ldots \quad \ldots \quad \ldots \quad \ldots 134$

C. casta, Ridl. .. .. $\quad$. $\quad \ldots \quad \ldots \quad \ldots 130$

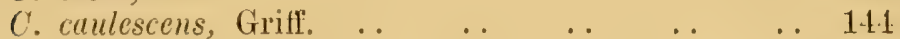

C. Cumuigi, Lindl. $\quad \ldots \quad$.

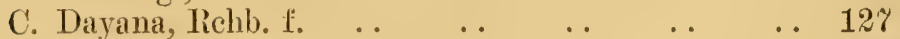

C. densiflora, Ridl. $\quad \ldots \quad \ldots \quad \ldots \quad \ldots \quad \ldots 128$

C. Foerstermannii, Rehb. fil. . . . . . . $\quad$.. 130

C. imbricata, Rchb. fil. $\quad \ldots \quad \ldots . \quad \ldots \quad \ldots 135$

C. Khasiyana, Rchb. fil. _. $\quad \ldots \quad \ldots \quad \ldots 136$

C. Kingii, Hook, fil. ... . . . $\quad \ldots \quad \ldots 130$

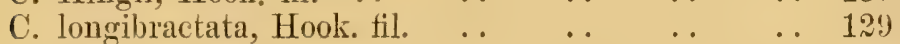

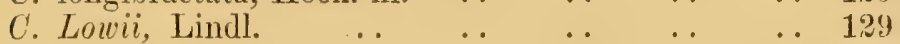

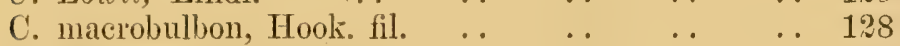

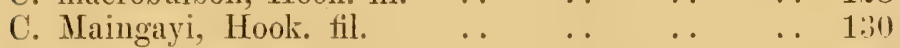

C. Massangeana, Rehb. fil. $\quad \ldots \quad \ldots \quad \ldots \quad \ldots 1 \%$.

C. Mayeriana, lichb. fil. . $\quad \ldots \quad \ldots \quad \ldots \quad \ldots 130$

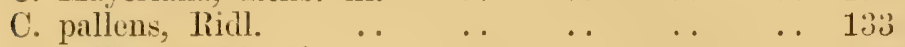

C. pallida, Rehb. fil. . . . . . . $\quad \ldots \quad \ldots 13 \tilde{}$

C. pandurata, Lindl. .. $\quad \ldots \quad \ldots \quad \ldots \quad \ldots \quad \ldots 131$

C. prasina, Ridl. $\ldots \quad \ldots \quad \ldots \quad \ldots \quad \ldots 133$

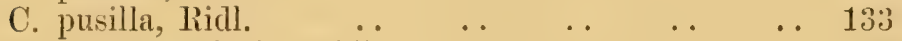

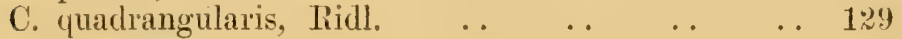

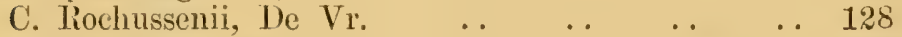

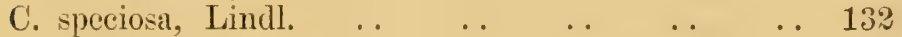

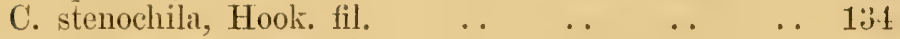

C. sulphurea, Richb. fil. _. $\quad \ldots \quad \ldots \quad \ldots 132$

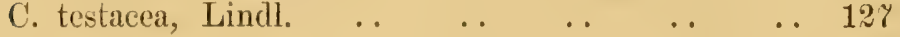

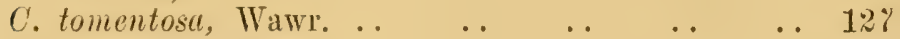

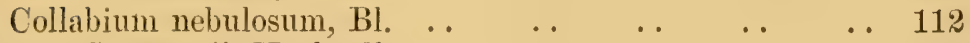

C. Wrayii, Hook. fil. .. $\quad \ldots \quad \ldots \quad \ldots \quad \ldots \quad \ldots 189$

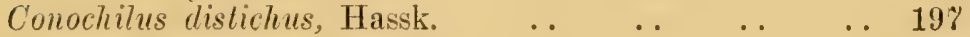

Corymbis brevistylis, Hook. fil. . . $\quad \ldots \quad \ldots \quad \ldots \quad \ldots 208$

C. longiflora, Hook, fil. . $\quad \ldots \quad \ldots 208$

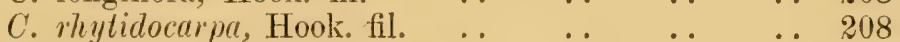

Corysanthes fornicata, Lindl. . . $\quad \ldots \quad \ldots \quad \ldots 204$

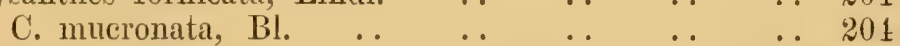

C. picta, Lindl. $\quad \ldots \quad \ldots \quad \ldots \quad \ldots \quad \ldots 204$

Cryptoglottis serpyllifolia, Rehb. fil. $\quad \ldots \quad \ldots \ldots 193$

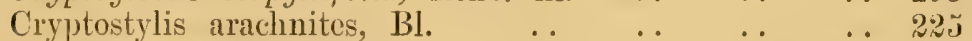

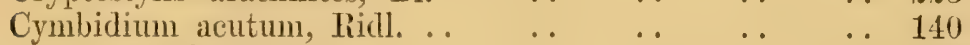

C. alatum, Roxb. $\quad . . \quad \ldots \quad \ldots \ldots 190$

C. bambusifolium, Roxb. . . . . . . . 124 
C.. Finlaysonianum, Lindl.

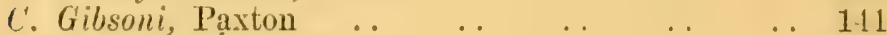

$\begin{array}{llllll}\text { C. Huttoni, Hook. fil. .. } & \text {. } & \text {.. } & \text {.. } & \text {.. } & 111\end{array}$

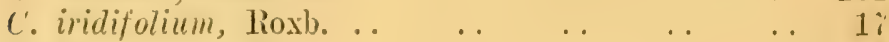

$\begin{array}{lllllll}\text { C. Javanicum, Bl. } & \text {.. } & \text {.. } & \text {.. } & \text {. } & \text {. } & 111\end{array}$

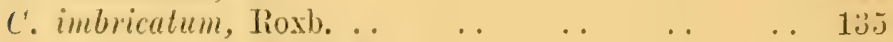

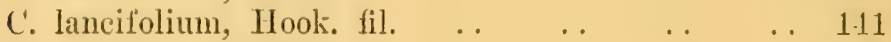

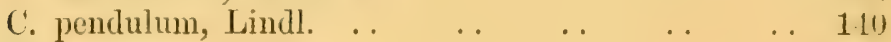

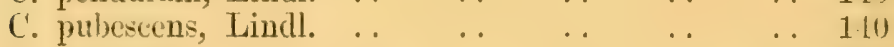

$\begin{array}{llllllll}\text { C. scriptum, Sw. } & \ldots & \ldots & \ldots & \ldots & \ldots & 113\end{array}$

$\begin{array}{lllllll}\text { C. s'lephensi, Ridl. } & \ldots & \ldots & \ldots & \ldots & \ldots & 141\end{array}$

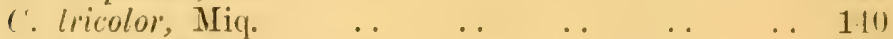

$\begin{array}{llllllll}\text { C. triste, Willd. } & \text {. } & \text {. } & \text {.. } & \text {.. } & \text {.. } & 118\end{array}$

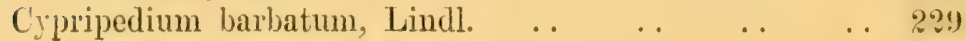

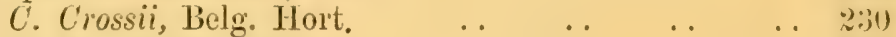

$\begin{array}{lllllll}\text { C. cruciforme, } \mathrm{Loll} . & \ldots & \ldots & \ldots & \ldots & \ldots & 230\end{array}$

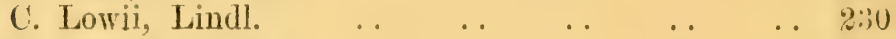

C. nivem, Rehb.

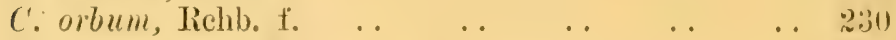

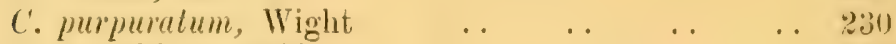

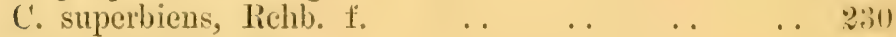

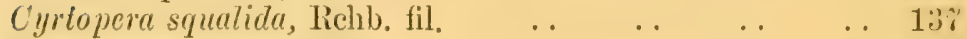

$\begin{array}{lllllll}\text { Cystorchis aphylla, Ridl. } & \ldots & \ldots & \ldots & \ldots & \ldots & \text { 21: }\end{array}$

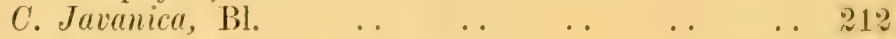

$\begin{array}{lllllll}\text { C. rariegata, Bl. } & \ldots & \ldots & \ldots & \ldots & \ldots & 21 \%\end{array}$

$\begin{array}{lllllll}\text { Cyrlosia altissima, Bl. } & \ldots & \ldots & \ldots & \ldots & \ldots & 90 \%\end{array}$

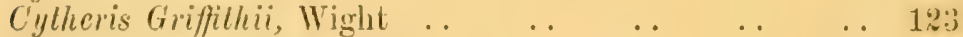

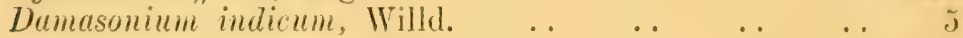

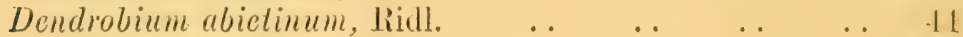

D. acerosum, Lindl. $\quad \ldots \quad$.

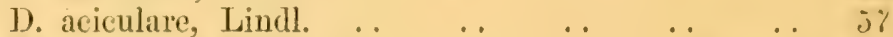

D. aduncum, Hook. fil. . $\quad \ldots \quad \ldots, \ldots . j 6$

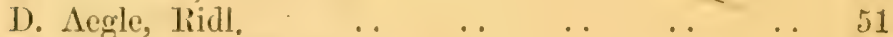

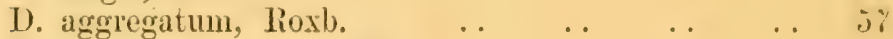

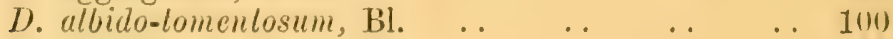

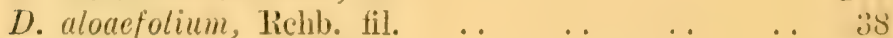

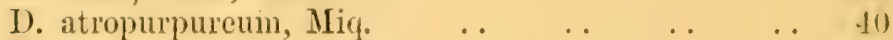

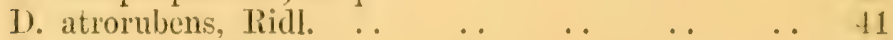

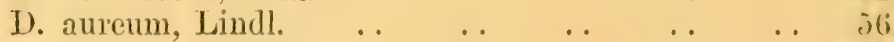

I), bilarim, Lindl. $\quad$.

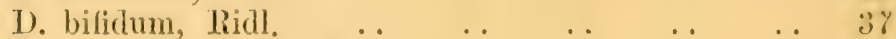

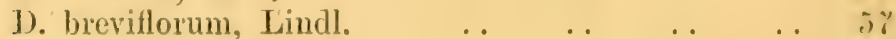

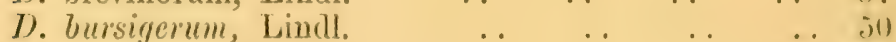

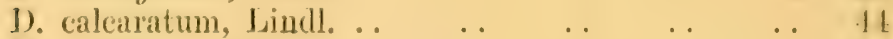

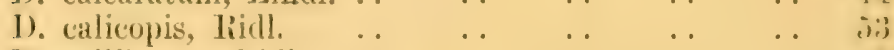

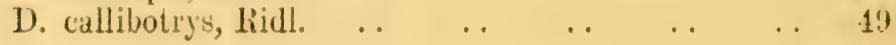




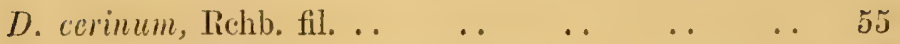

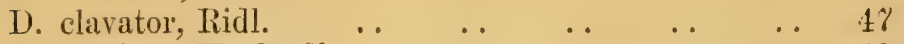

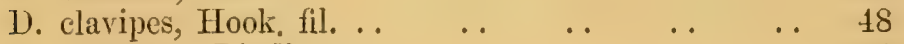

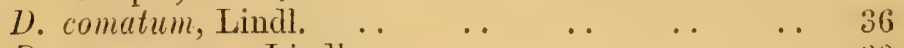

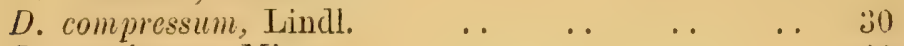

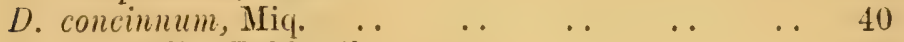

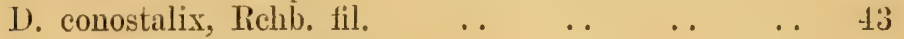

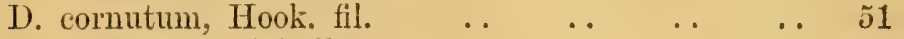

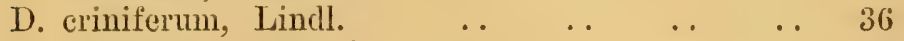

D. crocatum, Hook. fil. $\quad \ldots \quad$.

D. crumenatum, Sw.

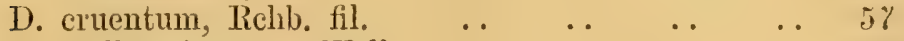

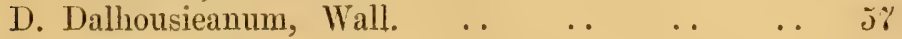

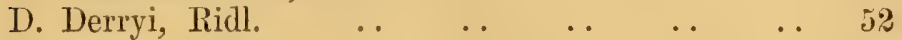

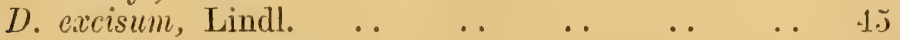

$\begin{array}{llllll}\text { D. eulophotum, Lindl. } & \ldots & \ldots & \ldots & \ldots & 39\end{array}$

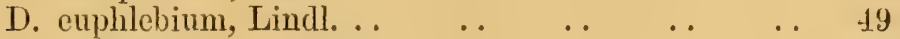

D. Flabellum, Rehb. fil. $\quad \ldots \quad$.

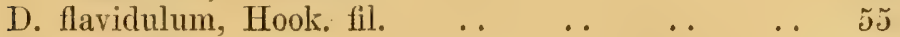

$\begin{array}{lllllll}\text { D. flexile, Ridl. } & \text {. } & \text {. } & \text {. } & \ldots & \ldots & 42\end{array}$

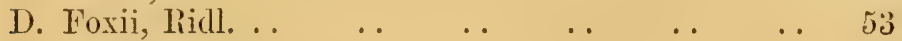

D. gemellum, Lindl. $\quad \ldots \quad$.

D. geminatum, Hook. fil. $\quad \ldots \quad$.

D. gracile, Lindl. $\quad \ldots \quad$.

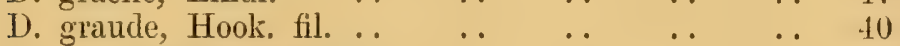

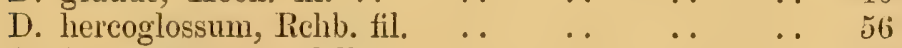

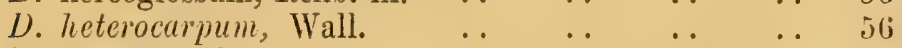

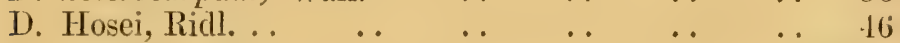

D. Hughii, Rehb. fil. . . . $\quad$. $\quad \ldots \quad$.

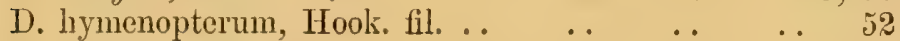

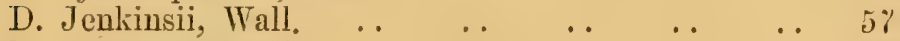

D. junceum, Lindl. $\quad \ldots \quad$.

D. Kelsallii, Ridl. $\quad \ldots \quad$.

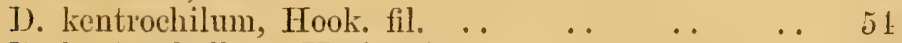

1. kentrophyllum, Hook. fil. .. $\quad$. $\quad$. $\quad$.. 4 4?

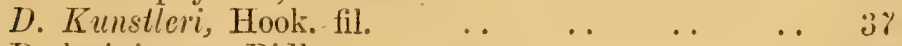

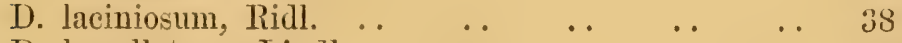

$\begin{array}{llllll}\text { D. lamellatum, Lindl. } & \ldots & \ldots & \ldots & \ldots & 50\end{array}$

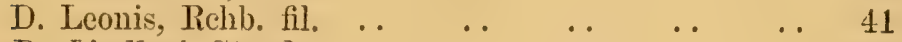

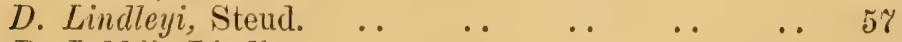

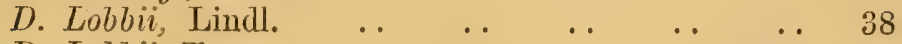

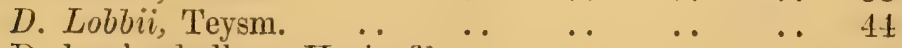

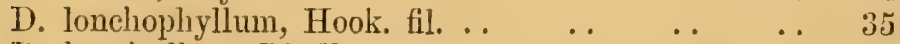

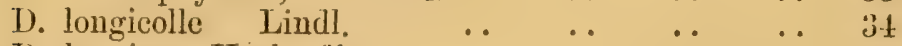

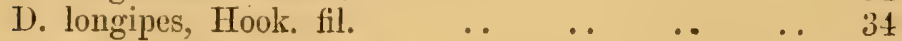

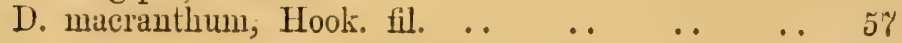


1). macropodum, Hook. fil. . $\quad \ldots \quad \ldots \quad \ldots \quad \ldots \quad 31$

D. macrophyllum, Lindl. $\quad \ldots \quad \ldots \quad \ldots \quad \ldots \quad$ ji

D. Hanillense, Schau.

D. Mannii, Ridl.

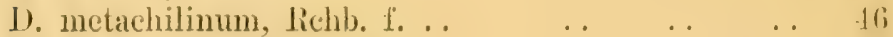

1) megaceras, Hook. fil. . $\quad \ldots$.. $\quad \ldots$ jt

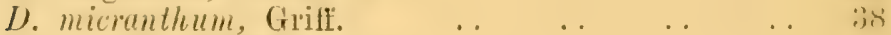

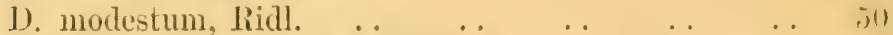

D. pachyglossum, Par. \& lichb. fil. . . . . . . 11

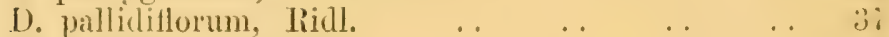

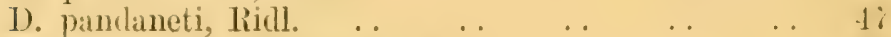

D. menduriferum, var. Hook, fil. . . . . . j?

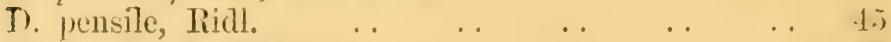

1). perakense, Hook. fil. . . . . . . $\quad \ldots$ (9!)

D. pumilum, Hook. fil. . . . . . . . jis

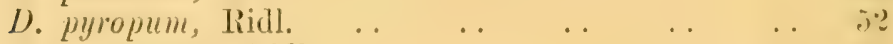

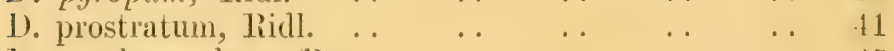

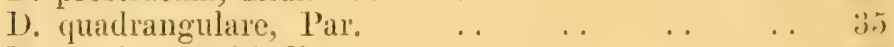

I). revolutum, Lindl. $\ldots \begin{array}{llllll} & \ldots & \ldots & \ldots & \ldots & 16\end{array}$

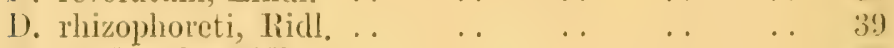

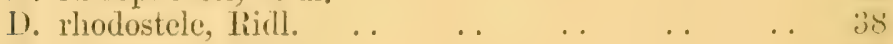

D. rombeum, Lind. .. $\quad \ldots \quad \ldots \quad \ldots \quad \ldots$ it;

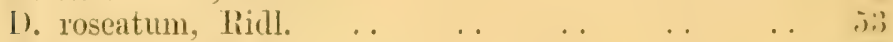

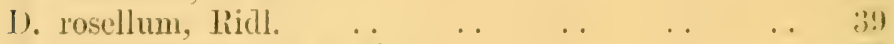

1). roseopunctatum, lidal. $\ldots \quad \ldots \quad \ldots \quad \ldots 3 \%$

1). sanguinolentum, Lindl. $\quad \ldots \quad \ldots \quad \ldots \quad \ldots \quad \ldots$,.j

1). Scortechinii, Hook. fil. . . . . . . . ji

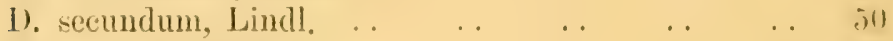

1). serpens, Hook. fil. . . . $\quad \ldots \quad \ldots \quad \ldots \quad \ldots$ i)

1). serra, Lindl.

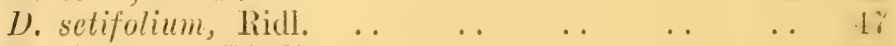

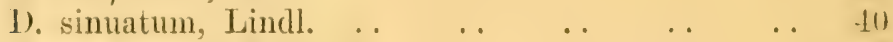

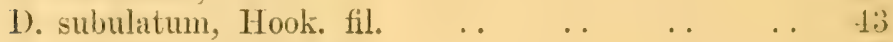

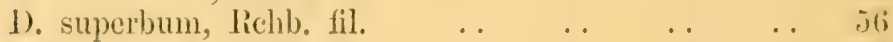

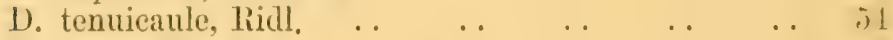

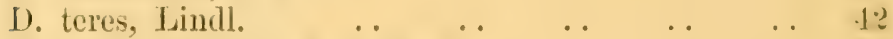

1). terminale, Par, \& lichb. fil. $\quad \ldots \quad \ldots \quad \ldots \quad$.j,

1). tropoeolitlorum, Hook. fil. . . . . . . . . il

1). tuberiferum, Hook, fil. . . . . . . . . 18

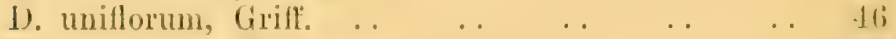

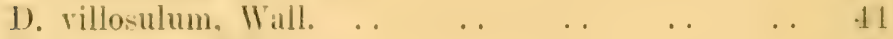

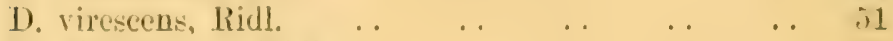

D. viridicatum, lídl. . $\quad \ldots \quad \ldots \quad \ldots \quad \ldots l$ i.j

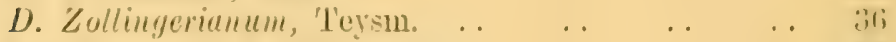

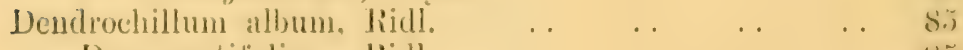

D. angustilolium, lídl. $\ldots \quad \ldots \quad \ldots \quad \ldots \quad$ s 
Materials for a Flora of the Malayan Peninsula.

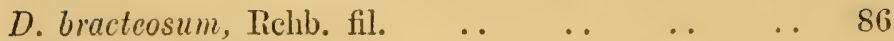

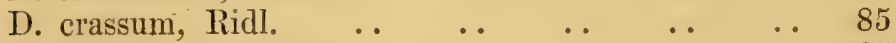

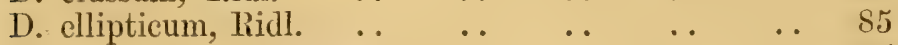

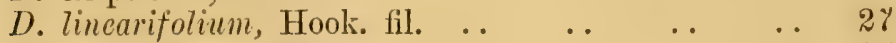

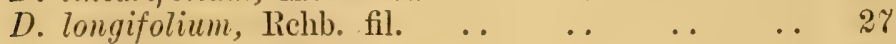

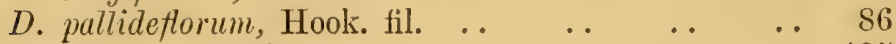

Dendrocolla alba, Ridl. $\quad \ldots \quad$.

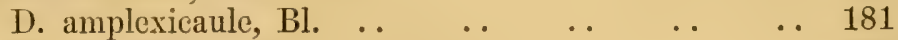

$\begin{array}{lllllll}\text { D. arachnites, Bl. } & \text {. } & \text {. } & \text {. } & \text {. } & \text {. } & 183\end{array}$

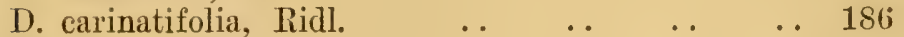

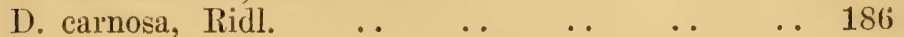

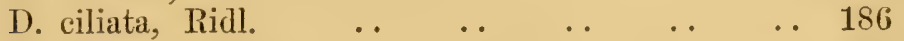

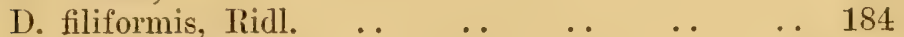

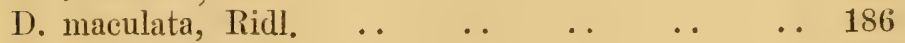

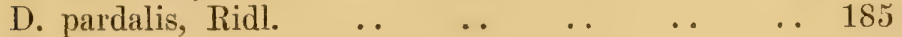

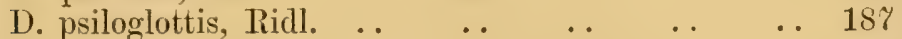

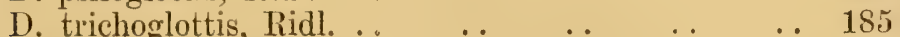

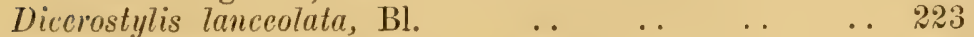

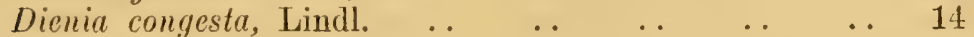

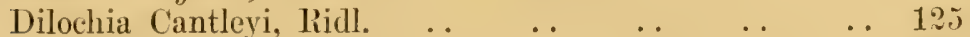

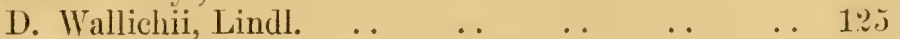

$\begin{array}{lllllll}\text { Diphyes capitata, BI. } & \ldots & \ldots & \ldots & \ldots & \ldots & 9\end{array}$

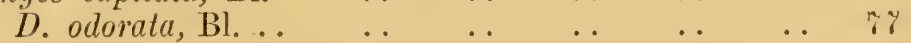

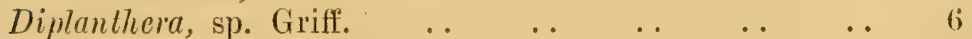

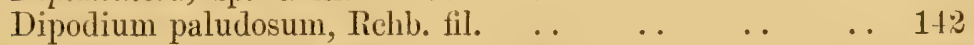

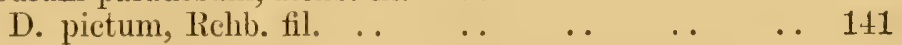

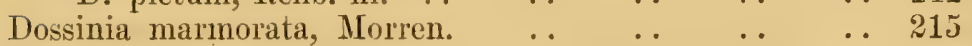

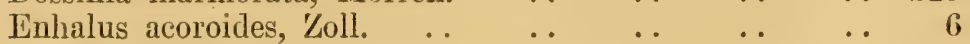

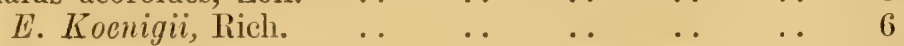

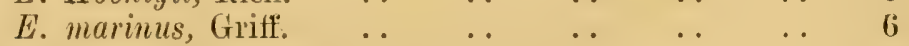

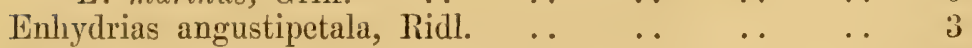

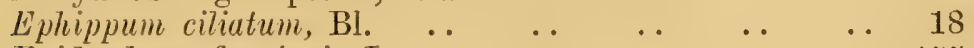

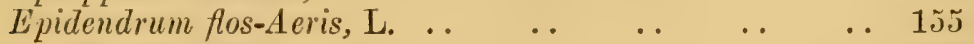

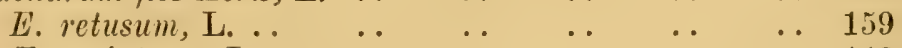

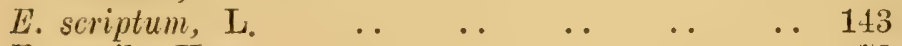

$\begin{array}{lllllll}\text { E. sessile, Koen. } & \ldots & \ldots & \ldots & \ldots & \ldots & \text { \%3 }\end{array}$

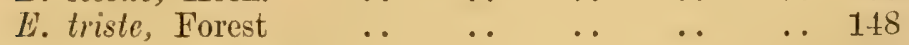

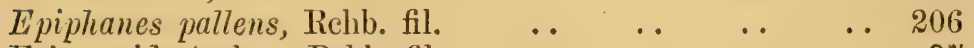

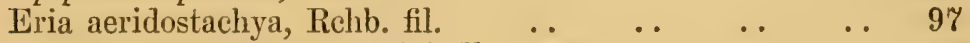

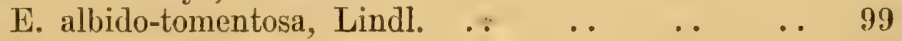

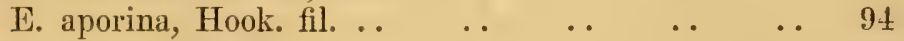

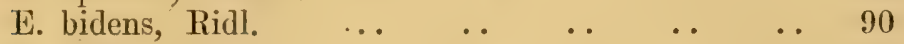

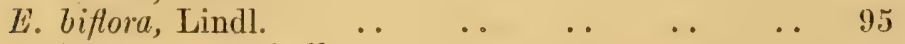

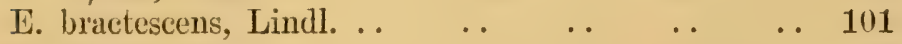

$\begin{array}{lllllll}\text { E. brunea, Ridl. } & \ldots & \ldots & \ldots & \ldots & \ldots & 98\end{array}$

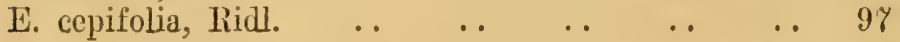




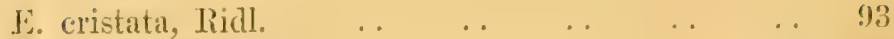

E. dasystachys, Ridl. .

$\begin{array}{lllllll}\text { 1. densa, Ridl. } & \ldots & \ldots & \ldots & \ldots & \ldots & 96\end{array}$

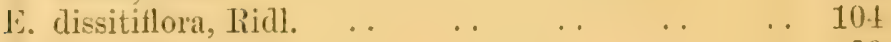

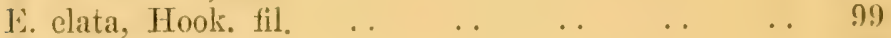

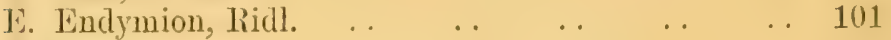

E. ferox, Bl. $\ldots$.

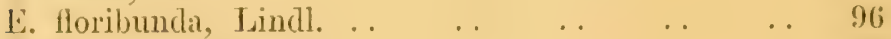

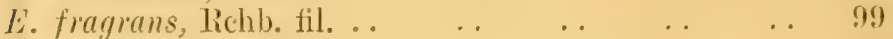

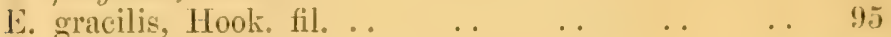

$\begin{array}{llllll}\text { E. hispidissima, Ridl. } & \ldots & \ldots & \ldots & \ldots & 94\end{array}$

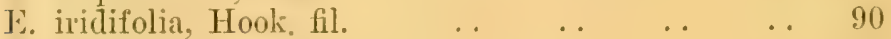

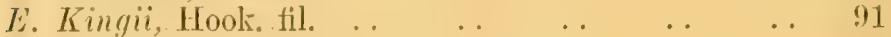

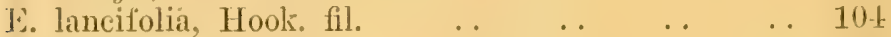

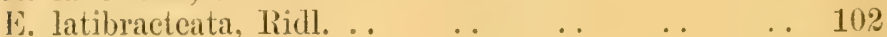

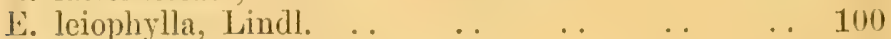

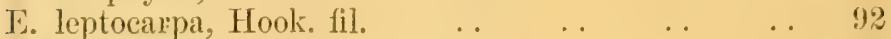

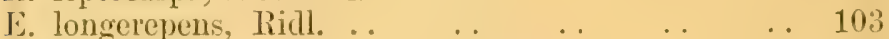

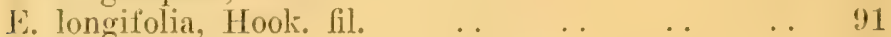

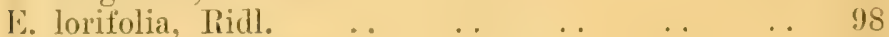

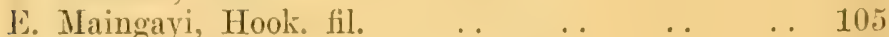

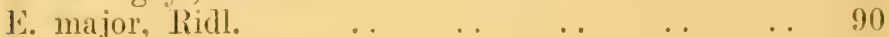

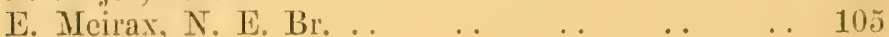

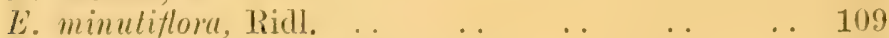

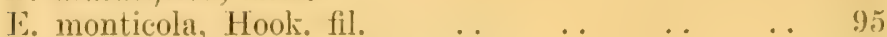

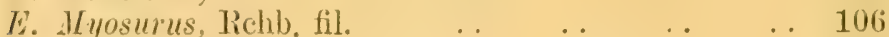

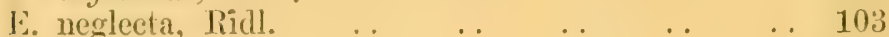

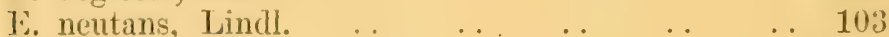

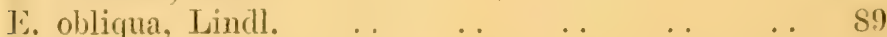

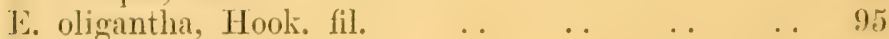

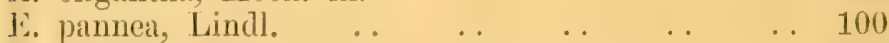

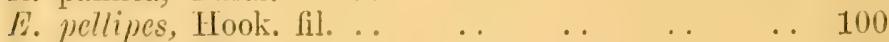

$\begin{array}{lllllll}\text { T. pendula, Ridl. } & \text {. } & \text {. } & \text {.. } & \text {.. } & \text {.. } & 92\end{array}$

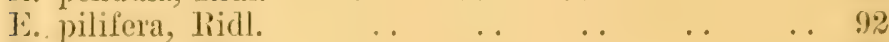

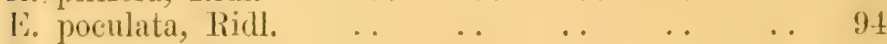

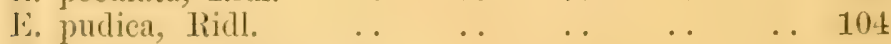

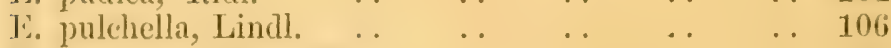

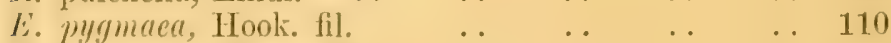

li. recurvata, Hook. fil. $\quad$. . . . $\quad$.. $\quad$.. 102

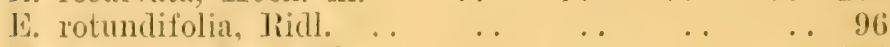

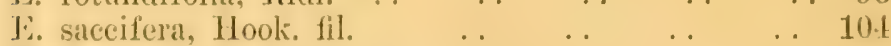

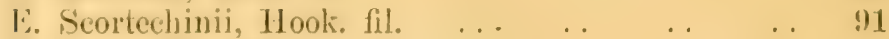

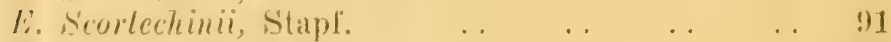

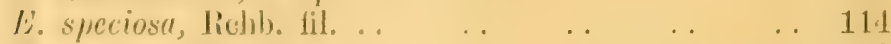

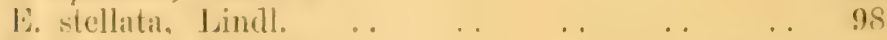

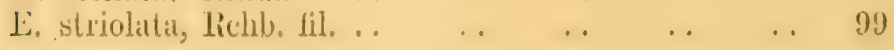


Materials for a Flora of the Malayan Peninsula.

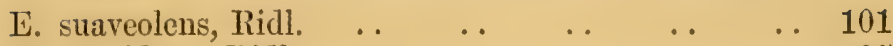

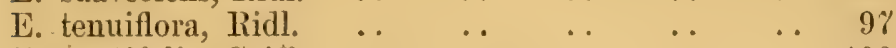

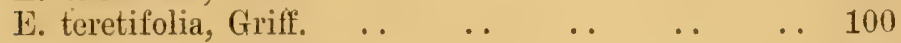

E. tuberosa, Hook. fil. $\quad$.

$\begin{array}{lllllll}\text { E.. velutina, Lindl. } & \ldots & \ldots & \ldots & \ldots & \ldots & 94\end{array}$

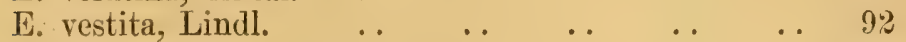

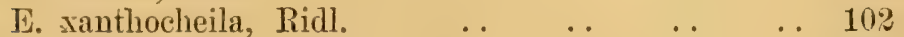

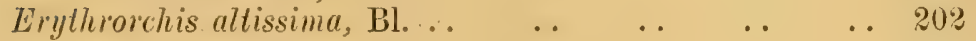

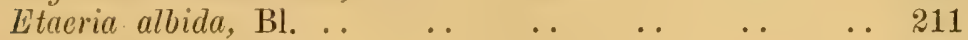

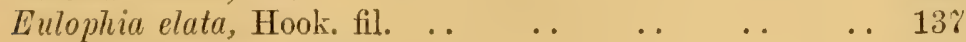

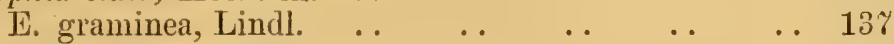

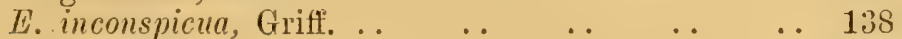

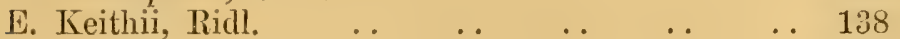

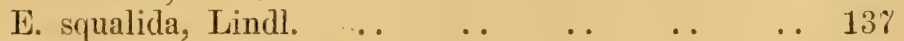

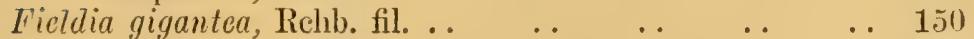

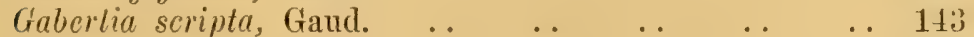

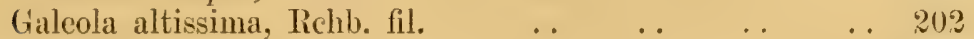

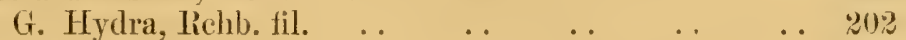

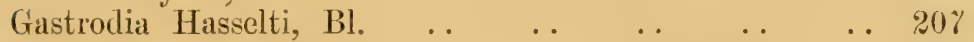

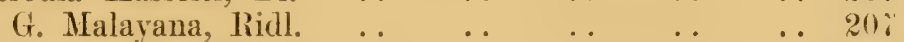

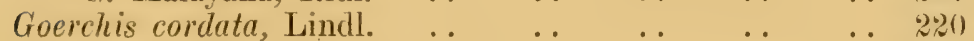

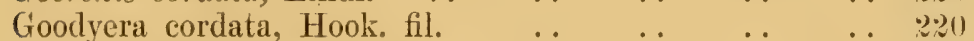

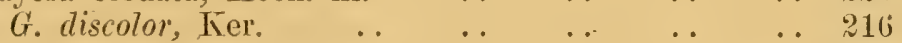

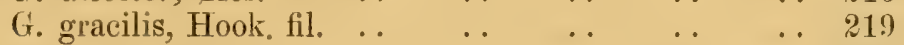

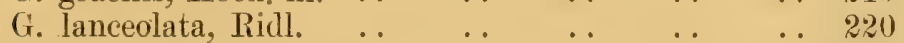

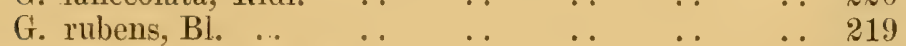

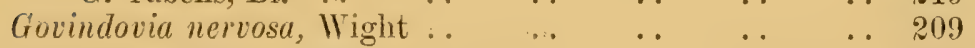

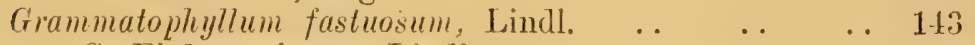

G. Finlaysonianum, Lindl. . . $\quad$. . $\quad$. . $\quad$. . 144

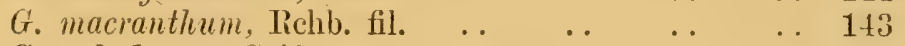

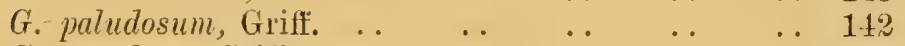

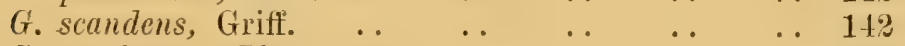

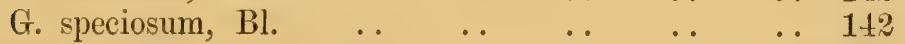

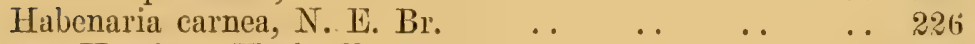

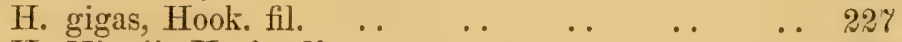

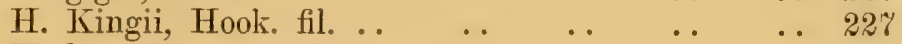

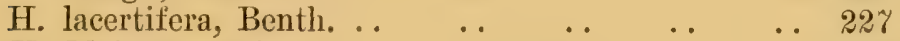

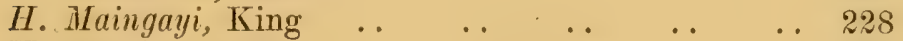

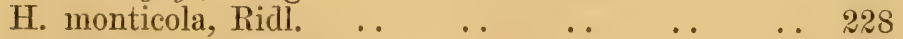

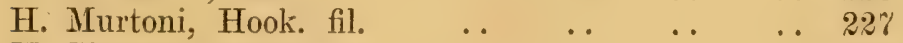

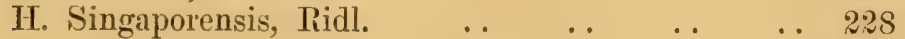

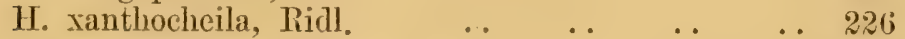

H. zosterostyloides, Hook. fil. $\quad$. $\quad$. $\quad$. . 226

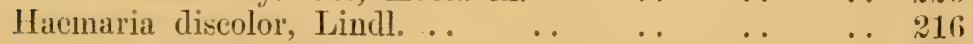

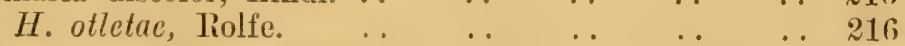

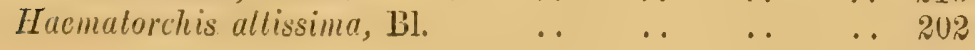


Halophila ovalis, Hook. fil. . .

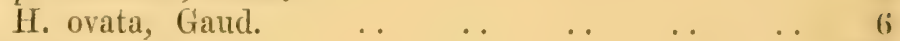

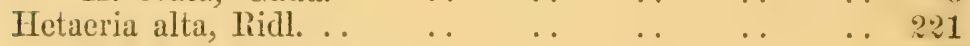

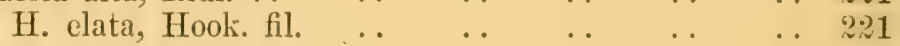

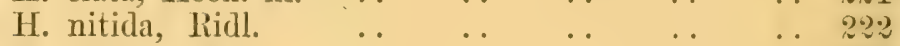

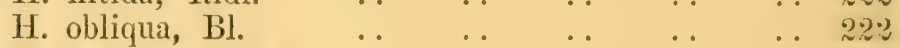

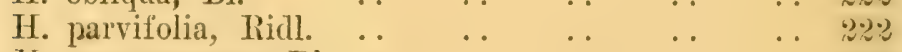

H. purpurascens, Bl. .. $\quad \ldots \quad$. . $\quad \ldots \quad$. . 218

Hydrilla verticillata, Presl. $\quad \ldots \quad \ldots \quad \ldots \quad \ldots \quad \ldots \quad$;

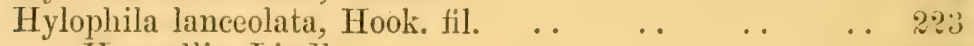

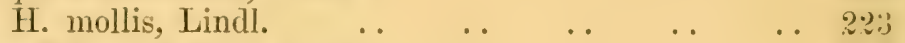

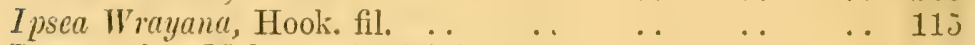

Lecanorchis Malaccensis, Ridl. . . $\quad \ldots \quad \ldots \quad \ldots \quad \ldots 206$

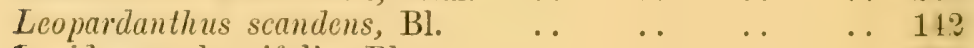

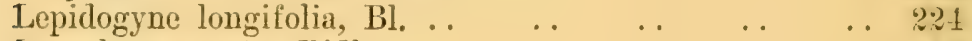

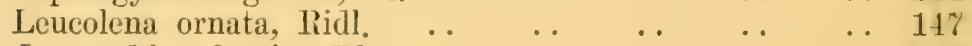

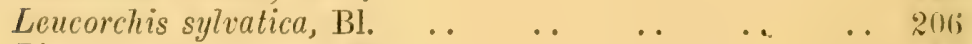

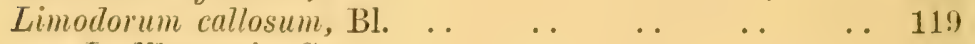

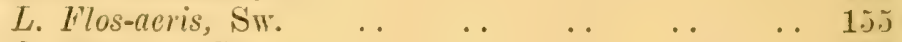

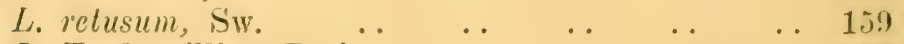

L. Tankervilliae, Roxb. _. . . . . $\quad \ldots 119$

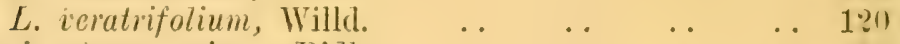

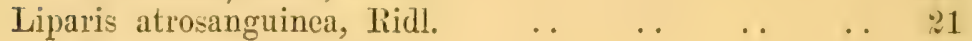

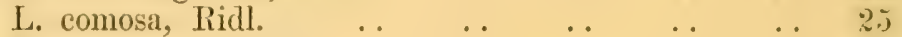

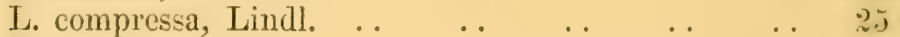

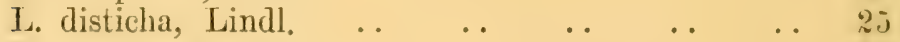

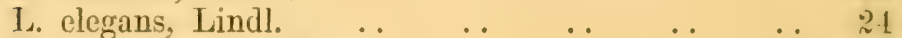

L. ferruginea, Lindl. $\ldots \quad \ldots \quad \ldots \quad \ldots \quad \ldots \quad \ldots$

L. flaccida, Richb. fil. $\quad \ldots \quad \ldots \quad \ldots \quad \ldots \quad \ldots \quad \ldots$

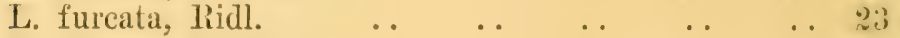

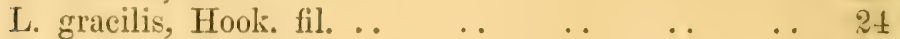

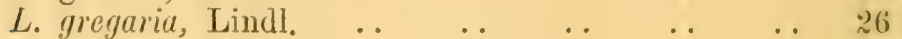

L. lacerata, Ridl. . $\quad \ldots \quad \ldots \quad \ldots \quad \ldots 2 t$

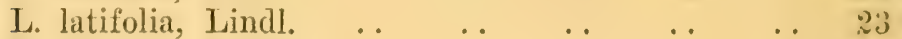

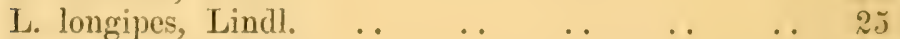

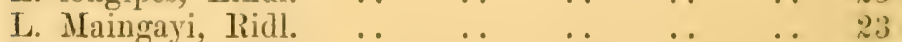

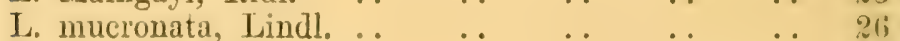

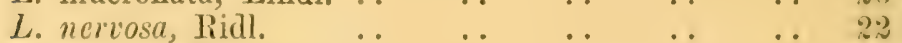

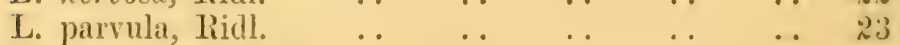

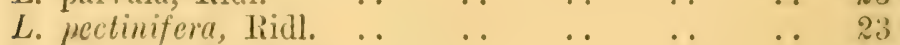

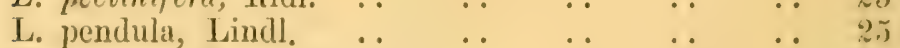

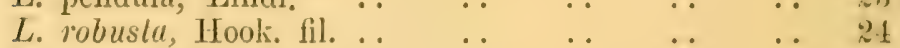

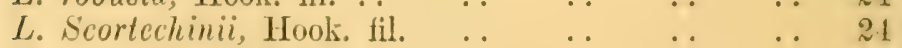

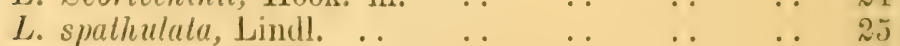

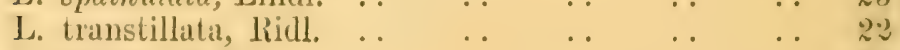

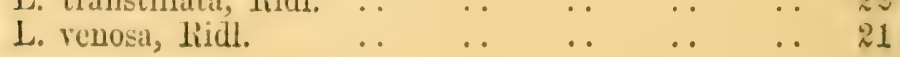


L. Wrayi, Hook, fil Ludisia discolor, Rich. Luisia antennifera, Bl.

L. bracbystachys, Bl. .

L. tristis, Hook. fil. Macodes Petola, Lindl.

Malaxis anceps, Rehb. $\mathrm{f}$

II. compressa, BI.

M. disticha, Thouars. .

M. iridifolia, Rehb. fil.

M. Tatifolia, Sm.

M. lunata, Bl. . .

II. miniata, Rchb. f. .

M. mucronata, Bl.

M. plicata, Roxb.

Mesoclastes brachystachys, Lindl. . Metachitum cyathiferum, Lindl.

Microsaccus javensis, Bl.

II. virens, Hook. fil.

Microstylis acutangula, Hook. fil. .

M. Bernaysii, F. Nuell

M. calophylla, Rehb. f.

M. furcata, Hook.

II. fusca, Rehb. fil.

M. Maingayi, Hook. fil.

M. micrantha, Hook. fil.

M. parvula, Hook. fil.

M. perakensis, Ridl.

M. plantaginea, Hook. fil.

M. Scottii, Hook. fil.

M. trilobulata, Kurz. .

Mitopetalum speciosum, Bl.

Monochilus affine, LindI.

. . . . .

.. 216

.. 148

.. 147

.. 148

.. 215

.. 18

.. 25

.. 26

.. 17

.. 14

.. 16

.. 18

.. 26

.. 14

. 148

.. 193

.. 172

.. 149

.. 14

.. 14

.. 13

.. 23

.. 14

.. 23

.. 14

.. 23

. 13

.. 14

. 13

. 14

. 114

. 218

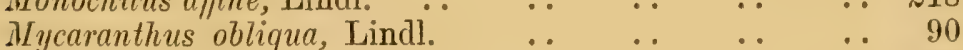

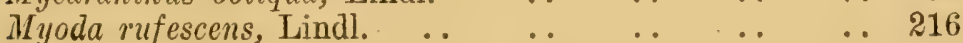

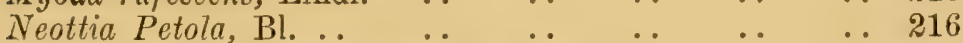

Nephelaphyllum grandiflorum, Hook. fil. $\quad \ldots \quad \ldots 2115$

$\begin{array}{lllllll}\text { N. pulchrum, Bl. } & \ldots & \ldots & \ldots & \ldots & \ldots & 116\end{array}$

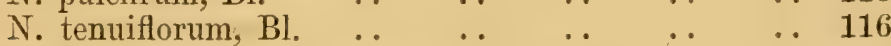

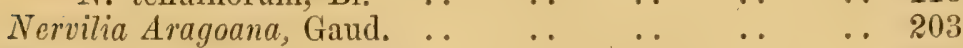

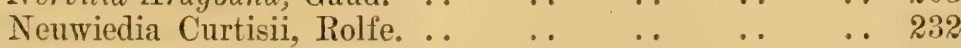

N. Griffithii, Rehb. f. .. $\quad$.. $\quad$.. $\quad$.. $\quad$.. 231

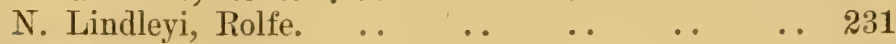

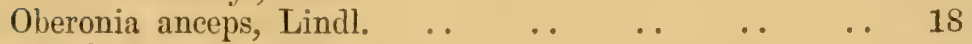

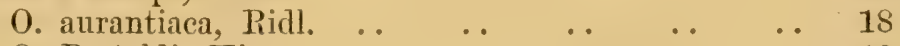

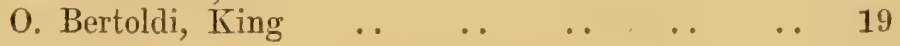


O. biaurita, Hook. fil. .. . . . . . . . . " 16

O. brunnescens, Ridl. . $\quad \ldots \quad \ldots \quad \ldots \quad \ldots \quad \ldots 19$

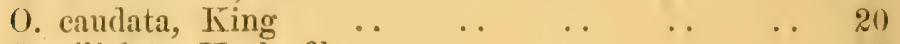

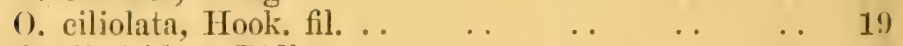

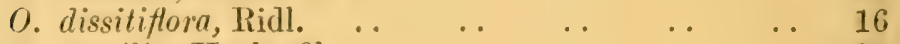

O. gracilis, Hook. fil. .. $\quad \ldots \quad \ldots \quad \ldots \quad \ldots \quad \ldots 20$

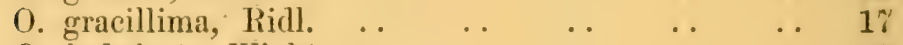

O. imbricata, Wight .. $\quad \ldots \quad \ldots \quad \ldots \quad \ldots \quad \ldots \quad 18$

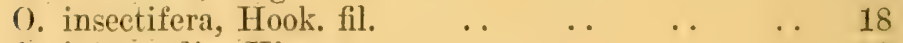

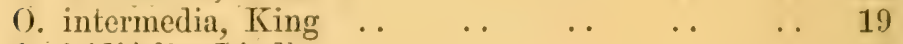

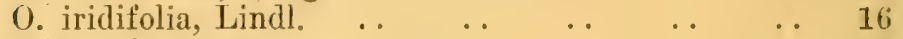

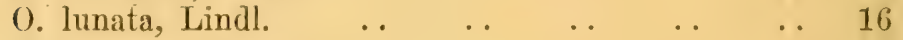

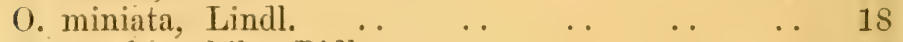

O. porphyrochila, Ridl. $\quad$. $\quad \ldots \quad \ldots \quad \ldots \quad \ldots \quad 19$

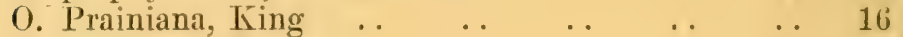

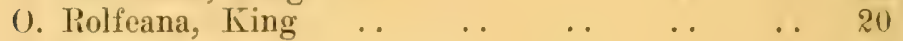

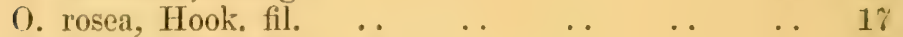

(). Scortechinii, Hook. fil. . $\quad \ldots \quad \ldots \quad \ldots \quad \ldots 1 \%$

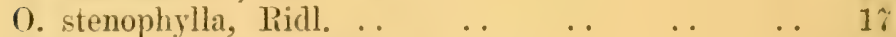

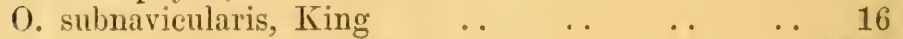

Odontochilus brevistylis, Hook. fil. _ . . $\quad \ldots \quad \ldots 214$

O. calcaratus, Hook. fil. . . . . . . . 214

O. macranthus, Hook. fil. . . . . . . 215

O. pectinatus, Hook. fil. $\quad \ldots \quad \ldots \quad \ldots \quad \ldots \quad \ldots 214$

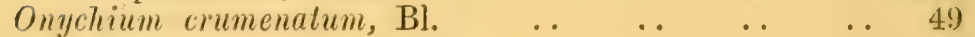

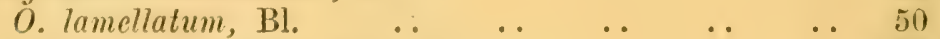

Orsidice amplexicaulis, Rehb. f. $\quad \ldots \quad$. . $\quad \ldots \quad \ldots \quad \ldots 181$

O. lilacinus, Rehb. fil. . $\quad \ldots \quad \ldots \quad \ldots \quad \ldots \quad \ldots 181$

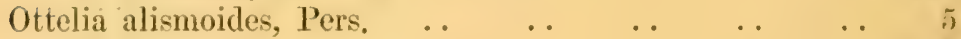

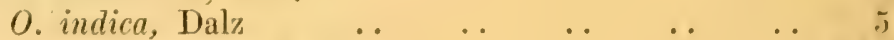

Oxyanthera carinata, Schlecht $\quad \ldots \quad \ldots \quad \ldots \quad \ldots \quad \ldots 200$

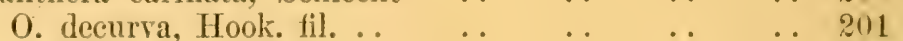

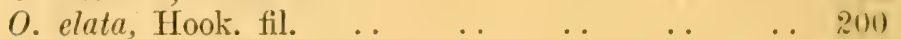

O. mierantha, Brngn. .. $\quad \ldots \quad \ldots \quad \ldots \quad \ldots \quad \ldots 200$

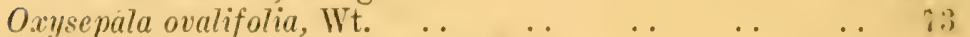

Oxystophyllum atropurpureum, Bl. $\quad \ldots \quad \ldots \quad \ldots \quad \ldots \quad$. 10

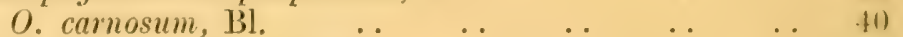

O. macrostoma, Hassk. $\ldots$. . $\quad \ldots \quad \ldots$ is

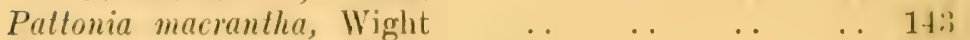

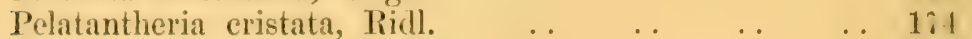

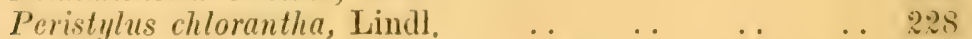

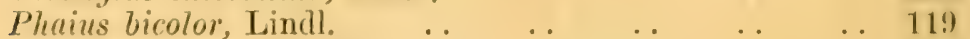

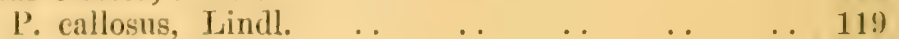

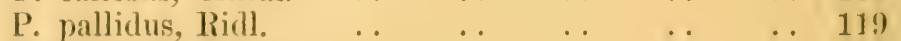

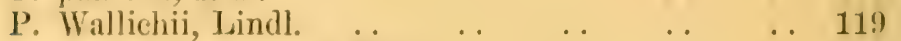

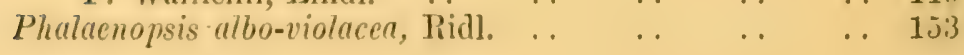


Materials for a Flora of the Matayan Peninsula.

$P$. antennifera, Rechb. fil.

P. Buyssoniana, Rehb. fil.

. 153

P. cornu-cervi, Par.

P. Denisiana, Coyn. ..

P. Esmeralda, Rehb. fil.

P. fugax, Kranzl.

P. muscicola, Ridl.

P. sumatrana, Korth. .

P. valentini, Rehb. fil.

$\mathrm{P}$. violacea, Teysm.

P. zebrina, Teysm.

Phalidota articulata, Lindl.

$P$. decurva, Ridl.

$\mathrm{P}$. gibbosa, De $\mathrm{Vl}$.

P. Griffithii, Hook. fil.

P. imbricata, Lindl.

P. Khasiana, Rehb. fil.

P. micrantha, Hook. fil.

$P$. pallida, Lindl.

Phreatia listrophora, Ridl.

P. minutiflora, Lindl...

P. myosurus, Lindl.

P. nana, Hook. fi

Physurus latifolius, Bl.

Platyclinis gracilis, Hook. fil.

P. Kingii, Hook. fil. . .

P. linearifolia, Ridl. ...

P, linearis, Ridl. . . .

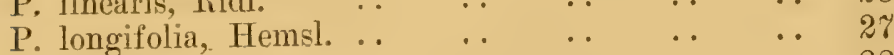

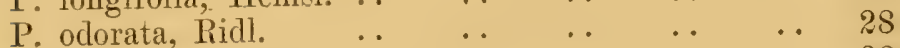

$\begin{array}{llllll}\text { P. Sarawakensis, Ridl. } & \ldots & \ldots & \text {. } & \text {. } & 28\end{array}$

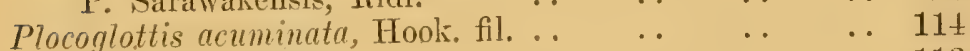

$\begin{array}{lllllll}P \text {. foetida, Ridl. } & \text {. } & \text {. } & \text {. } & \ldots & \ldots & 113\end{array}$

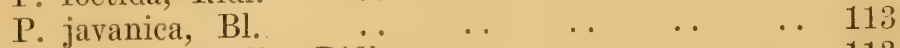

P, porphyrophylla, Ridl. . $\quad$. $\quad$. $\quad$. 113

Poaeophyllum pauciflorum, Ridl. .. $\quad$.. $\quad$.. $\quad$.. 109

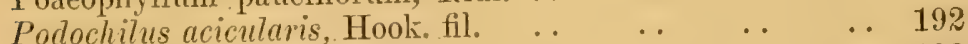

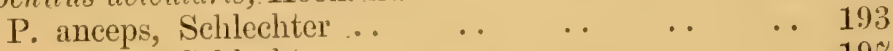

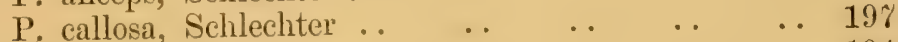

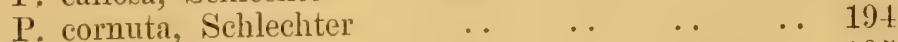

P. densifolia, Ridl. .

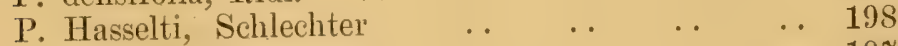

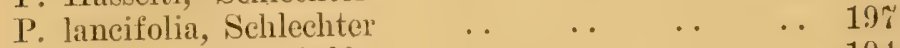

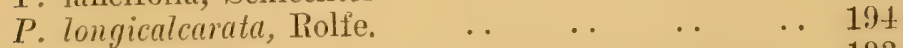

$\begin{array}{lllllll}\text { P. lucescens, Bl. } & \text {. } & \text {. } & \text {. } & \text {. } & \text {. } & 193\end{array}$

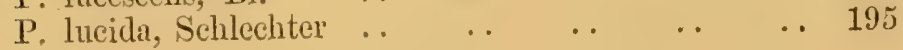


P. microphyllus, Hook. fil.

. 193

P. muricata, Schlechter

. 196

P. pendula, Schlechter

P. reflexa, Schlechter ..

P. rupestris, Schlechter

P. sciuroides, Rehb. fil.

P. tenuis, Lindl.

P. torta, Schlechter

P. uncata, Ridl.

$\begin{array}{llll}\ldots & \ldots & \ldots & \\ \cdots & \ldots & \ldots & \end{array}$

$\begin{array}{lllll}\ldots & \ldots & \ldots & \ldots & 196 \\ \ldots & \ldots & \ldots & \ldots & 196\end{array}$

P. unciferus, Hook. fil.

P. undulatus, Schlechter

Pogonia crispata, Wight

P. flabelliformis, Lindl.

P. punctata, Bl.

-

..

. 194

. 192

.. 192

.. 197

. . 195

.. 194

. 194

. 203

P. striata, Rid

Polychilus Cormu-cervi, Breda. Polystachya penangensis, Ridl.

P. siamensis, Ridl.

P. singaporensis, Ridl.

Porpax Meirax, King

$\cdots$

..

$\cdots \quad$

.

.

.

.

$\cdots$

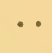

..

.

.

Preptanthe vestita, Richb. fil.

Psychechilus purpurascens, Bl.

Pterygodium sulcatum, Roxb.

Ptilocnema bracteatum, Don.

Renanthera alba, Ridl.

R. Arachnites, Lindl. . .

R. elongata, Lindl.

R. Flos-aeris, Rehb. fil.

$R$. histrionica, Rchb. fil.

R. Maingayi, Ridl.

R. matutina, Lindl

R. micrantha, Bl.

Renantherella histrionica, Ridl.

Rhynchostylis gigantea, Ridl.

R. Gurwalica, Rehb. fil.

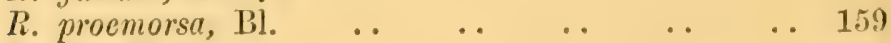

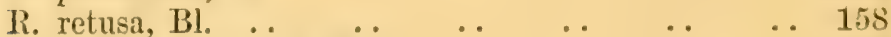

Saccolabium Angroecum, Ridl. . $\quad \ldots \quad \ldots \quad \ldots \quad \ldots 172$

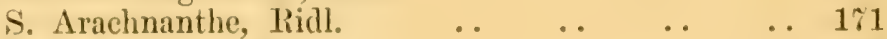

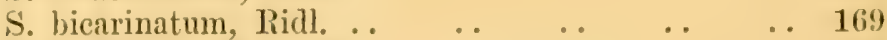

S. bigiblsum, Hook. fil. . $\quad \ldots \quad \ldots \quad \ldots 16+$

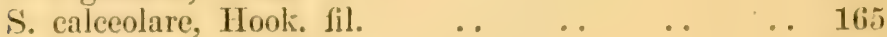

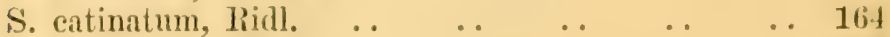

S. cornigerum, Ridl. .. $\quad \ldots \quad \ldots \quad \ldots \quad \ldots \quad \ldots 162$

S. cortinatum, Ridl, .. $\quad \ldots \quad \ldots \ldots$.. $\quad \ldots \quad 165$ 
Materials for a Flora of the Malayan Peninsula.

S. densiflorum, Lindl.

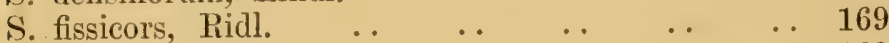

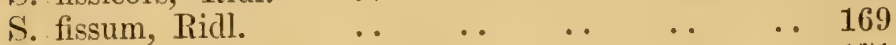

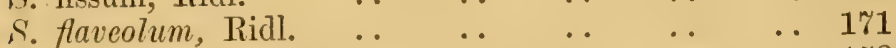

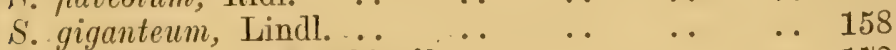

S. Griffithii, Par. \& Rchb. fil. .. $\quad$. $\quad$.. $\quad$.. 172

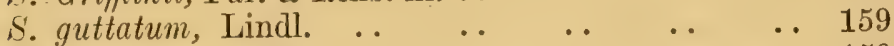

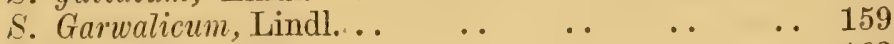

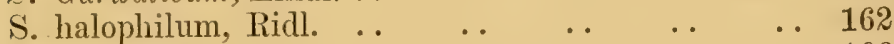

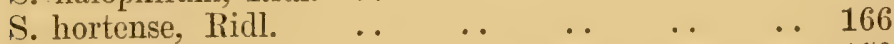

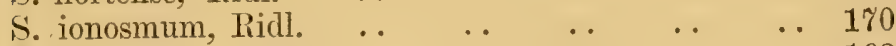

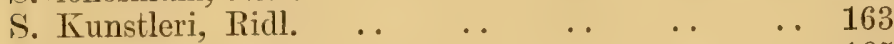

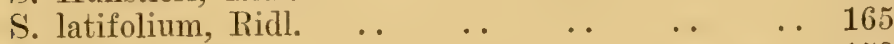

S. littorale, Rchb. f. $\quad$. $\quad$. . $\quad$. $\quad$. . $\quad$.. 159

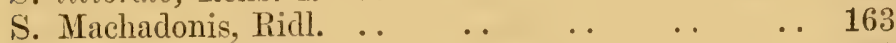

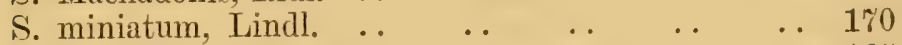

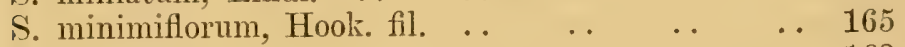

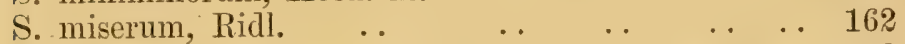

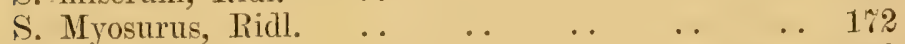

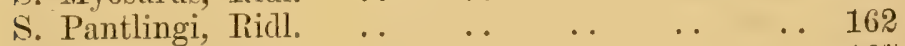

S. parvum, Ridl. $\quad \begin{array}{llllll} & \ldots & \ldots & \ldots & \ldots & 167\end{array}$

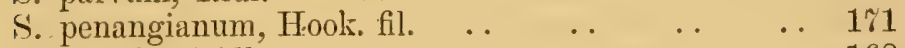

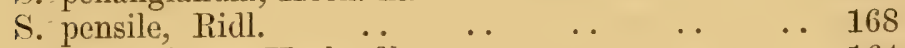

S. perpusillum, Hook. fil. . . $\quad$. $\quad$. $\quad \ldots \quad 164$

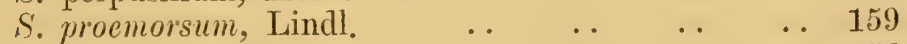

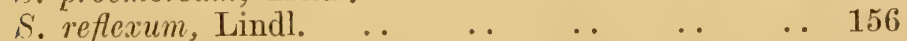

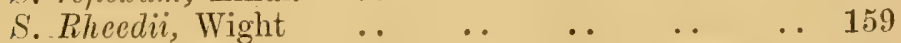

S. retusum, Flor. des Serres. .. $\quad$. $\quad$. . $\quad$.. 159

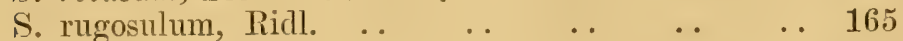

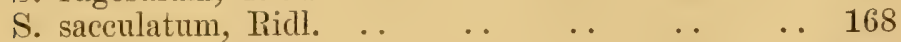

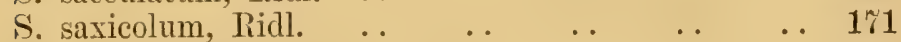

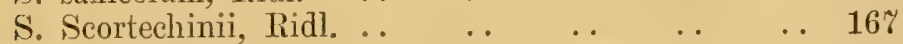

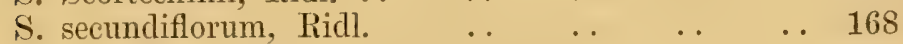

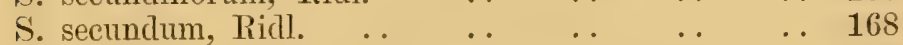

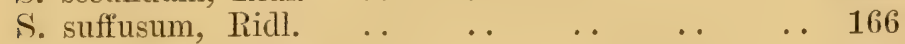

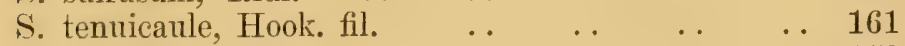

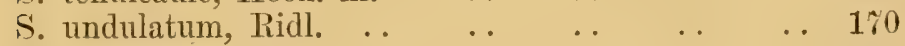

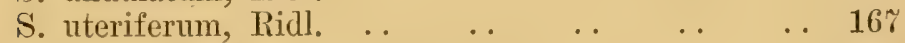

$\begin{array}{lllllll}\text { S. validum, Ridl. } & \ldots & \ldots & \ldots & \ldots & \ldots & 166\end{array}$

Sarcanthus densiflorus, Par. \& Rehb. f. $\quad$. . $\quad$. . $\quad$. 168

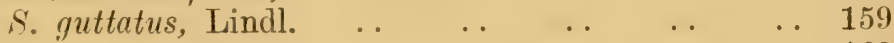

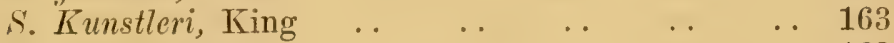

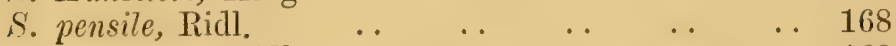

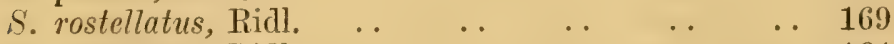

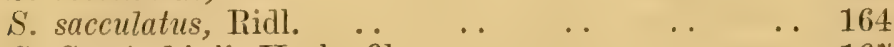

S. Scortechinii, Hook. fil. .. $\quad$. $\quad \ldots \quad$.. 167 
S. sccundus, Griff. _. $\quad \ldots \quad \ldots \quad \ldots \quad \ldots \quad \ldots 168$ Sarcochilus adnatus, Ridl. . $\quad \ldots \quad \ldots \quad \ldots \quad \ldots \quad \ldots \quad \ldots 1 \hat{\gamma}$

S..arachnites, Rehb. f. _ . $\quad \ldots \quad \ldots \quad \ldots 18: 3$

S. aureus, Hook. fil. . . . $\quad \ldots \quad \ldots . \quad \ldots$ 1\%

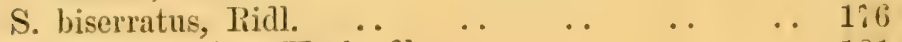

S. Urachystachys, Hook. fil. . . $\quad \ldots \quad \ldots \ldots$.. 181

S. brachyglottis, Hook. f. . $\quad \ldots \quad \ldots \quad \ldots \quad \ldots 182$

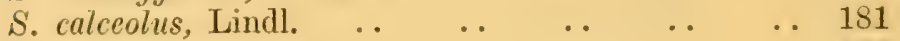

S. caligaris, Ridl. $\quad \ldots \quad \ldots \quad \ldots \quad \ldots \quad \ldots \quad \ldots 1 \%$

S. carinatifolius, Ridl. $\quad \ldots \quad \ldots \quad \ldots \quad \ldots \quad \ldots 187$

S. cladostachys, Hook. fil. . . $\quad \ldots \quad \ldots \quad \ldots \quad \ldots 175$

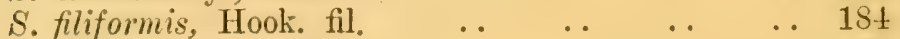

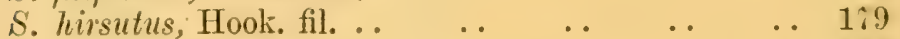

S. hirtutus, Hook. fil. .. $\quad \ldots \quad \ldots \quad \ldots \quad \ldots \quad \ldots \quad 1$. .

S. lilacinus, Griff. $\quad \ldots \quad \ldots \quad \ldots \quad \ldots \quad \ldots \quad \ldots 181$

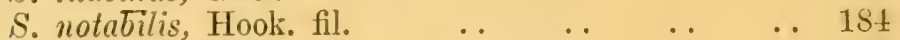

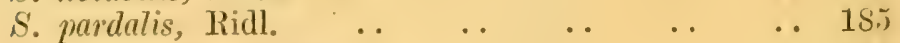

S. pauciflorus, Hook. fil. . $\quad \ldots \quad \ldots \quad \ldots 18$.

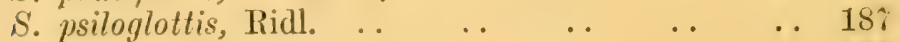

S. recurvus, Hook. fil. $\quad \ldots \quad \ldots \quad \ldots \quad \ldots 185$

S. Scopa, Hook, fil. .. . . $\quad \ldots \quad \ldots \quad \ldots \quad \ldots 183$

S. Scortechinii, Hook. fil. . $\quad \ldots \quad \ldots \quad \ldots$ 18:3

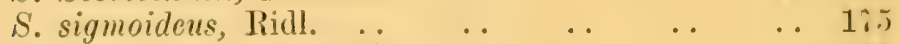

S. stenoglottis, Hook. fil. . $\quad \ldots \quad \ldots \quad \ldots 1$. .

S. tanyphyllus, Ridl. . $\quad \ldots \quad \ldots \quad \ldots \quad \ldots 1 ; 6$

S. trichoglottis, Hook. fil. . . . . . . $\quad$.. 185

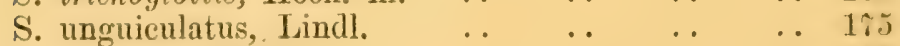

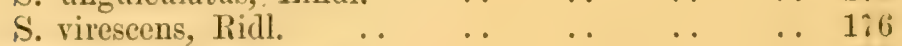

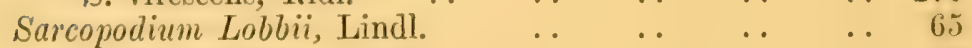

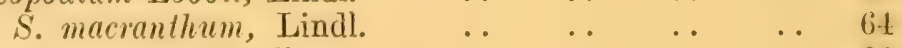

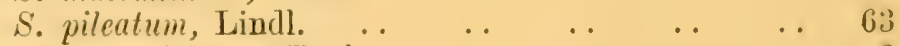

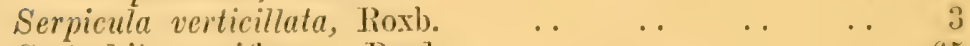

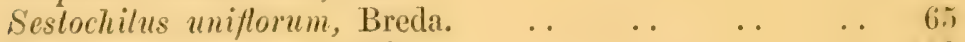

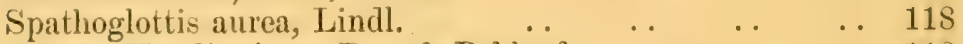

S. Handingiana, Par. \& Rehb. f. . . . . . 118

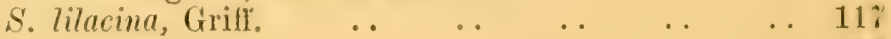

S. plicata, Bl. . $\quad \ldots \quad \ldots \quad \ldots \quad \ldots \quad \ldots \quad \ldots \quad \ldots 11$;

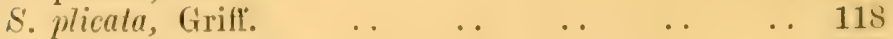

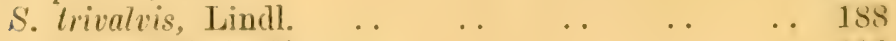

S. Wrayi, Hook. fil. .. $\quad \ldots \quad$.. $\quad \ldots \quad$.. 118

Stratiotes acoroides, Linn. fil. $\quad \ldots \quad$.

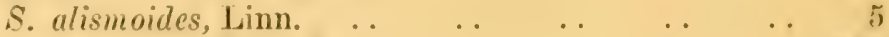

S'louritis violacea, Rehb. 1. . $\quad \ldots \quad \ldots \quad \ldots \quad \ldots . \quad \ldots \quad 151$

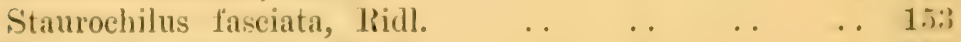

Stauropsis gigantea, Benth. _. $\quad \ldots \quad \ldots \quad \ldots 1$.

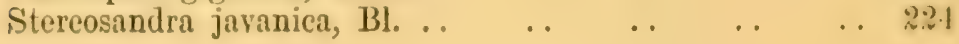


Taeniophyllum rubrum, Ridl.

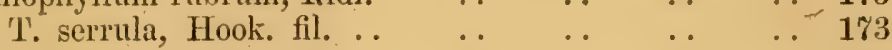

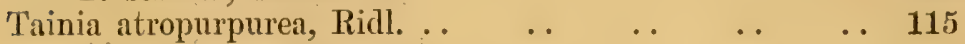

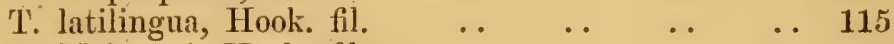

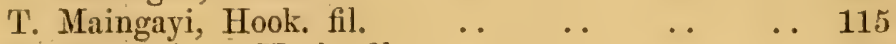

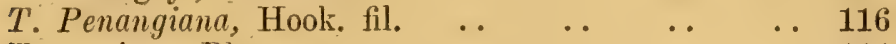

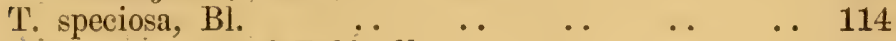

Thecostele alata, Par. \& Rehb. fil. . . $\quad$. $\quad$. . $\quad$. 190

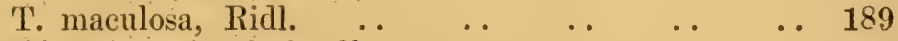

T. Maingayi, Hook. fil. . . . . . . . $\quad$. $\quad$.. 190

T. quinquefida, Hook, fil. $\quad$. $\quad$. $\quad$.. $\quad$.. 191

$\begin{array}{lllllll}\text { T. secunda, Ridl. } & \text {. } & \text {. } & \text {.. } & \text {.. } & \text {.. } & 190\end{array}$

T. Zollingeri, Rehb. fil. . . . . $\quad$. . . . 190

$\begin{array}{lllllll}\text { Thelasis capitata, Bl. } & \ldots & \ldots & \ldots & \ldots & \ldots & 199\end{array}$

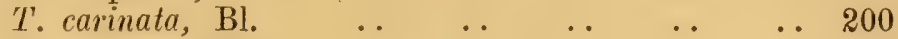

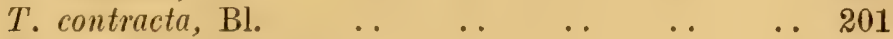

T. decurva, Hook. fil. . . . . . . $\quad$. . $\quad$.. 201

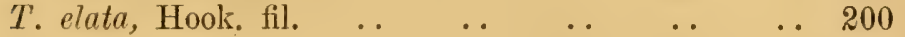

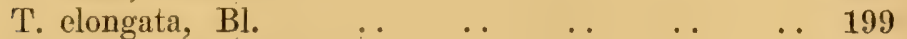

T. hongkongensis, Rolfe _. $\quad$. $\quad$. . $\quad$. . 199

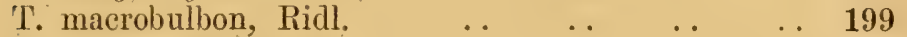

T. triptera, Rchb. fil. . . . . . $\quad$. . $\quad$.. 199

T. Zollingeri, Rchb. fil. ... . . $\quad$.. $\quad$.. 199

Thrixspermum Arachnites, Rehb. fil. $\quad \ldots \quad \ldots \quad \ldots 181$

$\begin{array}{llllll}\text { T. brachyglottis, Ridl. } & \text {. } & \text {. } & \text {. } & \text {.. } & 182\end{array}$

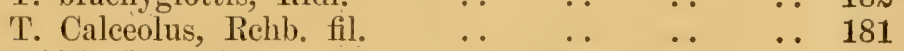

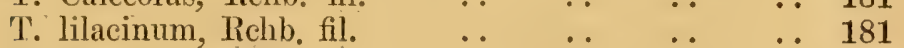

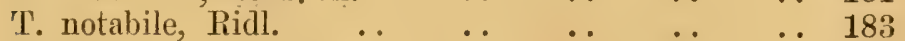

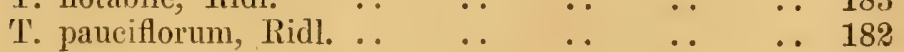

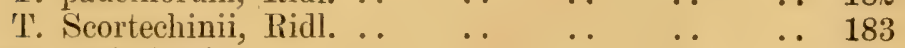

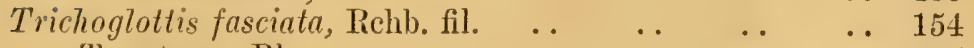

T. retusa, Bl. $\begin{array}{lllllll} & \ldots & \ldots & \ldots & \ldots & \ldots & 157\end{array}$

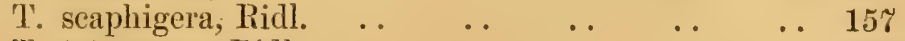

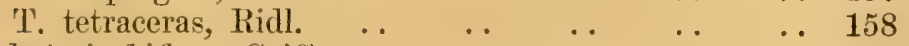

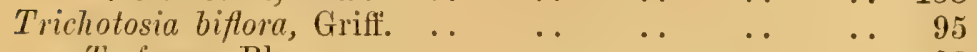

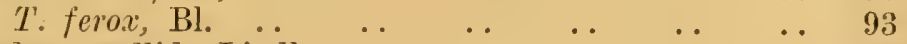

$\begin{array}{lllllll}\text { Tripleura pallida, Lindl. } & \ldots & \ldots & \ldots & \ldots & \ldots & 27\end{array}$

$\begin{array}{lllllll}\text { Tropidia angulosa, } \mathrm{Bl} . & \ldots & \ldots & \ldots & \ldots & \ldots & 209\end{array}$

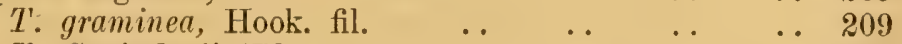

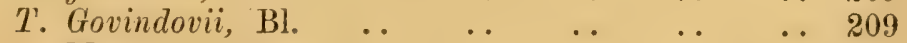

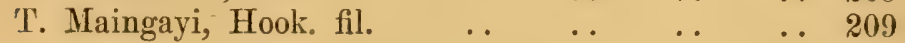

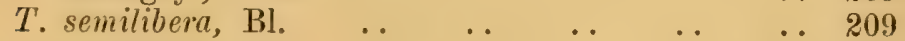

$\begin{array}{lllllll}\text { T. squamata, Bl. } & \text {. } & \ldots & \ldots & \ldots & \ldots & 209\end{array}$

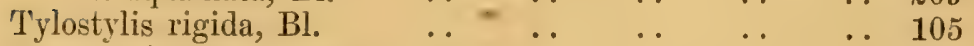

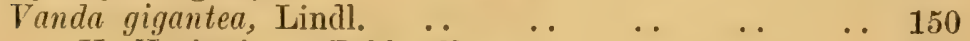

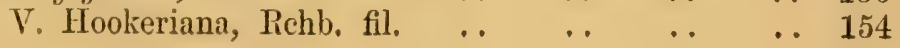




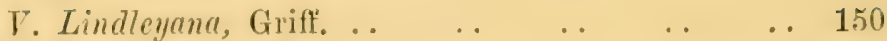

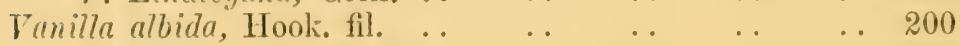

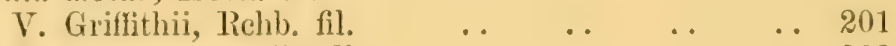

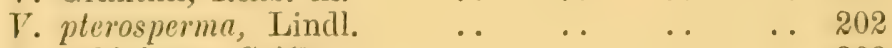

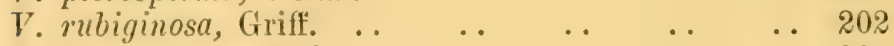

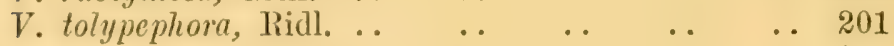

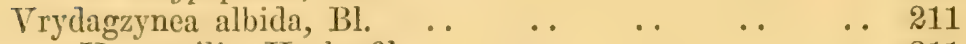

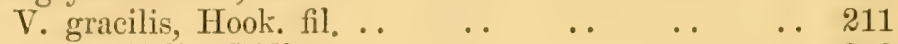

$\begin{array}{lllllll}\text { V. lancifolia, Ridl. } & \ldots & \ldots & \ldots & \ldots & \ldots & 210\end{array}$

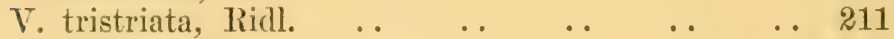

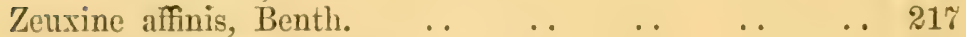

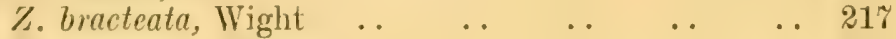

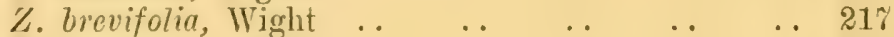

\%. clandestina, Bl. $\quad \ldots \quad$.

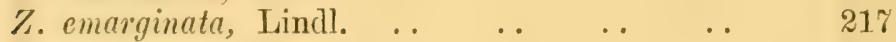

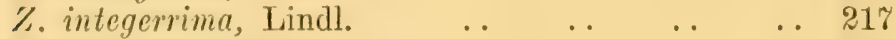

\%. membranacea, Lindl. . . $\quad$. $\quad$.. $\quad$.. 218

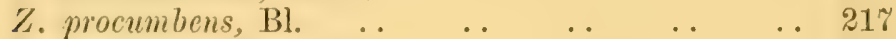

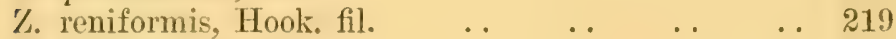

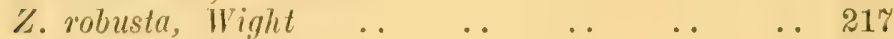

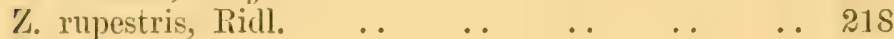

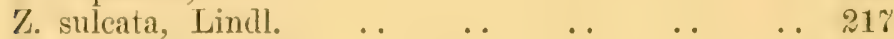

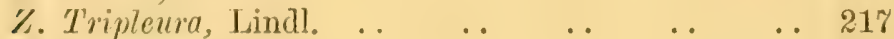

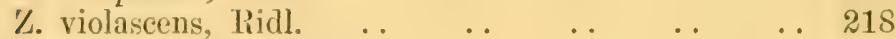

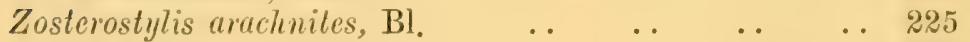

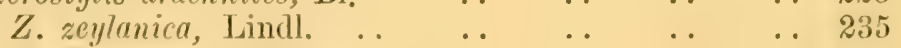

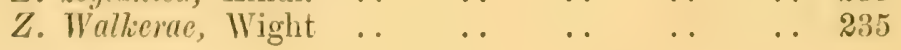






QK $354.7^{\text {New }}$ York Botanical Garden Library

Ridley, Henry Nicho/Materials for a flor

|| || || || || || || || || || || ||||||||||||||||||||||||||||||

35185000602167 
